Zentrum für Technologiefolgen-Abschätzung
Centre d'évaluation des choix technologiques

Centro per la valutazione delle scelte tecnologiche

Centre for Technology Assessment
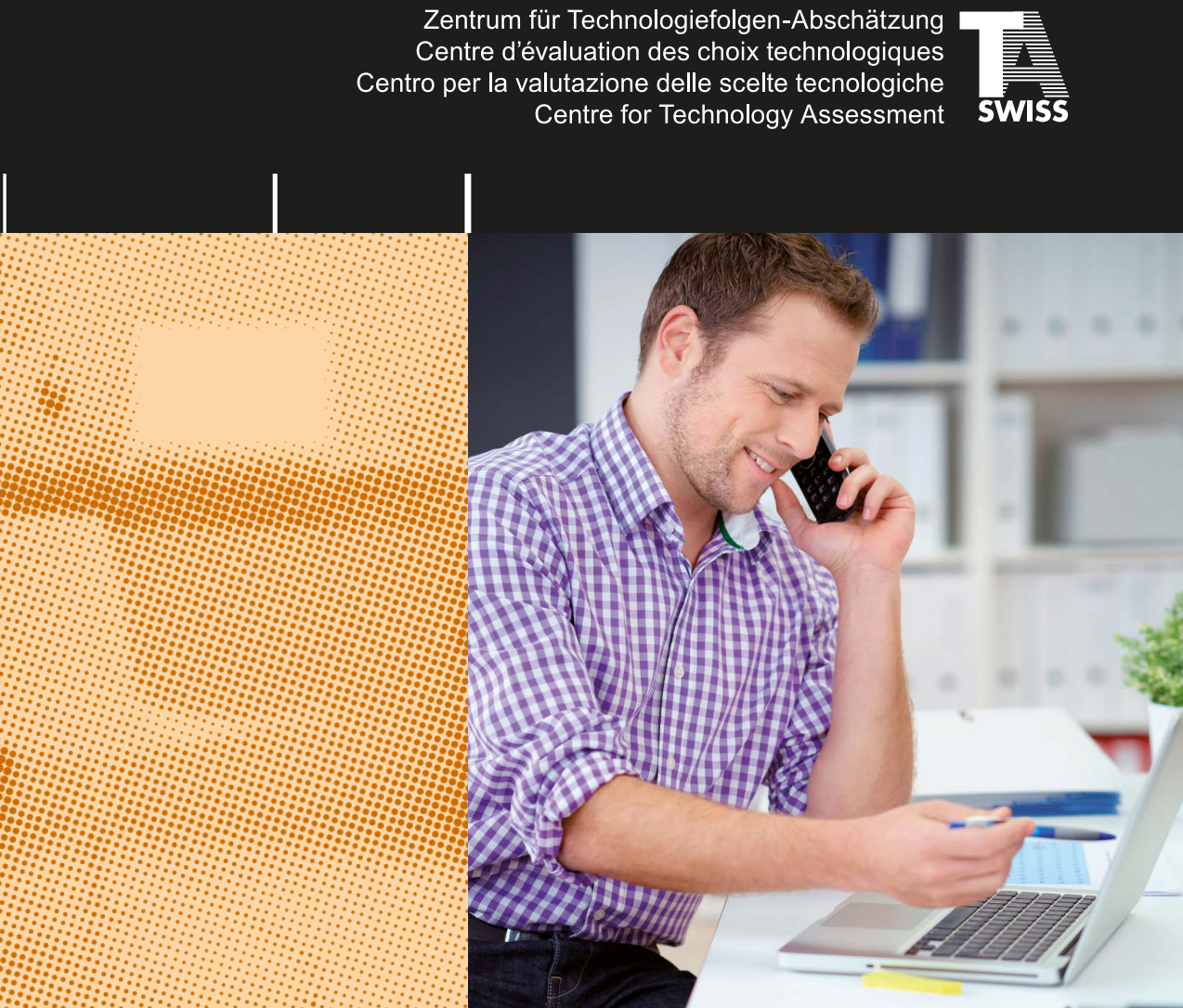

Jens O. Meissner, Johann Weichbrodt, Bettina Hübscher, Sheron Baumann, Ute Klotz, Ulrich Pekrubl, Leila Gisin und Alexandra Gisler

\title{
Flexible neue Arbeitswelt
}

Eine Bestandsaufnahme auf gesellschaftlicher und volkswirtschaftlicher Ebene 
Bibliografische Information der Deutschen Nationalbibliothek Die Deutsche Nationalbibliothek verzeichnet diese Publikation in der Deutschen Nationalbibliografie; detaillierte bibliografische Daten sind im Internet über http://dnb.d-nb.de abrufbar.

This work is licensed under creative commons license CC BY-NC-ND $2.5 \mathrm{CH}$.

\section{(c)}

SOME RIGHTIS RESERVED

Coverabbildungen:

(C) Robert Kneschke - fotolia.com (linkes Bild)

(C) contrastwerkstatt - fotolia.com (rechtes Bild)

(C) 2016 vdf Hochschulverlag AG an der ETH Zürich

ISBN 978-3-7281-3770-8 (Printausgabe)

Download open access:

ISBN 978-3-7281-3771-5 / DOI 10.3218/3771-5

www.vdf.ethz.ch

verlag@vdf.ethz.ch 


\section{Inhaltsverzeichnis}

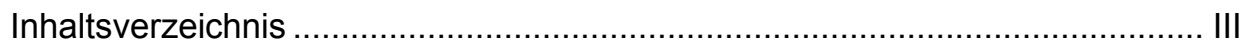

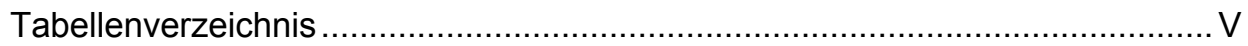

Abbildungsverzeichnis ..........................................................................

Abkürzungsverzeichnis...................................................................... VII

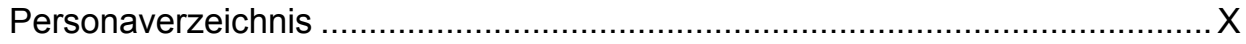

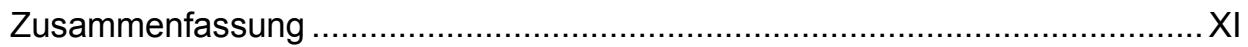

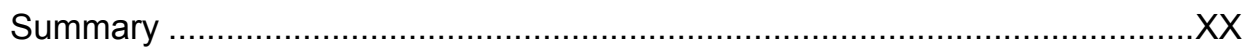

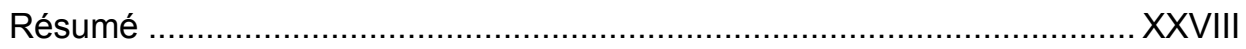

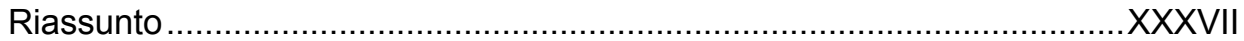

Methodischer und inhaltlicher Überblick .................................................. XLVII

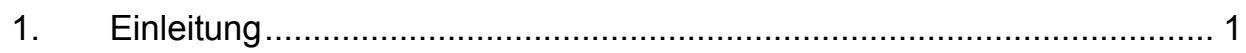

1.1 Projektauftrag und Fragestellung ............................................... 3

1.2 Gliederung des Berichts .......................................................... 4

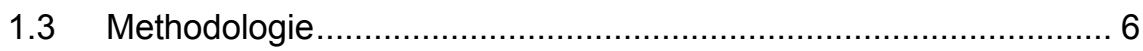

1.4 Zielgruppen ....................................................................... 7

2. Formen der Arbeitsflexibilisierung und Veränderung des Stellenwerts

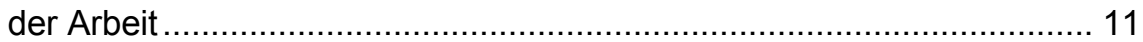

2.1 Charakteristika moderner Arbeit ................................................ 12

2.2 Flexibilisierung der Arbeit: Formen und ihre

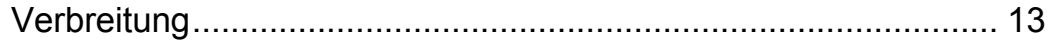

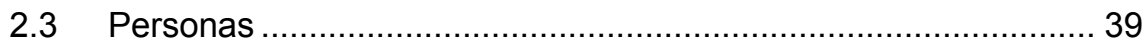

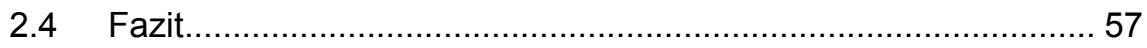

3. Ausprägungen mobil-flexibilisierter Arbeit und ihre spezifischen Merkmale unter Berücksichtigung der rechtlichen Dimension ................ 63

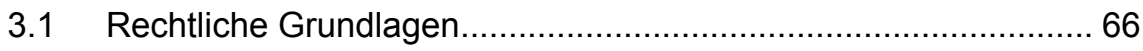


3.2 Einzelarbeitsvertrag, Werkvertrag, Auftrag und

Freelancingvertrag 69

3.3 Charakteristika von Telearbeit, Heimarbeit und

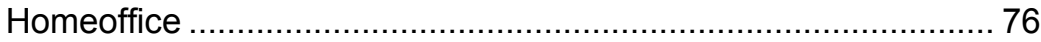

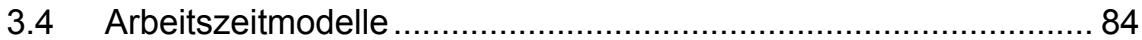

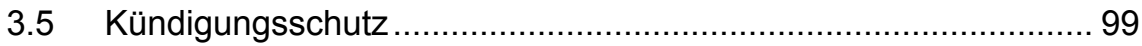

3.6 Gesundheit und soziale Absicherung..................................... 101

3.7 Vereinbarkeit von Arbeit mit anderen Lebensbereichen

3.8 Virtuelle Arbeit (Crowdsourcing) .............................................. 125

3.9 Schlussfolgerungen zu den Spannungsfeldern zwischen mobilen Arbeitsformen und rechtlichen

Grundlagen.

4. Mögliche Folgen der personabasierten Arbeitswelten auf gesamtwirtschaftlicher Ebene

4.1 Zugrunde liegende Annahmen und kurze Darstellung der Arbeitswelten

4.2 Resultate der Folgeabschätzung

4.3 Erläuterung zu den möglichen Auswirkungen auf die Indikatoren

5. Gesamtbeurteilung und Empfehlungen ........................................... 203

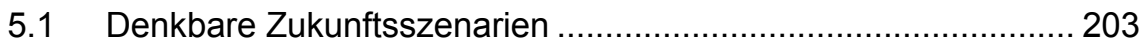

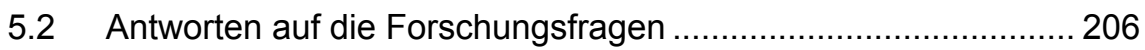

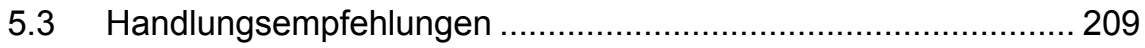

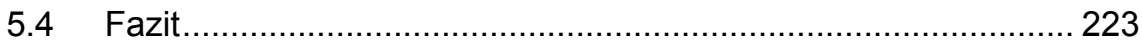

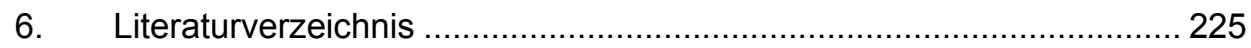

7. Anhang I: Methodisches Vorgehen ................................................ 253

8. Anhang 2: Die Elemente der Decent-Work-Agenda .......................... 271

9. Anhang 3: Beteiligte Personen und Institutionen ............................... 277 


\section{Tabellenverzeichnis}

Tabelle 1: Vor- und Nachteile der Arbeit im Homeoffice 24

Tabelle 2: $\quad$ Systematisierung verschiedener Angebote im Crowdsourcing . 29

Tabelle 3: Auswirkungen von Crowdsourcing auf die Anbieterinnen und Anbieter von Arbeitskraft .................................................. 31

Tabelle 4: $\quad$ Morphologischer Kasten zu Formen der Arbeitsflexibilität ........ 34

Tabelle 5: Matrix flexible Arbeitsformen ..................................................... 36

Tabelle 6: Übersicht rechtlicher Bestimmungen bezüglich Arbeitszeit in diversen Gesamtarbeitsverträgen ........................................ 116

Tabelle 7: Zusammengefasste Resultate der gesamtwirtschaftlichen Auswirkungen

Tabelle 8: $\quad$ Überblick der Handlungsempfehlungen zur flexiblen Arbeitswelt 


\section{Abbildungsverzeichnis}

Abbildung 1: $\quad$ Gliederung des Berichts $\ldots \ldots \ldots \ldots \ldots \ldots \ldots \ldots \ldots \ldots \ldots \ldots \ldots \ldots \ldots \ldots$

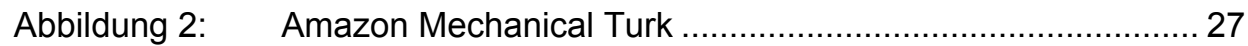

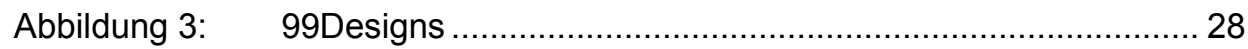

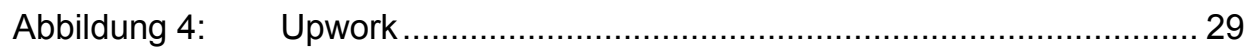

Abbildung 5: $\quad$ Personas in der Matrix flexible Arbeitsformen ..................... 38

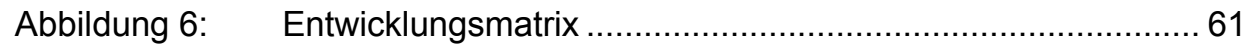

Abbildung 7: Die fünf Personas in der Entwicklungsmatrix verortet ......... 62

Abbildung 8: $\quad$ Gestaltung des schweizerischen Arbeitsrechts ................6 67

Abbildung 9: $\quad$ Übersicht über die Verträge auf Arbeitsleistung ................. 70

Abbildung 10: WorkAnywhere Fokus Mobilität, SBB AG und Swisscom (Schweiz) AG ...................................................... 85

Abbildung 11: $\quad$ Aspekte betreffend Gesundheit .............................. 102

Abbildung 12: $\quad$ Neugründungen und Beschäftigte in der Schweiz (Zahlenquelle: BFS Statistik der Unternehmensdemografie, eigene Berechnungen und Darstellung) ........................ 182

Abbildung 13: Quote der Erwerbstätigen mit zwei Tätigkeiten (Zahlenquelle: Eurostat).

Abbildung 14: Neugründungen und Veränderung der Beschäftigung nach Fritsch (2008, S. 8)

Abbildung 15: Ausbildungsniveauadäquanz der Hochschulabsolvent/innen 2003-2013 (Zahlenquelle: BFS (2013b)).

Abbildung 16: Erwerbslosenquote gemäss ILO der Hochschulabsolvent/ innen 2003-2013 (Zahlenquelle: BFS (2013b))............... 187

Abbildung 17: Weltweite Bitcoin-Transaktionen

(Zahlenquelle: Blockchain, 2016)........................... 193

Abbildung 18: Die fünf Personas in der Entwicklungsmatrix verortet........ 204 


\section{Abkürzungsverzeichnis}

\begin{tabular}{|c|c|}
\hline AGB & Allgemeine Geschäftsbedingungen \\
\hline $\mathrm{AHV}$ & Alters- und Hinterlassenenversicherung \\
\hline ALV & Arbeitslosenversicherung \\
\hline $\operatorname{ArG}$ & Arbeitsrecht (SR 822.11) \\
\hline ArGV 1 & Verordnung 1 zum Arbeitsgesetz (SR 822.111) \\
\hline ArGV 3 & Verordnung 3 zum Arbeitsgesetz (SR 822.113) \\
\hline AVG & Arbeitsvermittlungsgesetz (SR 831.10) \\
\hline AZR & Arbeitszeitrichtlinie \\
\hline BGE & Bundesgerichtsentscheid \\
\hline BIP & Bruttoinlandsprodukt \\
\hline bspw. & beispielsweise \\
\hline BVG & $\begin{array}{l}\text { Bundesgesetz über die berufliche Alters-, Hinterlassenen- } \\
\text { und Invalidenvorsorge (SR 831.40) }\end{array}$ \\
\hline BYOD & Bring-your-own-Device \\
\hline bzw. & beziehungsweise \\
\hline ca. & circa \\
\hline d.h. & das heisst \\
\hline DSG & Bundesgesetz über den Datenschutz (SR 235.1) \\
\hline EO & Erwerbsersatzordnung \\
\hline evtl. & eventuell \\
\hline f. & folgende \\
\hline ff. & fortfolgende \\
\hline GAV & Gesamtarbeitsvertrag \\
\hline ggf. & gegebenenfalls \\
\hline GIG & Gleichstellungsgesetz (SR 151.1) \\
\hline
\end{tabular}


GYDFF Get your device for free

HIT Human Intelligence Tasks

i.V.m. in Verbindung mit

IKT Informations- und Kommunikationstechnologie

ILO Internationale Arbeitsorganisation der Vereinten Nationen

inkl. inklusive

IV Invalidenversicherung

KAPOVAZ kapazitätsorientierte variable Arbeitszeit

KMU kleine und mittlere Unternehmen

MDM Mobile Device Management

mind. mindestens

NAV Normalarbeitsvertrag

o.J. ohne Jahr

o.Ä. oder Ähnliche

OR Obligationenrecht (SR 220)

RAV Regionale Arbeitsvermittlungszentren

resp. respektive

Rz. Randziffer

S. Seite

SECO Staatssekretariat für Wirtschaft

StGB Strafgesetzbuch (SR 311.0)

u.a. unter anderem

u.Ä. und Ähnliche

usw. und so weiter

u.U. unter Umständen

UVG Bundesgesetz über die Unfallversicherung (SR 832.20)

vgl. vergleiche 
WBF Departement für Wirtschaft, Bildung und Forschung

Z. Zeile

z.B. zum Beispiel

ZGB Zivilgesetzbuch (SR 210)

ZPO Zivilprozessordnung 


\section{Personaverzeichnis}

Persona A: Roland Müller, Finanzleiter im KMU, „Homeoffice in der Festanstellung"

Persona B: Sandra Könitz, Projektmanagerin, „Mobiles Arbeitskraftunternehmertum“

Persona C: Ursula Meyerhans, Redaktorin, „Portfoliowork auf mehreren Standbeinen“

Persona D: Noah Schmid, Informatiker, „Entrepreneurship“

Persona E: Andrea Burri-Lötscher, Küchenhilfe, „Mehrfachanstellungen und Minijobs“ 


\section{Zusammenfassung}

Die technischen Entwicklungen der letzten Jahrzehnte und insbesondere die Digitalisierung führen zu tief greifenden Umwälzungen in der Arbeitswelt. Sie gestatten ein zunehmend flexibles Arbeiten - und das gleich in mehrerer Hinsicht:

- Welchen Stellenwert besitzt die Arbeit (bezahlt/unbezahlt) in der Gesellschaft heute und in Zukunft?

- $\quad$ Dank Internet und Handy können Angestellte auch von zu Hause oder von einem anderen Standort aus auf Firmendaten zugreifen und mobil tätig sein (örtliche Flexibilität),

- $\quad$ anstelle fester Bürozeiten treten Modelle für Jahres- oder Vertrauensarbeitszeit (zeitliche Flexibilität),

- $\quad$ statt in einem über Jahrzehnte gleichbleibenden Arbeitsverhältnis engagiert man sich vermehrt in wechselnden Teams für Projekte von kürzerer Dauer (organisatorische Flexibilität).

- $\quad$ Betriebe wiederum nutzen die Möglichkeiten, ihre Belegschaft an das Arbeitsvolumen anzupassen und gegebenenfalls Aufgaben auszulagern (numerische Flexibilität).

Die vorliegende Studie hat zum Gegenstand, welche Folgen diese Entwicklungen für den Stellenwert der Erwerbsarbeit im gesellschaftlichen Leben zeitigen, inwiefern die Eigenarten der neuen Arbeitsformen mit der aktuellen Rechtslage kompatibel sind und welche volkswirtschaftlichen Konsequenzen sich daraus ergeben.

Folgende Punkte stellen die Kernergebnisse der Studie dar:

1. Der Stellenwert der Arbeit unterliegt einer gefährlichen Trendkombination zwischen „zeitlich-räumlicher Flexibilisierung“ und „Arbeitskraftunternehmertum".

2. Der rechtliche Rahmen ist generell gegeben. Abwägung zwischen weiteren gesetzlichen Regelungen und Selbstkontrolle der Sozialpartner sind aber notwendig. Darüber hinaus sollten virtuelle Vermittlungsplattformen genauer betrachtet werden. 
3. Als volkswirtschaftlicher Sicht stehen wenige leichte Verbesserungen relativ vielen (leichten und deutlichen) Verschlechterungen gegenüber.

4. Für die Flexibilität der Arbeit ist das sozialpartnerschaftliche Prinzip als notwendige Rahmenbedingung zukunftsweisend.

5. Auf dem Radar der Monitoring- und Kontrollsysteme fehlen gewisse Teilaspekte sowie vorausschauende Daten.

\section{Unterschiedlich weit fortgeschrittene Formen der Flexibilisierung}

Hinsichtlich der zeitlichen Arbeitsflexibilität hat sich die Teilzeitarbeit mit 37 Prozent der Schweizer Erwerbstätigen in 2014 längst etabliert und belegt einen europäischen Spitzenrang. Teilzeitarbeitende berichten oft von einer besseren Vereinbarkeit der Arbeit mit dem Privatleben (Life-Domain-Balance), schätzen aber andererseits ihre Karrierechancen oft schlechter ein als Vollzeitangestellte. Rund 61 Prozent der Schweizer Angestellten nutzen flexible Arbeitszeitmodelle wie etwa Gleitzeit. Diese Form wird geschätzt, kann aber problematisch werden, wenn auf die Arbeitszeiterfassung komplett verzichtet wird.

Was die örtliche Flexibilität betrifft, so arbeiten rund ein Viertel der Schweizer Erwärbstätigen anteilig im Homeoffice - auch das ein Spitzenwert in Europa. Zunehmend entwickelt sich auch die mobile Arbeit, die nebst der Tätigkeit zuhause das Arbeiten bei Kunden oder von unterwegs umfasst. Darüber hinaus setzen Betriebe vermehrt auf Desk-Sharing und sind damit auch innerhalb der Firmeninfrastruktur flexibel. Unter der Bedingung, dass das Arbeiten im Homeoffice auf Wunsch der Angestellten und nicht zusätzlich zur regulären Arbeit im Büro stattfindet, gehen mit dieser Form der Arbeitsflexibilität viele Vorteile wie höhere Produktivität, Zufriedenheit und bessere Life-Domain-Balance einher.

Die mengenmässige Flexibilisierung der Gesamtbelegschaft (numerische Flexibilität) konkretisiert sich beispielsweise in befristeten Arbeitsverhältnissen, Honorarprojekten oder neuen Formen der Auftragsvergabe wie etwa dem Crowdsourcing auf Internetplattformen. Rund 7 Prozent der Schweizer Arbeitsverträge sind befristet. Das „klassische“ Arbeiten auf Abruf ist in den letzten 15 Jahren relativ stabil bei 5 Prozent geblieben. Für die relativ junge Erscheinung des Crowdsourcing bzw. Crowdworking gibt es dagegen keine verlässlichen Daten, doch dürfte diese Art der Auftragsvermittlung künftig an Bedeutung gewinnen: Insbesondere in der Informatik- und Kommunikationsbranche ist es denkbar, dass Unternehmen in den nächsten Jahren Teile ihrer festen Belegschaft abbau- 
en. Die zusätzlich nötigen Arbeitskräfte bezieht man dann als "Liquid Talents“ aus der „Human Cloud“ - dem Pool von über das Internet rekrutierbaren Fachleuten. Generell lässt sich bei diesen neuen Arbeitsformen vermuten, dass Arbeitende mit höherer Qualifikation die Vorteile eher nutzen können und geringqualifizierte Arbeitende mit starken Nachteilen zu rechnen haben.

Neben diesen klar definierten Formen zeitlicher, örtlicher und numerischer Flexibilisierung setzt sich der Trend zur Hierarchieverflachung in Organisationen fort. Statt genaue Weisungen der Vorgesetzten befolgen zu müssen, geniessen Arbeitnehmende einen erweiterten Handlungsspielraum. Vorgängig vereinbarte Ziele sollen in eigener Verantwortung erreicht werden und sind häufig relevant für die variablen Lohnanteile. Auf diese Weise wird unternehmerisches Risiko auf Angestellte übertragen. In der sozialwissenschaftlichen Literatur wurde für diese Vermischung der klassischen Rollen zum einen der Begriff „Arbeitskraftunternehmer/in" geschaffen, andernorts ist auch von der Subjektivierung der Arbeit die Rede. Für Mitarbeitende kann diese Zunahme an Handlungsspielraum ein Gewinn darstellen. Es besteht aber auch die Gefahr der Selbstausbeutung, wenn sich die Life-Domain-Balance zunehmend stärker zu Gunsten der Arbeit verschiebt.

\section{Erhebliche Zunahme an beruflicher Vielfältigkeit}

Neben der Zunahme der Arbeitskraftunternehmenden ist künftig zu erwarten, dass Erwerbstätige eine oder mehrere Teilzeitanstellungen mit Einzelaufträgen kombinieren. Diese Form der (Teil-)Selbständigkeit kann berufliche Alternativen eröffnen und auch zu einer besseren Entfaltung der eigenen Interessen und Potenziale beitragen. Diese Art von hochflexibler Arbeit birgt aber auch grosse Tücken. Insbesondere besteht die Gefahr, dass die Beschäftigten in prekäre Verhältnisse abgleiten. Sind Teilzeitangestellte beispies/weise befristet beschäftigt und schwankt das Arbeitsvolumen, so sind Einkommen und Beschäftigungslage nicht mehr verlässlich kalkulierbar. Bei der Vermittlung über CrowdworkingPlattformen können Auftragssuchende Einkünfte und Belastung sehr schlecht planen. Zudem erfordert diese Art der Selbständigkeit viel Eigenverantwortung und Verhandlungskompetenz. Die Entwicklung hängt hier daher in erster Linie von der Qualifikation ab: Gutausgebildete und Selbständighandelnde vermögen aufgrund ihres gesuchten Profils ihre finanziellen Ansprüche und Arbeitsbedingungen gegenüber Mandanten durchaus durchzusetzen. Geringqualifizierte hin- 
gegen können in eine prekäre Abwärtsspirale aus beruflicher Unsicherheit und einbrechendem Einkommen geraten.

Am meisten von der Flexibilisierung profitieren Erwerbstätige in einer Festanstellung. Insbesondere Qualifizierte in führender Position gewinnen erheblichen Handlungs- und Entscheidungsspielraum. Das kann sich in einer höheren Zufriedenheit und Motivation zeigen, sofern der zunehmende Leistungsdruck gut bewältigt wird.

\section{Neue rechtliche Fragen zur Flexibilisierung}

Das Arbeitsrecht soll sicherstellen, dass die Interessen der Sozialpartnerinnen und Sozialpartner ausgewogen umgesetzt werden. In einer flexibilisierten Arbeitswelt wird es künftig darum gehen, soziale Errungenschaften zu schützen und die Chancen der flexiblen Arbeit zum Vorteil aller zu nutzen. Das Normalarbeitsverhältnis baut auf dem normalen Einzelarbeitsvertrag bzw. dem Gesamtarbeitsvertrag nach Obligationenrecht (OR) auf. Einzel- bzw. Gesamtarbeitsvertrag regeln zentrale Aspekte im Verhältnis der Sozialpartnerinnen und Sozialpartner. Dies betrifft sowohl die zu erbringenden Leistungen der Angestellten als auch den Lohn und die Schutzpflichten der Arbeitgebenden.

Es ist zunächst festzuhalten, dass das Schweizer Arbeitsrecht im europäischen Vergleich insbesondere mit Blick auf die soziale Sicherheit gut abschneidet. Doch diese Errungenschaft ist keineswegs für alle Zeiten gewährleistet. So bringen neue Arbeitsformen rechtliche Herausforderungen mit sich: Von zentraler Bedeutung ist die Unterscheidung zwischen dem Einzelarbeitsvertrag und anderen Vertragstypen, vor allem dem Auftrag und dem Werkvertrag. Während beim Einzelarbeitsvertrag der zeitliche und sachliche Kündigungsschutz gilt, kann ein Auftrag jederzeit widerrufen werden. Zentral bei der Unterscheidung ist auch, wie stark Arbeitnehmende den Weisungsrechten im Betrieb unterliegen. Dieses Kriterium entscheidet darüber, ob eine Person angestellt oder selbständig ist. In den durch die Flexibilisierung entstehenden Zwischenformen sind einzelne Erwerbstätige zwar nur noch ansatzweise in den Betrieb eingebunden, wirtschaftlich aber dennoch von ihm abhängig. Diese „Scheinselbständigen“ haben dann keinerlei Anspruch auf Arbeitslosenentschädigung, berufliche Vorsorge, obligatorische Unfallversicherung oder Absicherung bei Berufskrankheiten. 
Auch das Crowdsourcing liegt in einer rechtlichen Grauzone. Externe Crowdworker sind Selbständige, wodurch die Anwendung aller arbeitsrechtlichen Bestimmungen (Mindestlöhne, Ferien, Lohnfortzahlungen etc.) des Einzelarbeitsvertrags entfällt. Da Arbeitsbeziehungen über Crowdsourcing-Plattformen oft über die Landesgrenzen hinweg stattfinden, müssen auch Geltung und Anwendung ausländischen Rechts im Einzelfall aufwendig geprüft werden. Ungelöst ist zudem, wie die Abgabe von Einkommens- und Umsatzsteuer durchgesetzt werden kann. Die Crowdsourcing-Plattformen agieren zudem als Vermittler und damit Partner von Auftraggebenden und Auftragnehmenden. Die allgemeinen Geschäftsbedingungen der Plattform bilden dabei den rechtlichen Rahmen. Das oft ausgesprochene Kontaktverbot zu externen Auftraggebenden verletzt zudem das allgemeine Persönlichkeitsrecht und auch das Prinzip der Wirtschaftsfreiheit.

Aber nicht nur atypische Beschäftigungsverhältnisse werfen rechtliche Fragen auf. Die Flexibilisierung der Arbeit kann auch im Rahmen eines Normalarbeitsvertrags juristische Präzisierungen erfordern. Unklar ist, wie Arbeitsmittel und Vorleistungen entschädigt werden sollen, für welche die Angestellten mit eigenen Mitteln aufkommen. Aktuell sind Spesen zwingend zu vergüten, nicht aber der Einsatz privater Arbeitsgeräte.

Wenn vermehrt mobil oder von zuhause aus gearbeitet wird, stellt sich auch die Frage nach der Haftung für Schäden. Im Unternehmen haften die Arbeitgebenden. Wenn jedoch der Computer zu Hause aussteigt und Daten verloren gehen, ist die Lage unklar. Ein Unternehmen sollte abklären, ob die Betriebshaftpflichtversicherung spezielle Risiken (bspw. Datenverlust) des Heimbüros oder auch anderer mobiler Arbeitsformen deckt. Umgekehrt erhöht sich das Risiko für betriebliche Schäden, die durch berufliche Nutzung privater Geräte entstehen. Der Produktivität kommt dieses „Bring Your Own Device“ entgegen, weil die Arbeitnehmenden die innen vertrauten Geräte nutzen können und der Beschaffungsaufwand zum Teil wegfällt. Es gilt aber zu klären, wer bei einem Gerät, das dem Mitarbeitenden gehört, für Software und Support aufzukommen hat. Gesamthaft gesehen birgt es erhebliche Risiken für beide Seiten, sofern keine arbeitsvertragliche Regelung getroffen wurde. 


\section{Flexibilisierung nur bei ausreichender Qualifikation gesamtwirtschaftlich positiv}

Die Folgen flexibilisierter Arbeit auf gesamtwirtschaftlicher Ebene lassen sich ebenfalls nicht eindeutig prognostizieren. Verschiedene Rahmenbedingungen beeinflussen die Folgen der Flexibilisierung. Eine Schlüsselgrösse stellt dabei die Ausbildung der Arbeitskräfte dar: Je höher der Anteil Gutqualifizierter ist, desto grösser ist die Chance, dass flexible Arbeit dazu beiträgt, die gesamtwirtschaftliche Produktivität zu steigern und damit Potenziale für höhere Löhne und Steuererträge zu eröffnen. Herausforderungen stellen sich indes beim Selbstmanagement: Erreichbarkeit rund um die Uhr, wegfallende Trennung von Arbeitsund Freizeit und nicht immer optimal eingerichtete Arbeitsplätze gefährden die physische und psychische Gesundheit, während die örtliche und zeitliche Flexibilität andersherum aber auch genutzt werden kann, um die Arbeit an die eigenen Bedürfnisse (eigener Biorhythmus, Familie, Hobbys etc.) anzupassen.

Für Geringqualifizierte bringt die Flexibilisierung der Arbeit dagegen erhebliche Risiken mit sich. Da es die Digitalisierung erleichtert, Routinetätigkeiten in Länder mit tieferen Personalkosten zu verlagern, reduzieren sich für Geringerqualifizierte die Chancen auf eine Stelle. Oft bleibt nur Arbeit in Branchen der unmittelbaren persönlichen Dienstleistungen. Geringqualifizierte müssen zudem überdurchschnittlich oft Schichtarbeit oder Arbeit auf Abruf leisten, laufen stärker Gefahr, körperlich und psychisch zu erkranken, weisen eine tiefere Arbeitszufriedenheit auf und sind insbesondere dann, wenn sie nur befristet angestellt sind oder ihr Arbeitspensum tief ist, einem höheren Risiko der Arbeitslosigkeit ausgesetzt.

\section{Empfehlungen}

Die wichtigste Botschaft dieses Berichts lautet: Die individuellen und gesellschaftlichen Folgen flexibilisierter Arbeit sind ambivalent und sollten daher durch Massnahmen auf verschiedenen Ebenen umsichtig in eine positive Richtung gelenkt werden. Eine qualifizierte Grund- und Fachausbildung verbessert die Chancen erheblich, dass Arbeitnehmende die positiven Potenziale der Flexibilisierung nutzen können. In welche Richtung das Pendel in Zukunft ausschlägt, wird nicht technisch oder ökonomisch vorherbestimmt, sondern ist abhängig von der Gestaltung neuer Arbeitsformen auf betrieblicher und überbetrieblicher Ebene. Hier empfehlen wir: 
1. Einen weiten Rahmen für den Umgang mit zeitlicher und örtlicher Entgrenzung der Arbeit zu setzen: Gesetzliche Rahmenbedingungen, die gegebenenfalls auch sanktioniert werden, sind nötig - sollten sich aber nur auf das Ausschliessen von Extremformen beschränken und somit den Charakter von „Leitplanken“ haben.

2. Für die Subjektivierung der Arbeit (Stichwort „Arbeitskraftunternehmer/in“) zu sensibilisieren: Eine „Stärkung der Subjekte“ ist nötig, um Erwerbstätige besser darin zu befähigen, mit der flexibilisierten Arbeitswelt umzugehen. Hier sind insbesondere Institutionen der Ausund Weiterbildung gefragt, allenfalls bietet sich aber auch eine Chance für Gewerkschaften, ihren Aufgabenbereich auszuweiten.

3. Die betriebliche Flexibilität als partizipative Organisationsentwicklung zu planen: Innerhalb der gesetzlichen Leitplanken sollen die Sozialpartner die Möglichkeit haben, lokale und angepasste Lösungen gemeinsam zu auszuarbeiten.

4. Die betriebliche Mitwirkung und Selbstorganisation zu stärken: Insbesondere Arbeitgebende sind gefordert, auf Bedürfnisse nach mehr Flexibilität (oder bestimmte Ausprägungen davon) zu reagieren.

Die Flexibilisierung der Arbeitswelt hat zahlreiche rechtliche Konsequenzen. Verbesserungen und Überprüfungen sind wichtig hinsichtlich:

5. der Arbeitszeitregelungen, da das Arbeitsrecht beispielsweise Mindestruhezeiten und Maximalarbeitszeiten vorschreibt, die auf abweichende Arbeitszeitmodelle schwer anwendbar sind;

6. der Sozialversicherungsdefizite bei Kurz-Arbeitseinsätzen, weil sich schwankende Einkommen negativ auswirken können, u.a. auf die Invalidenrente;

7. dem Umgang mit (noch) undefinierten Arbeitsformen, da diese sich in rechtlichen Graubereichen bewegen;

8. der Klärung von Fragen zu Arbeitsort sowie Scheinselbständigkeit, denn diese stehen im Zusammenhang mit dem Auslagenersatz bei auswärtigen Arbeitsorten, steuerlichen Abzügen und Versicherungsmöglichkeiten;

9. der Personalvermittlung, da sich Anbieter von Crowdsourcing-Plattformen mit ihrem Firmensitz und ihren Websites dem schweizerischen Rechtssystem gänzlich entziehen können; 
10. der Rechtsdurchsetzung, da kleinere Organisationen, in denen neue Arbeitsformen zum Zug kommen, schwerer von den Arbeitsinspektoraten überprüft werden können als Grossbetriebe;

11. der Regelungen zur Nacherwerbsphase, weil diese sehr stark von der Erwerbstätigkeit abhängen (Äquivalenzprinzip) und

12. der Erstellung und Pflege einer Gesamtübersicht aller relevanten Rechtsnormen.

Die bestehenden Erhebungen der öffentlichen Statistik und die Periodizität ihrer Veröffentlichung lassen eine Beurteilung der aktuellen und kurzfristig zukünftigen Lage in vielen Fällen nicht zu. Dies ist anzupassen und zwar in folgenden Bereichen:

13. der beruflichen Mobilität der Arbeitnehmenden,

14. der Schattenwirtschaft und

15. der Arbeitsbedingungen der Arbeitskraftunternehmenden.

Da die Dynamik auf dem Arbeitsmarkt zunehmen dürfte, müsste das Monitoring vorausschauender werden, damit effiziente Massnahmen auf verschiedenen Ebenen ergriffen werden können. Es sollten hier mehr und zielgenauere Indikatoren mit Frühwarnfunktion entwickelt und entsprechende Daten zur besseren Massnahmensetzung erhoben werden.

Das Phänomen der flexiblen Arbeitswelt bleibt am Ende unscharf, d.h. es handelt sich dabei um ein bisher unzureichend definiertes Problem. Als solches ist es eine Herausforderung, deren Bewältigung einen echten Unterschied machen kann - einen Unterschied hin zu einer widerstandsfähigen, resilienten Schweizer Arbeitswelt, die ihre weltweite Spitzenposition erhalten oder sogar noch ausbauen kann. Dies ist keine Selbstverständlichkeit, sondern bedarf einer gemeinschaftlichen Entwicklungsleistung aller beteiligten Parteien. Nur dann wird es gelingen, aus dieser momentan eher unübersichtlichen, sehr komplexen, aber auch interessanten und chancenreiche Ausgangslage das Beste machen zu können.

Studienautorinnen und -autoren: Jens O. Meissner, Johann Weichbrodt, Bettina Hübscher, Sheron Baumann, Ute Klotz, Ulrich Pekruhl, Leila Gisin und Alexandra Gisler 
Rückfragen an:

Prof. Dr. Jens O. Meissner

Hochschule Luzern - Wirtschaft

Institut für Betriebs- und Regionalökonomie IBR

Zentralstr. 9

$\mathrm{CH}-6002$ Luzern

jens.meissner@hslu.ch

Dr. Johann Weichbrodt

Fachhochschule Nordwestschweiz

Hochschule für angewandte Psychologie

Institut für Kooperationsforschung und -entwicklung IfK

Riggenbachstr. 16

$\mathrm{CH}-4600$ Olten

johann.weichbrodt@fhnw.ch 


\section{Summary}

This allows for increasingly flexible working arrangements with regard to several aspects:

- Thanks to the internet and mobile phones employees can access company data from home or any other location and work on a mobile basis (location-al flexibility).

- $\quad$ Fixed daily working hours are replaced by models for annual and trustbased working time (temporal flexibility).

- Instead of committing oneself to the same job for decades, work is increasingly done in shorter term projects with changing teams (organizational flexibility).

- In turn companies use the possibilities to adjust their personnel to the volume of work and outsource tasks, if necessary (numerical flexibility).

This study aims to clarify what the consequences for the status of gainful employment in society are, to what extent the characteristics of the new forms of work are covered by the present legal situation and what macroeconomic consequences are to be expected and should be systematically observed in the future.

The following points represent the core results of the study:

1. The status of work is under threat from a trend to combine temporalspatial flexibility and employee entrepreneurship.

2. The legal situation is generally clear and adequate. However, balancing between additional regulations and voluntary self-regulation of the social partners is necessary. Furthermore, virtual work-exchange platforms should be scrutinized.

3. As a macroeconomic consequence, few slight improvements are accompanied by relatively many (slight to considerable) deteriorations.

4. For the flexibility of work, the principle of social partnership is a necessary and sustainable condition.

5. The monitoring and controlling systems miss certain aspects and lack in anticipatory data. 


\section{Varying degrees and forms of flexibilization}

Regarding flexibility in working hours, part-time work has long been established and is a reality for 37 percent of the Swiss labor population which translates into a European top rank. Part-time workers often report a better life-domain-balance, but rate their career opportunities lower than full-time employees. Roughly 61 percent of Swiss employees make use of flexible models of work time such as flexitime. This particular form is appreciated, but poses problems when timekeeping is waived.

With respect to locational flexibility, a quarter of all Swiss employees work at least partially from home which is also a top ranking percentage in the European context. Also increasingly observable are mobile forms of work that encompass work at home, at the customer's location or during commute. Furthermore, companies increasingly rely on desk-sharing and are consequently flexible with regards to their infrastructures. Provided that working from home is chosen voluntarily by employees and does not come in addition to regular work in the office, this form of flexible work offers many advantages such as higher productivity and satisfaction and a better life-domain-balance.

Numeric flexibility, i.e. the flexible size of the workforce, is exemplified by temporary work contracts, freelancer-based projects or new forms of procurement like crowdsourcing on internet platforms. About 7 percent of all Swiss work contracts are temporary. At 5 percent, the "traditional" on-call work has remained stable over the last 15 years. For the extent of the relatively recent phenomenon of crowdsourcing, respectively crowdwork, there is no reliable data yet. However, this form of procurement is likely to gain importance in the near future: Particularly in the IT and communications industries a reduction of the permanent workforce in the coming years is foreseeable. Additional necessary manpower will be recruited as "liquid talents" out of the "human cloud" - i.e. specialists that can be hired through the internet. In general, these new forms of work give rise to the assumption that workers with higher qualifications will benefit while the ones with low qualifications have to expect considerable draw-backs.

Along with these clearly definable forms of temporal, spatial and numeric flexibilization the trend to flatter hierarchies in organizations continues. Instead of strictly following instructions from superiors, employees are granted greater freedom of action. Predetermined goals can be reached on one's own authority and are often relevant for variable components of the salary. In this way, entrepreneurial 
risk is transferred to the employee. In the social sciences this blending of traditional roles has been termed employee-entrepreneur or entreployee. Elsewhere, it is known as the subjectivization of work. This increased leeway can be seen as an improvement by some employees. However, there is also a risk for selfexploitation, if the life-domain-balance gradually shifts in favor of work.

\section{Substantial increase in occupational diversity}

In addition to the increase in the number of employee-entrepreneurs, a growing portion of the labor force is expected to combine one or more part-time jobs with freelancing activities. This form of (partial) self-employment can lead to the realization of professional alternatives and a better self-fulfillment. However, there are also some pitfalls with this highly flexible mode of working. In particular, there is the risk that employees slide into precarious conditions. When part-time employees are temporarily employed and have fluctuating volumes of work, their income and employment situation are no longer reliably predictable. The acquisition of work orders using crowdsourcing platforms hardly permits a dependable planning of income and workload. Moreover, this form of self-employment requires a lot of individual responsibility as well as, for example, negotiation skills. Therefore the development of this work form mainly depends on personal qualifications: welltrained and self-reliant actors are quite able to push through their financial demands and terms vis-à-vis their clients thanks to their sought-after profiles. The low-skilled, however, might enter a precarious downward spiral due to occupational uncertainty and collapsing income.

The permanently employed benefit the most from flexibilization. Especially qualified personnel in executive positions gain considerable freedom of action and leeway in decision-making. This can result in higher motivation and satisfaction, if the increased pressure to perform can be coped with.

\section{New legal questions arising from flexibilization}

Labor legislation is intended to ensure that the interests of the social partners are accounted for in a balanced manner. In a more flexible world of work, it will be about protecting social achievements and making the benefits of flexible work available to everyone. The standard employment relationship is based on the normal individual employment agreement or the collective employment agree- 
ment according to the Swiss Code of Obligations. The individual and collective agreements regulate core aspects of the relationship between the social partners. This concerns the performance to be effected by the employed as well the obligations regarding pay and the duty to care on the employer's side.

Firstly, it should be noted that Swiss labor legislation compares favorably with its European counterparts, especially with regard to social security. However, this accomplishment is not guaranteed for all time and new forms of work entail legal challenges: Of central importance is the differentiation between the individual employment agreement and other forms of contracts, in particular the work order and the contract for work and services. While the individual employment agreement offers temporal and objective protection from dismissal, a work order can be revoked anytime. Also essential for the differentiation is the extent to which employees are subject to directives by management. This criterion determines whether a person is employed or self-employed. In the intermediate forms of work contracts arisen from flexibilization, individual workers are only rudimentarily integrated in corporate structures of employers, but economically fully reliant on them. These dependent contractors do not have legal access to unemployment benefits, occupational pension funds, compulsory accident insurance or protection from occupational diseases.

Crowdsourcing is also situated in a legal grey area. External crowdworkers are self-employed which renders all labor regulations (minimal pay, paid leave, continuation of payments, etc.) nonapplicable. Since these kinds of work relationships often cross national borders, the applicability of foreign legislation has to be clarified in a costly manner in each individual case. Presently also unresolved is the question how income and sales tax claims can be enforced. Furthermore, crowdsourcing platforms act as intermediaries and therefore as partners of principals and agents. The general terms and conditions of the platform hereby function as the legal framework. The often observable ban on contracts with external principals violates personal rights and the principle of economic freedom.

However, not only atypical work relationships pose legal problems. Flexible work within the scope of a standard employment relationship might also require legal clarifications. It is unclear how the means of labor and intermediate inputs paid for by the employees are to be compensated. Currently, the compensation for expenses is mandatory, but not the use of private working equipment. 
The continued growth of the importance of working from home and on a mobile basis also raises the question of who is liable for damages. In corporations, the employer has to assume liability. However, if the computer at home breaks down and data is lost, the situation is unclear. A company should clarify whether its business liability insurance covers special risks such as the loss of data in the home office or in other mobile forms of work. Vice-versa, the risk for damages to the company increases with the professional use of private equipment. Productivity benefits from this "bring-your-own-device" practice, because employees can use the means they are accustomed to and the costs of procurement are partially eliminated. But for all that, it is imperative to clarify who has to pay for software and support for devices owned by employees. In sum, there are considerable inherent risks for both sides, if no contractual agreement has been reached.

\section{Macroeconomic benefits of flexibilization depend on adequate qualifi- cations}

The macroeconomic consequences of flexible work cannot be predicted unambiguously, either. Different determining factors influence the outcome of flexibilization. Training and education of the workforce play a key role: The higher the portion of the highly qualified, the better the chances for flexibilization to increase overall productivity and subsequently unlock the potential for higher wages and tax revenue. Challenges can be found with regard to self-management: Roundthe-clock availability, waning separation of work and leisure and not always ideally arranged workplaces endanger the physical and psychological health. On the other hand, spatial and temporal flexibility can also be helpful in finding a better fit of work and one's own needs (biorhythm, family, hobbies, etc.).

For the low- and unskilled workers flexibilization holds considerable risks, however. Since the digital revolution facilitates outsourcing of routine activities to low wage countries, the chances of finding a job are diminished for workers with low or no skills. Often the only work left is in the personal service industries. Additionally, low-skilled workers on average have to perform more shift-work or work on-call, have a higher risk of falling physically or psychologically ill, have a lower work-satisfaction and are at a higher risk of becoming unemployed, especially if they work on a temporary basis or have a low quota of working hours. 


\section{Recommendations}

The most important message of this report is that the individual and societal consequences of flexible work are ambiguous and should be steered through careful measures on different levels in order to reach a positive outcome. A qualified basic educational and vocational training enhances the chances that employees can benefit from the positive potentials inherent in flexibilization. Which direction the development can take is not technically or economically predetermined, but depends on the shaping of new forms of work on the company and supracompany levels. Here we recommend:

1. to set a broad framework for the handling of the erosion of temporal and spatial barriers of work: Legal provisions that are enforced if need be, are necessary. However, they should only exclude extreme forms and represent legal guidelines.

2. to sensitize the public to the subjectivization of work ("employeeentrepreneurship"): A strengthening of individuals is necessary in order to enable workers to cope with a flexible world of work. Especially institutions offering basic and advanced training could step into the void here, but it is also an opportunity for labor unions to expand their range of activities.

3. to plan company flexibility as a participatory organizational development: Social partners should have the possibility to work out local and adapted solutions together within the legal framework.

4. to strengthen employee participation and self-organization in companies: Particularly employers are required to react to demands for more flexibility or certain forms thereof.

The flexibilization of work has numerous legal consequences. Improvements and reassessments are important with regard to:

5. working time regulation, because labor law regulates, e.g., minimal rest periods and maximum working hours that are difficult to apply to deviant working time models;

6. deficiencies in the social insurances concerning short periods of employment because variable incomes can affect, e.g., disability benefits;

7. the handling of (yet) undefined forms of work because they are situated in legal grey areas; 
8. the clarification of questions regarding the place of work as well as dependent contractors because they are connected to the compensation of expenses resulting from external places of work, tax deductibility and insurance options;

9. personnel placement because operators of crowdworking sites can elude the Swiss legal system with the help of registered business addresses and websites abroad;

10. the enforcement of regulations, because smaller organizations making use of new forms of work are harder to control by the Labor Inspection Authority than large firms;

11. the regulations concerning the retirement phase because this strongly depends on the previous occupation; and

12. the compilation of a general survey of all relevant legal forms and its maintenance.

The existing surveys by the official statistical bodies and the periodicity of their publications do not permit a current and short-term future assessment in many cases. This is especially relevant in the cases of:

13. the professional mobility of employees,

14. the shadow economy, and

15. the working conditions of the employee entrepreneurs.

As the dynamics of the labor market are expected to increase, a more forwardlooking monitoring is required in order be able to take efficient measures on different levels. The development of more and more accurate indicators with an early warning function and the associated appropriate data collection are desirable.

In conclusion, the phenomenon of a flexible world of work remains diffuse, i.e. it is an ill-defined problem. As such, it is a challenge the successful tackling of which can make a real difference towards a robust and resilient Swiss work environment able to keep or even expand its international top position. This is by no means a trivial task, but rather a collective achievement by all participants in order to be able to act in this currently fairly unclear, complex, but also interesting and promising situation.

Authors of the study: Jens O. Meissner, Johann Weichbrodt, Bettina Hübscher, Sheron Baumann, Ute Klotz, Ulrich Pekruhl, Leila Gisin und Alexandra Gisler 


\section{Contact:}

Prof. Dr. Jens O. Meissner

Hochschule Luzern - Wirtschaft

Institut für Betriebs- und Regionalökonomie IBR

Zentralstr. 9

$\mathrm{CH}-6002$ Luzern

jens.meissner@hslu.ch

Dr. Johann Weichbrodt

Fachhochschule Nordwestschweiz

Hochschule für angewandte Psychologie

Institut für Kooperationsforschung und -entwicklung IfK

Riggenbachstr. 16

$\mathrm{CH}-4600$ Olten

johann.weichbrodt@fhnw.ch 


\section{Résumé}

Au cours des dernières décennies, les développements technologiques, et en particulier la digitalisation, ont eu un impact important sur le monde du travail. Le mode de travail devient de plus en plus flexible, et cela à plus d'un titre :

- l'accès à Internet, également depuis les téléphones portables, permet aux employés de gérer les données de leurs entreprises depuis leur domicile ou en déplacement (flexibilité spatiale),

- des modèles d'horaires annualisés ou reposant sur la confiance remplacent peu à peu les modèles traditionnels qui impliquent des heures de bureau fixes (flexibilité temporelle),

- les travailleurs s'engagent dans des équipes qui gèrent des projets de durée réduite et ne gardent ainsi plus la même place de travail pendant des décennies (flexibilité organisationnelle),

- $\quad$ les entreprises adaptent leurs effectifs à la charge de travail et, le cas échéant, externalisent certaines charges (flexibilité numérique).

Le but de cette étude est de montrer les conséquences de ces différents développements sur la place du travail rémunéré dans notre société, mais également d'examiner dans quelle mesure les particularités de ces nouvelles formes de travail sont compatibles avec le droit actuel ainsi que les conséquences politicoéconomiques qui en découlent.

Les résultats principaux de l'étude sont résumés ci-après.

1. La place du travail dans notre société sous-tend une combinaison dangereuse entre une "flexibilisation spatio-temporelle » et l'émergence des « travailleurs-entrepreneurs ».

2. Le cadre juridique est généralement connu. II est toutefois nécessaire de trouver un juste équilibre entre davantage de règlementations légales et une autorégulation des partenaires sociaux. Néanmoins, les bourses aux emplois en ligne devraient être examinées de façon plus approfondie.

3. D'un point de vue politico-économique, quelques améliorations mineures s'opposent à de nombreuses détériorations, qu'elles soient légères ou conséquentes. 
4. A l'avenir, le partenariat social sera une condition cadre nécessaire à la flexibilité du travail.

5. Sur le radar des systèmes de monitoring et de contrôle, différents éléments tels que les données prédictives sont encore absents.

\section{Les différents types de flexibilisation progressent de manière variable}

Flexibilité temporelle

Le travail à temps partiel est établi depuis longtemps et, en $2014,37 \%$ des travailleurs suisses avaient choisi cette forme de travail. Ce taux se situe dans le haut du classement européen. Ces travailleurs sont souvent d'avis qu'ils parviennent ainsi à mieux concilier leur vie professionnelle et leur vie privée (LifeDomain-Balance). Toutefois, ils estiment également que leurs chances de carrière sont souvent plus faibles que celles des travailleurs à temps plein. Environ $61 \%$ des travailleurs suisses utilisent des modèles de temps de travail flexible, tel que l'horaire mobile. Cette flexibilité est très appréciée mais elle peut devenir problématique, lorsque l'employé renonce complètement à l'enregistrement de son temps de présence.

\section{Flexibilité spatiale}

Environ un quart des travailleurs suisses bénéficient du Home Office (travail à la maison) : la Suisse se situe ainsi également en haut du classement européen. Le travail mobile se développe de plus en plus ; il inclut, outre le travail à la maison, le travail chez les clients et le travail en déplacement. Parallèlement, les entreprises se tournent de plus en plus vers le Desk-sharing qui leur permet de bénéficier d'une infrastructure informatique plus flexible. Pour autant que le Home Office se fasse à la demande de l'employé et non en plus du travail régulier au bureau, cette forme de flexibilité du travail offre plusieurs avantages : une productivité augmentée, une satisfaction plus importante et un meilleur équilibre possible entre vie professionnelle et vie privée. 
Flexibilité numérique

La flexibilité en terme de volume de personnel se traduit, entre autres, par le travail temporaire, par des contrats liés à des projets spécifiques ou par de nouvelles formes d'externalisation comme le crowdsourcing via des plateformes en ligne. Environ $7 \%$ des contrats de travail en Suisse sont temporaires. Durant les 15 dernières années, le travail sur appel "classique " est resté relativement stable, à $5 \%$. II n'existe pas de données fiables sur le phénomène relativement récent du crowdsourcing, et en particulier le paid crowdsourcing. Toutefois, ce type des services devrait gagner en importance dans le futur : dans les domaines de l'informatique et de la communication, les entreprises réduiront probablement, dans les prochaines années, le nombre d'employés fixes. La main-d'œuvre supplémentaire sera recrutée parmi les « liquid talents » dans le « human cloud » : il s'agit d'une main-d'œuvre spécialisée qui peut être recrutée sur Internet. Ces nouvelles formes de travail devraient bénéficier aux personnes très qualifiées.

Parallèlement à ces formes de flexibilisation clairement identifiées, il y a une tendance à un aplanissement de la hiérarchie dans les organisations. Au lieu de suivre simplement les directives de leurs supérieurs, les collaborateurs ont une marge de manœuvre plus importante. Ils sont ainsi responsables de l'atteinte des objectifs qui prennent alors une grande importante pour la détermination de la part variable des salaires. Dans ce sens, les risques entrepreneuriaux sont assumés par les employés. Dans la littérature des sciences sociales, cette confusion des rôles classiques fait partie de la notion de " travailleur-entrepreneur ", ailleurs on parle également d'une subjectivation du travail. Pour les collaborateurs, cet accroissement du champ d'action peut constituer un avantage. II existe toutefois aussi le risque d'auto-exploitation, lorsque l'équilibre entre vie professionnelle et vie privée est sacrifié en faveur du travail.

\section{Forte augmentation de la versatilité professionnelle}

A l'avenir le nombre de «travailleurs-entrepreneurs" augmentera. Ces personnes combineront ce travail sur mandat et un ou plusieurs emplois temporaires. Cette forme d'autonomie partielle peut offrir des alternatives en termes d'emploi et permettre également le développement des intérêts propres et du potentiel de chacun. Cependant, ce travail très flexible comporte aussi des risques. Ainsi, les conditions de vie des employés peuvent devenir précaires. Si les travailleurs à temps partiels sont occupés de manière temporaire et par un 
volume de travail qui varie continuellement, alors les revenus sont difficiles à calculer et la situation de l'emploi est difficile à apprécier. Cette forme d'autonomie exige une grande responsabilisation individuelle ainsi que d'autres compétences, telles que des compétences de négociation. L'évolution professionnelle dépend alors avant tout des qualifications du travailleur: une personne bien formée qui travaille de manière indépendante réussira, en raison de son profil recherché, à faire valoir ses revendications en matière de salaire et de conditions de travail. Une personne moins qualifiée pourra tomber dans une spirale d'insécurité au niveau professionnel et ainsi faire face à de faibles revenus.

Au final, ce sont les employés avec un poste fixe qui profitent le plus de la flexibilisation. En particulier, les personnes qualifiées qui occupent des postes de direction jouissent d'une grande liberté d'action et de décision. Leur satisfaction au travail s'en trouve améliorée et leur motivation augmente, à condition toutefois que la pression induite par les obligations de rendement soit bien gérée.

\section{Des nouvelles questions juridiques concernant la flexibilisation}

Le droit du travail devrait garantir que les intérêts des partenaires sociaux soient représentés de manière équitable. Dans un monde du travail flexibilisé, il s'agira de valoriser des pratiques socialement responsables et de faire profiter chacun des chances du travail flexible. Les rapports de travail se basent sur un contrat individuel de travail ou le cas échant, sur des conventions collectives de travail basées sur le code des obligations (CO). Les contrats individuels ou les conventions collectives régularisent les aspects centraux des relations entre les partenaires sociaux: les prestations des employés, le salaire ou encore les obligations de protection de l'employeur.

Avant tout, il faut mettre en évidence qu'en matière de sécurité sociale, le droit du travail en Suisse fait bonne figure au regard des autres pays européens. Toutefois, cet accomplissement n'est pas garanti car les nouvelles formes de travail engendrent de nouveaux défis juridiques : il est essentiel de distinguer plus clairement les contrats individuels de travail des autres types de contrat, en particulier les mandats et les contrats d'entreprise. Alors que le contrat individuel de travail garantit la protection contre le licenciement, un mandat peut être révoqué à tout moment. II est aussi important d'identifier à quel point les employés ont le droit de donner des instructions au sein de l'entreprise : ce critère définit si une personne est employée ou indépendante. Avec la flexibilisation apparaissent des 
formes intermédiaires qui font que les employés sont seulement encore partiellement intégrés dans l'entreprise, mais toujours dépendants économiquement. Ces « faux indépendants " n'ont pas droit aux indemnités de chômage, à la prévoyance professionnelle, à l'assurance accident obligatoire ou à une assurance contre les maladies professionnelles.

Le paid crowdsourcing ou l'externalisation ouverte est aussi situé dans une zone d'ombre juridique. Les crowdworkers externes sont indépendants, ce qui compromet l'application des règles générales relatives au contrat de travail individuel (salaires minimaux, maintiens des paiements de salaires, etc.). Comme les relations professionnelles sur les plateformes de crowdsourcing se développent souvent au-delà des frontières nationales, la portée et l'application du droit étranger doivent aussi être examinées au cas par cas. Reste aussi à déterminer comment assurer le prélèvement des impôts sur le revenu et sur le chiffre d'affaires. Les plateformes de crowdsourcing agissent comme un médiateur entre mandataire et mandant. Les conditions générales de la plateforme constituent donc le cadre juridique. L'interdiction de contact avec des employeurs tiers, qui fait souvent partie intégrante de ces conditions, viole les droits généraux de la personnalité ainsi que le principe de la liberté économique.

Toutefois, les relations de travail atypiques ne sont pas les seules à soulever des questions juridiques. La flexibilisation du travail peut aussi exiger des mises au point d'ordre juridique pour des contrats de travail traditionnels. II est difficile de savoir comment indemniser les employés pour les outils de travail qu'ils ont développés eux-mêmes ou pour des paiements anticipés qu'ils ont également euxmêmes effectués. Actuellement, ce sont les frais qui doivent être remboursés et non l'utilisation du matériel de travail.

Quand on travaille en déplacement ou depuis la maison, la question se pose de savoir qui est responsable en cas de dommages. Dans une entreprise, l'employeur assume cette responsabilité. Si, par exemple, l'ordinateur s'éteint au domicile de l'employé et que des données sont perdues, la situation est floue. Une entreprise doit clarifier si, dans ce cas particulier, son assurance responsabilité civile couvre les risques spécifiques liés aux bureaux à domicile (par ex. perte de données) ou aux autres formes de travail en dehors du bureau. Inversement, l'utilisation des appareils privés au travail augmente le risque de dommages causés par des activités liées à l'entreprise. Mais le phénomène «bring your own device » a un impact positif sur la productivité, du fait que les employés peuvent se servir des dispositifs électroniques qui leur sont familiers et que 
l'entreprise économise sur l'achat de ces appareils. En outre, les questions du paiement du software et du support pour les appareils des employés doivent être clarifiées. Dans l'ensemble, les deux parties courent des risques importants si des dispositions adéquates n'ont pas été établies dans le contrat de travail.

\section{L'effet macroéconomique positif de la flexibilisation dépend des qualifications professionnelles}

II n'est pas encore possible de prédire les conséquences de la flexibilisation du travail au niveau macroéconomique car diverses conditions ne sont pas remplies. Une condition clé est la formation du personnel; plus la main-d'œuvre qualifiée est importante, plus la flexibilisation du travail contribue à une augmentation de la productivité. Ceci implique alors une hausse des salaires et des recettes fiscales. Cependant, des défis se posent quand il est question d'autogestion : d'une part, lorsque les employés ne distinguent plus leur temps de travail de leur temps de loisir et lorsque les places de travail ne sont pas aménagées de façon optimale, la santé mentale et la santé physique sont mises en danger. D'autre part, la flexibilité spatio-temporelle contribue parfois à satisfaire certains besoins spécifiques (biorythme, famille, loisirs, etc.).

La flexibilisation du travail engendre toutefois un risque important pour les employés moins qualifiés. Puisque que la flexibilisation facilite le transfert de certaines tâches courantes dans des pays où la main-d'œuvre est moins chère, les chances d'obtenir un poste diminuent pour les travailleurs moins qualifiés. Souvent, leur choix en matière d'emploi se limite aux prestations personnelles. De plus, les employés moins qualifiés sont souvent soumis à des horaires de travail irrégulier ou des travaux sur appel. Ils sont, de ce fait, plus exposés aux maladies mentales et physiques et leur satisfaction au travail est très faible. Finalement, en tant que travailleurs temporaires, ils encourent un risque plus élevé de se retrouver sans emploi.

\section{Recommandations}

Le message principal qu'il faut retenir de ce rapport est le suivant : les conséquences individuelles et sociales de la flexibilisation du travail sont ambivalentes et des mesures doivent être prises à plusieurs niveaux pour diriger ce changement dans la bonne direction. Une formation de base et une formation spéciali- 
sée de haut niveau augmentent considérablement les chances des employés de profiter des avantages de la flexibilité. La direction que prendra cette flexibilisation à l'avenir n'est pas déterminée par des éléments techniques ou économiques, mais dépend de la création de nouvelles formes de travail au niveau des entreprises et des industries. A ce sujet, nous émettons les recommandations suivantes:

1. Mise en place d'un cadre généreux qui permet un décloisonnement temporel et spatial du travail. Un cadre légal, incluant des sanctions, est nécessaire. II devrait toutefois se limiter à l'exclusion des abus extrêmes et agir comme " fil conducteur».

2. Sensibilisation des personnes concernées par la subjectivation du travail (mot-clé travailleur-entrepreneur) : une « affirmation du sujet » est nécessaire afin que ces travailleurs soient capables de faire face à la flexibilisation du travail. Les institutions de formation de base et de formation continue sont particulièrement nécessaires, et les syndicats pourraient en profiter pour étendre leur champ de compétences ;

3. Planification de la flexibilité opérationnelle en tant que développement organisationnel participatif: les partenaires sociaux devraient avoir la possibilité de développer des solutions personnalisées, au niveau local et en accord avec les directives légales

4. Renforcement de la participation dans l'entreprise et de l'organisation autonome : ce sont principalement les employeurs qui sont tenus de répondre aux demandes relatives à une plus grande flexibilité, ou tout au plus à certaines formes de flexibilité.

La flexibilisation du monde du travail a de nombreuses conséquences juridiques. Des améliorations et des contrôles sont nécessaires et ils concernent :

5. La réglementation du temps de travail : le droit du travail prescrit, entre autres, les périodes minimales de repos et le nombre d'heures maximales de travail, mais du fait qu'il y a différents modèles, il est difficile à appliquer;

6. Les déficits de sécurité sociale dans les engagements de courte durée: ceux-ci peuvent avoir des effets négatifs sur la fluctuation des revenus et ainsi, par exemple, sur la rente d'invalidité; 
7. La gestion des formes de travail (encore) indéfinies, du fait qu'elles sont juridiquement floues;

8. La clarification des questions touchant au lieu de travail ainsi que le « faux travail indépendant » : ces questions sont liées au remboursement des frais des employés quand ils sont appelés à travailler en dehors de leur lieu de travail, aux déductions fiscales et aux diverses possibilités d'assurances ;

9. Le recrutement du personnel : les prestataires de plateformes de crowdsourcing peuvent échapper au système juridique suisse lors les sièges sociaux et les sites web sont situés à l'étranger;

10. L'application du droit: les petites organisations utilisent aussi de nouvelles formes de travail mais elles sont plus difficiles à contrôler pour les inspecteurs du travail;

11. Les réglementations concernant la période après l'emploi, car elles dépendent fortement de l'emploi (principe d'équivalence);

12. La création et le maintien d'une vue d'ensemble comprenant toutes les normes juridiques pertinentes.

Les statistiques officielles existantes ainsi que leurs périodicités ne permettent pas une évaluation de la situation actuelle et de la situation sur le court terme. Elles doivent être adaptées dans les domaines suivants :

13. La mobilité professionnelle des salariés,

14. L'économie souterraine,

15. Les conditions de travail des travailleurs-entrepreneurs.

Du fait que la dynamique sur le marché de travail a tendance à s'accélérer, la surveillance doit être anticipée afin de prendre des mesures efficaces à différents niveaux. Il devrait y avoir plus d'indicateurs d'alerte rapide et les données nécessaires devraient être prélevées, pour améliorer les mesures.

Le phénomène de la flexibilisation du travail reste encore flou, il s'agit donc d'un problème mal défini. Toutefois, en tant que tel, nous sommes confrontés un véritable défi, qui, une fois surmonté, peut faire une véritable différence pour le monde du travail en Suisse, qui, résistant et résiliant aujourd'hui, pourrait occuper une position de pointe au niveau mondial ou tout au mieux faire croître son potentiel actuel. Ce n'est pas toujours évident, mais un effort collectif est nécessaire pour développer ces nouvelles formes de travail. C'est la condition pour 
réussir à faire évoluer au mieux la situation actuelle qui est encore confuse et complexe, mais également captivante et pleine d'opportunités.

Les auteurs de l'étude:

Jens O. Meissner, Johann Weichbrodt, Bettina Hübscher, Sheron Baumann, Ute Klotz, Ulrich Pekruhl, Leila Gisin et Alexandra Gisler

\section{Pour toute information complémentaire:}

Prof. Dr. Jens O. Meissner

Hochschule Luzern - Wirtschaft

Institut für Betriebs- und Regionalökonomie IBR

Zentralstr. 9

$\mathrm{CH}-6002$ Luzern

jens.meissner@hslu.ch

Dr. Johann Weichbrodt

Fachhochschule Nordwestschweiz

Hochschule für angewandte Psychologie

Institut für Kooperationsforschung und -entwicklung IfK

Riggenbachstr. 16

$\mathrm{CH}-4600$ Olten

johann.weichbrodt@fhnw.ch 


\section{Riassunto}

Le evoluzioni tecniche degli ultimi decenni, e in particolare la digitalizzazione, stanno producendo profonde trasformazioni nel mondo del lavoro. Esse consentono l'affermazione di un modello lavorativo sempre più flessibile da diversi punti di vista:

- $\quad$ grazie a Internet e ai cellulari, i dipendenti possono accedere ai dati aziendali anche da casa o da altri luoghi ed essere operativi anche fuori sede/in viaggio (flessibilità spaziale)

- $\quad$ il rigido orario d'ufficio lascia il posto a un orario di lavoro flessibile e/o basato sulla fiducia (flessibilità temporale)

- $\quad$ i lavoratori, anziché essere inquadrati in un rapporto lavorativo immutato per decenni, collaborano sempre più all'interno di team che si avvicendano in progetti di durata più breve (flessibilità organizzativa)

- $\quad$ le aziende sfruttano a propria volta le opportunità di adattare la quantità di personale alla quantità di lavoro ed eventualmente esternalizzano determinati compiti (flessibilità numerica).

II presente studio mira ad esaminare le conseguenze di questi sviluppi sul valore riconosciuto al lavoro retribuito nella vita sociale, la compatibilità delle nuove forme di lavoro con l'attuale quadro normativo e gli esiti che ne derivano a livello macroeconomico.

I seguenti punti ne riassumono i risultati essenziali:

1. il valore riconosciuto al lavoro è sottoposto alla pericolosa azione congiunta di due tendenze, "flessibilizzazione spazio-temporale" e comparsa degli "entreployees" o "impre-lavoratori"

2. il quadro giuridico è nel complesso adeguato. Ciò nonostante va valutato se sia più opportuno varare ulteriori normative o affidarsi all'autoregolamentazione delle parti sociali. Inoltre andrebbero esaminate con più attenzione le borse-lavoro virtuali

3. a livello di economia nazionale, a fronte di pochi e lievi miglioramenti si riscontrano parecchi (lievi e netti) peggioramenti 
4. il principio di coinvolgere le parti sociali nella strutturazione della flessibilità del lavoro è lungimirante

5. i sistemi di monitoraggio e controllo ignorano determinati aspetti parziali nonché dati indispensabili per le previsioni.

\section{Forme di flessibilità diversamente avanzate}

Per quanto riguarda la flessibilità temporale del lavoro, il part-time si è da tempo affermato nella realtà svizzera: raggiungendo il 37 per cento dei dipendenti nel 2014, il nostro Paese è ormai ai vertici europei. I lavoratori a tempo parziale riferiscono spesso di riuscire a conciliare meglio il lavoro con la vita privata (lifedomain-balance), ma suppongono di avere prospettive di carriera inferiori a quelle degli impiegati a tempo pieno. Circa il 61 per cento dei dipendenti svizzeri si avvale di modelli di lavoro a tempo più "morbido", come per esempio l'orario flessibile. Questa soluzione è considerata valida ma può risultare problematica se si rinuncia del tutto alla registrazione del tempo-lavoro.

Quanto alla flessibilità spaziale, quasi un quarto dei dipendenti svizzeri lavora parzialmente da casa (home office); anche in questo caso il dato è in vetta alla classifica europea. Sono in crescita anche forme di lavoro mobile che comprendono, oltre all'attività svolta da casa, quella esercitata presso i clienti o in viaggio. Inoltre le aziende puntano sempre più sul desk sharing, incrementando la flessibilità all'interno dell'infrastruttura aziendale. Questa forma di flessibilità lavorativa presenta molti vantaggi, tra cui una maggiore produttività, una maggiore soddisfazione personale e un migliore equilibrio tra lavoro e vita privata, a condizione che il lavoro in modalità home office sia una scelta volontaria del dipendente $\mathrm{e}$ non vada ad aggiungersi al monte-ore regolarmente svolto in ufficio.

La flessibilizzazione quantitativa del personale complessivo (flessibilità numerica) si manifesta concretamente per es. nei contratti a tempo determinato, nei contratti a progetto e in nuove forme di reclutamento come il crowdsourcing sulle piattaforme online. Circa il 7 per cento dei contratti di lavoro svizzeri sono a tempo determinato. Negli ultimi quindici anni il "classico" lavoro a chiamata è rimasto sostanzialmente stabile sul 5 per cento. Sui fenomeni relativamente recenti del crowdsourcing e del crowdworking finora mancano invece dati attendibili, ma si suppone che questa modalità di reclutamento sia destinata a crescere per importanza: soprattutto nel settore dell'informatica e della comunicazione si ipotizza 
che nei prossimi anni le aziende rinunceranno a parte del personale fisso. II resto del personale necessario verrà reclutato tra i "liquid talents" della "cloud umana", i professionisti ingaggiabili via Internet. In generale si presume che saranno i lavoratori più qualificati a trarre vantaggio da questi nuovi modelli di lavoro, mentre i meno qualificati subiranno svantaggi notevoli.

Accanto a queste forme di flessibilità temporale, spaziale e numerica, prosegue la tendenza al livellamento della gerarchia all'interno delle organizzazioni. Invece di ricevere precise istruzioni dai superiori, i dipendenti godono di un più ampio margine di autonomia operativa. A loro sta raggiungere gli obiettivi concordati sotto la propria responsabilità, spesso con ripercussioni rilevanti sulla quota variabile della retribuzione. Cosifacendo il rischio imprenditoriale si trasferisce ai dipendenti. Nelle scienze sociali per questo rimescolamento dei ruoli tradizionali è stato coniato il termine di "entreployee" alias "impre-lavoratore", in altri contesti si parla anche di soggettivazione del lavoro. Per i dipendenti la maggiore autonomia può costituire un vantaggio, ma non va sottovalutato il rischio di autosfruttamento se il life-domain-balance si sbilancia in misura crescente a favore del lavoro.

\section{Notevole incremento della varietà professionale}

Oltre all'aumento nel numero degli impre-lavoratori, ci si aspetta che in futuro i dipendenti associno uno o più lavori a tempo parziale ad attività indipendenti. Questa forma di lavoro (parzialmente) autonomo può offrire alternative professionali e contribuire a una migliore espressione dei propri interessi e potenzialità. Tuttavia un modello di lavoro altamente flessibile cela anche considerevoli insidie, prima fra tutte il rischio che i dipendenti scivolino verso condizioni lavorative precarie. Se, per esempio, i lavoratori part-time sono assunti a tempo determinato e il volume di lavoro è fluttuante, il loro reddito e la loro posizione occupazionale non sono più predeterminabili con attendibilità. Le borse-lavoro basate sulle piattaforme di crowdworking consentono solo malamente ai loro utenti di pianificare il reddito e il carico di lavoro che li aspetta. Inoltre questo tipo di attività autonoma richiede grande senso di responsabilità individuale e capacità di negoziazione. L'esito dipende pertanto innanzitutto dal livello di qualificazione: chi dispone di una buona formazione e sa agire in autonomia può senz'altro, grazie a un profilo professionale richiesto sul mercato, ottenere che siano soddisfatte le sue aspettative economiche e condizioni di lavoro adeguate. Chi è poco qualifi- 
cato può invece precipitare in una spirale fatta di precarietà professionale e reddito in calo.

A trarre i maggiori vantaggi dalla flessibilizzazione sono i dipendenti con il posto fisso. I quadri qualificati, in particolare, ne guadagnano un considerevole margine di manovra nel processo decisionale, che può tradursi a sua volta in soddisfazione e motivazione maggiori, a patto di riuscire a gestire la pressione crescente.

\section{Nuovi problemi normativi legati alla flessibilizzazione}

II diritto del lavoro deve assicurare che gli interessi delle varie parti sociali vengano soddisfatti in modo equilibrato. Nel mondo lavorativo flessibilizzato si tratterà di difendere le conquiste sociali e di sfruttare le opportunità del lavoro flessibile a vantaggio di tutti. II rapporto normale di lavoro si basa sul contratto di lavoro normale individuale o collettivo ai sensi del Codice delle obbligazioni (CO). I contratti di lavoro individuali o collettivi regolano aspetti centrali del rapporto tra le parti sociali, sia quanto alle prestazioni dei dipendenti, sia quanto al compenso e agli obblighi di tutela che fanno capo ai datori di lavoro.

Va detto, per cominciare, che il diritto svizzero del lavoro è ben posizionato nel panorama europeo, soprattutto quanto a tutela della sicurezza sociale. Questo risultato, tuttavia, non è da ritenersi conquistato per l'eternità. Le nuove forme di lavoro pongono nuove sfide normative: di fondamentale importanza è la distinzione tra il contratto individuale di lavoro e altri tipi di contratto, in particolare il mandato e l'appalto. Mentre il contratto individuale di lavoro prevede tutele dal licenziamento che ne regolano tempi e cause, un mandato può essere revocato in qualsiasi momento. Essenziale ai fini della distinzione è anche il grado di subordinazione dei dipendenti alle direttive aziendali. Questo criterio è dirimente per stabilire se un lavoratore è subordinato o autonomo. Nelle forme intermedie prodotte dalla flessibilizzazione i singoli lavoratori sono solo debolmente integrati nella struttura aziendale, ma economicamente ne dipendono. Questi "falsi autonomi" non hanno quindi diritto all'indennità di disoccupazione, alla previdenza professionale, all'assicurazione obbligatoria contro gli infortuni né alla tutela in caso di malattia professionale.

Legalmente anche il crowdsourcing è in una zona d'ombra. I crowdworker esterni sono lavoratori autonomi e di conseguenza non godono delle tutele previste nei contratti individuali di lavoro (salario minimo, ferie, continuazione del pagamento 
dello stipendio ecc.). Dato che i rapporti di lavoro negoziati sulle piattaforme di crowdsourcing scavalcano spesso i confini nazionali, è indispensabile esaminare di volta in volta l'applicabilità del diritto straniero e le sue modalità. Non è ancora chiaro, inoltre, come ottenere che vengano correttamente versate l'imposta sul reddito e l'IVA. Le piattaforme di crowdsourcing fungono da mediatrici e pertanto da controparte sia per i datori di lavoro che per i lavoratori. Il quadro giuridico di questi rapporti è definito dalle condizioni generali di contratto della piattaforma. II divieto di contratto con committenti esterni che spesso vi si ritrova viola il diritto della personalità e anche il principio di libertà economica.

Non sono però soltanto i rapporti di lavoro atipici a suscitare questioni legali. L'aumento della flessibilità può richiedere precisazioni normative anche nell'ambito del contratto normale di lavoro. Occorre chiarire come risarcire gli strumenti di lavoro e le prestazioni fornite anticipatamente dai lavoratori con dispiego di mezzi propri. Attualmente le spese devono essere obbligatoriamente rimborsate, ma non l'impiego dei propri strumenti di lavoro privati.

La maggiore possibilità di lavorare da casa o comunque fuori ufficio pone anche la questione della responsabilità per danni. In azienda ne risponde il datore di lavoro. Se però è il computer domestico a rompersi con conseguente perdita di dati, la questione si complica senza risposte univoche. Le aziende dovrebbero chiarire se l'assicurazione contro la responsabilità civile aziendale copra o meno determinati rischi (es. di perdita di dati) presenti anche nel lavoro da casa e in altre forme di lavoro mobile. Viceversa cresce il rischio di danni all'azienda legati all'utilizzo lavorativo degli apparecchi privati. La mentalità del "bring your own device" favorisce la produttività perché i dipendenti possono usare apparecchi che conoscono e i costi di acquisizione degli stessi in parte vengono meno. Va chiarito però chi debba accollarsi i costi di software e assistenza sostenuti per gli apparecchi privati dei dipendenti. Nel complesso, se queste questioni non sono disciplinate dal contratto, entrambe le parti si espongono a rischi considerevoli.

\section{Flessibilizzazione positiva per l'economia solo se associata a sufficiente qualificazione}

Nemmeno le conseguenze del lavoro flessibilizzato sul sistema economico complessivo sono difficili da pronosticare con chiarezza. I fattori di contorno che le influenzano sono molti. Uno dei principali è la formazione dei lavoratori: maggiore è la quota di personale ben qualificato e maggiori sono le probabilità che il 
lavoro flessibilizzato contribuisca ad accrescere la produttività complessiva, con conseguente possibilità di salari più elevati e maggiori entrate fiscali. La flessibilizzazione mette però a dura prova la gestione individuale del tempo e delle energie: essere sempre raggiungibili, senza una chiara separazione fra lavoro e tempo libero, e postazioni di lavoro non sempre adeguatamente allestite minacciano la salute fisica e psichica, benché d'altro canto la flessibilità spaziale e temporale possano essere sfruttate per adattare il lavoro alle proprie necessità (bioritmo, famiglia, hobby, ecc.).

Per i lavoratori meno qualificati la flessibilizzazione comporta invece rischi significativi. La digitalizzazione facilita infatti il trasferimento delle attività più ripetitive $\mathrm{e}$ standardizzate in paesi dove i costi del personale sono inferiori, riducendo le opportunità di impiego dei meno qualificati. Spesso rimangono soltanto occupazioni nei settori che offrono servizi a diretto contatto con la persona. I lavoratori poco qualificati sono inoltre costretti a svolgere più spesso della media lavori a turni o su chiamata, sono meno soddisfatti del proprio impiego e sono più esposti sia al rischio di ammalarsi fisicamente o psichicamente sia a quello della disoccupazione, soprattutto se assunti a tempo determinato o con un monte-ore ridotto.

\section{Raccomandazioni}

II nocciolo del presente resoconto è che le conseguenze del lavoro flessibilizzato per il singolo e la società sono ambivalenti e andrebbero quindi prudentemente incanalate in una direzione positiva tramite provvedimenti a vari livelli. Una formazione di base e professionale qualificata accresce notevolmente le probabilità che i lavoratori possano sfruttare le potenzialità positive della flessibilizzazione. II futuro di questo sviluppo non può essere predeterminato sul piano tecnico o economico, ma dipende piuttosto dalla strutturazione di nuove forme di lavoro a livello aziendale e sovraziendale. Raccomandiamo quindi:

1. di introdurre una cornice che delimiti la flessibilizzazione temporale e spaziale del lavoro, altrimenti illimitata: è necessario un inquadramento legislativo, che può eventualmente prevedere anche sanzioni, ma si limiti in ogni caso a escludere le forme estreme e abbia quindi il carattere di un "argine di contenimento";

2. di sensibilizzare sul fenomeno della soggettivazione del lavoro (ossia degli "impre-lavoratori"): è necessario rafforzare i soggetti per renderli in grado di meglio affrontare il mondo del lavoro flessibilizzato. Chiamate in 
causa sono soprattutto le istituzioni che offrono istruzione di base e avanzata, benché anche ai sindacati si offre un'occasione di espandere il proprio campo di competenza;

3. di strutturare la flessibilità aziendale come sviluppo partecipato dell'impresa: entro gli argini posti dalla legge, le parti sociali devono avere la possibilità di elaborare soluzioni congiunte adatte alle esigenze locali e specifiche;

4. di rafforzare la partecipazione e l'autorganizzazione all'interno dell'azienda: i datori di lavoro, in particolare, sono invitati a trovare risposte alle esigenze di maggiore flessibilità (o a determinate forme delle stesse).

La flessibilizzazione del mondo lavorativo ha numerose conseguenze legali. Miglioramenti e riesami sono importanti a proposito di:

5. regolamentazione del tempo-lavoro, poiché ad es. la legislazione vigente prescrive tempi minimi di riposo e tempi massimi di lavoro difficilmente applicabili ai modelli alternativi;

6. lacune delle assicurazioni sociali negli impieghi a breve termine, perché le oscillazioni di reddito possono avere ripercussioni negative ad es. sulla pensione di invalidità;

7. trattamento delle forme di lavoro (ancora) non definite, che legalmente parlando vengono a trovarsi in una zona d'ombra;

8. chiarimento di questioni legate al luogo di lavoro e al "finto lavoro autonomo", in quanto connesse a quella del rimborso spese per lavori fuori sede, deduzioni fiscali e opzioni assicurative;

9. borse-lavoro, perché i gestori dei siti di crowdworking possono eludere totalmente il sistema legale svizzero situando all'estero la sede aziendale e il sito internet;

10. applicazione delle leggi, perché gli ispettorati del lavoro hanno maggiori difficoltà a controllare le realtà più piccole, in cui stanno prendendo piede le nuove forme di lavoro, rispetto alle grandi aziende;

11. riesame del trattamento nella fase post-lavorativa, poiché al momento esso è fortemente legato alla fase di attività lucrativa (principio di equivalenza);

12. redazione e aggiornamento di un censimento generale di tutte le normative in materia. 
In molti casi i censimenti disponibili degli enti pubblici di statistica e la periodicità della loro pubblicazione non consentono una stima a breve termine aggiornata della situazione futura, come sarebbe invece assai auspicabile nei seguenti campi:

13. mobilità professionale dei lavoratori dipendenti,

14. economia sommersa e

15. condizioni di lavoro degli impre-lavoratori.

Dato che si prevede un incremento del dinamismo del mercato del lavoro, il monitoraggio dovrebbe essere più lungimirante affinché si possano adottare misure efficaci a vari livelli. Occorre sviluppare un maggior numero di indicatori-spia più mirati e raccogliere i relativi dati per poter poter adottare meglio i provvedimenti richiesti.

I contorni del fenomeno della flessibilizzazione lavorativa restano comunque vaghi, nel senso che si tratta di un problema finora non sufficientemente definito. Rappresenta pertanto una sfida che, se affrontata con successo, può portare a cambiamenti decisivi in favore di un mondo del lavoro svizzero resistente e resiliente, capace di mantenere o persino migliorare la propria posizione di punta a livello internazionale. Questo traguardo non va dato per scontato ma, al contrario, richiede un contributo comune allo sviluppo da parte di tutte le parti coinvolte. Soltanto così sarà possibile trarre il meglio da una situazione che attualmente si presenta piuttosto confusa e molto complessa, ma anche interessante e ricca di opportunità.

Autrici e autori dello studio:

Jens O. Meissner, Johann Weichbrodt, Bettina Hübscher, Sheron Baumann, Ute Klotz, Ulrich Pekruhl, Leila Gisin und Alexandra Gisler

\section{Per chiarimenti rivolgersi a:}

Prof. Dr. Jens O. Meissner

Hochschule Luzern - Wirtschaft

Institut für Betriebs- und Regionalökonomie IBR

Zentralstr. 9

$\mathrm{CH}-6002$ Lucerna

jens.meissner@hslu.ch 
Dr. Johann Weichbrodt

Fachhochschule Nordwestschweiz

Hochschule für angewandte Psychologie

Institut für Kooperationsforschung und - entwicklung IfK

Riggenbachstr. 16

$\mathrm{CH}-4600$ Olten

johann.weichbrodt@fhnw.ch 


\section{Methodischer und inhaltlicher Überblick}

Die vorliegende Studie geht den folgenden Fragen zum Thema der Flexibilisierung der Arbeitswelt nach:

- Welchen Stellenwert besitzt die Arbeit (bezahlt/unbezahlt) in der Gesellschaft heute und in Zukunft?

- $\quad$ Sind neue Arbeitsformen mit den rechtlichen Bestimmungen vereinbar?

- Was für volkswirtschaftliche Folgen haben neue Arbeitsformen?

- Welche Rahmenbedingungen müssten herrschen, damit möglichst viele Personen von mobil-flexiblem Arbeiten profitieren können (ohne dabei mit negativen Folgen konfrontiert zu sein)?

- Wie könnten Monitoring- und Kontrollsysteme aussehen, damit die bestehende soziale Absicherung und deren staatliche Finanzierung nicht durch mobil-flexibles Arbeiten unterlaufen werden können?

Zur Beantwortung dieser Fragen wurde in einem interdisziplinär besetzten Forschungsteam die vorliegende Studie realisiert. Sie umfasst entsprechende Arbeitspakete gemäss den Forschungsfragen 1-3, um die beiden letzten Fragen schlussendlich beantworten zu können. Das Projekt verfolgte den Forschungsansatz der qualitativen Sozialforschung, basierte wesentlich auf Literaturrecherchen und Gruppenworkshops mit dem Projektteam sowie der Begleitgruppe seitens TA-SWISS, und dauerte 18 Monate.

\section{Stellenwert der Arbeit}

Im ersten Kapitel wird die Frage nach dem Stellenwert der Arbeit behandelt, daneben werden verschiedene Formen und Ausprägungen flexibler Arbeit identifiziert und analysiert. Als Ausgangspunkt wird dabei das „Normalarbeitsverhältnis" mit einer unbefristeten Vollzeitanstellung, festen Arbeitszeiten und -orten sowie sozialer Absicherung verwendet. Insbesondere die numerische, zeitliche und örtliche Flexibilisierung dieses Arbeitsverhältnisses werden diskutiert.

Numerische Flexibilität bedeutet die flexible Anpassung der Anzahl Beschäftigten seitens eines Unternehmens. Dies kann z.B. realisiert werden durch befristete 
Arbeitsverhältnisse, Honoraraufträge oder neue Formen wie das Crowdsourcing. Der Anteil solcher Arbeitsverhältnisse liegt in der Schweiz grösstenteils im einstelligen Prozentbereich. Bei der zeitlichen Arbeitsflexibilität ist die Teilzeitarbeit am meisten verbreitet: 2014 arbeiteten 37 Prozent der Erwerbstätigen in Teilzeit, was im europäischen Vergleich der zweithöchste Wert ist. Die grosse Mehrheit der Teilzeiterwerbstätigen sind hierbei Frauen. Etwa 7 Prozent sind allerdings unterbeschäftigt, arbeiten also unfreiwillig in Teilzeit. Flexible Arbeitszeiten in Form von Gleitzeit oder noch flexibleren Modellen haben etwa 61 Prozent der Schweizer Angestellten, etwa 17 Prozent erfassen dabei ihre tatsächliche Arbeitszeit nicht. Örtliche Flexibilität kann sehr unterschiedliche Formen annehmen; am stärksten verbreitet ist wohl die anteilige Arbeit im Homeoffice (etwa ein Viertel der Angestellten). Neben diesen klar definierten Formen von Arbeitsflexibilisierung wird auch die eher schwieriger fassbare Subjektivierung von Arbeit diskutiert. Hierbei handelt es sich um die Übertragung von unternehmerischem Risiko sowie der entsprechenden Verantwortung auf Angestellte. Dies zeigt sich z.B. in der Verknüpfung von variablen Lohnbestandteilen mit dem Unternehmenserfolg, aber auch in der verstärkt zielorientierten Führungsweise. Soziologen prägten hierfür den Begriff „Arbeitskraftunternehmende“.

Um die Verknüpfung verschiedener Formen von Arbeitsflexibilität zu verdeutlichen, werden anschliessend zwei konkrete Formen flexibler Arbeit im Detail untersucht, und zwar die anteilige Arbeit im Homeoffice sowie das „Crowdsourcing for paid Work“. Hierbei wird insbesondere auf die Vor- und Nachteile aus Sicht der Arbeitenden eingegangen. Die Komplexität der verschiedenen Ausprägungen der "flexiblen neuen Arbeitswelt“" wurde innerhalb der Forschungsgruppe zunächst erweitert mittels eines morphologischen Kastens und anschliessend verdichtet auf eine $4 \times 3-$ Matrix. Diese beinhaltet jeweils drei Ausprägungen in einer rechtlichen Dimension (Anstellungsformen), einer physischen (zeitliche und örtliche Arbeitsflexibilität) sowie in zwei sozialen Dimensionen (ökonomische Rolle des Individuums und soziale Funktion von Arbeit).

Auf der Grundlage dieser Matrix wurden anschliessend fünf Personas gebildet, d.h., es wurden konkrete, fiktive Personen erdacht, die jeweils eine bestimmte Merkmalskonfiguration in der Matrix abbilden. Um sowohl positive als auch negative Seiten der flexiblen Arbeit abzubilden, wurde jeweils ein guter Tag und ein schlechter Tag beschrieben, den die Personas erleben. Persona A trägt den Namen Roland Müller, ist Finanzleiter eines KMU und steht für das Arbeiten im „traditionellen“ Arbeitsverhältnis mit nur geringen Anteilen von Flexibilität - ins- 
besondere die (tageweise) Arbeit im Homeoffice. Persona B hat den Namen Sandra Könitz bekommen und ist Projektmanagerin. Sie personifiziert insbesondere das Phänomen der „Arbeitskraftunternehmerin“, also einer angestellten Person, die aber in vielen Punkten so handelt wie eine Selbstständige. Gleichzeitig arbeitet sie örtlich und zeitlich sehr flexibel. Persona C, genannt Ursula Meyerhans, ist Journalistin und steht für das Phänomen „zwei Standbeine“, d.h., sie kombiniert eine Teilzeit-Festanstellung mit selbstständiger Erwerbsarbeit, zum Teil vermittelt über Crowdsourcing-Plattformen. Auch sie arbeitet örtlich und zeitlich sehr flexibel. Persona D namens Noah Schmid ist Informatiker und verkörpert den Unternehmer in der digitalen Arbeitswelt, d.h., er arbeitet voll selbstständig und nutzt dafür die volle zeitliche und örtliche Flexibilität. Aufträge werden insbesondere online/digital vermittelt. Schliesslich betreibt auch Persona E mit Namen Andrea Burri-Lötscher ein Modell mit zwei Standbeinen. Sie arbeitet als Küchenhilfe und steht dabei insbesondere für die Möglichkeiten und Herausforderungen gering qualifizierter Arbeitnehmerinnen und Arbeitnehmer.

Zum Abschluss des ersten Kapitels wird die Frage nach dem Stellenwert der Arbeit und seinem Wandel beantwortet, indem die zuvor ausgearbeiteten Facetten der "flexiblen neuen Arbeitswelt" auf zwei Phänomene komprimiert werden: die physische Entgrenzung von Arbeit sowie die Subjektivierung von Arbeit. Dies stellt Erwerbstätige, neben den vielen Vorteilen wie beispielsweise der Zeitsouveränität und der flexiblen Lebensgestaltung, grundsätzlich vor zwei Herausforderungen: Erstens müssen sie neue (zeitliche und räumliche) Grenzen ziehen zwischen Arbeit und anderen Lebensbereichen, was auch unter dem Stichwort „Boundary-Management" diskutiert wird. Dieses Management der eigenen Grenzen kann gelingen oder scheitern. Zweitens müssen sie als „Arbeitskraftunternehmer/innen“ eine Balance finden zwischen unternehmerischem Risiko, Eigenverantwortung und sozialer Absicherung. Auch diese Ausbalancierung kann wiederum gelingen oder scheitern. Diese beiden Dimensionen werden abschliessend in einer Entwicklungsmatrix einander gegenübergestellt und die Personas in ihr verortet.

\section{Rechtliche Bestimmungen}

Das Kapitel 3 behandelt die Ausprägungen mobil-flexibilisierter Arbeit und ihre spezifischen Merkmale unter Berücksichtigung der rechtlichen Dimension. Dabei werden zuerst die rechtlichen Grundlagen dargelegt und die zu berücksichtigen- 
den Rechtsquellen herausgearbeitet. In Bezug auf die mobil-flexibilisierte Arbeit bildet das Individualarbeitsrecht die Basis. Zentral für die Bearbeitung der rechtlichen Dimensionen ist es herauszufinden, welcher Vertragstyp jeweils zur Anwendung gelangt. Diese Unterscheidung und Qualifikation zwischen den Vertragsverhältnissen wie dem Einzelarbeitsvertrag, dem Auftrag und dem Werkvertrag ist wichtig und wird im Spannungsfeld I genauer analysiert. Für diese Qualifikation müssen Hauptmerkmale herausgearbeitet werden, um in einem zweiten Schritt zu sehen, welche Vertragsnormen sich auf die verschiedenen Arbeitsformen anwenden lassen. Wichtig ist hierbei, dass jeder Sachverhalt, sprich jede Arbeitssituation, im Detail geprüft werden muss, da es keine Pauschallösung gibt. Gleichzeitig kristallisieren sich auch gesetzliche Lücken heraus und es wird ersichtlich, wo allenfalls eine analoge Anwendung von gesetzlichen Bestimmungen Sinn macht.

Bei den verschiedenen Arbeitsformen wie Telearbeit, Heimarbeit, Homeoffice und Freelancing spielen auch spezifische rechtliche Aspekte wie beispielsweise Geheimhaltungspflicht, Lohnfortzahlung oder Datenschutz eine Rolle. Wie im Spannungsfeld II aufgezeigt wird, müssen diese Themen im Zusammenhang mit flexiblen Arbeitsformen geklärt werden. Einen durchaus politisch brisanten Schwerpunkt bildet die Vertrauensarbeitszeit und die damit zusammenhängende Arbeitszeiterfassung. Hierbei wurde ein weiteres Spannungsfeld identifiziert. Im Weiteren werden auch flexible Arbeitszeitmodelle wie Gleitzeit, Jahresarbeitszeit oder Arbeit auf Abruf behandelt. Grundsätzlich bringen sowohl die neuen Arbeitsformen als auch die Arbeitszeitmodelle positive Aspekte mit sich. Es kann eine Win-win-Situation für den Arbeitnehmenden und Arbeitgebenden entstehen. Wichtig zum Vermeiden einer negativen Entwicklung im Hinblick auf die rechtliche Dimension ist aber, dass die Rahmenbedingungen klar definiert und vertraglich oder in einem Reglement festgehalten werden.

Die sowohl räumliche als auch zeitliche Flexibilisierung der Arbeit bringen in Verbindung mit dem eher starren Modell der sozialen Sicherheit Schwierigkeiten mit sich. So fehlen beispielsweise bei Formen wie dem Crowdworking oder dem Freelancing Möglichkeiten von versicherungsrechtlicher Absicherung. Auch bei Kurzzeiteinsätzen stellen sich Fragen bezüglich der Lohnfortzahlung bei Arbeitsverhinderung des Arbeitnehmenden oder der Kündigungssperre. Zusätzlich werden diese Randgruppen auch nicht unter den Schutz des kollektiven Arbeitsrechts, die Gesamtarbeitsverträge, fallen, d.h., Risiken, wie die fehlende BVGAbsicherung oder auch das Risiko der Arbeitslosigkeit, werden vollständig auf 
den Arbeitnehmenden überwälzt. Diese Problematik wird in Spannungsfeld IV und $\mathrm{V}$ näher erläutert.

Die Spannungsfelder VI und VII befassen sich mit dem Gesundheitsschutz. Dabei wird aufgezeigt, dass dieser von beiden Sozialpartnern angestrebt werden sollte. Die Arbeitgeberin oder der Arbeitgeber ist zum Schutz der Gesundheit am Arbeitsplatz verpflichtet. Der oder die Arbeitnehmende trägt aber eine erhebliche Mitverantwortung. Dieser Thematik ist sich auch der Gesetzgeber bewusst, was unter dem Begriff der Stresshaftung erläutert wird. Ist die Arbeitnehmende von zu Hause aus für ihre Arbeitgeberin bzw. ihren Arbeitgeber tätig, so liegt es auf der Hand, dass sie dazu neigt, ihre eigenen Geräte für die Ausführung ihrer Arbeit zu benutzten. Der Begriff dafür lautet Bring-your-own-Device (BYOD). Dieses Verhalten ist aber aus rechtlicher Sicht nicht ganz ungefährlich (Spannungsfeld VIII). Das letzte Spannungsfeld IX ergibt sich im Zusammenhang mit Crowdsourcing. Denn obwohl dieses in den vergangenen Jahren auch in der Schweiz immer mehr an Bedeutung gewonnen hat, fehlen jedoch die rechtlichen Grundlagen weitgehend.

Die mithilfe der Spannungsfelder identifizierten Punkte sind sicherlich in absehbarer Zeit zu klären und Lösungen dazu zu entwickeln. Hierbei liefern diverse bereits umgesetzte Modelle in umliegenden Ländern Ideen und Möglichkeiten, auf welchen aufgebaut werden kann, oder es sind bereits Erfahrungen vorhanden, wie die Umsetzung eben nicht funktioniert. Zentral ist, dass die schweizerische Arbeitsrechtslandschaft, die heute schon durch die vielen gesetzlichen Grundlagen komplex ist, nicht durch viele zusätzliche Regelungen noch unübersichtlicher wird. Vielmehr soll versucht werden, durch analoge Anwendungen von bestehenden gesetzlichen Bestimmungen des Einzelarbeitsvertrags auf andere flexible Arbeitsformen die unklaren Situationen zu klären und für die Arbeitnehmenden abzusichern, sodass die Vorteile der flexiblen Arbeit am Ende auch überwiegen bzw. nicht zunichte gemacht werden.

\section{Volkswirtschaftliche Auswirkungen}

Für jede Arbeitswelt, die verschiedene mögliche Ausprägungen einer sich weiterentwickelnden Flexibilisierung der Arbeit darstellt, und den damit in Bezug stehenden prägenden Arbeitsformen erfolgt eine Abschätzung möglicher Folgen auf gesamtwirtschaftlicher Ebene. Die Auswirkungen werden aufgrund von Rechercheresultaten bestimmt und anhand einer Reihe von Indikatoren, die in $\mathrm{Zu}$ - 
sammenarbeit mit der Begleitgruppe von TA-SWISS ausgewählt wurden, veranschaulicht. Sie umfassen Produktivität, Erwerbseinkommen, den kollektiven Organisationsgrad der Beschäftigten, physische und psychische Gesundheitsrisiken, Arbeitsbedingungen und -zufriedenheit, Vereinbarkeit von Beruf mit anderen Lebensbereichen, Arbeitslosigkeit, Beschäftigte, Steuererträge, intransparente Zahlungsströme sowie die Situation der Systeme der sozialen Absicherung.

Eine Vielzahl von Befunden aus wissenschaftlichen Studien, journalistischen Beiträgen und öffentlichen Statistiken, welche im Wesentlichen innerhalb der letzten zehn Jahre publiziert wurden und in relevanter Weise mit den oben aufgeführten Indikatoren in Zusammenhang stehen, wurden systematisch gesucht, zusammengetragen und den fünf personabasierten Arbeitswelten zugewiesen. Aus den zahlreichen Einzelsachverhalten wurde anschliessend induktiv nach dem Grundsätzlichen gesucht und dieses, unter der Annahme, dass die darin enthaltenen Arbeitsformen gesamtwirtschaftliche Relevanz erreichen, zu einer möglichen Folge für den Indikator auf gesamtwirtschaftlicher Ebene extrapoliert. So konnten aus der aktuellen Entwicklung für die verschiedenen Indikatoren Prognosen mit einem Zeithorizont von zehn bis 15 Jahren gefolgert werden. 


\begin{tabular}{|c|c|c|c|c|c|}
\hline \multirow{2}{*}{$\begin{array}{l}\text { Indikator/ } \\
\text { Messgrösse }\end{array}$} & \multicolumn{5}{|c|}{ Stärke und Richtung der Auswirkung in 10 bis 15 Jahren in der Arbeitswelt von } \\
\hline & $\begin{array}{l}\text { Roland Müller / } \\
\text { Homeoffice } \\
\text { in der Fest- } \\
\text { anstellung }\end{array}$ & $\begin{array}{c}\text { Sandra Könitz / } \\
\text { Mobiles } \\
\text { Abeitskraftunter- } \\
\text { nehmertum }\end{array}$ & $\begin{array}{c}\text { Ursula } \\
\text { Meyerhans / } \\
\text { Portfoliowork auf } \\
\text { mehreren } \\
\text { Standbeinen }\end{array}$ & $\begin{array}{l}\text { Noah Schmid / } \\
\text { Entre- } \\
\text { preneurship }\end{array}$ & $\begin{array}{l}\text { Andrea Burri- } \\
\text { Lötscher / } \\
\text { Mehrfach- } \\
\text { anstellungen } \\
\text { und Minijobs }\end{array}$ \\
\hline Produktivität & $\sim \downarrow$ & $\uparrow$ & O & $\sim \downarrow$ & $\sim \uparrow$ \\
\hline $\begin{array}{l}\text { Erwerbsein- } \\
\text { kommen }\end{array}$ & O & $\sim$ & $\downarrow$ & $\downarrow$ & $\downarrow$ \\
\hline $\begin{array}{l}\text { Kollektiver Orga- } \\
\text { nisationsgrad der } \\
\text { Beschäftigten }\end{array}$ & O & O & $\sim \downarrow$ & $\downarrow \downarrow$ & $\downarrow \downarrow$ \\
\hline $\begin{array}{l}\text { Physische und } \\
\text { psychische } \\
\text { Gesundheit }\end{array}$ & $\sim \downarrow$ & $\downarrow$ & $\downarrow \downarrow$ & $\downarrow$ & $\downarrow$ \\
\hline $\begin{array}{l}\text { Arbeitsbedin- } \\
\text { gungen und } \\
\text {-zufriedenheit }\end{array}$ & $\uparrow$ & $\uparrow$ & $\sim \downarrow$ & $\sim \downarrow$ & $\downarrow$ \\
\hline $\begin{array}{l}\text { Vereinbarkeit } \\
\text { von Beruf mit } \\
\text { anderen Le- } \\
\text { bensbereichen }\end{array}$ & $\uparrow$ & $\sim \downarrow$ & $\sim$ & $\uparrow$ & $\sim \uparrow$ \\
\hline Erwerbsquote & 0 & 0 & $\sim \downarrow$ & $\downarrow$ & $\downarrow$ \\
\hline Beschäftigte & $\sim \uparrow$ & 0 & $\sim \downarrow$ & $\downarrow$ & $\sim$ \\
\hline Steuererträge & O & $\sim \uparrow$ & $\sim \downarrow$ & $\sim \downarrow$ & $\downarrow$ \\
\hline $\begin{array}{l}\text { Transparenz der } \\
\text { Zahlungsströme }\end{array}$ & 0 & $\sim$ & $\downarrow$ & $\downarrow \downarrow$ & $\downarrow$ \\
\hline $\begin{array}{l}\text { Situation der } \\
\text { Systeme der } \\
\text { sozialen Absi- } \\
\text { cherung }\end{array}$ & 0 & 0 & $\downarrow$ & $\downarrow \downarrow$ & $\downarrow \downarrow$ \\
\hline
\end{tabular}

Zeichenerklärung:

$\uparrow \uparrow$ deutlicher Anstieg bzw. deutliche Verbesserung der Situation; $\uparrow$ leichter Anstieg bzw. leichte Verbesserung der Situation; $\downarrow \downarrow$ deutlicher Rückgang bzw. deutliche Verschlechterung der Situation; $\downarrow$ leichter Rückgang bzw. leichte Verschlechterung der Situation; $\bigcirc$ keine oder unwesentliche Veränderung; $\sim$ Ergebnis sehr unsicher; $\sim \uparrow$ Ergebnis unsicher, Tendenz steigend; $\sim \downarrow$ Ergebnis unsicher, Tendenz fallend 
Die Auswirkungen der in den fünf Arbeitswelten herrschenden Bedingungen auf die ausgewählten Indikatoren ergeben zusammengefasst ein heterogenes Bild. Einerseits gibt es Unterschiede bei den Intensitäten und Richtungen der Auswirkungen, andererseits bei der Sicherheit, mit welcher ihr Eintritt beurteilt werden kann.

Die Arbeitswelten von Roland Müller (Persona A, „Homeoffice in der Festanstellung“) und Sandra Könitz (Persona B, „mobile Arbeitskraftunternehmerin“) sind heute bereits für eine bedeutende Anzahl Beschäftigter verwirklicht oder weisen nur geringe Unterschiede zu zeitgenössischen Arbeitsverhältnissen auf. Ihre Auswirkungen auf die Arbeitslosigkeit bzw. die Beschäftigung sind unwesentlich und auch bezüglich der anderen Indikatoren gibt es kaum Verschlechterungen zu erwarten. Die beiden Arbeitsformen verursachen gesamtwirtschaftlich am ehesten physische und psychische Probleme. Das liegt vor allem daran, dass der oder die Beschäftigte bzw. Arbeitnehmende ein Selbstmanagement entwickeln oder soweit verbessern muss, dass sie oder er mit den Arbeitsbedingungen zurechtkommen kann.

Dafür bieten die zwei Arbeitswelten viel Potenzial für bessere Arbeitsbedingungen und -zufriedenheit, insbesondere wenn Beschäftigte und Arbeitnehmende eine Präferenz für räumlich und zeitlich flexible Arbeitsformen haben. Die Entwicklung in Richtung der beiden Arbeitswelten schreitet indessen voran, da die objektiven Voraussetzungen nach Harabi, Schoch und Hespeler (2001) für diese Art der Flexibilisierung gegeben sind. Da in den Arbeitswelten von Roland Müller und der erfolgsbeteiligten Sandra Könitz immer noch Festanstellungen mit einem traditionellen Verhältnis zwischen Arbeitgebenden und Arbeitnehmenden dominieren, sind sie bezüglich intransparenter Zahlungsströme und der Auswirkungen auf die Systeme der sozialen Absicherung am absehbarsten und mit wenig gravierenden Folgen verbunden.

Die Arbeitswelt von Ursula Meyerhans (Persona C, „Portfoliowork auf mehreren Standbeinen“) steht exemplarisch für mögliche Entwicklungen, die einige Aspekte der Prekarisierung und den Verlust von Sicherheiten für eine breitere Masse der Beschäftigten mit sich bringen könnten. Die grössere Verbreitung und häufigere Kombination von Befristung und Teilzeit verschlechtern die Arbeitsplatzund Beschäftigungssicherheit, und die Einkommenssicherheit kann durch schwankende Arbeitsvolumina beeinträchtigt werden. Wenn Beschäftigte durch die Anstellungspraxis der Unternehmen unterbeschäftigt sind, könnten sie durch den zu niedrig ausfallenden Lohn folglich in der Existenzsicherheit bedroht sein. 
Ähnlich bedroht sind auch Auftragssuchende, die wenig erfolgreich oder schlecht bezahlt auf Crowdworking-Plattformen tätig sind.

Ursula Meyerhans und ihresgleichen sind ausserdem grösseren Risiken psychischer und physischer Art ausgesetzt, als es bei heutigen Normalarbeitsverhältnissen der Fall ist. In ihrer Arbeitswelt ist das traditionelle Verhältnis von Arbeitgebenden und -nehmenden aufgeweicht und die Arbeitgeberpflichten können durch die Outsourcingmethoden umgangen werden. Die zunehmend einfacheren Möglichkeiten für Ursula Meyerhans, aber auch für ihre Auftraggebenden, nicht rückverfolgbare Zahlungen tätigen zu können, vergrössert die Wahrscheinlichkeit intransparenter Zahlungsströme. Der dadurch entstehende kurz- und mittelfristige „Vorteil“ liegt im Umgehen von staatlichen Abgaben wie den Beiträgen der Arbeitgebenden und -nehmenden, welche die Systeme der sozialen Absicherung finanzieren. $\mathrm{Da}$ in dieser Arbeitswelt auch die Steuererträge aller Staatsebenen zurückgehen dürften, ist im Vergleich zu heute und den zwei vorangegangenen Arbeitswelten mit einer Verschlechterung der Situation dieser Systeme zu rechnen.

In der Arbeitswelt des selbstständigen Softwareentwicklers Noah Schmid (Persona D, „Entrepreneurship“) und sogar noch stärker in derjenigen von Andrea Burri-Lötscher (Persona E, „Minijobs und Mehrfachanstellungen“) treten Probleme auf gesamtwirtschaftlicher Ebene auf, die seit Längerem mit atypischer Beschäftigung in Zusammenhang stehen. Während die Beschäftigungssituation von Noah Schmid zu einem gewissen Grad als selbstgewählt bezeichnet werden kann, trifft dies auf die geringqualifizierte Andrea Burri-Lötscher nicht zu. Beide Personas leiden unter Arbeitsplatz- und Beschäftigungsunsicherheit, was sich u.a. gesamtwirtschaftlich durch höhere Arbeitslosenquoten bemerkbar macht. Kurzfristig schwankende Einkommen, das Resultat von häufig wechselnden Arbeitspensen und hohen variablen Anteilen am Lohn, bedrohen in beiden Arbeitswelten die Einkommenssicherheit. Mindestens im Fall von Andrea BurriLötscher tritt eine Existenzunsicherheit ein, da sie infolge der Unterbeschäftigung zu wenig Einkommen erzielen kann. Im Weiteren verschlechtern sich in beiden Fällen die Arbeitsbedingungen und es muss mit einer Zunahme der physischen und psychischen Gesundheitsrisiken durch die Arbeit gerechnet werden. Heute noch selbstverständliche Schutzbestimmungen verlieren ihre Wirkung, weil gesetzliche, kollektiv vereinbarte oder durch sozialpartnerschaftliche Gewohnheiten garantierte Bestimmungen fehlen oder umgangen werden. 
Die Systeme der sozialen Sicherheit können in den beiden letzten Arbeitswelten ihre Aufgabe im heutigen Sinn nicht mehr erfüllen. Die gesamtwirtschaftlich tieferen Erwerbseinkommen sowie die grössere Verbreitung und Akzeptanz auf der Nutzendenseite der Kryptowährungen verschärfen das Problem noch. Die auf Beiträgen von Arbeitgebenden und -nehmenden basierenden Systeme der sozialen Absicherung werden so infrage gestellt.

Der Schlussteil der Studie umfasst die Entwicklung von vier denkbaren Szenarien („Lebensweltlicher Ausgleich“, „Gelingende Integration“, „Verschärfende Wirtschaftsdominanz“, und „Abschmelzung des Lebensstandards“), deren Konsequenzen kurz umrissen werden. Die leitenden Forschungsfragen werden kurz beantwortet und Handlungsempfehlungen gegeben. Die zentralen Handlungsempfehlungen sind in der folgenden Tabelle enthalten:

\begin{tabular}{|l|l|l|}
\hline Nr. & Handlungsempfehlung & Zielgruppen \\
\hline 1 & $\begin{array}{l}\text { Einen weiten Rahmen für den Umgang } \\
\text { mit zeitlicher und örtlicher Entgrenzung } \\
\text { der Arbeit setzen }\end{array}$ & $\begin{array}{l}\text { Sozialpartnerin / Sozialpart- } \\
\text { ner auf Ebene Betrieb } \\
\text { Parlament }\end{array}$ \\
\hline 2 & $\begin{array}{l}\text { Sensibilisierung zur Subjektivierung } \\
\text { der Arbeit }\end{array}$ & $\begin{array}{l}\text { Bildungspolitik } \\
\text { Bildungspolitikanbieterinnen } \\
\text { und -anbieter } \\
\text { Gewerkschaften } \\
\text { Start-up-Initiativen }\end{array}$ \\
\hline 3 & $\begin{array}{l}\text { Betriebliche Flexibilitätsplanung als } \\
\text { partizipative Organisationsentwicklung }\end{array}$ & $\begin{array}{l}\text { Sozialpartnerin / Sozial- } \\
\text { partner }\end{array}$ \\
\hline 4 & $\begin{array}{l}\text { Breite Mitwirkung als gemeinsames } \\
\text { Interesse der Sozialpartnerinnen / } \\
\text { Sozialpartner stärken }\end{array}$ & $\begin{array}{l}\text { Sozialpartnerin / Sozial- } \\
\text { partner } \\
\text { Arbeitnehmendenverbände }\end{array}$ \\
\hline 5 & $\begin{array}{l}\text { Regelungen zu modernen Arbeitszeit- } \\
\text { systemen weiter anpassen }\end{array}$ & \begin{tabular}{l} 
Parlament \\
\hline 6
\end{tabular} \\
$\begin{array}{l}\text { Sozialversicherungsdefizite bei Kurz- } \\
\text { Arbeitseinsätzen gewährleisten }\end{array}$ & $\begin{array}{l}\text { Sozialpartnerin/Sozialpartner } \\
\text { Sozialversicherungen }\end{array}$ \\
\hline
\end{tabular}




\begin{tabular}{|c|c|c|}
\hline Nr. & Handlungsempfehlung & Zielgruppen \\
\hline & & Parlament \\
\hline 7 & $\begin{array}{l}\text { Arbeitsrechtliche Praxis im Umgang mit } \\
\text { noch undefinierten Arbeitsformen regeln }\end{array}$ & $\begin{array}{l}\text { Parlament } \\
\text { Parteien eines Arbeits- } \\
\text { vertrags }\end{array}$ \\
\hline 8 & $\begin{array}{l}\text { Aus dem Arbeitsort abgeleitete An- } \\
\text { sprüche klären }\end{array}$ & Parlament \\
\hline 9 & $\begin{array}{l}\text { Den rechtlichen Rahmen der Schein- } \\
\text { selbstständigkeit präzisieren }\end{array}$ & $\begin{array}{l}\text { Parlament } \\
\text { Arbeitgebende } \\
\text { Arbeitgebendenverbände }\end{array}$ \\
\hline 10 & $\begin{array}{l}\text { Gesetzgebung zur Personalvermittlung } \\
\text { überprüfen }\end{array}$ & Parlament \\
\hline 11 & $\begin{array}{l}\text { Mechanismen der Rechtsdurchsetzung } \\
\text { überprüfen }\end{array}$ & $\begin{array}{l}\text { Kantonale Verwaltungen } \\
\text { Arbeitsinspektorate } \\
\text { Sozialpartnerin/Sozialpartner }\end{array}$ \\
\hline 12 & $\begin{array}{l}\text { Soziale Sicherung in der Nacherwerbs- } \\
\text { phase erforschen und wenn nötig } \\
\text { politisch regeln }\end{array}$ & $\begin{array}{l}\text { Forschungsförderung } \\
\text { Forschende } \\
\text { Fachverbände }\end{array}$ \\
\hline 13 & $\begin{array}{l}\text { Gesamtübersicht über rechtliche } \\
\text { Aspekte und soziale Absicherung } \\
\text { fördern }\end{array}$ & $\begin{array}{l}\text { Parlament } \\
\text { Arbeitgebendenverbände } \\
\text { Schweizerischer Gewerk- } \\
\text { schaftsbund } \\
\text { Fachverbände }\end{array}$ \\
\hline
\end{tabular}




\begin{tabular}{|c|c|c|}
\hline 14 & Berufliche Mobilität zeitnaher erfassen & $\begin{array}{l}\text { Mitglieder der Bundes- } \\
\text { statistikkommission } \\
\text { Mitglieder von Fedestat und } \\
\text { Regiostat } \\
\text { Expertengruppen für Wirt- } \\
\text { schaftsstatistik } \\
\text { Forschungsinstitutionen } \\
\text { Politik }\end{array}$ \\
\hline 15 & $\begin{array}{l}\text { Virtuelle Schattenwirtschaft und } \\
\text { Schwarzarbeit erfassen }\end{array}$ & $\begin{array}{l}\text { Mitglieder der Bundes- } \\
\text { statistikkommission } \\
\text { Mitglieder von Fedestat und } \\
\text { Regiostat } \\
\text { Expertengruppen für Wirt- } \\
\text { schaftsstatistik } \\
\text { Forschungsinstitutionen } \\
\text { Politik }\end{array}$ \\
\hline 16 & $\begin{array}{l}\text { Kontinuierliches Monitoring der Berufs- } \\
\text { bedingungen von "Arbeitskraftunter- } \\
\text { nehmenden“ }\end{array}$ & $\begin{array}{l}\text { Mitglieder der Bundes- } \\
\text { statistikkommission } \\
\text { Mitglieder von Fedestat } \\
\text { und Regiostat } \\
\text { Expertengruppen für Wirt- } \\
\text { schaftsstatistik } \\
\text { Forschungsinstitutionen } \\
\text { Politik }\end{array}$ \\
\hline
\end{tabular}

Das Verständnis der traditionellen Büro- und Fabrikarbeit mit Vorgesetzten, welche die Arbeit anweisen und überwachen, scheint im Zuge der voranschreitenden Digitalisierung und strukturellen Veränderung der Arbeitswelt nicht mehr aktuell und angemessen. Und doch ist nicht ausgeschlossen, dass die weitreichende, globale Arbeitsteilung (tayloristisches Prinzip) in ihren negativen 
Ausprägungen im Bereich der mobilen Arbeit prominenter hervortreten könnte als bisher angenommen. Dieser Entwicklung ist (pro)aktiv zu begegnen.

Das Phänomen der flexiblen Arbeitswelt bleibt am Ende ein „unscharfes“ Problem, d.h. ein schlecht definiertes, ein „echtes“ Problem. Als solches ist es eine Herausforderung, deren erfolgreiche Bewältigung einen deutlichen Unterschied machen kann, einen Unterschied hin zu einer widerstandsfähigen, resilienten Schweizer Arbeitswelt, die ihre weltweite Spitzenposition erhalten und gar noch ausbauen kann. Dies ist keine Selbstverständlichkeit, sondern eine gemeinschaftliche Entwicklungsleistung aller beteiligten Parteien. Die vorliegende Studie leistet einen klaren Beitrag zur Orientierung in dieser momentan unübersichtlichen, komplexen, aber auch interessanten und an Chancen reichhaltigen Ausgangslage. 



\section{Einleitung}

Die Arbeitswelt befindet sich in einem tiefgreifenden Wandel, welcher zu grossen Teilen (aber nicht ausschliesslich) durch die schnelle Verbreitung von Informations- und Kommunikationstechnologien bedingt ist. Immer leistungsfähigere Geräte, Netzwerke und Software durchdringen das Wirtschafts- und das Arbeitsleben und treiben die Digitalisierung unserer Gesellschaft voran. Immer mehr Geschäfts- und Arbeitsprozesse werden damit nach und nach von Papier und Gebäuden losgelöst. Eine Konsequenz dieser Entwicklung ist die örtliche und zeitliche Flexibilisierung von Arbeit: Für mehr und mehr Arbeitstätigkeiten, insbesondere in der Büro- und Wissensarbeit, reichen ein Computer oder ein Smartphone mit Internetverbindung aus. So wird bspw. die Arbeit aus dem Homeoffice für einen zunehmenden Anteil der Beschäftigten zu einer Option.

Die Flexibilisierung von Arbeit findet allerdings noch in weiteren Dimensionen statt: So ermöglicht die Digitalisierung von Arbeitsprozessen häufig auch ein "Outsourcing“, d.h. eine Verlagerung der Arbeitstätigkeiten aus dem Unternehmen heraus - nicht nur physisch, sondern auch organisatorisch. Ein klassisches Beispiel hierfür ist der externalisierte telefonische Kundenservice. Neue Formen sind z.B. das "Crowdsourcing for paid Work", also die Ausschreibung bestimmter Aufgaben oder Dienstleistungen auf Crowdsourcing-Plattformen.

Darüber hinaus sind auch eine finanzielle Flexibilisierung von Arbeit (z.B. in Form von leistungsorientierter Entlöhnung) sowie flexible Karrieremodelle beobachtbar. In der Soziologie wird der Begriff „Arbeitskraftunternehmer“ bzw. „Arbeitskraftunternehmerin“ gebraucht, um das Phänomen zu beschreiben, dass mehr und mehr Angestellte in Abkehr vom Taylorismus heutzutage selbst verantwortlich sind für die Strukturierung, Überwachung und Rationalisierung ihrer Arbeit und damit letztlich, zumindest teilweise, die Verantwortung des Managements übernehmen und so die (paradoxe) Rolle der Unternehmerin oder des Unternehmers im eigenen Unternehmen innehaben.

Alle diese Formen von Arbeitsflexibilisierung sind für sich genommen nicht revolutionär - so haben bspw. Lehrer und Lehrerinnen schon immer teilweise zu Hause gearbeitet und auch leistungsorientierte Entlohnung ist kein neues Phänomen -, in der Summe tun sich hier allerdings tiefgreifende Veränderungen auf. Sichtbar wird dies auch an der Vielzahl von Studien, Tagungen oder Zeitungs- 
artikeln, in denen derzeit über Stichworte wie „Zukunft der Arbeit“, „Smart Working“ oder „Arbeit 4.0“ diskutiert wird (für eine Übersicht siehe z.B. Birk, Nelsen, Lackerbauer und Kirchner, 2015).

Diese vielschichtigen Veränderungen werden zum Teil äusserst kontrovers diskutiert. In den Extrempositionen wird die „New World of Work“ wahlweise entweder glorifiziert oder verteufelt: Auf der einen Seite werden mit der flexiblen Arbeit positive Effekte, wie Produktivitätssteigerungen, mehr Arbeitszufriedenheit oder eine bessere Life-Domain-Balance verbunden. Von Anhängerinnen und Anhängern erhofft und von „Evangelistinnen und Evangelisten“ versprochen werden Freiheit, Erfolg, Innovation, Mobilität, Entrepreneurship, die Verschmelzung von Arbeit mit anderen Lebensbereichen und eine höhere Sinnhaftigkeit der Arbeit. Auf der Gegenseite werden die negativen Auswirkungen an die Wand gemalt: Befürchtet wird die Entgrenzung von Arbeit und anderen Lebensbereichen, Stress aufgrund ständiger Erreichbarkeit, steigende Fallzahlen von Burnout und anderen psychischen Krankheiten (mit den entsprechenden Kosten für das Gesundheitssystem), die Erosion der bestehenden sozialen Sicherungssysteme kurz gesagt eine fortschreitende (Selbst-)Ausbeutung der Arbeitnehmenden.

Zusammengefasst lässt sich sagen, dass sich die Arbeitswelt in einem vielschichtigen Wandel befindet, aber eine hohe Unsicherheit darüber herrscht, ob dies nun zum Guten oder zum Schlechten geschieht. Mit dieser Erkenntnis tun sich grundsätzlich zwei Fragen auf. Zum einen stellt sich die Frage, welche Auswirkungen diese Flexibilisierung der Arbeit nun tatsächlich auf Individuen, Gesellschaft und Wirtschaft hat. Welche Chancen und welche Risiken tun sich damit auf - abseits der predigenden bzw. warnenden Stimmen? Die zweite Frage, die sich im Anschluss daran stellt, ist: Wie können diese Auswirkungen so gesteuert werden, dass die positiven Effekte möglichst gefördert und die negativen Effekte vermieden oder zumindest abgefedert werden? Welche (politischen, gesellschaftlichen, wirtschaftlichen und/oder individuellen) Weichen müssen jetzt gestellt werden? Insbesondere gilt es zu überprüfen, ob das derzeitige rechtliche Gefüge aus gesetzlichen Bestimmungen und sozialpartnerschaftlichen Verträgen auch für neue, flexiblere Arbeitsformen geeignet ist oder ob hier Anpassungen nötig sind.

Diesem Fragenkomplex widmet sich diese Studie. Ziel ist also zum einen, eine Standortbestimmung bezüglich der Flexibilisierung von Arbeit vorzunehmen und dabei aus einer neutralen Perspektive heraus positive wie negative Auswirkungen neuer, flexibler Arbeitsformen abzubilden (insbesondere für Arbeitende). Des 
Weiteren soll eine Bestandsaufnahme des derzeitigen Arbeitsrechtssystems gemacht werden im Hinblick auf mögliche Unzulänglichkeiten. Darüber hinaus sollen auf der Makroebene die volkswirtschaftlichen Folgen der Arbeitsflexibilisierung skizziert werden. Zum Schluss werden die gesammelten Erkenntnisse verwendet, um daraus konkrete Handlungsempfehlungen für Politik, Wirtschaft und Arbeitende abzuleiten.

\subsection{Projektauftrag und Fragestellung}

Kerninhalte des Projektberichts sind die Themen Stellenwert der bezahlten und unbezahlten Arbeit, die Ausprägungen neuer Arbeitsformen inkl. deren rechtliche Bestimmungen und die volkswirtschaftlichen Auswirkungen mobil-flexibler Arbeitsformen. Das übergeordnete Ziel ist, anhand einer detaillierten Analyse das Risiko- und Chancenpotenzial dieser aktuellen Entwicklungen darzustellen. Schliesslich erfolgt eine Gesamtbeurteilung mit abgeleiteten Empfehlungen, sodass Entscheidungsträgerinnen und Entscheidungsträger eine wissenschaftlich fundierte Dokumentation zum Thema flexible neue Arbeitswelt zur Verfügung steht.

Der durchgeführten Studie lagen die folgenden Forschungsziele zugrunde:

Ziele des Projektes zum Stellenwert der Arbeit:

- $\quad$ Eine Systematik von Dimensionen mobil-flexibler Arbeit ist erstellt und die Verortung der neuen Arbeitsformen in diesen Dimensionen ist vorgenommen.

- $\quad$ Die gegenwärtige und zukünftige Entwicklung des Stellenwerts von Arbeit sowie Vor- und Nachteile der neuen Arbeitsformen für Beschäftigte sind aufgezeigt.

Ziele des Projektes zu neuen Arbeitsformen und deren rechtlichen Bestimmungen:

- $\quad$ Arten der praktizierten mobil-flexiblen Arbeitsformen sind zusammengetragen.

- $\quad$ Die gesetzlich relevanten Rahmenbedingungen für mobil-flexible Arbeitsformen sind erfasst (national/international). 
- $\quad$ Zentrale Spannungsfelder sind dargestellt. Aus der Recherche der erfassten gesetzlichen Grundlagen und aus der Gegenüberstellung zu den praktizierten mobilen Arbeitsformen sind Widersprüche aufgezeigt.

Ziele des Projektes zu volkswirtschaftlichen Folgen der mobil-flexiblen Arbeit:

- $\quad$ Belastbare Aussagen in Form von drei Szenarien sind abgeleitet, d.h., es werden wissenschaftlich fundierte Schlussfolgerungen über die Potenziale und Realisierungsraten mobil-flexibler Arbeitsformen auf Basis der gewonnenen Daten und identifizierten Faktoren aufgezeigt.

- $\quad$ Die Darstellung der durch die Flexibilisierung der Arbeitsformen begünstigten Entwicklung intransparenter Finanzflüsse ist erstellt.

- $\quad$ Eine szenariobasierte Darstellung der Auswirkungen von mobil-flexiblen Arbeitsformen und den Folgen intransparenter Finanzflüsse auf ausgewählte gesamtwirtschaftliche Grössen, wie der Arbeitslosigkeit, ist erstellt.

- Die Darstellung der zur Erfassung der szenariobasierten Entwicklungen notwendigen Daten und allfälliger Erhebungslücken ist erfolgt.

Ziele über das gesamte Projekt:

- Rahmenbedingungen sind aufgezeigt, wie möglichst viele Personen von mobil-flexiblem Arbeiten profitieren können (ohne dabei mit negativen Folgen konfrontiert zu sein, wie bspw. Stress aufgrund der Entgrenzung von Arbeit, Konflikten in der Familie wegen fehlender Trennung von Arbeit/Familienleben oder des Verlustes von sozialer Absicherung).

- $\quad$ Monitoring- und Kontrollsysteme sind aufgezeigt, damit die bestehende soziale Absicherung und deren staatliche Finanzierung nicht durch mobil-flexibles Arbeiten unterlaufen werden.

- $\quad$ Eine Gesamtbeurteilung mit Handlungsempfehlungen für Entscheidungstragende liegt vor.

Die aufgeworfenen Fragen und die damit zusammenhängenden Zielsetzungen des Forschungsprojektes zeigen auf, dass neben neuen Forschungsschwerpunkten auch neue Betrachtungsweisen das Thema mobil-flexible Arbeit bereichern. Besonders interessant ist die interdisziplinäre Verschmelzung von volkswirtschaftlichen, betriebswirtschaftlichen, psychologischen, soziologischen, sozialwissenschaftlichen und juristischen Überlegungen in einem Forschungsprojekt. 


\subsection{Gliederung des Berichts}

Zum Erreichen der Zielsetzungen wurde das Projekt in vier Arbeitspakete gegliedert, deren inhaltliche Strukturen mit den Kapiteln des vorliegenden Berichts in weiten Teilen übereinstimmen (siehe Abbildung 1).

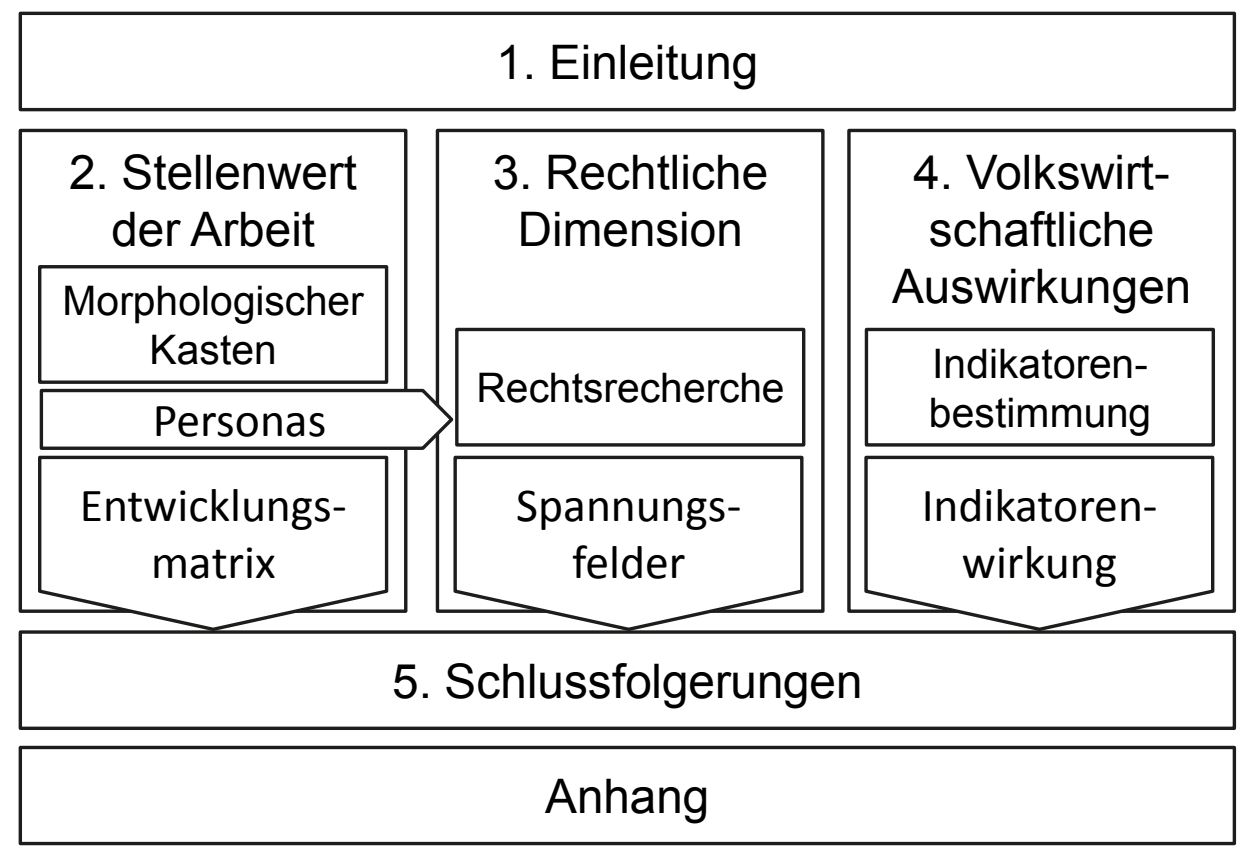

Abbildung 1: Gliederung des Berichts

Das erste Themengebiet (Kapitel 2) konzentriert sich auf den Stellenwert der Erwerbsarbeit. Freiwilligenarbeit oder ähnliche Tätigkeiten mit nicht unmittelbarem Bezug zum Erwerbsleben werden nur dann berücksichtigt, wenn hier zukünftig relevante Entwicklungen zu erwarten sind. In diesem Teil sind auch die Vor- und Nachteile der mobil-flexiblen Arbeitsformen integriert. Im Kapitel zwei erhält die Entwicklung eines morphologischen Kastens zur Bestimmung verschiedener Flexibilisierungsformen eine zentrale Bedeutung. Der Kasten wird dazu genutzt, unterschiedliche Personenprofile (Personas) zu entwickeln, die 
dann zur weiteren Bearbeitung in den Folgekapiteln zur Verfügung stehen. Die Personas werden je in einem Steckbrief vorgestellt, der mit der Beschreibung eines guten und schlechten Tages endet. Ziel dieser Methode ist es, die gesellschaftliche Einbettung der Personas deutlich zu machen und darauf hinzuweisen, dass eine gelingende oder scheiternde gesellschaftliche Dynamik die Situation der Persona entsprechend verbessern oder verschlechtern kann. Die Personenprofile kennzeichnen die Zukunftsthemen, die dann in den Überlegungen zum Stellenwert der Arbeit in einer Entwicklungsmatrix berücksichtigt werden.

Kapitel drei umfasst als zweites Themengebiet die rechtliche Komponente der flexiblen Arbeitswelt. Dieses Themengebiet beinhaltet eine Vielzahl an Fragen, welche höchst relevant für Arbeitnehmende, Arbeitgebende sowie auch selbstständig Erwerbstätige sind. Das Kapitel bildet die durchgeführte, sehr umfangreiche Rechtsrecherche ab und hebt die zentralen Spannungsfelder hervor. Diese Felder sind Ansatzpunkte für mehrere Handlungsempfehlungen und beschreiben die aktuelle Lage zwischen Rechtsetzung und -durchsetzung. Die damit verbundenen schwierigen Abwägungen im Rechtssystem sind zentral für die Bewältigung der Flexibilisierungsherausforderungen.

Als letztes Themengebiet werden im vierten Kapitel die gesamtwirtschaftlichen Auswirkungen der unterschiedlichen Personas genauer untersucht. Neben klaren Vorteilen der Flexibilisierung, welche in vielen anderen Projekten bereits untersucht wurden (bspw. betriebswirtschaftliche Einsparpotenziale durch mobilflexibles Arbeiten), gibt es bei den Personas auch zu erwartende Verschlechterungen, bspw. häufig im Bereich der sozialen Absicherung. Auf der Grundlage dieser Ausführungen werden Handlungsempfehlungen formuliert.

Der Bericht wird eingefasst durch diese Einleitung und durch das Kapitel fünf mit den Schlussfolgerungen. Die Schlussfolgerungen gehen auf die gestellten Forschungsfragen ein und geben den ableitbaren Handlungsempfehlungen entsprechenden Raum. Ein Anhang mit vertiefenden Informationen rundet das Informationsangebot des vorliegenden Dokuments ab.

\subsection{Methodologie}

Hinter dem Vorgehen der vorliegenden Studie stehen die methodischen Gütekriterien der qualitativen Sozialforschung. In dieser wird besonderer Wert auf die 
Dokumentation und Nachvollziehbarkeit der Argumentation gelegt, insbesondere hinsichtlich der Transparenz des Forschungsprozesses. Die Herausforderung lag darin, die ausserordentlich vielfältige Fragestellung dieser Studie zur flexiblen neuen Arbeitswelt einer gewissen „Messbarkeit“ zuzuführen, um Trendaussagen über gewichtige und relevante Themen plausibel ableiten zu können.

Diese Zuführung wurde über die volkswirtschaftlichen Konsequenzen der Veränderungen vorgenommen. Die Verlagerung des Fokus von der Themenvielfalt hin zur Beschreibung anhand von Kennzahlen erfolgte über die Entwicklung der Personas als Akteure der Zukunftsszenarien und Personenprototypen. Im Folgenden erläutern wir die methodologischen Komponenten des Studienprozesses. Hierzu gehören die Erläuterung von Herangehensweise und Methode, das gewählte Vorgehen sowie die Diskussion von Kriterien und Güte des verwendeten Designs. Eine komplette Darlegung der Methodologie wäre an dieser Stelle des Berichts zu umfangreich und ist daher im Anhang des Berichts zu finden.

\subsection{Zielgruppen}

Wie bereits thematisiert, wirkt sich mobil-flexibles Arbeiten auf die individuellen Arbeitnehmerinnen und Arbeitnehmer sowie Arbeitgeberinnen und Arbeitgeber aus. Es hat aber auch auf der kollektiven Ebene der Gesellschaft und des Staates weitreichende Konsequenzen. Daher gelten für das Forschungsprojekt „Flexible neue Arbeitswelt - eine Bestandsaufnahme" in erster Linie drei primäre Zielgruppen: mobil-flexible Arbeitnehmende an sich, Unternehmen/Wirtschaftsorganisationen und gesellschaftliche Anspruchsgruppen. Ferner profitieren auch Bildungsinstitutionen von mobil-flexiblem Arbeiten. Schliesslich gelten die Handlungsempfehlungen massgeblich für politische Akteurinnen und Akteure, denen eine bedeutende Gestaltungsrolle bezüglich der rechtlichen und volkswirtschaftlichen Faktoren zugewiesen wird. Der Nutzen für die genannten Zielgruppen lässt sich wie folgt umreissen.

\subsubsection{Nutzen für mobil-flexible Arbeitnehmende}

Personen, welche in einem mobil-flexiblen Angestelltenverhältnis arbeiten oder selbstständig (z.B. Crowdsourcing for paid Work) sind, kennen durch die Resultate des Forschungsprojektes neben den Vor- und Nachteilen neuer Arbeitsfor- 
men auch deren rechtlichen Aspekte. Dadurch erfolgt eine Befähigung dieser Personen, sodass der primäre Nutzen für diese Zielgruppe im Wissenstransfer besteht.

\subsubsection{Nutzen für Unternehmen und Wirtschaftsorganisationen}

Vorsitzende, Geschäftsleitungsmitglieder, Kaderpersonen und Fachverantwortliche erhalten eine Grundlage für die Diskussion von mobil-flexibler Arbeit im Unternehmen. Neben den Vor- und Nachteilen sowie den rechtlichen Bestimmungen und Problemen bei mobil-flexiblen Anstellungsverhältnissen kennen sie diese Themenbereiche auch bei Auslagerung von gewissen Aufgaben an Dritte (z.B. Crowdsourcing, Prosumer, Turker, Click- or Microworker). Weiter zeigen Trends bezüglich des Stellenwerts der Arbeit mögliche Entwicklungsbereiche für Unternehmen auf, die diese in ihre Strategieüberlegungen einfliessen lassen können (z.B. Neugestaltung der Arbeitsverhältnisse, Ressourcenoptimierung, Entwicklung von Dienstleistungen für neue Kundengruppen (mobil-flexibel arbeitende Personen) wie Beratungsangebote, Versicherungsschutz).

\subsubsection{Nutzen für gesellschaftliche Anspruchsgruppen}

$\mathrm{Zu}$ dieser Zielgruppe gehören primär Verbände, NGOs und politische Parteien sowie Regierungsmitglieder. Für die Weiterentwicklung der gesetzlichen Grundlagen in der Schweiz (und u.U. auch international) kann diese Zielgruppe auf wichtige Fakten und Zahlen zurückgreifen, sodass dem Trend der Flexibilisierung in der Arbeitswelt aus gesellschaftspolitischer Sicht genügend Rechnung getragen werden kann. Dabei dienen die zugrunde liegenden Zahlen und Fakten nicht nur arbeitsrechtlichen Aspekten der Gesetzgebung, sondern auch der volkswirtschaftlichen Ausgestaltung von Steuern und Abgaben (z.B. AHV/IV, Einkommenssteuern, Sozialleistungen wie Prämienverbilligungen). Weiter können dank der aufgezeigten Datenlücken diese zukünftig geschlossen werden.

\subsubsection{Nutzen für Bildungsinstitutionen}

Bildungsinstitutionen profitieren von diesem Forschungsprojekt, indem eine weitere Wissenslücke zum Thema mobil-flexibles Arbeiten geschlossen wird (siehe Kapitel Problemstellung und Zielsetzungen) und so ein Wissenstransfer zwischen Forschungsprojekt, Bildungsinstitutionen und letztlich den Kunden in der 
Aus- und Weiterbildung erfolgt. So könnten bspw. Ergebnisse in Unterrichtssequenzen zum Thema Personalführung und -management, Datenschutz und -sicherheit, Geschäftsgeheimnisse, Projektmanagement oder Arbeitsorganisation einfliessen. Ferner bildet das Forschungsprojekt eine umfassende Grundlage für weitere Forschungsthemen im Gebiet der mobil-flexiblen Arbeit; ein Forschungsgebiet, welches aufgrund der aufgezeigten Entwicklungen (Kapitel Ausgangslage, Kapitel Problemstellung) in den nächsten Jahren sicherlich ein grosses öffentliches Interesse wecken wird.

\subsubsection{Nutzen für politische Entscheidungsträgerinnen und Entschei- dungsträger}

Schliesslich sind die politischen Entscheidungsträgerinnen und Entscheidungsträger eine ausserordentlich wichtige Zielgruppe des vorliegenden Berichts. Die vielen recherchierten Informationen zeigen, wie umfangreich die Fragestellung ist und gefasst werden kann. Das siebenköpfige, interdisziplinär besetzte Projektteam widmete der Recherche sowie dem Aufbereiten und Zusammenführen von verschiedensten Informationen im Rahmen dieses Projektes Monate. Es ist schlicht nicht zu erwarten, dass Politikerinnen und Politiker im Rahmen ihrer Ratstätigkeiten oder anderer politischer Ämter die Zeit und Ressourcen zur Verfügung haben, auch nur annähernd umfassende Recherchen zu realisieren. Weil das Thema Flexibilisierung von seiner gesellschaftlichen Tragweite her ausserordentlich wichtig und gleichermassen reich an politischen Regulierungen ist, sind auch die Handlungsempfehlungen für diese Zielgruppe entsprechend umfangreich. 



\section{Formen der Arbeitsflexibilisierung und Veränderung des Stellenwerts der Arbeit}

Kurt Lewin, der als einer der Pioniere der Sozial- und Organisationspsychologie gilt, beschrieb die bekannten „Zwei Gesichter der Arbeit“" (Lewin, 1920, S. 11 f.). Arbeit sei zum einen „Mühe, Last, Kraftaufwand“ und letztlich nicht mehr als ein Mittel zum Zweck: „Arbeit ist unentbehrliche Voraussetzung zum Leben, aber sie ist selbst noch nicht wirkliches Leben." Aus dieser Perspektive ist eine „möglichst ökonomische Gestaltung des Arbeitsprozesses“ das einzig vernünftige Ziel. Fortschritt, im Sinne von besseren Arbeitsbedingungen, bedeutet hier also, die Erwerbsarbeit angenehmer, leichter und vor allem kürzer zu machen - sie also wenn irgend möglich abzuschaffen.

Demgegenüber beschreibt Lewin das andere Gesicht der Arbeit, wonach Arbeit einen inhärenten Wert hat, nämlich die Sinnstiftung: „Diese Fähigkeit der Arbeit, dem individuellen Leben Sinn und Gewicht zu geben, wohnt irgendwie jeder Arbeit inne [...]. Weil die Arbeit selbst Leben ist, darum will man auch alle Kräfte des Lebens an sie heranbringen und in ihr auswirken können." Aus dieser Sichtweise betrachtet bedeutet bessere Arbeit also gerade nicht die Verkürzung oder gar Abschaffung derselben, sondern eher eine Ausweitung - mit dem Ziel einer Steigerung und Intensivierung des Erlebens von Sinn in der Arbeit.

Die zwei Gesichter der Arbeit, die Mühe und Last auf der einen Seite und das Sinnerleben und die persönliche Entfaltung auf der anderen Seite, hat wohl jeder arbeitende Mensch bereits kennengelernt. Sie sind auch bald 100 Jahre nach Veröffentlichung des Textes aktuell - und werden uns wohl auch in Zukunft noch begegnen. In neuerer Zeit wurden diese beiden Seiten der Arbeit bspw. auch unter den Begriffen Entfremdung (z.B. Jaeggi, 2005) und Resonanzerfahrung (Rosa, 2012) sozialphilosophisch diskutiert.

Es erscheint daher unwahrscheinlich anzunehmen, dass die derzeitigen Veränderungen der Arbeit, insbesondere die Flexibilisierung, eines der beiden Gesichter vollständig abschaffen werden: Arbeit wird auch in Zukunft sowohl mühsam als auch sinnstiftend sein - die Frage ist nur, in welchem Ausmass. Interessant ist es daher, darauf zu schauen, unter welcher Perspektive zukünftige Arbeitsformen diskutiert werden, mit welchem Gesicht sie sich uns also zeigen. 


\subsection{Charakteristika moderner Arbeit}

Die Arbeitswelt hat sich in den letzten zwei bis drei Jahrzehnten in verschiedenen Dimensionen stark gewandelt. Unternehmen, aber auch Individuen, bewegen sich zunehmend auf globalen Märkten bzw. in globalen Kontexten. So wie der Handel in Laufe des letzten Jahrhunderts mehr und mehr den Globus umspannt, agieren auch Individuen zunehmend auf einer globalen Ebene, sei es durch zunehmende Fernreisen oder auch durch globale Initiativen, NGOs oder soziale Netzwerke. Die Veränderungen im Bereich der Informations- und Kommunikationstechnologien in den letzten Jahrzehnten sind womöglich am stärksten spürbar. Die alltägliche Nutzung von Computern, Smartphones und weiteren Geräten ist dabei nur die „Spitze des Eisbergs“. Mehr und mehr Arbeitsprozesse werden heutzutage selbst in kleinen Unternehmen von der Digitalisierung beeinflusst - vom simplen E-Mail- und Dokumentenablage-Server über Systeme für die Verwaltung von Geschäftsprozessen und Personal (wie z.B. SAP), bis hin zum Internetauftritt mit Webshop. Ein Ende der Ausbreitung in so gut wie alle Wirtschafts- und Arbeitsprozesse ist nicht absehbar. Für Arbeitende bedeutet die Einführung solcher Systeme oft grosse Herausforderungen, was die Anpassung angeht. Aber auch Punkte wie Kontrollierbarkeit der eigenen Arbeitsprozesse, Informationsflut und Überwachung spielen eine Rolle. Deutlich spürbar ist auch eine Zunahme der Wissensarbeit: Der Industriesektor, der in den 1960er-Jahren noch fast die Hälfte der Erwerbstätigen in der Schweiz beschäftigt hatte, verliert zunehmend an Bedeutung. Heute arbeitet die grosse Mehrheit der Erwerbstätigen im Dienstleistungssektor. Dieser Trend zeigt sich in allen fortgeschrittenen Industrieländern. Spürbar sind auch einige Schattenseiten des derzeitigen Wandels: Stresserleben, Burnout und andere psychische Beeinträchtigungen haben zugenommen und oftmals wird die Ursache der Arbeitswelt und den dortigen Veränderungen zugeschrieben. Gleichzeitig ist aber auch eine Gegenbewegung spürbar: Für viele Arbeitende ist die Vereinbarkeit von Beruf mit anderen Lebensbereichen (insbesondere der Familie) wichtiger als früher. Der "Generation Y“ wird nachgesagt, sie sei nicht mehr nur mit Geld zu locken, sondern lege bewusst Wert auf sinnhafte Arbeit und ein ausgeglichenes Leben. So wandeln sich moderne Arbeitnehmende in wissensintensiven Jobs auch vermehrt in Portfolioarbeiter (Clinton, Totterdell \& Wood, 2006; Platman, 2004) und schlüpfen in die Rollen hybrider Professionals (Meissner, 2016; 2009). 


\subsection{Flexibilisierung der Arbeit: Formen und ihre Verbreitung}

Ein weiterer, bereits seit Längerem andauernder Veränderungsprozess ist die Flexibilisierung von Arbeit. Hierunter ist ganz allgemein eine Auflösung bestehender "traditioneller" Grenzen und Rahmenbedingungen von Arbeit zu verstehen. Mit "traditionell“ ist hauptsächlich die Art und Weise gemeint, wie Arbeit in der späten Industrialisierung organisiert wurde: Als Ausgangspunkt der Arbeitsflexibilisierung - also als der Zustand, welcher flexibilisiert und damit verändert wird - kann das „Normalarbeitsverhältnis“ mit einer unbefristeten Vollzeitanstellung und festen Arbeitszeiten und -orten sowie sozialer Absicherung (Kranken-, Arbeitslosigkeits- und Rentenversicherung) angesehen werden. Dies ist allerdings eine künstliche Setzung, denn gewisse Formen der Arbeitsflexibilität waren schon früher vorhanden - bspw. haben Lehrerinnen und Lehrer schon immer "Homeoffice“ gemacht. Einen Zeitpunkt, zu welchem alle Arbeitskräfte eines Landes im Normalarbeitsverhältnis beschäftigt waren, hat es nie gegeben. Da jedoch eine Zunahme von Nicht-Normalarbeitsverhältnissen - sogenannten atypischen Beschäftigungsverhältnissen - in den letzten zwei Jahrzehnten beobachtbar ist, ist es dennoch sinnvoll, das Normalarbeitsverhältnis als Referenzpunkt anzunehmen. Wichtig ist die Abgrenzung zum Begriff „prekär“: Atypische Beschäftigungsverhältnisse sind nicht automatisch prekär (Brehmer \& Seifert, 2008). So stellt z.B. eine gut bezahlte 80-Prozent-Teilzeitanstellung als Projektleiterin in einer Beratungsfirma mit einem Tag Homeoffice in der Woche bereits ein sehr atypisches, flexibles Verhältnis dar, ist aber nicht als prekär anzusehen. Nach einer Definition der Internationalen Arbeitsorganisation der Vereinten Nationen (ILO) liegt eine prekäre Beschäftigung dann vor, wenn der Erwerbsstatus eine nur geringe Sicherheit des Arbeitsplatzes sowie wenig Einfluss auf die konkrete Ausgestaltung der Arbeitssituation gewährt, der arbeitsrechtliche Schutz lediglich partiell gegeben ist und die Chancen auf eine materielle Existenzsicherung durch die betreffende Arbeit eher schlecht sind (Rodgers, 1989).

Je nach Autorenschaft können ganz unterschiedliche Dimensionen der Flexibilisierung ein- oder ausgeschlossen werden und je nach Perspektive (z.B. Arbeitnehmende, Unternehmen oder der ganze Arbeitsmarkt) nimmt die jeweils diskutierte Arbeitsflexibilisierung sehr unterschiedliche Züge an. Um die folgenden Zahlen zur Verbreitung der verschiedenen Formen von Arbeitsflexibilität einordnen zu können, werden ein paar Basisfakten zur Erwerbstätigkeit in der Schweiz benötigt: Ende 2014 waren laut Bundesamt für Statistik (BFS) (2015a; 2015b) in der Schweiz 5 Millionen Menschen erwerbstätig. Das sind somit 
knapp 70 Prozent der ständigen Wohnbevölkerung. Zudem waren knapp 200'000 Personen erwerbslos (ILO-Definition); die Erwerbslosenquote betrug 4,1 Prozent. Von den 5 Millionen Erwerbstätigen sind etwa 650'000 (ca. 13 Prozent) selbstständig tätig. Dieser Anteil hat sich während der letzten 20 Jahre nur geringfügig geändert (Schwankungen von ca. 2 Prozentpunkten).

\subsubsection{Arten von Flexibilisierung der Arbeit}

Häufig werden die Begriffe interne und externe Flexibilität benutzt, um grundsätzliche Formen von Arbeitsflexibilität zu unterscheiden. Mit interner Flexibilität sind dabei alle Massnahmen gemeint, die die Arbeit innerhalb des Unternehmens flexibler machen (z.B. Teilzeit oder flexible Arbeitszeiten), während die Zahl der Angestellten nicht verändert wird. Externe Flexibilität meint dagegen eine variable Anpassung der Beschäftigtenzahl durch z.B. Outsourcing. Die Begriffe interne und externe Flexibilität werden oft in Bezug auf die Flexibilität des gesamten Arbeitsmarkts benutzt.

Reilly (1998) hat eine etwas differenziertere Kategorisierung vorgelegt, welche primär aus Arbeitgebersicht formuliert ist. Er unterscheidet dabei fünf verschiedene Arten:

- $\quad$ finanzielle Flexibilität

- funktionale Flexibilität

- $\quad$ numerische Flexibilität

- $\quad$ zeitliche Flexibilität

- $\quad$ örtliche Flexibilität

Finanzielle Flexibilität bedeutet für ein Unternehmen, dass das Einkommen variabel gehalten und an die Ertragssituation einer Organisation angelehnt werden kann. Dies ist sowohl über Umsatzbeteiligungen als auch über andere variable Lohnbestandteile möglich.

Die funktionale Flexibilisierung ermöglicht, die gleichen Arbeitskräfte an verschiedenen Arbeitsplätzen für verschiedene Aufgaben einzusetzen. Dadurch kann die Organisation ihre Reservekapazitäten verkleinern und Personalkosten senken. Beide Formen der Flexibilität werden in dieser Studie nicht weiter berücksichtigt. 


\subsubsection{Numerische Flexibilität}

Numerische Flexibilität „allows the numbers of staff used to vary according to the needs of the business" (Reilly, 1998, S. 9). Sie bezieht sich also auf die Veränderbarkeit der Anzahl der Beschäftigten. Ein Unternehmen kann numerische Flexibilität erzielen durch befristete Verträge, Honorarverträge, Arbeit auf Abruf oder andere flexible Formen der Anstellung. Auch ein niedriger Kündigungsschutz und eine „Hire-and-fire-Personalpolitik“ bedeutet für Unternehmen, dass sie numerisch flexibler sein können. Für Angestellte bedeutet numerische Flexibilität vor allem weniger Absicherung - andererseits kann eine befristete oder auf Honorar basierende Anstellung aber auch aufgrund der damit einhergehenden Freiheit gewünscht sein.

Die numerische Flexibilität auf dem Schweizer Arbeitsmarkt kann - relativ gesehen - als grundsätzlich erhöht eingeschätzt werden, und zwar aufgrund des vergleichsweise niedrigen gesetzlichen Kündigungsschutzes in der Schweiz. In konkreten Zahlen ausgedrückt zeigt sich allerdings, dass diese Form der Arbeitsflexibilität nicht so stark verbreitet ist wie andere Formen der Arbeitsflexibilität: Etwa 7 Prozent aller Arbeitsverträge in der Schweiz sind befristet; dieser Wert ist in den letzten 20 Jahren mit Schwankungen um etwa 2 Prozent relativ stabil geblieben. Von "Arbeit auf Abruf" (eine Mischform aus numerischer und zeitlicher Arbeitsflexibilität) waren in der Schweiz etwa 5 Prozent der Erwerbstätigen betroffen (bei nur geringen Schwankungen in den letzten 15 Jahren; BFS 2015j). In Grossbritannien ist diese Form unter den Namen „Zero-hours Contracts" bekannt (weil Arbeitsverträge zwar bestehen, aber keine garantierten Arbeitsstunden vereinbart sind). Insbesondere in Deutschland wird auch der Begriff KAPOVAZ gebraucht, welcher für „kapazitätsorientierte variable Arbeitszeit" steht (z.B. Pulte, 1987).

Eine neuere Form numerischer Flexibilität ergibt sich für Unternehmen in Form des "Crowdsourcing for paid Work“. Hierbei handelt es sich um das Ausschreiben von Arbeiten (z.B. das Redigieren von Texten, das Entwerfen eines Logos, aber auch komplexere Aufgaben) auf Crowdsourcing-Plattformen. Das Unternehmen „spart" sich somit die Anstellung der entsprechenden Person in einem festen Arbeitsverhältnis und kann je nach Bedarf viele oder wenige solcher Aufträge vergeben. Auf "Crowdsourcing for paid Work" wird in Abschnitt 2.2.7 noch vertieft eingegangen. 


\subsubsection{Zeitliche Flexibilität}

Zeitliche Arbeitsflexibilität kann viele Formen annehmen. An dieser Stelle werden zum einen die Teilzeitarbeit und zum anderen flexible Arbeitszeiten beschrieben. Teilzeitarbeit bedeutet die gleichzeitige anteilige Reduktion der Arbeitszeit sowie des Gehalts und ist in der Schweiz sehr weit verbreitet: 2014 arbeiteten 36 Prozent der Erwerbstätigen in Teilzeit, was im europäischen Vergleich der zweithöchste Wert ist. Der Anteil der Teilzeiterwerbstätigen hat in den letzten zehn Jahren deutlich (Steigerung um etwa 25 Prozent) zugenommen (BFS, 2014). Die grosse Mehrheit der Teilzeiterwerbstätigen sind Frauen: 59 Prozent aller erwerbstätigen Frauen, aber nur 16 Prozent aller erwerbstätigen Männer arbeiten Teilzeit. Teilzeitarbeit wird überwiegend aus familiären Gründen genutzt. Wie im vorherigen Abschnitt bereits erwähnt sind etwa 7 Prozent der Erwerbstätigen unterbeschäftigt, arbeiten also unfreiwillig in Teilzeit (BFS, 2015b). Verschiedene Studien zeigen, dass Teilzeitbeschäftigte allgemein eine höhere Zufriedenheit mit ihrer Life-Domain-Balance und weniger Stresserleben haben, allerdings ihre Arbeitsmarktfähigkeit und Karrierechancen geringer einschätzen als Vollzeitarbeitende (Grote \& Staffelbach, 2010; Johnson, Shannon \& Richman, 2008).

Neben der Teilzeitarbeit gehört auch die flexible Gestaltung der Arbeitszeit zur zeitlichen Arbeitsflexibilität. Dies kann z.B. als "Gleitzeit" organisiert sein, d.h., die Mitarbeitenden können Beginn und Ende ihrer Arbeit jeden Tag rund um eine fest vorgegebene Kernarbeitszeit frei wählen (auch Flextime oder Flexitime genannt). Je nach Modell muss dann die Sollarbeitszeit entweder an jedem einzelnen Tag oder aber pro Woche, pro Monat oder im gesamten Jahr erreicht werden (Wochen-, Monats- oder Jahresarbeitszeit). Unter dem Begriff „Vertrauensarbeitszeit" wird eine noch weitergehende Flexibilität verstanden, also die theoretisch völlig freie Wahl der Arbeitszeit ohne Erfassung der tatsächlichen gearbeiteten Stunden. Zur Verbreitung flexibler Arbeitszeit in der Schweiz lassen sich, je nach Methode der Erfassung, unterschiedliche Zahlen finden. Das BFS (2015b) gibt den Anteil der Arbeitnehmenden mit 45 Prozent an; der HRBarometer (Grote \& Staffelbach, 2010) ermittelte, dass 52 Prozent der Arbeitnehmenden Gleitzeit nutzen können; und Dorsemagen, Krause, Lehmann \& Pekruhl (2012) fanden gar einen Anteil von 61 Prozent der Angestellten, die flexible Arbeitszeiten haben. Sie fanden ferner, dass 17 Prozent der Angestellten zeitlich flexibel arbeiten, ohne dass ihre Arbeitszeit erfasst wird („Vertrauensarbeitszeit“) und dass die Nichterfassung der Arbeitszeit mit einer markant höheren geleisteten Arbeitszeit einhergeht als vertraglich vereinbart. 


\subsection{4 Örtliche Flexibilität}

Örtliche Arbeitsflexibilität beschreibt Reilly (1998, S. 12) als "the various ways of using employees outside the normal workplace, from mobile through partly home based, to full outworkers or teleworkers". Der Begriff und die Idee von Telearbeit wurde zwar bereits in den 1970er-Jahren eingeführt (Nilles, 1976). In den 1980er- und 1990er-Jahren wurden vermehrt Experimente und Pilotstudien zu Telearbeit und anderen Formen der Arbeitsflexibilität durchgeführt. Aber erst mit der massenhaften Verbreitung von mobilen Kommunikations- und Arbeitsgeräten wie Laptops und Smartphones sowie schnellen Internetverbindungen konnte sich die „Telearbeit" von einer Nischenexistenz hin zum „mobilen Arbeiten" als einem weitverbreiteten Phänomen entwickeln.

„Homeoffice“, auch genannt „alternierende Telearbeit“, kann als eine der am meisten verbreiteten Varianten örtlicher Arbeitsflexibilität gesehen werden. Dabei können Mitarbeitende einen Teil ihrer Arbeit von zu Hause aus leisten, wobei innen aber grundsätzlich ein Arbeitsplatz im Unternehmen zur Verfügung steht. Abzugrenzen ist daher die „Teleheimarbeit“, bei der die Mitarbeitenden vollständig zu Hause arbeiten und somit keine Flexibilität mehr haben. Der Anteil der Arbeitnehmenden, die derzeit manchmal oder überwiegend von zu Hause aus arbeiten, lag 2012 in der EU im Durchschnitt bei etwa 10 Prozent (Brenke, 2014). In der Schweiz waren es etwa ein Viertel (Grote \& Staffelbach, 2010; Weichbrodt, 2014). Diese Zahlen stammen aus Befragungen von Erwerbstätigen. Perch-Nielsen et al. (2014) fanden allerdings in einer Befragung von Arbeitgeberinnen und Arbeitgeber sehr viel niedrigere Werte (4 Prozent der Beschäftigten). Der „wahre“ Wert wird also wahrscheinlich dazwischen liegen - die Diskrepanz kann aber auch darin begründet sein, dass Unternehmen womöglich nicht sehr gut darüber Bescheid wissen, wer von ihren Mitarbeitenden eigentlich wie flexibel arbeitet. Aktuelle Metaanalysen (Gajendran \& Harrison, 2007; Allen, Golden \& Shockley, 2015) zu den Effekten von Homeoffice zeigen, dass diese Art von örtlicher Arbeitsflexibilität grösstenteils positive Effekte für Mitarbeitende hat - meist in Form von höherer Arbeitszufriedenheit und besserer Life-Domain-Balance. Negative Effekte zeigen sich im Erlebnis von sozialer Isolation sowie in schlechteren Karrierechancen aufgrund der verringerten „Sichtbarkeit“. Gisin et al. (2013) kamen daher auch zum Schluss, dass ein bis maximal zwei Homeoffice-Tage pro Woche als gute Balance angesehen werden können.

Über das Homeoffice hinaus lassen sich noch viele weitere Varianten der örtlichen Arbeitsflexibilität unter dem Stichwort „mobiles Arbeiten“ zusammenfassen. Ein Phänomen ist die zusätzliche Arbeit zu Hause am Abend oder am Wo- 
chenende, die durch mobile Kommunikationstechnologien ermöglicht und auch unter dem Stichwort „arbeitsbezogene erweiterte Erreichbarkeit“ diskutiert wird. Hierfür werden vermehrt negative Effekte berichtet (Pangert \& Schüpbach, 2015). Arbeit im Zug, im Café, bei Kundinnen und Kunden oder an sonstigen weiteren Orten lässt sich ebenso unter dem Begriff „mobile Arbeit“ zusammenfassen. Weichbrodt (2014) fand, dass "nur" 77 Prozent aller Arbeitsstunden der Schweizer Erwerbstätigen am üblichen Unternehmensstandort geleistet werden. Weitere Orte sind: zu Hause (8 Prozent), bei der Kundin bzw. beim Kunden (7 Prozent), unterwegs (3 Prozent) oder auch ein weiterer Unternehmensstandort (2 Prozent). Als ein Nischenphänomen der mobilen Arbeit lassen sich die "Coworking-Spaces“ identifizieren: Über das relativ fixe Modell der Bürogemeinschaft (mehrere, meist selbstständig Arbeitende teilen sich eine Bürolokalität) bieten sie auch spontane und sporadische Arbeitsplätze, die entweder auf Stundenbasis oder in einem „Abo“ verrechnet werden. Zudem steht hier der Austausch untereinander im Vordergrund.

Ein weiteres Phänomen, welches der örtlichen Arbeitsflexibilität zugerechnet werden kann, ist das Prinzip des „Desk Sharing“. Dabei wird in dem Sinne „mobil" gearbeitet, dass Mitarbeitende keinen festen, fixen Arbeitsplatz haben, sondern je nach Tätigkeit und Situation sich den am besten geeigneten Ort suchen. Oftmals wird auch die Möglichkeit, zu Hause zu arbeiten, angeboten und die Büroräume im Unternehmen werden bewusst zur Förderung von Austausch und Begegnungen gestaltet. Bekannte aktuelle Unternehmensbeispiele sind die beiden neuen Hauptstandorte der Schweizerischen Post sowie der SBB in Bern-Wankdorf. Weichbrodt et al. (2015) fanden in solchen hochflexiblen Unternehmen das Phänomen, dass das mentale Modell von Arbeitsmobilität gleichsam kippt: Aus der Vorstellung, dass man normalerweise im Büro anzutreffen ist und Homeoffice die Ausnahme darstellt, wird die Annahme, dass grundsätzlich alle mobil arbeiten und man die gemeinsame Präsenz (für formellen sowie informellen Austausch) bewusst organisieren muss. Je mobiler gearbeitet wird, desto mehr spricht für die einzelnen Mitarbeitenden dafür, auch mobil zu arbeiten - Rockman und Pratt (2015) sprechen in diesem Sinne auch vom „ansteckenden“ mobilen Arbeiten.

\subsubsection{Subjektivierung von Arbeit und das Phänomen „Arbeitskraftunter- nehmende"}

Die Dimensionen der Arbeitsflexibilität von Reilly helfen, eine Ordnung zu finden in der Vielfältigkeit der Flexibilisierung. Sie stellen relativ gut messbare Dimensionen dar. Daneben existieren aber noch andere Phänomene, die mit der Fle- 
xibilisierung von Arbeit zu tun haben, allerdings nicht so gut erfassbar sind. Eins dieser Phänomene wird in der soziologischen Literatur als Subjektivierung der Arbeit beschrieben (u.a. Voß \& Pongratz, 1998; Böhle, 2010; Minssen, 2012). Diese neuen Organisationsprinzipien umfassen die Verflachung von Hierarchien, unternehmerisches Denken, die Steuerung über Kontext (z.B. Vorgabe des Budgets), einen erweiterten Dispositions- und Handlungsspielraum bei gleichzeitiger Festlegung der Bedingungen, unter denen die Verantwortung wahrzunehmen ist, sowie die Projektorganisation zur Flexibilisierung von Unternehmen. Arbeitskräfte übernehmen also zunehmend mehr unternehmerische Verantwortung und tragen vermehrt unternehmerisches Risiko, was mit dem Begriffskonstrukt „Arbeitskraftunternehmer“ bzw. „Arbeitskraftunternehmerin“ ausgedrückt werden kann. An „Arbeitskraftunternehmer/innen“ werden fundamental neue Anforderungen gestellt: U.a. müssen sie über mehr Selbstdisziplin und Eigenverantwortung verfügen. Die zentrale Veränderung besteht laut Böhle (2010) darin, dass die Verantwortung für die Begrenzung der Arbeitsleistung sowie die Erhaltung der Arbeitsfähigkeit, welche zuvor zwischen Unternehmen und Beschäftigten geteilt wurde, vermehrt das Individuum allein tragen muss. Hornberger (2006) bemerkt daher auch, dass die entscheidende Frage in solchen Arbeitskontexten darin besteht, zu entscheiden, wie viel Verantwortung auf die Beschäftigten übertragen werden soll und welche Verpflichtungen (z.B. was Einhaltung von Arbeitszeiten, Gesundheitsschutz, Vermeidung von Überarbeitung etc. angeht) zwingend beim Arbeitgeber bleiben müssen.

Auch der Philosoph Byung-Chul Han (2010) beschreibt diese Veränderung in seinem bekannten Essay „Müdigkeitsgesellschaft“, in welchem er die Arbeitsparadigmen „Disziplin“ und „Leistung“ gegenüberstellt: In der „,alten Arbeitswelt“ war Disziplin das entscheidende Bewertungskriterium und diese konnte gezeigt werden, indem man pünktlich und arbeitsam war. Der Erfolg der Arbeit war zwar ein wichtiges, aber kein notwendiges Zielkriterium - solange man die vereinbarte Arbeitszeit mit disziplinierter Arbeit füllte, war man eine „gute Arbeitskraft“. In der "neuen Arbeitswelt“ genügt dies hingegen nicht mehr, sondern es ist Leistung in Form von Ergebnissen, Output gefragt. Dieses Zielkriterium ist aber laut Han letztlich niemals vollständig erfüllbar - man kann ja immer noch mehr Leistung bringen - und führt daher zu vermehrtem Stresserleben und Burnout in der Gesellschaft.

Peters (2011) hat diese Praxis des ziel- und ergebnisorientierten Führens als indirekte Steuerung bezeichnet. Für Geführte bedeutet dies, dass sie das Wann, Wo und Wie ihrer Arbeit vermehrt selber bestimmen können, sowie auch das „Wie viel“ - solange die Ziele erreicht werden. Es leuchtet ein, dass dies zu einer problematischen Situation führen kann, wenn seitens der Arbeitgebenden 
bzw. der Führungskräfte nur noch an diesen „Zielschrauben“ gedreht wird und die tatsächliche Auslastung der Mitarbeitenden ignoriert wird. Problematisch kann aber auch das Verhalten der Mitarbeitenden selbst sein, wenn sie eigenmotiviert ihre eigenen Grenzen überschreiten und damit „interessierte Selbstgefährdung" zeigen (Krause, Dorsemagen \& Peters, 2013; Krause et al., 2014). Das Phänomen „Arbeitskraftunternehmer/in“ ist somit ein Produkt von Arbeitgeber- sowie von Arbeitnehmerverhalten und -interessen und sollte nicht einseitig bei der einen oder anderen Partei verortet werden.

Subjektivierung von Arbeit lässt sich also als eine Verschiebung der ökonomischen Rolle des Individuums beschreiben: Von der passiven Arbeitskraft, die in fest ausgehandelten Grenzen ihre Ressourcen der Arbeitgebenden zur Verfügung stellt, hin zum „Arbeitskraftunternehmer“ (bzw. zur Arbeitskraftunternehmerin), der oder die proaktiv im Unternehmen tätig wird und damit Risiko, aber auch Verantwortung übernimmt und somit unternehmerähnlich agiert. Diese Verschiebung bringt auch die Frage nach der Bedeutung von Arbeit mit sich: Für eine reine Arbeitskraft kann Arbeit nur in sehr seltenen, glücklichen Fällen Selbstverwirklichung ermöglichen. Stattdessen geht es wohl den meisten „Arbeitskräften" darum, mit der Arbeit ein Auskommen zu erzielen, also die Arbeit als Mittel zu nutzen für den Zweck eines möglichst guten Lebens. Parallel dazu hat Arbeit aber schon immer auch eine Gemeinschaft stiftende Funktion. Es lässt sich schlussfolgern, dass tendenziell wohl das Gemeinschaftserleben in der Arbeit geringer werden wird, je mehr als „Arbeitskraftunternehmende“ gearbeitet wird.

\subsubsection{Fokus Homeoffice: Beschreibung und Übersicht der Vor- und Nachteile}

Zur punktuellen Vertiefung wurden für diesen Bericht zwei konkrete Formen flexibler Arbeit untersucht, und zwar zum einen das anteilhafte Arbeiten zu Hause (Homeoffice) im Rahmen eines Normalarbeitsverhältnisses und zum anderen das "Crowdsourcing for paid Work". Diese werden im Folgenden in den Fokus gestellt.

Der Begriff "Homeoffice“ meint das anteilhafte Arbeiten im Privaten zu Hause und unterscheidet sich demnach von der "Teleheimarbeit“, welche das ausschliessliche Arbeiten von zu Hause aus beschreibt.

Die Möglichkeit, einen Teil der vertraglich vereinbarten Arbeitsleistung in den heimischen vier Wänden zu erbringen, bringt eine Reihe an Vor- und Nachteilen für die Nutzenden mit sich, welche sich grösstenteils in die Kategorien Leis- 
tungserbringung (arbeitsspezifische Vor- und Nachteile) und individuelle Lebensgestaltung (Life-Domain-Balance betreffende Vor- und Nachteile) einteilen lassen (vgl. Tabelle 1). Beim genaueren Betrachten dieser Vorzüge und Fallstricke der Homeoffice-Arbeit fällt auf, dass die Art und Weise, wie die jeweilige Homeoffice-Situation gelöst wird, ausschlaggebend dafür ist, ob sich diese vorteilig oder nachteilig auf Arbeitsqualität und Lebensgestaltung auswirkt.

Wird die Homeoffice-Situation adäquat gelöst, kann die Produktivität durch bessere Konzentration bei ungestörtem Arbeiten gesteigert werden (Gisin, Schulze, Knöpfli \& Degenhardt, 2013; Gajendran \& Harrison, 2007; Harpaz, 2002). Dies bedingt, dass eine entsprechende Arbeitsatmosphäre im Homeoffice hergestellt wird und die dazu passenden Arbeiten für die Arbeit im Homeoffice ausgewählt werden. Weiter berichten unzählige Studien über die positive Auswirkung der zeitlichen und örtlichen Flexibilisierung der Arbeit (Greer \& Payne, 2014; Gisin et al., 2013; Gajendran \& Harrison, 2007). Dadurch, dass die Arbeit anteilig flexibel gestaltet werden kann, entsteht bei vielen Betroffenen die Möglichkeit, mindestens einen Arbeitstag regelmässig im Homeoffice zu erbringen. Diese Flexibilität unterstützt die Entwicklung und Wahrnehmung von Selbstständigkeit wie auch Autonomie und steigert durch flexible Zeiteinteilung an HomeofficeTagen das Gefühl, den beruflichen Anforderungen besser gewachsen zu sein. Zusätzlich werden im Homeoffice der Entbindung von unternehmensspezifischen Formalitäten, wie es bspw. der Dresscode darstellt, und der verbesserten Privatsphäre stressmindernde Eigenschaften zugesprochen, was wiederum die Produktivität positiv beeinflusst. Diese arbeitsspezifischen Vorzüge der Arbeit im Homeoffice führen sodann zu höherer Arbeitszufriedenheit und Eigenmotivation (Gisin et al., 2013; Hill, Ferris \& Märtinson, 2003).

Die Möglichkeit, im Homeoffice zu arbeiten, welche durch mobil-flexible Arbeitsbedingungen entsteht, hat sich im Weiteren als gesundheits- und erholungsförderlich erwiesen (Gisin et al., 2013; Moore, 2006; Hill et al., 2003). Es wird von besserer emotionaler Abgrenzung und verbesserter Erholungsfähigkeit durch mehr Schlaf, Abwechslung und Sport berichtet. Diese Vorzüge entstehen nicht zuletzt durch die hinzugewonnene Zeitautonomie. Die Reisezeit zum Main Office entfällt bspw. und kann somit eingespart werden. Es kann vermutet werden, dass durch das Wegfallen der Pendelreise, welche oft mit Stress verbunden ist, überdies Energie eingespart wird, welche wiederum in die Arbeit reinvestiert werden kann. Die Teilnehmenden an den Homeoffice-Day-Befragungen gaben bezüglich der Vorzüge der Arbeit im Homeoffice am häufigsten an, dass die Arbeit und das Privatleben besser vereinbar seien. Dank dem Homeoffice kann mehr unter einen Hut gebracht werden (bspw. Arzttermine, Handwerkerin oder Handwerker). Es kann so aber auch eine bessere Betreuung von schul- 
pflichtigen Kindern und Haustieren gewährleistet werden und darüber hinaus ist das Arbeitsmodell besser vereinbar mit Weiterbildung und Hobbys. Während das Homeoffice durch örtliche Nähe eine bessere Vernetzung nach aussen ermöglicht - sei dies geschäftlich mit Kundinnen und Kunden oder privat mit Familie, Freundinnen und Freunden sowie Bekannten -, kann die Vernetzung nach innen in die Unternehmung unter zu häufiger oder falscher HomeofficeNutzung leiden (Weichbrodt et al., 2013).

Die Schattenseiten der Arbeit im Homeoffice kommen zum Vorschein, wenn diese ungünstig gelöst wird, sei dies durch zu häufiges, unangebrachtes Zurückziehen ins Homeoffice oder durch unüberlegte Organisation der Homeoffice-Tage. Die arbeitsspezifischen Nachteile, welche dabei entstehen, können grob in die Bereiche Connectedness, sozialer Druck und Zusatzaufwände unterteilt werden (Greer \& Payne, 2014; Hill et al., 2003; Harpaz, 2002). Von unzureichender Connectedness wird gesprochen, wenn durch die Arbeit im Homeoffice der informelle Austausch mit Arbeitskolleginnen und -kollegen fehlt, die Zusammenarbeit erschwert wird und/oder der Teamgeist als solches leidet. Es wird von regelmässigen Homeoffice-Nutzenden in den Homeoffice-DayBefragungen 2012 und 2013 (Gisin et al., 2013; Degenhardt, Gisin \& Schulze, 2014) diesbezüglich berichtet, dass die im Homeoffice arbeitende Person im Betrieb vor Ort vergessen geht und nicht zuletzt dadurch auch nicht alle für sie relevanten Informationen erhält. Dies kann sich negativ auf die Karriereentwicklung auswirken (Leslie et al., 2012). Zusatzaufwände hingegen entstehen, wenn der Organisation des Homeoffice-Tages und dem Homeoffice selber zu wenig Beachtung geschenkt wird. Es wird hier von mangelhafter Infrastruktur, hohem Selbstorganisationsaufwand, hohem Koordinations- und Organisationsaufwand wie auch von monetären Kosten berichtet, welche selber getragen werden müssen (bspw. Miete, Strom oder Druckpapier). Schliesslich kann sozialer Druck aufkommen, wenn das Arbeiten im Homeoffice unzureichend in der Organisationskultur verankert ist und Unsicherheit darüber besteht, was Arbeit im Homeoffice bedeutet und beinhaltet. In solchen Fällen berichten HomeofficeNutzende bspw. von „Beweispflicht“, welche unnötig Druck schafft und den Eindruck vermittelt, dass im Homeoffice mehr und härter gearbeitet werden muss, um dieses zu rechtfertigen (Gisin et al., 2013).

Im Bereich der Lebensgestaltung führt eine nachteilig gelöste HomeofficeSituation zu Gesundheits- und Erholungsgefährdung, wenn eine saubere Abgrenzung zwischen Arbeit und Privat misslingt, eine klare Tendenz besteht, dass im Homeoffice wesentlich mehr gearbeitet wird als verlangt und/oder das Abschalten nach der Arbeit misslingt, weil kein eindeutiger Abschluss des Homeoffice-Tages vollzogen wird (Gisin et al., 2013). Dann wird von sozialen 
Einbussen berichtet, welche durch Isolation und Einsamkeit bei schlechter Vernetzung und übertriebener „Finkenkultur" (nicht mehr aus dem Haus gehen) im Homeoffice entstehen können (Gisin et al., 2013; Cooper \& Kurland, 2002). Auch die Vereinbarkeit von Arbeit mit Privatem weist ihre Grenzen auf und kann schnell von einer vorteilhaften zu einer nachteiligen Komponente umschlagen, wenn versucht wird, Homeoffice und Kleinkinderziehung unter einen Hut bringen zu wollen und/oder Homeoffice-Nutzende sich von der heimischen Umgebung zu sehr ablenken lassen. Allenfalls entstehen überdies Zusatzaufwände für die im selben Haushalt lebenden Familienmitglieder durch die Anwesenheit der sonst ausser Haus arbeitenden Person. Zudem entsteht durch die Möglichkeit der Arbeit im Homeoffice für das Gesamtteam Suchaufwand, weil im ungünstigen Fall nicht immer klar ist, wer sich gerade wo befindet, und das damit zusammenhängende Wrong-Place-Problem, wenn der Aufenthaltsort nicht zur Arbeitsaufgabe passt (Weichbrodt et al., 2013).

Es können nur begrenzt allgemeine Empfehlungen für eine adäquate Lösung der Homeoffice-Situation abgegeben werden, da die konkrete Ausgestaltung der jeweiligen Homeoffice-Nutzung individuell und typenspezifisch angegangen werden muss. Die Boundary-Theorie (Nippert-Eng, 1996) liefert hierzu einen geeigneten Bezugsrahmen. In der Homeoffice-Arbeit können der BoundaryTheorie zufolge drei Boundary-Typen unterschieden werden (Gisin, 2014). Während die Segmentierenden ein grosses Bedürfnis zeigen, zwischen Arbeit und Privat abzugrenzen, und die Integrierenden ein ebenso grosses, entgegengesetztes Bedürfnis zeigen, Arbeit und Privat ineinander zu integrieren, fallen die Mischtypen dadurch auf, dass sie je nach Präferenz und wahrgenommener Wichtigkeit Inhalte aus Arbeits- und Privatleben wahlweise segmentieren (als unwichtig Wahrgenommenes abgrenzen) oder integrieren (als wichtig Wahrgenommenes einflechten). Hier gilt es, den eigenen persönlichen Boundary-Typ zu eruieren und dementsprechend passende Boundary-Taktiken (Kreiner, Hollenspe \& Sheep, 2009) anzuwenden, um die gewünschte Grenzkongruenz herzustellen, welche wiederum hilft, das Homeoffice vorteilhaft zu erleben (Gisin, 2014).

Die in der wissenschaftlichen Literatur diskutierten Vor- und Nachteile der Arbeit im Homeoffice werden nachstehend in Tabelle 1 zusammenfassend dargestellt. 
Tabelle 1: Vor- und Nachteile der Arbeit im Homeoffice

\begin{tabular}{|c|c|}
\hline \multicolumn{2}{|c|}{ Arbeitsspezifisch } \\
\hline+ & - \\
\hline 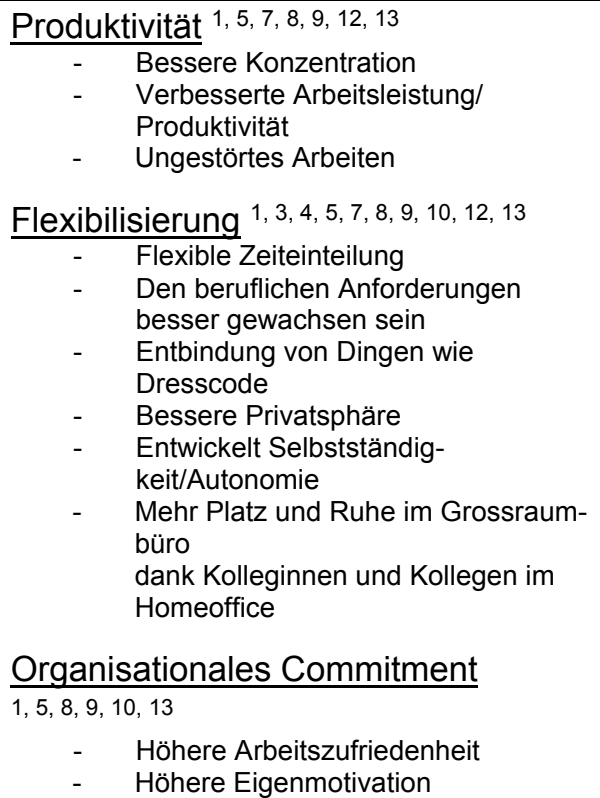 & 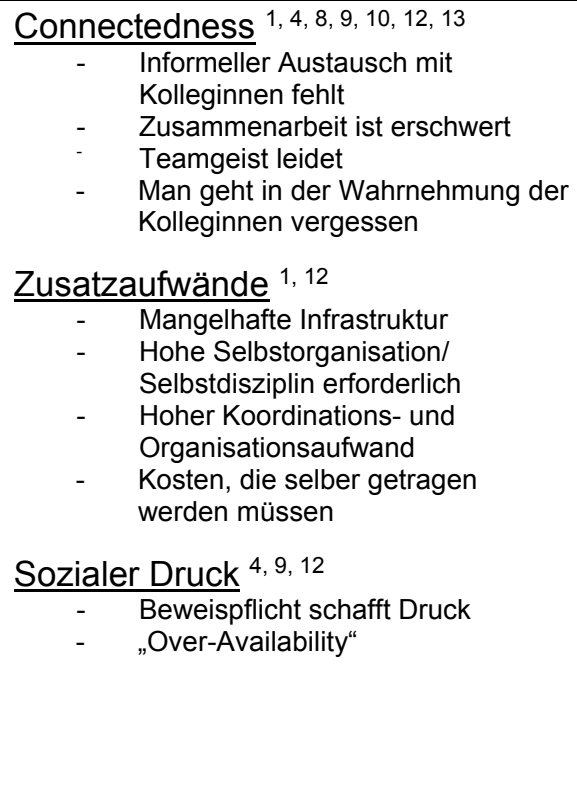 \\
\hline
\end{tabular}




\begin{tabular}{|c|c|}
\hline \multicolumn{2}{|c|}{ Life-Domain-Balance betreffend } \\
\hline+ & - \\
\hline 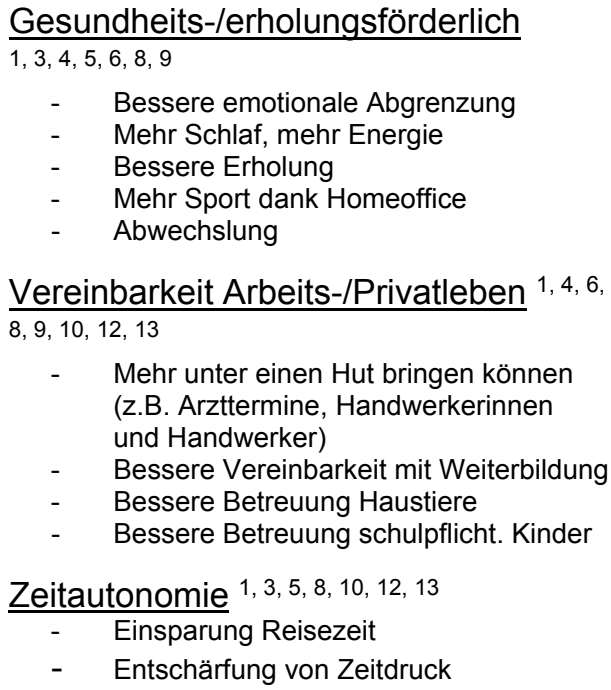 & 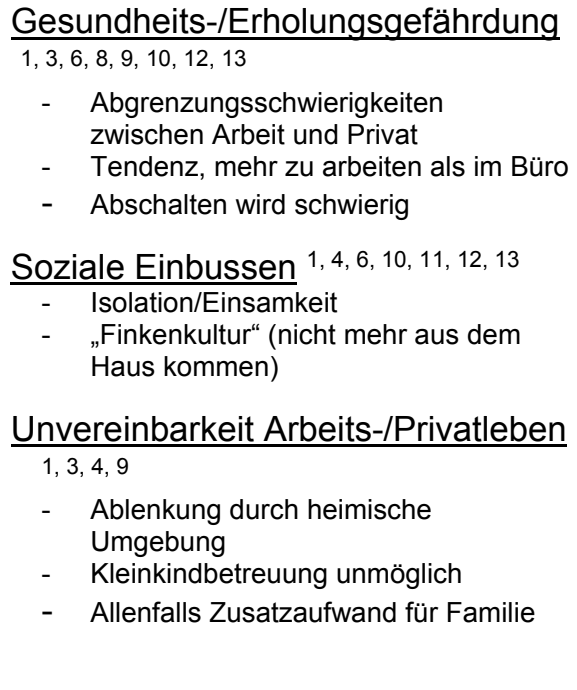 \\
\hline \multicolumn{2}{|c|}{ Boundary-Typen betreffend ${ }^{2}$} \\
\hline+ & - \\
\hline $\begin{array}{l}\text { Integrierer: Arbeit und Privat kann besser } \\
\text { verwoben werden, kommt dem ausgepräg- } \\
\text { ten Flexibilitätsbedürfnis entgegen, Zeit } \\
\text { kann optimal genutzt werden (durch Weg- } \\
\text { fall unnötiger Strukturen kann mehr in den } \\
\text { Tag gepackt werden) } \\
\text { Segmentierer: Einsparung Reisezeit } \\
\text { (ergo mehr Freizeit), Entschärfung Zeit- } \\
\text { druck }^{2}\end{array}$ & $\begin{array}{l}\text { Integrierer: Muss lernen, sich minimale } \\
\text { Struktur zu geben im Homeoffice } \\
\text { (Segmentation), Tendenz, sich bei un- } \\
\text { liebsamen Arbeiten zu verzetteln (andere } \\
\text { Arbeiten vorziehen) })^{2} \\
\text { Segmentierer: Muss lernen, minimal zu } \\
\text { integrieren, um Vorzüge des Homeoffice } \\
\text { optimal nutzen zu können, muss Rituale } \\
\text { entwickeln, um in Arbeitsenergie zu } \\
\text { kommen }{ }^{2}\end{array}$ \\
\hline \multicolumn{2}{|c|}{ Generelle Vor- und Nachteile der mobilen Arbeit allgemein } \\
\hline+ & - \\
\hline $\begin{array}{ll}- & \text { Bessere Vernetzung nach aussen } \\
- & \text { Besserer Beziehungs- und Vertrauens- } \\
\text { aufbau zu Kundinnen und Kunden durch } \\
\text { örtliche Nähe }\end{array}$ & $\begin{array}{ll}- & \text { Suchaufwand (wer ist wo unterwegs?) } \\
\text { - } & \text { Wrong-Place-Problem } \rightarrow \text { Aufenthaltsort } \\
& \text { passt nicht immer zu Arbeitsaufgabe }\end{array}$ \\
\hline
\end{tabular}

$1=$ Gisin et al., 2013; ${ }^{2}=$ Gisin, 2014; ${ }^{3}=$ Weichbrodt et al., 2013; ${ }^{4}=$ Degenhardt, Gisin \& Schulze, 2014; 5 = Gajendran \& Harrison, $2007 ;{ }^{6}=$ Moore, $2006 ;{ }^{7}=$ Bélanger, $1999 ;{ }^{8}=$ Hill, Weiner \& Colihan, 1998; ${ }^{9}=$ Hill, Ferris \& Märtinson, $2003 ;{ }^{10}=$ Schweitzer \& Duxbury, 2006; ${ }^{11}=$ Cooper \& Kurland, 2002; ${ }^{12}=$ Harpaz, 2002; ${ }^{13}=$ Greer \& Payne, 2014 


\subsubsection{Fokus „Crowdsourcing for paid Work“: Beschreibung und Über- sicht der Vor- und Nachteile}

Jeff Howe, Chefredaktor des renommierten Magazins Wired, prägte 2006 den Begriff Crowdsourcing als Kunstwort, indem sich die Begriffe Outsourcing und Crowdfounding verbinden: Unternehmen lagern Tätigkeiten aus an Leistungserbringende, die im weltweiten Netz rekrutiert werden. Howe definiert Crowdsourcing als "the act of taking a job traditionally performed by a designated agent (usually an employee) and outsourcing it to an undefined, generally large group of people in the form of an open call". Barnes et al. (2013), die eine umfassende Literaturstudie zum Thema vorgelegt haben, definieren Crowdsourcing etwas umfassender als:

"An internet-enabled exchange through which individuals (the workers, supply-side) can seek paid employment and organisations (the employers, demand-side) can reach a larger pool of workers to outsource tasks by utilizing online intermediaries or vendors (those providing an online platform in which tasks are advertised)." (S. 20)

Ganz ähnlich Kaganer et al. (2013), die in Anlehnung an die in der modernen IT unvermeidliche "Cloud“ hier von der „Human Cloud“ sprechen: „A thirdgeneration sourcing ecosystem (...) the human cloud is centred on an online middleman that engages a pool of virtual workers that can be tapped on demand to provide a wide range of services to any interested buyer" (S. 23); ein unerschöpfliches Reservoir an Arbeitskräften also, das je nach Belieben angezapft werden kann.

Es gibt keine Zahlen dazu, wie viele Menschen weltweit (oder in der Schweiz) auf diese Weise ihre Arbeitskraft verkaufen oder wie viele Unternehmen diese Quelle bisher bereits nutzen. Aber es gibt Anhaltspunkte dafür, dass es sich um eine Entwicklung handelt, die in absehbarer Zeit gravierende Auswirkungen auf die Arbeitsmärkte haben wird. So geht der HR-Chef von IBM davon aus, dass von den ca. 430'000 Beschäftigten seines Unternehmens im Jahr 2017 nur 100 '000 noch fest angestellt sein werden. Die zusätzlich nötigen Arbeitskräfte werden in der Form von "Liquid Talent" - auch noch ein schöner Begriff - aus der Human Cloud bezogen werden (Wirtschaftswoche 2012). Kaganer et al. (2013) finden einen explosionsartigen („skyrocketing“) Anstieg der Umsätze in diesem Geschäft und stellen fest, dass diese Entwicklung „[will] reshape established business processes, redraw organisational boundaries and, most importantly, profoundly change the global labour market" (S. 24).

Eines der ältesten und bekanntesten Beispiele für diese Form der Arbeitsvermittlung ist Amazon Mechanical Turk (vgl. Abbildung 2): 
Unternehmen bieten einfache und einfachste Tätigkeiten an, die sich (noch) nicht automatisieren lassen, die aber Menschen am Computer erledigen können. ${ }^{1}$ Bei diesen sogenannten HITs - Human Intelligence Tasks - handelt es sich bspw. um die Beurteilung von Fotos: Lächeln die Personen auf diesen Fotos, oder schauen sie eher grimmig drein? Ein weiteres Beispiel wäre die Erfassung von Kaufbelegen, die so unterschiedlich sind, dass sie durch OCRScanner nicht erfasst werden können. Weitere HITs aus dem aktuellen Angebot (Oktober 2015) wären z.B.:

- $\quad$ Describe the picture in a complete sentence using 10 words or more. Use descriptive adjectives.

- $\quad$ Find blog email addresses of American Universities.

- $\quad$ Enter information about a forum discussion thread in which a vehicle is being built, rebuilt, or restored.

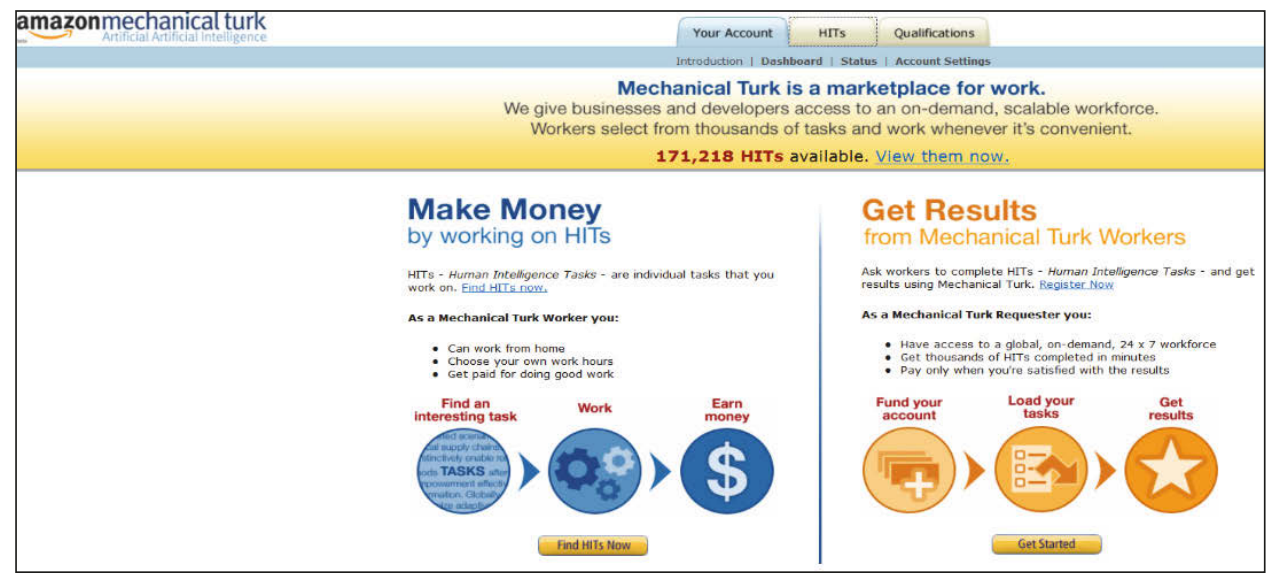

Abbildung 2: Amazon Mechanical Turk

Die Vergütung pro HIT ist niedrig, in den oben genannten Beispielen zwischen 0,01 und 0,25 US-Dollar. Dies ist jedenfalls das Angebot der Auftraggebenden; wenn sich für diesen Lohn kein „Turker” findet, der oder die den Auftrag übernehmen möchte, erhöht der Auftraggebende das Angebot sukzessive, bis der Job übernommen wird. Die Qualitätskontrolle ist einfach und wirkungsvoll: Die

${ }^{1}$ Der Begriff „Mechanical Turk“ erinnert an jenen Schachautomaten im 18. Jh., in dessen Inneren sich ein Mensch verbarg und mit „Human Intelligence“ die Züge des scheinbaren Automaten ausführte. 
Aufgaben werden von mehreren „Turkern“ parallel erledigt und nur wenn der oder die Einzelne zu 95 Prozent im Mainstream liegt, d.h. die gleiche Beurteilung, Lösung usw. eingibt wie die Mehrheit, wird das Entgelt ausbezahlt. Barnes et al. (2013) gehen davon aus, dass weltweit etwa 200'000 Menschen für den Mechanical Turk arbeiteten.

Auf der anderen Seite gibt es Portale, über die anspruchsvolle Aufgaben vermittelt werden. Bei 99Designs (vgl. Abbildung 3) suchen Auftraggeberinnen und Auftraggeber nach einem Logo für ihr Unternehmen, nach einem gut gestalteten Webauftritt o.Ä. und publizieren diesen Auftrag auf der Website von 99Designs. Dann beginnt ein Wettbewerb zwischen interessierten Designerinnen und Designern, die ihre Vorschläge einreichen. Der Auftraggebende sucht den aus seiner Sicht gelungensten Entwurf aus und muss nur diesen bezahlen.

\section{Professionelles Grafikdesign in wenigen Schritten}

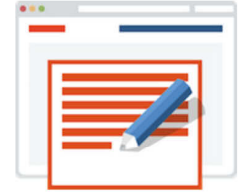

Beschreiben Sie, was

Sie brauchen

Füllen Sie das Design-Briefing aus und legen Sie den Preis fest, den Sie bezahlen wollen.

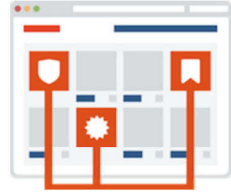

Design-Vorschläge erhalten und bewerten

Designer reichen Ideen in Ihren DesignWettbewerb ein und erhalten von Ihnen Feedback.

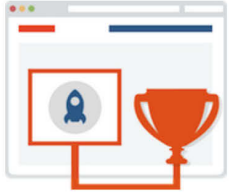

Wählen Sie Ihr Gewinner-Design

Innerhalb von 7 Tagen erstellen die Designer Ihr Wunschdesign.

\section{Abbildung 3: 99Designs}

Eine weitere prominente Crowdsourcing-Plattform ist upwork (vormals elance). Upwork (Abbildung 4) verspricht den Unternehmen eine "cloud of talents [in einem] pool of over 10 million skilled freelancers in $180+$ countries to build a private bench of pros hand-picked by you and your colleagues" (www.upwork.com). Ähnlich wie 99Designs wendet sich dieser Provider an typische Freelancer oder jene, die es werden wollen (oder müssen: siehe IBM). Damit ist auch schon angedeutet, dass die Chancen und Probleme, die sich für Beschäftigte im Crowdsourcing ergeben, viele Parallelen mit jenen aufweisen, die für selbstständig Beschäftigte seit jeher gelten. 


\section{The Earth-sized talent pool for enterprise}

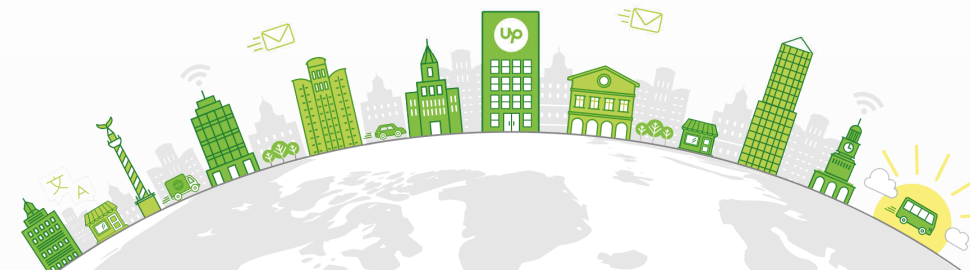

\section{Abbildung 4: Upwork}

Diese Beispiele machen deutlich, dass die Spannbreite der Angebote, die unter das Label "Crowdsourcing" fallen, gross ist. Genauso gross ist die Unterschiedlichkeit bei der Form der Vermittlung zwischen Beschäftigten und Unternehmen, die Art der Bezahlung und vor allem auch die Höhe des potenziellen Verdienstes. Während der Mechanical Turk in der Schweiz bestenfalls für Randgruppen des Arbeitsmarkts interessant sein dürfte, weist die Endung „..ch“ bei der Website von 99Designs schon darauf hin, dass sich hier auch Schweizer Kreative beteiligen.

Tabelle 2: Systematisierung verschiedener Angebote im Crowdsourcing

\begin{tabular}{l|l|l}
\hline Task Type & Characteristics & Examples \\
\hline Micro Tasks & $\begin{array}{l}\text { Generally very high volume, } \\
\text { extremely low pay rate per } \\
\text { task, and heavily automated } \\
\text { throughout the process }\end{array}$ & $\begin{array}{l}\text { - find email addresses or } \\
\text { company websites } \\
\text { - translate a product descrip- } \\
\text { tion to another language } \\
- \text { find prices for competitive } \\
\text { products } \\
\text { - choose a category from a } \\
\text { new catalog structure }\end{array}$ \\
\hline Macro Tasks & $\begin{array}{l}\text { Generally high volume, low } \\
\text { rate of pay, mostly automat- }\end{array}$ & $\begin{array}{l}\text { - write a product review } \\
\text { - test this website and pro- } \\
\text { vide feedback } \\
\text { - fill In the missing research } \\
\text { citations in this report } \\
\text { - build a list of universities } \\
\text { conducting energy research }\end{array}$ \\
& & \\
& &
\end{tabular}




\begin{tabular}{l|l|l}
\hline $\begin{array}{l}\text { Simple Pro- } \\
\text { jects }\end{array}$ & $\begin{array}{l}\text { Low volume or single tasks } \\
\text { with moderate rate of pay, } \\
\text { often requiring some direct } \\
\text { contact with the worker. }\end{array}$ & $\begin{array}{l}\text { - design a branded website } \\
\text { - prepare an outline for a } \\
\text { conference presentation } \\
\text { - contact all confirmed at- } \\
\text { tendees for an event }\end{array}$ \\
\hline $\begin{array}{l}\text { Complex } \\
\text { Projects }\end{array}$ & $\begin{array}{l}\text { Single project with high rate } \\
\text { of pay, typically requiring a } \\
\text { substantial amount of time } \\
\text { and direct interaction with } \\
\text { the worker. }\end{array}$ & $\begin{array}{l}\text { - program a software module } \\
\text { - design a new edible adhesive } \\
\text { - develop a new security al- } \\
\text { gorithm } \\
\text { - develop an ecommerce web- } \\
\text { site } \\
\text { - inbound/outbound calls } \\
\text { (sales, market research, sup- } \\
\text { port) }\end{array}$ \\
\hline
\end{tabular}

Eine Systematisierung verschiedener Angebote im Crowdsourcing nimmt Frei (2009) vor (vgl. Tabelle 2). Angesichts dieser Heterogenität von CrowdsourcingPlattformen und der dort vermittelten Jobs fällt die Bewertung der Auswirkungen dieser Entwicklungen auf die Beschäftigten (in der Schweiz) ambivalent aus. Eine synoptische Zusammenfassung bisher vorliegender Untersuchungen ${ }^{2}$ zeigt eine starke Ambivalenz (Tabelle 3).

2 Barnes et al., 2013; Green et al., 2014; Curtarelli, 2013; Saxton et al., 2013; Frei, 2009; Kaganer et al., 2013; Afuah \& Tucci, 2012; Lehdonvirta \& Mezier, 2013; Walker et al., 2010; Pekruhl, 2013; Howe, 2006; Kittur et al., 2013; Kawalec \& Menz, 2013. 
Tabelle 3: Auswirkungen von Crowdsourcing auf die Anbieterinnen und Anbieter von Arbeitskraft

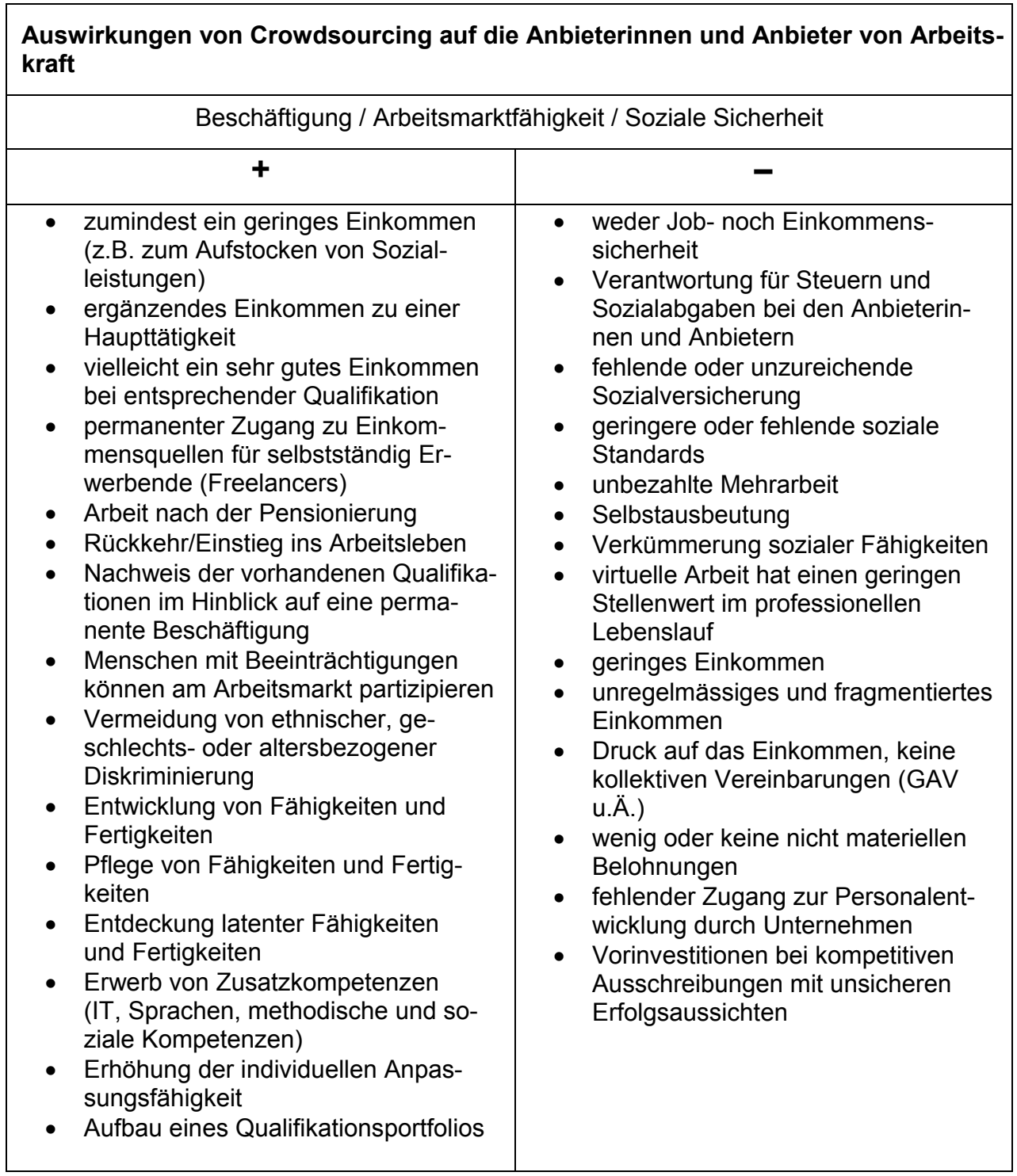




\begin{tabular}{|c|c|}
\hline \multicolumn{2}{|c|}{ Flexibilität / Soziales Leben } \\
\hline+ & - \\
\hline $\begin{array}{ll}\text { - } & \text { Work-Life-Balance (allgemein) } \\
\text { - } & \text { Vereinbarkeit von Berufs- } \\
\text { - } & \text { Heimarbamilienarbeit } \\
\text { - } & \text { geografische Flexibilität } \\
\text { - } & \text { freie Wahl der Ferientermine } \\
\text { - } & \text { Erwartungen von Generation Y } \\
\text { - } & \text { nerden erfüllt } \\
\text { - } & \text { freie Wahl der Arbeit und der Arbeits- } \\
& \text { aufgaben } \\
\text { - Handeln als Mitglied einer grossen } \\
\text { Gemeinschaft }\end{array}$ & $\begin{array}{ll}\text { - } & \text { soziale Isolation } \\
\text { - } & \text { mangelnder persönlicher Austausch } \\
\text { - } & \text { ginnendende Unterstützung durch Kolle- } \\
\text { - } & \text { mangelndes Feedback } \\
\text { - } & \text { unsichere zeitliche Planungen } \\
\text { - } & \text { Mehrarbeit zur Erreichung festgeleg- } \\
\text { - } & \text { ter Termine } \\
\text { - } & \text { und Prenzung zwischen Arbeits- } \\
\text { - Stress und Gesundheitsrisiken }\end{array}$ \\
\hline \multicolumn{2}{|c|}{ Arbeitsinhalte } \\
\hline+ & - \\
\hline 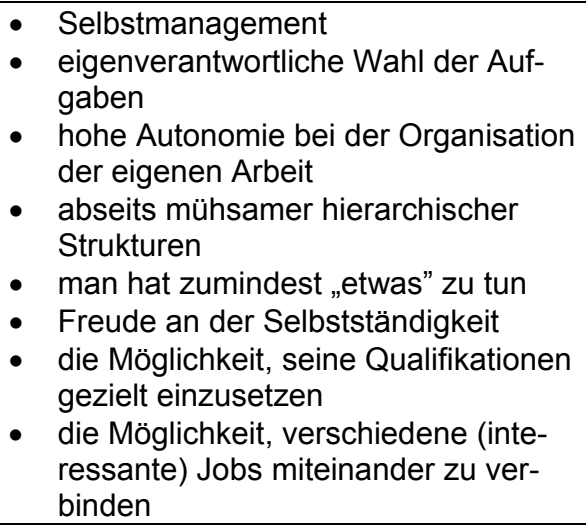 & $\begin{array}{ll}\text { - } & \text { hoch taylorisierte Arbeit / Rückkehr } \\
\text { - } & \text { des Taylorismus } \\
\text { - } & \text { repetitive Teilarbeit } \\
\text { - } & \text { Akkordarbeit } \\
\text { - } & \text { Dequalifizierung } \\
\text { - } & \text { De-Humanisierung des Arbeitsle- } \\
\text { - } & \text { bens } \\
\text { unmoralische Jobangebote } \\
\text { (z.B. erfundene positive Produkt- } \\
\text { rezensionen für Bewertungsportale) }\end{array}$ \\
\hline
\end{tabular}

Angesichts der hier zusammengefassten potenziellen Vor- und Nachteile von Crowdsourcing wird deutlich, dass es viele Parallelen zu der Situation von „klassischen“ (d.h. nicht über Crowdsourcing-Provider vermittelten) Selbstständigen/Freelancern gibt, und dass die Probleme und Chancen einer „Entgrenzung der Arbeit" (Homeoffice, Vertrauensarbeitszeit usw.) hier voll zum Tragen kommen. Weitere offensichtliche Gemeinsamkeiten gibt es zu bestimmten Formen der Zeitarbeit, in der Zeitarbeitsfirmen zwischen Beschäftigten und Arbeitgebenden vermitteln. Diese Brokerrolle nehmen beim Crowdsourcing die Betreiberinnen und Betreiber der entsprechenden Internet-Plattformen wahr. Und genauso wie bei der Zeitarbeit hängt die soziale Sicherheit der Beschäftigten nicht zuletzt davon ab, wie diese Intermediäre ihre Aufgabe definieren. Bei Mechanical Turk werden die Beschäftigten in dieser Hinsicht völlig allein gelassen, 
Amazon stellt lediglich den Kontakt zwischen den Parteien her. Bei Vermittelnden wie der Schweizer Jobbörse Staff Finder sind die Beschäftigten hingegen sozialversichert, darüber hinaus sorgt Staff Finder dafür, dass Schweizer Sozial- und Lohnstandards eingehalten werden.

Abschliessend soll noch eine Entwicklung im Bereich Crowdsourcing erwähnt werden, die künftig möglicherweise gravierende Auswirkungen auf Form und Inhalt von Arbeit haben wird. Schon jetzt sind die sogenannten "Micro Tasks" wie die HITs bei Mechanical Turk hochgradig taylorisierte Arbeitsaufgaben: kurzzyklisch, anspruchslos, fremdbestimmt, massiv kontrolliert, Motivation entsteht allein durch extrinsische Anreize (quasi Akkordsystem) und ohne jeden Blick auf eine ganzheitliche Arbeitsaufgabe. Nun besteht die Gefahr, dass eine solche Taylorisierung der Arbeit auch anspruchsvollere Aufgaben erfasst. Einige Crowdsourcing-Provider arbeiten mit Hochdruck an Algorithmen, die es erlauben, komplexe Tätigkeiten - etwa im Bereich der Softwareentwicklung, aber auch anspruchsvolle Konstruktionsaufgaben - automatisch in simple Teiltätigkeiten zu zerlegen, diese weltweit verteilt bearbeiten zu lassen und die Fragmente dann wiederum automatisch zusammenzufügen. Taylorisierung 4.0 sozusagen.

\subsubsection{Auslegeordnung der Formen der Arbeitsflexibilität}

Die beiden Beispiele „Homeoffice“ sowie "Crowdsourcing for paid Work" zeigen auf, wie vielfältig auf flexible Weise gearbeitet werden kann und welche Vorund Nachteile dies jeweils mit sich bringt. Es handelt sich dabei allerdings nur um zwei konkrete Varianten - denkbar sind natürlich viele mehr. Aus diesem Grund wurde in der Projektgruppe ein gemeinsames Brainstorming durchgeführt, um die Komplexität der flexiblen Arbeitsformen sichtbar zu machen. Dafür wurde die Methode des "morphologischen Kastens“ verwendet (Ritchey, 2011; Zwicky, 1989). Gemeinsam wurden möglichst viele unterschiedliche Formen und Ausprägungen flexibler Arbeit gesammelt und diese in einer Tabelle zusammengestellt. In Tabelle 4 sind die Formen (oder auch Dimensionen) flexibler Arbeit in der linken Spalte und die jeweiligen Ausprägungen in der entsprechenden Zeile beschrieben. Die Tabelle ist als Ergebnis eines Brainstormings im Sinne der Komplexitätserweiterung und somit als Zwischenschritt zu verstehen und erhebt keinen Anspruch auf Vollständigkeit. 
Tabelle 4: $\quad$ Morphologischer Kasten zu Formen der Arbeitsflexibilität

\begin{tabular}{|c|c|c|c|c|c|c|c|}
\hline & & & 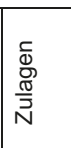 & & & 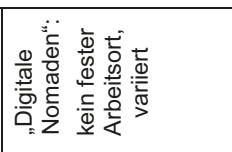 & 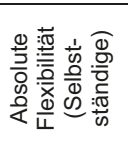 \\
\hline & & $\begin{array}{l}\text { 蛋 } \\
\text { 妾 }\end{array}$ & 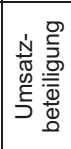 & & & 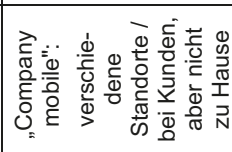 & 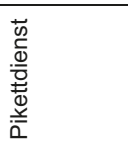 \\
\hline & 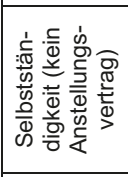 & 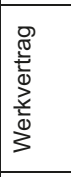 & 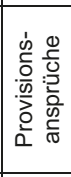 & 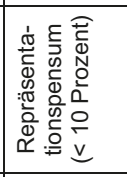 & 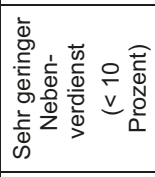 & 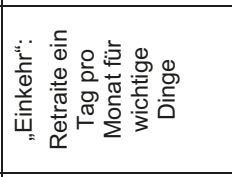 & 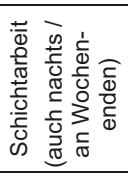 \\
\hline & 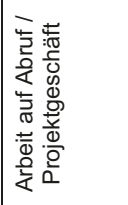 & 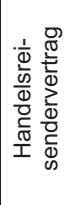 & 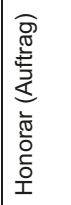 & 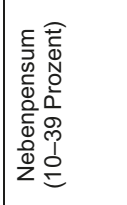 & 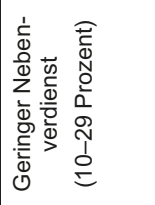 & 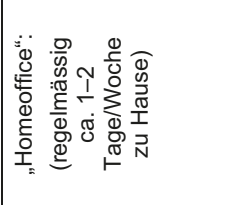 & 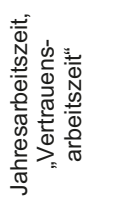 \\
\hline & 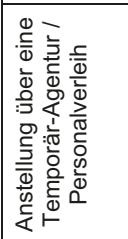 & 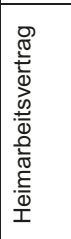 & 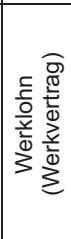 & 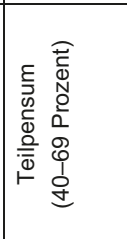 & 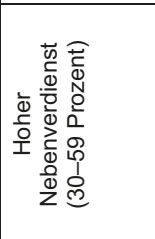 & 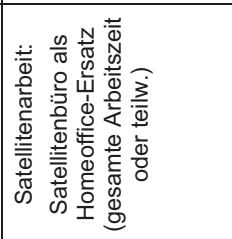 & 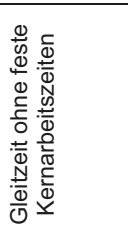 \\
\hline & 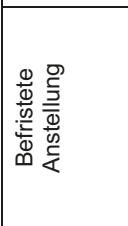 & 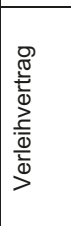 & 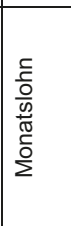 & 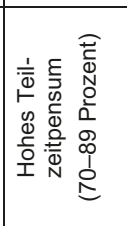 & 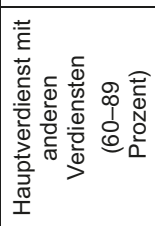 & 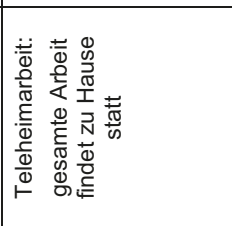 & 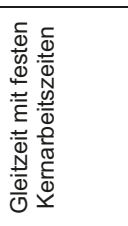 \\
\hline 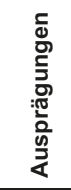 & 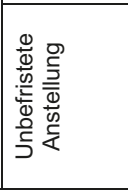 & 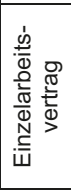 & 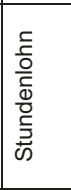 & 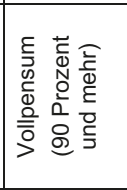 & 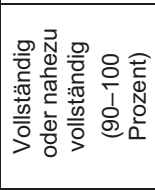 & 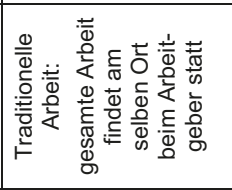 & 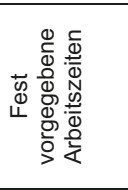 \\
\hline 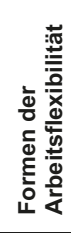 & 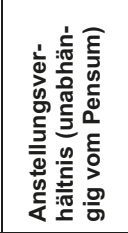 & 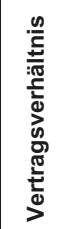 & 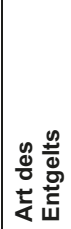 & 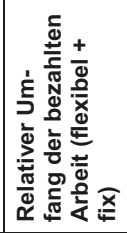 & 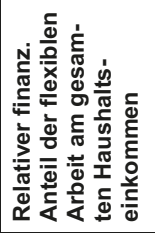 & 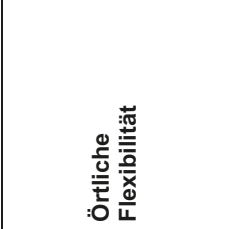 & 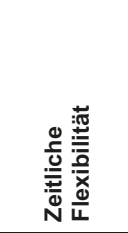 \\
\hline
\end{tabular}




\begin{tabular}{|c|c|c|c|c|}
\hline 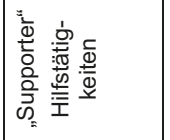 & 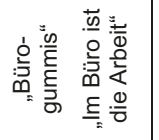 & 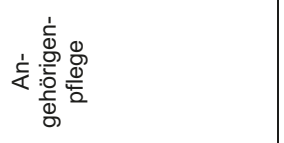 & & \\
\hline 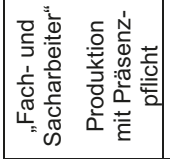 & 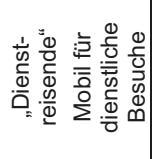 & 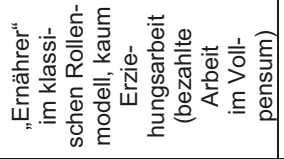 & & \\
\hline 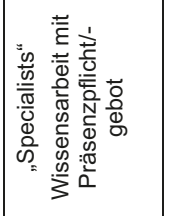 & 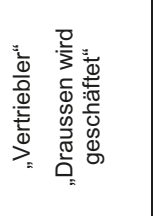 & 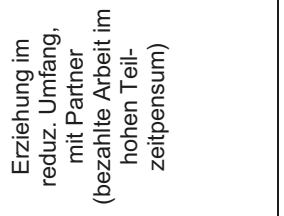 & 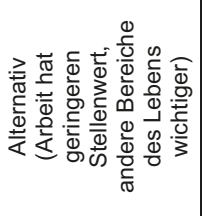 & 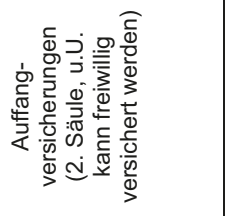 \\
\hline 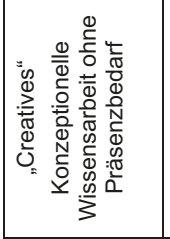 & 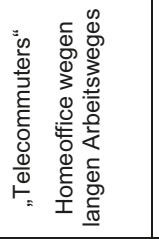 & 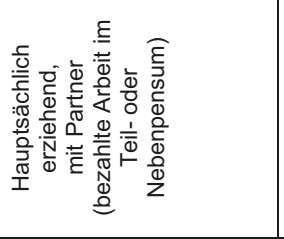 & 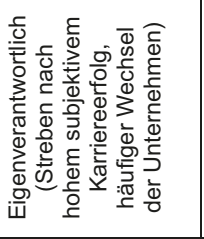 & 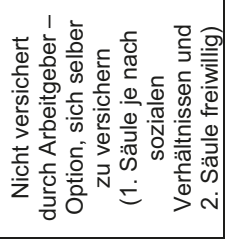 \\
\hline 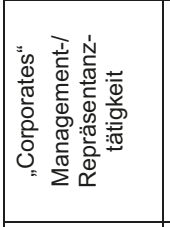 & 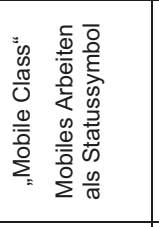 & 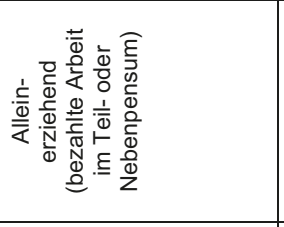 & 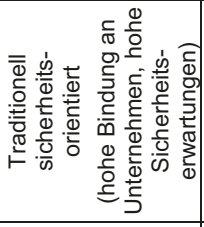 & 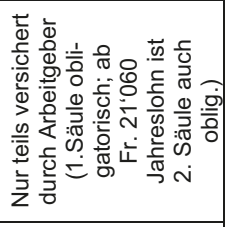 \\
\hline 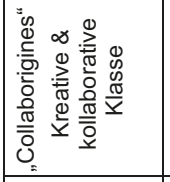 & 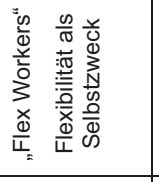 & 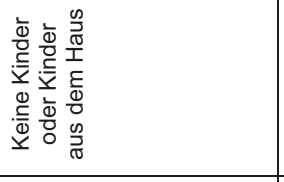 & 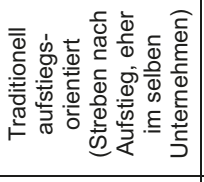 & 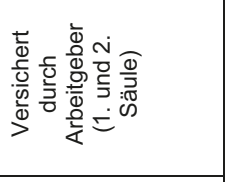 \\
\hline 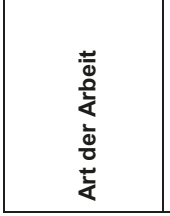 & 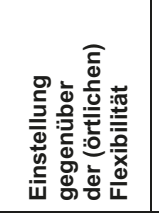 & 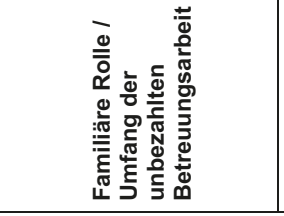 & 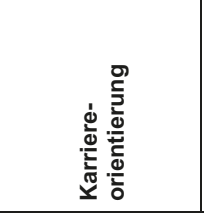 & 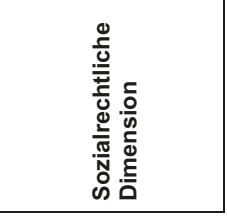 \\
\hline
\end{tabular}


Die Varianten des morphologischen Kastens machen deutlich, wie vielfältig flexible Arbeitsformen sein können. Um aus diesem Möglichkeitsraum eine Grundlage für die weitere Arbeit zu bilden, wurde der morphologische Kasten komprimiert und eine Matrix mit vier Dimensionen in je drei Ausprägungen abgeleitet. Die Dimensionen sollen die vier wichtigsten Bereiche der Arbeitsflexibilisierung erfassen: eine rechtliche Dimension (Anstellungsverhältnis), eine physische (zeitliche/örtliche Flexibilität) sowie zwei Dimensionen, die gemeinsam die soziale Ebene fassen sollen (ökonomische Rolle des Individuums sowie soziale Funktion von Arbeit).

Tabelle 5: Matrix flexible Arbeitsformen

\begin{tabular}{|c|c|c|c|}
\hline Dimension & \multicolumn{3}{|l|}{ Ausprägungen } \\
\hline $\begin{array}{l}\text { Anstellungs- } \\
\text { und Vertrags- } \\
\text { verhältnis } \\
\text { (rechtliche Di- } \\
\text { mension) }\end{array}$ & $\begin{array}{l}\text { (1) Fest ange- } \\
\text { stellt } \\
\text { üblicherweise } \\
\text { Vollzeit, auf } \\
\text { jeden Fall unbe- } \\
\text { fristet und mit } \\
\text { allen Sicherhei- } \\
\text { ten }\end{array}$ & $\begin{array}{l}\text { (2) Mischformen } \\
\text { z.B. befristete } \\
\text { Stellen, Honorar- } \\
\text { basis, Teilzeit- } \\
\text { anstellung plus } \\
\text { selbstständiger } \\
\text { Zuverdienst (,zwei } \\
\text { Standbeine“) ... }\end{array}$ & $\begin{array}{l}\text { (3) Voll selbst- } \\
\text { ständig } \\
\text { „Vollzeit“ selbst- } \\
\text { ständig; soziale } \\
\text { Absicherung in } \\
\text { Eigenverantwortung }\end{array}$ \\
\hline $\begin{array}{l}\text { Örtliche und } \\
\text { zeitliche Gren- } \\
\text { zen von Arbeit } \\
\text { (physische Di- } \\
\text { mension) }\end{array}$ & $\begin{array}{l}\text { (1) Feste Gren- } \\
\text { zen } \\
\text { traditionell strikte } \\
\text { Trennung von } \\
\text { Arbeit und ande- } \\
\text { ren Lebensbe- } \\
\text { reichen }\end{array}$ & $\begin{array}{l}\text { (2) Bandbreiten } \\
\text { Arbeit teilweise } \\
\text { von anderen Orten } \\
\text { und zu unüblichen } \\
\text { Zeiten, aber ge- } \\
\text { wisse Regelmäs- } \\
\text { sigkeiten noch } \\
\text { erkennbar }\end{array}$ & $\begin{array}{l}\text { (3) Entgrenzt } \\
\text { Arbeit und andere } \\
\text { Lebensbereiche } \\
\text { voll vermischt }\end{array}$ \\
\hline
\end{tabular}




\begin{tabular}{|c|c|c|c|}
\hline $\begin{array}{l}\text { Ökonomi- } \\
\text { sche Rolle } \\
\text { des Indivi- } \\
\text { duums } \\
\text { (soziale } \\
\text { Dimension 1) }\end{array}$ & $\begin{array}{l}\text { (1) „Arbeitskraft““ } \\
\text { Arbeit ist Zeit } \\
\text { (physische Anwe- } \\
\text { senheit) gegen } \\
\text { Geld; unterneh- } \\
\text { merisches Risiko } \\
\text { liegt bei den Ar- } \\
\text { beitgebenden }\end{array}$ & $\begin{array}{l}\text { (2) „,Arbeitskraft- } \\
\text { unternehmer/in““ } \\
\text { Eigenverantwor- } \\
\text { tung in der Anstel- } \\
\text { lung; teilweise } \\
\text { Übernahme des } \\
\text { unternehmerischen } \\
\text { Risikos (z.B. leis- } \\
\text { tungsorientierter } \\
\text { Lohn; unbezahlte } \\
\text { Überstunden; ...) }\end{array}$ & $\begin{array}{l}\text { (3) „Unternehmer/in“ } \\
\text { Risiko und Verant- } \\
\text { wortung voll beim } \\
\text { Individuum }\end{array}$ \\
\hline $\begin{array}{l}\text { Soziale } \\
\text { Funktion } \\
\text { von Arbeit } \\
\text { (soziale } \\
\text { Dimension 2) }\end{array}$ & $\begin{array}{l}\text { (1) Arbeit als } \\
\text { Auskommen } \\
\text { Arbeit als Mittel } \\
\text { zum Zweck; ge- } \\
\text { sellschaftliche } \\
\text { Teilhabe findet } \\
\text { eher in anderen } \\
\text { Bereichen statt; } \\
\text { „Man arbeitet, um } \\
\text { zu leben.“ }\end{array}$ & $\begin{array}{l}\text { (2) Arbeit als } \\
\text { Gemeinschaft } \\
\text { Arbeitsplatz = } \\
\text { Platz in der Ge- } \\
\text { sellschaft; der Be- } \\
\text { trieb als Halt ge- } \\
\text { bendes Kollektiv }\end{array}$ & $\begin{array}{l}\text { (3) Arbeit als } \\
\text { Selbstverwirkli- } \\
\text { chung } \\
\text { Arbeiten, um „etwas } \\
\text { zu erreichen“; gesell- } \\
\text { schaftliche Teilhabe } \\
\text { als erfolgreiches Indi- } \\
\text { viduum; „Man lebt, } \\
\text { um zu arbeiten.“ }\end{array}$ \\
\hline
\end{tabular}

Alle Dimensionen sind dabei so formuliert, dass sie jeweils zwei Extrempole abbilden und eine Kategorie für Mischformen beinhalten. Daher sind die Dimensionen nicht voneinander unabhängig. Gewisse Kombinationen sind nicht plausibel oder logisch (z.B. kann man nicht als „Arbeitskraft“ voll selbstständig arbeiten). Die Ausprägungen lassen sich nutzen, um Profile abzubilden (z.B. 12-1-2).

Aus dieser in der Komplexität reduzierten und verdichteten Matrix wurden anschliessend fünf Personas abgeleitet. Dabei wurde nach folgenden Leitsätzen vorgegangen: Zum einen wurde das Ziel gesetzt, in den Personas Extreme abzubilden. Zwei Personas arbeiten eher nur wenig flexibel (Persona A „Homeoffice in der Festanstellung" - mit dem Profil 1-2-1-2, sowie Persona E „Mehrfachanstellungen und Minijobs“ - mit dem Profil 2-1-1-1), eine Persona dagegen äusserst flexibel (Persona D - „Entrepreneurship“ - mit dem Profil 3-33-3). Das Extrem „überhaupt nicht flexibel“ (1-1-1-1) wurde bewusst ausgelas- 
sen, da es das „Normalarbeitsverhältnis“ widerspiegelt und keinen Erkenntnisgewinn bringt. Zwei weitere Personas wurden gebildet, welche Variationen zwischen diesen Extremformen abbilden, um die Bandbreite der Möglichkeiten aufzuzeigen. Allgemein wurde dabei nach den Prinzipien der Plausibilität und Gegenstandsnähe vorgegangen - also möglichst Kombinationen gebildet, die vermutlich weit verbreitet sind, während von allzu unrealistischen Kombinationen abgesehen wurde, auch wenn sie in der "neuen Arbeitswelt“ theoretisch möglich sind. Daher erfolgte der Entscheid für die folgenden zwei weiteren Personas: zum eine fest angestellte Person, welche aber flexibel als „mobile Arbeitskraftunternehmerin" arbeitet (Persona B mit dem Profil 1-2-2-3); dieser Typ sollte häufig anzutreffen sein in Beratungsfirmen, im Verkauf, in Banken und Versicherungen; zum anderen eine Persona, die das Modell „Portfoliowork auf mehreren Standbeinen“ verkörpert (also teils angestellt und teils selbstständig, Persona C mit dem Profil 2-3-2-1); dieser Typ dürfte in kreativen oder in ITBerufen oft vorkommen. Abbildung 5 zeigt die Profile der fünf Personas in der Matrix flexible Arbeitsformen.

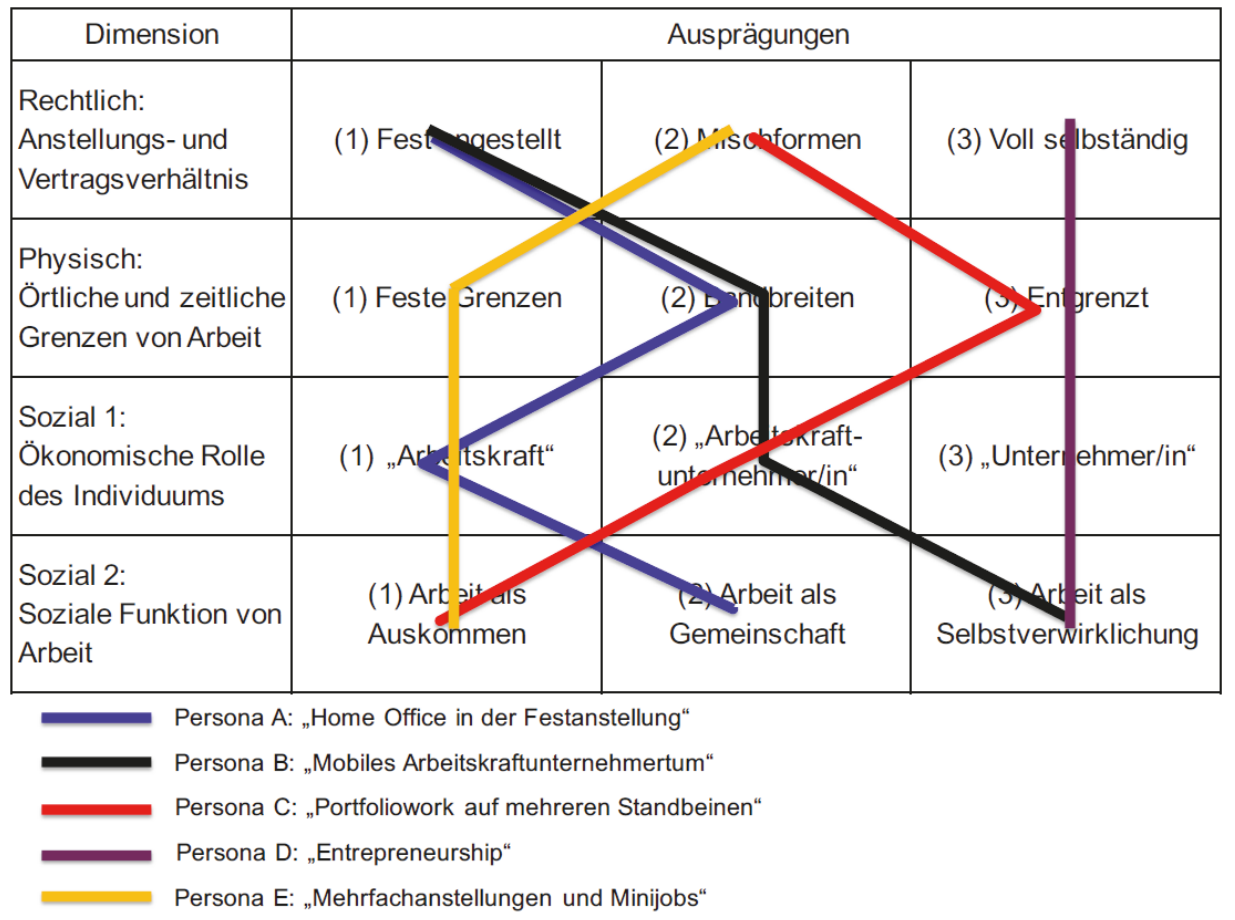

Abbildung 5: Personas in der Matrix flexible Arbeitsformen 
Jede der Personas hat zudem eine „Kernbotschaft“, soll also ein Bündel gewisser Aspekte der flexiblen Arbeitswelt verkörpern. Persona A steht dabei für das Arbeiten im „traditionellen“ Arbeitsverhältnis mit nur geringen Flexibilitätsanteilen - insbesondere die (tageweise) Arbeit im Homeoffice. Die Grenzen der Arbeit sind hier also nur minimal "aufgeweicht" und die soziale Absicherung ist kein Problem. Persona B personifiziert das Phänomen der „Arbeitskraftunternehmenden“", also einer angestellten Person, die aber in vielen Punkten so handelt wie eine Selbstständige. Dies zeichnet sich dadurch aus, dass auf der einen Seite ein grosser unternehmerischer Druck (z.B. durch Gewinnbeteiligung und harte Zielvorgaben), auf der anderen Seite relativ viel Freiheiten und Eigenverantwortung (insbesondere die Auflösung von traditionellen „physischen“ Grenzen der Arbeit) herrscht. Problematisch ist also eher das Thema Überarbeitung/Burnout, dagegen ist die soziale Absicherung hier kein Problem. Persona C steht für das Phänomen „zwei Standbeine“ (oder gar mehrere), d.h., es gibt eine Festanstellung in Teilzeit, aber gleichzeitig arbeitet die Person auch selbstständig. In beiden Standbeinen kann zeitlich und örtlich flexibel gearbeitet werden, daher herrscht eine grosse Vermischung. Problematisch kann die soziale Absicherung werden, weil aufgrund der (unsicheren) Teilzeit-Festanstellungen nur wenig in die AHV bzw. Pensionskassen eingezahlt wird. Persona D verkörpert die Unternehmenden in der digitalen Arbeitswelt, d.h., sie arbeitet voll selbstständig und nutzt dafür die volle zeitliche und örtliche Flexibilität. Aufträge werden insbesondere online/digital vermittelt. Die soziale Absicherung kann hier zum Problem werden, da sie voll in der Eigenverantwortung liegt. Persona $\mathrm{E}$ betreibt auch ein Zwei-Standbeine-Modell, steht aber insbesondere für die Möglichkeiten und Herausforderungen gering qualifizierter Arbeitnehmer/innen. Die Problematiken sind hier: geringes Einkommensniveau, wenig Aufstiegschancen im „klassischen“ Arbeitsmarkt, soziale Absicherung kritisch.

\subsection{Personas}

Bei der Ausformulierung der Personas wurde folgendes Vorgehen angewandt: Zunächst wird eine neutrale Beschreibung der Situation (Art des flexiblen Arbeitens, Hintergrundinfos zur Person) formuliert. Anschliessend werden die positiven Auswirkungen dieses Typs flexibler Arbeit in Form eines "guten Tages“, und die negativen in Form eines "schlechten Tages" beschrieben, welche die Personas erleben. Das bedeutet, dass die einzelnen Personas weder zu den „Gewinnern“ noch zu den „Verlierern“ der Flexibilisierung gehören. So gibt es 
z.B. keine Persona, die per se „prekär“ lebt, sondern das Prekäre zeigt sich in den „schlechten Tagen“ der Persona.

\subsubsection{Persona A: Roland Müller - „Homeoffice in der Festanstellung“}

- männlich

- 46 Jahre alt

- verheiratet, 2 Kinder

- Eidg. Dipl. Betriebswirtschafter

- Angestellter, Leiter Finanzen in KMU

- Arbeit vorwiegend vor Ort beim Arbeitgeber. $1 \mathrm{HO}-\mathrm{Tag} /$ Woche

- Karrieretyp: traditionell / aufstiegsorientiert

- finanziell gut abgesichert
Roland Müller arbeitet seit 18 Jahren zu 100 Prozent in der Buchhaltung eines mittelgrossen Unternehmens (120 Angestellte), wo er als Fachkraft eingestiegen und über die Jahre zum Abteilungsleiter aufgestiegen ist. Das Unternehmen ist nach wie vor fest in den Händen der Gründerfamilie, die eine traditionelle Unternehmenskultur pflegt. Aus der Produktionshistorie gewachsen, erfasst ein Grossteil der Belegschaft die Arbeitszeit via Stempeluhr. Roland Müller ist als Kadermitglied davon entbunden und kann infolgedessen auch keine Überstunden geltend machen. Diese werden bereits als Lohnbestandteil antizipiert und abgegolten.

Die Arbeit von Roland Müller setzt sich zusammen aus „Daily Business“, welches viel Kooperation mit dem geschäftlichen Umfeld erfordert, und aus strategischen Aufgaben, welche konzentrierte Einzelarbeit bedingen. Seit rund drei Jahren ist es den Mitgliedern des Kaders erlaubt, einen Teil ihres Arbeitspensums im Homeoffice zu erbringen, was Roland Müller - trotz einiger Bedenken zu Beginn - nun gerne regelmässig wahrnimmt.

Privat ist Roland Müller seit 15 Jahren verheiratet und Vater zweier Kinder im Alter von 6 und 12 Jahren. Seine Freizeit widmet er vor allem seiner Familie und seinen Hobbys (Eishockey im Winter, Fussball im Sommer - aktiv im Klub und passiv als Fan).

\begin{tabular}{|l|c|c|c|}
\hline & \multicolumn{3}{|c|}{ Typ 1-2-1-2 } \\
\hline Anstellungsverhältnis & $\begin{array}{c}\text { (1) fest } \\
\text { angestellt }\end{array}$ & (2) Mischformen & $\begin{array}{c}\text { (3) voll selbst- } \\
\text { ständig }\end{array}$ \\
\hline $\begin{array}{l}\text { Örtliche und zeitliche } \\
\text { Grenzen der Arbeit }\end{array}$ & $\begin{array}{c}\text { (1) feste } \\
\text { Grenzen }\end{array}$ & (2) Bandbreiten & (3) entgrenzt \\
\hline $\begin{array}{l}\text { Ökonomische Rolle } \\
\text { des Individuums }\end{array}$ & $\begin{array}{c}\text { (1) „Arbeits- } \\
\text { kraft“ }\end{array}$ & $\begin{array}{c}\text { (2) „Arbeitskraft- } \\
\text { unternehmer/in“ }\end{array}$ & $\begin{array}{c}\text { (3) „Unter- } \\
\text { nehmer/in“ }\end{array}$ \\
\hline
\end{tabular}




\begin{tabular}{|l|c|c|c|}
\hline $\begin{array}{l}\text { Soziale Funktion } \\
\text { von Arbeit }\end{array}$ & $\begin{array}{c}\text { (1) Arbeit als } \\
\text { Auskommen }\end{array}$ & $\begin{array}{c}\text { (2) Arbeit als Ge- } \\
\text { meinschaft }\end{array}$ & $\begin{array}{c}\text { (3) Arbeit als } \\
\text { Selbst- } \\
\text { verwirklichung }\end{array}$ \\
\hline
\end{tabular}

\section{Ein guter Tag im Leben von Roland Müller}

Roland Müller ist seit jeher ein Frühaufsteher. An Arbeitstagen fährt er jeweils gegen 6.45 Uhr zur Arbeit los. Diese beginnt er nach einer rund 45-minütigen Autofahrt um 7.30 Uhr. Seit gut drei Jahren versucht Roland jeweils donnerstags Homeoffice zu machen, wenn es die Termine zulassen. Diese Woche ist es ihm gelungen, den Donnerstag für das Homeoffice frei zu halten. Auch seine Homeoffice-Tage beginnt er regelmässig pünktlich um 6.45 Uhr. Nach einem kurzen Frühstück setzt er sich also direkt in sein Arbeitszimmer und beginnt mit der Budgetplanung - eine Aufgabe, die Ruhe und Konzentration erfordert, beides findet er im hektischen Büroalltag nur selten. Dank den neuen technologischen Gegebenheiten im Unternehmen kann er jetzt diese Arbeit in der Stille seines Homeoffices erledigen. Da die Anfahrt ins Büro entfällt, nutzt Roland die gewonnene Stunde gegen 9 Uhr für einen ausgiebigen Spaziergang mit seiner Frau und dem Familienhund. Seit die Kinder da sind, findet das Ehepaar nur noch selten Zeit füreinander, was diese Spaziergänge besonders wertvoll macht. Nach der Bewegung an der frischen Luft setzt sich Roland gut gelaunt und sichtlich erholt nochmals für rund 2,5 Stunden konzentriert an den Budgetplan. Um 12.30 Uhr gibt es dann bei Familie Müller Mittagessen. Roland unterhält sich rege mit seiner Familie, bevor er sich mit Kaffee und Zeitung aufs Sofa zurückzieht. Eine weitere Homeoffice-Routine, die er sehr lieb gewonnen hat.

Mit seinem Team hat Roland vereinbart, dass sie ihn an Homeoffice-Tagen per Telefon kontaktieren, falls etwas Dringendes anstehen sollte. Da alles ruhig zu verlaufen scheint, setzt sich Roland um 13.30 Uhr direkt wieder an den Budgetplan. An diesem arbeitet er mit Ausnahme einiger Kurzpausen konzentriert bis 17.30 Uhr. Roland ist sehr erleichtert darüber, mit dieser wichtigen Aufgabe ein grosses Stück vorangekommen zu sein, und beendet seinen Homeoffice-Tag zufrieden mit der Lektüre seiner E-Mails als Vorbereitung auf den kommenden Tag im Büro. Erfreut über das schöne Wetter und den Wegfall der manchmal mühseligen Heimfahrt im Feierabendverkehr nutzt Roland die Gelegenheit und mäht den Rasen im Garten, bis es Zeit fürs Abendessen ist.

Den Abend lässt er dann, nachdem er die Kinder zu Bett gebracht hat, mit seiner Frau vor dem Fernseher ausklingen. Dieser Homeoffice-Tag hat die ansonsten sehr stressige und fordernde Woche sehr entlastet. Jetzt freut sich 
Roland auf die morgige Arbeit mit seinem Team vor Ort. Der Budgetplan ist gut unterwegs und kann bis nächsten Donnerstag ruhen.

\section{Ein schlechter Tag im Leben von Roland Müller}

Der Wecker klingelt wie gewohnt um 5.45 und Roland Müller startet seinen Arbeitstag im Homeoffice wie sonst auch um 6.45 nach dem kurzen Frühstück. Heute ist allerdings irgendwie der Wurm drin. Der jüngste Sohn ist krank und geht den dritten Tag in Folge nicht zur Schule. Roland Müllers Frau hat bereits ihre Unzufriedenheit darüber geäussert, dass heute auch noch ihr Mann zu Hause ist. Auch wenn die Tür zu seinem Arbeitszimmer geschlossen ist, fühle sie sich an solch stressigen Tagen zusätzlich belastet durch seine Anwesenheit - so muss sie z.B. dem kranken Julius erklären, dass er nicht mit dem Vater spielen kann, obwohl der doch gleich nebenan ist. Roland Müller muss sich also nicht nur physisch abgrenzen, sondern auch emotional damit umgehen, dass an seinem heutigen Homeoffice-Tag die sonst sehr geschätzte familiäre Anbindung mehr Problem als Annehmlichkeit ist. Er versucht also, sich so gut es geht auf die Arbeit zu konzentrieren: Heute will er an der Präsentation für das nächste Quartalsmeeting weiterarbeiten. Gleich zu Beginn stellt Roland Müller allerdings mit Schrecken fest, dass er die am Vorabend im Büro vorbereiteten Folien falsch abgespeichert und nun keinen Zugriff darauf hat. Was nun? Er könnte seine Arbeitskollegin bitten, inm die Folien zu schicken, doch sie ist so früh sicherlich noch nicht im Büro. Roland Müller ist ziemlich entmutigt. Andere Aufgaben stehen entweder nicht an oder inm fehlt auch hier das nötige Arbeitsmaterial. Gefrustet ruft Roland Müller den Onlineauftritt seiner Lieblingszeitung auf und beginnt zu surfen. Die Selbstdisziplin, die er sonst meist problemlos aufbringen kann, geht inm heute ab. Erst nach fast eineinhalb Stunden unproduktivem Surfen schafft er es, sich aufzuraffen und sich wieder auf die Arbeit zu konzentrieren. Er ruft seine Kollegin im Büro an, welche über die Unterbrechung nicht sonderlich begeistert ist. Nun kann Roland Müller mit viel Verspätung endlich seinen Arbeitstag beginnen und kommt tatsächlich auch einigermassen gut voran. Der inm so lieb gewonnene Spaziergang mit seiner Frau zur „ZnüniPause" muss allerdings heute ausfallen. In solchen Momenten allein im Arbeitszimmer wird inm klar, wie sehr er den spontanen Plausch mit den Arbeitskollegen bei einem Kaffee vermisst.

Das Mittagessen heute ist kurz, und er realisiert, wie sehr seine Frau mit den heutigen Aufgaben im Haushalt plus Betreuung des kranken Julius im Stress ist. Sie bittet inn, am Nachmittag ein bis zwei Stunden auf Julius aufzupassen, während sie die Einkäufe erledigt. Roland Müller fühlt sich hin- und hergerissen: 
Einerseits will er natürlich gerne seine Frau entlasten und auch für seinen kranken Sohn da sein. Andererseits sieht er bereits den Stress vor sich, den er am morgigen Tag haben wird, wenn er heute mit seiner Präsentation nicht fertig wird. Auch die Vorstellung, am Homeoffice-Tag einfach so zwei Stunden nicht produktiv zu arbeiten, gefällt inm nicht: Auch wenn Roland Müllers Arbeitgeber hier sehr tolerant ist, das Gewissen nagt trotzdem - nicht zuletzt, weil er heute Morgen bereits Zeit verbummelt hat. Letztendlich willigt er ein und kümmert sich nach dem Mittagessen um Julius.

Als seine Frau gegen 15 Uhr zurückkommt, kann er sich endlich wieder seiner Arbeit widmen. Allerdings begeht er den „Fehler", statt sich direkt wieder in seine Präsentation zu vertiefen, zunächst einmal seine E-Mails abzurufen. Da sieht er eine E-Mail von der Personalabteilung, in dem es um die Kündigung eines Mitarbeiters aus seiner Abteilung geht. Auch wenn Roland Müller die Gründe für die Kündigung einsieht, belastet inn die Vorstellung dennoch. Gerne würde er jetzt persönlich bei der Personalleiterin vorbeigehen, um die Angelegenheit zu besprechen. Per Telefon scheint inm das aber nicht geeignet, also muss es bis morgen warten. Bedrückt versucht er nun endlich wieder, sich um seine Präsentation zu kümmern, doch die Ablenkungen - im Job wie im Privatleben - sind zu gross. Entmutigt kümmert sich Roland Müller nur noch darum, die wichtigsten E-Mails zu beantworten und macht dann um 17 Uhr unzufrieden Feierabend. Morgen muss er es mit neuer Energie erneut versuchen, die Präsentation fertig zu bekommen.

\subsubsection{Persona B: Sandra Könitz - „Mobiles Arbeitskraftunternehmer- tum"}

- weiblich

- 35 Jahre

- unverheiratet, in einer festen Beziehung

- $\quad$ zwei Kinder, 3 und 5 Jahre

- fest angestellt

- arbeitet immer und überall

- Karrieretyp: alternativeigenverantwortlich

- Arbeitsmobilität: sehr flexibel, zielorientiert
Sandra Könitz ist stets gut ausgelastet. Sie ist eine anspruchsvolle, fordernde, smart vernetzte Person, die in konkreten Spezialinhalten als angesehene Expertin gilt und dementsprechend „in der obersten Liga“ mitspielt. Nach ihrem Bachelorstudium in Wirtschaft und Recht schloss sie später einen berufsbegleitenden Master in Politikwissenschaften an der London School of Political Science ab und arbeitet nun für eine kleine Beratungsunternehmung als Projektmanagerin.

Sandras E-Mail-Eingang ist stets voll und wird von ihr gewissenhaft und zielstrebig abgearbei- 
tet. Oberste Priorität haben die gut zahlenden Kunden. Sandras Arbeitstempo ist sehr hoch, aber in ihrer beruflichen Position darf man sich auch keine Schwäche erlauben, sondern muss auf Dauer die Flughöhe halten. Sandra steht im Unternehmen kurz vor dem Teilhaberschritt, verdient ausserordentlich gut und profitierte mit 40 Prozent Bonusanteil am Gesamtgehalt auch sehr gut vom letzten Jahresgewinn.

Ihre direkte Arbeitsumgebung ist Sandra besonders wichtig. Sie muss repräsentativ und für eine professionelle Leistung geeignet sein. Ihr beruflicher Erfolg hängt massgeblich an ihrer eigenen Flexibilität, Mobilität und Erreichbarkeit. Es liegt häufig an ihr zu entscheiden, wie oft sie zur Akquisition neuer Geschäfte bei der Kundin vor Ort ist. In der Regel muss sie aufgrund der vielen Anfragen aber nur bestimmen, was sie nicht macht respektive wo die grösste Rendite verborgen liegt. Obwohl Sandra gerne arbeitet, natürlich ohne Zeiterfassung und auf Vertrauensbasis, macht sich der Akquisedruck bemerkbar. Sandras Arbeitszeiten sind flexibel. Die meiste Zeit verbringt sie im Büro oder bei Kundinnen und Kunden, wobei E-Mails checken am Abend für Sandra normal ist.

Sandra lebt in einer festen Beziehung mit ihrem Freund Tobias, selber Rechtsanwalt und viel unterwegs. Die beiden haben zwei gemeinsame Kinder im Alter von drei und fünf Jahren. Deren Betreuung erfordert eine penible Organisation: Drei Tage in der Woche verbringen die Kinder in einer Krippe, an den anderen beiden Tagen springen die Grosseltern ein. Sandra verbringt zugunsten ihrer Karriere nicht sehr viel Zeit mit ihren Kindern.

\begin{tabular}{|l|c|c|c|}
\hline & \multicolumn{3}{|c|}{ Typ 1-2-2-3 } \\
\hline Anstellungsverhältnis & $\begin{array}{c}\text { (1) fest ange- } \\
\text { stellt }\end{array}$ & (2) Mischformen & $\begin{array}{c}\text { (3) voll selbst- } \\
\text { ständig }\end{array}$ \\
\hline $\begin{array}{l}\text { Örtliche und zeitliche } \\
\text { Grenzen der Arbeit }\end{array}$ & $\begin{array}{c}\text { (1) feste } \\
\text { Grenzen }\end{array}$ & (2) Bandbreiten & (3) entgrenzt \\
\hline $\begin{array}{l}\text { Ökonomische Rolle } \\
\text { des Individuums }\end{array}$ & $\begin{array}{c}\text { (1) „Arbeits- } \\
\text { kraft“ }\end{array}$ & $\begin{array}{c}\text { (2),Arbeitskraft- } \\
\text { unternehmer/in“ }\end{array}$ & $\begin{array}{c}\text { (3) „Unter- } \\
\text { nehmer/in“ }\end{array}$ \\
\hline $\begin{array}{l}\text { Soziale Funktion } \\
\text { von Arbeit }\end{array}$ & $\begin{array}{c}\text { (1) Arbeit als } \\
\text { Auskommen }\end{array}$ & $\begin{array}{c}\text { (2) Arbeit als Ge- } \\
\text { meinschaft }\end{array}$ & $\begin{array}{c}\text { (3) Arbeit } \\
\text { als Selbst- } \\
\text { verwirklichung }\end{array}$ \\
\hline
\end{tabular}




\section{Ein guter Tag im Leben von Sandra Könitz}

6 Uhr, der Wecker klingelt. Sandras Tag ist durchgeplant. Nachdem sie die Kinder bei ihrer Mutter abgegeben hat, ist sie wie immer früh im Geschäft. Gestern und die letzte Woche hatte sie vorgearbeitet, weshalb bereits alle Pendenzen erledigt sind. Heute Morgen sichtet sie nochmals die Unterlagen für ein Kundenmeeting, um alles bereit zu haben. Sandra legt grossen Wert auf eine perfekte Leistung. Das bedeutet sehr viel Vorbereitung und erfordert ein strukturiertes Vorgehen. Gesetzliche Arbeitszeiten sind dabei eher ein Hindernis. Sandra ist froh, dass sie die Zeiten im Rahmen ihrer Projektverrechnung erfassen kann, denn so geht es um verrechenbare Stunden. Diese widerspiegeln ihre wahre Produktivität, nicht die gesetzliche Regelung. Überhaupt bedeuten Sandra solche Regelungen wenig, sie sind gut für andere, sie selber entscheidet gern selbst über den Rahmen ihrer Arbeit.

Auf dem Weg zum Meeting geht Sandra mit Petra, ihrer Teammitarbeiterin, nochmal alles durch, diskutiert die letzten Details, wiederholt die Argumentation und tauscht sich mit ihr über die nächsten Projekte aus, welche bereits ,in der Pipeline" sind. Im Gespräch läuft alles rund, einige kleine Ärgernisse bereitet einzig, dass Sandra weiss, dass der vom Kunden geäusserte Wunsch durch ihre Firma wieder einmal nicht angemessen abgedeckt werden kann. Dennoch wird es ein schönes Projekt werden.

Aufgrund ihrer flexiblen Arbeitszeiten kann Sandra eine etwas ausgedehntere Mittagspause einlegen, welche sie bei ihrer Mutter verbringt, die heute auf die Kinder aufpasst. Für Sandra sind diese gemeinsamen Mittagessen sehr wichtig. Sie geniesst die Zeit mit der Familie. Auch kommt sie dadurch etwas zur Ruhe und kann das Büro für einen kurzen Moment vergessen.

Gut gelaunt und mit frischer Energie kommt Sandra am Nachmittag zur Teamsitzung. Die neuen Projekte werden besprochen, Verzögerungen diskutiert und Hindernisse aus dem Weg geräumt. Kurz vorher hat Sandra per Mobiltelefon noch Konzerttickets für sich und ihre Mutter bestellt. Nach dem Meeting geht sie heute etwas früher, um ihre Mutter abzuholen und den als Geburtstagsgeschenk seit Langem versprochenen Konzertbesuch einzulösen. Sandras Freund, Tobias, wird die Kinder später bei ihrem Vater abholen. An diesem Tag hat Sandra bisher genügend erreicht und die wichtigen Dinge wird sie sich im kurzen E-Mail-Check am späteren Abend noch anschauen. An manchen Tagen, so denkt Sandra, läuft es wirklich schön rund. Sie kann selber bestimmen, fühlt sich akzeptiert, ihr wird Wertschätzung entgegengebracht und sie kann zudem ihre sozialen Verpflichtungen wahrnehmen. 
Spätabends im Bett ist Sandra zwar in Gedanken schon wieder beim nächsten Tag, kann aber schnell zufrieden einschlafen.

\section{Ein schlechter Tag im Leben von Sandra Könitz}

Heute ist wieder einer dieser Tage. 4.30 Uhr, der Wecker klingelt noch lange nicht. Den braucht Sandra auch nicht, denkt sie sarkastisch, wenn sie ohnehin jede dritte Nacht zu früh aufwacht, weil ihr Tausend Dinge im Kopf herumspuken. Der Job fordert, ihrer Mutter und ihrem Partner Tobias wird Sandra nur unzureichend gerecht, ebenso wie ihren Freunden, geschweige denn ihren Kindern. Manchmal fragt sie sich wirklich, warum sie das alles macht.

Die üblen Gedanken verdrängend, steht Sandra auf und setzt sich mit einem Kaffee an den Küchentisch, um die ersten „Deliverables“ des Tages zu leisten. Wenn sie sich anstrengt, sind diese fertig, noch bevor sie die Kinder bereitmachen muss und ins Geschäft fährt. An diesem Tag klappt das jedoch mal wieder gar nicht, weil sie noch wichtige Informationen von Petra, ihrer Mitarbeiterin, benötigt. Die hat die Daten gestern Abend zwar geliefert, aber in zu schlechter Qualität. Sandra spült ihren Ärger mit Kaffee runter und fordert Petra auf, ihr bis am späten Vormittag eine überarbeitete Version zu senden, damit die Unterlagen für das Kundenmeeting um 11 Uhr fertig sind.

Anschliessend schnell umziehen, die Kinder fertigmachen, ab ins Auto und ins Geschäft. Im Radio verfolgt Sandra eine Sendung zum Thema „flexibles Arbeiten", in welcher die Vorzüge gepriesen werden. Es würde eine bessere Wahrnehmung der Unternehmensverantwortung ermöglichen, so die Sprecherin. Die Sendung stösst ihr bitter auf. Sie denkt: „Flexibilität ist ja gut und schön, aber wenn es aufgrund des starken Arbeitsdrucks einfach nur bedeutet, dass man immer mehr arbeitet, wären ein paar feste Grenzen vielleicht doch angebracht." Aber gleichzeitig weiss sie, dass ihr beruflicher Erfolg im Grunde an ihrer eigenen Flexibilität, Mobilität und Erreichbarkeit hängt. Es liegt häufig an ihr zu entscheiden, wie oft sie für Akquisition neuer Geschäfte bei der Kundin vor Ort ist. Das ist meist sehr stressig und bedeutet, „zehn verschiedene Bälle in der Luft zu halten“, wie Sandra so gern sagt. In den letzten Monaten sind ihr dabei aber zu viele davon heruntergefallen. Sandra muss auch beim Teammeeting am Nachmittag genau auf die Kalkulationen schauen, denn im Geschäft ist der Preisdruck auf die Stundenlöhne enorm - grosse Teile der Routinearbeit werden heute von Indien aus erledigt. Vor einigen Jahren waren die Löhne noch fast doppelt so hoch. 
Der Akquisedruck macht sich zurzeit wieder sehr stark bemerkbar, und auch der Tinnitus, welcher seit sechs Jahren ihr ständiger Begleiter ist, stört Sandra heute gewaltig. Bereits im letzten Herbst war Sandra am Rand der Erschöpfung, als sich eine Häufung der Projektdeadlines mit dem Ausfall zweier ihrer besten Mitarbeiterinnen (und einer weiteren ungeplanten Kündigung) kreuzte. Zudem wurde auch noch ihre Tochter krank. Zum Glück schaffte sie es bis Weihnachten durchzuhalten. Immerhin gibt ihr das Hoffnung, auch den heutigen Tag durchzustehen.

Am Abend dann das Konzert mit ihrer Mutter. Sie hat Karten besorgt, musste allerdings länger im Büro bleiben, steht jetzt im Stau und kommt wie vermutet zu spät. Ihre Mutter kennt das. Da die Einladung allerdings ein nachgeholtes Geburtstagsgeschenk sein sollte, reagiert sie nur enttäuscht und nicht mehr verärgert. Nach dem Konzert fragt sie: „Wie lange willst du das noch mitmachen, das macht dich doch krank?"Sandra hasst Fragen wie diese, aber sie kommen immer wieder vor. Dabei würde sie die Antwort doch selber gern wissen. Sandra verdrängt die Grübelei, als sie am Abend ihre überlaufende Mailbox bearbeitet und danach erschöpft ins Bett fällt.

\subsubsection{Persona C: Ursula Meyerhans - „Portfoliowork auf mehreren Standbeinen"}

- 57 Jahre alt

- feste Partnerschaft, keine Kinder

- Studium der Germanistik

- Redaktorin/Journalistin mit mehreren Standbeinen:

○ Teilzeitanstellung bei einer Zeitung $(60 \%)$

- Selbstständige Tätigkeit als Texterin
Ursula Meyerhans arbeitet als Teilzeitredaktorin. Nach der Matura hat sie Germanistik und Anglistik studiert, dann als freie Journalistin für in- und ausländische Zeitungen und Zeitschriften gearbeitet. Und wann immer möglich hat sie eine inrer zahlreichen Reisen unternommen. Seit fast 15 Jahren arbeitet sie in der Redaktion einer kleinen, nationalen Zeitung, die auf gesellschaftliche und soziale Themen mit teilweise aufwendigen Recherchearbeiten spezialisiert ist. Sie hat eine Festanstellung auf Teilzeitbasis (60 Prozent), und ihre Aufgabe liegt in erster Linie darin, eine sehr grosse Gruppe von „Citizen Journalists“ zu koordinieren. Das macht ihr richtig Spass. Die Redaktion hatte vor einigen Jahren aus Zeit- und Kostengründen beschlossen, gesellschaftliche und soziale Themen, die eine zeitintensivere Recherchearbeit voraussetzen, auf der zeitungseigenen Website auszuschreiben und Citizen Journalists dafür anzuwerben. Und über die Jahre hat sich ein 
gutes Netzwerk entwickelt, das sich auch teilweise über Mund-zu-MundPropaganda weiterentwickelt hat. Ursula Meyerhans hat es geschafft, ein seit Jahren eingespieltes, nahezu professionelles Team aufzubauen. Das sieht sie und das sehen auch all ihre Kolleginnen und Kollegen als ihre ganz persönliche Leistung an.

Das Reisen ist ihr nicht mehr so wichtig wie früher, und deshalb hat sie vor fünf Jahren beschlossen, neben ihrem Teilzeitjob auch noch selbstständig zu arbeiten und sich auf die Erstellung von Werbebroschüren für komplexe, technische Produkte zu spezialisieren. Ihr Lebenspartner hat sie in diesem Vorhaben stark unterstützt. Nach einer Durststrecke von zwei bis drei Jahren hat sie es geschafft, mit mehreren Unternehmen langfristige Verträge abzuschliessen. Das gibt ihr finanzielle Sicherheit und auch die Möglichkeit, in kurzer Zeit genügend Ersparnisse aufzubauen.

Von ihren Freundinnen, Arbeitskolleginnen und Bekannten wird sie als zielorientiert, sehr hilfsbereit, intelligent und "mit beiden Beinen auf dem Boden“ beschrieben.

Sie freut sich, dass sie beruflich etabliert ist, geniesst ihren Erfolg und ihren sehr grossen Freundes- und Bekanntenkreis. Ihre mittlerweile 85-jährigen Eltern leben rund 300 Kilometer von ihr entfernt und sind pflegebedürftig. Ihr Bruder und seine Ehefrau leben in der Nähe und übernehmen zusammen mit einem professionellen Pflegedienst die Betreuung ihrer Eltern. Dafür ist sie den beiden sehr dankbar.

\begin{tabular}{|l|c|c|c|}
\hline & \multicolumn{3}{|c|}{ Typ 2-3-2-1 } \\
\hline Anstellungsverhältnis & $\begin{array}{c}\text { (1) fest ange- } \\
\text { stellt }\end{array}$ & (2) Mischformen & $\begin{array}{c}\text { (3) voll selbst- } \\
\text { ständig }\end{array}$ \\
\hline $\begin{array}{l}\text { Örtliche und zeitliche } \\
\text { Grenzen der Arbeit } \\
\text { Grenzen } \\
\text { desonomische Rolle }\end{array}$ & $\begin{array}{c}\text { (1) „Arbeste } \\
\text { kraft“ }\end{array}$ & $\begin{array}{c}\text { (2) Bandbreiten } \\
\text { unternehmer/in“ }\end{array}$ & $\begin{array}{c}\text { (3) entgrenzt } \\
\text { nehmer/in“ }\end{array}$ \\
\hline $\begin{array}{l}\text { Soziale Funktion } \\
\text { von Arbeit }\end{array}$ & $\begin{array}{c}\text { (1) Arbeit als } \\
\text { Auskommen }\end{array}$ & $\begin{array}{c}\text { (2) Arbeit als Ge- } \\
\text { meinschaft }\end{array}$ & $\begin{array}{c}\text { (3) Arbeit } \\
\text { als Selbst- } \\
\text { verwirklichung }\end{array}$ \\
\hline
\end{tabular}




\section{Ein guter Tag im Leben von Ursula Meyerhans}

Jetzt ist es 6 Uhr. Ursula Meyerhans hört sich im Bett liegend noch die Nachrichten im Radio an, dann geht der Tag los. Duschen, einen starken, schwarzen Kaffee zum Frühstück, dazu erste Online-Nachrichten und erste E-Mails auf dem Tablet. Um 7.30 Uhr macht sie sich auf den Weg in die Redaktion, knappe 10 Minuten zu Fuss, um dann die ersten Entwürfe der Citizen Journalists zu lesen. Sie hatte schon heute Morgen ein gutes Gefühl, aber jetzt hat es sich bestätigt. Ihre sehr grosse Gruppe von Citizen Journalists hat Unglaubliches geleistet. Die eingereichten Artikel sind nicht nur sehr gut recherchiert, nein, sie sind auch auf einem sehr hohen journalistischen Niveau. Sie kann jetzt sehen, dass sich die vielen Stunden gelohnt haben, in denen sie jeder einzelnen Kollegin und jedem einzelnen Kollegen ein differenziertes Feedback gegeben hat. Sie wird deshalb zwei der Artikel bei einem internationalen Citizen-JournalistsWettbewerb einreichen, und sie hofft, dass damit ihre Kolleginnen und Kollegen aus der Anzeigenabteilung bessere Chancen haben, neue und alte Anzeigenkunden zu gewinnen und weitere Abonnements abschliessen zu können. Sie selbst ist als Mitglied des Redaktionsteams am Umsatz des Zeitungsverlages beteiligt, zwar nur in bescheidenem Umfang, aber doch so, dass sie neben ihrer Arbeit als Teilzeitredaktorin auch an die Umsatzzahlen denken muss. Es ist ihr aber auch wichtig, an ihre Kolleginnen und Kollegen zu denken, Solidarität zu zeigen und sich selbst als Teil einer Gemeinschaft, eines Teams zu sehen.

Sie schaut auf die Uhr, Mittagszeit. Jetzt muss sie sich aber beeilen, sie trifft sich mit ihrer langjährigen Freundin Irene zum Business Lunch an einer kleinen Imbissbude, 30 Minuten von ihrer Redaktion entfernt. Ja, sie ist richtig neugierig. Ihre Freundin, die ebenfalls Journalistin ist, hatte schon immer eine Nase für gute Geschäftsmöglichkeiten, und dieses Mal will sie ihr erzählen, wie sie auf Crowdsourcing-Plattformen zusätzliches Geld verdienen kann. Ursula Meyerhans ist begeistert von den neuen Möglichkeiten. Sie weiss jetzt genau, auf welchen Plattformen welche Aufträge zu welchem Betrag und unter welchen Bedingungen vergeben werden. Sie nimmt sich vor, diese CrowdsourcingPlattformen am Wochenende auszuprobieren.

Und weiter geht es. Der Tag ist noch nicht zu Ende. Sie muss jetzt schnell nach Hause. Sie möchte noch mit zwei neuen Kunden telefonieren. Diese wollten einfache, aber umfassende Werbetexte, die ihre Haushaltsroboter beschreiben. Bei Neukunden braucht man immer etwas mehr Zeit, um die Anforderungen und die Erwartungshaltung abzuklären. Aber nach guten zwei Stunden ist alles geklärt. Sie soll bis in zwei Wochen die ersten Entwürfe abgeben. Darüber kann sie sich so richtig freuen: genügend Zeit, um die Arbeit seriös erledigen zu kön- 
nen, eine sehr gute Bezahlung, und zudem kann sie heute zusammen mit ihrem Lebenspartner einen gemütlichen Abend geniessen. Mittlerweile ist es 24 Uhr: Zeit zum Schlafen. Es war ein rundherum gelungener Tag.

\section{Ein schlechter Tag im Leben von Ursula Meyerhans}

Es ist jetzt 4 Uhr morgens. Ursula Meyerhans muss am Schreibtisch eingeschlafen sein. So ein Ärger aber auch, dass ihr das gerade jetzt passiert. Sie hätte bis Mitternacht für zwei Werbebroschüren das Gut zum Druck erteilt haben sollen, aber stattdessen ist sie vor Übermüdung eingeschlafen. Sie könnte heulen. Weitere Aufträge wird es von diesem Kunden wohl nicht mehr geben: In diesem Punkt war er sehr deutlich. An solchen Tagen hasst sie die selbstständige Tätigkeit. Schlafen kann sie nicht mehr, aber vielleicht schon die ersten Nachrichten und E-Mails anschauen, und dann das übliche Programm: $6 \mathrm{Uhr}$ aufstehen, duschen, Kaffee trinken und dann um 7.30 Uhr zu Fuss in die Redaktion gehen.

Sie wirft noch einen Blick in den Briefkasten und findet dabei eine ausserordentlich hohe Rechnung für die professionelle Betreuung ihrer pflegebedürftigen Eltern. Das fehlte gerade noch! Sie hat zwar Ersparnisse, aber diese sind eigentlich für die Pensionierung vorgesehen. Im Büro angekommen, erwartet sie schon die nächste schlechte Nachricht. Um $11 \mathrm{Uhr}$ erfolgt eine kurzfristig angesetzte Mitarbeitendenversammlung, an der allen mitgeteilt wird, dass der Lohn der ganzen Belegschaft zwischen fünf und zehn Prozent gekürzt wird. In all den letzten Jahren ist es aus ihrer Sicht immer schlimmer geworden: zuerst Entlassungen, dann Lohnkürzungen, Zusatzaufgaben und jetzt eine weitere Lohnkürzungsrunde. Sie versucht die anfallenden Arbeiten so gut wie möglich zu erledigen und verlässt dann gegen $13 \mathrm{Uhr}$ die Redaktion. Vielleicht trifft sie diese Lohnkürzung auch deshalb so stark, weil die Redaktion ein zentraler Punkt in ihrem Leben ist. Aber ganz auf die feste Stelle verzichten und allein von der Selbstständigkeit leben - diese Unsicherheit ist ihr dann doch zu gross. Sie geht nach Hause, isst eine Kleinigkeit und setzt sich dann wieder an die Arbeit. Sie hat angefangen, auf verschiedenen Crowdworking-Plattformen journalistische Auftragsarbeiten zu übernehmen. Sie schaut gerade auf die Uhr, $20.15 \mathrm{Uhr}$, es ist spät geworden, aber trotzdem, sie muss noch eine Weile arbeiten, so lange bis der Auftrag fertig ist. Wenn sie diese Auftragsarbeit früher abgibt, erhält sie eine kleine Prämie. Gerade ist eine E-Mail angekommen. Eine erledigte Auftragsarbeit ist ohne Bezahlung abgelehnt worden. Ähnliches ist ihr auf dieser Crowdsourcing-Plattform bereits zwei Mal passiert: Da hatte sich eine potenzielle Auftraggebende verschiedene Texte für Werbebroschüren vor- 
schlagen lassen - um am Ende festzustellen, dass sie nicht wusste, was sie wollte. Und so gingen alle leer aus. In letzter Zeit kommt es auch häufiger vor, dass sie vor lauter Müdigkeit viele Flüchtigkeitsfehler macht. Und dabei war sie früher immer so stolz auf ihre einwandfreie Arbeit. Ursula ist gar nicht zufrieden. Sie muss etwas ändern. Radikal. Sie weiss bloss nicht was. Und wirklich Zeit, um darüber nachzudenken, findet sie schon seit Monaten nicht.

\subsubsection{Persona D: Noah Schmid - „Entrepreneurship“}

- männlich

- 31 Jahre alt

- unverheiratet, in fester Beziehung, keine Kinder

- Informatiker ETH

- selbstständiger Softwareentwickler

- arbeitet zu Hause, im Coworking-Space, oder „überall, wo es WLAN gibt"

- Karrieretyp: eigenverantwortlich
Noah Schmid ist selbstständiger Softwareentwickler mit mehreren Standbeinen. Zum einen programmiert er Webshops für kleine Unternehmen, was ihm ein einigermassen regelmässiges Einkommen sicherstellt. Daneben nimmt er immer wieder weitere Aufträge im Bereich Webdesign an. Viele von diesen werden über eine Crowdsourcing-Plattform ausgeschrieben. Neben dieser Auftragsarbeit träumt Noah Schmid aber auch davon, mit einem eigenen Produkt Erfolg zu haben: Zusammen mit einem Studienfreund entwickelt er eine Bezahl-App, mit der über das Smartphone kleinere Geldbeträge ausgetauscht werden können.

Einen festen Arbeitsplatz hat er nicht. Am ehesten ist das noch der CoworkingSpace, in dem er mehrere Tage in der Woche arbeitet und seine Kolleginnen und Kollegen, Freundinnen und Freunde trifft. Daneben arbeitet er auch oft zu Hause, in einem Café oder überall, wo es WLAN gibt.

Auch zeitlich ist er flexibel: Mal arbeitet er bis spät in die Nacht, wenn ein Auftrag für einen Kunden oder eine Kundin noch dringend fertig werden muss. Mal gibt es aber auch schon um 16 Uhr ein Feierabendbier mit den Kolleginnen aus dem Coworking-Space oder es ergibt sich die Gelegenheit zu einem spontan Nachtessen. Grenzen zwischen Arbeit und Privatem gibt es für Noah praktisch nicht.

Er ist seit drei Jahren in einer festen Beziehung und wohnt mit seiner Freundin Sandra zusammen. Sie arbeitet in einem Reisebüro.

Als selbstständig Erwerbender zahlt Noah Schmid nur in die erste Säule der Sozialversicherung ein. Daneben überweist er in unregelmässigen Abständen immer wieder etwas in seine private Altersvorsoge (Säule 3a) bei einer Bank. 


\begin{tabular}{|l|c|c|c|}
\hline & \multicolumn{3}{|c|}{ Typ 3-3-3-3 } \\
\hline Anstellungsverhältnis & $\begin{array}{c}\text { (1) fest ange- } \\
\text { stellt }\end{array}$ & (2) Mischformen & $\begin{array}{c}\text { (3) voll selbst- } \\
\text { ständig }\end{array}$ \\
\hline $\begin{array}{l}\text { Örtliche und zeitliche } \\
\text { Grenzen der Arbeit }\end{array}$ & $\begin{array}{c}\text { (1) feste } \\
\text { Grenzen }\end{array}$ & (2) Bandbreiten & (3) entgrenzt \\
\hline $\begin{array}{l}\text { Ökonomische Rolle } \\
\text { des Individuums }\end{array}$ & $\begin{array}{c}\text { (1) „Arbeits- } \\
\text { kraft“ }\end{array}$ & $\begin{array}{c}\text { (2),Arbeitskraft- } \\
\text { unternehmer/in“ }\end{array}$ & $\begin{array}{c}\text { (3) „Unter- } \\
\text { nehmer/in“ }\end{array}$ \\
\hline $\begin{array}{l}\text { Soziale Funktion } \\
\text { von Arbeit }\end{array}$ & $\begin{array}{c}\text { (1) Arbeit als } \\
\text { Auskommen }\end{array}$ & $\begin{array}{c}\text { (2) Arbeit als Ge- } \\
\text { meinschaft }\end{array}$ & $\begin{array}{c}\text { (3) Arbeit } \\
\text { als Selbst- } \\
\text { verwirklichung }\end{array}$ \\
\hline
\end{tabular}

\section{Ein guter Tag im Leben von Noah Schmid}

Noah Schmid lässt diesen Tag gemütlich angehen: Ohne einen Wecker gestellt zu haben, wacht er von alleine gegen 7.30 Uhr auf. Nach dem Frühstück setzt er sich zu Hause an den Computer und arbeitet an einem Kundenauftrag weiter. Die Verwaltung seiner Gemeinde hat inn beauftragt, eine Webshop-Lösung für die Gemeindehomepage zu entwickeln, mit der Dienstleistungen der Gemeinde, wie z.B. Betreibungsauskünfte, zukünftig online bestellt und per Kreditkarte bezahlt werden können. Weil genau dies zu seinen Kompetenzen zählt und die Gemeinde auch noch gut zahlt, macht inm der Auftrag Spass. Zudem freut er sich, einen Beitrag zur Verbesserung der Kundenfreundlichkeit seiner Gemeinde leisten zu können.

Gegen 10 Uhr macht er eine kleine Pause und stellt fest, dass das Wetter überraschend angenehm ist. Er beschliesst daher, auf dem Balkon weiterzuarbeiten. Es tut gut, ein bisschen Sonne abzubekommen.

Für das Mittagessen trifft er sich in einem Restaurant mit seiner Freundin Sandra. Sie arbeitet zwar mehrheitlich im Büro, geniesst aber ebenfalls einige Freiheiten, was die Arbeitszeitgestaltung angeht. Sie sprechen mal wieder darüber, eine Familie zu gründen. Beide möchten gerne Kinder bekommen und die Betreuung gemeinsam wahrnehmen. Sobald es soweit ist, so planen sie, wird Sandra Teilzeit arbeiten und Noah sich ebenfalls einen „Vatertag“ pro Woche frei nehmen für das Kind.

Am Nachmittag möchte er lieber etwas anderes in Angriff nehmen, und da der Auftrag für die Gemeinde noch nicht dringend ist, entscheidet er sich, an seinem Lieblingsprojekt weiterzuarbeiten: die Entwicklung einer App für das Be- 
zahlen kleinerer Geldbeträge. Hierfür geht Noah in den Coworking-Space im Nachbarquartier, wo er für einen monatlichen Beitrag die Infrastruktur nutzen kann. Er trifft dort seine „Arbeitskolleginnen und -kollegen“ - selbstständige „Entrepreneurs" in einer ähnlichen Situation wie er. Es tut gut, diesen Austausch zu haben und nicht immer nur alleine vor sich hin zu arbeiten. Gegen 17 Uhr entscheiden Noah Schmid und ein paar Kollegen sich, die Arbeit am PC ruhen zu lassen und sie gehen auf ein Bier in eine nahe gelegene Bar. Dort diskutieren sie angeregt über Potenziale und Risiken ihrer Projekte.

Am Abend ist er alleine zu Hause, weil seine Freundin im Ausgang ist. Per EMail wird er über eine neue Offertenanfrage auf einer Crowdsourcing-Plattform benachrichtigt: Ein mittelgrosses Unternehmen aus den USA möchte eine Webshop-Lösung umgesetzt haben. Das Unternehmen würde den Auftrag in Bitcoins zahlen, was Noah Schmid sehr recht wäre. So tauchen die Einkünfte nirgends in seinen Unterlagen auf und er kann sie bei der Steuer "vergessen“. Noah Schmid macht sich sofort an die Arbeit und erstellt eine Offerte. Nach eineinhalb Stunden ist sie fertig und er schickt sie ab. Müde, aber zufrieden geht er ins Bett.

\section{Ein schlechter Tag im Leben von Noah Schmid}

Für Noah Schmids Verhältnisse ist ein Wecker um 6 Uhr morgens äusserst früh. Aber heute hat er keine andere Wahl, es stehen einfach noch zu viele Dinge auf seiner To-do-Liste. Nach einem schnellen Frühstück setzt er sich bereits um $7 \mathrm{Uhr}$ noch in der Küche an den Laptop und arbeitet ein paar kleinere Pendenzen ab. Die E-Mails haben sich angehäuft: Die Gemeinde will wissen, wie er im Zeitplan liegt für die Entwicklung der Webshop-Lösung. Gleich zwei Auftraggeber haben inm Änderungswünsche für ihre Aufträge geschickt. Und dann ist da noch dieses nervige Problem mit der Bewertung auf dem Crowdsourcing-Portal. Ein früherer Auftraggeber hat inm für seine Arbeit eine sehr schlechte Bewertung gegeben, die Noah aber ungerechtfertigt findet. Jetzt versucht er, bei den Betreibern der Plattform zu intervenieren, doch die stellen sich stumm. Dies sei allein Sache zwischen den beteiligten Parteien, sagen sie. Dass es somit aber keine Möglichkeit für Noah gibt, unfaire Bewertungen infrage zu stellen, lässt sie kalt. Solche Turbulenzen mit Plattformen im Internet, welche inn bares Geld kosten, hat er schon zuhauf erlebt. Zwar hat er das Gefühl, es langsam im Griff zu haben, aber es treten immer wieder neue aufreibende Konflikte auf.

Nach gut einer Stunde macht er sich ans Programmieren. Doch der Stress wegen der schlechten Bewertung sitzt inm im Nacken und er kann sich nicht so 
gut konzentrieren wie sonst. In solchen Momenten wünscht er sich, „richtige“ Arbeitskolleginnen und -kollegen zu haben, mit denen man gemeinsam über unfaire Verhältnisse im Betrieb klagen und anschliessend über Fussball reden kann. Sicher, er hat die Leute im Coworking-Space, doch die haben alle auch ihre eigenen Sorgen.

Trotz Ärger kommt er dann doch in einen Arbeitsmodus beim Programmieren, sodass er fast das Mittagessen vergisst. Schnell sucht er sich etwas aus dem Kühlschrank, bevor er losspurten muss zum Meeting mit den Gemeindevertretern. Denen muss er die neueste Vorabversion seiner Arbeit zeigen. Sie sind nur mässig zufrieden und haben ihre Gestaltungswünsche mal wieder geändert, sodass Noah einen Teil der bisherigen Arbeit umsonst gemacht hat.

Am frühen Abend kommt er in seine Wohnung zurück, wo er mit Sandra zu Abend isst. Sie reden über das Thema Kinder, und es fällt innen mal wieder auf, wie schwierig alles werden könnte. Denn mit ihrem Teilzeitgehalt und Noahs sehr variablen Einkünften könnte es problematisch sein, sich finanziell über Wasser zu halten. Am ehesten würden sie wohl Kürzungen machen bei der Altersvorsorge, aber eigentlich gefällt innen diese kurzfristige Denkweise überhaupt nicht. Es bleibt innen nichts anderes übrig, als auf den grossen Erfolg mit seiner App zu hoffen. Getrieben durch diese Überlegungen setzt sich Noah noch für ein bis zwei Stunden an dieses Projekt. Eigentlich ist es sein Lieblingsprojekt, aber jetzt würde er auch wirklich gern einen gemütlichen Abend mit seiner Freundin verbringen. Erschöpft und etwas gefrustet landet er spät im Bett und schläft mehr schlecht als recht ein.

\subsubsection{Persona E: Andrea Burri-Lötscher - „Mehrfachanstellungen und Minijobs“"}
- weiblich
- 43 Jahre alt
- verheiratet
- zwei Kinder
- erlernter Beruf: abgebrochene Aus- bildung als Köchin
- arbeitet Teilzeit als Küchenhilfe im Pflegeheim
- zusätzlich diverse kleinere Tätigkeiten

Andrea Burri-Lötscher arbeitet als Küchenhilfe in einem Pflege- und Altersheim. Ihre Anstellung ist unbefristet, ihr Teilzeitpensum beträgt 50 Prozent. Nach der obligatorischen Schulausbildung hat sie eine Ausbildung zur Köchin angefangen, diese aber abgebrochen. Seit ihre Kinder grösser sind, versucht sie noch zusätzliches Geld zu verdienen, in dem sie Arbeiten gegen Dienstleistungsschecks (so genannte Voucher-based Work) und auf Crowdsourcing-Plattformen übernimmt und stundenweise gegen Barzahlung arbeitet. 
Alle ihre Freundinnen leben in ähnlichen beruflichen und privaten Verhältnissen. Ihr Ehemann Urs meint, sie müsste entschiedener auftreten und von ihrem Vorgesetzten eine Lohnerhöhung verlangen. Aber seit zwei ihrer Kolleginnen entlassen worden waren, nachdem sie sich über die Arbeitsverhältnisse beklagt haben, ist sie noch vorsichtiger und zurückhaltender geworden.

Ihre mittlerweile 75-jährigen Schwiegereltern leben bei innen im Haushalt. Ihr Ehemann Urs und sie haben das so entschieden, nachdem sie feststellen mussten, dass sich ihre Schwiegereltern nicht mehr selbst versorgen können. Sie haben deshalb eine 4,5-Zimmer-Wohnung bei einer sozialen Wohnbaugenossenschaft gemietet und Andrea Burri-Lötscher hat ihr Arbeitspensum von 40 Prozent auf 50 Prozent erhöht und weitere Anstrengungen unternommen, um ihren Zusatzverdienst zu erhören. Ansonsten könnten sie sich diese Wohnung gar nicht leisten. Ja, es ist manchmal eng in der Wohnung und Spannungen aufgrund des engen Zusammenlebens bleiben nicht aus. Aber ihre Schwiegereltern haben ihnen früher geholfen, und jetzt helfen sie ihnen.

\begin{tabular}{|l|c|c|c|}
\hline & \multicolumn{3}{|c|}{ Typ 2-1-1-1 } \\
\hline Anstellungsverhältnis & $\begin{array}{c}\text { (1) fest ange- } \\
\text { stellt }\end{array}$ & (2) Mischformen & $\begin{array}{c}\text { (3) voll selbst- } \\
\text { ständig }\end{array}$ \\
\hline $\begin{array}{l}\text { Örtliche und zeitliche } \\
\text { Grenzen der Arbeit }\end{array}$ & $\begin{array}{c}\text { (1) feste } \\
\text { Grenzen }\end{array}$ & (2) Bandbreiten & (3) entgrenzt \\
\hline $\begin{array}{l}\text { Ökonomische Rolle } \\
\text { des Individuums }\end{array}$ & $\begin{array}{c}\text { (1) „Arbeits- } \\
\text { kraft“ }\end{array}$ & $\begin{array}{c}\text { (2) „Arbeitskraft- } \\
\text { unternehmer/in“ }\end{array}$ & $\begin{array}{c}(3), \text { Unter- } \\
\text { nehmer/in“ }\end{array}$ \\
\hline $\begin{array}{l}\text { Soziale Funktion } \\
\text { von Arbeit }\end{array}$ & $\begin{array}{c}\text { (1) Arbeit als } \\
\text { Auskommen }\end{array}$ & $\begin{array}{c}\text { (2) Arbeit als Ge- } \\
\text { meinschaft }\end{array}$ & $\begin{array}{c}\text { (3) Arbeit } \\
\text { als Selbst- } \\
\text { verwirklichung }\end{array}$ \\
\hline
\end{tabular}

\section{Ein guter Tag im Leben von Andrea Burri-Lötscher}

Heute Morgen ist Andrea Burri-Lötscher um 5.30 Uhr aufgestanden, hat das Frühstück für ihren Ehemann und die Kinder gemacht und nachdem alle aus dem Haus sind, hat sie sich um ihre Schwiegereltern gekümmert und dann zusammen mit innen gefrühstückt. Ihre Schicht im Pflege- und Altersheim beginnt erst um 13.30 Uhr. Ihre Schwiegereltern werden den ganzen Tag im nahe gelegenen Gemeindehaus verbringen. Die private Stiftung „Eltern \& Co.“ organisiert immer wieder halbe oder ganze Tage, an denen ältere Personen kostenlos, professionell und zuverlässig betreut und die Familienangehörigen entlastet 
werden. Diese Tage helfen ihr, sich um weitere Möglichkeiten für einen Zusatzverdienst zu kümmern. Um 9 Uhr wird sie in einem Privathaushalt putzen, waschen und kochen. Jetzt muss sie natürlich noch zeitlich und organisatorisch alles unter einen Hut bringen. Aber ihre beiden Kinder, 10 und 12 Jahre alt, sind sehr selbstständig. Sie wissen, dass von innen mehr Eigenverantwortung, Selbstständigkeit und Verantwortung erwartet wird als von ihren gleichaltrigen Freunden. Um 12 Uhr geht es dann nach Hause, vorkochen für die Kinder, selbst etwas essen, umziehen und dann ab zur Arbeit. Den Abwasch übernehmen ihre beiden Kinder. Um 13.30 Uhr beginnt sie zu arbeiten, die Arbeit geht ihr leicht von der Hand und kurz vor Schichtende bittet sie ihr Vorgesetzter zu sich ins Büro. Er fragt sie tatsächlich, ob sie nicht die Schichtleitung übernehmen will. Sie kann es nicht fassen. Das muss heute ihr Glückstag sein. Natürlich bedeutet es mehr Verantwortung, aber wie sie gerade erfahren hat, auch mehr Geld. Sie sagt ohne zu überlegen zu. Das Geld können sie so gut brauchen, denn die Rente der Schwiegereltern deckt ihre Betreuungskosten und die zusätzlichen Mietkosten nicht ab.

Um 21 Uhr geht Andrea Burri-Lötscher zwar müde, aber zufrieden nach Hause. Ihr Ehemann, die Schwiegereltern und ihre Kinder sind alle da. Sie erzählt innen die Neuigkeiten. Sie geniesst die wenigen Minuten des persönlichen Gesprächs mit ihrem Ehemann, bevor sie einschläft.

\section{Ein schlechter Tag im Leben von Andrea Burri-Lötscher}

Jetzt ist es 5.00 Uhr. Andrea Burri-Lötscher muss notfallmässig die Frühschicht um 6 Uhr übernehmen. Sie hasst den Personalmangel im Heim, der immer wieder solche Hauruck-Aktionen erfordert. Früher war das besser. Bevor sie losfährt, weckt sie noch ihren Ehemann und sagt ihm, dass er sich um seine Eltern kümmern müsse. Sie will jetzt nicht darüber nachdenken, wie sich die zusätzlichen Belastungen mit Schwiegereltern und erhöhtem Arbeitspensum auf ihre Ehe auswirken. Sie arbeitet unkonzentriert, macht viele Fehler und fühlt sich überfordert. Aufgrund ihrer mangelnden Belastbarkeit teilt inr Vorgesetzter sie jetzt immer öfters am Samstag und Sonntag ein. Der Zeitdruck ist dann zwar etwas geringer, aber inr Privatleben leidet unter dieser ständigen Abwesenheit. Hinzu kommt, dass sie seit der Privatisierung des Pflege- und Altersheims nicht nur einen neuen, sondern auch einen schlechteren Arbeitsvertrag bekommen hat. Das macht ihr zu schaffen. Sie muss viel darüber nachdenken, und an einem Tag wie heute hat sie sehr grosse Angst, ihren Arbeitsplatz zu verlieren. Sie hält deshalb ihren Mund. 
Um 13.30 Uhr geht Andrea Burri-Lötscher eilig nach Hause, ihre Schwiegereltern und ihre Kinder warten auf sie. Eigentlich müsste sie sich dringend einmal Zeit nehmen für einen Arzttermin: Der Husten, der sie bereits seit mehreren Wochen plagt, geht einfach nicht weg. Doch die Zeit dafür zu finden neben der ganzen Arbeit, der Familie und den Schwiegereltern ist schwierig.

Um 15 Uhr hat Andrea Burri-Lötscher bereits ihren nächsten Termin. Über eine Crowdsourcing-Plattform hat sie einen Auftrag gewinnen können: für eine Treuhandgesellschaft soll sie einen Apéro organisieren und betreuen. Um 14 Uhr erreicht sie die Nachricht, dass der Apéro aus Krankheitsgründen abgesagt wurde. So ein Pech aber auch. Damit erhält sie auch kein Geld und die Zeit kann sie kurzfristig auch nicht mit anderen bezahlten Arbeiten füllen. Sie versucht deshalb, die Zeit zu nutzen, um weitere Aufträge auf Crowdsourcing- oder Tausch-Plattformen zu finden. Nach etwa drei Stunden kann sie immerhin ein Tauschgeschäft (mehrere Kuchen backen gegen Steuererklärung ausfüllen) aushandeln. Normalerweise kann sie in der gleichen Zeit zwei Tauschgeschäfte und zwei Crowdworking-Aufträge abschliessen. Aber heute scheint es keine passenden Aufträge für geringqualifizierte Arbeitskräfte wie sie zu geben. Wenn man zur Berechnung des Stundenlohns die Zeit der Arbeitssuche auch dazu nimmt, so liegt der Lohn oftmals unter dem angedachten Mindestlohn. Das macht sie traurig und wütend zugleich. Sie war jetzt zwar den ganzen Nachmittag zu Hause, aber Zeit für ihre Kinder hatte sie trotzdem nicht. Heute gibt es auch kein gemeinsames Abendessen, was sie schade findet, aber durch die Unregelmässigkeiten inzwischen viel zu oft vorkommt. Ein Gespräch mit ihrem Ehemann Urs ist nur noch im Schlafzimmer kurz vor dem Einschlafen möglich. Aber selbst dazu reicht es heute nicht. Sie fühlt sich matt und leer und schläft erschöpft ein.

\subsection{Fazit}

Um die Frage, wie sich der Stellenwert der Arbeit in der Gesellschaft aufgrund der bisher diskutierten Phänome verändert, abschliessend beantworten zu können, muss eine weitere Vereinfachung und "Komprimierung“ der Themen vorgenommen werden. Eine Variante hierfür ist, die Veränderungen in der Arbeitswelt in zwei Dimensionen zu begreifen: eine Dimension der zunehmenden physischen Entgrenzung von Arbeit und eine Dimension der zunehmenden Subjektivierung von Arbeit. 


\subsubsection{Die physische Entgrenzung von Arbeit}

Die Fabrik in der frühen Industrialisierung kann als Metapher für die nicht entgrenzte Arbeitswelt herangezogen werden. Auch wenn in früheren Zeiten die wöchentlichen Arbeitszeiten der Arbeitenden durchaus höher waren, so waren sie dennoch klar begrenzt: Arbeit beginnt, wenn man die Fabrik betritt, und sie hört auf, wenn man das Gelände verlässt. Sie beginnt und endet zu festgelegten Zeiten - und zwischendrin läutet die Sirene die Mittagspause ein. Aufgrund dieser physikalischen Erfassbarkeit der Grenzen von Arbeit sind diese denn auch sehr gut verhandelbar. Dieses „Fabrikmodell der Arbeit" hat die Arbeitswelt über viele Jahrzehnte hinweg geprägt, doch mit der Digitalisierung beginnt es nach und nach an Bedeutung zu verlieren. Arbeit ist jetzt theoretisch immer und überall möglich. Die Aushandlung der Grenzen von Arbeit wird damit zunehmend schwieriger - zumindest auf einer allgemein gültigen, kollektiven Ebene. Ein Zeichen hierfür ist der Konflikt um die Neuregelung der gesetzlichen Pflicht zur Zeiterfassung. Die Aufgabe der Grenzziehung wird also zunehmend auf das Individuum übertragen - und taucht hier unter dem Stichwort „Boundary-Management“ in der psychologischen Literatur auf.

Das Management der Grenzen zwischen verschiedenen Lebensbereichen (Arbeit, Familie, Hobbys, Erholung, soziale Teilhabe, Aus- und Weiterbildung, Freiwilligenengagement etc.) kann gelingen oder scheitern - wobei diese Begriffe nicht so zu verstehen sind, dass die Gründe für das Gelingen oder Scheitern einzig beim Individuum zu finden sind. Denn selbstverständlich bedingt das Aushandeln von Grenzen ein Gegenüber, mit dem man aushandelt - die Arbeitgeberin, die Familie, die Ausbildungsinstitution, der Verein etc. - und dieses Gegenüber kann ausgewogene Grenzziehungen hindern oder fördern. Im Fall der Begrenzung von Arbeit liegt hier ein gehöriger Spielraum bei der Arbeitgeberin: Wenn beispielsweise „flexibles Arbeiten“ angeboten wird, aber eigentlich damit die Erwartung verbunden ist, dass dies nur eine einseitige Ausweitung der Arbeit in andere Lebensbereiche bedeutet (z.B. E-Mails bearbeiten am Abend), wird es für das Individuum schwer, gesunde und ausgewogene Grenzen zu ziehen. Wenn im Folgenden also von gelingendem oder scheiterndem Boundary-Management die Rede ist, dann ist damit ein Aushandlungsprozess mit Beteiligung vieler verschiedener Stakeholder gemeint. Gelingendes Boundary-Management zeigt sich in einer subjektiv stimmigen Ausgewogenheit zwischen Arbeit und allen anderen Lebensbereichen (deutlich sichtbar z.B. am "guten Tag" von Persona A oder Persona D). Hierbei kann es individuell sehr unterschiedlich sein, „wie viel“ Arbeit als angenehm angesehen wird: Wer einen Beruf mit hohem subjektiven Sinngehalt hat, in welchem er oder sie sich entfalten kann, der oder die ist womöglich glücklich mit einer 50-Stunden-Woche 
(z.B. Persona B und Persona D). Im Gegensatz ist es für Personen, für die Arbeit hauptsächlich der finanziellen Absicherung dient, wahrscheinlich sehr wichtig, den Umfang der Arbeit klar begrenzen und anderen Lebensbereichen genügend Platz geben zu können (z.B. Persona E). Ein scheiterndes BoundaryManagement drückt sich dementsprechend darin aus, dass andere Lebensbereiche stark und dauerhaft zugunsten einer Überpriorisierung der Arbeit vernachlässigt werden (am häufigsten wohl die Erholung und Gesundheit sowie das Familienleben).

\subsubsection{Die Subjektivierung von Arbeit}

Die zweite Dimension, auf der sich eine Veränderung des Stellenwerts von Arbeit feststellen lässt, ist die Frage, wie sehr Individuen bei ihrer Arbeit unternehmerische Risiken auf sich nehmen. Auch hier kann zur Kontrastierung das Fabrikmodell herangezogen werden: In der Fabrik sind die Arbeitenden nicht verantwortlich für das Erzielen bestimmter Verkaufszahlen oder die Erschliessung von Absatzmärkten. Zwar hängen ihre Arbeitsplätze am unternehmerischen Erfolg der Fabrik, doch gibt es mit den Sozialversicherungen und weiteren Errungenschaften der sozialen Marktwirtschaft zumindest eine grundlegende Absicherung. Eine Veränderung ist in den letzten Jahrzehnten dahin gehend zu beobachten, dass von Arbeitnehmenden zunehmend erwartet wird, sich proaktiv für den Unternehmenserfolg einzusetzen und anteilweise unternehmerische Verantwortung zu übernehmen. Auch enthalten Arbeitsmodelle mit selbstständiger (z.B. Persona D) oder teilselbstständiger Arbeit (z.B. Persona C) natürlich unternehmerische Aspekte. Auch wenn bei der Zahl der selbstständig Erwerbstätigen derzeit in der Schweiz keine Zunahme erkennbar ist, hat dennoch die Idee von Unternehmertum („Entrepreneur-ship“) in letzter Zeit deutlich Aufwind bekommen (sichtbar u.a. an der verstärkten Start-upFörderung an Universitäten und Fachhochschulen). Flexible Anstellungsverhältnisse (Honorarbasis, Freelancing, Teilzeitarbeit) gehen oft mit reduziertem Einkommen einher - zumindest aber mit der Gefahr desselben. Anders ausgedrückt: Wer Teilzeit arbeiten möchte, um z.B. für die eigenen Kinder zu sorgen, muss sich dies auch leisten können - für Niedriglohnempfänger ist dies letztlich nur mit hohen Einbussen möglich. In diesem Sinne sind also derzeit verschiedene gesellschaftliche Trends oder Phänomene erkennbar, die in Richtung einer zunehmenden ökonomischen Eigenverantwortung und einer eigenständigen ökonomischen Absicherung gehen. 
Diese ökonomische Absicherung des Individuums kann ebenso wie das Boundary-Management gelingen oder scheitern - wobei auch hier die Begriffe nicht als monokausal beim Individuum verortet zu verstehen sind. Eine gelungene ökonomische Eigenverantwortung drückt sich darin aus, dass mittels der (flexibilisierten) Arbeit ein ausreichendes Einkommen erwirtschaftet werden kann und eine ausreichende soziale Absicherung besteht. Dies kann ganz unterschiedliche Formen annehmen: Die Personas A und B z.B. erreichen eine soziale Absicherung überwiegend durch die Einzahlung in die sozialen Sicherungssysteme (1. und 2. Säule) sowie auch durch das Aufsparen eines gewissen Vermögens. Persona $D$ hingegen versucht, durch die Umsetzung einer klugen, erfolgreichen Geschäftsidee mittelfristig Vermögen und damit eine finanzielle Absicherung zu erreichen. Entscheidend für eine gelingende ökonomische Absicherung ist also auch das (unternehmerische) Risiko, das eingegangen wird, und die zugehörigen Erfolgschancen. Persona E und in gewissem Masse auch Persona $C$ können als Beispiel dafür dienen, dass sie zwar ein hohes Mass an Eigenverantwortung bei der Organisation ihrer Arbeit zeigen, sie jedoch nur geringe Chancen haben (insbesondere Persona E), mit diesen eigenverantworteten Jobs eine nachhaltige finanzielle Absicherung zu erzielen.

\subsubsection{Boundary-Management und ökonomische Absicherung in der Entwicklungsmatrix}

Die beiden Phänomene der physischen Entgrenzung sowie der Subjektivierung von Arbeit lassen sich in einem Vier-Felder-Schema miteinander kombinieren (siehe Abbildung 6).

In der Abbildung ist rechts oben ein Idealzustand beschrieben, in welchem Individuen die Vorzüge der flexibilisierten Arbeitswelt im Sinne einer ausgeglichenen Life-Domain-Balance bei gleichzeitiger ökonomischer Absicherung geniessen können. Im Feld rechts unten ist ein Lebens- und Arbeitsentwurf beschrieben, wie er womöglich heutzutage oft in gut bezahlten Führungsjobs zu finden ist: Arbeit dominiert das Leben auf Kosten anderer Lebensbereiche und/oder der Gesundheit, aber die finanzielle Absicherung ist - auch aufgrund des hohen Engagements - mehr als gegeben. Das Feld links oben beschreibt einen Lebensentwurf, der womöglich etwas weniger verbreitet ist, aber dennoch denkbar ist: Die Vorzüge von Arbeitsmodellen ausserhalb des Vollzeit-Normalarbeitsverhältnisses werden genossen (z.B. beschränkt man sich auf eine Teilzeitarbeit oder nur sporadische bezahlte Jobs mit längeren Pausen dazwischen) und es herrscht eine mehr als ausgewogene Balance zwischen Arbeit und anderen Lebensbereichen. Die finanziellen Einbussen, die damit einhergehen, werden in 
Kauf genommen, es herrscht aber langfristig die Gefahr einer mangelnden sozialen Absicherung. Das Feld links unten schliesslich stellt den "Worst Case“ der flexibilisierten Arbeitswelt dar: Flexible Anstellungsverhältnisse ohne soziale Absicherung und mit schlechtem Lohn nehmen einen dominanten Platz im Leben der Personen ein, sodass andere Lebensbereiche wie Familie, Erholung etc. langfristig zu kurz kommen. Die Extremvariante dieser prekarisierten Arbeit erfüllt die Kriterien der ILO für „Decent Work“ (ILO, 2012) nicht mehr.

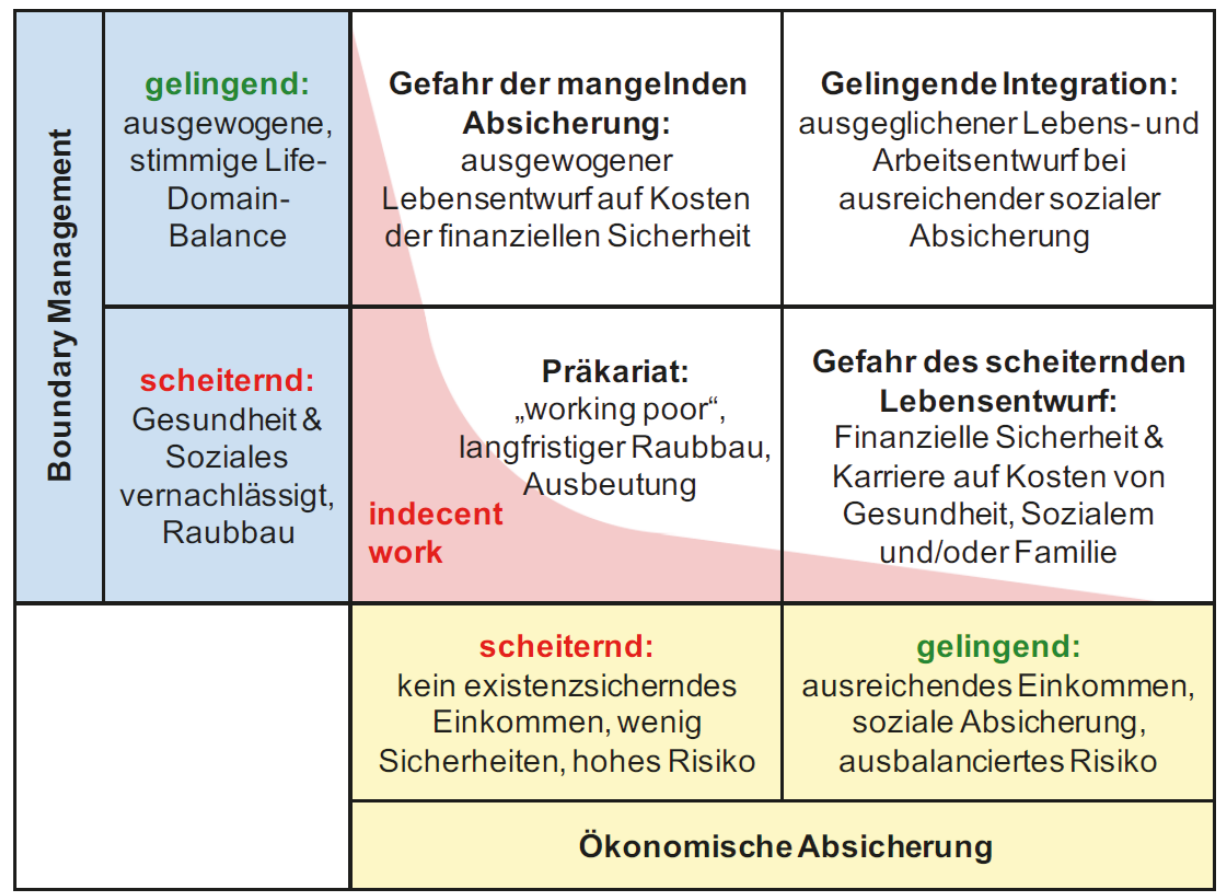

Abbildung 6: Entwicklungsmatrix

In diesem Vier-Felder-Schema können abschliessend die Personas grob verortet werden. In Abbildung 7 sind alle fünf Personas markiert, und zwar jeweils mit ihrem guten und ihrem schlechten Tag (z.B. A+ bzw. A-). Diese Einordnung ist natürlich nur annäherungsweise möglich und ist daher einzig zur Veranschaulichung sowohl der Personas als auch der Entwicklungsmatrix zu verstehen. 


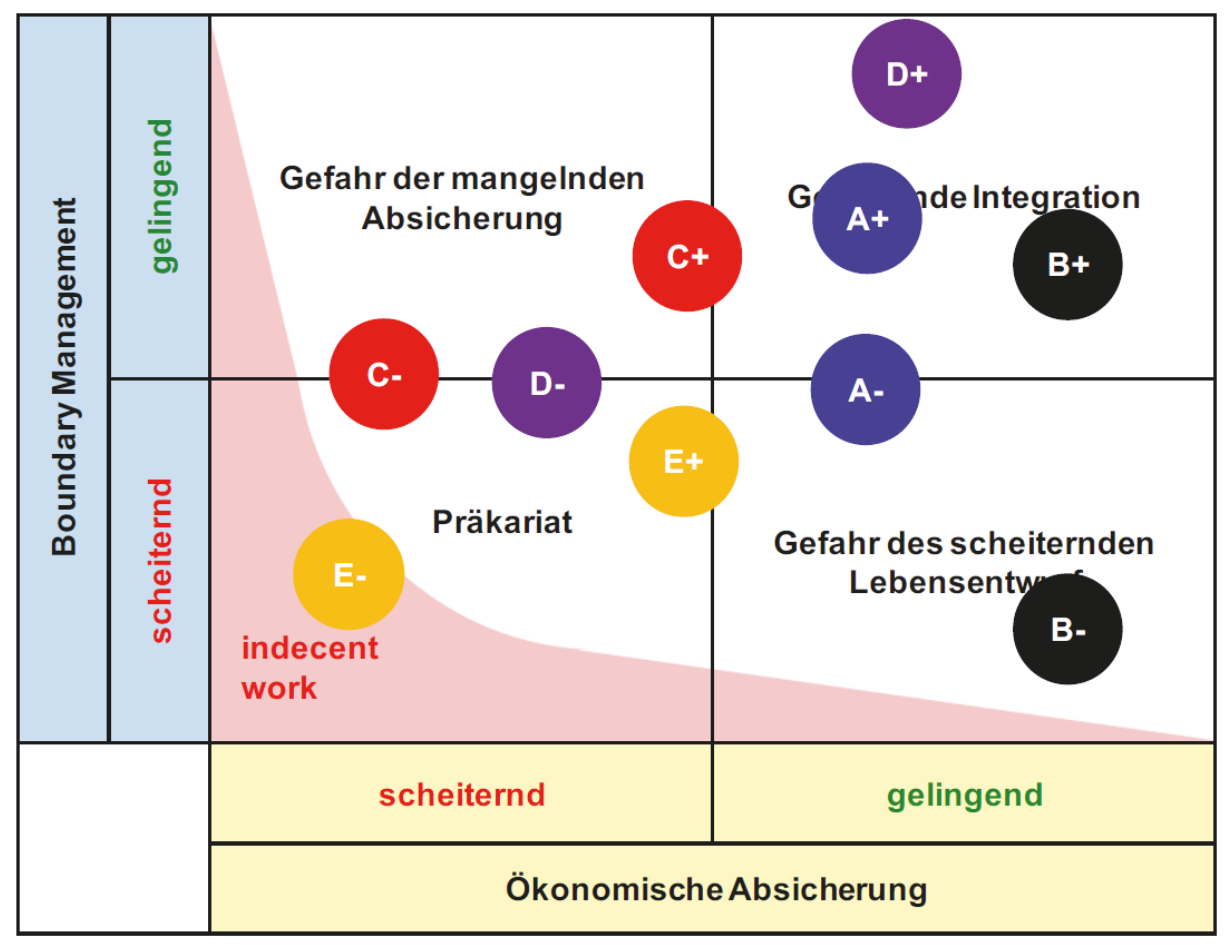

Abbildung 7: Die fünf Personas in der Entwicklungsmatrix verortet

Die Gegenüberstellung der zwei Dimensionen physische Entgrenzung sowie Subjektivierung der Arbeit bietet eine kompakte Übersicht über die zwei deutlichsten Phänomene der "flexiblen neuen Arbeitswelt" und ermöglicht somit eine Veranschaulichung der Verschiebung des Stellenwerts von Arbeit. 


\section{Ausprägungen mobil-flexibilisierter Arbeit und ihre spezifischen Merk- male unter Berücksichtigung der rechtlichen Dimension}

Das Arbeitsverhältnis wurde bereits im Jahre 1881 im Obligationenrecht (OR; SR 220) gesetzlich geregelt. Damals war die Vertragsfreiheit das zentralste Element des Arbeitsrechts, weshalb es gerade mal 12 Artikel zum Thema gab. Relativ bald wurde festgestellt, dass diese gesetzgeberische Zurückhaltung die Arbeitnehmenden stark benachteiligte. Deshalb wurde 1911 das OR bereits zum ersten Mal revidiert und in das Dienstvertragsrecht überführt. Obwohl die Vertragsfreiheit noch immer das oberste Prinzip war, wurden viele zwingende Vorschriften sowie Sondervorschriften für verschiedene Arten von Dienstverträgen entwickelt. Auch der Normalarbeitsvertrag und der Gesamtarbeitsvertrag (GAV) fanden in dieser Zeit ihre Anfänge. Einzige Schwachstelle des neuen Dienstvertragsrechts war die Regelung bezüglich der Lohnzahlungspflicht der Arbeitgebenden bei unverschuldeter Verhinderung an der Dienstleistung.

1960 bereitete eine Expertenkommission eine Totalrevision des Dienstvertragsrechts vor. Das damit zusammenhängende Differenzbereinigungsverfahren zwischen den beiden Räten gehört zu einem der längsten und intensivsten der Schweizer Geschichte. Die Neuregelung wurde erst am 1. Januar 1972 vom Bundesrat in Kraft gesetzt. In der gesamten Zeit zwischen 1911 bis 1972 wurden nur wenige Änderungen vollzogen. Speziell hervorzuheben ist die Einführung des öffentlich-rechtlichen Arbeitsgesetzes vom 1. Februar 1966 und mit inm drei neue Normen im OR, u.a. eine zwingende Vorschrift über die Mindestdauer von Ferien. Bereits zu jener Zeit gab es vom OR abweichende Spezialerlasse, die das Arbeitsvertragsverhältnis regelten. Dazu gehört auch das Bundesgesetz vom 12. Dezember 1940, welches die Heimarbeit betraf.

Nach der Totalrevision ordnete das OR von 1972 vor allem den Einzelarbeitsvertrag eingehend. Dies führte dazu, dass lediglich noch der Lehrvertrag, das Anstellungsverhältnis des Handelsreisenden und des Heimarbeitnehmenden als besondere Arten von Arbeitsverträgen separat geregelt waren. Das Arbeitsrecht umfasste nun 114 Artikel. Neu waren vor allem Regelungen des Einzelarbeitsvertrages, wobei eine Begriffsdefinition enthalten war. Im Weiteren wurden Pflichten der Arbeitnehmenden und der Arbeitgebenden sowie Normen über die 
Personalvorsorgeeinrichtungen darin festgehalten. Der Übergang des Arbeitsverhältnisses auf einen neuen Arbeitgebenden und auch dessen Beendigung waren neu gesetzlich geregelt. Zudem wurde nun auch der Heimarbeitsvertrag im OR aufgenommen. Schliesslich enthielt der vierte Abschnitt eine Auflistung der beidseitig und einseitig zwingenden Normen.

In den darauffolgenden Jahren erfuhr das OR immer wieder einzelne Änderungen, wie bspw. die Totalrevision der Kündigungs- und Kündigungsschutzbestimmungen im Jahre 1989, wobei es hauptsächlich um den Tatbestand einer missbräuchlichen Kündigung ging.

Heute ist die Tendenz eher dahin gehend, dass wichtige Bereiche des Arbeitsverhältnisses zunehmend durch Spezialgesetze geregelt werden, welche neben das OR treten und dieses ergänzen. Dies ist nicht zuletzt darauf zurückzuführen, dass das Arbeitsrecht, wie kaum ein anderes Rechtsgebiet, einem ständigen Wandel unterliegt. Die rasche Zunahme moderner Kommunikationsmittel sowie der vielseitige Wunsch nach Flexibilität und danach, die Familie und die Arbeit unter einen Hut zu bringen, führen dazu, dass die gesetzlichen Regelungen mit den tatsächlichen Gegebenheiten nicht mehr vollständig übereinstimmen. Das heute geltende Arbeitsrecht hat eigentlich jene Arbeitnehmende vor Augen, die pünktlich um 8 Uhr morgens am Arbeitsplatz sitzt, während 8 Stunden eine ihr klar vorgegebene Arbeit verrichtet, punkt 12 Uhr eine Mittagspause einlegt und schliesslich um 17 Uhr das Büro verlässt. Den Rest des Tages gestaltet sie nach ihren Bedürfnissen und Wünschen, ihre Gedanken fernab von der Arbeit. In der Realität sieht die Arbeitswelt im Jahre 2015 jedoch etwas anders aus. Viele Arbeitnehmende müssen oder wollen mehr Flexibilität und Verantwortung übernehmen. Die damit verbundenen Risiken wie die Entgrenzung und der Gesundheitsschutz werden plötzlich zu allgegenwärtigen Herausforderungen. Das vorliegende Kapitel 3 untersucht die rechtliche Komponente von mobil-flexiblen Arbeitsformen. Themen wie Individualisierung, Gesundheit und Entgrenzung werden aus juristischer Sicht beleuchtet und sowohl auf Gefahren wie auch auf Chancen wird hingewiesen. Es sind diverse Stakeholder auf dem Markt, die diese Flexibilität promoten, gleichzeitig aber sind auch Gegnerinnen und Gegner vorhanden, die einer Ausuferung der Arbeitsmodelle entgegenhalten. Ein Beispiel dafür ist die aktuell lancierte Syna-Kampagne zum Thema „meine Arbeit - meine Zeit“, welche eine mit dem Wandel im Arbeitsrecht zusammenhängende Problematik aufgreift, nämlich die dauernde Erreichbarkeit und die Überstundenarbeit. 
Es ist kein Geheimnis, dass die Regulierung des Arbeitsmarktes und die aktuelle Arbeitspolitik die Flexibilisierungsformen der Arbeitswelt beeinflussen und dadurch mit verantwortlich sind für das Ausmass und die Form der „Ausdehnung des Normalarbeitsverhältnisses". Nach und nach wird jede Branche von der Digitalisierung erfasst - von der Produktion bis zur einzelnen Dienstleistung. Dieser technische Fortschritt kann ein besseres, angenehmeres Leben verheissen, bedroht aber zugleich auch etablierte Geschäftsmodelle - Fluch und Segen (Frick et al., 2014, S. 29). Diese Fortschritte weisen oft eine grosse Dynamik auf, was sehr schnelle Änderungen mit sich bringt und eine rechtzeitige Anpassung des Regulators verunmöglicht.

Im Zusammenhang mit der immer wieder thematisierten Arbeitslosigkeit ist auch die Bedeutung der saisonalen Arbeitsstellen in der Schweiz nicht unberücksichtigt zu lassen. So verlieren aktuell rund 25'000 Personen monatlich ihren Job und etwa gleich viele finden wieder eine Stelle. Weiter mit diesem Wandel zu beobachten ist, dass der Anteil der Sozialbezügerinnen und Sozialbezüger im Alter zwischen 46 und 65 Jahren jährlich ansteigt. Gelangen also die älteren Beziehenden in die Sozialhilfe, ist es schwierig für sie, wieder auf dem Arbeitsmarkt Fuss zu fassen. In diesem Zusammenhang ist zu hinterfragen, ob die mit steigendem Alter zunehmenden Beiträge an die zweite Säule noch zeitgemäss sind. Dadurch verteuert sich der Faktor Arbeit, und im Umkehrschluss sinken für die Arbeitgebenden die Anreize, ältere Arbeitnehmende zu halten oder einzustellen (Müller, 2014).

Solche Entwicklungen können das Recht schnell an seine Grenzen bringen, sodass ein allfälliger Handlungsbedarf geprüft werden muss. Dieser Problematik widmet sich sodann auch das vorliegende Kapitel. Anhand einer umfassenden Literaturrecherche und dem Zusammentragen der gesetzlichen Grundlage wurde eine erste Auslegeordnung vollzogen. Hierbei wurden auch politische Entwicklungen aus dem Inland wie auch von grenznahen Ländern mit einbezogen. Nebst den gesetzlichen Grundlagen wurden auch Gesamtarbeitsverträge untersucht, um zu prüfen, ob und in welchem Umfang dort die flexiblen Arbeitsformen bereits berücksichtigt wurden. Basierend darauf wurden Spannungsfelder evaluiert und diese wiederum auch mit den Personas in Bezug gebracht. Zusammenfassend zeigt das vorliegende Kapitel 3 den juristischen Umgang mit der zunehmenden Flexibilisierung auf und weist auf Spannungsfelder sowie auch bestehende Schwachstellen oder gar Lücken hin. 


\subsection{Rechtliche Grundlagen}

Die Flexibilisierung der Arbeitsorganisation findet gegenwärtig ihre Grundlagen vor allem in den atypischen Arbeitsverhältnissen und vorwiegend in zeitlicher Hinsicht. Als aus rechtlicher Sicht betrachtet atypische Arbeitsverhältnisse werden Arbeitsformen bezeichnet, welche vom Normalarbeitsverhältnis abweichen. Das Normalarbeitsverhältnis gilt dabei als stabile, soziale sowie durch Mindestbestimmungen und kollektivvertragliche Regelungen abgesicherte Version. Die flexibleren Versionen sind demgegenüber atypische Arbeitsverhältnisse wie Telearbeit, Heimarbeit, befristete Beschäftigung, Leih- oder Temporärarbeit, Wochenend-, Abend- und Schichtarbeit sowie Arbeit auf Abruf. Welche gesetzlichen Grundlagen hier zur Anwendung gelangen, wird im folgenden Abschnitt ansatzweise aufgezeigt.

Das Arbeitsrecht gliedert sich inhaltlich in drei grosse Normenkomplexe: Individualarbeitsrecht (Arbeitsprivatrecht), öffentliches Arbeitsrecht und kollektives Arbeitsrecht (Rehbinder, 2002, Rz. 10). Die nachstehende Abbildung 8 verdeutlicht diese Struktur.

Das Individualarbeitsrecht unterliegt dabei verschiedenen Rechtsquellen (Rehbinder, Schweiz. Arbeitsrecht, Rz. 30-38):

1. Die gesetzliche Regelung findet sich in den Art. 319-362 des Obligationenrechts, die im Jahre 1971 grundlegend revidiert wurden, und daneben in einigen Sondergesetzen, z.B. Arbeitsvermittlungsgesetz (AVG; SR 831.10) betreffend den Personalverleih oder im Gleichstellungsgesetz (GIG; SR 151.1).

2. Die arbeitsrechtlichen Rechtsverordnungen betreffen in der Regel das (öffentlich-rechtliche) Arbeitsschutzrecht (siehe Rz. 426 ff.), z.B. die vier Verordnungen zum Arbeitsgesetz. Das Arbeitsschutzrecht hat aber gemäss Art. 342 Abs. 2 OR in der Regel auch privatrechtliche Wirkung. Rechtsverordnung ist ferner die Allgemeinverbindlicherklärung eines Gesamtarbeitsvertrages gegenüber den Aussenseitern (siehe Rz. 558) sowie der Normalarbeitsvertrag (siehe sogleich unter 3). 


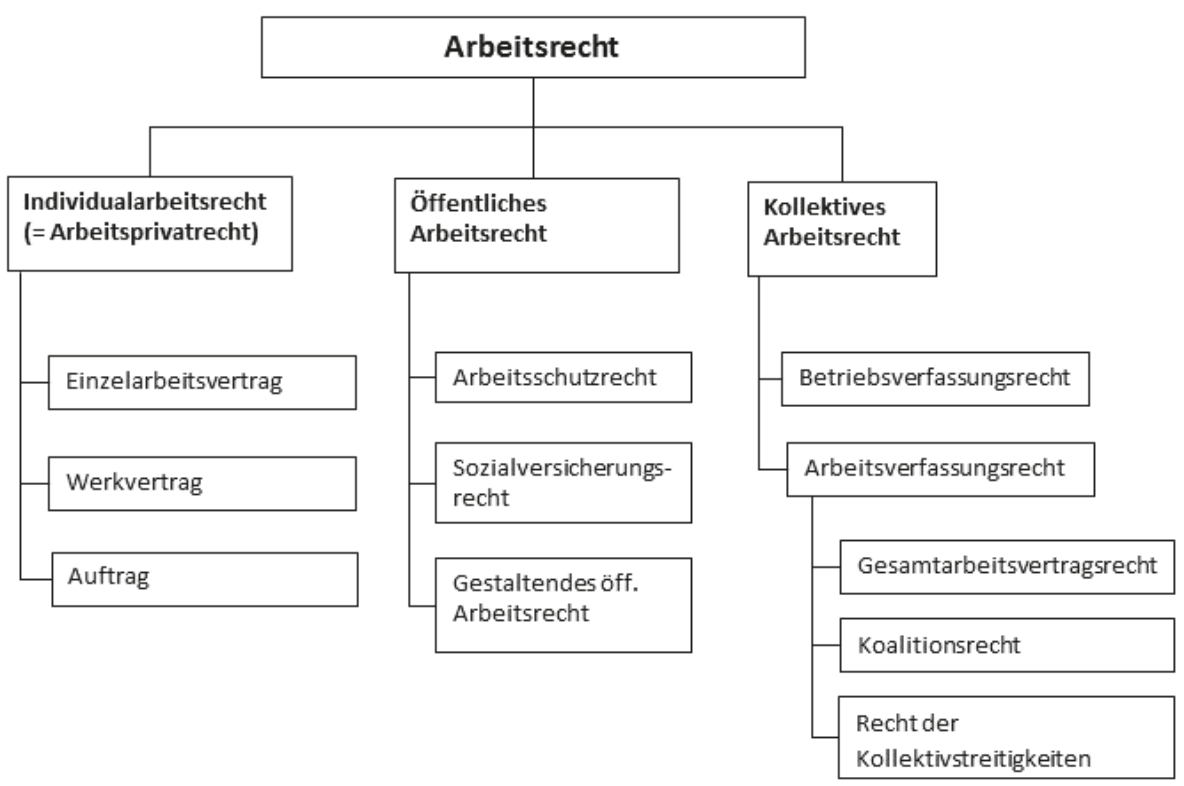

Rechtsquellen:

GesetzlicheRegelungen

Rechtsverordnungen

Normalarbeitsvertrag

Einzelvereinbarung

Direktionsrecht/Weisungsrecht
Betriebliche Übung

Betriebsordnung

Gesamtarbeitsvertrag

Abbildung 8: Gestaltung des schweizerischen Arbeitsrechts

3. Der Normalarbeitsvertrag ist eine besondere Form der Rechtsverordnung, nämlich ein Erlass des Bundes oder der Kantone, der dispositives Vertragsrecht für bestimmte Typen von Arbeitsverhältnissen setzt. Inhalt des Normalarbeitsvertrages sind Bestimmungen über den Abschluss, den Inhalt und die Beendigung einzelner Arten von Arbeitsverhältnissen (Art. 359 Abs. 1 OR).

Die praktische Bedeutung der Normalarbeitsverträge liegt dort, wo sich der Gesamtarbeitsvertrag noch nicht durchsetzen konnte.

4. Die Einzelvereinbarung kann zwischen Arbeitgebenden und Arbeitnehmenden schriftlich oder mündlich, ausdrücklich oder stillschweigend erfolgen. Nur in einigen besonders geregelten Fällen ist für die Gültigkeit der Vereinbarung die Schriftform vorgeschrie- 
ben (vgl. Rz. 81). In die Einzelvereinbarung können betriebseinheitliche Reglemente (sog. Allgemeine Arbeitsbedingungen, entsprechend den Allgemeinen Geschäftsbedingungen im Wirtschaftsleben) einbezogen werden (vgl. Rz. 56).

5. Das Direktionsrecht oder Weisungsrecht ermöglicht dem Arbeitgebenden, die Leistungspflicht der Arbeitnehmenden zu konkretisieren (Art. 321d Abs. 1 OR, vgl. Rz. 166 ff.).

6. Die betriebliche Übung ist eine besondere Form stillschweigender Kollektivvereinbarung, bei der aus der faktischen Handhabung im Betrieb nach einer gewissen Dauer Rechtsansprüche entstehen können (z.B. der Anspruch auf Weihnachtsgratifikation, vgl. Rz. 181).

7. Die Betriebsordnung ist eine kollektive Regelung der Arbeitsverhältnisse eines Betriebes durch einseitigen Erlass oder durch Vereinbarung mit der Arbeitnehmendenvertretung (Betriebskommission). Sie ist für industrielle Betriebe zwingend vorgeschrieben (Arbeitsgesetz; ArG SR 822.11, vgl. Rz. 621 ff.).

8. Der Gesamtarbeitsvertrag schliesslich ist die kollektive Vereinbarung von Arbeitsbedingungen zwischen den Gewerkschaften und einzelnen Arbeitgebenden oder deren Verbänden (Art. 356-358 OR, vgl. Rz. 509 ff.).

Um die Vielfalt der rechtlichen Gestaltungsfaktoren des Einzelarbeitsverhältnisses zu verstehen, ist die Kenntnis ihrer Hierarchie notwendig. Nur damit ist es möglich, die massgebliche Regelung festzustellen, falls verschiedenartige Normen einander widersprechen. Der Gesetzgeber hat grundsätzlich folgende Rangordnung vorgeschrieben (BSK OR I- Wolfgang Portmann, Art. 319 ff. $\mathrm{N} 8$ f.):

1. Rang zwingendes Verfassungs-, Gesetzes- und Verordnungsrecht (Art. 358, Art. 359 Abs. 3, Art. 361 f. OR, Art. 38 Abs. 3 ArG)

2. Rang Gesamtarbeitsvertrag (Art. 357, Art. 38 Abs. 3 ArG)

3. Rang Betriebsordnung (Art. 39 Abs. 2 ArG)

4. Rang Einzelarbeitsvertrag

5. Rang Normalarbeitsvertrag (Art. 360 Abs. 1 OR)

6. Rang dispositives Gesetzesrecht

7. Rang Weisungen der Arbeitgebenden (Art. 321d OR) 
Diese Rangordnung wird durch das Günstigkeitsprinzip abgeschwächt. Es besagt, dass die rangschwächere Regelung der ranghöheren vorangeht, wenn sie zugunsten des Arbeitnehmenden ist.

\subsection{Einzelarbeitsvertrag, Werkvertrag, Auftrag und Freelancingvertrag}

Im Zusammenhang mit den mobil-flexiblen Arbeitsformen ist die Abgrenzung des Einzelarbeitsvertrages zu anderen Vertragstypen von grosser Bedeutung. Weshalb dies so ist, wird in diesem Kapitel und mit den Verweisen auf die Persona exemplarisch aufgezeigt.

Der Einzelarbeitsvertrag gehört genau wie der Werkvertrag, der Auftrag und die Innominatverträge (nicht im Gesetz geregelte Verträge) zu den Verträgen über Arbeitsleistung (Geiser/Müller, Rz. 99). Damit ein Vertragsverhältnis richtig charakterisiert und eine saubere Abgrenzung der verschiedenen Formen vorgenommen werden kann, weist jede Vertragsart ihre eigenen Merkmale auf. Es ist jedoch anzumerken, dass es stets einer Gesamtbetrachtung aller Umstände bedarf, um festzustellen, ob es sich beim infrage stehenden Vertragsverhältnis um einen Arbeitsvertrag nach OR handelt (Streiff et al., Art. 319 N 2). Die alleinige Bezeichnung eines Vertragsverhältnisses als Einzelarbeitsvertrag genügt nicht, wenn die einzelnen Vertragsmerkmale nicht eingehalten sind.

Eine klare Abgrenzung der verschiedenen Verträge auf Arbeitsleistung ist auch deshalb sehr wichtig, weil insbesondere das Arbeitsvertragsrecht über zahlreiche zwingende Normen (bspw. Art. 361 und 362 OR) verfügt und wichtige Normen zum Sozialschutz und den Zuständigkeiten von Spezialgerichten enthält (Streiff et al., Art. 319 N 4).

Wie erwähnt, kennt das Gesetz verschiedene Verträge auf Arbeitsleistung, wobei der gängigste wohl der Arbeitsvertrag nach Art. 319 OR ist. Einen Überblick über alle Verträge auf Arbeitsleistung zeigt die nachstehende Abbildung 9: 


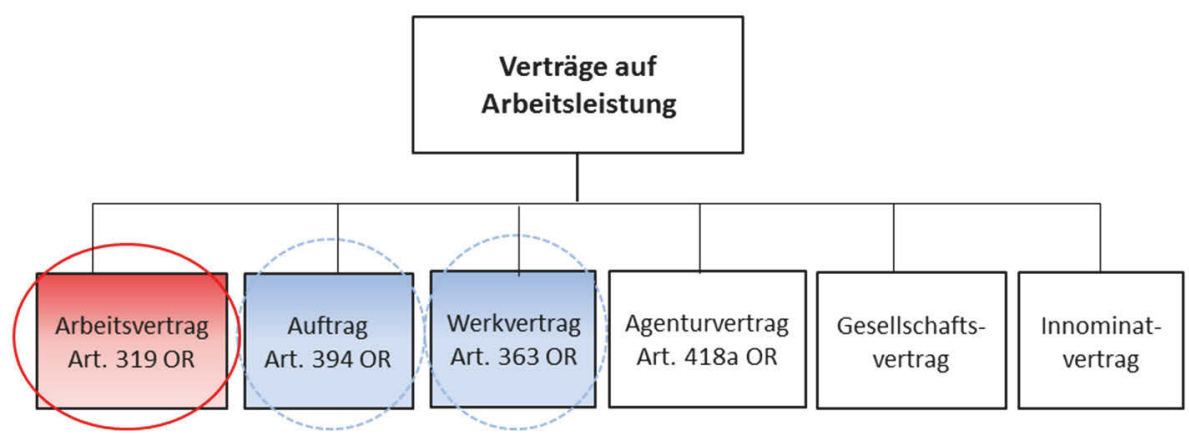

Abbildung 9: Übersicht über die Verträge auf Arbeitsleistung

Bei den mobilen Arbeitsformen steht die Abgrenzung zwischen dem Arbeitsvertrag (Einzelarbeitsvertrag), dem Auftrag und dem Werkvertrag im Fokus. Auf diese drei Arten von Verträgen auf Arbeitsleistung wird deshalb im Folgenden näher eingegangen, wobei einleitend jeweils die zentralen Vertragsbestandteile erläutert werden.

\subsubsection{Der Einzelarbeitsvertrag}

Der Einzelarbeitsvertrag als privatrechtlicher Schuldvertrag ist in Art. 319 OR verankert. Aus dem Gesetz ergeben sich die vier begriffsnotwendigen Elemente (Geiser/Müller, 2015, Rz. 100):

- $\quad$ Angebot einer Arbeitsleistung

- $\quad$ bestimmte oder unbestimmte Zeit (Dauerschuldverhältnis)

- $\quad$ Eingliederung in eine fremde Arbeitsorganisation (Subordinationsverhältnis)

- $\quad$ Entgeltlichkeit

Die Arbeitnehmende ist zunächst verpflichtet, eine positive Leistung zu erbringen, d.h., ihre Arbeitskraft in Form einer körperlichen oder geistigen Tätigkeit zur Verfügung zu stellen. Darunter fallen auch kaum wahrnehmbare Tätigkeiten wie z.B. das Überwachen oder Kontrollieren von Maschinen (Geiser/Müller, Rz. 101). Das Arbeitsverhältnis muss sodann auf bestimmte oder unbestimmte Zeit begründet sein, sodass von einem Dauerschuldverhältnis gesprochen werden kann. Als drittes Element muss ein Abhängigkeits- oder Subordinationsverhältnis vorliegen, welches durch den Eintritt in das Arbeitsverhältnis entsteht. Die Arbeitnehmende steht persönlich, organisatorisch, zeitlich und wirtschaftlich unter der Direktionsgewalt der Arbeitgeberin (Geiser/ 
Müller, Rz. 108). Als letztes Merkmal des Einzelarbeitsvertrages ist die Entgeltlichkeit zu erwähnen. Es gehört zu den Pflichten der Arbeitgebenden, der Arbeitnehmenden im Rahmen des Arbeitsvertrages einen angemessenen Lohn zu entrichten (Rehbinder, Rz. 53). Nach Art. 327a OR ist auch der Auslagenersatz geregelt, wonach die Arbeitgebende der Arbeitnehmenden sämtliche durch die Ausführung der Arbeit notwendigen entstehenden Auslagen zu ersetzen hat. Eine Ausdehnung entsteht beim auswärtigen Arbeitsort, wo auch alle für den Unterhalt erforderlichen Aufwendungen zu entschädigen sind. Die Übernahme der Auslagen gilt als Ausprägung der allgemeinen Fürsorgepflicht der Arbeitgebenden.

Im Zusammenhang mit der Mobilität rückt auch die Bedeutung des Arbeitsorts ins Zentrum und auch hier lassen sich gesetzliche Vorgaben finden. Jede Mitarbeitende, die sich zur Leistung von Arbeit verpflichtet, verfügt situativ über einen oder mehrere Arbeitsorte. Der Ort der Arbeitsleistung bildet in der Regel ein inhaltliches Element des Arbeitsvertrags, wobei die Eingliederung in die Arbeitsorganisation zentral ist. Um die Flexibilisierung zu regeln, können im Arbeitsvertrag sogenannte "Mobilitätsklauseln" vereinbart werden. Diese erteilen der Arbeitgebenden das Recht, den Arbeitnehmenden ausserhalb des vertraglichen Arbeitsorts einzusetzen. Zu beachten ist jedoch, dass eine Klausel, welche besagt, dass der Arbeitsort stets dort sein soll, wo ein Einsatz bei einer Kundin bzw. einem Kunden zu leisten ist, eine Umgehung von Art. 13 ArGV 1 darstellt. Die Reisezeit muss dann als Arbeitszeit berechnet werden.

Mit dem Arbeitsort verbunden ist auch die Auswirkung auf den Gerichtsstand. Art. 34 Zivilprozessordnung (ZPO) regelt die Zuständigkeit für Klagen aus arbeitsrechtlichen Verträgen. Es handelt sich um eine teilzwingende Norm, womit die Arbeitnehmende weder zum Voraus noch durch Einlassung auf diese Gerichtsstände verzichten kann. Der Begriff der arbeitsrechtlichen Klage ist weit zu verstehen. Der Wahlgerichtsstand am Wohnsitz bzw. Sitz der beklagten Partei oder am gewöhnlichen Arbeitsort steht beiden Parteien zur Verfügung (BSK ZPOKaiser, Art. $34 \mathrm{~N}$ 14). Es fragt sich, was unter dem Begriff des gewöhnlichen Arbeitsortes zu verstehen ist. Gemäss herrschender Lehre und Rechtsprechung befindet sich dieser dort, wo sich der tatsächliche Mittelpunkt bzw. der geografische Schwerpunkt der Berufstätigkeit der Arbeitnehmenden befindet. Dieser fällt, häufig mit dem Unternehmenssitz oder dem Betriebsort zusammen, wobei nicht erforderlich ist, dass die Arbeitgebende eine Organisationseinheit mit festen Einrichtungen auf längere Zeit an einem bestimmten Ort angelegt hat. Somit kann auch die Baustelle ein gewöhnlicher Arbeitsort sein oder bei der Telearbeit die eigene Wohnung. Entscheidend ist folglich die relative Zeitdauer im Vergleich zur Dauer des Arbeitsverhältnisses und der anderen Arbeitsorte, nicht etwa die abso- 
lute Zeitdauer. Auch bei Aussendienstmitarbeitenden kann die eigene Wohnung der gewöhnliche Arbeitsort sein, wenn sie die Arbeiten vorwiegend von zu Hause aus erledigt (BSK ZPO-Kaiser, Art. $34 \mathrm{~N}$ 16).

Ein anderer Arbeitsort ist etwa das Coworking-Space, das auch von Noah Schmid in seiner Tätigkeit als Informatiker genutzt wird. Dabei handelt es sich um historisch neue, örtlich und räumlich institutionalisierte Formen des Arbeitens nebeneinander, ohne dass in der Regel miteinander gearbeitet wird. Angestellte oder Selbstständige, die einen Arbeitsplatz innehaben, ohne über einen vorgegebenen oder festen Platz zum Arbeiten zu verfügen und somit in der Regel einen hohen Mobilitätsgrad aufweisen, können in Coworking-Spaces arbeitend sesshaft werden, indem sie sich an „Fix Desks" einmieten und dafür einen durch die Betreiberinnen und Betreiber von Coworking-Spaces festgelegten Geldbetrag leisten. Zentrales Merkmal bei Coworking-Spaces ist, dass die Nutzerinnen und Nutzer immer ihrer Arbeit mit einem Laptop nachgehen (Bender, 2013, S. 25 ff.). Die Nutzung von Synergieeffekten, die Vermittlung von Aufträgen sowie das Kontaktknüpfen unter Kolleginnen und Kollegen sind die grossen Vorteile eines Coworking-Offices (Waser, 2010, S. 12). Festzustellen ist, dass die Anzahl der Coworking-Spaces stark zugenommen hat (Rothenberger, 2013, www.startwerk.ch).

Bezüglich des Festlegens der Arbeitszeiten sind die Arbeitsvertragsparteien nicht völlig frei. Vielmehr sieht das Arbeitsgesetz Höchstarbeitszeiten vor, regelt die Massnahmen und deren Überschreitung und enthält Vorschriften bezüglich der Nacht- und Sonntagsarbeit. So schreibt das Arbeitsgesetz eine wöchentliche Höchstarbeitszeit von 45 Wochenstunden für Arbeitnehmende in industriellen Betrieben, Büropersonal, Angestellte und Verkaufspersonal von Grossbetrieben vor. 50 Wochenstunden gelten für alle übrigen Arbeitnehmenden, insbesondere solche des Kleingewerbes. Diese Grenzen können um höchstens vier Stunden verlängert werden, solange sie im Jahresdurchschnitt nicht überschritten werden. Allerdings gelten strenge Anforderungen, wann eine Verlängerung um volle vier Stunden möglich ist (vgl. Verordnung $1 \mathrm{ArGV}$ ).

Wenn man von Höchstarbeitszeit spricht, muss vorab geklärt werden, was überhaupt als Arbeitszeit gilt. Das Arbeitsgesetz schweigt dazu. Jedoch legt die Verordnung 1 fest, dass Arbeitszeit die Zeit ist, während der sich der Arbeitnehmer oder die Arbeitnehmerin zur Verfügung stellt (Weka, 2014, S. 69 ff.).

Weiter wichtig ist die Unterscheidung zwischen selbstständig und unselbstständig erwerbend. Das Arbeitsvertragsrecht bietet der Arbeitnehmenden einen weitgehenden Sozialschutz. Dieser kann auch bei Innominatverträgen zum Tragen kommen, nicht aber bei der unselbstständigen Tätigkeit. Diese Unter- 
scheidung ist nicht immer einfach zu vollziehen. Aus Lehre und Rechtsprechung hat sich eine Vielzahl von Kriterien herausgebildet, diese sind aber für sich alleine oft nicht entscheidend. Es bedarf entsprechend immer einer Gesamtbetrachtung (Geiser, 2007).

Gemäss Streiff et al. (Art. 319 N3) wird in der Praxis häufig die Wegleitung zum massgebenden Lohn (WML) in der AHV, IV und EO des Bundesamtes für Sozialversicherung zur Unterscheidung beigezogen. Es geht dabei vor allem um die Abgrenzungskriterien der betriebswirtschaftlichen bzw. arbeitsorganisatorischen und wirtschaftlichen Abhängigkeit und das fehlende Unternehmerrisiko, wobei auch hier eine Gesamtbetrachtung erforderlich ist. Zentrale Elemente im Zusammenhang mit der Abgrenzung zwischen selbstständiger und unselbstständiger Erwerbstätigkeit sind die frei gewählte Organisation und das Tragen des Unternehmerrisikos seitens des Selbstständigen. Gemäss Reich (2002) kann die selbstständig Erwerbende nämlich die Gestaltung der innerbetrieblichen Abläufe, die Auswahl ihrer Mitarbeitenden sowie die Pflege der Geschäftsbeziehungen grundsätzlich unabhängig erledigen und sie kann über ihre Zeit beliebig verfügen.

Besonders dann, wenn sich die Arbeitnehmende nicht in eine fremde Arbeitsorganisation eingliedert, aber trotzdem wirtschaftlich stark von der Vertragspartnerin bzw. vom Vertragspartner abhängig ist, gelangen die zwingenden Vorschriften des Arbeitsrechtes zur Anwendung. Solche Scheinselbstständigen sind z.B. freie Mitarbeitende der Medien, wie Ursula Meyerhans als Redaktorin, Franchisenehmende oder Tankstellenpächterinnen und Tankstellenpächter. Bei diesen arbeiternehmerähnlichen Personen gelangen bspw. die Vorschriften über den Kündigungsschutz des Obligationenrechts zur Anwendung (Rehbinder, Rz. 25).

\subsubsection{Der Werkvertrag}

Beim Werkvertrag steht im Gegensatz zum Arbeitsvertrag der Erfolg, d.h. das Abliefern des bestellten Werks, im Vordergrund. Dabei wird bei Vertragsabschluss meist ein zum Voraus festgelegter Pauschalpreis vereinbart. Zudem erfolgt keine Eingliederung in eine Arbeitsorganisation. Klares Indiz für das Vorliegen eines Werkvertrags ist somit der Umstand, dass die Unternehmerin bzw. der Unternehmer die Arbeitszeiten selber festlegt und nur der Zeitpunkt der Ablieferung des Werkes fixiert ist. Die freie Einteilung der Arbeitszeit ist jedoch nicht begriffsnotwendig (Streiff et al., Art. 319 N 4). 


\subsubsection{Der Auftrag}

Das entscheidende Unterscheidungsmerkmal beim Auftrag ist das Mass der Unterordnung bzw. das Subordinationsverhältnis, also die rechtliche Unterordnung in persönlicher, zeitlicher und organisatorischer Hinsicht. Charakterisierendes Element des Auftrags ist aber das besondere Vertrauensverhältnis, welches zwischen den Parteien besteht.

Der Auftrag sieht eine jederzeitige Auflösung des Vertragsverhältnisses vor, im Gegensatz zur Kündigungsfrist und zum Kündigungsschutz des Arbeitsvertrags (Geiser, 2007).

\subsubsection{Der Freelancingvertrag}

Eine Person gilt als Freelancer, wenn sie für ein Unternehmen Aufträge ausführt, ohne dabei in das Unternehmen eingegliedert zu sein. Ein Freelancer wird auch als freie oder freischaffende Mitarbeitende bezeichnet. Sie gilt grundsätzlich als Selbstständigerwerbende, die eine bestimmte Dienstleistung aus einem Auftrag und nach Instruktion der Auftraggebenden, jedoch unter Verwendung der eigenen Infrastruktur erbringt. Die Schweizer Rechtsordnung kennt keinen gesetzlich geregelten Vertragstyp „Freelancer“. Der Freelancingvertrag ist ein Innominatvertrag und somit nicht im OR geregelt. Je nach Ausprägung kann er aber ein Werkvertrag sein, nämlich wenn vom Freelancer ein bestimmtes Resultat geschuldet ist, oder ein Auftrag, wenn er eine sorgfältige Dienstleistung erbringen muss.

In der Praxis muss ein besonderes Augenmerk auf die Scheinselbstständigkeit geworfen werden. Das massgebliche Unterscheidungsmerkmal zwischen dem Freelancingvertrag und einem herkömmlichen Arbeitsvertrag ist die Frage, ob es sich beim vorliegenden Verhältnis effektiv um eine Zusammenarbeit zwischen Selbstständigen handelt oder ob doch ein Arbeitsverhältnis vorliegt. Ist ein vermeintlicher Freelancer in eine betriebliche Arbeitsstruktur eingebunden, werden inm Arbeitsmittel durch das Unternehmen zur Verfügung gestellt, ist er an fixe Arbeitszeiten gebunden, erhält er eine regelmässige Bezahlung und ist seine Aufgabe nicht auf ein Projekt beschränkt, dann ist die Wahrscheinlichkeit gross, dass es sich um einen Arbeitsvertrag handelt, auch wenn der Vertrag als Freelancingvertrag deklariert ist. Diese Unterscheidung zwischen einem Arbeitsvertrag und einem Freelancingvertrag ist von grosser Bedeutung, weil beim Arbeitsvertrag die entsprechenden Regeln des Arbeitsvertragsrechts zur Anwendung gelangen. Eine Arbeitgebende hat zudem Sozialversicherungsbeiträ- 
ge für ihre Mitarbeitenden zu bezahlen, jedoch nicht an eine Freelancerin bzw. an einen Freelancer (WEKA, 2014; Bürgi Nägeli Rechtsanwälte, 2014).

\subsubsection{Spannungsfeld I: Abgrenzung der einzelnen Arbeitsleistungen gegenüber dem Einzelarbeitsvertrag}

In Bezug auf mobile Arbeitsformen ist die Abgrenzung zwischen einem Einzelarbeitsvertrag einerseits und einem Auftragsverhältnis, einem Werkvertrag bzw. einem Freelancingvertrag andererseits von zentraler Bedeutung. Der Einzelarbeitsvertrag ist in Art. 319 OR definiert und weist vier begriffsnotwendige Elemente auf: Es muss eine Arbeitsleistung angeboten werden, ein Dauerschuldverhältnis, also eine Arbeit auf bestimmte oder unbestimmte Zeit, sowie ein Subordinationsverhältnis bestehen und die Arbeitsleistung erfolgt gegen ein Entgelt (Geiser/Müller, 2012, Rz. 100).

Im Rahmen von mobilen Arbeitsformen muss somit immer sorgfältig geprüft werden, ob ein Einzelarbeitsvertrag, ein Werkvertrag oder ein Auftrag besteht. Kernpunkt dieser Frage ist, wie die betreffende Person in die Organisation eingebunden und wie das Weisungsrecht der Arbeitgebenden ausgestaltet ist. Die Unterscheidung zwischen Einzelarbeitsvertrag, Werkvertrag und Auftrag ist auch in Bezug auf die Kündigung wichtig. Während beim Einzelarbeitsvertrag der zeitliche und sachliche Kündigungsschutz gilt, kann ein Auftrag grundsätzlich jederzeit frei widerrufen werden (vgl. Abschnitt 3.5).

Beim Einzelarbeitsvertrag ist zudem zwischen selbstständiger und unselbstständiger Erwerbstätigkeit zu unterscheiden. Dabei geht es vorwiegend um Abgrenzungskriterien wie betriebswirtschaftliche bzw. arbeitsorganisatorische Abhängigkeit sowie fehlendes Unternehmerrisiko (Streiff et al., Art. 319 N 3). Es kann also festgehalten werden, dass, wenn die vier einleitend genannten Merkmale (Arbeitsleistung; Dauerschuldverhältnis; Subordinationsverhältnis und Entgeltlichkeit) vorhanden sind, demzufolge ein Einzelarbeitsvertrag vorliegt und entsprechend auch die Schutzbestimmungen des Arbeitsrechts zur Anwendung gelangen (Geiser/Müller, Rz. 134a). Diesen Merkmalen unterliegen Roland Müller, Finanzleiter im KMU, und Sandra Könitz, Projektmanagerin, eindeutig, wie auch Ursula Meyerhans, Redaktorin, und Andrea Burri-Lötscher, Küchenhilfe, welche in einem Teilzeitverhältnis angestellt sind. Die prozentuale Auslastung hat jedoch keine Auswirkung auf die Anwendung der einzelarbeitsvertragsrechtlichen Bestimmungen. Sie kommen genauso bei einer hundertprozentigen Anstellung wie bei Roland Müller zur Anwendung. 
Die Frage, die sich stellen wird, ist, wie weit die Abweichung vom typischen Einzelarbeitsvertrag bei der Entwicklung der mobil-flexibilisierten Arbeitsformen künftig gehen wird und welche Normen hier zur Anwendung gelangen. Eine gewisse Anpassung von Rahmenbedingungen wird dabei wohl unumgänglich sein, wie sich im Abschnitt 3.8 zeigt.

\subsection{Charakteristika von Telearbeit, Heimarbeit und Homeoffice}

Wie Geiser bereits 1997 feststellte, ist die arbeitsvertragliche Wirklichkeit vielseitiger geworden. Das Bedürfnis nach höherer Flexibilität hat sowohl im persönlichen Bereich wie auch in der Wirtschaft zum Wunsch nach einer individuelleren Gestaltung der Arbeitszeit und anderen dienstlichen Präsenzen geführt (Geiser, 1997, S. 787). Klar ist, dass dieses Bedürfnis in den letzten Jahren noch grösser geworden ist und die Umsetzung solcher flexibler Arbeitsformen bereits Einzug gehalten hat. Die verschiedenen „modernen Arbeitsformen“ wie z.B. Mobile Work, Homeoffice und Coworking-Spaces kristallisieren sich dabei heraus, und eine Beleuchtung der unterschiedlichen Arbeitsformen aus rechtlicher Sicht wird unumgänglich sein, wenn Unklarheiten vermieden werden sollen.

Bei diesen modernen Arbeitsformen sind im Zusammenhang mit der häufig vorkommenden Mehrfachbeschäftigung auch Themen wie Konkurrenzverbot, die Einhaltung der Arbeits- und Ruhezeiten, Ansprüche auf AHV und Unfallversicherung, BVG sowie bei der ALV zu regeln und zu klären. Die Qualifikation des Vertragsverhältnisses ist nicht nur für arbeitsrechtliche, sondern auch für sozialversicherungsrechtliche Fragen ein entscheidendes Kriterium.

In den nachstehenden Unterkapiteln werden die Charakteristika der einzelnen Arbeitsformen wie Telearbeit, Heimarbeit und Homeoffice erläutert und deren Herausforderungen im rechtlichen Kontext aufgezeigt.

\subsubsection{Telearbeit, Heimarbeit und Homeoffice}

Das schweizerische Recht enthält keine besonderen Bestimmungen über die Telearbeit. Das Begriffsmerkmal der Telearbeit ist die Verrichtung der Arbeit aus der Ferne. Das heisst, der Arbeitsplatz wird aus dem zentralen Betrieb ausgelagert. Arbeitet die Telearbeitende permanent an ihrem Wohnort, kom- 
men Bestimmungen über die Heimarbeit gemäss Art. $351 \mathrm{ff}$. OR und ergänzend die Bestimmungen über den Einzelarbeitsvertrag zur Anwendung.

Grundsätzlich liegt bei der Telearbeit im Normalfall ein Einzelarbeitsvertrag vor. Bei der isolierten Telearbeit ist je nach dem Ausmass der Weisungsgebundenheit und der Kontrolle der Arbeitnehmenden durch die Arbeitgeberin ein gewöhnlicher Arbeitsvertrag oder ein Heimarbeitsvertragsverhältnis gegeben (Geiser, 1997, S. 792). Die fehlende Eingliederung der Arbeitenden in die fremde Arbeitsorganisation der Arbeitgebenden lässt die Frage aufkommen, inwiefern bei der Telearbeit eine Selbstständigkeit oder die Gefahr einer Scheinselbstständigkeit vorliegt. Von Scheinselbstständigkeit wird gesprochen, wenn die Parteien keinen Arbeitsvertrag beabsichtigt haben, das eingegangene Rechtsverhältnis trotzdem alle essenziellen Elemente eines Arbeitsvertrags erfüllt und von Behörden und Gerichten im Fall einer Auseinandersetzung auch als Arbeitsvertrag qualifiziert wird. Telearbeitende sind zu einer persönlichen Arbeitsleistung verpflichtet und dadurch wirtschaftlich von den Arbeitgebenden abhängig. Die Arbeitnehmende läuft hier Gefahr, den vom Gesetzgeber angestrebten Sozialschutz zu verlieren, obschon sie aufgrund des Abhängigkeitsverhältnisses darauf angewiesen ist. Die Gefahr der Scheinselbstständigkeit ist bei der Telearbeit durchaus vorhanden.

So wurde im März 2014 im Nationalrat eine Motion (14.3127) eingereicht mit dem Titel „OR. Regelung der Vergütung für Homeoffice- und Telearbeit“. Hierbei wurde der Bundesrat beauftragt, Art. 327 OR so anzupassen, dass Arbeitnehmende für ihre im Rahmen von Homeoffice und Telearbeit für die Erfüllung eines Auftrags eingesetzten Mittel eine Vergütung erhalten. Diese sei nicht als Lohnbestandteil zu betrachten. Stellen die Arbeitgebenden Arbeitsgeräte zur Verfügung, so dürfen sie dafür keine Entschädigung verlangen. Diese Forderung ging mit der Begründung ein, dass der Nutzen der flexiblen Arbeitsformen kaum mehr umstritten sei und diese auch von verschiedener Seite gefördert würden. Die rechtlichen Grundlagen jedoch hinkten demgegenüber hinterher. Weiter wird erwähnt, dass für die gewerbliche und industrielle Heimarbeit in Art. 5 HArG die Frage von Auslagenersatz sowie die Entschädigung des Arbeitsgeräts geregelt seien. Für das durchaus vergleichbare Homeoffice bzw. die mobile Telearbeit gelte das HArG jedoch nicht, und im Arbeitsgesetz fänden sich keine analogen Bestimmungen. Dafür regeln die Art. 327 und 327a im Obligationenrecht die Frage von Arbeitsgerät und Auslagen, allerdings widersprüchlich: Während Spesen zwingend zu vergüten sind (Art. 327a OR), gilt dies nicht für den Einsatz von privaten Arbeitsgeräten für die Ausführung von 
Arbeiten zu Hause oder von unterwegs. Daraus ergibt sich eine Rechtsunsicherheit, die es zu vermeiden und zu regeln gilt. ${ }^{3}$

Ein Blick über die Grenze zeigt, dass das Landesgericht Düsseldorf sich im Falle einer einseitigen Aufhebung einer Telearbeitsvereinbarung klar für die analoge Anwendung des Aufhebungsvertrags im Einzelarbeitsvertragsrecht eingesetzt hat. Eine Arbeitgebende wollte diese Telearbeitsvereinbarung einseitig aufheben. Dabei hat das Gericht nicht gesagt, dass eine Telearbeitsvereinbarung nie wieder zu kündigen ist. Aber die Arbeitgebende darf sie nicht einseitig aufheben, ohne die Interessen der Mitarbeitenden zu berücksichtigen.

Die Heimarbeit ist keine Neuerscheinung. Seit 1941 gibt es in der Schweiz das Heimarbeitsgesetz. Die Besonderheit beim Heimarbeitsvertrag besteht darin, dass die Arbeitnehmende die vom Arbeitgebenden übertragenen Arbeiten in ihrer Wohnung oder in einem anderen von ihr selbst bestimmten Arbeitsraum ausführen kann (dazu näher Art. 351-354 OR), (Rehbinder/Stöckli, Art. 319 N 41).

Der Heimarbeitsvertrag ist von der Arbeit zu Hause, die heute z.B. bei Informatikerinnen und Informatikern verbreitet ist und durch die neuen Kommunikationsformen über das Internet begünstigt wird, zu unterscheiden (Geiser/Müller, Rz. 168). Dies wird damit begründet, dass der für den Heimarbeitsvertrag typische und nach dem Bundesgericht notwendige Prozessablauf „Ausgabe von Arbeit - Verarbeitung - Rückgabe der Arbeit" meistens fehlt. Die allgemeinen Vorschriften über den Einzelarbeitsvertrag sind gemäss Art. 355 OR ergänzend anwendbar. Besonders tritt bei der Heimarbeit das Merkmal der Unterordnung und Eingliederung in die Arbeitsorganisation der Unternehmung gemäss Art. 319 OR in den Hintergrund. Bei der Heimarbeit verbleibt der Arbeitgebenden ein eingeschränktes Weisungsrecht, welches ihr aber nicht erlaubt, Weisungen bezüglich des Ortes der Arbeitsleistung zu erlassen, da dies dem Wesen der Heimarbeit widersprechen würde. Daraus ergibt sich, dass die Heimarbeitnehmende selber bestimmt, wo sie ihre Arbeit verrichten will (Streiff et al., Art. $351 \mathrm{~N} 2$ ).

\footnotetext{
${ }^{3}$ Der Bundesrat teilt die Auffassung der Motionärin, dass es bei der Telearbeit und beim Homeoffice verschiedene klärungsbedürftige Rechtsfragen gibt. Er hat deshalb das Postulat Meier-Schatz 12.3166, „,Rechtliche Folgen der zunehmenden Flexibilisierung des Arbeitsplatzes“, am 16. Mai 2012 zur Annahme beantragt.
} 


\subsubsection{Herausforderungen im Homeoffice}

Im Zusammenhang mit dem Homeoffice können spezielle Fragen im Bereich des Arbeitsrechts auftauchen. Diese betreffen folgende Punkte:

- $\quad$ die Geheimhaltungspflicht

- $\quad$ die Arbeitszeit und die Überstunden

- $\quad$ die Lohnfortzahlung

- das Weisungsrecht und Kontrollrechte der Arbeitgebenden

- $\quad$ die Haftung für Schäden

- $\quad$ die Arbeitsmittel und die Vergütungen

- $\quad$ die Risiken bei Informations- und Kommunikationstechnologie (IKT)

- den Datenschutz und die Datensicherheit

- $\quad$ steuerrechtliche Abzüge

Die meisten der heute bestehenden Arbeitsverträge bieten zu wenig Rechtssicherheit in Bezug auf die flexiblen Arbeitsformen, denn die Verträge sind noch auf die klassischen Arbeitsverhältnisse zugeschnitten. Dieser Umstand sollte sich jedoch nicht negativ auf die Arbeitnehmenden auswirken. Deshalb ist es wichtig, detaillierte Regelungen in einem Reglement oder einer Weisung zusätzlich zum Arbeitsvertrag festzuhalten. Damit können Unsicherheiten und Risiken minimiert werden.

\subsubsection{Die Geheimhaltungspflicht}

Gemäss Art. 321a Abs. 4 OR „Fabrikations- und Geschäftsgeheimnisse“ dürfen geheim zu haltende Tatsachen weder während noch nach Beendigung des Arbeitsverhältnisses mitgeteilt werden. Dazu kommt der durch das Datenschutzgesetz gewährleistete Schutz von Personendaten vor unbefugter Bearbeitung. Bei Homeoffice und auch anderen mobilen Arbeitsformen ist gerade das Risiko der Verletzung von Geheimhaltungspflichten sowie des Datenschutzes massiv erhöht, weil bspw. eine Mitarbeitende ein wichtiges Telefonat im Zug führt oder ihre Arbeitsunterlagen bei sich herumliegen lässt, für die übrigen Familienmitglieder oder den Besuch leicht einsehbar. Es empfiehlt sich auch hier eine Klausel im Sinne einer Konventionalstrafe im Arbeitsvertrag aufzunehmen, auch wenn der Strafgesetzbuchartikel „Berufs- und Amtsgeheimnisverletzung" (Art. 162 StGB) für Heimarbeit ebenfalls gilt (Kaufmann, 2013). Eine Konventionalstrafe kann zusätzlich zur Sensibilisierung der Mitarbeitenden führen. 
Solche Geheimhaltungspflichten finden sich zusätzlich zum OR auch im Sozialversicherungsrecht, Fernmelderecht, Strafrecht oder Bankenrecht. Die Unternehmerin bzw. der Unternehmer hat sicherzustellen, dass all diese Pflichten auch im Rahmen der mobilen Tätigkeiten vollends umgesetzt werden (Sury, 2011, S. 29).

\subsubsection{Die Arbeitszeit und die Überstunden}

Für privatrechtliche Arbeitsverhältnisse muss nach Art. 321 OR die vertraglich übernommene Arbeit geleistet werden. Ansonsten enthält das Gesetz keine weiteren Regeln über diese vertraglich zwischen der Arbeitgebenden und der Arbeitnehmenden vereinbarte Arbeitszeit, welche grundsätzlich nach Wochenstunden gemessen wird. Wird die vereinbarte oder betriebsübliche Arbeitszeit jedoch überschritten, leistet eine Arbeitnehmende also Überstunden, greift das Gesetz in Art. 321c Abs. 1 OR mit einer Geldkompensation samt 25 Prozent Zuschlag ein. In Absprache zwischen den Parteien ist auch eine Kompensation mit Freizeit von gleicher Dauer möglich. Im Weiteren ist es auch zulässig, dass die Überstunden wegbedungen werden, was allerdings der Schriftlichkeit bedarf. Geleistete Überstunden sind ohne anderslautende Vereinbarung zu kompensieren oder zu entschädigen (Art. 321c Abs. 4 OR) (Weka Fachkongresse, S. $69 \mathrm{ff}$.).

Im Zusammenhang mit der Arbeitszeit besteht für die Arbeitgebende die Pflicht, die geleistete Arbeitszeit ihrer Mitarbeitenden zu erfassen (Art. 46 ArG). Eine solche Erfassung ist aber insbesondere beim Homeoffice schwierig, weil die Arbeitnehmenden nicht am Arbeitsplatz anwesend sind. Die Pflicht zur Zeiterfassung kann in einem solchen Fall an die Mitarbeitende übertragen werden. Dabei ist die Art und Weise, wie dies erfolgen soll, detailliert zu regeln. Eine schriftliche Tabelle ist das Minimum. Die Übertragung der Pflicht zur Zeiterfassung impliziert bereits, dass das Arbeiten im Homeoffice ein grosser Vertrauensbeweis des Unternehmens ist. Aus diesem Grund sind für das Unternehmen verschiedene Kontrollmechanismen denkbar. So können bspw. die monatlichen Stundenabrechnungen dazu dienen, die geleistete Arbeitszeit zu überprüfen. Auch die Auswertung der Anzahl oder Dauer von Serverzugriffen ist eine zusätzliche Kontrolle, allerdings ist diese nur beim konkreten Verdacht auf Fehlverhalten zulässig und im Arbeitsvertrag bzw. in der Weisung festzuhalten (Kaufmann, 2013). 


\subsubsection{Die Lohnfortzahlung}

Gemäss Art. 324a OR ist die Arbeitgebende, sofern die Heimarbeitende unverschuldet von der Arbeit verhindert ist, nur bei der ununterbrochenen Heimarbeit zur Lohnfortzahlung verpflichtet. Diese Pflicht entfällt in der Situation bei der unterbrochenen Arbeit gemäss Art. 353b OR.

\subsubsection{Das Weisungsrecht und das Kontrollrecht der Arbeitgebenden}

Die Arbeitgebende hat nach Art. 321d OR das Recht, Weisungen und Kontrollen zur Ausführung der Arbeit und zum Verhalten am Arbeitsplatz zu erteilen. Homeoffice oder auch andere mobile Arbeitsformen können die Ausübung dieses Rechts verunmöglichen. Dauerüberwachungen der Heimarbeitenden sind nicht zulässig. Nach einer Vorinformation dürfen allerdings Stichproben gemacht werden. Das Weisungsrecht der Arbeitgebenden beinhaltet z.B. die Möglichkeit, den Arbeitsort zu bestimmen. Wird aber überwiegend zu Hause gearbeitet, kann es sinnvoll erscheinen, nicht mehr die Geschäfts-, sondern die Privatadresse als Arbeitsort einzutragen. In diesem Fall gilt dann die Anreisezeit zu Sitzungen am Unternehmenssitz als Arbeitszeit. Zudem ist, wie bereits erläutert wurde, der Arbeitsort für die Bestimmung des Gerichtsstands bei arbeitsrechtlichen Klagen von Bedeutung (Kaufmann, 2013).

\subsubsection{Die Haftung bei Schäden}

Bei Schäden haftet primär gemäss Art. 321e OR die Arbeitgebende. Grundsätzlich ist es aber so, dass die Mitarbeitenden für Schäden, welche sie der Arbeitgebenden vorsätzlich oder fahrlässig zugefügt haben, haften (Art. 321e OR). Tritt der Schaden bei einem Dritten ein, haftet primär die Arbeitgebende, jedoch kann sie auf die Mitarbeitenden nach Art. 55 OR Regress nehmen. Für die Haftungsfrage gegenüber Dritten ist es unerheblich, ob die Arbeit im Betrieb oder zu Hause erledigt wurde. Steigt aber der Computer zu Hause aus und gehen damit Daten verloren, ist unklar, wer für diesen Schaden verantwortlich ist. Für solche Fälle tut ein Unternehmen gut daran, abzuklären, ob die Betriebshaftpflichtversicherung spezielle Risiken (bspw. Datenverlust) bei der Arbeit im Homeoffice oder auch anderen mobilen Arbeitsformen deckt (Kaufmann, 2013). Es ist häufig so, dass genau solche Risiken ausgeschlossen sind. Wie später aufgezeigt wird, ist dies auch bei Bring-your-own-Device (BYOD) ein Thema (bspw. Datenleck; Attacken aufs Unternehmen etc.). Die Mitarbeitende kann sich durch einen Sorgfaltsnachweis der Haftung entziehen. Die Beweisbarkeit ist nach Art. 321a OR aber oft schwierig. Die Haftung der Arbeitnehmenden 
widerspricht grundsätzlich dem Schutzgedanken des Arbeitsrechts, aber hier ist wohl ein Gerichtsentscheid notwendig, welcher diesen Graubereich klärt.

\subsubsection{Die Arbeitsmittel und die Vergütung}

Ein weiterer zu nennender Punkt in diesem Zusammenhang mit Homeoffice ist die Bereitstellung von Arbeitsmitteln. Auch im Falle von Homeoffice hat die Bereitstellung der Arbeitsmittel grundsätzlich durch die Arbeitgebenden zu erfolgen. Mit dem Einverständnis der Arbeitgebenden ist es möglich, private Geräte zu gebrauchen.

Diese Punkte sind in Art. 327 Abs. 2 OR geregelt. Beim Einsatz von privaten Geräten ist dafür eine Entschädigung geschuldet, ebenfalls für Spesen nach Art. 327a OR. Bei Homeoffice ist zu prüfen, ob zusätzlich ein Anteil an Strom und Miete übernommen wird. Da das Gesetz eine Entschädigung für Arbeitsmittel und weitere Aufwendungen vorsieht, insbesondere für notwendige Räumlichkeiten, ist es ratsam, die Entschädigung explizit im Arbeitsvertrag zu regeln. Gemäss Art. 327a Abs. 2 OR kann ein Pauschalbetrag (monatlich, quartalsweise oder jährlich) vereinbart werden. Eine Entschädigung für Arbeitsmittel kann aber auch vertraglich wegbedungen werden.

Um spätere Unklarheiten bezüglich der Arbeitsmittel zu verhindern, empfiehlt es sich, eine Inventarliste zu erstellen. Wichtig ist hier, dass neben der gesetzlichen Rückgabepflicht auch im Vertrag geregelt ist, dass sämtliche Informationen und auch Dokumentationen an die Arbeitgebende zurückzugeben sind. Diese Pflicht kann mit einer Konventionalstrafe ergänzt werden. Wenn eine Rückgabe nicht möglich ist, müssen Daten gelöscht werden. Zudem ist auch sicherzustellen, dass die Daten am richtigen Ort archiviert werden. Weiter ist der Aspekt des GYDFF (Get your Device for free) zu berücksichtigen, wenn private Daten der Arbeitnehmenden auf den Geräten der Unternehmung bearbeitet und gespeichert werden. Dadurch besteht das Risiko, dass die notwendigen Sicherheitsvorschriften nicht eingehalten werden und unsichere Daten auf einer Betriebssoftware sind, die entsprechend Probleme verursachen können.

\subsubsection{Die Risiken bei der Informations- und Kommunikationstechnologie} (IKT)

Als Hauptrisiken der flexiblen Arbeitsformen und der Nutzung der modernen IKT gilt der unberechtigte Zugriff auf Daten. Sämtliche Informations- und Kommunikationstechnologien werden bei den flexiblen Arbeitsformen zusätzlich gefor- 
dert. Darunter fallen Telefonie, E-Mail, Laptops, Tablets und mobile Telefongeräte, Schadsoftware, Abhörangriffe mit Spionage-Apps, Überwachung durch GPS-Module etc. Weitere Herausforderungen bezüglich Datenschutz-, Datenund Informationssicherheit treten bereits beim Transport von Dokumenten und Datenträgern zwischen der Unternehmung und externen Stellen auf. Darunter ist bspw. der Verlust, der Diebstahl, das Mitlesen oder die Manipulation von Daten unterwegs zu verstehen. Besonders grosser Schaden entsteht, wenn es sich bei den Informationen um Unikate oder vertrauliche Daten handelt. Weitere Risiken bestehen bei der Entsorgung von Dokumenten oder Datenträgern ausserhalb der Unternehmung. Wichtige Informationen können durch eine falsche Entsorgung in die falschen Hände geraten (Rieder et al., 2011).

\subsubsection{Der Datenschutz und die Datensicherheit}

Der steigende Anteil der Mobilität und Flexibilität bringt den Einsatz von EDV automatisch mit sich. Mobile Arbeit hinterlässt also eine Datenspur, welche die Anforderungen an die technischen und organisatorischen Massnahmen an den Datenschutz erhöht.

- $\quad$ Sind die Haftungsrisiken für die flexible Tätigkeit ausreichend und zumutbar geregelt?

- $\quad$ Sind die Eigentumsverhältnisse der eingesetzten Hilfsmittel geklärt?

Im Rahmen der Datensicherheit und des Datenschutzes ist die Unternehmerin bzw. der Unternehmer verpflichtet, die sichere Bearbeitung der Daten technisch wie organisatorisch zu gewährleisten. Darunter fällt auch, dass sie die Daten vor unberechtigten Zugriffen Dritter schützt. Um die Zugriffsberechtigungen zu klären, sollte ein Konzept erstellt werden. Diese Berechtigungen sind mit Identifikation und Verifikation zu prüfen. Da der Zugriff oft extern passiert, ist technisch sicherzustellen, dass die Daten sicher übertragen werden. Dabei können Daten zudem klassifiziert (bspw. vertraulich, intern, öffentlich) werden und es kann definiert werden, dass vertrauliche Dokumente nicht mobil verwendbar sind. Dies bedarf einer klaren Regelung und Schulung der Mitarbeitenden, damit alle ihre Pflichten in Bezug auf den Datenschutz und die Datensicherheit auch wahrnehmen können. Im Rahmen der Mobilität werden Daten häufig von einem Datenträger auf den anderen kopiert. Dies erhöht das Risiko, dass Daten verändert oder beschädigt werden. Durch eine automatisierte Kontrolle kann dem entgegengewirkt werden (Sury, 2011, S. 30). 
Klar ist aber, dass die Unternehmung in allen Fällen die Hauptverantwortung für die Sicherheit der Daten innehat. Deshalb ist zu empfehlen, die notwendigen technischen und organisatorischen Massnahmen konkret festzulegen und diese auch konstant zu überprüfen und bei den Mitarbeitenden Kontrollen durchzuführen.

\subsubsection{Steuerrechtliche Abzüge}

Bei den flexiblen Arbeitsformen gilt es auch, die steuerrechtliche Komponente zu prüfen. Wenn die Heimarbeitnehmende für die Räumlichkeiten oder das Arbeitsmaterial von der Arbeitgebenden eine Rückvergütung erhält, kann sie diese bei den Steuern nicht von ihrem Einkommen abziehen. Die Freelancerin im Gegenzug kann ihre Aufwendungen vollumfänglich von den Steuern abziehen, soweit sie für die geschäftliche Tätigkeit notwendig sind (Grüter, 2011, S. 26).

\subsubsection{Spannungsfeld II: Herausforderungen und Risiken bei mobiler Arbeit}

Es lässt sich abschliessend festhalten, dass den in Abschnitt 3 aufgezeigten Risiken oder Ungenauigkeiten in unserem Schweizer Rechtssystem Beachtung zu schenken ist. Zahlreiche dieser Herausforderungen lassen sich mit vertraglichen Vereinbarungen oder Reglementen klären. Häufig fehlt jedoch beim Zeitpunkt der Verhandlungen die Sensibilität bezüglich dieser Punkte und sie werden somit nicht diskutiert und geregelt. Mit einfachen Hilfsmitteln wie Checklisten könnte diesem Umstand Sorge getragen werden.

\subsection{Arbeitszeitmodelle}

Der klassische Achteinhalbstundentag wird aufgrund flexibler Arbeitsformen immer seltener und es sind zahlreiche Arbeitszeitmodelle bereits heute umgesetzt. Wie sich aus der WorkAnywhere-Studie der SBB und der Swisscom AGStudie entnehmen lässt, ergeben sich aus der Kombination von Arbeitsinhalt und Arbeitsort eine Vielzahl von Arbeitstagtypen (Weichbrodt et al., 2013). 


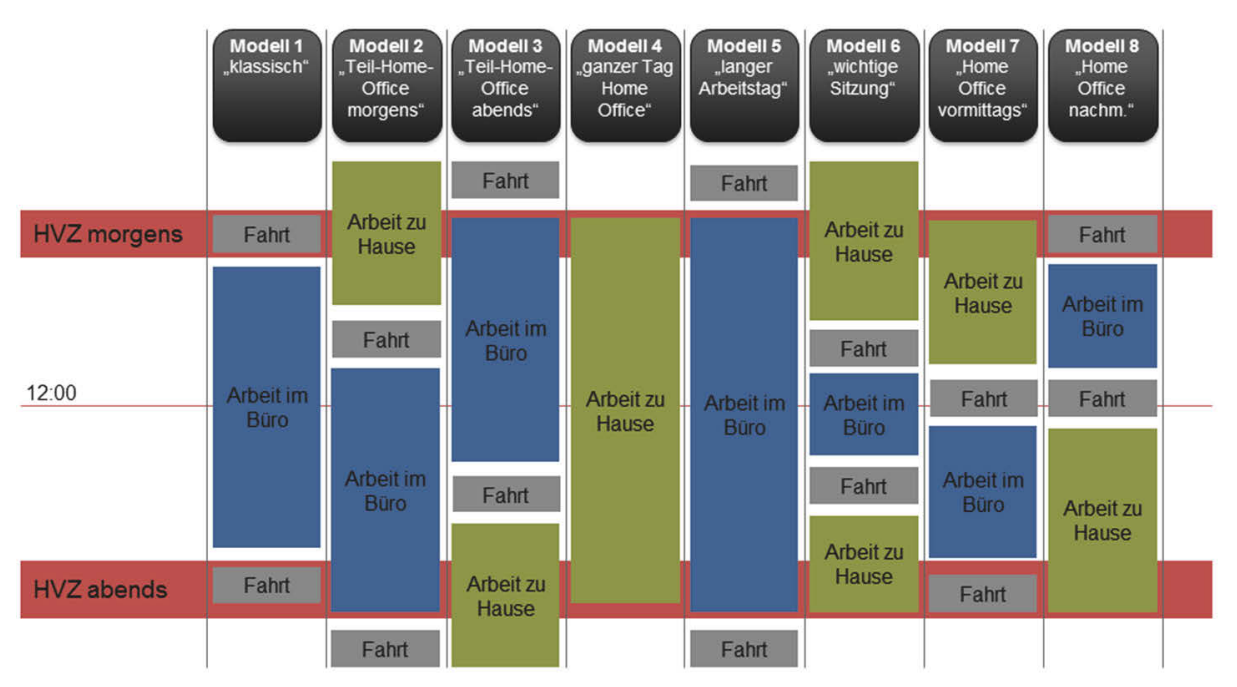

Abbildung 10: WorkAnywhere Fokus Mobilität, SBB AG und Swisscom (Schweiz) AG

Ein Modell, das zunehmend Anklang findet, ist das Modell der Jahresarbeitszeit. Bei gelungener Umsetzung kann dies für die Arbeitgebende wie auch für die Arbeitnehmende zu einer Win-win-Situation führen. Normalerweise werden die Arbeitszeiten dem jeweiligen Personalbedarf und der aktuellen Situation in der Organisation angepasst. Dabei können verschiedene Variationen sinnvoll erscheinen. Diese werden im vorliegenden Abschnitt erläutert.

Einher mit den Arbeitszeitmodellen geht ebenfalls die Arbeitszeiterfassung, die gesetzlich vorgeschrieben ist. Es stellt sich die Frage: Wird durch die mobilen Arbeitsformen dieses Controlling-Instrument ausgeschaltet, nach dem Motto „aus den Augen aus dem Sinn“?

Mit jedem Smartphone kann heute auf einfache Weise mit einer ZeiterfassungsApp die Arbeitszeit erfasst werden, unabhängig davon, wo sich die Mitarbeitende gerade befindet. Weiter gibt es unternehmensangepasste SoftwareLösungen, die auf firmeneigenen Laptops installiert werden können. Aus der Arbeitnehmendenperspektive ist auch daran nichts auszusetzen. Diese Modelle der Zeiterfassung sind zwingend von den Arbeitgebenden zu fordern und umzusetzen, wie es bereits gesetzlich festgehalten ist. Denn flexible Arbeitszeiten können zur Vermischung von Freizeit und Arbeit führen, weshalb hier die Erfassung der Arbeitszeit grosse Wichtigkeit erlangt. Nur wenn ein Überblick über die 
geleistete Arbeit vorhanden ist, können die gesetzlich vorgeschriebenen Ruheund Erholungszeiten eingefordert werden (Cirigliano, 2013).

\subsubsection{Arbeitszeit und Arbeitszeiterfassung}

Die Arbeitszeit ist ein im europäischen und internationalen Recht verankertes Grundrecht. In der Europäischen Union wurden diese Rechte in der Charta der Grundrechte und in Verträgen festgeschrieben. Artikel 31 der Charta behandelt das Thema "gerechte und angemessene Arbeitsbedingungen" und schreibt vor, dass jede Arbeitnehmerin und jeder Arbeitnehmer das Recht auf gesunde, sichere und würdige Arbeitsbedingungen hat. Weiter ist festgehalten, dass alle Arbeitnehmenden das Recht auf eine Begrenzung der Höchstarbeitszeit, auf tägliche und wöchentliche Ruhezeiten sowie auf bezahlten Jahresurlaub haben. Die Ausführungen gehen weiter in Artikel 151 des Vertrags über die Arbeitsweise der Europäischen Union (AEUV), der besagt, dass die Förderung der Beschäftigung und die Verbesserung der Lebens- und Arbeitsbedingungen als Ziele gesetzt werden.

Weiter steht in der Präambel der IAO-Verfassung zur Gründung der internationalen Arbeitsorganisation von 1919, dass die Arbeitszeit, Höchstdauer des Arbeitstages sowie der Arbeitswoche festzulegen sind. 39 IAO-Normen betreffen die Arbeitszeit.

Die Arbeitszeitlichtlinie (AZR), die in der Richtlinie 2003/88/EG vereinbart wurde, legt weitere Mindestvorschriften zur Sicherheit und zum Gesundheitsschutz fest mit folgendem Wortlaut: „Die Verbesserung von Sicherheit, Arbeitshygiene und Gesundheitsschutz der Arbeitnehmenden bei der Arbeit stellen Zielsetzungen dar, die keinen rein wirtschaftlichen Überlegungen untergeordnet werden dürfen" (ETUC; Die Arbeitszeitrichtlinie und Grundrechte).

Die Richtlinien regeln:

- die wöchentliche Höchstarbeitszeit von durchschnittlich 48 Stunden, einschliesslich Überstunden

- $\quad$ einen Mindesturlaub von 4 Wochen

- $\quad$ eine Ruhepause bei einer täglichen Arbeitszeit von mehr als 6 Stunden

- Mindestruhezeiten und eine Regelung für Nachtarbeit (Richtlinie 2003/ 88/EG).

Aber auch diese Regelungen werden weiterentwickelt, diskutiert und es laufen auf europäischer Ebene Verhandlungen mit den Sozialpartnerinnen und Sozial- 
partnern, bspw. darüber, die wöchentliche Höchstarbeitszeit auf 40 Stunden zu senken.

Verlassen wir nun den internationalen Kontext und fokussieren uns auf die Bestimmungen im Schweizer Recht. Die Arbeitnehmende erfüllt ihre Arbeitspflicht nicht durch Lieferung eines Arbeitsergebnisses, sondern durch Arbeitsleistung nach Zeit (Rudolph/von Kaenel, 2012, S. 197). Im Zusammenhang mit dieser Leistung von Arbeitszeit, vor allem im Zusammenhang mit mobiler Arbeit, ergeben sich daher immer wieder Fragen bezüglich Arbeitsweg, Pausen, Höchstarbeitszeit, Leistung von Überstunden und deren Erfassung sowie Erreichbarkeit der Arbeitnehmenden.

Im OR und im ArG finden sich keine Definitionen in Bezug auf Arbeitszeit. Allerdings ist in der Verordnung 1 zum Arbeitsgesetz (ArGV 1; SR 822.111) eine Erläuterung vorhanden: „Die Zeit, während der sich der Arbeitnehmer oder die Arbeitnehmerin zur Verfügung des Arbeitgebers zu halten hat." (Art. 13 Abs. 1 ArGV 1) Jede Zeitspanne, die die Arbeitnehmende mit Willen der Arbeitgebenden in deren hauptsächlichen Interesse verbringt, ist Arbeitszeit. Zu diesem Schluss kommen auch Streiff et al.: „Arbeitszeit ist diejenige Zeitspanne, während der ein Arbeitnehmer inner- oder ausserhalb des Betriebes für die Bedürfnisse des Arbeitgebers zur Verfügung zu stehen hat und auch tatsächlich steht und damit über seine Zeit nicht frei verfügen kann." Nicht zur Arbeitszeit gehört hingegen der Arbeitsweg (Streiff et al., Art. $321 \mathrm{~N}$ 9). „Wird der Arbeitnehmer dagegen an einem anderen Arbeitsort als dem vertraglichen eingesetzt, so ist die dadurch entstehende Verlängerung des Arbeitsweges Arbeitszeit (für das öffentliche Recht Art. 13 Abs. 2 ArGV 1)“ (Rudolph/von Kaenel, 2012, S. 198). Des Weiteren regeln das OR wie auch das ArG den Umgang bezüglich Überstunden sowie der Höchstarbeitszeiten.

Um diese Situation bezüglich Überstunden und Überzeit prüfen zu können, besteht nach Art. 46 ArG und Art. 73 ArGV 1 die Pflicht der Arbeitgebenden, die Arbeitszeit zu erfassen und zu dokumentieren (Rudolph/von Kaenel, 2012, S. 204). Diese Unterlagen müssen allenfalls den Vollzugs- und Aufsichtsbehörden zur Verfügung gestellt werden können. „Für die Erfassung ist die Arbeitgeberin verantwortlich, wobei sie die Dokumentation auch an den Arbeitnehmenden weiter delegieren kann" (Geiser/Müller, Rz. 955). Die Mitwirkung der Arbeitnehmenden ist vor allem bei flexiblen Arbeitszeitsystemen erforderlich. Wie einleitend bereits festgehalten, gibt es heute technische Möglichkeiten, welche diese Arbeitszeiterfassung ermöglichen. Bezüglich höheren leitenden Angestellten (Art. 3 ArG) gilt die Ausnahmeregelung, dass sie den Höchstarbeitszeitvorschriften nicht unterstellt sind. 
Flexible Formen der Arbeitszeitgestaltung, welche die Deklaration der Arbeitszeiten ganz- oder teilweise den Arbeitnehmenden überlassen und sich an vom ArG abweichenden Zeitintervallen orientieren (z.B. Gleitzeit, monatliche Sollarbeitszeit, Jahreszeit), entbinden die Arbeitgebenden allerdings nicht von der Pflicht, die Einhaltung der Vorschriften des ArG über die täglichen und wöchentlichen Höchstarbeitszeiten zumindest stichprobenweise zu kontrollieren. Widerhandlungen gegen Art. 46 ArG, vor allem die Unterlassung der Dokumentation und die Verweigerung der Herausgabe der Dokumente, sind nicht strafbar. Sie unterliegen aber dem Verwaltungszwang nach Art. 51 und Art. 52 ArG. Dieser kann mit Androhung von Ungehorsamsstrafe nach Art. 292 StGB verbunden werden, sodass indirekt doch eine strafrechtliche Sanktion möglich ist (Rudolph/von Kaenel, 2012).

Wichtig im Zusammenhang mit der Erfassung der Arbeitszeit ist noch die Frage der Beweislast im Falle von Streitigkeiten. Denn gemäss Gesetz hat diejenige, die einen Anspruch geltend machen will, diesen auch zu beweisen (vgl. Art. 8 Zivilgesetzbuch: ZGB, SR 210). Wenn nun eine Arbeitgebende ihre Pflicht zur Dokumentation unterlässt, kann sich das negativ für die klagende Arbeitnehmende auswirken. Die Beweislast für den Nachweis der Überstunden bzw. Überzeit ist trotz der Unterlassung der Dokumentation und lückenhaften Führung derselben bei der Arbeitnehmenden. Hingegen können und sollen solche Pflichtverletzungen der Arbeitgebenden in die Beweiswürdigung durch das $\mathrm{Ge}-$ richt einfliessen (Rudolph/von Kaenel, 2012).

\subsubsection{Politische Diskussion um Arbeitszeiterfassung}

Bezüglich des jahrelangen Konflikts um die Arbeitszeiterfassung sind schon diverse Optionen politisch diskutiert worden. Die Sozialpartnerinnen und Sozialpartner, das Staatssekretariat für Wirtschaft (SECO) sowie das Parlament suchen seit 2009 nach Anpassungsmöglichkeiten bei der Arbeitszeiterfassung. Denn in den vergangenen Jahren hat sich die Diskrepanz zwischen Pflicht zur detaillierten Arbeitszeiterfassung und Realität des Arbeitsalltags vergrössert und immer mehr Mitarbeitende arbeiten zeitlich und örtlich flexibel wie auch die in Kapitel 2 beschriebenen Personas.

Eine Revisionsvorlage für die Verordnung 1 zum Arbeitsgesetz im Jahre 2012 sah eine Lockerung der Pflicht zur Arbeitszeiterfassung vor, diese wurde wegen der zu weit auseinanderliegenden Positionen der Sozialpartnerinnen und Sozialpartner bezüglich des vorgeschlagenen Modells aber verworfen. Eine im Jahre 2013 eingereichte Motion sah Ähnliches vor. Sie verlangte, die Verordnung 1 dahin gehend anzupassen, dass Mitarbeitende von gewissen Branchen die 
Möglichkeit haben sollten, im Zusammenhang mit der Arbeitszeiterfassungspflicht eine Verzichtserklärung zu unterschreiben (Opting-out). Insbesondere im Investmentbanking und in der Beratungsbranche ist eine bürokratische Arbeitszeiterfassung belastend. Der Bundesrat bestätigte, dass manche Arbeitnehmende eigenverantwortlich konzipieren, planen und agieren, und zwar zum Teil ausserhalb von Bürogebäuden oder festen Bürozeiten. Deshalb erachtet er die Möglichkeit, bestimmte Arbeitnehmende von der Pflicht zur systematischen Arbeitszeiterfassung zu befreien, als eine denkbare Lösung. Dem Bundesrat erscheint es jedoch sinnvoller, Kriterien festzulegen, mit denen die Lockerung der Arbeitszeiterfassung auf diejenigen Personen beschränkt werden kann, welche objektiv über ausreichend Autonomie und Verantwortung verfügen. Eine solche Beschränkung wäre in Form einer Lohngrenze, kombiniert mit anderen Kriterien, möglich.

Im Juli 2013 hat das SECO einen Lockerungsvorschlag für Arbeitnehmende mit einem Lohn von über 175'000 Franken allerdings begraben. Die Positionen der Sozialpartnerinnen und Sozialpartner lagen damals zu weit auseinander: Die Wirtschaft wünschte auch bei tieferen Lohnklassen mehr Flexibilität, die Gewerkschaften sahen den Arbeitnehmerschutz ausgehöhlt.

Für Kaderleute mit Weisungsrecht und vollamtliche Projektleitende gilt seit dem 1. Januar 2014 eine vereinfachte Arbeitszeiterfassung. Von dieser Regelung profitiert Sandra Könitz, Projektmanagerin. Für alle übrigen Angestellten muss die Arbeitszeit derzeit lückenlos dokumentiert werden. Von der obligatorischen Arbeitszeiterfassung ausgenommen sind Topmanagerinnen und Topmanager.

Anfang 2015 wurde nun aber vom Bundesrat ein neuer Lösungsvorschlag präsentiert. Bei Arbeitnehmenden mit „sehr grosser Arbeitszeitsouveränität“ und Löhnen über 120'000 Franken soll die Erfassungspflicht gelockert werden können. Allerdings muss der Verzicht auf die Arbeitszeiterfassung im Rahmen eines Branchen- oder Unternehmensgesamtarbeitsvertrags erfolgen, wie das Departement für Wirtschaft, Bildung und Forschung (WBF) mitteilt. Die geplante Neuregelung betrifft vor allem Dienstleistungsunternehmen.

Diese Kompromisslösung bringt gemäss Daniel Lampart, Chefökonom des Schweizerischen Gewerkschaftsbundes, Vor- und Nachteile: „Positiv ist jetzt, dass die Kantone die Arbeitszeit bei den Normalverdienenden kontrollieren müssen. Negativ ist, dass ein Teil der Arbeitnehmerschaft ausgenommen ist. Dieser Teil, das sind vor allem Kaderleute, die zu einem grossen Teil selber bestimmen können, wie sie arbeiten wollen." Der Direktor des Schweizerischen Arbeitgeberverbands, Roland A. Müller, sieht die Lösung als ein Zeichen, dass 
Kompromisse zwischen den Sozialpartnerinnen und Sozialpartnern noch möglich sind. „Es ist aber auch ein Zeichen der Flexibilität. Nämlich, dass wir im Bereich der zeitsouveränen Arbeitnehmenden etwas deregulieren können.“

Aufgrund der gering zu erwartenden Opposition ist es das Ziel, den Einigungsvorschlag mit seinen beiden Elementen nun in die Verordnung 1 des Arbeitsgesetzes aufzunehmen. Damit dies rasch geschehen kann, wurde nur eine verkürzte Konsultation durchgeführt, sodass die Verordnung im dritten Quartal 2015 in Kraft gesetzt werden kann.

Der grösste Knackpunkt in der Umsetzung liegt dann in der GAV-Pflicht für jene Firmen und Branchen, die von der Lockerung der Zeiterfassung profitieren wollen. Es soll gemäss Erläuterungen des Bundes möglich sein, einen Mini-GAV zu diesem Thema abzuschliessen, der faktisch nur Regeln zur Arbeitszeiterfassung und zum Gesundheitsschutz enthält. Nicht zulässig wäre aber laut Bund die Gründung einer Ad-hoc-Gewerkschaft nur zwecks Abschluss eines GAV zur Arbeitszeiterfassung (www.admin.ch / www.nzz.ch).

Am 4. November 2015 hat der Bundesrat schliesslich beschlossen, die Arbeitszeiterfassung der heutigen Arbeitswelt anzupassen. Wie bereits erwähnt, wird er dazu die Verordnung 1 zum Arbeitsgesetz mit der Einführung von Art. 73a und Art. 73b ergänzen. Art. 73a ArGV 1 sieht sodann vor, dass künftig auf die Arbeitszeiterfassung gänzlich verzichtet werden kann, sofern ein Gesamtarbeitsvertrag und eine schriftliche Zustimmung des Arbeitnehmenden vorhanden ist. Von dieser Möglichkeit Gebrauch machen können ausschliesslich Arbeitnehmende, die über ein Bruttojahreseinkommen von mehr als 120'000 Franken verfügen.

Art. 73b ArGV 1 soll die Möglichkeit einer stark vereinfachten Arbeitszeiterfassung bieten, d.h., es soll nur noch die Gesamtdauer der täglichen Arbeitszeit erfasst werden. Ausnahme hierfür ist die Sonntags- und Nachtarbeit. Hier muss weiterhin Beginn und Ende des Arbeitseinsatzes dokumentiert werden. Um von der vereinfachten Arbeitszeiterfassung profitieren zu können, ist eine Vereinbarung zwischen Arbeitgebenden und der internen oder externen Arbeitnehmervertretung notwendig. Fehlt eine Arbeitnehmervertretung, so hat die Mehrheit der Arbeitnehmenden einer Unternehmung der Einführung zuzustimmen. Bei $\mathrm{KMU}$ mit weniger als 50 Arbeitnehmenden kann diese sogar auf einer individuellen Vereinbarung basieren.

Mit den beiden neuen Artikeln sollen sowohl die Rechtssicherheit wiederhergestellt und die Unternehmen administrativ entlastet werden. Die Revision der Verordnung 1 zum Arbeitsgesetz tritt per 1. Januar 2016 in Kraft (SECO, 2015). 


\subsubsection{Vertrauensarbeitszeit}

Das Modell der Vertrauensarbeitszeit, wie bei Sandra Könitz, Projektmanagerin, umgesetzt, würde eigentlich alle Vorteile der flexiblen Arbeitstätigkeit mit sich bringen, jedoch verstösst es gegen das geltende Recht. Gemäss Gesetz und Rechtsprechung ist eine Zeiterfassungskontrolle vorgeschrieben (Gutzwiller, 2010). Zudem geht die Arbeitgebende das Risiko von Geldforderungen ein. Sofern bei einer Kündigung eine Mitarbeitende die Mehrarbeit glaubhaft machen kann, muss die Arbeitgebende diese nachträglich ausbezahlen. Zusätzlich kann das Arbeitsinspektorat bei fehlenden Kontrollmechanismen bezüglich Arbeitszeiterfassung im Wiederholungsfall Bussen aussprechen. Charakteristisch für die Vertrauensarbeitszeit ist, dass bei dieser Form der Arbeitszeiterfassung statt auf Überwachung auf die Eigenverantwortlichkeit der Mitarbeitenden gesetzt und dabei auf eine Kontrolle der Arbeitszeit verzichtet wird (Chilles, 2012). Der Vertrauensarbeitszeit steht jedoch grundsätzlich nichts im Wege, sofern die Höchstarbeitszeit eingehalten wird.

Gemäss Art. 46 ArG i.V.m. Art. 73 ArGV 1 besteht die Pflicht, die Arbeitszeit zu erfassen und die entsprechenden Unterlagen und Verzeichnisse während mindestens fünf Jahren aufzubewahren. Mit flexiblen Arbeitsmodellen entsteht aber zunehmend der Wunsch nach flexiblen Arbeitszeiten. Dies kann zu Problemen bei der Erfassung der Arbeitszeit führen. Grundsätzlich ist die Arbeitgebende zur Erhebung und Dokumentation der entsprechenden Daten verpflichtet, jedoch kann dabei auch die Arbeitnehmende mitwirken. Gerade bei flexiblen Arbeitszeitsystemen, bei denen die Arbeitnehmende ihre Arbeitszeit selbst definiert, ist dies von Bedeutung. Es ist aber zu betonen, dass auch bei der Gleitzeit, monatlichen Sollarbeitszeit, Jahresarbeitszeit und anderen flexiblen Arbeitszeitsystemen die Pflicht zur Deklaration der Arbeitszeit und die zumindest stichprobenartige Kontrolle bei der Arbeitgebenden verbleibt und nicht an die Arbeitnehmenden übergeht. Die in Art. 73 ArGV 1 enthaltene, nicht abschliessende Aufzählung, stellt einen Minimalstandard der zu dokumentierenden Angaben dar. Mit dieser Regelung wird allerdings der Rechtswirklichkeit in Unternehmen nicht mehr Rechnung getragen. Vor allem in der Banken- oder Versicherungsbranche ist die Vertrauensarbeitszeit weit verbreitet und es wird oftmals ganz oder zumindest teilweise auf die Zeiterfassung bzw. deren Dokumentation verzichtet (Rudolph/von Kaenel, 2014). Möchte man allerdings dem öffentlich-rechtlichen Arbeitnehmerschutz nachkommen, ist ein Mindestmass an Kontrolle unabdingbar.

Das Dilemma zwischen Praxisnähe sowie administrativer Vereinfachung einerseits und ausreichender Kontrolle andererseits versuchte das SECO mit dem 
Pilotprojekt „Vertrauensarbeitszeit bei Banken“ in den Griff zu bekommen. Aus dem Projekt resultierte der Vorschlag, die Verordnung 1 zum Arbeitsgesetz mit einem neuen Art. 73a zu ergänzen. Dabei sollten Arbeitnehmende, welche über einen grossen Gestaltungsfreiraum beim Inhalt sowie bei der Organisation ihrer Arbeit verfügen und Verhandlungsmacht gegenüber ihren Arbeitgebenden geniessen, auf die gesetzliche Arbeitsaufzeichnung verzichten können. Dies wäre gestützt auf objektive Kriterien wie ein Jahreseinkommen von 175'000 Franken und einen Handelsregistereintrag möglich. Ein solches Absehen von der Arbeitszeiterfassung wäre mit der Arbeitnehmenden dieser Kategorie individuell und schriftlich zu vereinbaren und ist jederzeit widerrufbar (SECO, 2011, S. 19).

Der Vorschlag vom SECO wurde allerdings, wie im Abschnitt 3.4.2 geschildert, im Juli 2013 verworfen. Das SECO hat jedoch eine Weisung an die kantonalen Arbeitsinspektorinnen und Arbeitsinspektoren erlassen. Diese sieht vor, dass Mitarbeitende, welche ihre Aufgaben sehr autonom erfüllen und die Arbeitszeit selbstständig verwalten, ab 1. Januar 2014 nur noch ihre tägliche und wöchentliche Arbeitszeit erfassen müssen (Rudolph/von Kaenel, 2014). Voraussetzung dafür ist das Vorliegen einer schriftlichen Vereinbarung. Mit dieser „erleichterten Zeiterfassung“ wurde ein Mittelweg geschaffen zwischen der „vollen Zeiterfassung“, welche für die meisten Arbeitnehmenden besteht, und "gar keiner Zeiterfassung“, die gemäss Art. 3 lit. d ArG für höhere leitende Angestellte gilt (Rudolph/von Kaenel, 2014).

\subsubsection{Vertrauensarbeitszeit bei Gesamtarbeitsverträgen}

Die am meisten verbreitete Bestimmung bei Gesamtarbeitsverträgen (GAV) betrifft die wöchentliche Dauer der Arbeitsleistung (Stöckli, 1990). Konkrete Regelungen zur Zeiterfassung und -kontrolle sind lediglich in 6 Prozent der Verträge vorhanden (Stöckli, FN 19, 307). Wertvolle Beispiele für Bestimmungen im Zusammenhang mit flexibilisierter Arbeitszeit sind Art. 15 und 21 des L-GAV des Gastgewerbes sowie die Vereinbarung über die Anstellungsbedingungen der Bankangestellten 2006 (Müller/Oechsle, 2007, S. 852).

Weder aus dem Arbeitsgesetz noch aus den entsprechenden Verordnungen ist explizit ersichtlich, wer die Zeiterfassung durchführen muss. Demzufolge ist es auch möglich, die Pflicht zur Erfassung der Arbeitszeit mittels Einzelarbeitsvertrag oder Arbeitszeitreglement der Arbeitnehmenden zu übertragen (Müller/Oechsle, 2007, S. 852). Wichtig ist jedoch, dass die Verantwortung zur Zeiterfassung weiterhin bei der Arbeitgebenden liegt und die erwähnten Anforderungen an die Dokumentation bestehen bleiben (Müller/Oechsle, 2007, S. 852; Müller FN 27, N 2). Für eine Delegation an die Arbeitnehmenden sind einerseits 
die notwendigen formellen Voraussetzungen einzuhalten und andererseits muss die Qualität der Daten den Anforderungen des Arbeitsgesetzes entsprechen (Müller/Oechsle, 2007, S. 852).

Bei Arbeitszeitregelungen, die Gleitzeitarbeit oder Vertrauensarbeit vorsehen, kann hingegen die Einhaltung der arbeitsgesetzlichen Bestimmungen nicht immer gewährleistet werden. Das Arbeitsgesetz schliesst in Art. 3 einige Personen aus dem persönlichen Geltungsbereich aus, sodass die entsprechenden Bestimmungen auf sie nicht anwendbar sind. Dazu gehören u.a. „Personen, die eine höhere leitende Tätigkeit oder eine wissenschaftliche oder selbstständige künstlerische Tätigkeit ausüben“ (Art. 3 Bst. d ArG). Massgebend bei der Entscheidung, ob eine Person unter diese Bestimmung fällt, ist die Frage, ob sie weitreichende Entscheidungsbefugnisse im Betrieb innehat, wobei sich die Entscheide auf wesentliche Angelegenheiten zu beziehen haben und mindestens die Hauptteile der Struktur des Unternehmens nachhaltig bestimmen (Geiser, FN 31, N 22). Funktionsbezeichnungen oder hierarchische Stellungen dürfen dabei nicht relevant sein (Müller/Oechsle, 2007, S. 850).

Diese Ausnahmen gelten hingegen nicht absolut. Gemäss Art. 3a ArG sind für gewisse ausgenommene Personen die Bestimmungen über den Gesundheitsschutz anwendbar, so auch für Arbeitnehmende, die eine höhere leitende Tätigkeit, eine wissenschaftliche oder selbstständige künstlerische Tätigkeit ausüben (Art. 3a Bst. b ArG).

Eine im September 2014 eingereichte Motion verlangte eine Anpassung der Bestimmungen betreffend Arbeitszeiterfassung an die heutigen neuen Arbeitsmodelle. Dabei sollten Art. 73 Absätze c, d, e ArGV 1 nicht zur Anwendung gelangen, wenn eine Arbeitgebende sozialpartnerschaftlich mit einem Arbeitnehmendenverband durch separate Vereinbarung die Handhabung der Arbeitszeiterfassung regelt. Der Bundesrat anerkannte zwar, dass eine Anpassung der Modalitäten für die Arbeitszeiterfassung in Art. 73 ArGV 1 an die Entwicklung der Arbeitswelt notwendig ist, hielt jedoch fest, dass eine sofortige Anpassung der Verordnung nicht der richtige Weg sei, um dieses Ziel zu erreichen.

\subsubsection{Spannungsfeld III: Arbeitszeitregelung versus Flexibilität}

Die Arbeitszeit umschreibt das OR in der konkreten zeitlichen Beanspruchung des Arbeitnehmenden pro Tag, Woche, Monat oder Jahr. Wie diese Arbeitszeit geregelt wird, suchen wir vergebens im Gesetz. Hier gilt die Vertragsfreiheit, indem die zwei Parteien diese Rahmenbedingungen im Einzel-, im Gesamtarbeitsvertrag oder einfach im Personalreglement bilateral regeln können. Die 
Vertragsfreiheit wird allerdings durch zwingende Schutzvorschriften des Arbeitsgesetzes beschränkt. Insbesondere dessen Bestimmungen über die wöchentliche Höchstarbeitszeit, über Tages-, Abend-, Nacht- und Sonntagsarbeit sowie über die freien Tage und Pausen sind wie vorgeschrieben einzuhalten.

Da das Arbeitsgesetz öffentlich-rechtliches Arbeitsschutzrecht enthält, steht das Recht auf Einhaltung und Durchsetzung gegenüber der Arbeitgebenden dem Staat zu. Dieser setzt zur Überprüfung und Sanktionierung von Nichteinhalten wiederum Arbeitsinspektorate ein. Aber auch die Arbeitnehmenden direkt haben aufgrund von Art. 342 Abs. 2 OR einen zivilrechtlichen Anspruch gegenüber der Arbeitgebenden auf Einhaltung der Arbeitsschutzvorschriften des Arbeitsgesetzes. Weniger aufwendig, als einen Zivilprozess zu lancieren, ist es natürlich, dem Arbeitsinspektorat eine Meldung zu machen, das dann für die Einhaltung des Gesetzes besorgt ist.

\subsubsection{Flexible Arbeitszeitmodelle}

In den nachfolgenden Abschnitten werden die unterschiedlichen, bereits in der Praxis bewährten Arbeitszeitmodelle erläutert und immer wieder untersucht, inwieweit diese mit der Flexibilität vereinbar sind respektive wo wir rechtliche Bestimmungen einzuhalten haben.

\subsubsection{Gleitzeitmodell}

Das bekannteste Modell ist das Gleitzeitmodell. Hier kann eine hohe Flexibilität der Arbeitszeit erreicht werden. Die Modalitäten sind in einem Reglement schriftlich festzuhalten und zum Vertragsbestandteil zu erklären.

\subsubsection{Gestaltlose Arbeitszeit oder Arbeitszeitkonti/Jahresarbeitszeit}

Bei diesem Modell wird nur fixiert, wie viel Arbeit eine Mitarbeitende in einem gewissen Zeitraum zu erbringen hat. Wie lange und wann gearbeitet wird, ist nicht festgehalten. Dabei geht es um Wochen-, Monats-, Jahres- oder Lebensarbeitszeit. Besonders in den USA sind diese Modelle weit verbreitet. In der Schweiz findet man sie oftmals in der öffentlichen Verwaltung. Je grösser die Abrechnungsperiode gewählt wird, desto grösser wird die Flexibilität. So lassen sich Sabbaticals, Auszeiten oder Frühpensionierungen realisieren. Zu beachten ist, dass diese Mehrarbeit der Mitarbeitenden eine geldwerte Leistung darstellt 
und entsprechend als Schuld in der Bilanz deklariert werden muss (Gutzwiller, 2010, S. 19).

Auch bei schwankenden Arbeitseinsätzen bleibt der Lohn bei der Jahresarbeitszeit konstant gleich. Überstunden werden nicht ausbezahlt, sondern in der Regel durch Freizeit kompensiert. Dieses Modell eignet sich gut für Unternehmen, deren Produktivität schwankt (bspw. Saisonbetriebe), oder eben für Unternehmungen, die den Mitarbeitenden eine Flexibilität und dadurch eine bessere Vereinbarkeit von Familie, Freizeit und Beruf ermöglichen wollen (Gutzwiller, 2010, S. 19 / www.kvschweiz.ch).

Zudem können die Arbeitseinsätze laufend den Auftragsvolumen angepasst werden, welche durch die Flexibilität mit der bestehenden Belegschaft und nicht durch zusätzliche Temporärarbeitende gestemmt werden muss.

Die Probleme bei der praktischen Implementierung dürfen aber nicht unterschätzt werden. So wird häufig die Arbeitszeit als knappe Ressource verstanden. Darüber hinaus will diese optimal eingesetzt sein - ohne dabei zu einer ganz unregelmässigen und zugleich „Arbeit auf Abruf“ zu werden. Trotz Flexibilisierung muss das Arbeitspensum berechenbar, planbar und im Durchschnitt konstant bleiben. Nur eine gerechte Verteilung der Vorteile der Flexibilisierung zwischen Arbeitgebenden und Arbeitnehmenden führt nachhaltig zum Erfolg.

So kommen auch die Bestimmungen des ArG zum Zuge, denn auch die Jahresarbeitszeit ist nicht gleichbedeutend mit einem Freipass für vorübergehende unbeschränkte Arbeitsleistung und damit verbundene Überstunden oder eben gar Überzeit. Die gesetzlichen wöchentlichen Höchstarbeitszeiten sind auch hier einzuhalten.

Wichtige Punkte, die geregelt sein sollten (in Anlehnungen an Baua, 2013):

- Zugriffsrechte der Beschäftigten auf ihr Zeitguthaben

- $\quad$ Mitbestimmung über die Situation verlängerter Arbeitszeiten

- $\quad$ Mindestankündigungsfrist bei Arbeitszeitänderungen und Mehrarbeit

- Höchstarbeitszeiten

- Höchstgrenzen für Zeitkontostände und Regelungsmodus für Höchstgrenzen

- $\quad$ Regelung zur Personalaufstockung bei nicht abbaubaren Guthaben

- $\quad$ Flexibilitätsbonus als Zeitzuschlag

- $\quad$ Verzinsung von Zeitguthaben

- Modus für Konfliktregelung 


\subsubsection{Arbeit auf Abruf}

Bei der Arbeit auf Abruf arbeitet die Mitarbeitende nur dann, wenn Arbeit für sie anfällt. Dabei ist zwischen echter und unechter Arbeit auf Abruf zu unterscheiden. Während bei der echten Arbeit auf Abruf die Arbeitnehmende zum Einsatz verpflichtet ist, kann sie diesen bei der unechten Arbeit auf Abruf ablehnen. Die Lage der Arbeitszeit, deren Dauer oder gar beides ist bei der echten Arbeit auf Abruf nicht im Voraus bestimmt oder nicht bestimmbar. Bei der unechten Arbeit auf Abruf einigen sich die Parteien im Einzelfall über den Arbeitseinsatz, dessen Lage sowie Dauer. Darüber hinaus muss die Arbeitnehmende bei der echten Arbeit auf Abruf Bereitschaftsdienst für die Dauer der vereinbarten Arbeitszeit leisten, was bei der unechten Arbeit auf Abruf entfällt. Dieser Bereitschaftsdienst ist entschädigungspflichtig, allerdings zu einem tieferen Ansatz als zum Lohn für den effektiven Arbeitseinsatz (BGE 124 III 251). Des Weiteren wird für die unechte Arbeit auf Abruf grundsätzlich ein Rahmeneinsatzvertrag ausgearbeitet, in dem die Bedingungen für allfällige Arbeitseinsätze schriftlich festgehalten sind (WEKA, S. 67). Gewerkschaften sehen diese Art der Arbeit zwar nicht gerne, vom Bundesgericht ist sie jedoch für zulässig erklärt worden (Gutzwiller, 2010).

Weiter ist festzuhalten, dass durch die Abrufe nicht jedes Mal neue Arbeitsverträge zustande kommen wie bei der Gelegenheitsarbeit. Hier liegt ein fortdauerndes Arbeitsverhältnis vor, das aktive und latente Phasen aufweist. Häufig ist es in der Praxis jedoch mangels unklarer vertraglicher Abmachungen schwierig, genaue Unterscheidungen zu treffen (Nötzli, 2009).

\subsubsection{Spannungsfeld IV: Flexibilität der Arbeit auf Abruf versus soziale Sicherheit}

Für die Arbeitgebende kann sich die Arbeit auf Abruf als sehr attraktiv erweisen, weil sie ihre Kapazitäten und Bedürfnisse so optimal abdecken kann. Für die Arbeitnehmende ist diese Art von Arbeit ein Risiko, da der Lohn infolge des schwankenden Umfangs unter dem existenziellen Mindesteinkommen liegen kann. Zudem bleibt sie unflexibel, da sie sich dennoch für einen möglichen Einsatz bereithalten muss. Diese Situation kann abgefedert werden, indem eine Mindesteinsatzdauer im Vertrag festgelegt wird. Zudem muss eine Arbeitnehmende einen abrupten und erheblichen Einsatzverlust ihres gewohnten Pensums nicht hinnehmen. Das Bundesgericht befand (BGE 125 III 65), dass eine solche Reduktion einer Umgehung der Kündigung gleichkomme, weshalb der Arbeitnehmenden entsprechende Lohnforderungen, die dem Lohn während der Kündigungsfrist entsprachen, zugesprochen wurden. 


\subsubsection{Temporärarbeit}

Seit Januar 2011 sind alle Personalvermittlungsbüros dem Gesamtarbeitsvertrag unterstellt. Zudem sind etliche Verleihunternehmen nach Qualitätsstandards SQS zertifiziert. Gleichzeitig ist eine vermehrte Zusammenarbeit von RAV und privaten Arbeitsvermittelnden spürbar. Darüber hinaus wachsen die temporären Arbeitsverhältnisse an. Die steigende Anzahl zeigt, dass Temporäranstellungen vermehrt auch für Unternehmen attraktiv sind. Es gibt Unternehmen, die immer eine gleich hohe Anzahl Temporärpersonal beschäftigen und diesen Einsatz so auch budgetieren. Dies deutet darauf hin, dass nicht für die Überbrückung von Engpässen auf Temporärarbeitende gesetzt wird, sondern dass diese ein fester Bestandteil der Personalpolitik sind (Frossard, 2013). Zudem werden temporäre Arbeitende häufig dann eingesetzt, wenn es in kurzer Zeit mit spezifischem Know-how ein Problem zu lösen gilt. Dies verlangt seitens der Arbeitnehmenden Flexibilität, soziale Kompetenz und die Fähigkeit, sich in kürzester Zeit in eine neue Aufgabe hineinzudenken. Daraus resultieren häufig interessante Einsätze und es entsteht ein Netzwerk, aber es ist auch klar, dass diese Personen mit einer Festanstellung liebäugeln. Tatsächlich kommt es auch häufig vor, dass sich aus einer Temporäranstellung eine Festanstellung ergibt.

Weiter bietet sich die Temporärarbeit vor oder nach dem Studium, nach einer längeren Abwesenheit aufgrund eines Urlaubs, vor oder nach der Rekrutenschule oder auch bei plötzlichem Stellenabbau an. Es können so Lohnausfälle und Lücken bei den Sozialversicherungen vermieden werden. Ausserdem bleiben Temporärbeitende im Arbeitsprozess und in einer geregelten Tagesstruktur und können sich so Zeit lassen, eine geeignete Festanstellung zu suchen. Für eine solche Möglichkeit des „Zwischenverdienstes“ ist Temporärarbeit die einzige aktuell vorhandene Einsatzform (Felder, 2014).

\subsubsection{Altersteilzeit}

Dieses Modell bietet älteren Arbeitnehmenden die Möglichkeit, ihre Arbeitszeit nach Vollendung des 55. Lebensjahres um die Hälfte zu verringern. Die verbleibende Arbeitszeit kann flexibel verteilt werden. Gemäss der Studie von Baua war in den letzten Jahren erkenntlich, dass für viele Unternehmen der einzige Vorteil des Altersteilzeitmodells das „preiswerte Loswerden“ von älteren Mitarbeitenden zu sein scheint. Langsam und nicht zuletzt vor dem Hintergrund des demografischen Wandels wächst jedoch die Erkenntnis, dass mit den älteren Mitarbeitenden auch viel Know-how und Erfahrung verloren gehen. Deshalb wird es vielerorts nun das Ziel, diese Gruppe im Unternehmen zu erhalten. Auch hier hilft Flexibilität, denn so wird der veränderten Leistungsfähigkeit der 
Mitarbeitenden Rechnung getragen. In Deutschland gibt es hierzu das Altersteilzeitgesetz (Bundesministerium der Justiz und für Verbraucherschutz). Es ermöglicht der Mitarbeitenden ab dem 55. Lebensjahr, vorausgesetzt, dass sie die letzten fünf Jahre einer versicherungspflichtigen Beschäftigung nachgegangen ist, in Absprache mit der Arbeitgebenden eine Halbierung der Arbeitszeit. Die Mitarbeitende erhält 70 Prozent ihres letzten Nettolohns, der in der Regel von der Arbeitgebenden auf 85 Prozent aufgestockt wird. Hinsichtlich der Rentenversicherung wird sie so gestellt, als würde sie 90 Prozent arbeiten. Einen Rechtsanspruch auf eine Altersteilzeitvereinbarung haben weder Arbeitgebende noch Arbeitnehmende, sofern nicht ein Tarifvertrag einen solchen Anspruch einräumt. Es kann also festgehalten werden, dass auch die Unternehmen Vorteile aus der Altersteilzeitarbeit ziehen, denn innen bleiben Wissen, Können und Erfahrungen der älteren Arbeitnehmenden erhalten, wobei die Arbeitszeit nach den Bedürfnissen der Unternehmen sowie den Wünschen der Arbeitnehmenden verteilt werden kann.

\subsubsection{Spannungsfeld IV: Regulierung durch Rahmenbedingungen ver- sus genügend Freiraum für Flexibilität}

Wenn die aufgezeigten Herausforderungen der Planbarkeit und Zeiterfassung eingehalten werden, kann bezüglich flexibler Arbeitszeiten eine Win-WinFlexibilität für beide Parteien erreicht werden. Zumal hier auch gesetzliche Rahmenbedingungen zum Schutz der Arbeitnehmenden vorhanden sind, sehen wir hier den rechtlichen Auftrag als erfüllt an. Dass sich solche Ideen auch umsetzen lassen und in der Wirtschaft verfolgt werden, zeigt die Charta „Work Smart". Microsoft Schweiz, Die Mobiliar, Die Post, die SBB, die SRG, die Swisscom und der Bürokonzeptentwickler Witzig haben diese Charta gemeinsam unterzeichnet. Sie wollen flexibles Arbeiten in den eigenen Betrieben fördern und die Schweizer Wirtschaft bei der Einführung von flexiblen Arbeitsformen unterstützen. Auch das Modell der Teilzeitarbeit für Männer wurde vom Eidgenössischen Büro für Gleichstellung von Mann und Frau bis 2014 gefördert.

Welche Form auch immer umgesetzt wird, wichtig ist, dass klare Rahmenbedingungen geschaffen und definiert werden, bspw. bezüglich der Erreichbarkeit der flexiblen Arbeitnehmenden. Fehlen diese Abmachungen, droht gerade bei der Vertrauensarbeitszeit die Gefahr der entgrenzten Arbeit, weil Arbeit und Freizeit nicht mehr klar getrennt sind. Hier wird interessant sein, ob die vorgeschlagenen Änderungen von den Arbeitnehmer- und Arbeitgeberverbänden akzeptiert werden und wie sie dann zur Umsetzung gelangen. 


\subsection{Kündigungsschutz}

Einen Schutz gegen missbräuchliche Kündigungen gibt es in der Schweiz seit über 25 Jahren. Inzwischen mussten sich die Gerichte unzählige Male mit der Frage auseinandersetzen, wann eine Kündigung missbräuchlich ist und wann nicht. Zudem gibt es rechtliche Sperrfristen, die dem andernfalls ,jederzeitigen“ Kündigungsrecht beider Parteien Grenzen setzen. Die Relevanz bezüglich der flexiblen Arbeitswelt wird nachfolgend erläutert.

\subsubsection{Kündigungsschutz beim Einzelarbeitsvertrag}

Das Arbeitsprivatrecht des Obligationenrechts ist geprägt vom Grundsatz der Kündigungsfreiheit. Gemäss Rudolph bedeutet dies in formeller Hinsicht, dass das Arbeitsverhältnis von beiden Vertragsparteien jederzeit unter Beachtung der anwendbaren Kündigungsfristen und -termine aufgelöst werden kann. Inhaltlich zeigt sich die Kündigungsfreiheit darin, dass die Wirksamkeit einer Kündigung keine besondere sachliche Rechtfertigung voraussetzt. Eine Kündigung ist nach dem Obligationenrecht auch dann zulässig, wenn sie objektiv betrachtet nicht notwendig ist oder gar unvernünftig erscheint (Rudolph, 2011, Rz. 1).

Davon betroffen sind unbefristete wie befristete Arbeitsverhältnisse. Auch wenn gemäss Art. 334 Abs. 1 OR ein befristetes Arbeitsverhältnis ohne Kündigung endigt, sehen Streiff et al. (Art. 334, N2) die Möglichkeit, dass ein befristetes Arbeitsverhältnis vorzeitig durch Kündigung enden kann.

Allerdings sind in diesem Bereich auch Schutzbestimmungen vorgesehen. Das Prinzip der Kündigungsfreiheit, welches beiden Parteien gleichermassen zusteht, wird durch den sachlichen und zeitlichen Kündigungsschutz eingeschränkt. Gemäss Geiser \& Müller (Rz. 612) ist der Zweck des zeitlichen Kündigungsschutzes nach Art. 336c OR, der Arbeitnehmenden die Stelle zu erhalten, solange sie verhindert ist, einen neuen Arbeitsplatz zu suchen.

\subsubsection{Zeitlicher Kündigungsschutz}

Im Gesetz sind für die Kündigung durch die Arbeitgeberin vier Sperrzeiten vorgesehen (Art. 336c Abs. 1 OR):

- $\quad$ Leistung von Militär-, Schutz- oder Zivildienst

- $\quad$ Dienste für eine behördliche Hilfsaktion im Ausland

- unverschuldete Arbeitsverhinderung durch Krankheit oder Unfall

- $\quad$ Schwangerschaft der Arbeitnehmerin 
Gemäss Rudolph sind die Kündigungen, die während dieser Sperrfristen ausgesprochen werden, nichtig: „Tritt die Sperrfrist (z.B. eine Erkrankung) erst während der Kündigungsfrist ein, bleibt die Kündigung zwar gültig, doch ruht die Kündigungsfrist während dieser Zeit, was zu einer entsprechenden Erstreckung des Arbeitsverhältnisses führt“ (Rudolph, 2011, Rz. 2).

\subsubsection{Sachlicher Kündigungsschutz}

Beim sachlichen Kündigungsschutz geht es in erster Linie um die Gründe, aus denen das Arbeitsverhältnis gekündigt wird. Dabei ist es gleichgültig, ob die Arbeitgebende oder die Arbeitnehmende die Kündigung ausspricht (Rehbinder, Rz. 327). Missbräuchliche Kündigungsgründe sind sodann (Art. 336 Abs. 1 $\mathrm{OR})$ :

- $\quad$ Kündigung wegen persönlicher Eigenschaften, die weder einen Bezug zum Arbeitsverhältnis haben noch das Betriebsklima wesentlich beeinträchtigen, wie zum Beispiel: Rasse, Religion, Alter usw.

- Kündigung wegen der Ausübung verfassungsmässiger Rechte, bspw. politische Meinungsäusserungen

- $\quad$ Kündigung wegen Vereitelung von Ansprüchen

- $\quad$ Rachekündigung

- Kündigung wegen Zugehörigkeit oder Nichtzugehörigkeit der Arbeitnehmenden zu einer Gewerkschaft

Der Beweis einer Kausalität zwischen Grund und Kündigung obliegt im Allgemeinen der gekündigten Partei (Geiser/Müller, Rz. 626). Im Weiteren hat die Missbräuchlichkeit einer Kündigung nicht deren Ungültigkeit zur Folge, sondern führt vielmehr zu einer Entschädigungspflicht (Art. 336a Abs. 1 OR; Geiser/Müller, Rz. 630).

Ist die mobile Arbeitsform aufgrund der in Abschnitt 3.2.1 erläuterten Abgrenzungskriterien nicht als Arbeitsverhältnis nach Obligationenrecht zu deklarieren, kommt der Kündigungsschutz so nicht zur Anwendung. Nur wenn ein Arbeitsvertrag nach OR vorliegt, gelten auch der zeitliche und sachliche Kündigungsschutz. Handelt es sich hingegen um einen Auftrag oder Werkvertrag, kann dieser grundsätzlich jederzeit frei widerrufen werden. Hier sind Überlegungen zu tätigen, ob diese Schutzprinzipien auch bei neuen mobilen Arbeitsformen entweder durch Gesetzeserlasse oder aber durch Rechtsprechung zum Tragen kommen sollen. Erste Überlegungen sind bereits erfolgt, so merkt Rehbinder an, dass die Kündigungsschutzbestimmungen für Scheinselbstständige analog gelten (Rehbinder, Schweiz. Arbeitsrecht, Rz. 25). 


\subsubsection{Kündigung beim Werkvertrag}

Ein Werkvertrag kann gemäss Art. 375 bis 379 OR auf fünf Arten enden: Rücktritt wegen Überschreitung des Kostenansatzes, Untergang des Werkes, Rücktritt des Bestellers gegen Schadloshaltung, Unmöglichkeit der Erfüllung aus Verhältnissen des Bestellers oder Tod und Unfähigkeit des Unternehmers. Gemäss Zindel/Pulver kann nur der Besteller und nur gegen Ersatz des Erfüllungsinteresses jederzeit vom Werkvertrag zurücktreten (Gaudenz G. Zindel/Urs Pulver, BSK OR I, 4. Aufl., Vor Art. 363-379 N8). Demzufolge kann eine Unternehmerin bzw. ein Unternehmer nicht ohne Weiteres vom Vertrag zurücktreten. Im Unterschied zum Einzelarbeitsvertrag ist hier vor allem auf die einseitige Kündigungsmöglichkeit der Unternehmenden (bzw. Arbeitgebenden) hinzuweisen. Befände sich die mobile Arbeitnehmende in einem Werkvertragsverhältnis, hätte nur ihre Arbeitgebende ein Beendigungsrecht und das jederzeit.

\subsubsection{Kündigungsschutz beim Auftrag}

Gemäss Art. 404 OR kann der Auftrag von allen Vertragsparteien jederzeit gekündigt bzw. widerrufen werden. Fellmann schreibt dazu im Berner Kommentar, dass der Auftrag seitens der Auftraggebenden mittels Widerruf und seitens der beauftragten Person mittels Kündigung beendigt werden kann. „Die Besonderheit dieses Auflösungsrechtes liegt darin, dass sowohl der Auftraggeber wie auch der Beauftragte das Auftragsverhältnis grundsätzlich jederzeit, d.h. ohne die Einhaltung von Kündigungsterminen und fristlos, ohne Beachtung von Kündigungsfristen, beenden kann“ (Fellmann, 2010, Art. 404 OR, Rz. 19). Im Unterschied zum Einzelarbeitsvertrag ist hier die jederzeitige, fristlose Auflösungsmöglichkeit hervorzuheben. Wenn gemäss Abschnitt 3.2.3 der Einzelarbeitsvertrag nicht anwendbar ist, sondern ein Auftrag vorliegt, kann die Beauftragte (bzw. Arbeitnehmende) ihre Arbeit jederzeit ohne Frist verlieren.

\subsection{Gesundheit und soziale Absicherung}

Im Zusammenhang mit flexiblen Arbeitsformen stellt sich die Frage, wie die Arbeitgebende ihrer Fürsorgepflicht der Arbeitnehmenden gegenüber nachkommt oder welche anderen sozialen Absicherungen zum Zuge kommen. In diesem Kapitel geht es hauptsächlich um die gesundheitliche Komponente des Arbeitnehmerschutzes. Art. 328 OR regelt die Fürsorgepflicht der Arbeitgebenden. Diese Pflicht ergibt sich aus der Beziehung zwischen Arbeitnehmenden und Arbeitgebenden durch den Arbeitsvertrag. „Die Fürsorgepflicht ist ein wich- 
tiges Korrelat zur Weisungsgebundenheit und persönlichen Abhängigkeit des Arbeitnehmers" (Streiff et al., Art. 328 N 2).

Es muss allerdings noch kurz geklärt werden, was unter Gesundheit zu verstehen ist. Gemäss der Definition der WHO ist Gesundheit nicht nur das Fehlen von Krankheit und Gebrechen, sondern ein Zustand des vollständigen körperlichen, geistigen und sozialen Wohlergehens (WHO, 2014). Die nachstehende Abbildung 11 zeigt, dass eine Arbeitnehmende sowohl durch physische als auch durch psychische Faktoren während der Arbeit erkranken kann. Es hängt allerdings von der individuellen Situation der Arbeitnehmenden ab, welche Versicherung (Kranken- oder Unfallversicherung) für den Erwerbsausfall einspringt. Darüber hinaus kann nicht pauschal gesagt werden, welche Faktoren zu späteren IV-Fällen führen.

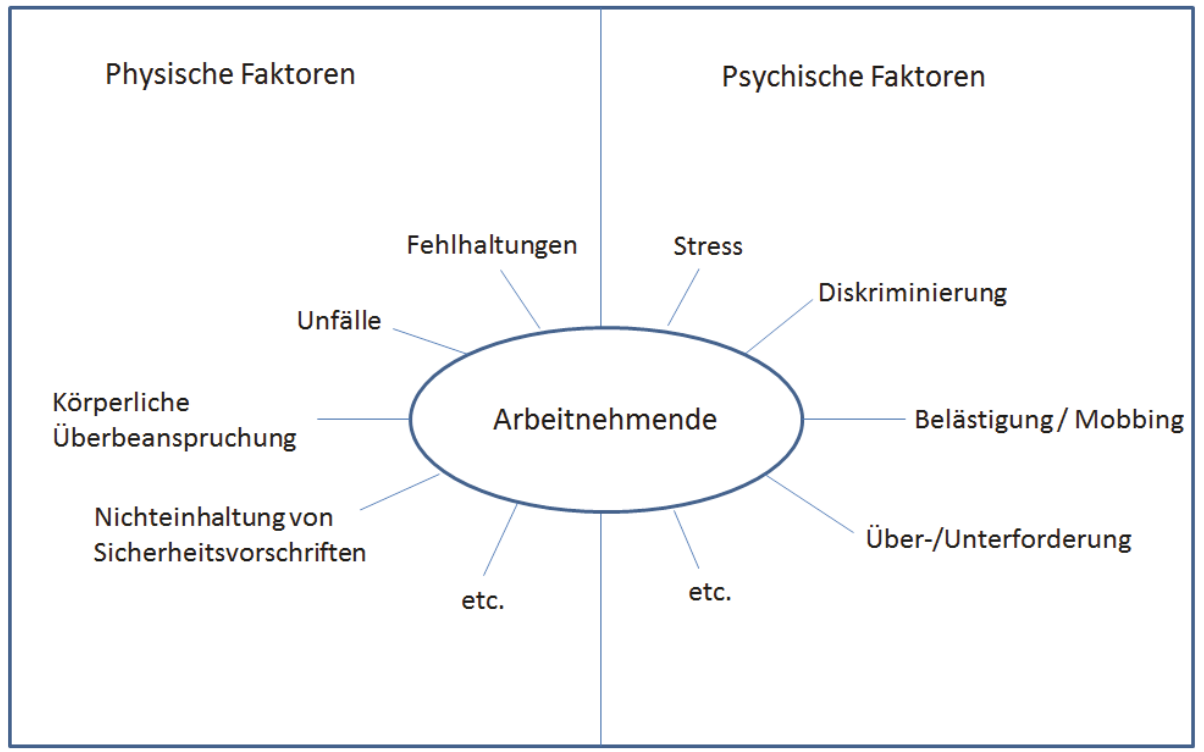

Abbildung 11: Aspekte betreffend Gesundheit

Wie bereits erwähnt, muss die Arbeitgebende dafür sorgen, dass die Arbeitnehmende keinen gesundheitlichen Schaden erleidet. Es wird nun aufgezeigt, welche rechtlichen Rahmenbedingungen zur Sicherstellung der sozialen Absicherung auch ausserhalb eines Einzelarbeitsvertragsverhältnisses zur Anwendung gelangen. 


\subsubsection{Bedeutung für die Schweiz}

Die Debatte in der Europäischen Union zeigt auf, dass die heutigen Sozialversicherungen in vielen europäischen Ländern nur ungenügend auf die zunehmende Flexibilität im Arbeitsmarkt zugeschnitten sind. Dies kann zu einer sozialen Unsicherheit der durch den Arbeitsmarkt flexibilisierten Personen führen. Daraus ist ein internationaler Forschungszweig entstanden, der nun die Flexibilität und die soziale Sicherheit zu regeln versucht (Marti et al., 2007).

\subsubsection{Berufliche Vorsorge BVG}

Wie deckt das schweizerische Sozialsystem den flexibilisierten Arbeitsmarkt $a b$ ? Gemäss dem Forschungsbericht ergeben sich Lücken vor allem bei der beruflichen Vorsorge nach BVG:

- $\quad$ Bei geringfügigen Beschäftigungen (bspw. Arbeit auf Abruf oder Teilzeit) - abgesehen von Ausnahmefällen von hochqualifizierter Arbeit ein geringes, d.h. nicht obligatorisch versichertes Einkommen.

- $\quad$ Bei befristeten Arbeitsverhältnissen bis max. 3 Monate - keine obligatorische Versicherung.

- $\quad$ Vorbezug der PK-Gelder für den Aufbau der Selbstständigkeit - bei mangelndem wirtschaftlichem Erfolg fehlt eine Absicherung durch die 2. oder 3. Säule.

\subsubsection{Arbeitslosigkeit ALV}

Weitere Lücken bestehen bei den Selbstständigerwerbenden im Falle einer Arbeitslosigkeit, da sie sich nicht bei der ALV gegen Arbeitslosigkeit versichern können. Zudem sind auch bei der Arbeit auf Abruf Nachteile vorhanden, wenn eine schleichende Abnahme des Arbeitspensums vorliegt. Und weiter ist bei schwankenden Pensen die Feststellung schwierig, ob ein Anspruch auf Arbeitslosenentschädigung besteht oder nicht. Auch ist der Grundsatz zu hinterfragen, dass sich bei Zwischenverdiensten der Taggeldanspruch trotz geringer Kompensationszahlungen in Tagen berechnet und sich also nicht im entsprechenden Verhältnis verlängert.

\subsubsection{Nichtberufsunfall und Berufskrankheit}

Das Risiko Nichtberufsunfall ist sehr gering, da auch bei minimaler Beschäftigung das Risiko via Krankenkasse aufgefangen werden kann. 
Das Risiko Berufskrankheit ist im Arbeitsalltag ebenfalls zentral. Arbeit ist aber keineswegs ein Gesundheitsrisiko, sondern bedeutet für viele Menschen Lebenssinn und Befriedigung, da eine erfüllte Tätigkeit vor sozialen Schwierigkeiten und Depressionen schützen kann. Gute Arbeitsbedingungen und Erfolge in der beruflichen Tätigkeit können demnach Quellen von psychischem und physischem Wohlbefinden sein, die Motivation und Arbeitsleistung steigern und somit durchaus gesundheitsfördernd sein. Andererseits gibt es spezifische berufliche Belastungen, die schwere Erkrankungen verursachen oder wesentlich zu deren Entstehung beitragen. Ungünstige Arbeitsbedingungen (organisatorischer, ergonomischer, physikalischer, chemischer oder biologischer Art) können Ursachen von gesundheitlichen Beschwerden sein (Läubli et al., 2014, S. 207).

Was sagt das Gesetz hierzu?

Gemäss dem OR ist die Arbeitgebende verpflichtet, die Gesundheit der Arbeitnehmenden zu schützen. Zudem, wie im nachfolgenden Abschnitt 3.6.8 beschrieben, wird der Gesundheitsschutz am Arbeitsplatz im Arbeitsgesetz in Art. 6 geregelt. Das ArG verpflichtet die Arbeitgebenden zu einer wirksamen Prävention der beruflich mitbedingten Erkrankungen und damit auch der Beschwerden des Bewegungsapparates. Die Verordnung 3 zum Arbeitsgesetz konkretisiert diese Anforderung und umschreibt im Grundsatz in Art. 2: „Der Arbeitgeber muss alle Massnahmen treffen, die nötig sind, um den Gesundheitsschutz zu wahren und zu verbessern und die physische und psychische Gesundheit der Arbeitnehmer zu gewährleisten." Lässt sich ein enger Zusammenhang zwischen beruflicher Belastung und einer Erkrankung nachweisen, wird diese gesetzlich als Berufskrankheit anerkannt (bspw. Sehnenscheidenentzündungen, Drucklähmungen der Nerven u.a.). Diese Krankheiten werden nach dem Unfallversicherungsgesetz abgewickelt (Jost, Pletscher, 2013).

Was alles fällt unter Berufskrankheit?

Alle in der Schweiz beschäftigten Arbeitnehmerinnen und Arbeitnehmer in der Schweiz sind obligatorisch UVG-versichert. Sie sind somit gleichzeitig auch gegen Berufskrankheiten versichert. Nach Art. 9 UVG gelten Krankheiten dann als Berufskrankheit, wenn sie bei der beruflichen Tätigkeit ausschliesslich oder vorwiegend durch schädigende Stoffe oder bestimmte Arbeiten verursacht worden sind. Die Liste der schädigenden Stoffe und Berufskrankheiten nach Art. 9 UVG ist im Anhang 1 der UVV aufgeführt.

Weil Art. 9 Abs. 1 UVG lückenhaft ist, wird er in Art. 9 Abs. 2 UVG durch eine Generalklausel ergänzt. Diese verlangt, dass die Erkrankung ausschliesslich oder stark überwiegend durch die berufliche Tätigkeit verursacht wurde. Dieser strenge Massstab hat zur Folge, dass die Generalklausel nur in seltenen Fällen 
die Anerkennung einer Berufskrankheit erlaubt. Wird ein Rückenleiden durch die berufliche Tätigkeit bloss ausgelöst und ist die berufliche Tätigkeit mithin nur Anlass und nicht Ursache des Leidens, so sind die Voraussetzungen gemäss Art. 9 Abs. 2 nicht erfüllt (BGE 116 V 144).

Eine Berufskrankheit muss, wie bereits erwähnt, „stark überwiegend“ durch die berufliche Tätigkeit verursacht sein. Es gibt eine Empfehlung dazu, wie „stark überwiegend" zu verstehen ist:

Stark überwiegend durch die berufliche Tätigkeit verursacht sind solche Krankheiten, deren Ursache zu mindestens 75 Prozent in der beruflichen Tätigkeit zu suchen ist. Für eine bestimmte berufliche Exposition muss die Erkrankungswahrscheinlichkeit nach der Rechtsprechung viermal höher sein als für die Allgemeinbevölkerung (vgl. BGE 116 V 136; BGE vom 31.12.1996).

Im März 2015 reichte der Neuenburger Ständerat Didier Berberat eine Interpellation beim Bundesrat ein. Darin verweist er darauf, dass immer mehr Erwerbstätige an stressbedingten Erkrankungen leiden, die nicht zu den Berufskrankheiten gemäss UVG gehören, und zeigt auf, dass eine deutlich bessere Betreuung der Patientinnen und Patienten möglich wäre, wenn diese psychischen Erkrankungen im UVG anerkannt würden. Dies würde auch die Akzeptanz solcher Erkrankungen in der Gesellschaft erhöhen. Aufgrund dieser Ausführung stellt Berberat dem Bundesrat die Frage, ob er hier Handlungsbedarf sehe.

In seiner Antwort vom Mai 2015 erklärt der Bundesrat, dass er keinen Anlass für eine Gesetzesänderung sehe. Ebenso wenig halte er es für angezeigt, den Katalog der Berufskrankheiten um ein „berufliches Erschöpfungssyndrom“ zu ergänzen, und zwar nicht zuletzt aufgrund der fehlenden Kausalität. Eine Erschöpfung könne auch durch familiäre oder finanzielle Umstände begründet werden. Diesen Ausführungen des Bundesrates kann insofern zugestimmt werden, als dass mit der Stresshaftung (vgl. Abschnitt 3.6.10) ein probates Mittel vorhanden ist, welches den Arbeitgebenden bei zu hoher Belastung der Arbeitnehmenden zur Rechenschaft zieht.

Diese Deklaration der Berufskrankheit entfällt bei allen Arbeitsformen (Auftrag, Werkvertrag, Freelancer etc.), die nicht als Einzelarbeitsvertragsverhältnis geregelt sind. Hier wird, analog zur bestehenden Rechtsanwendung, bestimmt werden müssen, inwieweit es "Berufsunfälle“ bei mobilen Arbeitsformen zu schützen gilt oder ob dieses Risiko vollständig die flexible Arbeitnehmende zu tragen hat bzw. ob dieses in zweiter und dritter Linie von ihre Krankenversicherung respektive der IV übernommen wird. 


\subsubsection{Spannungsfeld V: Erhöhung der Arbeitsflexibilität und der Marktwirtschaftlichkeit mit gleichzeitiger Einhaltung der sozialen Sicherheit der Arbeitnehmenden}

Die Ergebnisse des Forschungsberichtes (Flexicurity) hinsichtlich AHV/IV fallen relativ gut aus, da dank der auch für Nichterwerbstätige und Arbeitslose geltenden Beitragspflicht sowie der Einrichtung der Ergänzungsleistungen die tiefen Renten aufgefangen werden können. Bei Leih- und Temporär-arbeit gelten hohe Anspruchsvoraussetzungen bezüglich der Arbeitslosenentschädigung und es gibt bei Kurzarbeit und Schlechtwetterentschädigung keinen ALV-Versicherungsschutz. Bei neuer Selbstständigkeit besteht keine Versicherung gegen Arbeitslosigkeit, keine obligatorische berufliche Vorsorge und keine obligatorische Versicherung nach UVG. Gleiches gilt für die Scheinselbstständigkeit (Marti et al., 2007).

Das Anstreben punktueller Anpassungen bezüglich einzelner Sozialversicherungen ist sicherlich notwendig. Um die Lücken jedoch definitiv zu schliessen, bedarf es wohl gleichermassen einer Verbesserung der institutionellen Zusammenarbeit und der Stärkung des Case Managements sowie der Aktivierung des Arbeitsmarktes durch eine verbesserte Anreizwirkung in der Sozialhilfe und der Weiterbildung. Zudem ist nicht ausser Acht zu lassen, dass die soziale Sicherheit nicht nur dem Individuum eine Verwirklichungschance bietet. Indem sie die Arbeitnehmende schützt, nützt sie mit der Sicherstellung einer effizienteren Allokation der Mittel auch der Gesellschaft (Marti et al., 2007).

Die Realisierung eines ausgewogenen Verhältnisses zwischen Flexibilität und Sicherheit wird helfen, die Wettbewerbsfähigkeit der Unternehmen zu stärken und dadurch die Arbeitsplatzqualität sowie Arbeitsproduktivität zu steigern. Um dies zu erreichen, müssen die notwendigen Rahmenbedingungen vorhanden sein; gleichzeitig dürfen diese die Flexibilität nicht zu sehr einschränken. Ein Problem entsteht einerseits dann, wenn nötige strukturelle Anpassungen in einem Arbeitsfeld nicht durchgeführt werden können, weil die entsprechenden rechtlichen Rahmenbedingungen fehlen, wie in nachfolgenden Situationen:

- $\quad$ Durch die arbeitsrechtlichen Kündigungsschutzvorschriften ist die Kündigungsfreiheit für die Unternehmerin bzw. den Unternehmer eingeschränkt, weshalb er Neuanstellungen zurückhaltend handhabt.

- Die rechtlichen Einschränkungen von Schichtarbeit (Nachtarbeitsverbot) verlangen die Amortisation von Investitionskosten und mindern dadurch die internationale Konkurrenzfähigkeit.

- $\quad$ Hohe Sozialabgaben bei älteren Arbeitnehmenden reduzieren die Bereitschaft, ältere Personen überhaupt anzustellen (Marti et al., 2007). 
Andererseits kann die Flexibilität so weit gehen, dass die soziale Absicherung nicht genügend gewährleistet wird, wie bei nachfolgenden Situationen:

- $\quad$ Arbeit auf Abruf führt zu existenziellen Unsicherheiten bezüglich des Einkommens.

- $\quad$ Geringer Beschäftigungsgrad oder nur kurz befristete Arbeitsverhältnisse verhindern die BVG-Absicherung und verunmöglichen so eine Altersvorsorge (Marti et al., 2007).

Es wird eine Herausforderung sein, diese zwei Aspekte der Flexibilität und der Sicherheit künftig im Gleichgewicht zu regeln. Das bereits mehrstufige Modell (1.-3. Säule), welches im europäischen Vergleich ein sehr modernes und flexibles Instrument der sozialen Sicherheit darstellt, ist eine gute Basis für weitere Lösungen. Die Niederlande haben hierzu bereits 1999 ein Flexibilitäts- und Sicherheitsgesetz („Flexwet“) eingeführt, welches den gegensätzlichen Interesse der Arbeitnehmenden und Arbeitgebenden Rechnung trägt. Es ermöglicht den Arbeitgebenden, flexibel Temporärpersonal einzusetzen, und bietet den Arbeitnehmenden gleichzeitig Schutz. Auch Dänemark verfügt über einen der am wenigsten regulierten und sehr flexiblen Arbeitsmärkte. Es gibt ein geringes Mass an Arbeitnehmerschutz, dafür ist die Unterstützung bei Arbeitslosigkeit im europäischen Vergleich sehr grosszügig (Marti et al., 2007). Solche Modelle können Inputs liefern, wie künftig in der Schweiz der Arbeitsmarkt geregelt werden könnte.

\subsubsection{Wirtschaftsfreiheit}

Die Grundsätze der sozialen Sicherheit der Schweiz haben einen direkten Zusammenhang mit der Wirtschaftsfreiheit, die in der Bundesverfassung (SR 101) verankert ist. Die nachfolgenden Auszüge aus der Verfassung zeigen, wo überall die Erwerbstätigkeit und deren Zusammenhänge geregelt und verankert sind.

Art. 27 Wirtschaftsfreiheit

1 Die Wirtschaftsfreiheit ist gewährleistet.

2 Sie umfasst insbesondere die freie Wahl des Berufes sowie den freien Zugang zu einer privatwirtschaftlichen Erwerbstätigkeit und deren freie Ausübung. 


\section{Art. 41 Sozialziele}

1 Bund und Kantone setzen sich in Ergänzung zu persönlicher Verantwortung und privater Initiative dafür ein, dass:

d. Erwerbsfähige ihren Lebensunterhalt durch Arbeit zu angemessenen Bedingungen

bestreiten können;

2 Bund und Kantone setzen sich dafür ein, dass jede Person gegen die wirtschaftlichen Folgen von Alter, Invalidität, Krankheit, Unfall, Arbeitslosigkeit, Mutterschaft, Verwaisung und Verwitwung gesichert ist.

\section{Art. 94 Grundsätze der Wirtschaftsordnung}

1 Bund und Kantone halten sich an den Grundsatz der Wirtschaftsfreiheit.

2 Sie wahren die Interessen der schweizerischen Gesamtwirtschaft und tragen mit der privaten Wirtschaft zur Wohlfahrt und zur wirtschaftlichen Sicherheit der Bevölkerung bei.

3 Sie sorgen im Rahmen ihrer Zuständigkeiten für günstige Rahmenbedingungen für die private Wirtschaft.

4 Abweichungen vom Grundsatz der Wirtschaftsfreiheit, insbesondere auch Massnahmen, die sich gegen den Wettbewerb richten, sind nur zulässig, wenn sie in der Bundesverfassung vorgesehen oder durch kantonale Regalrechte begründet sind.

\section{Art. 95 Privatwirtschaftliche Erwerbstätigkeit}

1 Der Bund kann Vorschriften erlassen über die Ausübung der privatwirtschaftlichen Erwerbstätigkeit.

$2 \mathrm{Er}$ sorgt für einen einheitlichen schweizerischen Wirtschaftsraum. Er gewährleistet, dass Personen mit einer wissenschaftlichen Ausbildung oder mit einem eidgenössischen, kantonalen oder kantonal anerkannten Ausbildungsabschluss ihren Beruf in der ganzen Schweiz ausüben können. 
Art. 110 Arbeit

1 Der Bund kann Vorschriften erlassen über:

a. den Schutz der Arbeitnehmerinnen und Arbeitnehmer;

b. das Verhältnis zwischen Arbeitgeber- und Arbeitnehmerseite, insbesondere

über die gemeinsame Regelung betrieblicher und beruflicher Angelegenheiten;

c. die Arbeitsvermittlung;

d. die Allgemeinverbindlicherklärung von Gesamtarbeitsverträgen.

2 Gesamtarbeitsverträge dürfen nur allgemeinverbindlich erklärt werden, wenn sie begründeten Minderheitsinteressen und regionalen Verschiedenheiten angemessen Rechnung tragen und die Rechtsgleichheit sowie die Koalitionsfreiheit nicht beeinträchtigen.

\subsubsection{Soziale Sicherheit in der Schweiz}

In der Schweiz besteht ein engmaschiges Netz von Sozialversicherungen, das den hier lebenden und arbeitenden Menschen und ihren Angehörigen einen weitreichenden Schutz vor Risiken bietet, deren finanzielle Folgen sie nicht allein bewältigen können.

Das schweizerische Sozialversicherungssystem wird in fünf Bereiche unterteilt:

- $\quad$ die Alters-, Hinterlassenen- und Invalidenvorsorge (Drei-Säulen-System),

- $\quad$ der Schutz vor Folgen einer Krankheit und eines Unfalls,

- $\quad$ der Erwerbsersatz für Dienstleistende und bei Mutterschaft,

- die Arbeitslosenversicherung,

- $\quad$ die Familienzulagen (Bundesamt für Sozialversicherungen, 2015).

Diese Versicherungen gewähren Schutz, indem sie Leistungen wie Renten, Erwerbsersatz und Familienzulagen ausrichten oder indem sie Kosten bei Krankheit und Unfall tragen.

Die Leistungen der einzelnen Sozialversicherungszweige werden vorab durch Beiträge aus dem Erwerbseinkommen finanziert. In der Krankenversicherung zahlt jede versicherte Person eine Prämie ein. Bund und Kantone beteiligen sich in unterschiedlichem Umfang an der Finanzierung der Sozialversicherun- 
gen (AHV/IV) oder sie finanzieren sie entweder ganz (Ergänzungsleistungen, EL) oder helfen wirtschaftlich schwachen Personen bei der Prämienzahlung (Prämienverbilligung in der Krankenversicherung).

Es ist durchaus möglich, mit innovativen und flexiblen Arbeitszeitmodellen die verschiedenen Positionen so miteinander zu vereinen, dass gleichzeitig sowohl die Sicherheit und Gesundheit der Beschäftigten wie auch die Wettbewerbsfähigkeit der Organisation gesichert sind.

Als Basis hierzu gibt es das Arbeitszeitgesetz und dessen Verordnungen. Im Sinne von Sicherheit und Gesundheit legt das Arbeitszeitgesetz die Rahmenbedingungen für die Grenzen der Arbeitszeiten fest. Das Arbeitsgesetz bildet somit einen der Grundbausteine des Arbeits- und Gesundheitsschutzes. Zusätzlich gibt es hier zahlreiche weitere Richtlinien der SUVA oder EKAS sowie die Aufsichts- und Kontrollfunktion der jeweiligen Arbeitsinspektorate.

\subsubsection{Gesundheitsschutz}

Das Arbeitsgesetz ist per se ein Arbeitsschutzgesetz, welches in erster Priorität den Schutz der Gesundheit und danach allfällige finanzielle Ansprüche der Arbeitnehmenden regelt. Wie in Abbildung 11 aufgezeigt, können Arbeitnehmende sowohl durch physische als auch psychische Faktoren in ihrer Gesundheit beeinträchtigt werden.

Im Hinblick auf mobile Arbeit stellt sich unter anderem die Frage, inwiefern die Arbeitgebende ihren Pflichten nachkommt. Der Gesetzgeber regelt den Grundsatz der Pflicht sowohl in Art. 328 OR als auch in Art. 6 ArG / Art. 3. ArGV. Nicht anwendbar sind gemäss ArGV 3 die Bestimmungen über den Arbeitsschutz auf Arbeitnehmende, die vom persönlichen Geltungsbereich des Arbeitsgesetzes ausgenommen sind, wie beispielswese Heimarbeitende und Handelsreisende (Staatssekretariat für Wirtschaft SECO, 2014, S. 301-1).

Streiff et al. führen zur Erfüllung dieser Fürsorgepflicht aus, dass es ausreichend sei, wenn die Arbeitgebende die Arbeitnehmende im Detail auf Gefahren hinweist und ihr aufzeigt, wie sie sich richtig zu verhalten hat. Sie muss das Verhalten der Arbeitnehmenden zudem regelmässig überwachen. Es liegt aber im Bereich des Unmöglichen, die Arbeitnehmende vor sämtlichen Gefahren zu schützen (Streiff et al., Art. 328 N 15). „Er (der Arbeitgeber) muss darum nur jene Gefahren abwenden, die aus dem Wesen der Arbeit und aus dem bestimmungsgemässen Gebrauch der Anlagen erwachsen." 
Mit Massnahmen sind nicht nur Schutzmassnahmen wie etwa an Maschinen gemeint. Der Begriff Massnahmen erstreckt sich über die Arbeitsorganisation, Instruktion, Ergonomie und das Raumklima, bis hin zu Massnahmen gegen psychische Belastungen wie ausufernder Stress, Überbelastung, Mobbing usw. Die in diesem Zusammenhang verlangte Erfahrung über die Grenzen des Betriebs hinaus meint dabei insbesondere auch die Kenntnisse der Branche. Zudem sind auch der Stand der Technik oder etwa die SUVA-Richtlinien zu beachten.

Unter Stand der Technik wird verstanden, dass die Massnahmen den Kenntnissen einer Fachperson entsprechen müssen. Im Weiteren bedeutet dies, „dass alle anerkannten Regeln der Technik im fraglichen Fachgebiet, wie sie insbesondere in technischen Normen und Richtlinien der SUVA zum Ausdruck kommen, zur Anwendung kommen müssen“ (Streiff et al., Art. 328 N 4). Die genannten Punkte müssen unter Berücksichtigung der Verhältnisse des Betriebes angewendet werden. Dabei spielen sowohl technische wie auch wirtschaftliche Aspekte eine Rolle.

Gemäss Art. 23 ArGV 3 sind Arbeitsplätze, Arbeitsgeräte und Hilfsmittel nach ergonomischen Gesichtspunkten zu gestalten und einzurichten. Die Wegleitung des SECO zu den Verordnungen 3 und 4 zum Arbeitsgesetz konkretisiert darüber hinaus die ergonomischen Anforderungen an einen Arbeitsplatz. Die erwähnte Wegleitung zeigt allgemeine Grundsätze auf, die sowohl für Büroräumlichkeiten als auch für industrielle Arbeitsplätze gelten.

Entsprechend der Wegleitung zur Verordnung 3 zum Arbeitsgesetz soll die Arbeitshöhe der Körpergrösse angepasst sein. Darüber hinaus ist darauf zu achten, dass Sitz, Arbeitsfläche sowie Tische als Einheit gestaltet werden, damit der Arbeitnehmende eine bevorzugte Körperhaltung während der Arbeit einnehmen kann. Im Weiteren ist für Körperbewegungen genügend Freiraum zu lassen (Beinfreiheit von mindestens 60 Zentimetern). Auch für mobile Arbeit stellen Bildschirmgeräte ein zentrales Arbeitsmittel dar. „Von Bedeutung ist die sachgerechte individuelle Anpassung der Arbeitsmittel, deren Nutzung und ausreichende Bewegung des Körpers. Dazu braucht es Schulung und Mitarbeit, aber vor allem auch Selbstverantwortung der Arbeitnehmerinnen und Arbeitnehmer" (Staatssekretariat für Wirtschaft SECO, 2014, S. 323-4). Werden die ergonomischen Erkenntnisse in Bezug auf Arbeiten vor einem Bildschirm nicht eingehalten, so kann dies zu gesundheitlichen Beschwerden wie z.B. Augenermüdung und Kopfschmerzen führen. 
Diese Ausführungen sind sehr detailliert umschrieben und bieten umfassenden Schutz für die Arbeitnehmende. Die Frage, die sich hier aber stellt, ist, inwieweit die Umsetzung in den Unternehmungen erfolgt und wie sie überprüft wird. Zudem beziehen sich die Bestimmungen auf den Arbeitsplatz in der Unternehmung und nicht auf flexible, mobile Arbeitsorte.

Ein weiterer Punkt ist die Überalterung unserer Gesellschaft. Die Generation der 50- bis 65-Jährigen wird in den nächsten Jahren noch deutlich wachsen. Angesichts dieser Entwicklung stehen die Unternehmen vor der Aufgabe, die wirtschaftlichen Herausforderungen künftig mit durchschnittlich älteren Belegschaften zu bewältigen (Baua, 2013). Entsprechend sind sie gefordert, die Beschäftigungsfähigkeit ihrer Mitarbeitenden bis zum gesetzlichen Rentenalter zu erhalten, zu sichern und auszubauen. Dass hierbei eine flexible und auch menschenwürdige Arbeitszeitgestaltung eine wichtige Rolle spielt, ist offensichtlich.

\subsubsection{Spannungsfeld VI: Flexible Arbeit ja, aber nicht auf Kosten der Gesundheit}

Keine Frage, wenn es darauf ankommt, kann der Mensch auch mal zwölf Stunden durcharbeiten, ohne dass gleich die Gesundheit auf dem Spiel steht. Sobald dies jedoch zur Gewohnheit wird, sieht die Sache anders aus. Es gibt zwar individuelle Unterschiede, wie überlange Arbeitszeiten verkraftet werden, und auch die Art der Tätigkeit hat Einfluss auf die zu erwartenden Folgen für Sicherheit und Gesundheit. Aber grundsätzlich ist erwiesen, dass überlange Arbeitszeiten, vor allem in Kombination mit Schicht- und Nachtarbeit, zu Problemen in den Bereichen Gesundheit, Leistung und Sicherheit führen (Baua, 2013). Vor allem im Zusammenhang mit der Flexibilisierung ist deshalb darauf zu achten, dass die gesundheitsgerechte Gestaltung der flexiblen Arbeitszeiten und der Rahmenbedingungen berücksichtigt wird. Dieser Thematik ist sich auch die Judikative bewusst, was sich aus dem nächsten Kapitel ableiten lässt.

\subsubsection{Stresshaftung}

Sehr lange und atypisch gelagerte Arbeitszeiten werden nicht selten als Belastung empfunden. Zudem verstärkt die Erreichbarkeit ausserhalb der Arbeitszeit in vielen Fällen den subjektiv empfundenen Stress. Zeitdruck und Stress, insbesondere durch quantitative Überforderung, gehen mit vermehrten körperlichen und psychischen Beschwerden einher. Angehörige von Sozialberufen sowie Frauen sind überdurchschnittlich stark von Diagnosen wie dem Burnout betroffen (Eichhorst/Tobsch, 2014). 
Können die Mitarbeitenden den an sie gestellten Anforderungen nicht mehr gerecht werden, fühlen sie sich gestresst. Sie schaden nicht nur ihrer eigenen Gesundheit, ihre stressbedingten Ausfälle bedeuten nicht selten auch eine Mehrbelastung für die anderen Teammitglieder und einen Verlust von Knowhow, was sich wiederum negativ auf die Rentabilität des Unternehmens auswirkt und einen Produktivitätsabfall bedeuten kann (Sprenger et al., 2013). Stress am Arbeitsplatz birgt darüber hinaus rechtliche Risiken für die Arbeitgebende. Bei einem Ausfall besteht bspw. eine Lohnfortzahlungspflicht oder aber es entsteht ein Haftungsrisiko (Sprenger et al., 2013). So hat das Thema der Stresshaftung in den vergangenen Jahren auch das Bundesgericht vermehrt beschäftigt (4C. 24/2005). Einer Arbeitnehmerin wurde eine Genugtuung von 10 '000 Franken zugesprochen, weil sie sich am Arbeitsplatz überbeansprucht fühlte, in der Folge eine schwere Depression erlitt und schliesslich zur Arbeit nicht mehr fähig war (4C. 24/2005).

Damit juristisch von einer Stresshaftung gesprochen werden kann, müssen kumulativ vier Voraussetzungen erfüllt sein. Die Arbeitnehmende muss zunächst einen Schaden, also eine unfreiwillige Vermögenseinbusse infolge der Stresswirkung erleiden. Dies ist der Fall, wenn die Stresssymptome Krankheitswert erlangen und dadurch Behandlungskosten entstehen. Sodann ist eine Vertragsverletzung seitens der Arbeitgebenden notwendig. Diese hat nämlich die Persönlichkeit der Arbeitnehmenden im Arbeitsverhältnis zu achten und zu schützen und muss demzufolge auf die Gesundheit der Arbeitnehmenden Rücksicht nehmen. Bestehen in einem Unternehmen jedoch Arbeitsbedingungen, welche erhöhten Stress auslösen, stellt das einen Verstoss der Arbeitgebenden gegen ihre Fürsorgepflicht und somit eine Vertragsverletzung dar. Als dritte Voraussetzung muss zwischen dem erlittenen Schaden der Arbeitnehmenden und der eben dargelegten Vertragsverletzung ein natürlicher und adäquater Kausalzusammenhang bestehen. Und schliesslich ist ein Verschulden der Arbeitgebenden notwendig, wobei der Umstand massgebend ist, ob die stressbedingte Gesundheitsbeeinträchtigung für die Arbeitgebende vorhersehbar war. In diese Entscheidung werden vor allem Aspekte wie die Arbeitsorganisation und die konkreten Arbeits- und Überwachungsbedingungen mit einbezogen, aber auch die Kommunikation und Anzeichen von Stress wie bspw. häufige Absenzen. Von einem Verschulden wird regelmässig ausgegangen, wenn die Arbeitgebende die Gesundheitsgefährdung kannte oder aber hätte kennen können (Rohrer, 4/09).

Kommt das Gericht zum Schluss, dass alle vier Voraussetzungen erfüllt sind, heisst es die Klage gut und spricht eine Genugtuung mit der Begründung aus, dass der langanhaltende Arbeitsdruck zu einer Gesundheitsschädigung der 
Arbeitnehmenden führte und die Arbeitgebende ihrer Fürsorgepflicht nicht nachkam (Rohrer, 2009).

Im Arbeitsalltag ist es jedoch oft schwierig zu beurteilen, wann die Schwelle des zulässigen Arbeitsstresses überschritten ist. Aus diesem Grund wird die Stresshaftung auch immer aus subjektiver Sicht der betroffenen Arbeitnehmenden betrachtet und somit den Umständen des Einzelfalles Rechnung getragen. Um das Risiko einer Stresshaftung zu minimieren, sollte die Arbeitgebende frühzeitig Schutzmassnahmen wie bspw. die Errichtung einer Anlaufstelle oder die Durchführung von Stress-audits ergreifen (Rohrer, 2009). Schafft die Arbeitgebende keine Verhältnisse, die die Arbeitnehmende vom Stress entlasten, hat sie das Recht, ihre Arbeitsleistung zu verweigern, wenn die Stressbelastung derart hoch ist, dass sie als unzumutbar erscheint (Art. 324 OR) (Sprenger et al., 2013).

Es bleibt festzuhalten, dass die neuen Formen der Arbeit ein geeignetes Mittel gegen stressbedingte Ausfälle im Arbeitsalltag und die damit verbundenen rechtlichen und betriebswirtschaftlichen Risiken sein können. Wichtig dabei ist allerdings, dass das Unternehmen entsprechende Voraussetzungen dafür schafft und sich den Anliegen seiner Angestellten annimmt (Sprenger et al., 2013).

\subsubsection{Kollektives Arbeitsrecht - Gesamtarbeitsverträge}

Der GAV wird in Art. 356 Abs. 1 OR begrifflich beschrieben. Dabei handelt es sich um eine gemeinsame Regelung von einzelnen Arbeitsverhältnissen zwischen Arbeitgebenden oder deren Verbänden und Arbeitnehmerverbänden. Diese Verträge enthalten auch normative Bestimmungen. Da diese wie ein Gesetz wirken, sind sie auch wie ein solches auszulegen. Das Bundesgericht betont aber auch, dass der Wille der am Abschluss des Gesamtarbeitsvertrages beteiligten Personen zu beachten ist (Geiser, 2007). Im Weiteren kann der GAV von den zuständigen Behörden als allgemeinverbindlich erklärt werden. Mit dieser Allgemeinverbindlicherklärung wird der Geltungsbereich eines GAV auf sämtliche Arbeitnehmende einer bestimmten Branche ausgedehnt.

Basis für eine moderne Arbeitszeitgestaltung ist das Arbeitszeitgesetz, welches auch den Rahmen für die Vereinbarungen zwischen den Gewerkschaftspartnerinnen und Gewerkschaftspartnern bildet. Hierbei müssen sich die Parteien arbeitnehmerseitig wie arbeitgeberseitig treffen, um die Rahmenbedingungen für die flexible Arbeitszeit zu verbessern und dabei die Sicherheit und den Gesundheitsschutz der Arbeitnehmenden zu gewährleisten. 
Das Arbeitsgesetz und die Gesamtarbeitsverträge können die gleichen Materien, insbesondere die Arbeits- und Ruhezeit regeln. Dies geschieht mit unterschiedlicher Wirkung, einerseits als öffentliches Recht mit staatlicher Kontrolle (Arbeitsgesetz) und andererseits als Privatrecht, das von der einzelnen Arbeitnehmenden oder Arbeitgebenden durchgesetzt werden muss (GAV). Nach der vorherrschenden Meinung geht die gesamtarbeitsvertragliche Regelung vor, weil sie dem Willen der Sozialpartnerinnen und Sozialpartner entspricht und weil sie den örtlichen Gegebenheiten und den Verhältnissen in den einzelnen Branchen Rechnung tragen kann. Ihre Schwäche jedoch liegt im begrenzten Geltungsbereich und es zeigen sich Mängel bei der Durchsetzung. Deshalb hat ein Mindeststandard wie das Arbeitsgesetz, welches den Interessen der Persönlichkeit, der Gesundheit und der öffentlichen Ordnung Rechnung trägt, seine Bedeutung. Zudem ist die öffentlich-rechtliche Norm gesichert und wird durch staatliche Organe vollzogen.

Um offene Punkte der Flexibilität in der Arbeitswelt zu regeln, steht dem GAV die Möglichkeit zu, gewisse Punkte gegenüber den anderen gesetzlichen Bestimmungen detaillierter oder ergänzend zu definieren. Bspw. kann ein GAV einen Arbeitsort näher regeln, da dies zu den Inhaltsnormen gehört. Dabei handelt es sich um von den GAV-Parteien gesetztes objektives Recht, das für die beteiligten Parteien verpflichtend ist. So finden sich, um bei diesem Beispiel zu bleiben, häufig konkrete Regelungen zum Arbeitsort, der beispw. bei der Post explizit im Einzelarbeitsvertrag geregelt wird. Dazu kommen spezifische Bestimmungen zur Reichweite eines Arbeitsorts, zur Arbeitszeit und zu Spesenfragen. Auch der Landesgesamtarbeitsvertrag regelt den auswärtigen Arbeitsort, indem er auf einen bestimmten Kilometerradius abstellt.

Es wurde vorliegend überprüft, inwiefern Gesamtarbeitsverträge eine Regelung im Zusammenhang mit flexiblen Arbeitszeitmodellen enthalten. Grundsätzlich ist die Regelung der flexiblen Arbeitsmodelle in den GAV nur grob thematisiert. Immerhin verweisen einzelne GAV aber auf die Möglichkeit der Telearbeit. Dabei werden die Rahmenbedingungen für die mobile Arbeit aufgeführt, so beispw., dass Telearbeit auf Freiwilligkeit beruht und nur mit Zustimmung der Vorgesetzten erfolgen kann. Darüber hinaus wird festgehalten, dass die Arbeitgebende die technische Infrastruktur für die Telearbeit zur Verfügung stellt und kein Anspruch auf berufsbedingte Auslagen wie etwa die Nutzung von privaten Räumlichkeiten besteht. Allerdings verweist der GAV für die Regelung des Datenschutzes und der Einrichtung des Arbeitsplatzes zu Hause auf eine individuelle Vereinbarung. 
Die Möglichkeit zur Jahresarbeitszeit ist in den meisten der analysierten GAV enthalten. Teilweise wird die spezifische Ausgestaltung bereits dort ausgeführt. Bei einigen wird jedoch lediglich die Möglichkeit zur Jahresarbeitszeit eingeräumt und in individuellen Reglementen präzisiert. Ein Verweis auf das entsprechende Reglement ist im jeweiligen GAV enthalten. Auch erwähnen einige GAV die Möglichkeit von Langzeitkonti. Die dabei zu befolgenden Bedingungen sind ebenfalls im GAV ausgeführt. Auf eine spezifische Altersgrenze verweist jedoch nur ein einziger GAV, hält gleichzeitig aber fest, dass von dieser mittels individueller Vereinbarung abgewichen werden kann. ${ }^{4}$

Tabelle 6: Übersicht rechtlicher Bestimmungen bezüglich Arbeitszeit in diversen Gesamtarbeitsverträgen

\begin{tabular}{|c|c|c|c|c|c|}
\hline Bereich & Fenaco-GAV & $\begin{array}{l}\text { Im- } \\
\text { pressum }\end{array}$ & Post & $\begin{array}{l}\text { Maschi- } \\
\text { nen- } \\
\text { industrie }\end{array}$ & $\begin{array}{l}\text { Basler } \\
\text { Pharma }\end{array}$ \\
\hline $\begin{array}{l}\text { Arbeits- } \\
\text { zeit }\end{array}$ & $\begin{array}{l}\text { 11.1 Die durchschnittlichen, } \\
\text { wöchentlichen Höchstarbeitszei- } \\
\text { ten der dem GAV unterstellten } \\
\text { Firmen sind im Anhang zum } \\
\text { GAV festgehalten. } \\
\text { 11.2. Unter Berücksichtigung der } \\
\text { gesetzlichen Bestimmung und } \\
\text { der betrieblichen Bedürfnisse } \\
\text { werden sinnvolle flexible Ar- } \\
\text { beitszeitmodelle vereinbart. Die } \\
\text { Arbeitszeit kann auch als Jah- } \\
\text { resarbeitszeit berechnet werden. }\end{array}$ & $\begin{array}{l}\text { Art. 23: } \\
\text { wö- } \\
\text { chent- } \\
\text { liche } \\
\text { Arbeits- } \\
\text { zeit ge- } \\
\text { mäss } \\
\text { EAV }\end{array}$ & $\begin{array}{l}4041 \mathrm{~h} / \mathrm{W} \\
\text { Geeignete } \\
\text { Erfassung } \\
\text { (vgl. Anhang 3) } \\
\text { A3: } 20 \text { gleitende } \\
\text { Arbeitszeit und } \\
\text { Möglichkeit Jah- } \\
\text { resarbeitszeit } \\
\text { 23: Alternieren- } \\
\text { de Telearbeit }\end{array}$ & $\begin{array}{l}\text { Art. 12: } \\
\text { Arbeitszeit } \\
\text { Möglich- } \\
\text { keit eines } \\
\text { Langzeit- } \\
\text { kontos }\end{array}$ & $\begin{array}{l}\text { Art. 8: } \\
\text { Möglich- } \\
\text { keit zur } \\
\text { Jahres- } \\
\text { arbeits- } \\
\text { zeit (40 } \\
\text { h/w) mit } \\
\text { Verweis } \\
\text { auf } \\
\text { Regle- } \\
\text { ment }\end{array}$ \\
\hline
\end{tabular}

${ }^{4}$ Untersucht wurden:

Verband SCHWEIZER PRESSE (2000): Gesamtarbeitsvertrag 2000 für Journalistinnen/Journalisten und das technische Redaktionspersonal.

Gesamtarbeitsvertrag fenanco. Gütig ab 01.01.2013 (2013).

Post (2011): Gesamtarbeitsvertrag Post.

Gesamtarbeitsvertrag für Basler Pharma-, Chemie- und Dienstleistungsunternehmen. Gültig ab 1. Mai 2012 (2012).

Gesamtarbeitsvertrag der Maschinen-, Elektro- und Metall-Industrie. Vertragsperiode 1. Juli 2013 30. Juni 2018 (2013).

Verband SCHWEIZER PRESSE (2000): Gesamtarbeitsvertrag 2000 für Journalistinnen/Journalisten und das technische Redaktionspersonal.

Gesamtarbeitsvertrag fenanco. Gütig ab 01.01.2013 (2013).

Die Post (2011): Gesamtarbeitsvertrag Post.

Gesamtarbeitsvertrag für Basler Pharma-, Chemie- und Dienstleistungsunternehmen. Gültig ab 1. Mai 2012 (2012).

Gesamtarbeitsvertrag der Maschinen-, Elektro- und Metall-Industrie. Vertragsperiode 1. Juli 2013 30. Juni 2018 (2013). 


\subsubsection{Spannungsfeld VII: Wie setzen wir einen „mobilen“ Gesundheits- schutz um?}

Zusammenfassend kann festgehalten werden, dass der Gesetzgeber die Pflicht zum Gesundheitsschutz einerseits auf die Arbeitgebende überträgt, indem diese angehalten wird, alle Massnahmen zu ergreifen, um die Arbeitnehmende vor gesundheitlichen Gefährdungen am Arbeitsplatz zu schützen. Andererseits wird aber auch die Arbeitnehmende in die Pflicht genommen. Sie hat nämlich die Mittel, die die Arbeitgebende zur Verhinderung von gesundheitlichen Gefahren zur Verfügung stellt, ordnungsgemäss zu benützen. Wichtig und damit hervorzuheben ist aber die Pflicht der Arbeitgebenden, die Arbeitnehmende regelmässig auf die Gefahren hinzuweisen und zeitnah Mittel aufzuzeigen, diese Gefahren abzuwenden. Bei der mobilen Arbeit ist es klar, dass die Arbeitgebende nicht in dem Ausmass auf die Einrichtung des Arbeitsplatzes eingehen kann, wie bei einem festen Arbeitsplatz in ihren eigenen Büroräumlichkeiten. Punktuelle Änderungen können Lücken im Sozialversicherungssystem für gewisse Arbeitsverhältnisse sicherlich schliessen. Solche Änderungen erhöhen in der Regel aber auch die Sozialabgaben und dadurch die Kosten für den Faktor Arbeit.

\subsection{Vereinbarkeit von Arbeit mit anderen Lebens- bereichen}

Das gültige Arbeitsgesetz wird den heutigen Veränderungen hin zu einer digitalisierten und zunehmend entgrenzten Arbeitswelt nicht mehr gerecht, weshalb eine Anpassung notwendig ist. Die aktuell gesetzlich verankerte Fürsorgepflicht der Arbeitgebenden gegenüber der Arbeitnehmenden ist zwar nach wie vor auf physische Gefährdungen zu beziehen, aber zukünftig ebenfalls auf solche im psychosozialen Bereich (Syndicom Studie, 2015, S. 5).

\subsubsection{Entgrenzung der Arbeit}

Gemäss Letsch ist das bisherige Arbeitsmodell einer Tätigkeit, der man zwischen 8 Uhr morgens und 17 Uhr nachmittags nachgeht, nicht mehr vereinbar mit dieser Entgrenzung. Er hält darüber hinaus fest: „Begünstigt durch neue Informations- und Kommunikationstechnologien gewinnen Umzugs- und Pendelmobilität sowie Telearbeit an Bedeutung“ (Letsch, 2008, S. 36). 
Das Problem ist die Vermischung von zeitlichen und räumlichen Grenzen. Zeitlich gesehen ist die Arbeit immer weniger an die Normalarbeitszeit gebunden, was durch die Vertrauensarbeitszeit ermöglicht wird. Basis der Vertrauensarbeitszeit ist nicht die Anwesenheit im Büro, sondern es sind die Leistungsanforderungen, welche die Arbeitnehmenden zu erfüllen haben (Syndicom Studie, 2015, S. 7).

So ist die Entgrenzung gerade im Zusammenhang mit der Arbeitszeit von grosser Bedeutung. Es sind nicht mehr nur die Managerinnen und Manager sowie Spezialistinnen und Spezialisten, die immer und überall erreichbar sein müssen und dafür entsprechende Infrastrukturen benötigen. Heute fühlen sich Arbeitnehmende zunehmend verpflichtet, länger als vorgesehen zu arbeiten und auch in der Freizeit und während den Ferien ständig erreichbar zu sein. Die modernen, mobilen Kommunikationsmittel, der technologische Wandel sowie die Globalisierung der Märkte und ein steigender Wettbewerbsdruck unterstützen diese Tendenz (Waser, 2010, S. 12). Rein theoretisch lässt sich die Arbeitszeit klar von der Freizeit unterscheiden. Sie ist nämlich die Zeit, vom Beginn bis zum Ende der Arbeit, ohne Ruhepausen (Chilles, 2012, S. 125). Gemäss Art. 9 ArG liegt die Höchstarbeitszeit bei 45 bzw. 50 Stunden pro Woche. Zudem steht jeder Arbeitnehmenden nach Beendigung der Arbeit eine Ruhezeit von mindestens elf aufeinanderfolgenden Stunden zu, und Sonn- und Feiertage sind generell arbeitsfrei (Art. 15a, 18, 20a ArG). Diese gesetzlichen Vorschriften existieren zwar, stehen jedoch in manchen Fällen in grosser Diskrepanz zur Realität. Viele Arbeitnehmende checken am Abend nochmals kurz die E-Mails oder setzen sich am Wochenende an den Computer, so auch Sandra Könitz, Projektmanagerin. Die Gründe für ein solches Verhalten können vielfältig sein: Karrieredenken, Erwartungen seitens der Vorgesetzten etc. Fest steht allerdings, dass solche Entwicklungen gegen das geltende Arbeitsrecht verstossen (Chilles, 2012, S. 125). Gerade wenn der Urlaub durch ständige Anrufe und E-Mails unterbrochen wird, kann dies erhebliche Auswirkungen auf den Gesundheitszustand einer Arbeitnehmenden haben. Die benötigte Erholung bleibt in einem solchen Fall aus (Chilles, 2012, S. 125).

Unternehmensstrukturen sollten so ausgestaltet sein, dass sich die Regenerationsfähigkeit der Angestellten verbessert. Nur dadurch können eine bessere Lebensqualität für die Angestellten sowie eine höhere Leistungsfähigkeit fürs Unternehmen erzielt und gleichzeitig Ausfallzeiten und Gesundheitskosten gesenkt werden (Syndicom-Studie, 2015, S. 9).

In der erwähnten Studie werden verschiedene Handlungsempfehlungen abgegeben (Syndicom-Studie, 2015, S. 10 f.). Dabei geht es darum, die Mitbestim- 
mungsrechte von Personalvertretenden und Mitarbeitenden über die Arbeitsprozesse hinaus auszubauen und in die Gesamtarbeitsverträge zu integrieren. Diese Gesamtarbeitsverträge müssen mehr Erholungszeit, etwa in Form von zusätzlichen Ferientagen, Sabbaticals, bezahlten Urlaubstagen etc., vorsehen (Syndicom-Studie, 2015, S. 20) und allfällige Stressfaktoren in Mitarbeitendengesprächen lösungsorientiert ansprechen. Damit die Mitarbeitenden am von ihnen erwirtschafteten Mehrwert beteiligt sind, müssen die Löhne erhöht werden und die Produktivitätssteigerung darf nicht zu längeren Arbeitszeiten führen. Alle Leistungsziele sind so auszugestalten, dass sie während der Arbeitszeit erreicht werden, ohne Freizeit dafür einzusetzen. Überzeit sollte zudem möglichst schnell kompensiert werden. Es muss das Anliegen jedes Unternehmens sein, Governance-Leitlinien zu definieren, sodass den Mitarbeitenden die Trennung von Arbeit und Freizeit ermöglicht wird. Des Weiteren sind Unternehmen aufgefordert, technische Barrieren zu errichten, damit geschäftliche EMails lediglich während der ordentlichen Arbeitszeit eingesehen werden können. Die Arbeitgebenden müssen ihre Mitarbeitenden stets für psychische Belastungen und Symptome von psychischen Krankheiten sensibilisieren. Dies kann dazu führen, dass die Ausrichtung und Umsetzung des betrieblichen Gesundheitsmanagements mit den Sozialpartnerinnen und Sozialpartnern sowie den Personalvertretungen thematisiert und ggf. neu definiert werden. Zudem ist die Personenknappheit zu thematisieren, das Problem als solches zu erkennen und die strategische Personalplanung entsprechend anzupassen. Dazu gehören u.a. das konsequente Ersetzen von Abgängen sowie die Sicherstellung von Stellvertretungen und Einführungszeiten.

Gemäss der Syndicom-Studie litten im Jahr 201378 Prozent der Befragten eher häufig, sehr häufig oder sogar immer unter Zeitdruck (Syndicom-Studie, 2015, S. 18). Als Gründe wurden unrealistische Zielvorgaben, Personalknappheit, höhere Arbeitskomplexität, Vorgesetzte mit ungenügender Fach- und Sozialkompetenz, interne Bürokratie oder unklare Prozesse genannt. Möglich ist auch, dass sich einzelne Mitarbeitende selber zu hohe Ziele setzen und somit unter ihrem eigenen Druck leiden (Syndicom-Studie, 2015, S. 18). Solchen Schwachstellen in einem Unternehmen kann mittels angemessener Schulungen begegnet werden.

Flexible Arbeitszeitmodelle durchbrechen das traditionelle Arbeitszeitmodell mit einem Achtstundentag. Damit die zunehmende Arbeitsdichte bewältigt werden kann, leisten die Mitarbeitenden meist mehr Arbeit als vertraglich vereinbart (Syndicom-Studie, 2015, S. 29). Im Rahmen der Vertrauensarbeitszeit passen die Mitarbeitenden ihre individuelle Arbeitszeit den Bedürfnissen der Unternehmen sowie den Markterfordernissen an (Syndicom-Studie, 2015, S. 29). Dies 
hat eine ständige Erreichbarkeit der Mitarbeitenden sowohl gegenüber dem Unternehmen wie auch den Kundinnen und Kunden zur Folge (SyndicomStudie, 2015, S. 29).

Auf die Frage hin, wie oft sie ausserhalb des normalen Arbeitspensums geschäftliche E-Mails oder Anrufe beantworten, gaben in der Syndicom-Studie rund 47 Prozent der Befragten an, dies eher häufig, sehr häufig oder gar immer zu tun, und zwar obwohl es von innen nicht explizit erwartet worden wäre (Syndicom Studie, 2015, S. 32). Auch diesem Phänomen kann mit einer gezielten Schulung der Mitarbeitenden begegnet werden, indem sie lernen, dass sie ausserhalb der Arbeitszeiten nicht für das Unternehmen zur Verfügung stehen müssen.

Rund 36 Prozent gaben an, häufig oder sehr häufig in ihrer Freizeit geschäftliche Aufgaben zu erledigen. Dies wird jedoch oft als normal angesehen und die stattfindende Arbeitsentgrenzung dabei gar nicht wahrgenommen. Es ist anzunehmen, dass dieses Beantworten von geschäftlichen Anfragen in der Freizeit meist nicht als Arbeitszeit erfasst wird. Das ist ein Zeichen dafür, dass das Vertrauensarbeitszeitmodell tendenziell zulasten der Arbeitnehmenden und zugunsten der Unternehmen geht (Syndicom-Studie, 2015, S. 39). Kadermitarbeitende, von denen permanente Erreichbarkeit oft quasi erwartet wird, erhalten zumindest eine höhere Entlöhnung dafür.

Vertrauensarbeitszeit braucht eine gewisse Kontrolle und Unternehmen müssen deshalb Richtlinien erlassen, die eine klare Trennung zwischen Arbeit und Freizeit ermöglichen (Syndicom Studie, 2015, S. 40). Wollen Unternehmen an der Vertrauensarbeitszeit festhalten, müssen diese sicherstellen, dass dies nicht zulasten der Mitarbeitenden geht. Zudem sollten neue Mitarbeitende bereits bei ihrer Einführung darauf hingewiesen werden, dass Arbeit in der Freizeit nicht der Philosophie des Unternehmens entspricht und deshalb nicht erwünscht ist, sondern dass die Freizeit der Erholung dienen soll (Syndicom-Studie, 2015, S. 40). Es ist jedoch anzumerken, dass all diese Ansätze bereits im OR geregelt sind.

Ein weiterer wichtiger Punkt der Syndicom-Studie ist die Verbesserung des Gesundheitsmanagements. Dabei sollte die Umsetzung des betrieblichen Gesundheitsmanagements mit den Sozialpartnerinnen und Sozialpartnern sowie der Personalvertretung vertieft diskutiert und neu definiert werden. Zudem empfiehlt es sich, eine Sensibilisierungskampagne durchzuführen, um zu erreichen, dass die Arbeitnehmenden im Krankheitsfall zu Hause bleiben. Bei der Einstellung und Weiterbildung von Vorgesetzten sollte die Sozialkompetenz künftig 
stärker gewichtet werden. Das Gesundheitsmanagement in den Betrieben gilt es also zu überarbeiten (Syndicom-Studie, 2015, S. 54).

Ein zusätzlicher Aspekt der Entgrenzung der Arbeit ist, dass die Arbeit in Zukunft vermehrt in Projekten organisiert wird. Mitarbeitende springen dann von einem Arbeitsprojekt zum anderen und erleben dabei immer neue Konstellationen und Teamzusammensetzungen. Dabei müssen einerseits die Mitarbeitenden eine grosse Konfliktfähigkeit, Lernbereitschaft, Flexibilität und Kommunikationsfähigkeit mitbringen. Andererseits muss auch das Büro als Arbeitsort umstrukturiert werden und künftig vermehrt auf die Ermöglichung von Kommunikation und Kooperation ausgerichtet sein, um individuelles Arbeiten zu ermöglichen (Waser, 2010, S. 14 ff.).

\subsubsection{Erreichbarkeit}

Das Thema der Erreichbarkeit der Mitarbeitenden wird oft diskutiert. In Bezug auf das Arbeitsrecht ergeben sich vor allem Probleme im Zusammenhang mit Höchstarbeitszeit, der Nacht- und Sonntagsarbeit sowie dem Ferienbezug. Dabei sind zwei Aspekte zentral:

1. Als Arbeitszeit ist auch die Zeit einzustufen, in der die Arbeitnehmende im Rahmen ihrer Tätigkeit für die Arbeitgebende einen Anruf entgegennimmt und ein Gespräch führt, eine E-Mail liest und beantwortet oder an einer Videokonferenz teilnimmt etc.

2. Ebenfalls als Arbeitszeit einzustufen ist die Zeit, in der die Arbeitnehmende so häufig durch Anrufe und E-Mails gestört wird, dass sie diese nicht mehr sinnvoll als Freizeit nutzen kann. Diese Zeit ist sodann als Bereitschaftszeit zu deklarieren und wird eindeutig im Interesse der Arbeitgebenden verbracht.

Vom zweiten Fall zu unterscheiden ist die Abrufbereitschaft. Die Zeit kann im privaten Interesse genutzt werden und steht daher nicht im Interesse der Arbeitgebenden. In Analogie dazu kann demnach auch die Zeit, in der eine Arbeitnehmende in Bereitschaft für einen Anruf ihr Handy mit sich trägt oder ihren Laptop zur Beantwortung allfälliger E-Mails einschaltet, nicht als Arbeitszeit gelten. Die Frage nach der Entschädigung ist allerdings getrennt von der Qualifikation als Arbeitszeit zu betrachten und ist Sache der Privatvereinbarung. Fehlt eine solche Vereinbarung, kann an die Praxis des Bundesgerichts zur Arbeit auf Abruf angeknüpft werden: Danach ist auch die blosse Rufbereitschaft zu entschädigen, wenn auch nicht gleich wie die Haupttätigkeit (Rudolph et al., 2012). 


\subsubsection{Bring-your-own-Device}

BYOD bedeutet, dass eine Mitarbeitende ihr privates Gerät wie einen Laptop, ein Smart- oder Mobilephone, ein Tablet ect. geschäftlich nutzt, also für ihre Arbeitgebende und innerhalb von deren Infrastruktur. Von dieser Arbeitsform unbedingt abzugrenzen ist die Nutzung von unternehmenseigenen mobilen Geräten unter der technischen Kontrolle der Arbeitgebenden während und nach der Arbeit. Diese Nutzung fällt nicht unter den Begriff BYOD, weil das Gerät nicht Eigentum der Arbeitnehmenden ist.

Warum aber sollte ein Unternehmen die Arbeitsform BYOD einführen?

Das Bedürfnis dafür besteht vor allem auf Seite der Mitarbeitenden. Mit den gewohnten Geräten zu arbeiten und gleichzeitig private Informationen und Daten nutzen zu können, verschafft der Mitarbeitenden und dem Unternehmen gleichermassen mehr Effizienz und erhöht daneben die Mitarbeitendenzufriedenheit. Auch kann aus BYOD ein finanzieller Nutzen hervorgehen, denn mit weniger eigenen Geräten entstehen auch weniger Anlagekosten für die Arbeitgebende. Hauptgründe zur Einführung von BYOD sind jedoch Effizienz und Produktivität. Verfügt eine Mitarbeitende privat über das neueste technische Gerät, möchte sie am Arbeitsplatz kein altes Gerät verwenden. Mit BYOD werden also auch Erwartungen der Mitarbeitenden erfült und es fördert die Effizienz sowie die Freude an der Arbeit (Beranek Zanon, 2012).

Die Verwendung von BYOD wirft jedoch viele juristische Fragen auf. Insbesondere geht es dabei um Datenschutzfragen, arbeitsrechtliche Fragen sowie Fragen bezüglich der Compliance, also des rechtskonformen Verhaltens. Weitere Aspekte sind neben Rechten an Immaterialgütern auch Support- und Haftungsfragen. Diese Themen werden im Folgenden aufgegriffen und soweit möglich beantwortet.

BYOD sollte im Arbeitsvertrag oder in einem Zusatz geregelt sein. Ggf. kann eine entsprechende Regelung auch in einem Arbeitsreglement aufgenommen werden. In einem solchen Fall muss der Arbeitsvertrag jedoch einen Verweis darauf enthalten. Wird eine BYOD-Vereinbarung bei laufendem Arbeitsverhältnis aufgezwungen, um das Arbeitsverhältnis aufrechtzuerhalten, kann je nach Inhalt von einer Änderungskündigung gesprochen werden. Stützt diese sich nicht auf wirtschaftliche oder betriebliche Veränderungen, ist sie unter Umständen missbräuchlich. Eine Arbeitgebende tut somit gut daran, wenn sie BYOD als Option nur für jene Mitarbeitenden einführt, die dies auch wollen. BYOD kann sicherlich nicht als Gesamtlösung für ein ganzes Unternehmen gesehen werden, sondern ist für jede Mitarbeitende individuell als Arbeitsform zu prüfen. Ohne das Vorliegen der Einwilligung der Arbeitgebenden darf eine Arbeitneh- 
mende nach derzeitigem Schweizer Recht nämlich nicht einfach ihre privaten Geräte einsetzen. Macht sie dies trotzdem, verletzt sie eventuell ihre Sorgfaltsund Treuepflicht gegenüber der Arbeitgebenden. Sind jedoch die Grundsätze der Datenaufbewahrung und deren Verwendung im Vertrag geregelt, ist die arbeitsrechtliche Situation bei einem allfälligen Verstoss gegen die Bestimmungen klarer. In einem solchen Fall kommen arbeitsrechtliche Instrumente wie Abmahnungen, Sperrungen des Internetzugriffs, Schadenersatzforderungen, Lohnkürzungen oder Versetzungen infrage. Im Wiederholungsfall kann sogar eine ordentliche Kündigung ausgesprochen werden. In Extremfällen, in denen der Arbeitgebenden die Fortsetzung des Arbeitsverhältnisses nach Treu und Glauben nicht mehr zugemutet werden kann, ist sogar eine fristlose Entlassung denkbar.

Aber auch die Interessen der Arbeitgebenden sind mit einer schriftlichen Regelung besser geschützt. Um das private Device der Mitarbeitenden systematisch mit dem Mobile-Device-Management (MDM) zu verwalten, braucht die Arbeitgebende nämlich deren Einwilligung. Eine Regelung für den Einsatz einer MDM-Software ist alleine schon für den Fall sinnvoll, dass die Arbeitnehmende das mobile Endgerät verliert oder das Arbeitsverhältnis beendet wird. Liegt allerdings keine Einwilligung der Mitarbeitenden vor, verletzt die Arbeitgebende nicht nur Art. 328b OR (Schutz der Persönlichkeit des Arbeitnehmenden), sondern auch Bestimmungen des Datenschutzgesetzes und des Fernmeldegeheimnisses nach Art. 13 Abs. 1 BV.

Durch die Verwendung von BYOD treten auch Fragen bezüglich der Kosten auf. Grundsätzlich ist die Arbeitgebende von Gesetzes wegen verpflichtet, der Arbeitnehmenden Arbeitsgeräte und -mittel zur Verfügung zu stellen. Von diesen Kostenübernahmepflichten kann durch spezielle Vereinbarungen mit der Mitarbeitenden abgewichen werden.

Wie erwähnt nutzt die Mitarbeitende bei BYOD ihr eigenes, privates mobiles Gerät für geschäftliche Zwecke. Besonders zu erwähnen sind deshalb die Eigentumsverhältnisse. Das Eigentum liegt bei BYOD bei der Mitarbeitenden. Es muss also geklärt werden, wer Software und Apps beschafft und finanziert und wem sie gehören.

Die Verwendung von BYOD wirft weiter datenschutzrechtliche Fragen auf, v.a. bei der Bearbeitung von Mitarbeiter- und Kundendaten oder auch im Zusammenhang mit IP-Adressen, da es sich dabei um Personendaten handelt (Art. 3 lit. a DSG). Bei der Bearbeitung von Personendaten müssen die datenschutzrechtlichen Grundprinzipien beachtet werden. Die Bearbeitung muss demnach nach Treu und Glauben erfolgen, verhältnismässig sein und eine gesetzliche 
Grundlage oder die Zustimmung der Kundin bzw. des Kunden muss vorliegen. Einer Mitarbeitenden sollte mit BYOD deshalb nur so viel Zugriff auf Daten gegeben werden, wie dies für die Erledigung ihrer Aufgaben notwendig ist, und das Unternehmen muss insbesondere sicherstellen, dass die Daten über ihre Kundinnen und Kunden nicht in unzulässiger Weise bearbeitet werden. BYOD stellt also besondere Herausforderungen in Bezug auf den Datenschutz dar. Um diesen einzuhalten, hat das Unternehmen am besten einen Remote-Zugriff auf das Gerät der Mitarbeitenden.

Auch im Zusammenhang mit der Datensicherheit, also die Sicherstellung der Integrität, Verfügbarkeit und Vertraulichkeit der Daten, müssen angemessene technische und organisatorische Massnahmen gegen unbefugtes Bearbeiten von Daten getroffen werden. Zu solchen Massahmen zählt insbesondere das Löschen von Inhalten auf USB-Sticks, die Verschlüsselung des Inhalts einer Kommunikation, die Einschränkung von Zugriffsberechtigungen oder die Protokollierung und Überwachung von Zugriffen von aussen. Zudem sollte die Mitarbeitende bedacht darauf sein, das private Gerät nie unbeaufsichtigt in der Öffentlichkeit liegen zu lassen.

Des Weiteren sind die gesetzlichen und vertraglichen Geheimhaltungsvorschiften einzuhalten. Ein Geheimnisbruch kann Konventionalstrafen oder strafrechtliche Konsequenzen nach sich ziehen. Auch das Berufs- und Amtsgeheimnis können verletzt werden.

Dies wirft Fragen bezüglich des Supports und insbesondere der Haftung auf. Wie bietet das Unternehmen den Mitarbeitenden Support bei technischen Problemen? Dabei gibt es die Möglichkeit des Full-Supports, des virtuellen UserSelf-Service oder sogar keiner Unterstützung. Diese Frage muss ebenfalls Bestandteil der vertraglichen Regelung sein.

Was passiert jedoch, wenn die Mitarbeitende ihr mobiles Endgerät verliert oder dieses gestohlen wird? Dies kann erhebliche Auswirkungen auf das Unternehmen haben: Datendiebstahl, Manipulation, Angriffe auf die Gesamtinfrastruktur etc. Hat die Mitarbeitende einem Mobile-Device-Management zugestimmt, beschränkt dieses die grössten technischen Risiken. Die Mitarbeitende ist dabei haftbar, wenn sie die Sicherheitsvorschriften nicht beachtet hat.

Fehlt ein solches Mobile-Device-Management, haftet die Mitarbeitende nur, wenn sie nicht beweisen kann, dass sie die gebotene Sorgfalt angewendet und die technischen Anweisungen befolgt hat. Dieser Beweis ist jedoch schwierig zu erbringen. Der Transfer der Haftung für das Betriebsrisiko von der Arbeitgebenden zur Arbeitnehmenden widerspricht eigentlich dem Schutzgedanken des 
Arbeitsrechts. Die Frage, ob eine solche Vorgehensweise zulässig ist, wurde bisher von der Rechtsprechung noch nicht abschliessend beantwortet.

\subsubsection{Spannungsfeld VIII: Risiken der IT-Nutzung}

Gemäss der vorangegangenen Ausführungen ist festzuhalten, dass die ITPolitik BYOD erhebliche Risiken für die Arbeitgebende mit sich bringt, sofern sie keine arbeitsvertragliche Regelung mit den Mitarbeitenden trifft. Ein entsprechender Vertragszusatz ist deshalb unerlässlich und fördert gleichzeitig das Bewusstsein für die Problematik. Eigentumsverhältnisse, Kosten sowie Verantwortlichkeiten beim Datenschutz, Lizenzen, Support und Haftung müssen unbedingt kommuniziert werden und die Anwendung eines MDM ist sinnvoll.

\subsection{Virtuelle Arbeit (Crowdsourcing)}

Crowdsourcing-Plattformen, auch Projektbörsen genannt, haben in den vergangenen Jahren auch in der Schweiz erheblich an Bedeutung gewonnen. Ob Selbstständige, Beratende, Studierende, Teilzeitarbeitende oder gar Rentnerinnen und Rentner, die Plattform steht allen Interessentinnen und Interessenten offen (Zaugg, 2013). Auch Ursula Meyerhans, Redaktorin, und Andrea BurriLötscher, Küchenhilfe, machen vom Angebot der verschiedenen Plattformen Gebrauch. Der Boom der Crowdsourcing-Plattformen wird wohl in den kommenden Jahren weiter wachsen, wie auch die „Solo-Selbstständigkeit“. Grosse Konzerne überlegen sich, Stammbelegschaften zu reduzieren und entlassene Mitarbeitende im Modell Crowdsourcing in Subunternehmen auszugliedern. Dadurch können Gebäudekosten, Rentenansprüche sowie Kosten für das Gesundheitswesen eingespart werden.

Wieweit sich diese junge "Disziplin“ in die rechtlichen Rahmenbedingungen einfügt, wird im vorliegenden Kapitel aufgezeigt.

Zentrale Fragen hierbei sind:

- Was für ein Vertragsverhältnis liegt vor, zwischen welchen Parteien?

- Welche rechtlichen Bestimmungen kommen auf diese Vertragsverhältnisse zur Anwendung?

- Welche Herausforderungen werden erwartet? 


\subsubsection{Rechtliche Bedeutung von Crowdsourcing}

Die Plattformen agieren in der Regel als Vermittelnde. Sie sind also Partner der beiden Seiten, der Auftraggebenden und der Crowdworkerin. Es entsteht somit kein direktes Vertragsverhältnis zwischen diesen zwei letztgenannten Parteien. Die externe Crowdworkerin wird als selbstständige Person wahrgenommen, wodurch die Anwendung aller arbeitsrechtlicher Bestimmungen (Mindestlöhne, Ferien, Lohnfortzahlungen etc.) des Einzelarbeitsvertrags per se nicht zwingend ist. Die wirtschaftliche Abhängigkeit sowie ein Subordinationsverhältnis zwischen Vermittelnden und Crowdworkern sind ebenfalls nicht vorhanden, was gegen das Vorliegen eines Arbeitsverhältnisses spricht. Diese Situation kann sich aber ändern (Konicz, 2014), denn grossteils befindet sich das Crowdsourcing noch in einer rechtlichen Grauzone. Wann bspw. eine Virtual-Workerin unter die Definition einer Arbeitnehmenden fällt, ist noch nicht in allen Rechtsordnungen klar ausgearbeitet (Leimeister/Zogaj, 2013, S. 72 ff.).

Den rechtlichen Rahmen für Crowdsourcing-Plattformen bilden regelmässig die allgemeinen Geschäftsbedingungen (AGB) der jeweiligen Plattform sowie allgemeine gesetzliche Vorschriften. Die AGBs definieren in der Regel, dass das Recht am Sitz der Unternehmung, sprich der Plattform zur Anwendung gelangt und dort auch der Gerichtsstand liegt.

Die Entlohnung der Crowdsourcees richtet sich nach Angebot und Nachfrage, wobei besondere Fähigkeiten höher entlohnt werden. Das ist für den Aufgabensteller besonders lukrativ, da er aus einer Vielzahl von Lösungen auswählen kann. Bezahlungen erfolgen in der Regel nur an den Ausgewählten, allenfalls noch an den Zweitplatzierten. Die anderen haben umsonst eine Arbeitsleistung erbracht.

Recherchen haben gezeigt, dass die Löhne je nach Arbeitsform und Art der Aufgabe stark variieren können. Auf manchen Plattformen wird die Belohnung nicht in Form von Geld ausbezahlt, sondern als Sachprämien oder Bonuspunkte (Leimeister/Zogaj, 2013, S. 72 ff.). Weiter gibt es in den USA laufende Verfahren, die mit der Crowdworkerinnen die Anwendbarkeit des Fair Labour Standard Act und der Mindestlöhne für ihre Arbeit durchsetzen wollen. Sollten diese Prozesse erfolgreich geführt werden, würden die Crowdworkerinnen nicht mehr als selbstständige Unternehmende, sondern als Arbeitnehmende angesehen, auf die der Fair Labour Standard Act zutreffen und mit Anrecht auf Mindestlöhne. Dadurch wäre ein Mindestschutz für die Crowdworkerinnen geschaffen. Auch die Geltung und Anwendung ausländischen Rechts muss situativ geprüft werden. Hier bestehen zurzeit noch offene Rechtsfragen. 
Zudem sehen die AGBs häufig Kontaktverbote vor wie z.B.: „Falls Sie von Dritten wegen Beschäftigungsmöglichkeiten angesprochen werden, müssen Sie den Vermittelnden sofort darüber informieren und sind nicht berechtigt, ausserhalb von der Plattform mit der Userin bzw. dem User in Kontakt zu treten." Solche Regelungen sind schon wegen der Verletzung des allgemeinen Persönlichkeitsrechts unwirksam und wären auch bezüglich der Grundrechte der Wirtschaftsfreiheit unzulässig. Um eine solche Ausnutzung zu verhindern, verlangen die Gewerkschaften, dass Crowdworkerinnen und Crowdworker in die sozialen Sicherungssysteme aufgenommen werden, um so die Umgehung arbeitsrechtlicher Schutzvorschriften zu verhindern (Konicz, 2014).

Ist das Crowdworking eine externe Nebentätigkeit, welcher die Arbeitnehmende zusätzlich zu ihrem Hauptarbeitsverhältnis nachgeht, ist dies grundsätzlich möglich. Die Nebentätigkeit wird dann unzulässig, wenn sie zu einer erheblichen Beeinträchtigung der Arbeit im Hauptarbeitsverhältnis oder zur Konkurrenzierung der Arbeitgebenden führt. Weiterhin sind Fragen des Wucherzinses sowie der Verpflichtung zur Abgabe von Einkommens- und Umsatzsteuer zu klären.

\subsubsection{Spannungsfeld IX: Rechtliche Rahmenbedingungen für Crowd- sourcing schaffen}

Im Rahmen der Literaturrecherche zum Crowdsourcing musste festgestellt werden, dass zahlreiche Erkenntnisse und Diskussionen aus dem amerikanischen Markt vorliegen. Diese sind jedoch nicht eins zu eins auf das schweizerische Recht anwendbar. Aus diesem Grund ist es schwierig, hier Aussagen über die rechtlichen Rahmenbedingungen von Crowdsourcing in der Schweiz zu machen. Wie kann Crowdsourcing in der Schweiz rechtlich reguliert werden? Haben Crowdworkerinnen ein Mitbestimmungsrecht? Die Beantwortung solcher und anderer zahlreicher offener Fragen im Zusammenhang mit den rechtlichen Auswirkungen des Crowdsourcings dürfte in Zukunft einige Expertinnen und Experten beschäftigen. 


\subsection{Schlussfolgerungen zu den Spannungsfeldern zwischen mobilen Arbeitsformen und recht- lichen Grundlagen}

Ob die Work-Life-Balance ausgewogen ist, entscheidet sich nicht zuletzt durch die Gestaltung der Arbeitszeit. Flexible Arbeitszeiten, die richtige Gestaltung vorausgesetzt, können also dazu beitragen, dass die Work-Life-Balance im Lot bleibt. Wer über Zeitsouveränität verfügt, wer seine individuellen Interessen bei der Verteilung der Arbeitszeit einbringen kann, dessen Arbeitszufriedenheit und Lebensqualität steigen (Baua, 2013).

Arbeit ohne zeitliche und räumliche Grenzen hat zur Folge, dass völlig neue Instanzen zur Regulierung aktiviert werden müssen. Wie weit soll nun die Selbststeuerung gehen und wo setzen die Regulierungsmechanismen ein? Die Veränderung der Arbeitswelt wird mit starren Regelungen kaum noch funktionieren können. Neben wirtschaftlichen Notwendigkeiten sind es dabei auch die veränderten Bedürfnisse und Werthaltungen vieler Arbeitnehmender, die einer Flexibilisierung Vorschub leisten.

Die Zukunft wird zeigen, ob der Entscheid des Bundesparlaments, keine weiteren gesetzlichen Bestimmungen zu schaffen, richtig ist. Es wird wohl notwendig sein, die vorhandenen Standards zu prüfen und diese allenfalls auch zu einem späteren Zeitpunkt anzupassen. Zentral ist dabei, dass die Handlungsmöglichkeiten für die Organisationen nicht eingeschränkt werden und die unternehmerischen Aktivitäten erfolgen können. Zugleich gilt es zu verhindern, dass der Arbeitsmarkt und die Arbeitswelt auf Kosten der Mitarbeitenden zersplittert werden. Einheitliche und flexible Rahmenbedingungen können dies ermöglichen.

Es bleibt festzuhalten, dass Sicherheit und Flexibilität ein Spannungsverhältnis bleiben, welches auch aus juristischer Sicht entsprechend aufgenommen und geregelt werden muss.

\subsubsection{Arbeitsrechtliche Herausforderungen im flexiblen Arbeitsmarkt}

Die vorangegangenen Kapitel haben verschiedene Spannungsfelder im Zusammenhang mit mobilen Arbeitsformen und dem schweizerischen Arbeitsrecht identifiziert und umschrieben:

Spannungsfeld I: Abgrenzung der einzelnen Arbeitsleistungen gegenüber dem Einzelarbeitsvertrag 
Spannungsfeld II: Herausforderungen versus Risiken bei mobiler Arbeit

Spannungsfeld III: Arbeitszeitregelung versus Flexibilität

Spannungsfeld IV: Regulierung durch Rahmenbedingungen versus genügend Freiraum für Flexibilität

Spannungsfeld V: Erhöhung der Arbeitsflexibilität und der Marktwirtschaftlichkeit mit gleichzeitiger Einhaltung der sozialen Sicherheit der Arbeitnehmenden

Spannungsfeld VI: Flexible Arbeit ja, aber nicht auf Kosten der Gesundheit

Spannungsfeld VII: Wie setzen wir „mobilen“ Gesundheitsschutz um?

Spannungsfeld VIII: Risiken der IT-Nutzung

Spannungsfeld IX: Rechtliche Rahmenbedingungen für Crowdsourcing schaffen

Diese Spannungsfelder zeigen jeweils auf, wo rechtlich gesehen Herausforderungen im Zusammenhang mit mobil-flexibler Arbeit bestehen. Diese Herausforderungen werden in den nachstehenden Abschnitten nochmals kurz umrissen und zusammengefasst.

Schutz basierend auf Gesamtarbeitsverträgen: Die Gesamtarbeitsverträge gehen oft über den rechtlichen Minimalschutz hinaus, wobei die Arbeitnehmende davon profitiert. Die nun bereits vorhandenen und neu entstehenden flexiblen Arbeitsformen sind gewerkschaftlich nicht oder nur schlecht organisiert, mit der Konsequenz, dass gerade Teile von „Arbeitnehmenden“, die den arbeitsrechtlichen Schutz benötigen würden, nicht von diesem Schutz profitieren können. Bereiche wie Mindestlohn, Arbeitszeiten und andere Rechte bleiben ungeregelt.

Regelung bezüglich Arbeitszeit: Das Arbeitsrecht führt bis heute keine Regelungen zu modernen Arbeitszeitsystemen wie Jahresarbeitszeit, Sabbatical, Langzeitkontos u.a. Die Rahmenbedingungen führen Mindestruhezeiten und Maximalarbeitszeiten aus, die von einer "normalen“ Arbeitswoche ausgehen. Bei davon abweichenden Arbeitszeitmodellen bestehen also rechtliche Unklarheiten und damit auch Unsicherheiten.

Nachteile bei Kurzarbeitseinsätzen: Diverse Regelungen im Gesetz (Lohnfortzahlung bei Arbeitsverhinderung, Art. 324a OR; gesetzliche Kündigungsfrist, Art. 335c OR; Kündigungssperre, Art. 336c OR) stehen nur der Arbeitnehmenden mit etlichen Dienstjahren zur Verfügung. Hier stellt sich insbesondere auch 
ein Problem bei Eintritt einer Invalidität, da sich die schwankenden Einkommenserträge negativ auf die Invalidenrente auswirken können (EDI, 2014). Gleiches gilt bei längeren Krankheiten, da die obligatorische Krankentaggeldversicherung das Risiko eines längerdauernden Arbeitsausfalls nicht deckt. Hier ist auch in Betracht zu ziehen, dass die GAV häufig befristete Arbeitsverhältnisse, Teilzeitarbeiten oder Arbeit auf Abruf aus ihrem Geltungsbereich ausschliessen.

Definition der Qualifizierung: Es gibt Personen in der Arbeitswelt, die weder Arbeitnehmende noch Selbstständigerwerbende sind, sondern etwas dazwischen wie bspw. die Crowdworkenden. Durch solche rechtlich nicht qualifizierten Arbeitsformen entstehen Schwierigkeiten in der Subordination der rechtlichen Normierung (vgl. Abschnitt 3.2). Besteht ein Einzelarbeitsvertrag oder doch ein Auftrag? Gibt es eine betriebliche Eingliederung oder eine Weisungsbefolgungspflicht? Solche Fragen müssen bei der Qualifizierung von Vertragsverhältnissen geklärt werden. Von dieser Qualifizierung hängt nicht zuletzt ab, ob eine Vertragspartei dem arbeitsrechtlichen Schutz untersteht oder nicht. Hier bestehen rechtliche Lücken, die geschlossen werden müssen, um Klarheit zu schaffen. Solche Regelungen sind aber nicht neu, es gibt schon heute für „atypische" Arbeitsverhältnisse wie für den Lehrvertrag, den Handelsreisenden oder den Personalverleih zusätzliche gesetzliche Vorschriften. Weitere atypische Arbeitsverhältnisse sowie deren Rechte und Pflichten könnten künftig auch unter bereits bestehende Normen subsumiert werden. Bis diese Klärung stattgefunden hat, bleiben jedoch auch hier in Lehre und Rechtsprechung Unsicherheiten bestehen.

Weiterbildungsansprüche: Gemäss Gesetz hat die Arbeitnehmende keinen Anspruch gegenüber ihrer Arbeitgebenden auf Weiterbildung. Gesamtarbeitsverträge haben solche Ansprüche teilweise definiert. Gemäss der FlexicurityStudie ist dieser Anspruch von fundamentaler Bedeutung für eine flexible und sozial abgesicherte Arbeitswelt. Hier ist zu prüfen, ob dieser Anspruch gesetzlich geregelt werden sollte.

Arbeitsort: Die Bestimmung des Arbeitsorts sollte im Sinne der Privatautonomie nach wie vor den Parteien überlassen werden. Eine begriffliche Normierung im Obligationenrecht oder in anderen Vorschriften erscheint nicht als empfehlenswert. Jedoch halten wir eine Konkretisierung von Art. 327a Abs. 1 OR für ratsam. Diverse Studien und Umfragen zeigen, dass der Auslagenersatz bei auswärtigen Arbeitsorten häufig ungenügend ausfällt. Dies kann davon abgeleitet werden, dass der Begriff „Unterhalt“ zu wenig konkret ist. Durch eine exemplarische Aufzählung könnte dieser Artikel transparenter gestaltet werden. 
"Scheinselbstständigkeit": Hier stellt sich die Frage, in welchen rechtlichen Rahmen diese Form sich einfügen soll. Fakt ist, dass keine Versicherungsmöglichkeiten gegen Arbeitslosigkeit, weder eine berufliche Vorsorge noch eine obligatorische Versicherung nach UVG besteht und auch das Thema Berufskrankheiten nicht abgedeckt ist. Es ist abzuklären, ob es in diesem Bereich gesetzliche Präzisierungen bräuchte, um Missverständnisse aus dem Weg zu räumen. Ansonsten sind Arbeitgebende und Arbeitnehmende dafür zu sensibilisieren, dass dieser nicht klar definierte Bereich vertraglich präzisiert und geregelt werden muss. Ein anderer Ansatz ist, dass der Gesetzgeber den Arbeitnehmendenbegriff ausdehnt und dadurch als Grundlage für das gesamte Arbeitsrechtfeld das Obligationenrecht zur Anwendung gelangt.

\subsubsection{Ausblick und Blick über die Grenzen}

Die Thematik von Homeoffice und längeren oder kürzeren Arbeitszeiten beschäftigt auch das nähere Ausland. Im vergangenen Juli verschaffte das niederländische Parlament den Arbeitnehmenden neben einem Rechtsanspruch auf Heimarbeit gleichzeitig einen Anspruch darauf, ihre Arbeitszeit weitgehend selbst festzulegen. Zwar muss ein derartiger Antrag von der Arbeitgebenden bewilligt werden, jedoch müssen bei dessen Ablehnung schwerwiegende betriebliche Interessen vorliegen. In jüngster Zeit wurden in Deutschland ähnliche Forderungen laut. Es wurde verlangt, dass in Unternehmen mit mehr als zehn Beschäftigten die Arbeitnehmenden von zu Hause arbeiten können sollen, sofern dem keine Sicherheitsanforderungen oder zwingende betriebliche Gründe entgegenstehen. Ob diese Anliegen politisch umgesetzt werden, muss sich noch zeigen. Ebenfalls abzuwarten bleibt, ob die Schweiz diese Debatte aufnimmt und ob Arbeitnehmerorganisationen ähnliche Vorstösse in der Schweizer Politik einreichen werden.

Es darf abschliessend aber auch festgehalten werden, dass die Schweiz im europäischen Vergleich vor allem mit Blick auf die soziale Sicherheit gut abschneidet. Gewisse Optimierungen der bestehenden Systeme sind sicherlich notwendig. Zudem müssen auch die genannten rechtlichen Lücken geprüft und allenfalls geschlossen werden. Gleichzeitig ist darauf hinzuweisen, dass das Arbeitsgesetz bereits heute viele Grundsätze beinhaltet, dazu kommen noch viel mehr Ausnahmen und zahlreiche Verordnungen. Diese Unübersichtlichkeit führt genauso häufig zu Unklarheiten oder Missverständnissen im Arbeitsalltag, wie es rechtliche Lücken tun. Es ist deshalb auch zu prüfen, ob durch eine Vereinfachung der Strukturen nicht bereits einige Erleichterungen erreicht und gleichzeitig bestehende Lücken geschlossen werden könnten. 



\section{Mögliche Folgen der persona- basierten Arbeitswelten auf ge- samtwirtschaftlicher Ebene}

Dieses Kapitel widmet sich den möglichen Folgen auf gesamtwirtschaftlicher Ebene einer sich weiterentwickelnden Flexibilisierung der Arbeit. Dazu werden, aufgrund von aus einer Literatur- und Datenanalyse gezogenen Erkenntnissen, die Auswirkungen auf gesamtwirtschaftliche Grössen dargestellt, welche in Zusammenarbeit mit der Begleitgruppe für diesen Bericht ausgewählt wurden. Eine grafische Zusammenfassung der Stärke und Richtung der diversen Auswirkungen findet sich in Abschnitt 4.3.12.

Für die Folgeabschätzung in diesem Kapitel wurden Befunde aus wissenschaftlichen Studien, Erkenntnisse aus journalistischen Beiträgen und Daten aus öffentlichen Statistiken, welche die Indikatoren betreffen, systematisch zusammengetragen und den jeweiligen personabasierten Arbeitswelten zugewiesen. Dabei wurde insbesondere auch auf die Aktualität der Quellen geachtet. So wurde bspw. nach Literatur gesucht, welche Aussagen zur Produktivität von flexibilisierten Arbeitsformen, wie dem Homeoffice, enthält. Aus einer grösseren oder kleineren Anzahl von Einzelsachverhalten wurde anschliessend induktiv nach dem Grundsätzlichen gesucht und in einem Abschnitt zur zukünftigen Auswirkung auf den jeweiligen Indikator berücksichtigt und zusammengefasst. Die Aussagen wurden sodann auf die verschiedenen Arbeitswelten bezogen und unter der Annahme, dass die darin enthaltenen Arbeitsformen gesamtwirtschaftliche Relevanz erreichen, zu einer möglichen Folge für den Indikator auf volkswirtschaftlicher Ebene extrapoliert. So entstanden Prognosen für die Entwicklung der Indikatoren, die auf Annahmen und ungesicherten Zahlen basieren, welche sich aus der aktuellen Entwicklung folgern lassen. Berücksichtigt wurde dabei ein Zeithorizont von zehn bis 15 Jahren.

Die in Abschnitt 4.2 dargestellten Auswirkungen stellen somit das Resultat einer Partialanalyse dar, die auch als ein auf das Wesentlichste beschränktes gedankliches ökonomisches Experiment bezeichnet werden kann. Aus den Sachzusammenhängen wurden nur die als besonders wichtig identifizierten Einflussgrössen einbezogen und die übrigen möglicherweise ebenfalls relevanten Faktoren konstant gehalten (ceteris paribus). Es liegt in der Natur von zukunftsbezogenen Aussagen, dass sie fast immer ein spekulatives Element bzw. Unsicherheiten enthalten. Da die Gewichtung einzelner Forschungsergebnisse bzw. 
deren Generalisierbarkeit einen Interpretationsspielraum offenlassen, sind für die Nachvollziehbarkeit der Aussagen zu den Auswirkungen die Quellen der eingeflossenen Sachverhalte im Text enthalten. Für einige Indikatoren (Arbeitslosigkeit, Beschäftigte, Steuererträge, Systeme der sozialen Absicherung) konnten kaum Literatur oder Daten gefunden werden und die Einschätzung der Auswirkungen erfolgte deduktiv von den Ergebnissen der übrigen Indikatoren auf die gesuchte Messgrösse.

\subsection{Zugrunde liegende Annahmen und kurze Darstellung der Arbeitswelten}

\subsubsection{Roland Müller - „Homeoffice in der Festanstellung“}

Die räumliche Flexibilisierung ist für die Mehrzahl der bürogestützten Arbeiten in allen Wirtschaftssektoren vollzogen, aber nicht überall gleich stark ausgeprägt. Insbesondere Manager sind bei der Wahrnehmung ihrer Funktionen, Koordination und Führung, auf den persönlichen Kontakt zu ihren Mitarbeitenden angewiesen. Arbeiten in den Funktionen Planung, Organisation und Kontrolle werden hingegen oft zu Hause oder ausserhalb der Betriebsliegenschaften erledigt. Die meisten Arbeitnehmenden sind weiterhin fest angestellt und haben ein Arbeitspensum von mindestens 80 Prozent, welches Mitarbeitende bereits ab den unteren Kaderstufen als Vertrauensarbeitszeit absolvieren. Mitarbeitende auf der untersten Hierarchiestufe müssen weiterhin ihre Arbeitszeit erfassen. Erfolgsbeteiligungen sind für das geschäftsführende Personal üblich, jedoch nicht weit verbreitet unter den Mitarbeitenden bis und mit der mittleren Kaderstufe.

Prägend: Homeoffice, Festanstellung, hohe Teilzeitpensen, Vertrauensarbeitszeit

\subsubsection{Sandra Könitz - „Mobiles Arbeitskraftunternehmertum“}

Die räumlich und zeitlich flexibilisierte Arbeit hat fast den ganzen Dienstleistungssektor erfasst und spielt auch im zweiten Sektor eine bedeutende Rolle. Homeoffice, mobiles Arbeiten und Büroarbeit sind also für eine Mehrheit der Arbeitnehmenden feste Bestandteile ihrer Arbeitswelt, wobei „ICT-based mobile Work" überwiegt. Die meisten Arbeitnehmenden sind weiterhin fest angestellt und haben ein Arbeitspensum von mindestens 80 Prozent, welches als Vertrauensarbeitszeit absolviert wird. Während der Anteil der Erwerbstätigen mit 
fixen Arbeitszeiten in den Jahren 2001 bis 2014 noch bei über 57 Prozent lag (BFS, 2015i), hat sich dieser Anteil auf ca. 20 Prozent verringert. Mitarbeitende ab der mittleren Kaderstufe sind grundsätzlich erfolgsbeteiligt und haben Vertrauensarbeitszeit. Bei der Bewältigung der unternehmerischen Aufgaben wird häufig Outsourcing betrieben und zunehmend auf standardisierte Prozesse gesetzt.

Prägend: Vertrauensarbeitszeit, Unternehmertum der Mitarbeitenden, erweiterte Erreichbarkeit, funktionale Flexibilität, räumliche Mobilität

\subsubsection{Ursula Meyerhans - „Portfoliowork auf mehreren Standbeinen“}

Die räumlich und zeitlich flexibilisierte Arbeit hat fast den ganzen Dienstleistungssektor erfasst und spielt auch im zweiten Sektor eine bedeutende Rolle. Viele Unternehmen verzichten darauf, Büros für alle Mitarbeitenden zu betreiben, wenn sie bürogestützte Aufgaben wie Personalmanagement, Rechnungswesen, Administration, Verkauf usw. überhaupt noch unternehmensintern wahrnehmen und sie nicht in irgendeiner Form ausgelagert haben. Bei verschiedenen Outsourcing-Methoden dominiert das Paid Crowdsourcing, welches sich seit seiner beginnenden Verbreitung Anfang der 2000er-Jahre weiterentwickelt hat. Dank den Fortschritten in der technischen und inhaltlichen Aufbereitung und der Fragmentierung der Arbeitsanforderungen sind heute fast alle Berufe in unterschiedlicher Intensität davon betroffen. Während KMU oft auf spezialisierte Crowdsourcing-Intermediäre setzen, die Aufträge von Unternehmen an Internetnutzer vermitteln und sich um Abwicklung, Besoldung und Projektcontrolling kümmern, haben Grossunternehmen häufig eine eigene Abteilung dafür. Als Folge haben Unternehmen ihre feste Belegschaft drastisch reduziert und beschäftigen nur noch eine Rumpfmannschaft. Dabei dominieren bis im mittleren Kader Teilzeit-Arbeitsverträge. Vollzeitstellen sind fast nur noch im oberen Kader zu finden. Um ihre Einkommen aufzubessern und berufliche Erfahrung zu sammeln, suchen viele Teilzeitangestellte Aufgaben über Crowdsourcing-Plattformen und erledigen sie zu Hause oder in den verschiedenen Arten von s.g. Coworking-Spaces. Kryptowährungen haben ihren Weg über bedienfreundliche Apps und Anbietende von Online-Zahlungsverkehr in den Alltag gefunden.

Prägend: (Solo-)Selbstständigkeit, geringfügige Beschäftigung, Portfoliowork, Teilzeit, befristete Arbeitsverhältnisse 


\subsubsection{Noah Schmid - „Entrepreneurship“}

Die technologischen Möglichkeiten zur Flexibilisierung der Arbeit werden von den Unternehmen voll genutzt. Neben der Anpassung der Arbeitsorganisation durch die räumliche und zeitliche Flexibilisierung der Arbeitsverhältnisse, ist die interne Flexibilisierung weit fortgeschritten. Während sich im zweiten Sektor die Umstellung auf neue Fertigungstechnologien (z.B. Roboter und vollautomatische Fertigungsstrassen) vollzieht, setzen die Unternehmen aller Branchen bei ihren Kernbelegschaften auf funktionale Flexibilität und beschäftigen nur noch Personal mit entsprechend breiten Qualifikationen. Bei den immer seltener werdenden Anstellungsverhältnissen dominieren Teilzeit- oder befristete Arbeitsverträge. Die Unternehmen nutzen über Werkverträge für die meisten Aufgaben Netzwerke von selbstständigen Fachkräften („Neue oder Solo-Selbstständige“5) bzw. Portfolioworkenden, die als virtuelle Organisationen funktionieren, sowie Shared Employees. Neben dem Dienstleistungssektor macht auch der industrielle Sektor nach vollzogener Tertiärisierung regen Gebrauch dieser neuen Organisationsformen, insbesondere für die sogenannten primären und sekundären Dienstleistungstätigkeiten (kaufmännische Tätigkeiten, Reinigungs-, Bewirtungs- und Lagertätigkeiten bzw. Forschungs- und Entwicklungstätigkeiten, Beratung und Management). Auch die öffentliche Hand setzt in zunehmendem Masse auf die neuen Formen der Arbeit.

Prägend: Teilzeit- oder befristete Arbeit, Werkverträge, Neue Selbstständige, Solo-Selbstständigkeit, Collaborative Self-Employment, Shared Employees

\subsubsection{Andrea Burri-Lötscher - „Mehrfachanstellungen und Minijobs“}

Die Arbeitskräfte sind stark in Hoch- und Geringqualifizierte polarisiert. Dabei werden Arbeitnehmende mit geringen Qualifikationen kaum noch Vollzeit angestellt. Oft müssen sie mehreren Jobs nachgehen, damit sie über die Runden kommen. Die Unternehmen, aber auch die öffentliche Hand und private Haushalte beschäftigen Geringqualifizierte häufig saisonal oder für kürzere Einsatzzeiten via Dienstleistungs- bzw. Beschäftigungsschecks. Private Vermittlungsdienste decken v.a. Haushalts- und Pflegedienste ab, während Gemeinden und regionale Arbeitsvermittlungszentren subventionierte Arbeitskräfte vermitteln, welche früher in Beschäftigungsprogrammen angestellt bzw. ausgesteuert wa-

\footnotetext{
${ }^{5}$ Gemäss Kelleter (2009) werden auch Freiberufler sowie Personen, die auf Basis eines Werkvertrages arbeiten, zur Gruppe der Solo-Selbstständigen gezählt.
} 
ren und wegen fehlender Computergrundkenntnisse kaum am Arbeitsmarkt teilnehmen können. Auch Höherqualifizierte müssen sich meistens mit Teilzeitarbeit zufrieden geben und bewegen sich zwischen Arbeitskraftunternehmertum, Solo-Selbstständigkeit und Mehrfachjobs.

Prägend: Teilzeit- oder befristete Arbeit, Mehrfachjobs (Zweit-, Nebenjobs), Minijobs, Voucher-based Work

\subsection{Resultate der Folgeabschätzung}

Tabelle 7 fasst die Auswirkungen der Bedingungen in den einzelnen durch die Personas exemplifizierten Arbeitswelten mithilfe von Symbolen, welche für die Stärke und Richtung der Auswirkung stehen, zusammen. Für das vollständige Eintreten der beschriebenen Auswirkungen wurde von einem Zeithorizont von ca. zehn bis 15 Jahren ausgegangen. Die Symbole zeigen also die Situation, wie sie bis im Jahr 2030 eintreten könnten im Vergleich zu heute. Die detaillierten Erläuterungen zu den möglichen Wirkungen der fünf Arbeitswelten auf die Indikatoren finden sich im nächsten Abschnitt.

Zur besseren Verständlichkeit wurden für die Tabelle einige Indikatoren anders gepolt, sodass alle Indikatoren positiv formuliert sind und ein Pfeil nach oben immer eine positive, wünschenswerte Entwicklung zeigt: physische und psychische Gesundheit statt Gesundheitsrisiken, Erwerbsquote statt Arbeitslosigkeit und Transparenz der Zahlungsströme statt intransparente Zahlungsströme. 
Tabelle 7: Zusammengefasste Resultate der gesamtwirtschaftlichen Auswirkungen

\begin{tabular}{|c|c|c|c|c|c|}
\hline \multirow[b]{2}{*}{ Indikator/Messgrösse } & \multicolumn{5}{|c|}{$\begin{array}{l}\text { Stärke und Richtung der Auswirkung in } 10 \text { bis } 15 \text { Jahren } \\
\text { in der Arbeitswelt von }\end{array}$} \\
\hline & $\begin{array}{c}\text { Roland } \\
\text { Müller / } \\
\text { Homeoffice in } \\
\text { der Fest- } \\
\text { anstellung }\end{array}$ & $\begin{array}{c}\text { Sandra } \\
\text { Könitz / } \\
\text { Mobiles } \\
\text { Arbeits- } \\
\text { kraftunter- } \\
\text { nehmertum }\end{array}$ & $\begin{array}{c}\text { Ursula } \\
\text { Meyerhans / } \\
\text { Portfoliowork } \\
\text { auf mehreren } \\
\text { Standbeinen }\end{array}$ & $\begin{array}{c}\text { Noah } \\
\text { Schmid / } \\
\text { Entrepre- } \\
\text { neurship }\end{array}$ & $\begin{array}{l}\text { Andrea } \\
\text { Burri- } \\
\text { Lötscher / } \\
\text { Mehrfach- } \\
\text { anstellun- } \\
\text { gen und } \\
\text { Minijobs }\end{array}$ \\
\hline Produktivität & $\sim \downarrow$ & $\uparrow$ & $\circ$ & $\sim \downarrow$ & $\sim \uparrow$ \\
\hline Erwerbseinkommen & ० & $\sim$ & $\downarrow$ & $\downarrow$ & $\downarrow$ \\
\hline $\begin{array}{l}\text { Kollektiver Organisationsgrad der } \\
\text { Beschäftigten }\end{array}$ & $\circ$ & $\circ$ & $\sim \downarrow$ & $\downarrow \downarrow$ & $\downarrow \downarrow$ \\
\hline Physische und psychische Gesundheit & $\sim \downarrow$ & $\downarrow$ & $\downarrow \downarrow$ & $\downarrow$ & $\downarrow$ \\
\hline $\begin{array}{l}\text { Arbeitsbedingungen und } \\
\text {-zufriedenheit }\end{array}$ & $\uparrow$ & $\uparrow$ & $\sim \downarrow$ & $\sim \downarrow$ & $\downarrow$ \\
\hline $\begin{array}{l}\text { Vereinbarkeit von Beruf mit anderen } \\
\text { Lebensbereichen }\end{array}$ & $\uparrow$ & $\sim \downarrow$ & $\sim$ & $\uparrow$ & $\sim \uparrow$ \\
\hline Erwerbsquote & $\circ$ & $\circ$ & $\sim \downarrow$ & $\downarrow$ & $\downarrow$ \\
\hline Beschäftigte & $\sim \uparrow$ & o & $\sim \downarrow$ & $\downarrow$ & $\sim$ \\
\hline Steuererträge & $\circ$ & $\sim \uparrow$ & $\sim \downarrow$ & $\sim \downarrow$ & $\downarrow$ \\
\hline Transparenz der Zahlungsströme & $\circ$ & $\sim$ & $\downarrow$ & $\downarrow \downarrow$ & $\downarrow$ \\
\hline $\begin{array}{l}\text { Situation der Systeme der sozialen } \\
\text { Absicherung }\end{array}$ & ० & o & $\downarrow$ & $\downarrow \downarrow$ & $\downarrow \downarrow$ \\
\hline
\end{tabular}

Zeichenerklärung:

$\uparrow \uparrow$ deutlicher Anstieg bzw. deutliche Verbesserung der Situation; $\uparrow$ leichter Anstieg bzw. leichte Verbesserung der Situation; $\downarrow \downarrow$ deutlicher Rückgang bzw. deutliche Verschlechterung der Situation; $\downarrow$ leichter Rückgang bzw. leichte Verschlechterung der Situation; o keine oder unwesentliche Veränderung; Ergebnis sehr unsicher; 个 Ergebnis unsicher, Tendenz steigend; $\downarrow$ Ergebnis unsicher, Tendenz fallend 


\subsection{Erläuterung zu den möglichen Auswirkungen auf die Indikatoren}

In diesem Abschnitt werden jeweils die einzelnen Indikatoren allgemein und kurz erläutert, bevor die möglichen Auswirkungen der verschiedenen Arbeitswelten detaillierter beschrieben werden.

\subsubsection{Produktivität}

Die Produktivität beschreibt grundsätzlich ein Input-Output-Verhältnis. Bei volkswirtschaftlichen Betrachtungen interessieren dabei meistens Arbeit und/ oder Kapital als Inputs und der erzielte Ertrag als Output bzw. Ergebnis. Je nach Ebene der Betrachtung sind die totale Produktivität (Bruttoinlandsprodukt im Verhältnis zur Einsatzmenge aller Faktoren) relevant oder partielle Produktivitäten, welche hauptsächlich für die eingesetzte Arbeit und das aufgewendete Kapital ermittelt werden. Im Folgenden wird vor allem auf die Arbeitsproduktivität fokussiert, die typischerweise als Ertrag pro eingesetzte Arbeitsstunde ausgewiesen wird. Sie hängt davon ab, wie stark Arbeitende mit physischem Kapital, Humankapital, natürlichen Ressourcen und technologischem Wissen ausgestattet sind und wie effektiv mit dieser Ausstattung umgegangen wird. Sie ist ausserdem wichtig für die Bestimmung von Löhnen und entscheidend für den Lebensstandard in einem Land.

Die Arbeit im Homeoffice, wie sie von Roland Müller praktiziert wird, hat gemäss einer aktuellen Studie des Bundesamtes für Energie (BFE, 2014) keinen statistisch signifikanten Einfluss auf die Produktivität der Arbeitnehmenden. Haribi (2001) konnte aber zeigen, dass es zumindest aufseiten der Arbeitgebenden Bedenken gibt bezüglich der Produktivität von Arbeitnehmenden, welche nicht vor Ort im Betrieb arbeiten. In seiner Befragung zu den Barrieren für die Einführung von Telearbeit äusserten sich 31 Prozent der Befragten in dieser Weise (S. 27). Durch eine Auswertung von 86 Fallstudien kommt der internationale Business-Center-Anbieter Regus ebenfalls zum Ergebnis, dass das Homeoffice nicht unbedingt der Arbeitsort mit der höchsten Produktivität ist (Regus, 2011). Arbeitnehmende, die zu Hause arbeiten, kämpfen demnach oft mit Problemen der Abgrenzung zwischen Privatem und der Arbeit und stellen in der Folge bezüglich ihrer Produktivität Probleme fest (ebd., S. 15 f.). Anstatt im Homeoffice zu arbeiten, bevorzugen sie die Arbeit an Orten in der Nähe ihres Wohnsitzes (ebd., S. 19). 
Gisin, Schulze, Knöpfli und Degenhardt (2013) untersuchen die subjektive Arbeitsproduktivität von erfahrenen Homeoffice-Arbeitenden und messen sie über das Gefühl, den beruflichen Anforderungen gewachsen zu sein, sowie der selbst wahrgenommenen Arbeitsleistung. Es zeigt sich, dass die beiden Grössen zunächst mit zunehmenden Stunden im Homeoffice ansteigen, dann jedoch zurückgehen und somit ein umgekehrter U-förmiger Zusammenhang zwischen der Intensität des Arbeitens im Homeoffice und der erlebten Arbeitsproduktivität besteht (S. 57). Der Produktivitätsverlust erfolgt bei zwei bis drei Tagen im Homeoffice (S. 57 ff.). Dies sollte zukünftig jedoch noch an grösseren Stichproben geprüft werden, um robustere Aussagen dazu machen zu können (S. 58).

Die Ergebnisse der analysierten Studien weisen nicht auf eine Produktivitätssteigerung durch die breite Anwendung von Homeoffice in Roland Müllers Arbeitswelt hin, sondern lassen eher auf einen leichten Rückgang der Arbeitsproduktivität schliessen.

Die Folgen der Vertrauensarbeitszeit und anderer flexibler Arbeitszeitmodelle auf die Produktivität von Beschäftigten, wie der leitenden und am Geschäftserfolg beteiligten Sandra Könitz, werden kontrovers diskutiert und sind nicht eindeutig. Aus Sicht der Unternehmen spricht für flexible Arbeitszeiten, dass sich Arbeitsanfall und Arbeitseinsatz besser abstimmen lassen und damit erhebliche Einsparungspotenziale realisiert werden können (Dorsemagen, Krause, Lehmann \& Pekruhl, 2012, S. 5). Unternehmen können also besser sicherstellen, dass der Arbeitseinsatz auch mit einer hohen Arbeitsproduktivität einhergeht. Aus älteren internationalen Studien ist bekannt, dass es einen Zusammenhang zwischen flexiblen Arbeitszeiten und positiven wirtschaftlichen Effekten gibt. Eine gross angelegte systematische Literaturanalyse von Janssen und Nachreiner (2004) kam zu diesem Resultat, ohne jedoch Genaueres über die kausalen Zusammenhänge herauszufinden. Mandl (Eurofound, 2015a) berichtet ebenfalls von Produktivitätsgewinnen in ihren EU-weiten Fallstudien aus Unternehmen, die mobil arbeitende Mitarbeitende beschäftigen, und belegt sie mit anekdotischer Evidenz (S. 80).

Spitzley (2007) geht davon aus, dass die Verdrängung der Anwesenheit (fixe Arbeitszeiten) durch das Erreichen von zuvor definierten Ergebnissen als Erfolgsmerkmal die Motivation und Leistungsfähigkeit der Mitarbeitenden steigert. Die erhöhte Selbstständigkeit, die eine Umstellung auf Vertrauensarbeitszeit oder andere flexible Arbeitszeitmodelle bringt, kann dabei als Grund für die erhöhte Motivation verstanden werden (ÖIF, 2011). Die Selbstständigkeit trägt aber gemäss Krieger et al. (2012) auch direkt positiv zur Leistung der Arbeitenden bei. Sie argumentieren, dass gemäss der Studien zu High Performance 
Work Systems die Leistungserbringung besser ausfällt, wenn sie in grosser Selbstständigkeit bzw. Autonomie erfolgt (S. 57). In der Schweiz ist das Mass an Autonomie am Arbeitsplatz generell sehr hoch und ermöglicht die Nutzung des produktiven Potenzials der Beschäftigten im europäischen Vergleich überdurchschnittlich gut (Krieger et al., 2012, S. 61 f.). Die Annahme, dass Vertrauensarbeitszeit und unternehmerische Spielräume die Produktivität der Arbeitnehmenden erhöht und dies auch in Zukunft so sein wird, ist also plausibel und wird durch die Ergebnisse aus BFE (2014) gestützt. Die Studie untersuchte die Effekte der räumlichen und zeitlichen Flexibilisierung auf die Produktivität in der Schweiz. Dabei wurde ein signifikanter, positiver Zusammenhang zwischen Arbeitsproduktivität und mobilem Arbeiten, jedoch kein signifikanter Zusammenhang mit Homeoffice gefunden. Unternehmen in modernen (wissensintensiven) Dienstleistungsbranchen, welche häufiger mobiles Arbeiten und Homeoffice anbieten, haben eine statistisch signifikant höhere Arbeitsproduktivität. Der Querschnittscharakter der in dieser Studie benutzten Daten lässt jedoch keine Aussagen über einzelne Branchen zu, eignet sich aber für gesamtwirtschaftliche, d.h. branchenübergreifende Betrachtungen (BFE, 2014).

Die verstärkte mobile Arbeitsweise in modernen Dienstleistungsbetrieben macht nicht nur einzelne Arbeitnehmende produktiver. Ein weiterer Grund für die höhere Produktivität in diesen Unternehmen könnte in der Verbreitung von Outsourcing-Praktiken liegen. So findet Schwörer (2012) einen statistisch signifikanten Zusammenhang zwischen dem Produktivitätswachstum bei Unternehmen und dem Vorhandensein von Offshoring, welches im Sample besonders oft bei multinationalen Unternehmen vorkommt. Olsen (2006) fasst für eine Vielzahl von Studien, die mit Daten aus den Jahren 1992 bis 2003 arbeiten, die Effekte des Outsourcings auf die Produktivität zusammen und identifiziert vor allem positive Zusammenhänge. Auch Balmer, Eichler, Wagner und Kunz (2013) suggerieren, dass „International Sourcing“ mit Produktivitätssteigerungen einhergehen und führen den Effekt auf verschiedene Transmissionsmechanismen zurück. Eine mögliche Folge der in der Personabeschreibung erwähnten Konflikte zwischen Mitarbeitenden, welche mehr Homeoffice fordern und ihren Vorgesetzten, die einen Kontrollverlust befürchten, könnte eine Zunahme der Outsourcingbemühungen sein, da hier die Überwachungsproblematik entfällt.

Obwohl der Zusammenhang zwischen räumlich flexibler Arbeit und höherer Produktivität robust scheint, könnte die erhöhte Arbeitsproduktivität auch nur ein Effekt der bei dieser Arbeitsform weniger konsequent erfassten Arbeitszeit sein. Bspw. wird in SECO (2012) erwähnt, dass es aus der Perspektive der Unternehmen und Organisationen ein starkes Argument für den Verzicht auf die Ar- 
beitszeiterfassung gibt, da durch eine Ausweitung nicht erfasster und nicht kompensierter Mehrarbeit die realen Stundenlöhne der Beschäftigten reduziert werden und damit einhergehend die Gesamtlohnkosten. Diese Praxis hat direkte Auswirkungen auf die Arbeitsproduktivität, da die Zeit im Nenner des Verhältnisses kleiner wird und somit die Produktivität steigt. 17 Prozent der Schweizer Arbeitnehmenden arbeiten, ohne dass ihre Arbeitszeit erfasst wird; der Anteil erhöht sich unter den hochqualifizierten und leitenden Angestellten auf 26 Prozent, während nur 11 Prozent der weisungsgebundenen Arbeitnehmenden mit niedrigen und mittleren Qualifikationen keine Arbeitszeiten erfassen (Dorsemagen, Krause, Lehmann \& Pekruhl, 2012).

Die Zahlen des Bundesamtes für Statistik zur Arbeitsproduktivität nach Branchen (BFS, 2014) zeigen, dass es im Zeitraum 1998-2012 gesamtwirtschaftlich ein mittleres jährliches Produktivitätswachstum von rund einem Prozent gegeben hat. Im gleichen Zeitraum hat sich die Flexibilisierung der Arbeit als Trend verfestigt. Branchen, die gemäss BFE (2014) relativ häufig räumlich-flexible Arbeitsformen anbieten, sind die IT-Branche, die Medienbranche, die Elektrotechnik, der Grosshandel, die technischen Unternehmensdienstleistungen und die Forschung und Entwicklung sowie die Pharmabranche (S. 17 f.). Sie zeigen jedoch nicht alle einheitlich ein Produktivitätswachstum. Während die Pharmabranche das Feld mit einem Produktivitätswachstum von 5,5 Prozent anführt, beträgt es für die anderen Branchen zum Teil erheblich weniger. So verzeichneten die Elektrotechnik mit 0,9 Prozent und der Grosshandel mit 3,7 Prozent Zunahmen, während die anderen Branchen durchschnittlich über den betrachteten Zeitraum Produktivitätsrückgänge zu verzeichnen hatten (IT-Branche - 2,3 Prozent, Forschung und Entwicklung - 2,4 Prozent, technische Unternehmensdienstleistung $-1,5$ Prozent, Medienbranche $-1,5$ Prozent). Ein weiterer Hinweis darauf, dass Produktivitätssteigerungen nicht zwangsläufig durch räumliche und zeitliche Flexibilisierung zustande kommen müssen, zeigt die Telekombranche. Sie weist im betrachteten Zeitraum ein mittleres jährliches Produktivitätswachstum von 5,3 Prozent auf. Die Unternehmen der Branche bieten jedoch relativ selten räumlich-flexible Arbeitsformen an (zwischen 15 und 20 Prozent bieten entweder Homeoffice oder mobiles Arbeiten an [BFE, 2014]).

Nicht unerwähnt bleiben sollte, dass flexible Arbeitszeitmodelle einen wichtigen Beitrag zum Erhalt der Produktivität leisten können. So können sie zur Verminderung von Produktivitätsverlusten beitragen, welche durch den Humankapitalverlust bei Pensionierungen auftreten, indem sie helfen, den Übergang in den Ruhestand gleitend zu gestalten (Mayrhuber \& Lutz, 2010). 
Grundsätzlich scheint es plausibel, dass die Arbeitsproduktivität in der Arbeitswelt von Sandra Könitz gesteigert wird, speziell in den wissensintensiven Dienstleistungsbranchen. Dies liegt besonders aus unternehmerischen Überlegungen nahe, empirisch kann dazu aber noch keine eindeutige Schlussfolgerung gezogen werden.

Blum (2002) identifiziert Chancen der Teilzeitarbeit insbesondere für Unternehmen mit schwankenden Auslastungen und solchen, die in einem besonders dynamischen Umfeld aktiv sind. Arbeitnehmende wie Ursula Meyerhans zeigen nämlich eine erhöhte Aufmerksamkeit und eine geringere Fehlerquote. Dadurch erhöhen sich die Motivation und die Loyalität zum Unternehmen, was zu einer sinkenden Fluktuation und einer erhöhten Produktivität führt.

Auf der Grundlage einer umfassenden Literaturanalyse folgert ÖIF (2011), dass aus betrieblicher Sicht grundsätzlich ein Zusammenhang zwischen Teilzeitbeschäftigung und Produktivitätssteigerung zu bestehen scheint, ohne dass jedoch der Wirkungszusammenhang klar ist. Die Annahme einer positiven Auswirkung der Teilzeitbeschäftigung auf die Produktivität ist durchaus plausibel, allerdings setzen produktivere Unternehmen eher flexible Massnahmen ein, während andere es sich nicht leisten können (ÖIF, 2011, S. 114 f.). Eine Analyse der EU-Statistik, welche die durchschnittlichen Arbeitszeiten pro Woche und die Produktivität pro Stunde in den EU-Mitgliedstaaten vergleicht, kommt auf Ebene der Länder zum gleichen Schluss. Kürzere Arbeitszeiten bewirkten gemäss dem Studienleiter einerseits eine höhere Arbeitsproduktivität, weil Leistungsfähigkeit und Konzentration höher seien und weniger Fehler gemacht würden, andererseits bestehe in Ländern mit höherer Produktivität mehr Spielraum für Teilzeit und insgesamt kürzere Arbeitszeiten (Hans Böckler Stiftung, 2007, S. 6). Die Aussagen wurden vor dem Hintergrund der Analyseresultate gemacht, welche zeigen, dass EU-Länder mit kurzen Arbeitszeiten eine hohe Arbeitsproduktivität haben (ebd., S. 6).

Die betriebliche Sicht der Teilzeitarbeit wurde auch in einer Befragung des deutschen Instituts für Arbeitsmarkt- und Berufsforschung (IAB) beleuchtet. So stuften 35 Prozent der Betriebe mit 43 Prozent der Beschäftigten die Produktivität der Teilzeitmitarbeitenden (Arbeitsproduktivität je Stunde) im Vergleich zu Vollzeitmitarbeitenden besser ein, während nur rund 7 Prozent der Betriebe mit 6 Prozent der Beschäftigten die Produktivität der Teilzeitmitarbeitenden schlechter einstuften als bei Vollzeitmitarbeitenden (IAB 2006, S. 3).

Der Zusammenhang zwischen der Befristung von Arbeitsverträgen, wie sie für Ursula Meyerhans zur Normalität geworden ist, und der Produktivität der Arbeitnehmenden ist aus der Literatur nicht eindeutig abzuschätzen (Nielen \& 
Schiersch, 2012). Es existieren zahlreiche Mechanismen, die in der Theorie eine Zu- oder Abnahme hervorrufen, während die meisten empirischen Untersuchungen einen negativen Effekt auf die Produktivität finden (Cappellari, Dell'Aringa \& Leonardi, 2012), wobei nicht immer klar ist, auf welche Art von Produktivität sich die Forscher beziehen. In einer mittlerweile schon älteren Studie, die auf Zahlen der Schweizer Arbeitskräfte Erhebung (SAKE) des Bundesamtes für Statistik basiert (Engellandt \& Riphahn, 2005), wurde versucht, den Einfluss von befristeten Arbeitsverträgen auf das Verhalten der Arbeitnehmenden zu berechnen. Gemäss den Resultaten haben befristet Angestellte im Vergleich zu unbefristet Angestellten, unabhängig vom Geschlecht, durchschnittlich eine 60 Prozent höhere Neigung, unbezahlte Überstunden zu leisten (Engellandt \& Riphahn, 2005, S. 297). Befristete Verträge, die eine Aufstiegsmöglichkeit bieten, gehen mit noch mehr Leistungsbereitschaft und somit einer höheren Arbeitsproduktivität einher als der Durchschnitt (ebd., S. 293). Dolado und Stucchi (2008, S. 18) kommen durch die Auswertung italienischer Daten zum ähnlichen Schluss, dass die Aussicht auf eine Umwandlung von befristeten zu unbefristeten Verträgen die Produktivität erhöht. Dieser Punkt wird auch von Nielen und Schiersch (2012) genannt, allerdings aus nicht empirischer Sicht. Sie führen aus, dass befristete Verträge grundsätzlich zwei Wirkungen auf die Arbeitsproduktivität auf betrieblicher Ebene haben können. Einerseits kann sie durch die höhere Flexibilität, die befristete Verträge bieten, gesteigert werden. Andererseits kann sie sinken, wenn man davon ausgeht, dass betriebsspezifisches Know-how, über das befristete Angestellte typischerweise weniger verfügen, sich eigentlich günstig auf die Arbeitsproduktivität auswirken sollte. Betreffend den Nettoeffekt der Befristung auf die Produktivität kommen Studien aus Südeuropa und Deutschland jedoch nicht zum gleichen Ergebnis wie Engellandt \& Riphan (2005). Die empirischen Ergebnisse von Dolado und Stucchi (2008), die auf spanischen Daten des zweiten Sektors beruhen, zeigen ebenfalls einen negativen Einfluss der Anzahl Arbeitnehmende mit befristeten Arbeitsverträgen auf die Produktivität der Unternehmen. Nachdem z.B. in den frühen 1990er-Jahren in Spanien Arbeitsmarktreformen durchgeführt wurden, welche die temporäre Beschäftigung erleichtert haben, verschlechterte sich dort die Produktivität (Dolado \& Stucchi, 2008, S. 18). Auch die empirische Untersuchung der Wirkung der Erleichterung von befristeten Arbeitsverträgen in Italien von Cappellari, Dell'Aringa und Leonardi (2012) weist eine Verringerung der Produktivität der Arbeitnehmenden aus. Für den zweiten Sektor in Deutschland können Nielen und Schiersch (2012, S. 16) jedoch keinen signifikanten Zusammenhang zwischen dem Anteil der befristet Beschäftigten und der Arbeitsproduktivität nachweisen. 
Zusätzlich zu den Auswirkungen der Teilzeitarbeit und der Befristung der Arbeitsverträge auf die Produktivität muss in Ursula Meyerhans' Arbeitswelt mit Effekten der Arbeitsplatzunsicherheit sowie der Fragmentierung der Arbeitsanforderungen gerechnet werden, die sich möglicherweise über die Arbeitsmotivation manifestieren.

Aufgrund der zu Beginn des Abschnitts beschriebenen positiven Effekte der Teilzeitarbeit auf die Produktivität sowie der empirischen Ergebnisse der Befristung aus der Schweiz und der theoretischen Überlegungen dazu, wird davon ausgegangen, dass sich die Arbeitsproduktivität unter den Bedingungen der Arbeitswelt von Ursula Meyerhans nur unwesentlich verändert.

Im internationalen Vergleich zeigt sich, dass eine hohe Selbstständigenquote, welche durch die Zunahme der Solo-Selbstständigen vom Typus eines Noah Schmid entstehen kann, eher mit einer geringen Leistungsfähigkeit einhergeht, wie z.B. in den Ländern Süd- und Osteuropas (Brenke, 2013, S. 5). SalasFumás und Sanches-Asin (2013) zeigen z.B., dass in den OECD-Ländern zwischen 1970 und 2007 der Anteil an Selbstständigen und die Produktivität negativ korreliert sind (S. 2356). Darüber hinaus folgern Van Praag und Versloot (2008) aus ihrer Literaturanalyse, dass Einzelunternehmende bezüglich Produktivität grösseren Unternehmen hinterherhinken (S. 120). Auch Ortega und Marchante (2010) zeigen modellhaft, dass in Spanien die Steigerung der Angestelltenquote auf Kosten der Selbstständigenquote zwischen 1987 und 2000 ein grösseres Produktivitätswachstum gebracht hätte. Pro Prozentpunkt Erhöhung der Angestelltenquote auf Kosten der Selbstständigenquote hätte demnach eine Erhöhung der gesamtwirtschaftlichen Produktivität um 0,4 Prozentpunkte zur Folge gehabt (ebd., S. 210). Dabei muss wohl zwischen Solo-Selbstständigen mit tiefer Wertschöpfung und geringeren Wachstumsmöglichkeiten und Selbstständigen mit Angestellten unterschieden werden (Broughton \& Ussher, 2013, S. 5). Das gilt insbesondere für Einpersonen-Betriebe, deren Motivation für die Selbstständigkeit nicht aus dem Verfolgen einer von innen wahrgenommenen Chance resultiert, sondern rein aus der Notwendigkeit, einer Erwerbstätigkeit nachgehen zu müssen (ebd., S. 6). Diese zweite Kategorie wird demnach auch als „unfreiwillige Selbstständigkeit“ bezeichnet und unterscheidet sich bezüglich Motivation, Grad der Eigenständigkeit und Ehrgeiz wesentlich von der ersten (Wennekers, van Stela, Carree \& Thurik, 2010). Ihr Anteil an der gesamten Selbstständigkeit bereitet Schwierigkeiten in der empirischen Messung, wird aber in OECD-Ländern als nicht vernachlässigbare Minderheit eingeschätzt (ebd., S. 27). 
Da unter den Bedingungen von Noah Schmids Arbeitswelt durch die Tertiärisierung mit einem Wachstum der neuen Selbstständigkeit gerechnet werden kann, muss von einer Verschlechterung der gesamtwirtschaftlichen Produktivität, zumindest von einem Wachstumsrückgang, ausgegangen werden.

Andrea Burri-Lötscher, die wegen ihrer Halbtagsstelle nur ein geringes stabiles Einkommen erzielt und zusätzlich für verschiedene Auftraggeber arbeiten muss, leidet unter ihren geringen Qualifikationen. In ihrer Arbeitswelt bringt die Polarisierung der Arbeitskräfte einerseits eine Verbesserung der Arbeitsproduktivität von Hochqualifizierten und gleichzeitig eine Stagnation oder Verschlechterung bei den Geringqualifizierten und dem Teil der Hochqualifizierten, die in die neue Selbstständigkeit oder in prekäre Arbeitsverhältnisse gedrängt werden. Dank der besseren Vermeidung von Schwarzarbeit durch Dienstleistungsschecks, s.g. Vouchers, kann ein leicht positiver Effekt auf die Arbeitsproduktivität auf gesamtwirtschaftlichem Niveau erwartet werden. Viele Tätigkeiten, die früher schwarz bezahlt wurden, werden dank der ordentlichen Abwicklung durch die Voucher-Agenturen über die Sozialversicherungen nun im BIP erfasst, auch wenn das Ausmass des Effekts der Vouchersysteme auf die Schattenwirtschaft noch kontrovers diskutiert wird (Marx \& Vandelannoote, 2014, S. 13). Allerdings ist wegen der gleichzeitigen Zunahme der Erwerbstätigen bzw. eingesetzten Arbeitsstunden und den geringen Löhnen der Zuwachs sehr gering.

\subsubsection{Erwerbseinkommen}

Das Erwerbseinkommen, das auch als Arbeitseinkommen bezeichnet wird, setzt sich aus Einnahmen in Form von Geld, Natural- oder Dienstleistungen zusammen, die einer Person aus der Ausübung ihrer beruflichen Tätigkeit in Form eines Lohns oder eines Ertrags aus der Selbstständigkeit entstehen. Der Schwerpunkt der Betrachtung in diesem Kapitel liegt dabei beim Einkommen in Geldform.

Nicht zum Erwerbseinkommen gehören Einnahmen aus anderen Quellen wie z.B. dem Vermögen, der Sozialhilfe oder Transferleistungen.

Aus der Literatur zu Homeoffice bzw. räumlich flexibilisierter Arbeit sind keine Ergebnisse bekannt, welche aufgrund der Arbeitsform eine Veränderung des Erwerbseinkommens beobachten, wie es etwa Kadermitglied Roland Müller erzielt.

Als Arbeitskraftunternehmerin und höheres Kadermitglied darf Sandra Könitz in ihrer Arbeitswelt mit einem steigenden Einkommen rechnen. Die grössere 
Verbreitung der Erfolgsbeteiligung bedeutet zwar, dass die Erwerbseinkommen der mittleren und oberen Kader stärker konjunkturellen Schwankungen ausgesetzt sind. Tendenziell sollten sie jedoch über längere Zeit aufgrund der verbesserten Leistungsfähigkeit steigen. Die Erwerbseinkommen der Mitarbeitenden, welche keine bzw. untere Kaderpositionen besetzen, bleiben hingegen von den Veränderungen, welche eine Umstrukturierung der Arbeitswelt erfordern, weitgehend unberührt. Dank der Verbesserung der Leistungsfähigkeit der Unternehmen durch Angestellte wie Sandra Könitz gibt es jedoch mehr Potenzial für Lohnerhöhungen. Mitarbeitende und Lernende ohne Kaderfunktion machen gemäss den Zahlen des Bundesamtes für Statistik zur beruflichen Stellung (BFS, 2015a) rund 60 Prozent der unselbstständigen Erwerbspersonen aus, 40 Prozent der Arbeitnehmenden halten eine Vorgesetztenposition inne. Ein Viertel davon ist in der Unternehmensleitung, während über die Verteilung auf untere und mittlere Kader nichts Genaues bekannt ist, was eine Quantifizierung der gesamtwirtschaftlichen Einkommenseffekte erschwert.

Die Höhe der Erwerbseinkommen von Arbeitskräften wie Ursula Meyerhans wird beeinflusst von einer Kombination aus Faktoren, welche aus den Bedingungen für befristete Arbeitsverträge, Crowdworking als Solo-Selbstständigkeit und Teilzeitarbeit resultieren. Diese drei Beschäftigungsformen sind atypisch und werden oft mit prekären Arbeitsbedingungen und Löhnen in Verbindung gebracht. Streuli (2002, S. 10) identifiziert für Schweizer Arbeitsplätze drei Variablen, die tendenziell mit prekären Bedingungen korrelieren, insbesondere wenn sie zusammen auftreten: Teilzeit, Befristung und flexible Arbeitszeiten. Gleichzeitig bei mehreren Arbeitgebenden in Teilzeitbeschäftigung angestellt zu sein, ist daher besonders mit dem Status der Working Poor verbunden (ebd., S. 66). Nur ein kleiner Teil der gesamten atypischen Beschäftigung erfüllt jedoch die Bedingungen für prekäre Verhältnisse (Walker, Marti \& Bertschy, 2008). Stark überdurchschnittlich gefährdet, unter solchen Bedingungen arbeiten zu müssen, sind Solo-Selbstständige (Selbstständige ohne Angestellte) (Streuli, 2002, S. 10). Auch wenn atypische Beschäftigungsformen wegen der erzielbaren Einkommenshöhe keine eigenständige wirtschaftliche Absicherung bilden und als prekär eingestuft werden können, gilt es festzuhalten, dass sie eine wichtige zusätzliche Einkommensquelle darstellen (Lutz und Mayrhofer, 2010), auf die die Haushalte nicht verzichten können.

Teilzeitarbeit ist eine der Arbeitsformen, die in der Schweiz häufig durch tiefe Löhne gekennzeichnet ist. Ausserdem kann sie "mit ungesicherten Arbeitsverhältnissen, schlechteren sozialen Absicherungen (z.B. bei der Pensionskasse) oder geringeren Weiterbildungsmöglichkeiten und Karrierechancen einhergehen" (BFS, 2013, S. 12 f.) Eine Untersuchung von Hirsch (2004) mit deutschen 
Daten hat gezeigt, dass es eine sogenannte „Part-time Wage Penalty“ gibt, die für Frauen etwas geringer ausfällt als für Männer, insgesamt aber eher klein ist. Diese "Teilzeitlohn-Strafe“ beschreibt den Umstand, dass Teilzeitangestellte auch unter Berücksichtigung verschiedener Faktoren bezüglich des Humankapitals und des Arbeitsplatzes weniger verdienen als Vollzeitangestellte. Eine Analyse des Statistischen Amts des Kantons Zürich (Statistik Zürich, 2011) bestätigt, dass es diese Strafe auch in der Schweiz gibt. So liegt die Differenz bei im Kanton Zürich arbeitenden Frauen und Männern bei 6 bzw. 16 Prozent (S. 8). Aus anderen Kantonen liegen keine Analysen zur "Teilzeitlohn-Strafe“ vor.

Zusätzlich zu den Unterschieden beim Lohn können Teilzeitangestellte weitere Nachteile bezüglich freiwillig erbrachter Leistungen und Sonderzahlungen der Arbeitgebenden haben, welche jedoch aufgrund der heterogenen Situation kaum quantifizierbar sind (Hirsch, 2004). Allgemein werden die Unterschiede im Lohnniveau und bei den Zusatzleistungen dadurch erklärt, dass es ein Überangebot an Personen gibt, die eine Präferenz für Teilzeitarbeit haben und einen tieferen Lohn in Kauf nehmen.

Wie Leimeister und Zogaj (2013) in ihrer Literaturstudie zu Crowdsourcing feststellen, spielen intrinsische Faktoren wie der soziale Austausch, die Möglichkeit zur Erweiterung der individuellen Fähigkeiten und die Freude an der (Crowd-) Arbeit eine wesentliche Rolle bei den Arbeitsanreizen. Die monetäre Entlohnung ist jedoch, wie im Fall von Ursula Meyerhans, der primäre Anreizfaktor für Crowdsourcees. Die erzielbaren Entgelte variieren dabei stark mit der Arbeitsform und der Art der Aufgaben, während manche Aufgaben (zumeist Mikroaufgaben) mit wenigen Eurocents entlohnt werden, gibt es auch zumeist wettbewerbsbasierte Crowdsourcing-Aufträge, im Rahmen welcher Preisgelder von bis zu 100'000 Euro bezahlt werden (Leimeister und Zogaj, 2013). Mit Mikroaufgaben, welche kein spezialisiertes Fachwissen benötigen, lässt sich auch gemäss Ipeirotis (2010b) nur ein geringes Einkommen erzielen. Er schätzt einen Stundenlohn von zwei US-Dollar. In seiner Analyse die Nutzenden der Crowdworking-Plattform „Mechanical Turk“, welche vor allem Mikroaufgaben vermittelt, kommt Ipeirotis (2010b) zum Schluss, dass Auftragnehmende, die ihr Einkommen hauptsächlich nicht mit Crowdsourcing for paid Work erarbeiten, weniger als einen Tag damit verbringen. Wenn ein Einkommen über 1000 USDollar erzielt werden soll, müssen die Crowdworkenden jedoch erheblich mehr Zeit investieren, und nur knapp 15 Prozent der untersuchten US-amerikanischen Auftragnehmenden geben an, dass Crowdsourcing for paid Work die Hauptquelle für ihr Einkommen darstellt (Ipeirotis, 2010b). Für europäische Crowdworkende hält Mandl (2015) fest, dass die Entlohnung eher bescheiden 
ausfällt. Beispiele von verschiedenen Plattformen aus Deutschland, der Tschechischen Republik und Portugal zeigen, dass je nach Qualifikationsniveau weniger als zehn Euro pro Stunde bezahlt wird (S. 115). Ein Grund für die tiefe Entlöhnung sehen Expertinnen und Experten im intensiven Wettbewerb unter den Auftragnehmenden (S. 115). Zusätzlich müssen selbstständige Crowdworkende im Vergleich zur Lohnarbeit auf wichtige und einkommenssichernde Schutzrechte verzichten. So gibt es bspw. keine staatliche Arbeitslosen- und Rentenversicherung, keine Lohnfortzahlungspflichten der Arbeitgebenden im Krankheitsfall, kein Lohnanspruch während den Ferien usw. Streuli (2002) berechnet in einer älteren Studie für das Bundesamt für Statistik, dass SoloSelbstständige stark überdurchschnittlich Working-Poor-gefährdet sind (S. 66) und maximal ein Einkommen von 60 Prozent des Medianeinkommens erzielen.

Gemäss Henneberger, Sousa-Poza und Ziegler (2004) sind die Löhne von befristet Beschäftigten in der Schweiz nicht per se geringer als diejenigen von unbefristet angestellten Personen (S. 43). Das Arbeitslosigkeitsrisiko ist bei dauerhaft Angestellten jedoch tiefer (S. 44). Befristet angestellte Personen sind also häufiger arbeitslos und müssen Einkommenseinbussen dadurch erleiden, dass die Arbeitslosenversicherung für die Zeit der Arbeitslosigkeit Personen mit Unterhaltspflicht, Invalidenrente oder tiefem Einkommen nur 70 bzw. 80 Prozent des versicherten Verdienstes bezahlt, welcher aus den letzten zwölf Beitragsmonaten berechnet wird. Hinzu kommt eine Obergrenze für den versicherbaren Lohn. Wenn sich befristete Beschäftigung und Phasen der Arbeitslosigkeit abwechseln, resultiert somit ein tieferes Einkommen als bei unbefristeten Angestellten, welche die gleichen Qualifikationen haben.

Zusammenfassend kann für die Arbeitswelt von Ursula Meyerhans festgehalten werden, dass das häufigere Vorkommen von Teilzeitarbeit, Befristung und Crowdwork bzw. Solo-Selbstständigkeit einen durchschnittlich eher negativen Effekt auf das Erwerbseinkommen hätte.

Der gesamtwirtschaftliche Effekt einer höheren Selbstständigenquote, wie sie in der Arbeitswelt des selbstständigen Softwareentwicklers Noah Schmid zu beobachten ist, auf die Erwerbseinkommen hängt stark davon ab, wie hoch die Einkommen von Selbstständigen im Vergleich zu denjenigen von Angestellten ausfallen. Gemäss dem Bundesamt für Statistik (BFS, 2012, S. 38-39) sind heutzutage Selbstständigerwerbstätige in 7,9 Prozent der Fälle arm, während das nur für 2,8 Prozent der Angestellten der Fall ist. Solo-Selbstständige sind mit 9,9 Prozent ausserdem doppelt so häufig von Armut betroffen wie Selbstständige mit Angestellten (ebd., S. 39). Da die Erfassung der Einkommen von Selbstständigen gemäss dem Bundesamt jedoch mit Problemen verbunden ist, 
müssen die Ergebnisse mit Vorsicht interpretiert werden (BFS, 2012, S. 39). Dazu geben auch die Ergebnisse von Åstebro und Chen (2013) Anlass. Einerseits finden sie in einer umfangreichen, internationalen Literaturstudie zahlreiche Studien, die auf Daten u.a. aus der OECD, Frankreich, Grossbritannien, Kanada und Spanien beruhen und zum Resultat kommen, dass Angestellte mehr verdienen als selbstständige Unternehmerinnen und Unternehmer, die beobachtbar ähnliche berufliche Eigenschaften haben. Andererseits finden die Autoren aber nicht weniger gegenteilige Evidenz.

Brenke $(2013$, S. 3) hat festgestellt, dass in Deutschland ein Teil der SoloSelbstständigen hohe Einkünfte erzielt, dass aber ihr Medianeinkommen unter demjenigen der Angestellten liegt (siehe auch Andersson, 2008, S. 221 f.; Siebecke \& Lisakowski, 2010, S. 123). Ein Grund dafür, dass Solo-Selbstständige teilweise relativ tiefe Einkünfte akzeptieren, könnte auch darin liegen, dass nicht alle auf das eigene Erwerbseinkommen angewiesen sind. Im Jahr 2009 waren es aber in Deutschland 88 Prozent (Brenke, 2013, S. 14), und es kann angenommen werden, dass dieser Anteil in Noah Schmids Arbeitswelt nicht wesentlich kleiner ist.

Fossen und Büttner (2013) untersuchen Bildungsrenditen von Selbstständigen und unterscheiden dabei zwischen den Kategorien, die bereits in Abschnitt 4.3.1 beschrieben worden sind; einerseits also Selbstständige, die aus der Wahrnehmung einer Opportunität selbstständig werden, und andererseits solche, die dies aus purer Notwendigkeit, einer Erwerbsarbeit nachgehen zu müssen, tun. Sie benutzen dazu Daten des deutschen Sozio-ökonomischen Panels (SOEP), welches u.a. Einkommen und Bildung repräsentativ für die deutsche Bevölkerung erhebt. Das Resultat zeigt klare Unterschiede bei der Bildungsrendite, welche über das Bruttoeinkommen gemessen wurde. So liegt die Rendite eines zusätzlichen Ausbildungsjahres bei den opportunitätsgetriebenen Unternehmern gleich hoch wie bei Angestellten bei 8,8 Prozent, während sie bei notwendigkeitsgetriebenen Unternehmern rund drei Prozentpunkte tiefer liegt (S. 80).

Bei der Beurteilung, welchen Effekt die Bedingungen der Arbeitswelt von Noah Schmid auf die Einkommen haben, spielt also die Unterscheidung, aus welchem Motiv die Selbstständigkeit gewählt wurde, eine bedeutende Rolle. Die Daten des Global Entrepreneurship Monitors (GEM, 2014) zeigen für die Schweiz eine tiefe Quote notwendigkeitsgetriebener Unternehmender. Im Durchschnitt betrug sie von 2002 bis 2014 12,2 Prozent der Neugründungen (GEM, 2014) und zeigt somit einen für wirtschaftlich stark entwickelte Länder typischen geringeren Wert als die opportunitätsgetriebene Neugründungsquote 
(GEM, 2014, S. 21). Zwar liegt Letztere in der Schweiz etwas höher als im Durchschnitt der innovationsbasierten Volkswirtschaften (GEM, 2014, S. 21), die Selbstständigenquote liegt aber hier tiefer als im umliegenden Ausland bzw. der Eurozone. Dies legt den Schluss nahe, dass Menschen, die in anderen Ländern aus Notwendigkeit die Selbstständigkeit ergreifen, dies unter Schweizer Bedingungen noch nicht tun müssen und eine Anstellung finden. Unter den Bedingungen der Arbeitswelt von Noah Schmid dürfte sich diese Quote jedoch erhöhen, insbesondere auch dadurch, dass die Unternehmen in einem tertiärisierten Umfeld mehr Outsourcing betreiben und weniger Angestellte haben. Da die Einkommen von notwendigkeitsgetriebenen Selbstständigen und SoloSelbstständigen tiefer liegen als bei Angestellten und freiwillig Selbstständigen (Fossen \& Büttner, 2013), kann davon ausgegangen werden, dass der gesamtwirtschaftliche Effekt dieser Arbeitswelt auf die Einkommenshöhe negativ ist. Hinzu kommt, dass man aufgrund von Beobachtungen von deutschen Startups, welche besonders stark auf flexible Arbeitsformen zurückgreifen und als wichtige Treiber für die Veränderungen in der Arbeitswelt gelten, weiss, dass solche Unternehmen statistisch signifikant tiefere Medianlöhne zahlen als Unternehmen, die bereits seit längerer Zeit im Markt aktiv sind (Koch \& Späth, 2009).

Die Erwerbseinkommen, die sich in Andrea Burri-Lötschers Arbeitswelt erzielen lassen, hängen, wie auch heute bereits, massgeblich vom Bildungsniveau ab. Dieses hat einen ausschlaggebenden Einfluss auf die Erwerbsbeteiligung und die Möglichkeiten zur Erzielung von Einkommen (BFS, 2012, S. 6). Das zeigt sich beispielsweise an der Armutsgefährdung in der Schweiz, welche im Jahr 2013 bei Personen bis Sekundarstufe I bei 16,3 Prozent, bis Sekundarstufe II bei 8,4 Prozent und bis Tertiärstufe bei 3,7 Prozent (BFS, 2015d) lag.

Die Polarisierung der Arbeitskräfte wird sich möglicherweise deutlicher als heute in zwei verschiedenen Lohnniveaus äussern, welche innerhalb von Unternehmen, aber auch gesamtwirtschaftlich wahrnehmbar sein werden. Während ein Kern von Angestellten und hochqualifizierte Selbstständige mit guter Bezahlung, Zulagen und Beschäftigungssicherheit den oberen Bereich bilden, sind geringqualifizierte Selbstständige und die in Unternehmen peripher, befristet und Teilzeitbeschäftigten im unteren Bereich.

Die Arbeitsformen, die in dieser Arbeitswelt eine wichtigere Rolle spielen, stehen bereits in der Gegenwart mit prekären bzw. nicht existenzsichernden Einkommen in Verbindung und dürften so auch in Zukunft und am unteren Pol der Einkommensverteilung angesiedelt sein. So bieten befristete Arbeitsverträge, kleine Unternehmen und Solo-Selbstständigkeit im Vergleich zu Normalarbeits- 
verhältnissen Arbeitsbedingungen, die häufig entweder eindeutig oder tendenziell als unsicher gelten und die Beschäftigten eher einem Armutsrisiko aussetzen (BFS, 2012, S. 6). Über die Hälfte aller Erwerbstätigen (50,4 Prozent) ist in einem Arbeitsverhältnis mit Wochenendarbeit, Nachtarbeit und/oder arbeitet auf Abruf und weist mit 3,4 Prozent eine höhere Armutsquote auf als Personen ohne atypische Arbeitsbedingungen, welche in 2,1 Prozent der Fälle von Armut betroffen sind (ebd. S. 39). Das gilt nicht nur für schweizerische Verhältnisse, sondern kann generell für bestimmte Arbeitsformen festgestellt werden. Bspw. sind gemäss einer Literaturstudie von Keller und Seifert (2011a) in Deutschland besonders geringfügig Beschäftigte und Temporärarbeitende beim Lohn benachteiligt, aber auch bei allen anderen atypischen Arbeitsformen lassen sich Tendenzen zu geringen Erwerbseinkommen feststellen (S. 141). In diese Richtung zeigen auch Hinweise journalistischer Art. Ein Artikel in der Süddeutschen Zeitung (Beitzer, 2015) erwähnt Architekten, die für monatlich 1500 Euro brutto Vollzeit arbeiten, selbstständige Grafikdesignerinnen und Grafikdesigner mit monatlichen Gewinnen von 200 Euro und Hochschulabsolventinnen und -absolventen, die für ein Praktikumsgehalt von 500 Euro in Werbeagenturen und Verlagen Kampagnen leiten.

Wie die Autoren einer US-amerikanischen Studie (Addison \& Surfield, 2007) argumentieren, gibt es durchaus Gründe auf der Seite des Arbeitsangebots für die im Vergleich zum Normalarbeitsverhältnis geringeren Einkommen, die atypischen Arbeitsformen anhaften. Bspw. sind Erwerbstätige, die stark in ausserberuflichen Lebensbereichen involviert sind, oder ältere Arbeitnehmende, die ihre normale Karriere bereits beendet haben, bereit, ein geringeres Einkommen zu akzeptieren, wenn die Arbeitsform eine grössere Flexibilität als ein Normalarbeitsverhältnis bietet (S. 1038). Die Einkommensunterschiede lassen sich aber dadurch nicht vollständig erklären (S. 1038) und ein Teil der Erklärung muss bei den Unternehmen sowie den Konsumentinnen und Konsumenten von Dienstleistungen liegen.

Auch wenn viele atypische Arbeitsformen mit tieferen Einkommen in Verbindung stehen, übernehmen sie dank der Flexibilität, die sie bieten, eine wichtige Funktion. Die Möglichkeit eines Zweitjobs kann für die einen lebenswichtig sein, um das geringe Einkommen aufzubessern, und für die anderen ein Nebenverdienst, der sogar das Haupteinkommen übersteigen kann (Bosch, Brügelmann, Breyer \& Holst, 2014, S. 236). Trotz dieses Potenzials muss jedoch in Andrea Burri-Lötschers Arbeitswelt gesamtwirtschaftlich von tieferen Erwerbseinkommen ausgegangen werden, als dies heute der Fall ist. 


\subsubsection{Kollektiver Organisationsgrad der Beschäftigten}

Typischerweise wird unter dieser Messgrösse der gewerkschaftliche Organisationsgrad verstanden. Da in einigen Arbeitswelten auch andere kollektive Akteurinnen und Akteure wichtig sind, wurde ein allgemeinerer Begriff gewählt. So wird z.B. auch auf die Konsequenzen für die Organisation von Selbstständigen eingegangen, sei es, weil sie als Scheinselbstständige eher Arbeitnehmende sind oder weil sie als Auftrags- und Werkvertragsnehmer an fairen Bedingungen interessiert sind.

In den Arbeitswelten der beiden Kadermitglieder Roland Müller und Sandra Könitz werden sich die Organisationsgrade der Beschäftigten im Vergleich zu heute kaum verändern. Aus dem europäischen Umfeld ist bekannt, dass die Mitarbeitendenvertretung nicht unbedingt unter der bisherigen Flexibilisierung der Arbeitsverhältnisse gelitten hat. Mehr als die Hälfte der entsprechend engagierten Angestellten darf die dafür nötigen Aufgaben während der Arbeitszeit erledigen, während nur rund 11 Prozent der Befragten dafür entweder keine oder zu wenig Arbeitszeit zur Verfügung gestellt erhält (Eurofound, 2015b, S. 101). Zwei Drittel der in der Studie erfassten Unternehmen setzen flexible Arbeitszeiten ein und 69 Prozent haben Teilzeitangestellte (Eurofound, 2015b, S. $69 \mathrm{ff}$.). Für die Schweiz gibt es keine vergleichbare Erhebung, es ist lediglich aus Krieger, Pekruhl, Lehmann und Graf (2012, S. 120) bekannt, dass rund 45 Prozent der abhängig Beschäftigten eine offizielle Mitarbeitendenvertretung in ihrem Betrieb haben; frühere Vergleichswerte fehlen.

Da die bereits stattgefundene Flexibilisierung der Arbeitsverhältnisse mit abnehmenden Mitgliederzahlen der Gewerkschaften korreliert, kann nicht angenommen werden, dass sich die Arbeitnehmenden im Durchschnitt durch die Veränderung der Arbeitswelt stärker veranlasst sehen, sich kollektiv zu organisieren. Auch die Tatsache, dass mehr Kaderpersonen wie Sandra Könitz erfolgsbeteiligt sind, könnte das Bedürfnis nach weniger unternehmerischen Sachzwängen, welche kollektive Mitarbeitervertretungen mit sich bringen können, breiter in der Bevölkerung verankern. Somit würde der kollektive Organisationsgrad der Beschäftigten in den Arbeitswelten der zwei Kaderangestellten Roland Müller und Sandra Könitz eher abnehmen.

Während für Ursula Meyerhans als fest angestellte Teilzeitmitarbeitende grundsätzlich die gleichen Anreize und Möglichkeiten wie für befristet Angestellte bestehen, sich kollektiv zu organisieren und ihre Interessen gegenüber den Arbeitgebenden vertreten zu lassen, sieht ihre Situation als Crowdworkerin anders aus. Felstiner (2011) zählt einige der Probleme der Crowdworkenden im Zusammenhang mit dem Aufbau einer Gewerkschaft oder ähnlichen Interes- 
senvertretung auf, insbesondere wenn diese global tätig sein wollen. So stehen die traditionellen Verhandlungspunkte wie Löhne, Arbeitsstunden und -bedingungen im Widerspruch zum Wesen des Crowdsourcings als offene Ausschreibung. Die Anzahl und Verschiedenheit der Crowdsourcing-Plattformen sind ausserdem zu gross, um verhandelbare Bedingungen durchzusetzen. Im Weiteren ist der traditionell physische Zugang von Gewerkschafterinnen und Gewerkschaftern zu ihren Mitgliedern am Arbeitsort kaum mehr möglich (Felstiner, 2011, S. 185).

Die deutsche Gewerkschaft IG Metall (Industriegewerkschaft Metall) hat diese Probleme erkannt und mit „FairCrowdWork Watch" eine Internetplattform für verschiedene Bedürfnisse von Crowdworkenden geschaffen. „FairCrowdWork Watch" erlaubt es z.B., Bewertungen zu den AGBs von CrowdsourcingPlattformen abzugeben und zu konsultieren, Arbeitsbedingungen zu bewerten und die Bezahlung zu vergleichen. Als weiteres Beispiel, sich kollektiv für bessere Arbeitsbedingungen einzusetzen, kann das Netzwerk „Turkopticon“ herangezogen werden. Es erlaubt über ein Browser-Plug-In Auftraggebende auf der Plattform Mechanical Turk hinsichtlich ihrer Zahlungszuverlässigkeit und -grosszügigkeit, Fairness bei der Bewertung der Arbeitsleistung sowie des Kommunikationsverhaltens zu bewerten (Hodson, 2013, o. S.). Durch die niedrigen Barrieren für eine Beteiligung an solchen Netzwerken könnte der kollektive Organisationsgrad von Crowdworkenden im Vergleich zu traditionellen Arbeitnehmenden sogar zunehmen. Über die Verhandlungsmacht solcher Netzwerke ist jedoch im Moment empirisch nichts belegt. Grundsätzlich sind virtuelle Umfelder, worin sich auch Crowdsourcing-Plattformen bewegen, von der Präsenz und Mitwirkung einer engagierten Gemeinschaft abhängig und empfänglich für die Anliegen einer gut organisierten, hauptsächlich online kommunizierenden Gruppe (Felstiner, 2011, S. 203). Crowdworkende könnten z.B. speziell missbräuchliche Auftraggebende boykottieren oder selbst auferlegte Standards übernehmen, welche es verbieten, Aufträge unterhalb einer bestimmten Höhe der Entlohnung anzunehmen (ebd., S. 203).

Trotz des Potenzials, welches Netzwerke von selbstständigen Auftragnehmenden bieten, muss aufgrund des seit Langem abnehmenden gewerkschaftlichen Organisationsgrades der Arbeitnehmenden in der Schweiz und der Schwierigkeiten, welche beim Organisieren von kollektivem Handeln bestehen (dazu z.B. Olson, 2004), davon ausgegangen werden, dass der kollektive Organisationsgrad unter den Bedingungen der Arbeitswelt von Ursula Meyerhans zumindest kurz- und mittelfristig deutlich abnimmt. 
Durch die Tertiärisierung wurden in Noah Schmids Arbeitswelt viele Arbeitsplätze, welche einst in grösseren Betrieben bestanden, in Branchen mit schwächeren Arbeitnehmervertretungen oder an Selbstständige ausgelagert. Die Berechnungen des Schweizerischen Gewerkschaftsbundes (SGB, 2011, S. 7), zeigen, dass gerade in Branchen wie den Informatikdiensten und den Dienstleistungen für Unternehmen eine geringe Abdeckung der Beschäftigten durch Gesamtarbeitsverträge besteht. Eine weitere Branche, die beschäftigungsmässig in dieser Arbeitswelt zulegen dürfte, ist die der persönlichen Dienstleistungen. Sie ist besonders schwach kollektiv organisiert, gemäss SGB unterliegen hier nur 2 Prozent der Beschäftigten einem GAV.

Das Ausmass, in welchem die neuen Selbstständigen, sei es als wirklich unabhängige Kleinunternehmende oder als Scheinselbstständige mit dem immer gleichen Auftraggebenden, sich kollektiv neu organisieren oder bestehenden Organisationen beitreten, wird sich stark auf den gesamtwirtschaftlichen kollektiven Organisationsgrad der Beschäftigten auswirken. Neben der Möglichkeit, welche innen Netzwerke bieten, sind Selbstständige heute und wohl auch in Zukunft oftmals in Berufsverbänden, aber nicht unbedingt in Gewerkschaften organisiert, auch wenn dies mittlerweile z.B. in Deutschland möglich ist (Ver.di, 2015). Gerade die wenig qualifizierten Arbeitsplätze in Verkauf und Gastgewerbe, der hochqualifizierte Finanzbereich und die Dienstleistungen an Unternehmen sind durch aktive Verbände geprägt, welche nicht dieselbe Verankerung besitzen wie die Gewerkschaften im Baugewerbe oder den öffentlichen Infrastrukturbetrieben (Oesch, 2007. S. 19).

Analog zu Candeias (2008), der den fehlenden bzw. schwachen kollektiven Organisationsgrad von Angestellten als Grund für eine Individualisierung der Entlohnungs- und Arbeitsbedingungen sieht, werden die Selbstständigen in Noah Schmids Arbeitswelt wenig kollektive Regelungen haben oder sogar wollen. Sie werden häufig als Konkurrentinnen und Konkurrenten auftreten und nur wenn sie sehr spezialisierte und seltene Fähigkeiten haben, ihre Interessen gegen die Auftraggebenden durchsetzen können. Es ist also anzunehmen, dass der kollektive Organisationsgrad unter den Bedingungen der Arbeitswelt von Noah Schmid kurz- und mittelfristig deutlich abnimmt.

In der Vergangenheit war in der Schweiz die kollektive Interessenvertretung für den typischen Normalarbeitnehmer, der männlich, relativ gut ausgebildet und in einem grösseren Betrieb angestellt war, ziemlich erfolgreich. Dies äussert sich in den generell guten Arbeitsbedingungen, die z.B. in Industrieunternehmen und dem öffentlichen Dienst geboten werden. Das Organisationspotenzial der Gewerkschaften leidet aber unter einer zunehmend heterogenen Zusammenset- 
zung der Beschäftigten. In der Arbeitswelt der geringqualifizierten Andrea Burri-Lötscher arbeiten mehr Personen, insbesondere Frauen, in der Dienstleistungsbranche, wo traditionell ein tiefer Organisationsgrad herrscht. Oesch (2007) sieht deshalb auch im Erlangen von Repräsentativität und Verhandlungsmacht ausserhalb ihrer traditionellen Kernbranchen eine Hauptherausforderung für die erfolgreiche Zukunft von Gewerkschaften und der kollektiven Gestaltung von Arbeitsverhältnissen in der Schweiz (S. 23).

Aber nicht nur der schlechte Organisationsgrad der Arbeitnehmenden hat Konsequenzen für die Wahrung ihrer Interessen. Der Schweizerische Gewerkschaftsbund weist z.B. für das Gastgewerbe, den Detailhandel, die persönlichen Dienstleistungen und die Angestellten in Privathaushalten besonders hohe Anteile an Niedriglohnempfängern aus (SGB, 2011, S. 8). Er macht jedoch für die beiden letztgenannten Gruppen das Fehlen von Arbeitgeberorganisationen als Verhandlungspartnerinnen und Verhandlungspartner mitverantwortlich für die Lohnsituation.

Ausser der Zunahme der Mitgliedszahlen der Berufsverbände (Oesch, 2007, S. 22), welche jedoch die oben genannten Niedriglohnbranchen wenig betreffen, gibt es kaum Gründe in der jüngsten Vergangenheit, die zur plausiblen Annahme verleiten, dass sich der kollektive Organisationsgrad der Beschäftigten in der Arbeitswelt von Andrea Burri-Lötscher verstärkt. Es muss aufgrund der Heterogenisierung der Beschäftigten eher mit einer leichten bis deutlichen Abnahme gerechnet werden.

\subsubsection{Physische und psychische Gesundheitsrisiken}

In diesem Abschnitt wird beurteilt, ob in den verschiedenen Arbeitswelten die physischen und psychischen Risiken für die Gesundheit der Beschäftigten zuoder abnehmen. Bestimmend sind dabei die Bedingungen der jeweils prägenden Arbeitsformen.

Flexibel arbeitende Kaderangestellte wie Roland Müller, die hohen arbeitsbezogenen Anforderungen ausgesetzt sind, wählen oft Bewältigungsstrategien zur Erfüllung von Zielen und Aufgaben, die negative Auswirkungen auf ihr Befinden und ihre Erholung haben (Krause, Dorsemagen, Stadlinger und Baeriswyl, 2012). Die Anwendung solcher Strategien ist z.B. in Jahresarbeitsmodellen mit Homeoffice-Anteilen besonders gut denkbar, da sie kaum kontrollierbar sind (Krause et al., 2015). Gisin et al. (2013) berichten jedoch in diesem Zusammenhang von einer geringen bis mittleren Tendenz zum Präsentismus bei Schweizer Arbeitnehmenden die über eine längere Erfahrung mit Homeoffice 
verfügen (S. 32). Dies deutet an, dass der richtige Umgang mit dieser Form der Flexibilisierung gefunden werden kann, auch wenn fast 20 Prozent der Befragten in Gisin et al. (2013) noch oft bis sehr oft keine oder verspätete Pausen machen (S. 35).

Dennoch stellen Grebner, Berlowitz, Alvarado und Cassina (2011, S. 5) fest, dass allgemein eine Zunahme des empfundenen Stresses bei Schweizer Erwerbstätigen stattgefunden hat. In ihrer Befragung von 2010 berichten 34 Prozent über häufigen oder sehr häufigen Stress, während es im Jahr 2000 noch 27 Prozent waren. Die Häufigkeit des Erlebens von Stress variiert auffällig zwischen den Altersgruppen. Die Gruppe der 55- bis 64-Jährigen gibt häufiger als der Durchschnitt an, sich nie gestresst gefühlt zu haben, während die jüngsten Erwerbstätigen (15- bis 24-jährig) und diejenigen im Alter von 25 bis 34 Jahren überdurchschnittlich oft berichten, sich häufig bzw. sehr häufig gestresst gefühlt zu haben. Die Gründe dafür, bspw. der Anstieg des Termindrucks, die Zunahme des Arbeitstempos und häufigere Unterbrechungen der Arbeit durch unvorhergesehene Aufgaben, könnten einen Zusammenhang mit der Flexibilisierung der Arbeit haben. Sie werden aber in der Studie (Grebner et al. 2011) nicht damit in Verbindung gebracht. Allerdings beobachteten Grebner et al. (2011) zwischen 2005 und 2011 einen Boom bei der Flexibilisierung der Arbeitszeiten (S. 73).

Aus Janssen und Nachreiner (2004) folgt, dass Arbeitnehmende, die keinen oder nur einen kleinen Einfluss auf die Lage und Dauer ihrer flexiblen Arbeitszeit haben, statistisch signifikant mehr über nervöse Beschwerden, MagenDarm-Beschwerden und Kopf- und Rückenschmerzen berichten. Die Autoren empfehlen deshalb, Gestaltungsspielräume für die betroffenen Arbeitnehmenden einzuräumen, da sie von besonderer Bedeutung für die physische und psychosoziale Befindlichkeit sind. So kann ein Gestaltungsspielraum bei der Arbeitszeit z.B. den Schlaf optimieren, was sich positiv auf die Produktivität und die Arbeitsmoral auswirkt (Gangwisch, 2014, S. 1159 f.). Auch Berndt (2015) stellt für deutsche Verhältnisse fest, dass das Auftreten von psychischen Erkrankungen wesentlich vom Verhalten der Führungskräfte abhängt, worunter insbesondere auch das Einräumen von Gestaltungsspielräumen gehört (Berndt 2015). Eichhorst und Tobsch (2014, S. 14) beobachten, dass physische und psychische Beschwerden in der zunehmend flexiblen deutschen Arbeitswelt zugenommen haben, und dass flexible Arbeitszeiten und -orte verbesserte Möglichkeiten bieten, um Arbeit und Privat- bzw. Familienleben miteinander zu vereinbaren. 
Grundsätzlich kann psychische Belastung durch die dafür erhaltenen Belohnungen, wie Anerkennung, Entlohnung, Aufstiegsmöglichkeiten und Arbeitsplatzsicherheit, in gewisser Weise entgolten werden (Gisin et al. 2013, S. 53). Bei den routinierten Homeoffice-Arbeitenden in der Schweiz haben Gisin et al. (2013) festgestellt, dass fast ein Drittel ein ungünstiges Verhältnis zwischen Belohnung und Belastung wahrnimmt und dadurch einem erhöhten Krankheitsrisiko ausgesetzt ist (S. 55).

Über physische Risiken im Zusammenhang mit der Arbeit im Homeoffice bzw. zu Hause ist wenig bekannt. Ein Hinweis auf Probleme der Arbeitsplatzgestaltung findet sich in Gisin et al. (2013). Fast ein Fünftel der erfahrenen Homeoffice-Beschäftigten gibt an, „keinen festen Arbeitsplatz zu haben und flexibel bspw. vom Esstisch oder Balkon aus zu arbeiten“ (S. 36).

Die in der Arbeitswelt von Roland Müller vorkommenden Elemente der Flexibilisierung können also je nach Ausprägung und Gestaltungsmöglichkeiten mit einem leichten Anstieg der psychischen Gesundheitsrisiken in Verbindung gebracht werden.

Die physischen und psychischen Risiken der arbeitsbedingten räumlichen Mobilität, wie sie von Sandra Könitz praktiziert wird, lassen sich nicht eindeutig feststellen, da auch die Arbeitstätigkeit per se, die weiteren Arbeitsbedingungen sowie die Erfahrungen und Einstellungen der Betroffenen einen wesentlichen Einfluss haben (Hupfeld et al. 2013, S. 8). Physische Gefahren erwarten die häufig unterwegs arbeitende Sandra Könitz in erster Linie durch die oft ungünstige oder schlecht gestaltete Arbeitsumgebung. Sie kann Fehl- und Zwangshaltungen fördern, die bei länger andauernden Belastungen zu verschiedenen Erkrankungen führen können (Hupfeld, et al., 2013, S. 17).

Psychisch belastend sind an Sandra Könitz' mobil-flexibler Arbeitsform der Stress und die Zeitknappheit (Ducki, 2009). Insbesondere begünstigen flexible Arbeitszeiten ohne Zeiterfassung den Präsentismus, der sich vor allem mittelund langfristig in gesundheitlicher Hinsicht bemerkbar macht (Dorsemagen et al., 2012) und sich beispielsweise in einer höheren Burnout-Gefahr auswirken könnte. Mobil Arbeitende neigen dazu, ständig online zu sein und zu arbeiten (Meissner 2007), zur Arbeit in der Nacht, an Wochenenden und während den Ferien (Krause \& Schulze, 2012). In der Schweiz hängt die Dauer der Arbeitszeit gemäss den Resultaten des EWCS 2010 bereits für beinahe drei Viertel der abhängig Beschäftigten davon ab, ob sie ihre Aufgaben und Ziele erreicht haben (Krieger et al., 2012, S. 120). Beschäftigte mit weisungsgebundener, ausführender Arbeit sind mit 62,3 Prozent etwas weniger häufig davon betroffen als hochqualifizierte bzw. leitende Beschäftigte mit 89,5 Prozent (ebd., S. 120). 
Entscheidende Faktoren für die Gesundheitsverträglichkeit von flexiblen Arbeitszeiten sind gemäss Janssen und Nachreiner (2004) der Einfluss, den Arbeitnehmende ausüben können, sowie die Vorhersehbarkeit und Variabilität bezüglich Lage und Dauer der Arbeitszeit (S. 34). Sind diese Faktoren nicht im Sinne des Arbeitnehmenden ausgestaltet, kann es zu ähnlichen gesundheitlichen Folgen kommen, wie sie aus der Schichtarbeitsforschung bekannt sind, insbesondere zu Schlaf- und Verdauungsproblemen sowie psychovegetativen Störungen (S. 128). Interessanterweise treten diese Effekte gemäss Janssen und Nachreiner (2004) auch bei Arbeitnehmenden, die selbst über ihre Flexibilität bestimmen können, relativ häufig auf. Die Ergebnisse einer Befragung mit einer Stichprobe von über 2000 deutschen abhängig Beschäftigten von Zok und Dammasch (2012) bestätigen die gesundheitlichen Risiken verschiedener Formen flexibler Arbeit. So stehen insbesondere die Arbeit an Wochenenden und Feiertagen und Änderungen von Freizeitaktivitäten wegen beruflicher Verpflichtungen in statistisch signifikantem Zusammenhang mit psychischen Beschwerden der Arbeitnehmenden (S. 44).

Die ständige Erreichbarkeit, die von Angestellten immer öfter erwartet wird, kann auch ein gesundheitliches Risiko darstellen. Dettmers, Vahle-Hinz, Friedrich, Keller, Schulz und Bamberg (2012) zeigen für deutsche Arbeitnehmende, die unter Rufbereitschaft arbeiten, dass sie ihr Befinden unabhängig davon, ob es tatsächlich zu einem Arbeitseinsatz kommt, statistisch signifikant schlechter einschätzen als an Tagen ohne Rufbereitschaft. So leiden die Befragten unter Schlafstörungen, nachmittäglichen Befindensbeeinträchtigungen und Irritationen am Abend (Dettmers et al., 2012). Auch als eine Folge von arbeitsbedingter erweiterter Erreichbarkeit gilt die emotionale Erschöpfung (Pangert \& Schüpbach, 2015). Sie kann ein solches Ausmass erreichen, dass Angestellte privaten Anforderungen und Bedürfnissen wegen Zeitmangels oder Energielosigkeit nicht mehr nachkommen können (Pangert \& Schüpbach 2015, S. 75 ff.).

Eine weitere Quelle für psychische Belastung kann durch die Verlagerung von unternehmerischen Risiken auf Arbeitnehmende entstehen; eine Entwicklung, die sich auch in der Schweizer Arbeitswelt von Sandra Könitz verstärkt, zurzeit aber nur in Nachbarländern empirisch belegt ist. Die Ergebnisse von Chevalier und Kaluza (2015) zeigen, dass z.B. ein leistungsabhängiger Lohn bei deutschen Angestellten die Work-Life-Balance sowie den Schlaf statistisch signifikant negativ beeinflusst (S. 8). Eichhorst und Tobsch (2012, S. 16) fanden für die Arbeitswelt in Deutschland „klare Anzeichen für eine stärkere und dauerhafte Verlagerung von unternehmerischen Risiken auf dauerhaft Beschäftigte, flexibel Beschäftigte und Zulieferer bzw. Dienstleister". Höge (2011) hat auch für österreichische Arbeitnehmende statistisch signifikante Zusammenhänge 
zwischen Flexibilitätsanforderungen im Zusammenhang mit dem Konzept der Arbeitskraftunternehmende und kognitiver Irritation gefunden. Dabei korrelieren Anforderungen betreffend Selbstorganisation, Karriereentwicklung, selbstgesteuertem Lernen und zeitliche Flexibilität statistisch signifikant mit kognitiver Irritation (Höge, 2011, S. 13).

Die Arbeit von Sandra Könitz ist also von verschiedenen physischen und psychischen Gesundheitsrisiken geprägt. Auch wenn sich davon viele durch ein gutes Selbstmanagement minimieren lassen, kann davon ausgegangen werden, dass die Risiken bei einer stärkeren Verbreitung der Bedingungen der Arbeitswelt von Sandra Könitz eher zunehmen.

Clasen (2012) stellt die Arbeits- und Gesundheitssituation von Freelancern dar und befragte dazu über 330 freie Mitarbeitende. Freelancer gelten als Prototyp der mobil-flexiblen Arbeitsorganisation (Krause et al., 2012) und wie Ursula Meyerhans arbeiten sie an den Grenzen zwischen Selbstständigkeit und Arbeitnehmertum bzw. Autonomie und Abhängigkeit (Clasen 2012). Die Studie fand bei einem Viertel der Befragten heraus, dass sie unter starkem psychischem Stress leiden und Faktoren wie Einkommensunsicherheit, auftragsbedingte Stressoren und Zeitdruck besondere Risiken darstellen (Clasen, 2012).

Bei Arbeitnehmenden, welche eine geringe Präferenz für ihre flexible Arbeitsform haben, kann ein Gefühl der Ungewissheit der Zukunft die Erfahrung der Arbeit negativ beeinflussen, insbesondere kann das bei freien Mitarbeitenden beobachtet werden (Dütschke \& Boerner, 2012, S. 177). Grundsätzlich gibt es einen Zusammenhang zwischen schlechtem Gesundheitszustand und Arbeitsplatzunsicherheit (Dixon, Woodman, Strazdins, Banwell, Broom \& Burgess, 2013). Von Letzterer sind z.B. Angestellte mit befristeten Arbeitsverträgen betroffen. Mewes et al. (2013) finden in ihrer für die deutsche Allgemeinbevölkerung repräsentative Stichprobe negative Effekte von unsicherer Beschäftigung auf die psychische Gesundheit (S. 142) und kommen zum Schluss, dass ihr Ergebnis in Übereinstimmung mit ähnlichen Untersuchungen steht. Den Zusammenhang hat auch Haupt (2010) an über 1900 deutschen Arbeitnehmenden untersucht. Er kommt zum Schluss, dass Personen mit häufiger Arbeitsplatzunsicherheit im Vergleich mit solchen ohne oder nur geringer Arbeitsplatzunsicherheit mehr psychosomatische Erkrankungen und depressive Verstimmungen haben, sich mehr und bessere soziale Kontakte wünschen, mehr rauchen und Alkohol konsumieren, häufiger das Gesundheitswesen beanspruchen und sich schlechter ernähren (S. 103-106).

Den Zusammenhang zwischen schlechter Ernährung und flexibilisiertem Berufsleben untersuchten auch Dixon et al. (2013) näher in einer Mehrgeneratio- 
nen-Studie. Sie kamen zum Schluss, dass flexibles Arbeiten den Arbeitnehmenden ein flexibles kulturelles Leben abverlangt und sie aus der vorherigen Zeitstruktur, bestehend aus Ritualen und Routinen, scheiden müssen. Die Arbeitnehmenden müssen sich einem neuen Umfeld anpassen, was häufig zu einer Entkoppelung von Arbeitszeiten und Essgewohnheiten und einer Verschlechterung der Gesundheit führen kann (Dixon et al., 2013).

Krieger und Graf (2009) stellen aufgrund der Daten der schweizerischen Gesundheitsbefragung fest, dass Teilzeitarbeitende häufiger über Rückenschmerzen, Einschlaf- und Durchschlafstörungen als Vollzeitarbeitende klagen. Vollund Teilzeitarbeitende berichten aber gleich häufig über gesundheitliche Probleme im Zusammenhang mit ihren Arbeitszeiten (S. 50).

Im Weiteren gibt es Hinweise darauf, dass Crowdsourcing-Beschäftigte, insbesondere sogenannte Microworkers, unter Identitätsproblemen und fehlender Anerkennung leiden können (Lehdonvirta \& Mezier, 2013).

Die Arbeitsbedingungen der Teilzeitredaktorin und selbstständigen Texterin Ursula Meyerhans sind durch Unsicherheiten geprägt, welche eindeutig mit psychischen Risiken in Verbindung gebracht werden können Bei einer gesamtwirtschaftlich bedeutenden Verbreitung der beschriebenen Arbeitsformen bzw. deren Kombination ist es plausibel, davon auszugehen, dass vor allem die psychischen Gesundheitsrisiken deutlich zunehmen.

Als „Ein-Mann-Unternehmen“ im IT-Bereich dürfte Noah Schmid gemäss einer deutschen Untersuchung (Siebecke \& Lisakowski, 2010) häufiger von Muskelund Skelettbeschwerden sowie psychischen Problemen betroffen sein als fest angestellte Arbeitnehmende. Das gilt ebenfalls für Ängste, Erschöpfungszustände und Regenerationsunfähigkeit, Folgen, die Siebecke und Lisakowski (2011) in einer Befragung von vorwiegend selbstständigen IT-Fachkräften identifiziert haben. Diese Ergebnisse bestätigen die schon für Freelancer identifizierten Risiken von Clasen (2012), die auch für die Arbeitswelt von Noah Schmid relevant sind.

Zu einem gegenteiligen Ergebnis kommt Oren (2011). In seiner Studie wurden insgesamt 308 Teilnehmende befragt, davon waren 149 Selbstständige und 159 fest angestellte Arbeitnehmende. Alle hatten eine Universitätsausbildung und unterschiedliche Berufe (S. 165). Es konnten bezüglich des allgemeinen Stressniveaus und der Arbeitsbelastung keine Unterschiede zwischen den Selbstständigen und den fest angestellten Arbeitnehmenden abgeleitet werden, wenn es auch Unterschiede bezüglich der einzelnen Stressfaktoren gab. Auch das Bundesamt für Statistik (2013b, S. 24) hat in seiner Befragung von Hochschulabsolventen und -absolventinnen keinen signifikanten Unterschied in der 
Zufriedenheit mit der intellektuellen und physischen Belastung zwischen angestellten und auf Honorarbasis arbeitenden Personen gefunden. In beiden Gruppen sind gut 63 bzw. 66 Prozent der Befragten zufrieden. Selbstständige, die ihr eigenes Unternehmen führen, sind jedoch im Vergleich noch einmal deutlich häufiger zufriedener (83,6 Prozent).

Ein Grund für die unterschiedlichen Ergebnisse zwischen Siebecke und Lisakowski (2011) und den Untersuchungen von Oren (2011) sowie des Bundesamtes für Statistik (2013b) könnte in den verschiedenartigen Samples liegen. Siebecke und Lisakowski (2011) befragten nicht ausschliesslich Hochschulabgängerinnen und Hochschulabgänger. Ihr Sample dürfte also mehr Individuen enthalten, die wegen der Notwendigkeit, einer Erwerbsarbeit nachgehen zu müssen, selbstständig wurden, als die beiden anderen Studien. Da dieses Sample repräsentativer für die Arbeitswelt von Noah Schmid ist, muss in ihr von gesamtwirtschaftlich höheren Gesundheitsrisiken ausgegangen werden.

Die Risiken für die Gesundheit von Andrea Burri-Lötscher ergeben sich hauptsächlich durch die in ihrer Arbeitswelt stärkeren Verbreitung von Tätigkeiten, die geringe Qualifikationen verlangen, und der Verdrängung von höher qualifizierten Personen in atypische und zum Teil prekäre Arbeitsverhältnisse.

Krieger und Graf (2009) haben im Rahmen der schweizerischen Gesundheitsbefragung festgestellt, dass Erwerbstätige, die über keinen Berufsabschluss verfügen, hohen psychischen Belastungen ausgesetzt sind. Ausserdem erledigen sie häufig Arbeiten, die langweilig und eintönig sind (S. 49-50). In der Arbeitswelt von Andrea Burri-Lötscher muss zwar nicht von einer Zunahme der Erwerbstätigen ohne Berufsabschluss ausgegangen werden, jedoch könnten viele mit eher geringen Qualifikationen unter vergleichbaren Bedingungen arbeiten müssen. Nebst diesem Effekt kommen die bereits in den anderen Arbeitswelten erwähnten Risiken der Teilzeit, Befristung, Selbstständigkeit sowie räumlich und zeitlich flexibler Arbeit hinzu, sodass sich ein Gesamtbild mit zunehmenden Gesundheitsrisiken ergibt.

\subsubsection{Arbeitsbedingungen und Arbeitszufriedenheit}

Unter Arbeitsbedingungen sind für die Zwecke dieses Berichts die für ein Arbeitsverhältnis wirksamen Konditionen zu verstehen. Sie können entweder direkt in einem Arbeitsvertrag geregelt sein, wie z.B. die Arbeitszeiten, oder können aus der Notwendigkeit der effizienten Aufgabenerfüllung für die Beschäftigten entstehen, wie z.B. ein starkes Selbstmanagement. 
Die Haltung der erwerbstätigen Personen gegenüber ihrer Arbeit wird hier als Arbeitszufriedenheit verstanden. Sie kann als das Resultat der Arbeitsbedingungen interpretiert werden bzw. als menschliche Reaktion auf die Situation, die durch die Bedingungen der verschiedenen flexibilisierten Arbeitsformen entsteht.

Die Möglichkeit, im Homeoffice o.Ä. zu arbeiten, wird von einer grossen Mehrheit von Angestellten wie Roland Müller begrüsst. Im europäischen Kontext wurde bei einer Befragung (Diena, 2013 zit. in Eurofound, 2015, S. 75) dazu festgestellt, dass 81 Prozent der Antwortenden gerne zu Hause oder in Telezentren arbeiten möchten. Für die Schweiz stellt Grote (2010) fest, dass rund zwei Drittel der Arbeitskräfte im Homeoffice-Modus arbeiten möchten, während nur 23 Prozent von innen auch tatsächlich von dieser Arbeitsform Gebrauch machen können. Die individuelle Motivation dazu kann zumindest teilweise in der Verbesserung der wahrgenommenen Zusammengehörigkeit mit dem Umfeld ausserhalb der Arbeit gefunden werden (Vitters $\varnothing$ et al., 2003). Schweizer Homeoffice-Routiniers haben eine mittlere Zufriedenheit mit ihrer Arbeitstätigkeit von 8,95 auf einer Skala von 1 (= vollkommen unzufrieden) bis 10 (= vollkommen zufrieden) (Gisin et al., 2013, S. 45). Die mittlere Zufriedenheit mit der Arbeit generell beträgt bei den Befragten ebenfalls hohe 8,94.

Die Zeitersparnis, welche räumlich flexible Arbeitsformen in Bezug auf das Pendeln zwischen Zuhause und Arbeitsplatz bietet, spielt bei der Wahrnehmung der Arbeitsbedingungen und der Entwicklung der Arbeitszufriedenheit möglicherweise eine wesentliche Rolle. Gisin et al. (2013) weisen den Zeitgewinn durch Wegfall der Reisezeit zusammen mit der Flexibilität denn auch als meistgenannten Vorteil bei erfahrenen Homeoffice-Beschäftigten aus (S. 48).

Gemäss den Daten des Schweizer Haushalt-Panels arbeiten rund 42 Prozent aller Erwerbstätigen in irgendeiner Form zu Hause, was auch das Homeoffice einschliesst. Auch wenn man die 6,7 Prozent Selbstständigen davon abzieht, arbeiten mehr als ein Drittel aller Arbeitnehmenden in irgendeiner Form von zu Hause aus. Dabei geben 92 Prozent an, mittel bis hochzufrieden mit den Arbeitsbedingungen zu sein, während dieser Anteil bei den Erwerbstätigen ohne Arbeit zu Hause 91 Prozent beträgt (SHB, 2015). Aus deutschen Studien ist bekannt, dass die körperlichen Arbeitsbedingungen (INQA, 2015) sowie bei flexiblen Arbeitszeiten deren Lage (Janssen \& Nachreiner, 2004) wichtige Einflussgrössen auf die gemessene Arbeitsbedingungen sind. Beide Faktoren scheinen in der Schweiz angesichts der hohen Zufriedenheit mit den Arbeitsbedingungen günstig ausgeprägt zu sein. 
Die Gestaltung der Arbeit zu Hause stellt jedoch hohe Anforderungen an das Selbstmanagement von Arbeitskräften wie Roland Müller damit sie z.B. in Zeiten erhöhter Anforderungen nicht zu einer verstärkten Belastung führt. So sehen Hupfeld, Brodersen und Herdegen (2013) die Gefahr der Selbstausbeutung, wenn die zeitliche Begrenzung der Arbeit fehlt und die Strukturierung den Beschäftigten überlassen wird (S. 18). Schweizer Homeoffice-Routiniers entscheiden oft selber darüber, welche Regeln im Homeoffice gelten, und arbeiten relativ häufig ausserhalb der regulären Arbeitszeiten (Gisin et al., 2013). So arbeiten 18,1 Prozent nachts von 22 bis 6 Uhr und knapp 40 Prozent in den Abendstunden von 18 bis 22 Uhr (S. 34). Beinahe die Hälfte (45,5 Prozent) der Befragten hat keinerlei Regeln zum Arbeiten im Homeoffice mit Vorgesetzten abgemacht (S. 43). Die Flexibilität und freie Zeiteinteilung im Homeoffice wird von den Befragten sehr geschätzt und als einer der meistgenannten Vorteile dieser Arbeitsform aufgezählt (S. 48). Schweizer Angestellte arbeiten jedoch im Vergleich zum EU-Durchschnitt etwas häufiger in ihrer Freizeit, um die Arbeitsanforderungen erfüllen zu können (Grebner et al., S. 75).

Die Vermischung von Arbeitszeit und Privatleben (Entgrenzung), die im Homeoffice, aber auch bei anderen mobilen Arbeitsformen droht und z.B. für Deutschland klar empirisch belegt ist (INQA 2015), scheint hierzulande nur für eine Minderheit ein Problem zu sein. Schweizer Homeoffice-Routiniers nennen die Entgrenzung nur vereinzelt als solches (Gisin et al., 2013, S. 84). Nur ein Prozent kann die Arbeit und das Private nicht miteinander vereinbaren, bei immerhin 17 Prozent klappt es auch nicht besonders gut (S. 44).

Die Wahrnehmung der Arbeitsbedingungen wird nicht zuletzt durch die hierarchische Stellung der Arbeitnehmenden bestimmt. Während die Hälfte der deutschen leitenden Angestellten ihre Arbeitsbedingungen als besonders gut bewertet, ist es bei den Arbeiterinnen und Arbeitern nur ein Viertel (INQA 2015, S. 34). Inwiefern ihre Stellung es den Arbeitnehmenden erlaubt, Einfluss auf ihre Arbeitsbedingungen zu nehmen, ist allerdings nicht geklärt. Ein Zusammenhang kann aber kaum ausgeschlossen werden.

Von einer generellen Verschlechterung der Arbeitsbedingungen durch die stärkere Verbreitung von Homeoffice kann aufgrund der ausgewerteten Literatur nicht ausgegangen werden. In der Schweiz hat sich trotz potenziell anspruchsvollerer Arbeitsbedingungen die bisher stattgefundene Flexibilisierung durch Home Office und ähnliche Arbeitsformen kaum negativ auf die Arbeitszufriedenheit ausgewirkt. Es scheint ausserdem aufgrund der hohen Präferenz plausibel anzunehmen, dass eine stärkere Ausweitung der Arbeitswelt von Roland Müller auf andere Personenkreise kaum gesamtwirtschaftlich relevante Ver- 
schlechterungen der Arbeitsbedingungen mit sich bringen würde, sondern eher leichte Verbesserungen.

Die grosse Nachfrage nach arbeitsplatzungebundenen Arbeitsformen kann zumindest teilweise auf die wahrgenommenen besseren Arbeitsbedingungen, die sie bieten, zurückgeführt werden. Arbeitnehmende, wie die erfolgsbeteiligte Projektmanagerin Sandra Könitz, die eine hohe Präferenz für flexible Beschäftigung zeigen, schätzen insbesondere die Autonomie, Entscheidungsfreiheit, Abwechslung und persönliche Weiterentwicklung, die flexibilisierte Arbeitsformen mit sich bringen können (Dütschke \& Boerner, 2012; Chevalier \& Kaluza, 2015).

Auch flexible Arbeitszeitenregelungen bringen Verbesserungen in den Arbeitsbedingungen, die allgemein in einer höheren Arbeitszufriedenheit resultieren (INQA 2015; Masuda et al. 2012) und seltener zu Kündigungsabsichten führen (Masuda et al. 2012). Im Schweizer Kontext ist empirisch gesichert, dass sich insbesondere die Berücksichtigung der Interessen von Beschäftigten an einer besseren Life-Domain-Balance sowie einer grösseren Selbstbestimmung im Arbeitsleben in einer höheren Zufriedenheit mit der Arbeitszeitgestaltung, mit der Arbeit im Allgemeinen und mit der Vereinbarkeit von Arbeit mit Privatleben niederschlägt (Dorsemagen et al. 2012). Flexiblere Arbeitszeiten und die Möglichkeit, ausserhalb des Büros zu arbeiten, führen in der Schweiz zu einer Steigerung der wahrgenommenen Lebensqualität bzw. -zufriedenheit (Gisin et al. 2013).

Auch Mandl (2015) berichtet von einer generell hohen Arbeitszufriedenheit von „Mobile Workers" in der EU und sieht den Grund dafür in der Art der Arbeitnehmenden, welche vor allem Manager und Spezialisten umfasst, die eine Präferenz und die Fähigkeit zum mobilen Arbeiten hätten (S. 80), also auch freiwillig räumlich ungebunden arbeiten würden. Mobile Arbeitnehmende müssen jedoch ein hohes Mass an Selbstorganisation mitbringen, um erfolgreich zu sein (Paridon \& Hupke, 2009, S. 12 f.). Zwei Drittel schätzten das an ihrer Arbeitsstelle, während das restliche Drittel diesem Aspekt inrer Arbeit indifferent gegenübersteht (Paridon \& Hupke, 2009).

Die Zufriedenheit der Arbeitskräfte scheint aber zumindest in der Schweiz auch davon beeinflusst zu sein, ob eine Zeiterfassung erfolgen muss. So ist für hochqualifizierte und leitende Beschäftigte ohne obligatorische Zeiterfassung die Zufriedenheit mit der Arbeit im Allgemeinen nicht höher als bei Beschäftigten mit fixen Arbeitszeiten, und die Zufriedenheit mit der Arbeitszeitgestaltung ist bei der ersten Gruppe sogar leicht tiefer als bei der zweiten (Dorsemagen et al. 2012). Es sind aber nicht nur Hochqualifizierte wie Sandra Könitz, die ihre 
Arbeitszeiten nicht mehr erfassen, sondern mit 16,7 Prozent ein bedeutender Anteil aller Schweizer Angestellten (Dorsemagen et al., 2012, S. 41). Nebst den Problemen des dadurch begünstigten Präsentismus, welche sich vor allem mittel- und langfristig in gesundheitlicher Hinsicht bemerkbar machen, gehen flexible Arbeitszeitenregelungen ohne Zeitaufschrieb mit einer deutlich geringeren Berücksichtigung von Mehrarbeit einher. So gilt bei den hochqualifizierten und leitenden Beschäftigten ohne Zeitaufschrieb gegenüber jenen mit Zeitaufschrieb bei ansonsten ebenfalls flexibler Arbeitszeitregelung (Dorsemagen et al., 2012, S. 21):

- $\quad$ Es wird statistisch signifikant häufiger Mehrarbeit geleistet (89,8 Prozent gegenüber 75,7 Prozent).

- $\quad$ Die Mehrarbeit wird im Betrieb aber statistisch hochsignifikant weniger häufig erfasst (18,6 Prozent gegenüber 73,8 Prozent).

- $\quad$ Arbeitgeber vergüten Mehrarbeit statistisch signifikant weniger häufig finanziell (14 Prozent gegenüber 31,3 Prozent) bzw. seltener mit Freizeit (54,5 Prozent gegenüber 69 Prozent).

Während die Flexibilisierung der Arbeitszeiten sich auf die Arbeitszufriedenheit auswirkt, spielt es in diesem Zusammenhang keine Rolle, ob in Voll- oder Teilzeit gearbeitet wird. Die Ergebnisse des Schweizer Haushalt-Panels (SHP, 2014) zeigen, dass beide Gruppen sehr hohe Anteile von mittlerer bis hoher Arbeitszufriedenheit haben (91 bzw. 92 Prozent).

Die variable Entlohnung, ein weiterer Aspekt flexibilisierter Arbeitsformen, ist in den EU-Mitgliedstaaten bereits stark verbreitet und für viele Erwerbstätige Realität. So benutzen 63 Prozent aller Unternehmen in der EU eine Art von variabler Entlohnung, wobei die leistungsabhängige Bezahlung die verbreitetste Form darstellt (Eurofound 2015b, S. 75). Erfolgsbeteiligung wird in rund 30 Prozent der Unternehmen praktiziert und ist besonders verbreitet in der Finanzindustrie (Eurofound 2015b, S. 75 ff.). Dabei gibt es einen positiven Zusammenhang zwischen Unternehmensgrösse und variabler Entlohnung, d.h., kleine Unternehmen verwenden durchschnittlich weniger variable Entschädigungen (S. 77). Interessanterweise findet die Untersuchung ebenfalls, dass die Arbeitsplatzzufriedenheit bei Unternehmen mit stärker ausgeprägten Formen von variabler Entlohnung höher ist als bei solchen mit weniger ausgeprägten Formen (S. 79).

Die Mobilitätsanforderungen mobiler Arbeit beeinflussen die wahrgenommene Arbeitsqualität der Beschäftigten stark. Paridon (2012) stellt die Ergebnisse einer Befragung von Fuchs (o.J.) für den Deutschen Gewerkschaftsbund (DGB) dar und folgert, dass alle Dimensionen des DGB-Indexes "Gute Arbeit" - ausser 
die der sinnvollen Arbeit - von mobilen Arbeitnehmenden schlechter beurteilt werden als von nicht mobilen (S. 82).

Weitere Anforderungen an die Flexibilität von Arbeitnehmenden können die Befindlichkeit negativ beeinflussen. Bahamondes Pavez et al. (2012) kamen bei einer Befragung von Arbeitnehmenden aus den deutschen IT- und Metallbranchen zum statistisch signifikanten Ergebnis, dass insbesondere die Unvorhersehbarkeit und die funktional-zeitliche Flexibilität zu Irritationen bei den Befragten führt (S. 175).

Ob die Bedingungen der Arbeitswelt von Sandra Könitz als positiv oder negativ wahrgenommen werden, hängt aufgrund der aus der Literatur verfügbaren Resultate stark von der Präferenz der Beschäftigten ab. Eine abschliessende Beurteilung der Auswirkungen einer starken Verbreitung der Arbeitsform von Sandra Könitz auf die gesamtwirtschaftlichen Arbeitsbedingungen ist deshalb schwierig durchzuführen. Leichte Verbesserungen scheinen plausibel, insbesondere wenn Arbeitnehmende und -gebende fair miteinander verhandeln.

Die Bedingungen der flexiblen Beschäftigung, unter denen Ursula Meyerhans täglich arbeitet, sprechen nicht alle Leute gleich an. Eine Präferenz für flexible Beschäftigung korreliert gemäss verschiedenen europäischen Studien positiv mit der Arbeitszufriedenheit (Dütschke \& Boerner, 2012; Ellingson, Gruys \& Sackett, 1998; Krausz, 2000; Silla, Gracia \& Peiro, 2005). Auch Böhne und Breutmann (2012) betonen, dass hochqualifizierte Mitarbeitende in modernen IT-Unternehmen das eigenverantwortliche Arbeiten bezüglich der Gestaltung von Arbeitszeiten und -ort grundsätzlich schätzen und tiefere Krankenstände sowie eine höhere Mitarbeiterzufriedenheit aufweisen (S. 25). Diese Tatsache hat dazu geführt, dass Unternehmen die Flexibilität als Teil des EmployerBranding einsetzen und auf den Effizienzgewinn setzen, der durch die wegfallenden Dienstreisen und Arbeitswege möglich wird (S. 25).

Wenn die flexible Beschäftigung jedoch unfreiwillig eingegangen werden muss, werden Arbeitsplatzsicherheit, fehlende Fortzahlung des Lohnes bei Krankheit bzw. Urlaub und ein fehlendes Einkommen zwischen zwei Beschäftigungsverhältnissen als Nachteile wahrgenommen, was bei Freiwilligkeit nur bedingt der Fall ist (Dütschke \& Boerner, 2012). Generell ist sozialvertragspflichtigen Arbeitnehmenden die Sicherheit der Beschäftigung besonders wichtig und der Aspekt trägt in hohem Mass zu ihrer Zufriedenheit bei (INQA 2015, S. 10). Beschäftigte von Crowdsourcing-Plattformen müssen typischerweise auf diese Sicherheit verzichten, und ihre Arbeitsbedingungen sind gemäss Eurofound (2015a) zusätzlich schlecht, da sie tiefe und unsichere Einkommen haben und 
Konflikte mit dem Auftraggebenden aufgrund der komplizierten Rechtslage wenig Aussicht auf Erfolg bieten (S. 115).

Die Analyse der Arbeitszufriedenheit von Henneberger et al. (2004) zeigt, dass ein befristeter Arbeitsvertrag zwar im Vergleich mit unbefristeten Angestellten und unabhängig vom Geschlecht eine statistisch signifikant höhere generelle Arbeitszufriedenheit bringt (S. 29). Die Zufriedenheit mit dem Lohn und dem Arbeitsklima ist bei befristet beschäftigten Männern jedoch geringer als bei anderen männlichen Arbeitskräften (S. 30f.). Befristet Beschäftigte profitieren gleich häufig von beruflichen Weiterbildungsmassnahmen wie andere Arbeitnehmende (S. 43). Die Befristung des Arbeitsvertrags hat gemäss Henneberger et al. (2004) also kaum einen negativen Einfluss auf die Arbeitsbedingungen und -zufriedenheit.

Die Wichtigkeit der Vereinbarkeit von Beruf und Familie bzw. Privatleben für die Arbeitszufriedenheit wird durch eine deutsche Studie (INQA 2015) bestätigt. Während deutsche Arbeitnehmende den Aspekt als sehr wichtig für die Zufriedenheit einstufen, zeigen die Ergebnisse, dass die Befragten ihre erlebte Zufriedenheit weniger hoch bewerten (INQA 2015, S. 14). Interessanterweise ist die Zufriedenheit der Frauen mit der Vereinbarkeit von Beruf und Familie höher als bei den Männern (INQA 2015, S. 16), was möglicherweise auf die viel höhere Teilzeitquote bei Frauen zurückzuführen ist. Sie beträgt in Deutschland für Männer gerade 7 Prozent, während sie bei Frauen 51 Prozent beträgt (INQA 2015, S. 26).

Wie für Sandra Könitz spielt auch für Ursula Meyerhans die individuelle Präferenz für flexible Arbeitsformen eine wichtige Rolle für die Beurteilung der Arbeitsbedingungen und der daraus resultierenden Arbeitszufriedenheit. Die Unsicherheiten, welche insbesondere die Befristung von Arbeitsverhältnissen und Crowdworking-Aktivitäten mit sich bringen, würden jedoch wohl für die Mehrheit der heute Beschäftigten zu einer Verschlechterung der (wahrgenommenen) Arbeitsbedingungen und der resultierenden Zufriedenheit führen.

Trotz der gesundheitlichen Auswirkungen, welche Siebecke und Lisakowski (2011) für Selbstständige (siehe vorangehenden Abschnitt) beschreiben, sehen Unternehmer und Unternehmerinnen wie Noah Schmid die starke bis sehr starke Leistungsorientierung mehrheitlich als positiv an. Für viele sind die hohen Freiheitsgrade ein Grund für die Wahl der Selbstständigkeit (S. 127).

Die Zufriedenheit von schweizerischen Selbstständigen mit ihrer Erwerbstätigkeit wurde vom Bundesamt für Statistik (2013b) im Jahr 2011 untersucht, allerdings nur für die Gruppe der Hochschulabsolventen und -absolventinnen. Auffallende Unterschiede zwischen Selbstständigen, die auf Auftrags- und Hono- 
rarbasis arbeiten, und Angestellten ergeben sich bei der Beurteilung der Einkommenssituation sowie bei der Sicherheit der Stelle. In beiden Fällen sind Erstere deutlich weniger oft zufrieden. Selbstständige, die ein Unternehmen gegründet oder übernommen haben, sind ausserdem mit der Sicherheit der Arbeitsstelle deutlich weniger häufig zufrieden als Selbstständige auf Auftragsund Honorarbasis. In Bezug auf die Arbeitszeiten und die selbstständige Organisation der Arbeit können kaum Unterschiede zwischen Angestellten und Auftragnehmenden beobachtet werden. Rund drei Viertel der Befragten in diesen Gruppen sind zufrieden damit, während selbstständige Unternehmerinnen und Unternehmer im Vergleich noch einmal fast zehn Prozentpunkte häufiger zufrieden sind.

Auswirkungen auf die Arbeitszufriedenheit und -bedingungen von Geringqualifizierten unter den Voraussetzungen der Arbeitswelt von Noah Schmid lassen sich kaum aus der Literatur ableiten. Sicher scheint, dass auch sie von der Flexibilisierung der Arbeit stark betroffen sein werden. So könnte eine bedeutende Zunahme der unfreiwillig Selbstständigen (siehe Abschnitt 4.6.2) stattfinden. Sie würde es Unternehmen erlauben, finanzielle Sachzwänge, die durch Arbeitsverträge entstehen, wie Lohnfortzahlung während Krankheit und konjunkturell bedingten Phasen geringer Betriebsauslastung oder Arbeitgeberverpflichtungen gegenüber Sozialversicherungen, zu vermeiden. Plausibel ist ebenfalls eine verstärkte Verbreitung von atypisch prekären Arbeitsverhältnissen wie befristeten Arbeitsverträgen und Arbeit auf Abruf, da diese den Unternehmen eine ähnliche Flexibilität bieten wie die Auslagerung an kleine Dienstleistungsunternehmen.

Obwohl eine bedeutende Gruppe von Erwerbstätigen, welche meistens eine tertiäre Bildung mitbringen und eine Präferenz für ein autonomes, selbstverantwortliches Erwerbsleben haben, die Arbeitsbedingungen in der Arbeitswelt von Noah Schmid vorteilhaft wahrnimmt, bringen sie für eine nicht weniger wichtige Gruppe kaum Verbesserungen gegenüber einem unbefristeten Vollzeitarbeitsvertag. Als durchschnittlicher Effekt dürfte sich auf gesamtwirtschaftlicher Ebene eine leichte Verschlechterung der Arbeitsbedingungen und -zufriedenheit einstellen.

Arbeitskräfte mit niedriger Qualifikation wie Andrea Burri-Lötscher sind in der Schweiz gemäss Krieger und Graf (2009) insgesamt deutlich weniger zufrieden mit ihrer Arbeitssituation als der Durchschnitt der Erwerbstätigen. Dieses Ergebnis dürfte auf die Arbeitsbedingungen und die damit verbundenen psychischen und physischen Gesundheitsrisiken von Geringqualifizierten zurückzuführen sein. 
Welchen Effekt dabei die Befristung von Arbeitsverträgen bei Geringqualifizierten hat, kann aus den vorliegenden Studien nicht eindeutig abgeleitet werden. Diese Tatsache ist möglicherweise mit der Heterogenität dieser Form von Arbeitsverträgen zu erklären (de Cuyper, de Jong, de Witte, Isaksson, Rigotti \& Schalk, 2008, S. 33). Eine Metaanalyse von Wilkin (2013) in diesem Zusammenhang berücksichtigt 72 Studien mit Stichproben aus verschiedenen Ländern und Kulturkreisen. Sie findet im Durchschnitt nur einen kleinen statistisch signifikanten Unterschied zwischen Festangestellten und Personen ohne permanente Arbeitsverträge, und zwar haben Letztere eine leicht geringere Arbeitszufriedenheit. Buddelmeyer, McVicar und Wooden (2015) untersuchten die Arbeitszufriedenheit von Personen mit Gelegenheitsbeschäftigungen und anderen befristeten Anstellungen. Sie können zeigen, dass Männer in Gelegenheitsjobs im Vergleich zu allen anderen eine 5 bis 7 Prozent tiefere Wahrscheinlichkeit haben, hohe Werte von Arbeitszufriedenheit zu melden, und eine um 1 bis 3 Prozent höhere Wahrscheinlichkeit für sehr tiefe (Buddelmeyer et al. 2015, S. 273). Wie die Autoren betonen, ist dieses Resultat bemerkenswert, da es mit einem Sample aus Australien zustande gekommen ist, wo andere Arbeitsmarktbedingungen herrschen als in Europa, und die europäischen Ergebnisse bestätigt. Im Weiteren konnte die Studie keine nennenswerten Korrelationen zwischen dem Qualifikationsniveau und der Arbeitszufriedenheit von atypisch Beschäftigten finden (S. 271), ausser bei gut ausgebildeten Frauen, die in prekären Arbeitsverhältnissen die geringsten Werte von Arbeitszufriedenheit aufweisen.

Die in verschiedenen Studien gefundene geringere Arbeitszufriedenheit von Gelegenheitsarbeitenden und anderen befristet Angestellten lässt für die Arbeitswelt von Andrea Burri-Lötscher einen sicheren, wenn auch eher leichten Rückgang der gesamtwirtschaftlichen Arbeitszufriedenheit plausibel erscheinen. Über die Arbeitsbedingungen per se lässt sich aus den Studienergebnissen wenig verallgemeinern. Wie die Ergebnisse von Dütschke und Boerner (2012) jedoch zeigen, werden bei unfreiwillig eingegangenen flexiblen Arbeitsverhältnissen, wie sie für prekarisierte Beschäftigte die Norm sein dürften, Arbeitsplatzsicherheit, fehlende Fortzahlung des Lohnes bei Krankheit bzw. Urlaub und ein fehlendes Einkommen zwischen zwei Beschäftigungsverhältnissen als Nachteile wahrgenommen. Werden solche Arbeitsverhältnisse über ein Vouchersystem eingegangen, so bieten Vermittlungsagenturen, welche als reguläre Arbeitgeber auftreten, generell bessere Arbeitsbedingungen als fragmentierte Modelle, die auf vereinzelten, nicht koordinierten und sozial nicht abgesicherten „Minijobs“ basieren. So sorgen sie für Ersatz, wenn Arbeitskräfte krank oder 
verhindert sind, und bieten Schulungen und andere Qualifizierungsmöglichkeiten (Angermann \& Eichhorst, 2013).

\subsubsection{Vereinbarkeit von Beruf mit anderen Lebensbereichen}

Dieser Indikator bestimmt, inwiefern die flexiblen Arbeitsformen eine Auswirkung auf die Kompatibilität von Beruf und verschiedenen Lebensbereichen haben und eine Balance ermöglichen. Für die Zwecke dieses Berichts wurde keine spezifische Einschränkung der Lebensbereiche vorgenommen. Diese können u.a. ehrenamtliche und freiwillige Tätigkeiten, die Familie oder die CareArbeit umfassen.

Da gemäss BFE (2014) die räumlich flexiblen Arbeitsformen, wie das Homeoffice von Roland Müller, nur sehr geringe Anteile an der gesamten geleisteten Arbeitszeit in der Schweiz ausmachen (0,9 Prozent Homeoffice resp. 0,8 Prozent mobile Arbeit), ist nur bei einer starken Zunahme von gesamtwirtschaftlich relevanten Problemen bei der Vereinbarkeit von Beruf und anderen Lebensbereichen auszugehen. Die Vereinbarkeit zwischen beruflichen und sozialen Verpflichtungen hat sich in der Schweiz zwischen 2005 und 2010 trotz der Zunahme der Arbeitsverhältnisse mit flexiblen Arbeitszeiten nicht verändert und 88 Prozent empfinden sie als gut (Grebner et al. 2012, S. 76). Da das Hauptmotiv für die Einführung räumlich flexibler Arbeitsformen der Wunsch der Mitarbeitenden ist (BFE, 2014, S. III), kann nicht davon ausgegangen werden, dass die Arbeitnehmenden davon grosse Nachteile erleiden. Die angenommene Verbesserung der Work-Life-Balance ist aber nicht unumstritten. Durch räumlich flexible Arbeit kann die Abgrenzung zwischen Arbeit und Freizeit zunehmend verwischen (Brandt, 2010) und der durch die IT ermöglichte, permanente Zugang zu Firmendaten und Managementinformationen führt gemäss Paridon und Hupke (2012) dazu, das Arbeitnehmende immer "online“ sind und ständig zum Arbeiten verleitet werden. Auch an Wochenenden, nachts oder im Urlaub gehen räumlich flexibel Arbeitende immer öfter der Arbeit nach (Krause \& Schulze, 2012). Da die Grenzen zwischen Arbeit und anderen Lebensbereichen nicht von den Unternehmen gezogen werden (können), müssen die Arbeitnehmenden die Herausforderung, Grenzen zu ziehen, selber meistern (Meissner 2007a). Um Konflikte zu vermeiden, ist ein hohes Mass an Selbstmanagement notwendig, damit der erhöhte Koordinations- und Entscheidungsbedarf im privaten Bereich der Arbeitnehmenden erfolgreich bewältigt werden kann (Hupfeld et al., 2013). Es ist jedoch in der Schweiz davon auszugehen, dass die Entgrenzung nicht nur vom Einsatz moderner Kommunikationsmittel abhängt. So nehmen 34 Prozent der Schweizer Arbeitnehmenden, die in irgendeiner Form zu 
Hause arbeiten, Beeinträchtigungen zwischen ihren privaten Aktivitäten bzw. Familienpflichten und der Arbeit wahr, während nur ein Viertel ihrer Kolleginnen und Kollegen, die nie zu Hause arbeiten, das gleiche Problem haben (SHP, 2014).

Neben dem erleichterten Zugang zur Arbeit wird auch die arbeitsbezogene Erreichbarkeit ein zunehmend wichtiger Grund für Konflikte zwischen beruflichen Angelegenheiten und dem Privatleben, insbesondere für Angestellte. Durch die Ergebnisse ihrer Befragung von deutschen Angestellten kommen Pangert und Schüpbach (2015) zum Schluss, dass eine hohe arbeitsbezogene erweiterte Erreichbarkeit mit Konflikten zwischen Arbeit und Privatleben sowie emotionaler Erschöpfung korreliert (S. 78) und fehlende Zeitpuffer als zentraler Faktor in der Erklärung der Konflikte sind. Die beiden Autoren (Pangert \& Schüpbach, 2015) haben im Weiteren 23 internationale Studien zum Problem der arbeitsbedingten Erreichbarkeit analysiert und kommen zum Schluss, dass die Befunde negative sowie positive Effekte der Erreichbarkeit zeigen. Einerseits können Beeinträchtigungen im Privatleben und Befindlichkeitsstörungen auftreten, je mehr Arbeitsangelegenheiten im Privatleben Einzug halten. Andererseits wird die Arbeit aber positiver bewertet, je ausgeprägter die arbeitsbedingte Erreichbarkeit ist (Pangert \& Schüpbach, 2015, S. 76).

Die Verlagerung der Arbeit vom Arbeitsplatz nach Hause hat nicht nur für die Arbeitenden Folgen. Vitters $\varnothing$ et al. (2003) fanden für ein europäisches Sample von Angestellten, die teilweise zu Hause arbeiten, heraus, dass die Zufriedenheit ihrer Partnerinnen und Partner mit zunehmender Anzahl der Tage, an denen zu Hause gearbeitet wird, abnimmt. Im Weiteren erkannten die Forscher (Vittersø et al., 2003), dass sich das Zusammengehörigkeitsgefühl bei den Partnerinnen und Partnern verschlechtert, wenn sich die Arbeitnehmenden zu Hause bei der Arbeit besser konzentrieren können.

Die Flexibilisierung des Arbeitsortes und der Arbeitszeit steht grundsätzlich mit einer Verbesserung der Vereinbarkeit von Beruf mit anderen Lebensbereichen bzw. sozialen Verpflichtungen in Verbindung, allerdings müssen vor allem abhängig Beschäftigte wie Roland Müller ein Selbstmanagement entwickeln, das es erlaubt, die Vorteile der Flexibilisierung zu nutzen.

Die Entgrenzung von Arbeit und Freizeit hat im Leben von Sandra Könitz ein beachtliches Ausmass angenommen, und ihre Work-Life-Balance ist regelmässig gestört. In einer deutschen Studie (BMAS 2015) sollten die Beschäftigten angeben, ob sie der Aussage „die modernen Kommunikationsmittel wie E-Mail, Handy oder Internet machen meine Freizeit häufig zur Arbeitszeit" zustimmen oder widersprechen. 17,2 Prozent stimmten uneingeschränkt zu, rund 10 Pro- 
zent zeigten sich unentschieden, während 73 Prozent nicht zustimmten (BMAS, 2015, S. 48). Inwiefern das auch für schweizerische Arbeitnehmende zutrifft, lässt sich durch Zahlen nicht belegen. Angesichts des hohen Ausmasses flexibler Arbeitsformen in der Schweiz muss aber davon ausgegangen werden, dass hier ein ähnlich hohes Mass an Entgrenzung herrscht, das sich in der Zukunft noch vergrössern wird. Gemäss Meissner (2007) neigen auch in der Schweiz zumindest mobil Arbeitende dazu, ständig online zu sein und zu arbeiten.

Als beständigstes familiäres Merkmal für die Vorhersage eines Ungleichgewichts bzw. einer Unvereinbarkeit in der „Work-Life-Balance“ nennen Tausig und Fenwick (2001) die Tatsache, ein Elternteil zu sein, während das beständigste berufliche Merkmal dafür die Anzahl Arbeitsstunden darstellt (S. 101). Auch BMAS (2015) nennt die wöchentliche Arbeitszeit und deren Lage als wichtige, empirisch belegte Quelle für Konflikte zwischen Arbeit und Privatleben.

Die im Vergleich mit dem europäischen Umfeld langen Arbeitszeiten in der Schweiz scheinen den Unternehmen eine gewisse Flexibilität zu verleihen, die es verhindert, dass die Arbeitsstunden gesamtwirtschaftlich weiter erhöht werden und die Vereinbarkeit von Beruf mit anderen Lebensbereichen unter erhöhten Arbeitszeiten leidet. So stellen Dorsemagen et al. (2012) fest, dass diese Entwicklung in der EU möglicherweise dadurch getrieben wird, dass die „benötigte Flexibilität in den Betrieben zunehmend über Mehrarbeit - und nicht über die Neueinstellung von Beschäftigten - erzielt wird“ (S. 67). Die Flexibilisierung der Arbeitszeiten und -verhältnisse birgt jedoch weiteres Potenzial, welches die sogenannte Work-Life-Balance aus dem Gleichgewicht bringen kann.

Das blosse Vorhandensein bzw. die Nutzbarkeit von flexiblen Arbeitszeitoptionen hat gemäss der quantitativen Studie von Tausig und Fenwick (2001) keinen Effekt auf die wahrgenommene Balance. Erst wenn die Angestellten auch die Kontrolle über den Arbeitszeitenplan haben, fühlen sie eine Verbesserung der Work-Life-Balance (S. 116). Tausig und Fenwick (2001) argumentieren im Weiteren, dass gerade unflexible, regelmässige Arbeitspläne es erleichtern, die Balance herzustellen, da solche die Planung erleichtern und dabei helfen, zwischen Arbeits- und Familienzeit zu unterscheiden. Als empirischen Beweis dafür wird das hohe wahrgenommene Gleichgewicht von Staatsangestellten genannt, welche typischerweise zu Standard-Bürozeiten arbeiten (Tausig \& Fenwick, 2001).

Einen - zumindest für Manager und Managerinnen - gegenteiligen Befund beschreiben Masuda et al. (2012). Sie untersuchten den Zusammenhang zwischen flexiblen Arbeitszeiten und Konflikten zwischen Familie und Arbeit bei Angestellten in Managementpositionen und fanden heraus, dass es in indivi- 
dualistischen Gesellschaften bei Vorhandensein von flexiblen Arbeitszeiten statistisch signifikant weniger Konflikte aus Günden zeitlicher und arbeitsbedingter Belastung gibt als bei festen Arbeitszeiten (S. 18 f.).

Aufgrund der aktuellen wissenschaftlichen Erkenntnisse aus Deutschland und der hohen durchschnittlichen wöchentlichen Arbeitszeit in der Schweiz muss bei einer bedeutenden Zunahme der Arbeitnehmenden, die unter den Bedingungen von Sandra Könitz tätig sind, von einer qualitativen und quantitativen Zunahme der Einschränkungen der Vereinbarkeit von Beruf mit anderen Lebensbereichen gerechnet werden.

Ursula Meyerhans gelingt es als Teilzeitarbeitende mit mehreren Standbeinen besser, Beruf und Familie miteinander zu vereinbaren, als Vollzeitarbeitenden. Aus den Ergebnissen der Befragung des Schweizer Haushalt-Panels im Jahr 2013 kann entnommen werden, dass nur 22 Prozent der Teilzeiterwerbstätigen mittlere bis hohe Beeinträchtigungen zwischen dem Privatleben und der Arbeit wahrnehmen, während dieser Wert für Vollzeiterwerbstätige 35 Prozent beträgt. Die Daten des Schweizer Haushalt-Panels zeigen ausserdem, dass hier gut ein Drittel (34,5 Prozent) der Teilzeitarbeitenden aus familiären Gründen nicht Vollzeit arbeitet (SHP, 2014), was die Wichtigkeit dieser Erwerbsform für die Vereinbarkeit von Beruf und Familie unterstreicht. Nur gerade 4,3 Prozent der Teilzeitarbeitenden finden keine Vollzeitarbeit und arbeiten deshalb Teilzeit (SHP 2014). Obwohl Teilzeitbeschäftigung mit ungesicherten Arbeitsverhältnissen, schlechteren sozialen Absicherungen (z.B. bei der Pensionskasse) oder geringeren Weiterbildungsmöglichkeiten und Karrierechancen einhergehen kann, bietet sie auch die Möglichkeit, neben der Erwerbsarbeit andere Aufgaben zu übernehmen wie Kinderbetreuung, informelle Hilfeleistungen und Hausarbeit (BFS, 2013, S. 12 f.).

Da Ursula Meyerhans nicht ausschliesslich auf Crowdsourcing-Aufträge angewiesen ist, steht es noch einigermassen gut um ihre Work-Life-Balance. Die voraussichtlich stärkere Verbreitung von Crowdsourcing for paid Work kann aber Bedingungen schaffen, die das Wahrnehmen von sozialen Aktivitäten erschwert, z.B. weil kurzfristig Aufträge angenommen werden müssen, durch die Zeitverschiebung beim Auftraggebenden zu Unzeiten gearbeitet werden muss oder der Aufwand für einen Auftrag falsch eingeschätzt wurde und andere Termine nicht mehr wahrgenommen werden können. Ipeirotis (2010b) kommt in seiner Analyse der Nutzerinnen und Nutzer der Crowdworking-Plattform „Mechanical Turk" zum Schluss, dass Auftragnehmende, die ihr Einkommen hauptsächlich nicht mit Crowdsourcing for paid Work erarbeiten, weniger als einen Tag damit verbringen. Wenn ein monatliches Einkommen von über 1000 US- 
Dollar erzielt werden soll, müssen die Crowdworker jedoch erheblich mehr Zeit investieren. Rund ein Drittel aller Crowdworkenden auf Mechanical Turk verbringt wöchentlich acht oder mehr Stunden auf der Plattform (Ipeirotis, 2010b). Die bisherige Forschung zu Crowdsourcing-Auftragnehmenden zeigt, dass sie im Allgemeinen repräsentativ sind für die internetnutzende Bevölkerung, mit dem Vorbehalt, dass sie eher ein bisschen jünger sind, ein tieferes Einkommen erzielen und kleinere Familien haben (Ipeirotis, 2010a).

Die Situation bezüglich der Vereinbarkeit von Beruf mit anderen Lebensbereichen bei Solo-Selbstständigen, die parallel für verschiedene Auftraggebende arbeiten, ist generell schlecht erforscht (Süss \& Sayah, 2013, S. 253). Grundsätzlich erzeugen die Anforderungen an die Flexibilität und die im Vergleich mit Festangestellten reduzierte Stabilität spezielle Herausforderungen, wenn das Berufs- mit dem Privatleben in Einklang gebracht werden kann (ebd., S. 251). Ein Hinweis auf die Schwierigkeiten enthält Siebecke (2010), welche die Arbeitsbedingungen von Solo-Selbstständigen, insbesondere die Vermischung von Arbeits- mit Freizeit, mit resultierendem Stress in Verbindung sieht.

Während befristete Arbeitsverträge und Teilzeitarbeit kaum mit einer Verschlechterung der Vereinbarkeit von Beruf mit anderen Lebensbereichen in Verbindung gebracht werden können, arbeiten Solo-Selbstständige oft unter Bedingungen, die als Voraussetzungen dafür gelten. Hinzu kommt die Tatsache, dass Solo-Selbstständige wie auch Selbstständige mit Angestellten vermehrt einer zweiten Erwerbsarbeit nachgehen, was kaum eine Verbesserung der Vereinbarkeit von Beruf mit anderen Lebensbereichen bringen dürfte. Ihre Zahl im Jahr 2014 hat sich seit 2006 um rund 7500 Personen erhöht (Eurostat, 2015). Auch bei den Personen mit mehr als einem Anstellungsverhältnis wurde im gleichen Zeitraum eine Erhöhung um 62'700 Fälle beobachtet (ebd., 2015). Bei einer gesamtwirtschaftlich starken Verbreitung der drei Arbeitsformen und deren Kombinationen, wie es für die Arbeitswelt von Ursula Meyerhans zu erwarten ist, sind die Auswirkungen für die Vereinbarkeit von Beruf mit anderen Lebensbereichen daher unsicher.

Obwohl Noah Schmid seine Selbstständigkeit wegen der vielen Freiheiten schätzt, kann es auch bei inm zu Konflikten zwischen der Arbeit und dem Privatleben kommen, wenn die Arbeitsbedingungen inn zu sehr einschränken. Gemäss einer qualitativen Studie von Süss und Sayah (2010, S. 254) ist das typisch für Freelancer in der IT- und Medienbranche (S. 259). Die Studienergebnisse sind bezüglich der Bewertung der Vereinbarkeit von Beruf mit anderen Lebensbereichen jedoch nicht eindeutig. So empfinden manche Freelancer die 
Arbeitsbedingungen als einschränkend, während andere diese als fördernd wahrnehmen (S. 259).

Die Ergebnisse der Befragung von Hochschulabsolventinnen und Hochschulabsolventen zu ihrer Erwerbstätigkeit durch das Bundesamt für Statistik (BFS, 2013b) zeigen ein klareres Bild. Hier zeigen sich fünf Jahre nach Studienabschluss die Selbstständigen ebenso zufrieden mit der Situation ihrer Erwerbstätigkeit in Bezug auf den Raum, den sie für ihr Privat- und Familienleben bietet, wie Angestellte. Einen weiteren Hinweis dafür, dass sich Selbstständigkeit gut mit anderen Lebensbereichen vereinen lässt, sieht das Bundesamt für Statistik darin, dass selbstständige Frauen mehr als doppelt so häufig von zu Hause aus arbeiten als selbstständige Männer (BFS, 2006, S. 10).

Dorsemagen et al. (2012, S. 19) geben zu bedenken, dass die Überlappung der beruflichen und privaten Sphären nicht per se zu negativen Folgen führen muss und insbesondere für die Generation $Y$ eine scharfe Trennung zwischen privaten und beruflichen Tätigkeiten scheinbar weder möglich noch gewünscht ist. Gàbor Jánszky (2010, S. 20) sieht die zukünftige Entwicklung der Arbeitsformen in diesem Zusammenhang positiv und beschreibt das Entstehen von Patchwork-Identitäten, in denen sich das private und berufliche Leben aus Mosaiksteinen zusammensetzen: Partnerinnen und Partner, Kinder, Wohnorte sowie Tätigkeiten und Projekte.

Die Vereinbarkeit von Beruf mit anderen Lebensbereichen in der Arbeitswelt des selbstständigen Noah Schmid, welche viele Elemente mit derjenigen der Teilzeitangestellten Ursula Meyerhans teilt, jedoch noch stärker individualistisch geprägt ist, verbessert sich im Vergleich zur Gegenwart auf gesamtwirtschaftlicher Ebene.

Andrea Burri-Lötscher befindet sich in einer Situation, bei der die sogenannte "Care-Arbeit" eine wichtige Rolle spielt und mit der Notwendigkeit, ein Erwerbseinkommen erzielen zu müssen, vereinbart werden muss. Gemäss dem Eidgenössischen Büro für die Gleichstellung von Mann und Frau (EBG) (2012) erhoffte man sich in den letzten Jahren vom grösseren Angebot an flexiblen Arbeitszeitmodellen, dass sich Care-Arbeit und Beruf besser miteinander vereinbaren lassen. Weil Frauen häufiger Care-Arbeit verrichten und das damit verbundene Armutsrisiko tragen, wäre ihre Entlastung besonders hoch (EBG, 2012). Wie aus den vorausgegangenen Abschnitten hervorgeht, ist unter zukünftigen Bedingungen, wenn überhaupt, nur eine eher geringe Verbesserung der Vereinbarkeit durch eine stärkere Verbreitung von flexiblen Arbeitsformen zu erwarten. Eine Verbesserung war auch bei der Einführung des Vouchersystems in Belgien eine beabsichtigte Wirkung für die Auftraggebenden (Marx \& Van- 
delannoote, 2014, S. 13). Voucher-Käuferinnen und -Käufer sollten mehr Zeit bei der Arbeit oder mit ihren Familien verbringen können, während vermittelte Auftragnehmende z.B. Haushaltsarbeiten übernehmen sollten. Nur rund 10 Prozent der Käuferinnen und Käufer von Dienstleistungschecks arbeiteten jedoch dank diesen auch mehr, während immerhin gut 40 Prozent mehr Zeit mit ihren Familien verbrachten oder anderen Freizeitaktivitäten nachgingen (ebd., S. 13). Somit subventioniert das System der Vouchers primär die Freizeit von Wohlhabenden. Inwiefern sich die Vereinbarkeit von Beruf mit anderen Lebensbereichen seitens der Beauftragten der Dienstleistungschecks verändert hat, wurde jedoch nicht untersucht. Angermann und Eichhorst (2013) kommen jedoch in diesem Zusammenhang zum Schluss, dass die Ausgestaltung der Gutscheinsysteme, insbesondere das Auftreten der Vermittlungsagenturen als reguläre Arbeitgeber, entscheidend ist.

\subsubsection{Arbeitslosigkeit}

Für die Abschätzung der Auswirkungen der personabasierten Arbeitswelten auf die Arbeitslosigkeit bzw. auf die Quote der Arbeitslosen wurden zwei Masse verwendet, einerseits die Arbeitslosigkeit gemäss der Definition der Internationalen Arbeitsorganisation der Vereinten Nationen (ILO) und andererseits die durch das Staatssekretariat für Wirtschaft (SECO) ausgewiesenen, bei Regionalen Arbeitsvermittlungszentren (RAV) registrierten Arbeitslosen. Gemäss der ILO-Definition ist eine Meldung beim RAV keine Voraussetzung, um als arbeitslos zu gelten, und sie umfasst somit auch ausgesteuerte Personen und solche, die sich noch nicht registriert haben. Die beiden Definitionen führen gemeinhin auch zu den Massen Erwerbslosenquote (ILO) und Arbeitslosenquote (SECO), wenn die absolute Zahl der Arbeitslosen in Beziehung zur Zahl der Erwerbspersonen gesetzt wird. Durch die Unterschiede in den beiden Konzepten ist die Erwerbslosenquote historisch gesehen immer grösser als die Arbeitslosenquote.

Dem fest angestellten Kadermitglied Roland Müller droht durch seine Arbeit im Homeoffice keine Arbeitsplatzunsicherheit. Flexibilitätsanforderungen können jedoch möglicherweise auf der individuellen Ebene als Bedrohung der Vollzeitanstellung oder genereller der Arbeitsplatzsicherheit empfunden werden, wenn Arbeitnehmende innen gegenüber eher negativ eingestellt sind. Die räumliche und zeitliche Flexibilisierung der Arbeit steht jedoch bei einer Betrachtung auf der Makroebene nicht in einem kausalen Zusammenhang mit der Arbeitslosigkeit und hat keine oder nur unwesentliche Folgen für sie. 
Aufgrund der starken Abhängigkeit zwischen Arbeitszufriedenheit und der Präferenz der Arbeitnehmenden für Arbeitsformen, wie Ursula Könitz sie als meist mobil arbeitende Projektmanagerin ausübt, ist es denkbar, dass es vermehrt zu Kündigungen durch Arbeitnehmende kommt, die sich den gestiegenen Anforderungen nicht mehr gewachsen fühlen. Auch Änderungskündigungen aufgrund einer flexibilisierungsbedingten Reduktion der Jahresarbeitszeit könnten vermehrt auftreten. Arbeitnehmende, die eine solche Reduktion akzeptieren, könnten zu einer höheren Teilarbeitslosigkeit führen. Inwiefern sich solche empirisch nicht gefestigten Phänomene gesamtwirtschaftlich auf die Arbeitslosigkeit auswirken, kann jedoch nicht ermittelt werden, und es wird somit von unwesentlichen Veränderungen ausgegangen.

Die stärkere Verbreitung von atypischen Arbeitsverhältnissen in der Arbeitswelt von Ursula Meyerhans bleibt für die auf gesamtwirtschaftlicher Ebene beobachtete Arbeitslosigkeit nicht ohne positive bzw. negative Folgen. Für die Schweiz zeigen Henneberger et al. (2004, S. 44), dass zumindest in den von innen untersuchten Jahren (1999-2001) rund ein Drittel aller Personen ohne Erwerbstätigkeit über einen befristeten Arbeitsvertrag in den Arbeitsmarkt eintreten. Ohne diese Eintritte erhöht sich prinzipiell die Arbeitslosigkeit, wie sie die ILO misst, und ihr Einfluss auf die Anzahl der registrierten Arbeitslosen kann sich vor allem durch eine geringere Arbeitslosigkeit bei Ausbildungsabgängerinnen und Ausbildungsabgängern bemerkbar machen.

Aus der Untersuchung von Keller und Seifert (2011a) in Deutschland ist bekannt, dass Übergänge aus atypischer Beschäftigung in Normalarbeitsverhältnisse seltener gelingen als Übergänge zwischen zwei Normalarbeitsverhältnissen. Auch Henneberger et al. (2004) erwähnen in diesem Zusammenhang gewisse Persistenzprobleme befristeter Beschäftigung in der Schweiz (S. 43). Die deutschen Forscher betonen, dass atypische Arbeitsverhältnisse folglich keine Brückenfunktion zu unbefristeten Vollzeitstellen erfüllen und tendenziell wieder in atypische Stellen münden oder sogar, wie in mindestens 40 Prozent der Fälle, in die Arbeitslosigkeit (Keller \& Seifert, 2011a, S. 142).

Die Beschäftigungsstabilität für befristete Beschäftigte, ob sie geringfügig, Volloder Teilzeit arbeiten, fällt ebenfalls ungünstiger aus als bei Normalarbeitsverhältnissen (Keller \& Seifert, 2011a, S. 141). Somit ist das Risiko, arbeitslos zu werden, für die Personen mit diesen Arbeitsformen höher als für Personen mit unbefristeten Vollzeitstellen. Während für unbefristete Teilzeitbeschäftigung das Gegenteil zutrifft, gelingt ein Übergang zu Vollzeit nur in zehn Prozent der Fälle (ebd., S. 141-142). 
Die Arbeitslosigkeit könnte weiter dadurch wachsen, dass in der Zeit der zunehmenden gesamtwirtschaftlichen Flexibilisierung vermehrt Personen in Normalarbeitsverhältnissen, die keine Pensenreduktion wollen, entlassen werden. Auch unfreiwillige Pensenreduktionen könnten über die Zunahme der Teilarbeitslosigkeit zu einer grösseren Arbeitslosigkeit und Belastung der Arbeitslosenversicherung führen.

Obwohl sich die Effekte kaum quantifizieren lassen, muss als Folge der Entwicklungen, die zur Arbeitswelt von Ursula Meyerhans führen, anhand der dargestellten Studienresultate mindestens mit einer tendenziell zunehmenden Arbeitslosigkeit gerechnet werden.

Nebst den bereits für Ursula Meyerhans beschriebenen Effekten, die eine grössere Verbreitung von Befristung, Teilzeit, geringfügige Beschäftigung und andere atypische Arbeitsverhältnisse auf die Arbeitslosigkeit haben, kommen in Noah Schmids Arbeitswelt noch die Auswirkungen der neuen Selbstständigkeit hinzu. Grundsätzlich käme es zu einer Verschiebung der Anteile zuvor Selbstständiger und Angestellter an den Arbeitslosen. Der Anteil der Selbstständigen ist zwar in der Schweiz historisch tief, unter den skizzierten zukünftigen Bedingungen wird dies aber kaum so bleiben. Durch die angenommene Zunahme der Selbstständigkeit muss unter Berücksichtigung der Überlebensrate von ca. 50 Prozent fünf Jahre nach der Unternehmensgründung (Meyer \& Sidler, 2009, S. 11) von einem wachsenden Anteil ehemals Selbstständigerwerbender an den Arbeitslosen ausgegangen werden. Die gescheiterten neuen Selbstständigen würden so direkt die Arbeitslosigkeit gemäss ILO und, falls sie sich beim RAV registrieren lassen, auch die Zahl der registrierten Arbeitslosen vergrössern. Da sie generell nicht zu einer Arbeitslosenentschädigung berechtigt sind, würden viele vor existenziellen Problemen stehen.

Durch die Strukturen des Arbeitsmarktes, auf dem sich Noah Schmid als SoloSelbstständiger bewegt, könnte ausserdem die Teilarbeitslosigkeit steigen, da wenig Vollzeitstellen angeboten werden, was einen weiteren Grund darstellt, von einer leicht steigenden Arbeitslosigkeit auszugehen.

Für Arbeitnehmende mit atypischer Beschäftigung wie Andrea Burri-Lötscher wird es gemäss Keller und Seifert (2011) zunehmend schwieriger, Wissen und Fertigkeiten an veränderte, aktuelle Qualifikationsanforderungen anzupassen. Sie sind beim Zugang zu betrieblich-beruflicher Weiterbildung gegenüber vergleichbaren Beschäftigten mit Normalarbeitsverhältnissen benachteiligt, wobei geringfügig Beschäftigte am meisten Diskriminierung in diesem Zusammenhang erleiden, gefolgt von befristet Beschäftigten und temporär Arbeitenden (S. 142). Die Flexibilisierung birgt je nach Ausgestaltung des atypischen Arbeitsverhält- 
nisses also die Gefahr der Dequalifizierung der Arbeitnehmenden (Marti et al., 2007, S. 18). Da es für die Arbeitgebenden zunehmend einfacher wird, geeignete Personen im Ausland zu suchen und Aufgaben auszulagern, werden mehr Personen länger in der Arbeitslosigkeit verweilen als unter heutigen Bedingungen. In der Folge kann es gesamtwirtschaftlich zu einem so genannten Hysterese-Effekt kommen, bei dem die Arbeitslosigkeit auf einem höheren Niveau verharrt (Marti et al., 2007, S. 18).

Von der Einführung von Dienstleistungschecks, etwa für die Verrichtung von persönlichen Dienstleistungen oder für einfache Arbeiten der öffentlichen Hand, kann aufgrund der empirischen Evidenz aus Belgien kein vermindernder Effekt auf die Arbeitslosigkeit erwartet werden. Personen, die durch das Vouchersystem Beschäftigungen finden, verdrängen oft andere, die im normalen Arbeitsmarkt eigentlich die gleichen Tätigkeiten ausführen bzw. anbieten (Marx \& Vandelannoote, 2014, S. 14). Dadurch nimmt die Zahl der registrierten Arbeitslosen zu, und zwar um Personen, deren Arbeitsmarktintegration sich in Andrea BurriLötschers Arbeitswelt eher schwierig gestaltet. Als Veranschaulichung dafür kann eine Befragung von Personalberatenden und Arbeitsvermittelnden der Arbeitsmarktbehörden im Jahr 2008 herangezogen werden (Amosa, 2008). Die drei meistgenannten Hindernisse bei der Integration von Geringqualifizierten in den Arbeitsmarkt waren Deutschkenntnisse, Fachkenntnisse und körperliche Gesundheit. Insbesondere Personalberatende, die viel Kontakt mit Arbeitgebenden hatten, betonten Sprachprobleme und Zuverlässigkeit als die Haupthindernisse bei der Stellensuche von Geringqualifizierten (S. 11).

\subsubsection{Beschäftigte}

Dieser Abschnitt bezieht sich auf die Veränderung bei der Anzahl der Arbeitskräfte, die mindestens einer Erwerbstätigkeit nachgehen bzw. eine Stelle besetzen. Dabei spielt es keine Rolle, wo die Arbeitsleistung erbracht wird, z.B. im Betrieb, zu Hause, beim Kunden, in einem Coworking-Space usw.

Mit Blick auf die Arbeitswelten der beiden Kaderangestellten Roland Müller und Sandra Könitz lassen sich keine Aussagen über den Zusammenhang zwischen Beschäftigten sowie räumlich und zeitlich flexibilisierter Arbeit von Festangestellten ableiten. Eine naheliegende und plausible Folge der verbesserten Vereinbarkeit von Beruf mit anderen Lebensbereichen durch die Arbeit von zu Hause (siehe Abschnitt 4.3.6) könnte sein, dass sich mehr nicht erwerbstätige Personen entscheiden könnten, eine Erwerbstätigkeit aufzunehmen, beispielsweise gut qualifizierte Frauen, die ihre Erwerbstätigkeit nach einer Mutterschaft aufgegeben haben. Ausserdem könnten Firmen möglicherweise eher dazu 
geneigt sein, Arbeitnehmende in kleineren Pensen anzustellen, wenn sie die Kosten der Arbeitsplatzinfrastruktur vermeiden bzw. sehr gering halten können.

Aus einer Studie des Bundesamtes für Energie (BFE, 2014) geht hervor, dass die Anzahl Mitarbeitende einer Unternehmung einen positiven Zusammenhang mit deren Angebot räumlich flexibler Arbeitsformen hat, die kausale Richtung der Wirkung wird in der Studie jedoch nicht untersucht. Grundsätzlich ist es denkbar, dass es grösseren Unternehmen leichter fällt, die Bedingungen für erfolgreiches räumlich flexibilisiertes Arbeiten zu bieten als Kleinunternehmen. Andererseits könnten aber auch die mit räumlich flexibilisierten Arbeitsformen in Verbindung stehenden Effizienzgewinne dafür sorgen, dass Unternehmen wachsen und mehr Mitarbeitende anstellen.

Unter den Bedingungen von Ursula Meyerhans' Arbeitswelt, insbesondere der stärkeren Verbreitung von geringfügiger Beschäftigung, Portfoliowork, Teilzeit und befristeten Arbeitsverhältnissen, kommt neuen Unternehmen eine besondere Bedeutung bei der Arbeitsnachfrage zu. Start-ups sind wichtige Treiber für qualitative Veränderungen in der Arbeitswelt und die Verbreitung von neuen Arbeitsformen (Koch, Pastuh \& Späth, 2013). Koch und Späth (2009) beschreiben für Deutschland, dass höhere Risiken mehr Flexibilität notwendig machen und Start-ups auf vergleichsweise grössere funktionale, numerische und finanzielle Flexibilität setzen als etablierte Unternehmen. Start-ups können diese erreichen, indem sie Höherqualifizierte in Teilzeit und anderen atypischen Arbeitsverhältnissen beschäftigen und ihre Löhne an ihre marginale Produktivität koppeln (Koch \& Späth, 2009). In Deutschland kann diese Dynamik empirisch mit quantitativen und qualitativen Daten belegt werden. Späth (2013) zeigt in einer quantitativen Untersuchung von Daten aus dem deutschen BetriebsHistorik-Panel (BHP), dass junge Firmen signifikant häufiger auf sogenannte „Midi Jobs“, Freelancer, befristete Anstellungen, Überstunden und Vertrauensarbeitszeit zurückgreifen als etablierte. Das bestätigt frühere Resultate von Koch und Späth (2009), die zeigen, dass junge Firmen numerisch flexibilisieren, indem sie mehr Teilzeit, mehr Arbeitnehmende mit weniger als 18 Arbeitsstunden pro Woche und mehr geringfügig Beschäftigte einsetzen, und funktional flexibilisieren, indem sie weniger Personal mit mittlerem und geringem Qualifikationsniveau beschäftigen.

Die Zahl der Beschäftigten und ihre Arbeitsbedingungen dürften somit auch in der Schweiz nicht unwesentlich vom Ausmass der neu gegründeten Unternehmen abhängen. Die Tatsache, dass die tertiäre Bildung das Unternehmertum fördert und Studierende vermehrt in der Unternehmensgründung ausgebildet werden, hat hierauf einen nicht zu vernachlässigenden Effekt. 
In der Schweiz setzen Unternehmen, die neu gegründet werden, häufig Teilzeitangestellte ein. Wie in Abbildung 12 dargestellt, ist bei neu gegründeten Unternehmen der Anteil der Teilzeitbeschäftigten an allen Beschäftigten hoch. Im Vergleich zu 2001 ist der Anteil im Jahr 2013 um 37,5 Prozent gestiegen und betrug knapp 38 Prozent. Im gleichen Zeitraum ging die Anzahl der Beschäftigten von Start-ups um beinahe 10 Prozent zurück, die Zahl der Vollzeitbeschäftigten sogar um gut 22 Prozent.

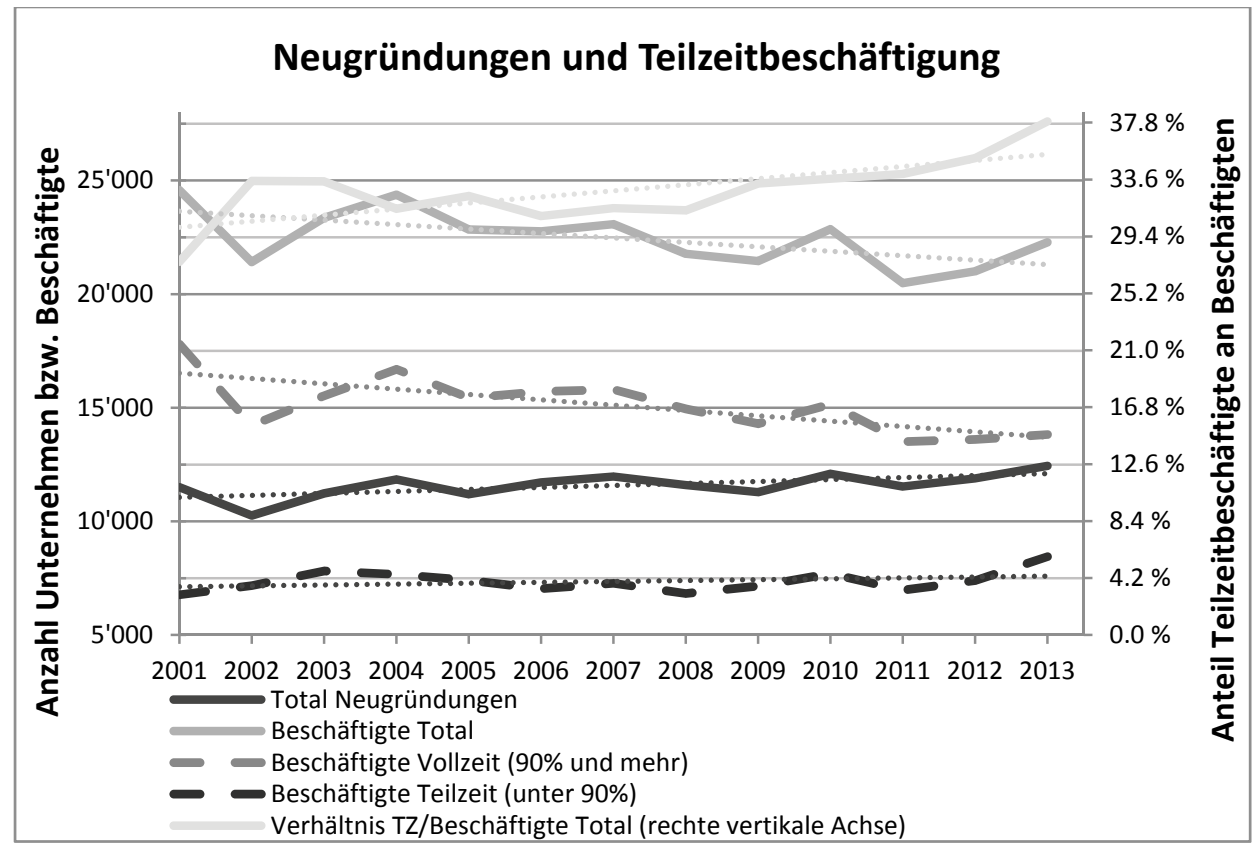

Abbildung 12: Neugründungen und Beschäftigte in der Schweiz (Zahlenquelle: BFS Statistik der Unternehmensdemografie, eigene Berechnungen und Darstellung). Gepunktete Linien: jeweiliger linearer Trend

Eine mögliche Folge der zunehmenden Anzahl Teilzeitbeschäftigter ist die Zunahme von Erwerbstätigen, die mehr als einer Erwerbstätigkeit nachgehen, z.B. weil das Einkommen von einer Arbeitsstelle nicht mehr ausreicht, um davon leben zu können. Abbildung 13 bildet die Quote solcher Personen an den jeweiligen nationalen Erwerbstätigenzahlen ab. In der Schweiz fand im Zeitraum von 15 Jahren eine Steigerung um 24 Prozent statt, was vergleichbar mit denjenigen im Euroraum und in Frankreich ist. Während in Österreich eine Abnahme zu beobachten war, stieg die Quote in Deutschland um 118 Prozent. 


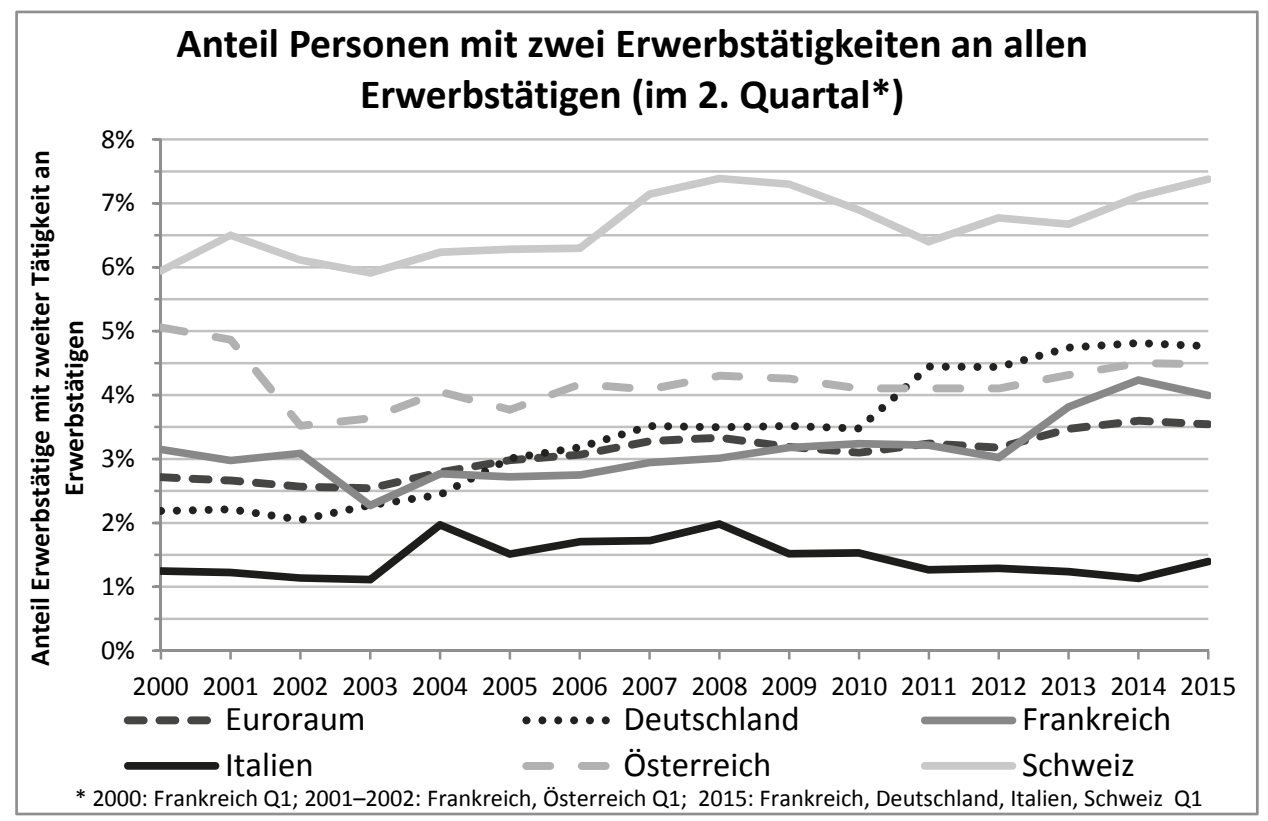

Abbildung 13: Quote der Erwerbstätigen mit zwei Tätigkeiten (Zahlenquelle: Eurostat)

Der Effekt, den die Befristung von Arbeitsverträgen auf die Beschäftigtenzahl hat, ist bisher wenig erforscht. Allgemein gelten befristete Arbeitsverhältnisse oft als Eintrittsmöglichkeit in die unbefristete und unselbstständige Beschäftigung. Ortega und Marchante (2010) konnten für Spanien empirisch nachweisen, dass ein Anstieg der befristeten Arbeitsverträge einen Rückgang bei der Selbstständigenquote nach sich gezogen hat. Ein positiver Nettoeffekt auf die Beschäftigung konnte aber nicht belegt werden.

Unter den Bedingungen der Arbeitswelt von Ursula Meyerhans scheint zumindest ein Anstieg der Teilzeitstellen plausibel. Die Zahl der Beschäftigten muss aber nicht zwangsläufig wachsen, da die Quote der Erwerbstätigen, die mehr als eine Stelle haben, seit Jahren ansteigt. Der gesamtwirtschaftliche Effekt auf die Beschäftigten ist daher unsicher.

Die Arbeitswelt des selbstständigen Noah Schmid ist stark durch die Tertiärisierung geprägt und der Anteil des Dienstleistungssektors an der Gesamtwirtschaft ist gegenüber heute noch grösser. Das ist für die Beeinflussung der Beschäftigung durch die Aufnahme der Selbstständigkeit insofern relevant, als dass die durchschnittliche Unternehmensgrösse kleiner ist als im zweiten Sek- 
tor und dass die Eintrittsbarrieren bedeutend tiefer sind (Wennekers et al., 2010, S. 18).

Der Effekt von Start-ups auf die Beschäftigung konnte durch Fritsch (2008) auf Basis westdeutscher Daten im Modell nachgewiesen werden. Gemäss seiner Interpretation (S. 8) gibt es zum Zeitpunkt der Neugründung einen direkten Beschäftigungszuwachs, der in Abbildung 14 durch die Fläche I gekennzeichnet ist und nach etwa einem Jahr stagniert. Die folgenden Jahre sind durch die Selektion des Marktes gekennzeichnet. Schon länger bestehende Unternehmen werden von den Neugründungen verdrängt und einige der Start-ups scheitern am Wettbewerb. Dieser Zeitabschnitt (Fläche II in Abbildung 14) ist von einem negativen Beitrag der Neugründungen auf die Beschäftigung gekennzeichnet. Erst nach ca. sechs Jahren dominieren dann Effekte auf der Angebotsseite, wie die verbesserte Wettbewerbsfähigkeit, die zu einer positiven Veränderung in der Beschäftigung durch Neugründungen führen (Fläche III in Abbildung 14), bis sie nach rund zehn Jahren nicht mehr nachweisbar sind.

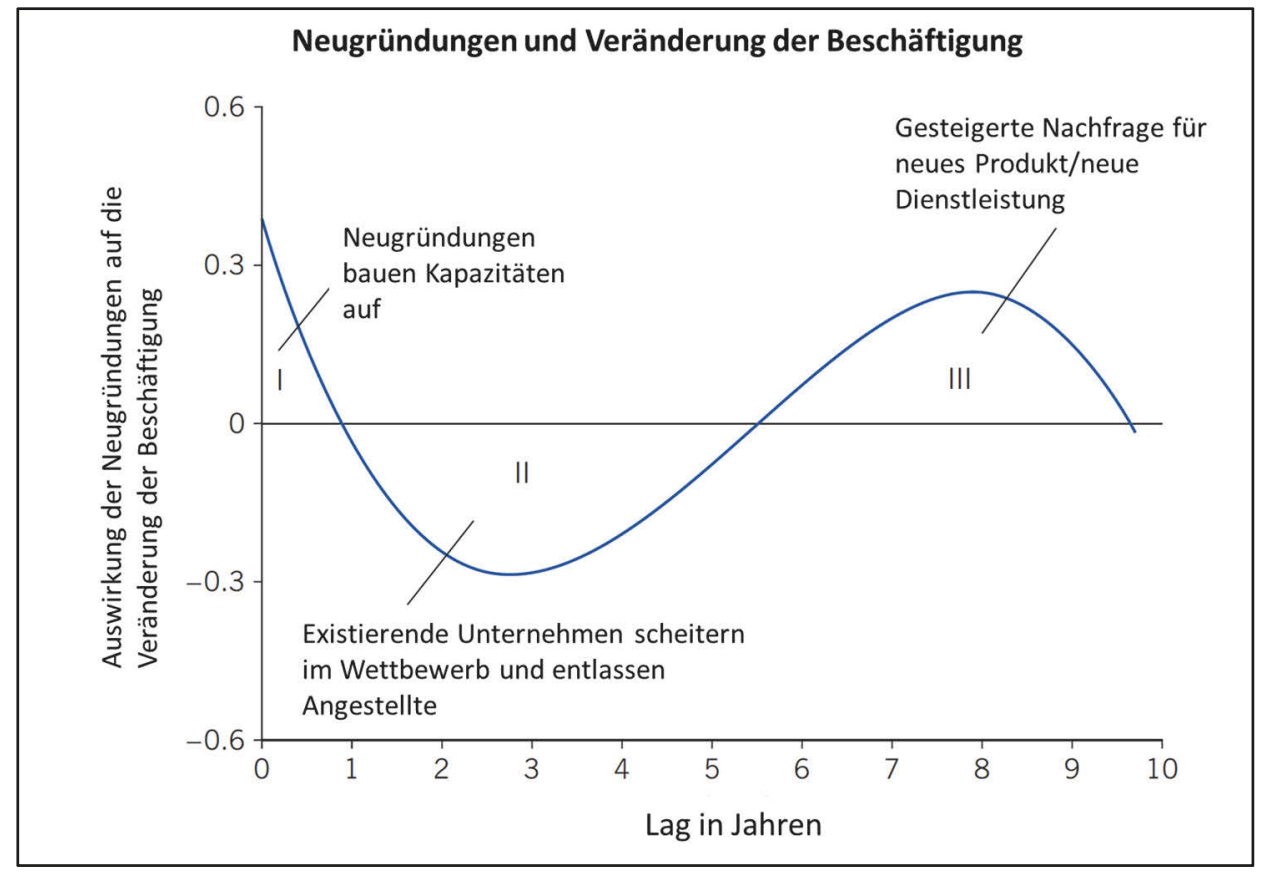

Abbildung 14: Neugründungen und Veränderung der Beschäftigung nach Fritsch $(2008$, S. 8) 
Van Praag und Versloot (2008) ergänzen Fritschs Befund und folgern aus ihrer Literaturanalyse, dass der wichtigere und langfristigere Effekt von neuen Unternehmen auf die Beschäftigung nicht nur durch ihre neu geschaffenen Stellen entsteht, sondern auch durch den zusätzlichen Wettbewerbsdruck, der die gesamte Nachfrage nach Arbeitskräften stimuliert.

Baumgartner, Schulz \& Seidl (2013) beurteilen den kurz- und mittelfristigen Einfluss des Unternehmertums auf die Beschäftigung in ländlichen Regionen der Schweiz, welche ca. einen Viertel der Schweizer Bevölkerung umfassen und kommen zum Schluss, dass ihr Modell den durch die Fläche II in Abbildung 14 beschriebenen Effekt bestätigt, auch wenn dieser statistisch nicht signifikant und eher klein ist. Die verwendeten Daten lassen aber keine Beurteilung des für Deutschland festgestellten längerfristigen Beschäftigungszuwachses zu (S. 235). Somit besteht Unsicherheit, ob eine Zunahme der Unternehmen in der ländlichen Schweiz langfristig auch zu mehr Beschäftigung führt. Diese Unsicherheit kann auch auf urbanere Regionen übertragen werden. Sie wird insbesondere durch die Tatsache genährt, dass Studien zu Start-ups und Unternehmertum generell zu wenig zwischen der grossen Anzahl wirtschaftlich marginaler, winziger und leistungsschwacher Unternehmen und sog. „Gazellen“, welche die positive Entwicklung der Wirtschaft vorantreiben, unterscheiden (Nightingale und Coad, 2013).

Die Beschäftigungseffekte, die durch die Zunahme der Selbstständigkeit in Noah Schmids Arbeitswelt entstehen, können somit aufgrund der vorliegenden Resultate aus der Literatur nicht abschliessend beurteilt werden. Ein mittelfristiger Rückgang scheint plausibel, nicht zuletzt auch durch die Praxis der Unternehmen bedingt, weniger Arbeitnehmende anzustellen und sich vermehrt nur noch auf die Kernkompetenzen zu konzentrieren. Ausserdem ist unter den Bedingungen der Arbeitswelt von einer Vielzahl Selbstständiger ohne Mitarbeitende auszugehen. Ehemals Angestellte oder neu in den Arbeitsmarkt tretende Menschen, denen der Schritt in die Solo-Selbstständigkeit nicht gelingt, könnten Schwierigkeiten haben, eine Beschäftigung zu finden. Der Umstand, dass Handwerkerinnen und Handwerker, unqualifizierte Angestellte sowie Lehrabgängerinnen und Lehrabgänger eine schwache Neigung zum Übergang in die Selbstständigkeit haben (BFS, 2006, S. 17), zeugt von den möglichen Problemen, welche diese Personen in Noah Schmids Arbeitswelt haben könnten.

Die gesellschaftlichen und demografischen Veränderungen, wie die Überalterung und die steigende Erwerbsquote bei Frauen, eröffnen für Geringqualifizierte wie Andrea Burri-Lötscher Beschäftigungspotenziale (Amosa, 2008, S. 8). So könnte die Zahl der beschäftigten Geringqualifizierten, welche infolge des technologischen Fortschritts und der Globalisierung durch Rationalisierungen, Au- 
tomatisierungen und neue Informations- und Kommunikationstechnologien unter Druck geraten sind (ebd., S. 8), in der Arbeitswelt von Andrea BurriLötscher wieder zunehmen.

Durch die Polarisierung der Nachfrage nach Arbeitskräften mit eher geringen und sehr hohen Qualifikationen könnte für Arbeitnehmende, die dazwischen liegen, eine schwierige Situation entstehen. Oesch und Rodriguez Menes (2010) konnten mit Daten der Schweizerischen Arbeitskräfteerhebung (SAKE) zeigen, dass dies im Zeitraum von 1991 bis 2008 in der Schweiz bereits der Fall gewesen ist und die Beschäftigung für die Gruppe der mittleren Einkommensempfängerinnen und -empfänger im Vergleich zu den anderen Gruppen stärker zurückgegangen ist (S. 528). Für viele der neuen Stellen sind Arbeitnehmende mit mittleren Qualifikationen entweder zu wenig oder zu hoch qualifiziert. Ihre Erwerbsquote könnte als kurz- und mittelfristige Folge sinken, einerseits weil sie sich freiwillig gegen die Aufnahme einer Erwerbstätigkeit entscheiden, da sie sich z.B. lieber der Familienarbeit widmen, als Jobs auszuüben, in denen sie sich unterfordert fühlen oder schlechte Arbeitsbedingungen herrschen. Dieser Umstand wird auch vom britischen Ökonomen Guy Standing beschrieben. Er hat verschiedene Hypothesen zu dieser neuen sozialen Klasse, die er Prekariat nennt, formuliert und untersucht. Gemäss seinen Beobachtungen ist diese Klasse die erste, welche höhere Qualifikationen haben muss, als für die Ausübung ihrer Arbeit nötig sind (Standing, 2014, S. 970). Andererseits könnten viele Personen mit mittleren Qualifikationen aufgrund ihrer höheren Lohnerwartungen und dem Überangebot von Geringqualifizierten kaum Chancen haben, einen der neuen Jobs im Bereich der persönlichen Dienstleistungen, im Detailhandel oder Gastgewerbe zu ergattern. Ein möglicher Ausweg besteht für sie in der Aufnahme der Selbstständigkeit.

Ob die Zunahme der Beschäftigung von Hochqualifizierten, wie sie seit Beginn der 1990er-Jahre bis 2008 beobachtet wurde (Oesch \& Rodriguez Menes, 2010), weitergehen kann, hängt stark davon ab, ob die Expansion von Nichtroutine und interaktionslastigen Aufgaben durch die technologischen und strukturellen Veränderungen gebremst wird. Einen Hinweis dazu, dass dies bereits heute geschieht, könnte die in Abbildung 15 und Abbildung 16 dargestellte abnehmende oder stagnierende Ausbildungsniveauadäquan $z^{6}$ und die Erwerbs-

6 BFS (2015): „Zur Berechnung der Ausbildungsniveauadäquanz wird die Frage ,Wurde für Ihre jetzige Haupterwerbstätigkeit von Ihrem Arbeitgeber ein akademischer Abschluss oder Fachhochschulabschluss verlangt?' herbeigezogen. Die Ausbildungsniveauadäquanz gibt den Anteil der Absolventinnen und Absolventen an, deren Erwerbstätigkeit einen Hochschulabschluss voraussetzt." 
quote gemäss ILO der Hochschulabsolventinnen und -absolventen ein Jahr nach Studienabschluss geben.

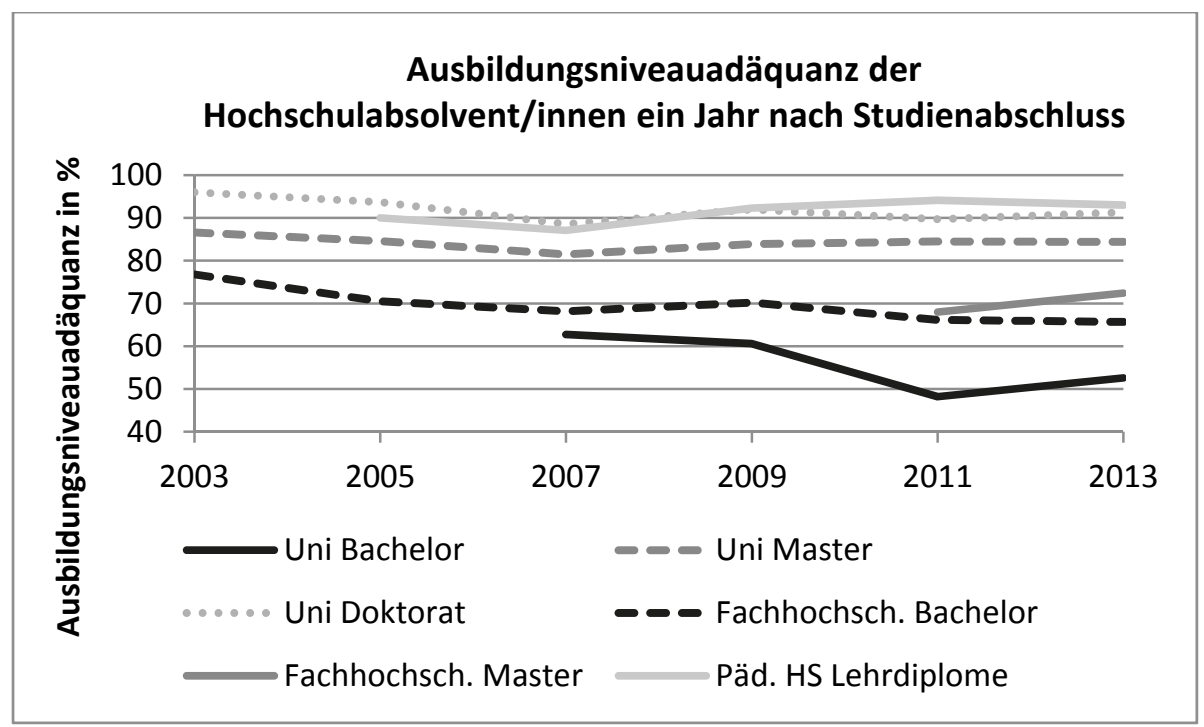

Abbildung 15: Ausbildungsniveauadäquanz der Hochschulabsolvent/innen 2003-2013 (Zahlenquelle: BFS (2013b))

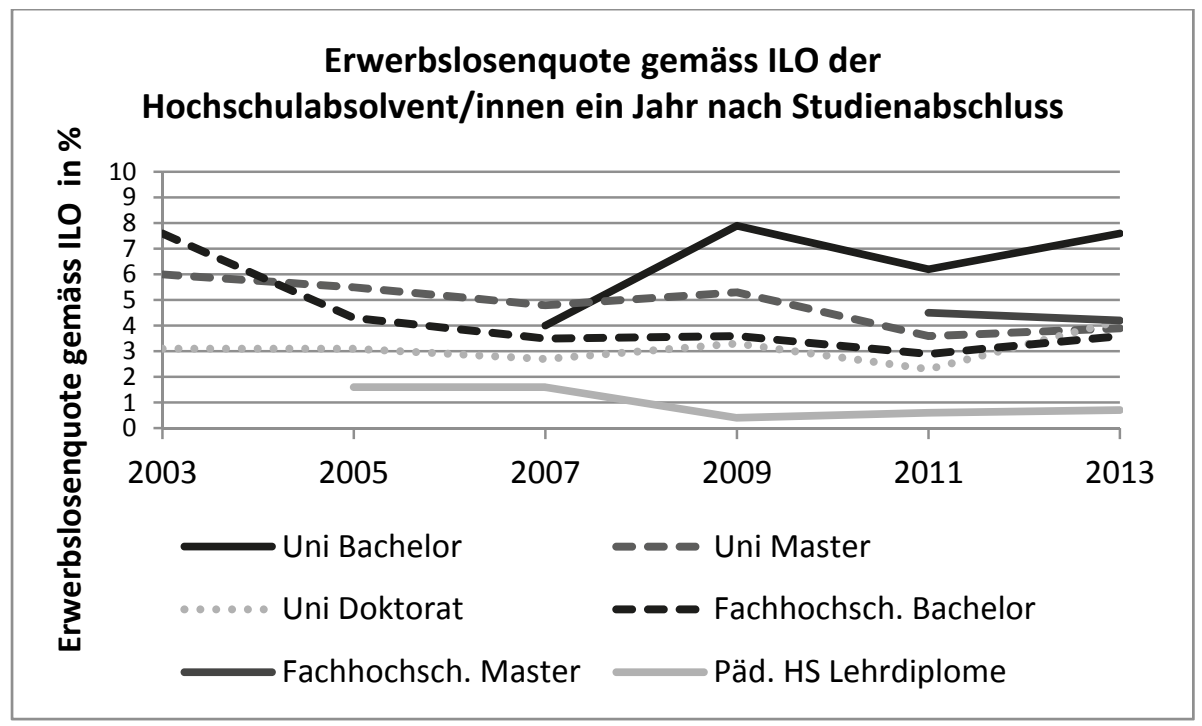

Abbildung 16: Erwerbslosenquote gemäss ILO der Hochschulabsolvent/innen 2003-2013 (Zahlenquelle: BFS (2013b)) 
Die Frage nach der Qualität der Beschäftigung wird möglicherweise in der Arbeitswelt von Andrea Burri-Lötscher für viele wichtiger sein als die Frage, ob überhaupt eine Beschäftigung gefunden werden kann.

\subsubsection{Steuererträge}

Dieser Indikator umfasst die Auswirkungen der Arbeitswelten hinsichtlich der Fiskalerträge auf allen Staatsebenen. Dabei wurden bei den direkten Steuern die Einkommens- und Vermögenssteuern für natürliche Personen und für juristische Personen die Gewinn- und Kapitalsteuern berücksichtigt. Als indirekte Steuer wurde die Mehrwertsteuer, die auf Bundesebene anfällt, mit einbezogen.

Da Homeoffice, das prägende Element in Roland Müllers Arbeitswelt, keine bedeutenden Auswirkungen auf das Einkommen der Arbeitnehmenden hat und die Unternehmen durch die Arbeitsform per se nicht profitabler werden, kann davon ausgegangen werden, dass sich die Erträge aus den Einkommens-, Vermögens-, Gewinn- und Kapitalsteuern dadurch nicht in gesamtwirtschaftlich relevanter Weise verändern. Kleine Veränderungen bei unselbstständig erwerbenden natürlichen Personen sind dadurch zu erwarten, dass häufiger Berufskostenabzüge, z.B. für ein privates Arbeitszimmer oder Informatikmittel, geltend gemacht werden und möglicherweise die Gewinnungskosten kleiner werden, z.B. weil weniger gependelt werden muss und weniger auswärtige Verpflegung konsumiert wird.

Wie bei einer stärkeren Verbreitung von Homeoffice sind auch bei vermehrter räumlicher und zeitlicher Flexibilisierung der Arbeit, wie sie Sandra Könitz praktiziert, kaum gesamtwirtschaftlich bedeutende Auswirkungen auf das Einkommen der Arbeitnehmenden und somit auf die Steuererträge bei natürlichen Personen absehbar. Die häufigere Erfolgsbeteiligung wird zwar individuell zu Veränderungen der Einkommenshöhe führen, dürfte jedoch nur in konjunkturell besonders schwachen bzw. starken Zeiten gesamtwirtschaftlich spürbar werden. Durch die verbesserte Arbeitsproduktivität der Mitarbeitenden (siehe Abschnitt 4.3.1) und die Vorteile der Auslagerung und der Standardisierung sollte sich die Leistungsfähigkeit der Unternehmen verbessern. Als Folge davon sollten die Gewinne steigen und somit auch die Erträge aus deren Besteuerung.

Die Arbeitswelt von Ursula Meyerhans, Teilzeitangestellte und Selbstständige mit verschiedenen Auftraggebern, zeichnet sich durch fallende Steuererträge bei natürlichen Personen aus. Da Teilzeit, Befristung, Solo-Selbstständigkeit und geringfügige Beschäftigung zugenommen haben, gehen die Einkommen zurück und parallel dazu die Erträge ihrer Besteuerung. Obwohl aus heutiger 
Sicht die zusätzlichen Einkommen, die durch Crowdsourcing erzielt werden können, als eher gering eingestuft werden, kommt hinzu, dass die Verbreitung von Crowdsourcing-Plattformen und auf Kryptowährung basierende Zahlungsmöglichkeiten mit ihrem Potenzial, die Schattenwirtschaft zu begünstigen, ebenfalls eine eher ertragsmindernde Auswirkung haben.

Andererseits sollten die Gewinne der Unternehmen durch die gewonnene Flexibilität zunehmen und somit höhere Steuereinnahmen resultieren. Da die Einnahmen der Einkommens- und Vermögenssteuern aller Staatsebenen seit 2008 rund zweieinhalb bis dreimal höher sind als diejenigen der Gewinn- und Kapitalsteuern, ist jedoch nicht mit einen positiven Nettoeffekt zu rechnen, sondern eher mit einem leicht negativen.

Die bereits in Abschnitt 4.3.8 erwähnten Modellberechnungen von Baumgartner, Schulz und Seidl (2013) versuchen auch den Einfluss des lokalen Unternehmertums auf die Einnahmen der Gewinnsteuer des Bundes zu bestimmen. Da die verwendeten Daten vor allem kleine Unternehmen umfassen, die auch in der Arbeitswelt von Noah Schmid eine wichtige Rolle spielen, sind die Resultate für die Beurteilung der Auswirkungen von Noah Schmids Arbeitswelt relevant. Baumgartner, Schulz und Seidl (2013) finden zwar einen positiven Zusammenhang zwischen dem Ausmass des Unternehmertums in ländlichen Gebieten und den Einnahmen der Gewinnsteuer des Bundes. Dieser ist jedoch klein und statistisch nicht signifikant (S. 234). Das Resultat dürfte durch die Tatsache beeinflusst sein, dass die ländlichen Kleinunternehmerinnen und -unternehmer tendenziell eher geringe Qualifikationen aufweisen und kaum über Managementerfahrung verfügen (S. 235). Ihre Selbstständigkeit könnte eher aus der Notwendigkeit, einer Erwerbstätigkeit nachgehen zu müssen, entstanden sein, als das Resultat von Opportunitäten durch Innovationsleistungen darzustellen. Solche Unternehmen sind von tiefer Wertschöpfung und kleinen Gewinnen gekennzeichnet (Fossen \& Büttner, 2013) und ihre Zunahme ist unter den Bedingungen von Noah Schmids Arbeitswelt plausibel. Zwar existieren mehr Selbstständige, sie erzielen tendenziell aber tiefere Einkommen bzw. ihre Unternehmen kleinere Gewinne mit weniger Kapital. Während also die Einnahmen des Staates durch Einkommens-, Gewinn- und Kapitalsteuern eher zurückgehen dürften, ist bei der Mehrwertsteuer aufgrund der zunehmenden Anzahl Transaktionen in einer tertiärisierten Wirtschaft eine Erhöhung der Einnahmen möglich. Welche Nettowirkung resultiert, kann nicht abschliessend beurteilt werden. Die Annahme einer gesamtwirtschaftlichen Abnahme der Erträge aufgrund eines stärkeren Effekts bei den Abgaben der neuen notwendigkeitsgetriebenen Selbstständigen und Solo-Selbstständigen als bei den Mehrwertsteuereinnahmen scheint jedoch plausibel. 
Da die Erwerbseinkommen in der Arbeitswelt von Andrea Burri-Lötscher tendenziell sinken, gehen auch die Erträge aus der Einkommenssteuer zurück. Die Einkommen der Höherqualifizierten in der polarisierten Arbeitskräftelandschaft werden nicht ausreichen, um die tiefen Einkommen der gering- und überqualifizierten Beschäftigten gesamtwirtschaftlich auszugleichen. Ein Grund dafür liegt in der Steuerprogression, welche tiefe Einkommen kaum oder gar nicht steuerlich belastet, ein anderer, dass in Andrea Burri-Lötschers Arbeitswelt genau die Branchen mehr Beschäftigte verzeichnen werden, welche bereits heute durch Niedriglöhne gekennzeichnet sind. Die Einführung eines Vouchersystems ändert an dieser Situation wenig, da es nur geringe Einkommen bzw. Zusatzeinkommen erlaubt. Ausserdem enthalten die Dienstleistungschecks ein Element der Subventionierung, welches seinerseits durch Steuereinnahmen finanziert werden muss.

Da die Polarisierung der Arbeitskräfte ein Resultat der Arbeitsnachfrage der Unternehmen ist, kann nicht ausgeschlossen werden, dass die daraus resultierende Arbeitsorganisation für sie effizienter ist und dadurch höhere Gewinne erwirtschaftet werden. Grosse neu gegründete oder betrieblich umstrukturierte Unternehmen mit volkswirtschaftlicher Bedeutung geniessen jedoch in vielen Kantonen während bis zu zehn Jahren Steuererleichterungen, die auch vom Bund gewährt werden können. Die Bestimmung eines Nettoeffekts seitens der juristischen Personen ist deshalb schwierig.

Unter den heutigen Voraussetzungen des Steuersystems würde die Arbeitswelt von Andrea Burri-Lötscher am ehesten durch die tieferen Einkommen bedingt niedrigere Steuererträge zur Folge haben.

\subsubsection{Intransparente Zahlungsströme}

Inwiefern die Bedingungen, unter denen die verschiedenen Persona arbeiten und entlohnt werden, sich auf das Ausmass der intransparenten Zahlungsströme auswirken, wird im folgenden Abschnitt abgeschätzt. Ausschlaggebend ist hierbei, ob die Schwarzarbeit als Teil der Schattenwirtschaft durch die flexiblen Arbeitsformen begünstigt wird. Eine Zunahme der intransparenten Zahlungsströme wirkt sich in der Folge u.a. auf die Steuererträge und die Systeme der sozialen Absicherung aus.

Homeoffice und andere Formen der räumlichen Flexibilisierung der Arbeit, die Roland Müllers Arbeitswelt prägen, begünstigen nicht per se intransparente Zahlungsströme. Ihr Ausmass, welches zu erfassen im Zusammenhang mit der Schattenwirtschaft bzw. der Schwarzarbeit versucht wird, ist umstritten. Das 
Beitragssystem für die schweizerischen Sozialversicherungen wird allgemein als geeignet dafür bezeichnet, Schwarzarbeit nicht zu fördern (Brechbühl, 2002). Ausserdem geniesst die Schweiz mit ihren im europäischen oder sogar globalen Vergleich tiefen Arbeitslosenzahlen einen gewissen Schutz gegen das Angebot von Schwarzarbeit. Gemäss Haigner, Jenewein, Schneider und Wakolbinger (2013) sind nämlich Arbeitslose sowie ehemals Arbeitslose mit grösserer Wahrscheinlichkeit dazu geneigt, Schwarzarbeit anzubieten, als Erwerbstätige.

Denkbar ist, dass im Homeoffice-Beschäftigte das Vertrauen ihrer Arbeitgebenden missbrauchen könnten, um zu Hause noch für andere Auftraggebende kleinere Aufgaben zu erledigen oder sich auch z.B. über CrowdsourcingPlattformen Aufträge zu sichern, welche dann nicht gesetzeskonform abgerechnet werden. Hierfür gibt es zurzeit aber keine empirischen Belege und das Ausmass der intransparenten Zahlungsströme in Roland Müllers Arbeitswelt unterliegt somit im Vergleich zu heute kaum Veränderungen.

Die räumlich und zeitlich flexibilisierte Arbeitswelt von Sandra Könitz ist geprägt von traditionellen, unbefristeten Arbeitsverträgen. Die dadurch ausgelösten Zahlungsströme, welche auch Bonizahlungen umfassen, sind in der Zukunft nicht stärker durch Intransparenz gekennzeichnet als heute. Es besteht jedoch die Möglichkeit, dass einheimische und ausländische Zuliefernde und Outsourcingnehmende (Mishra \& Makhija, 2013) zunehmend mit Kryptowährungen entschädigt werden oder dass im Handel mit Unternehmen aus Ländern mit unterentwickelten Finanzsystemen solche akzeptiert werden, da sie sichere Transaktionen ohne Banken ermöglichen (Surowiecki, 2011). Kryptowährungen können auch verwendet werden, um Spenden zu tätigen (Silverman, 2015). Zusätzlich könnten nicht monetäre Tauschgeschäfte und Entschädigungen, insbesondere im Zusammenhang mit dem Einsatz von verschiedenen Ausprägungen des Crowdsourcings, in ihrer Bedeutung zunehmen.

Das Potenzial für intransparente Zahlungsströme, die durch die Bedingungen in Ursula Meyerhans' Arbeitswelt hervorgerufen werden können, unterscheidet sich wenig im Vergleich zur Gegenwart. Die Ausnahme besteht in der Verwendung von Kryptowährungen für die Entlohnung von Arbeitsleistungen, die ausserhalb der festen (Teilzeit-)Arbeitsverhältnisse erbracht werden. Das elektronische Zahlungsmittel ist wegen der Chancen, die es der Masse der Unternehmen bietet (Conerly, 2015), zu einem breit akzeptierten Zahlungsmittel im E-Commerce wie auch im traditionellen Geschäft geworden. Es wird entweder direkt zur Zahlung verwendet („Peer-to-Peer“) oder über sogenannte „ThirdParty Payment Processors" zeitnah in traditionelle Währungen umgerechnet, 
zur Zahlung angenommen und dann den angeschlossenen Anbietenden in traditioneller Währung ausbezahlt bzw. überwiesen. Dadurch könnten Lohnzahlungen auch in Kryptowährungen beliebt werden, insbesondere wenn sie über nationale Grenzen hinweg gemacht werden. Es ist denkbar, dass im Crowdsourcing Kryptowährungen für Lohnzahlungen verwendet werden, die dann u.a. über die „Third-Party Payment Processors“ ausgegeben werden. Crowdarbeiter und -arbeiterinnen könnten auch in traditionellen Währungen bezahlt werden, die zuvor vom Auftraggebenden in Kryptowährung über einen „Third-Party Payment Processors“ überwiesen wurden. Vor allem die direkte Zahlung („Peer-to-Peer") kommt einer Zahlung in bar nahe. Bargeldzahlungen sind für schattenwirtschaftliche Tätigkeiten besonders wichtig, da sie vertraulicher sind als rückverfolgbare Zahlungsaufträge (Schaltegger \& Schneider, 2005, S. 2). Kryptowährungen erfüllen diese Bedingung noch besser als Bargeld, und Zahlungen können völlig anonym und elektronisch gemacht werden.

Schneider (2001, S. 426 f.) sieht die Ursachen für die Schattenwirtschaft und den damit verbundenen Zahlungsströmen im Zusammenhang mit der generellen Ausdehnung der Staatstätigkeit, in Verbindung mit strukturellen Veränderungen auf dem Arbeitsmarkt und dem Beschäftigungssystem sowie mit einem generellen Wertewandel in der Gesellschaft. Schneider und Bookmann (2015, S. 8-11) nennen u.a. als wichtige kausale Determinanten, die nach dem ökonomischen Verhaltensmodell die Schattenwirtschaft fördern, die Steuer- und Sozialversicherungsbeitragslast sowie die Selbstständigkeit. Beide Determinanten dürften sich in der Schweiz in den nächsten zehn bis 15 Jahren so entwickeln, dass die Schattenwirtschaft begünstigt wird. In Kombination mit der Möglichkeit, ganz einfach nicht rückverfolgbare Zahlungen mithilfe von Kryptowährungen tätigen zu können, bilden die beiden Determinanten die Basis für die Schlussfolgerung, dass intransparente Zahlungsströme in der Arbeitswelt von Ursula Meyerhans zunehmen werden. Da moderierende Faktoren bei der Vorhersage des Ausmasses der Schattenwirtschaft wie die Qualität von staatlichen Institutionen, die wirtschaftliche Freiheit, die Qualität und die Quantität von öffentlich bereitgestellten Gütern und Dienstleistungen, die Steuermoral sowie die Entwicklung der offiziellen Wirtschaft in der Schweiz günstig ausgestaltet sind, dürfte die Zunahme gering bleiben.

Grundsätzlich besteht in Noah Schmids Arbeitswelt für die Entstehung von intransparenten Zahlungsströmen ein ähnliches Potenzial wie in der Arbeitswelt von Ursula Meyerhans. Durch die höhere Zahl der Selbstständigen, welche als Folge der Tertiärisierung entsteht, vergrössern sich die Zahlungsströme und somit auch die Entscheidungen der Marktteilnehmenden, ob sie ordentlich abgewickelt werden oder intransparent sein sollen. Während gegenwärtig in man- 
chen Bereichen wie derjenigen der Produktionswirtschaft ausserhalb des Handwerks Schattenwirtschaft aufgrund der Gegebenheiten des Arbeitsprozesses wenig nachgefragt wird, ist sie in anderen z.B. haushaltsnahen Dienstleitungen heute eine relativ leicht verfügbare Option (Schneider \& Tübingen, 2015, S. 27). Die zunehmende Internationalisierung und Ausdehnung von Crowdsourcing-Methoden in vielen Branchen, die bereits beschriebene, einfache Möglichkeit, nicht rückverfolgbare Zahlungen tätigen zu können, und der Nutzen, welcher das Ausüben dieser Möglichkeit bietet, beeinflussen die Entscheidung. Mit zunehmender Zahl von Unternehmen, die Bitcoins und andere Kryptowährungen akzeptieren, steigt auch die Anzahl von Transaktionen.

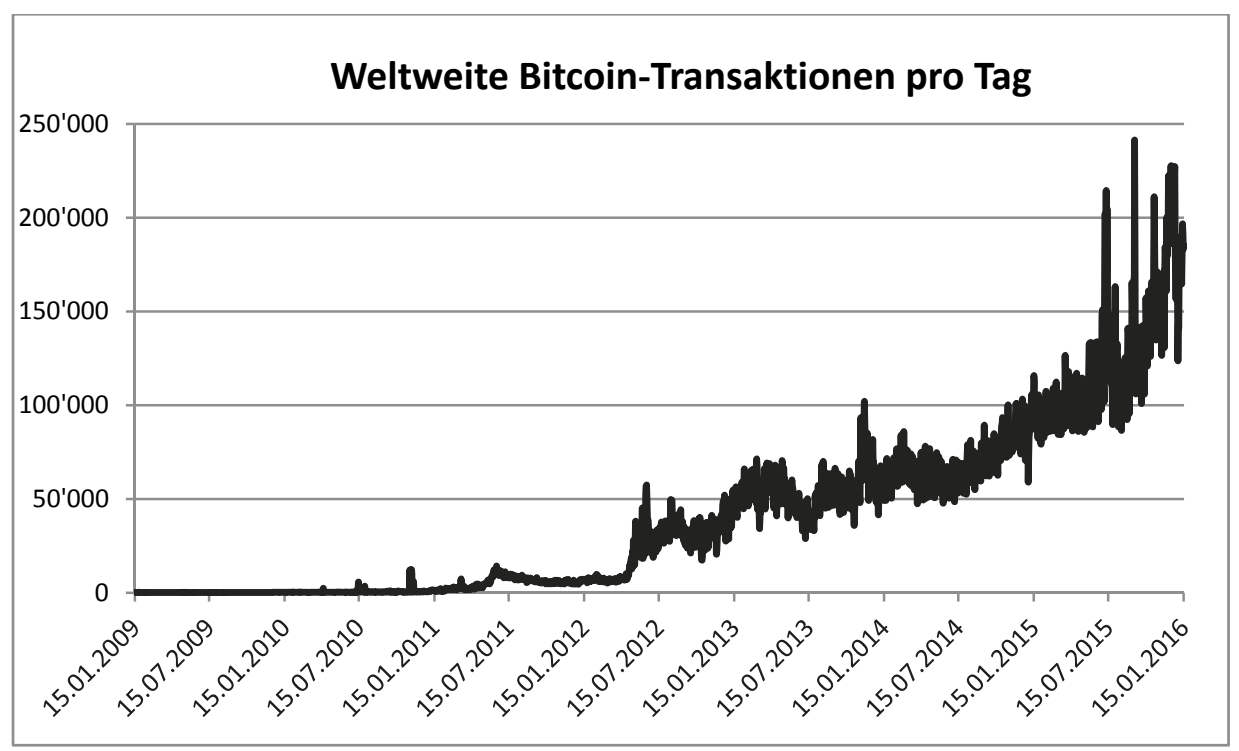

Abbildung 17: Weltweite Bitcoin-Transaktionen (Zahlenquelle: Blockchain, 2016)

Weltweit haben sie, wie in Abbildung 17 abgebildet, innerhalb von sieben Jahren ca. 200'000 Bitcoin-Transaktionen pro Tag erreicht. In der Schweiz dürften täglich einige Tausend davon stattfinden. Anfang September 2015 akzeptierten in der Schweiz ca. 80 Unternehmen Bitcoins (Bitcoin Suisse, 2016), wobei sie nur an einem kleinen Teil der täglich abgewickelten Zahlungen mit der Kryptowährung in der Schweiz beteiligt sein dürften. Bemerkenswert ist, dass diese Entwicklung in wenigen Jahren stattgefunden hat und sich in der Zukunft beschleunigen könnte. Neben Bitcoin gibt es zahlreiche weitere ähnliche Zahlungsmittel, die es erlauben, Zahlungen anonym auszulösen und entgegenzu- 
nehmen, was einen weiteren Faktor in der Beurteilung des Ausmasses von intransparenten Zahlungsströmen in Noah Schmids Arbeitswelt darstellt.

Aufgrund der im Vergleich zur Arbeitswelt von Ursula Meyerhans grösseren Selbstständigenquote und von deren statistisch hochsignifikanten positiven Einfluss auf die Schattenwirtschaft in OECD-Ländern (Schneider \& Tübingen, 2015) und des im internationalen Vergleich tiefen Verhältnisses von Schattenwirtschaft und offiziellem BIP wird in der Arbeitswelt des selbstständigen Softwareentwicklers Noah Schmid von einem deutlichen Anstieg der intransparenten Zahlungsströme ausgegangen.

Wie für die Arbeitswelt von Noah Schmid beschrieben, ist es plausibel anzunehmen, dass es auch in derjenigen der geringqualifizierten Andrea BurriLötscher ein erhöhtes Potenzial für intransparente Zahlungsströme gibt. Aufgrund der Voucher sollte jedoch ein Teil der geringfügigen Löhne am einen Ende der polarisierten Arbeitskräfte erfasst werden können, was in der Folge zu einer Verringerung der Schwarzarbeit führen könnte. In Österreich, Belgien, Frankreich, Griechenland und Italien wurde gerade in Branchen, wo oftmals Schwarzarbeit stattfindet, nämlich bei Haushaltsarbeiten und in der Landwirtschaft, eine Konzentration dieser Arbeitsform festgestellt (Eurofound, 2015a, S. 82). Schätzungen aus Belgien beziffern, dass durch die Einführung des Vouchersystems zwischen 10 bis 20 Prozent der Schwarzarbeit legalisiert worden ist (IDEA Consult, 2010, zit. in Eurofound, 2015). Zwar erreicht die Schwarzarbeit gegenwärtig in der Schweiz nur eine Höhe von ca. 6,5 Prozent des BIP, was im internationalen Vergleich tief ist, in der Zukunft könnten so aber dennoch Zahlungsströme in Milliardenhöhe legalisiert werden.

\subsubsection{Systeme der sozialen Absicherung}

Die in diesem Kapitel verwendete Definition der Systeme der sozialen Absicherung umfasst die Institutionen, welche während und nach der Erwerbsfähigkeit eine Absicherung der sozialen Risiken durch Krankheit, Arbeitsunfall, Berufskrankheit, Invalidität, Alter, Tod des Ernährenden, Arbeitslosigkeit, Familienlasten und Mutterschaft ermöglichen. Dies erfolgt über das bundesstaatliche Sozialversicherungssystem und wird durch kantonale und kommunale Systeme sowie individuelle Absicherungsmöglichkeiten ergänzt. Im Einzelnen umfassen diese die Familienzulagen, die Erwerbsersatzordnung (EO), die obligatorische Krankenversicherung, die Krankentaggeldversicherung, Berufs- und Nichtberufsunfallversicherungen, die AHV/IV, die Ergänzungsleistungen, die berufliche Vorsorge, die Selbstvorsorge und die Arbeitslosenversicherung. 
Die Ergebnisse der Eurofound-Studie zu neuen Formen der Arbeit (Eurofound, 2015a) bestätigen, dass durch die Informationstechnologie ermöglichtes, räumlich flexibilisiertes Arbeiten vor allem bei Normalarbeitsverträgen vorkommt. Diese Art von Arbeitsverhältnis, insbesondere in unbefristeter Form, wie es zwischen Roland Müller bzw. Sandra Könitz und ihren Arbeitgebern existiert, ist die Stützsäule der schweizerischen Sozialversicherungen (Caritas, 2001).

Die Systeme der sozialen Absicherung sind nicht direkt von Veränderungen in der Arbeitswelt des Finanzleiters betroffen. Die Form seiner Arbeitsverrichtung bzw. das Ausmass der räumlichen und zeitlichen Flexibilisierung der Arbeitsverhältnisse hat wenig Einfluss auf die Abgaben der Erwerbstätigen und Arbeitgebenden, welche im Wesentlichen die Finanzierung der bundesstaatlichen Sozialversicherungen ausmachen (Knöpfel, 2015).

Auch für die Systeme der sozialen Absicherung, welche durch die Kantone und Gemeinden getragen werden, wie z.B. die Ergänzungsleistungen, Krankenkassenprämienverbilligungen oder die Sozialhilfe, sind keine direkten Folgen abzusehen, solange räumlich-zeitlich flexibilisierte Arbeitsformen nicht mit einem Einkommensabbau in Verbindung stehen. Einzig durch die tendenziell kleineren Pensen könnten Auswirkungen auf die soziale Sicherheit nach dem Erwerbsleben festgestellt werden. Da die Renten aus der beruflichen Vorsorge und der AHV kleiner würden, könnte dadurch vermehrter Bedarf nach Ergänzungsleistungen entstehen.

Eine Studie des österreichischen Instituts für Wirtschaftsforschung (Mayrhuber \& Lutz, 2010) betont, dass die zunehmende Bedeutung der neuen Arbeitsformen grundsätzlich als positive Entwicklung für die Finanzierung der Sozialversicherungen zu sehen ist, wenn sie zusätzliche Beschäftigung induziert, d.h. zu mehr Beitragszahlenden führt und die Erwerbsquote steigert. Falls das Vollzeitarbeitsverhältnis aber durch neue Arbeitsformen substituiert wird, höhlen sie die Beitragsgrundlage zur sozialen Sicherheit jedoch aus (Mayrhuber \& Lutz, 2010). Dies gilt auch für die Schweiz, wo das Vollzeitarbeitsverhältnis nach wie vor die Hauptstütze der Systeme der sozialen Absicherung darstellt (Caritas, 2001).

Aufgrund der in der Arbeitswelt der Teilzeitredaktorin Ursula Meyerhans starken Verbreitung von geringfügiger Beschäftigung, die ein jährliches Einkommen von weniger als 2300 Franken pro Arbeitsverhältnis generiert, und von Auftragsverhältnissen mit Selbstständigen ist jedoch zu erwarten, dass die Einnahmen der Sozialversicherungen insbesondere durch AHV/IV/EO- und ALVBeiträge nicht im gleichen Ausmass ansteigen wie die Erwerbsquoten. Auch das Umgehen bzw. das Entfallen der Lohnfortzahlungspflicht im Krankheitsfall 
bei solchen Arbeitsverhältnissen können die Sozialversicherungsbeiträge verkleinern, da diese an das Einkommen gekoppelt sind, welches im Krankheitsfall sinkt.

Die Tatsache, dass Arbeitnehmende mit befristeten Verträgen aufgrund des niedrigeren Jahreseinkommens eine geringere Wahrscheinlichkeit haben, Ansprüche aus der zweiten Säule zu erwerben (Henneberger et al., 2004), führt zu weiteren Problemen bei der Altersvorsorge.

Durch die Zunahme von geringfügiger Beschäftigung und Solo-Selbstständigkeit sowie dem Rückgang des Medianeinkommens durch mehr Teilzeitund befristete Arbeitsverträge werden die AHV-Renten im Durchschnitt sinken. In Kombination mit den geringeren Leistungen aus der beruflichen Vorsorge können die minimalen Lebenskosten von einer wachsenden Zahl von Personen nicht mehr gedeckt werden, und es entsteht somit potenziell mehr Nachfrage nach (periodischen/jährlichen) Ergänzungsleistungen. Das Wachstum bei der Anzahl Bezügerinnen und Bezügern ist hier seit Jahren ziemlich stabil bei unter 3 Prozent (BSV, 2015, S. 1). Seit 2009 steigen die Bestände der Ergänzungsleistungen zur AHV jedoch stärker an als diejenigen zur IV (BSV, 2015, S. 2). Dies könnte ein Hinweis auf die Tatsache sein, dass die Flexibilisierung der Arbeitswelt schon in jüngster Vergangenheit eine Verschlechterung der Altersvorsorge mit sich gebracht hat. Das ist eine mögliche Entwicklung, die sich in Ursula Meyerhans' Arbeitswelt fortsetzt. Mit der Verschlechterung der Absicherung im Alter könnte sich auch der von Bütler (2014) beschriebene Nichtbezug von berechtigten sozialen Zuschlägen verringern. Die Hemmung, Sozialleistungen zu beanspruchen, stellt gesamtwirtschaftlich einen Entlastungsfaktor für die Systeme der sozialen Absicherung dar, der durch die Bedingungen der neuen Arbeitsformen zunehmend entfallen könnte.

Zusammen mit dem prognostizierten gesteigerten Ausgabenwachstum für die AHV aufgrund der gesteigerten Lebenserwartung sowie dem Eintritt der Babyboomer in den Ruhestand könnten die Folgen der neuen Arbeitswelt von Ursula Meyerhans weiteren Reformbedarf bei der AHV und der beruflichen Vorsorge auslösen.

Das selbstständige Kleinunternehmertum, wie es von Noah Schmid gelebt wird, hat gemäss Baumgartner, Schulz und Seidl (2013) in ländlichen Regionen der Schweiz einen leicht entlastenden Effekt auf die Zahl der Sozialhilfefälle. Die Beobachtung könnte darauf zurückzuführen sein, dass solche Unternehmen Geringqualifizierten Arbeitsplätze bieten. Dieser empirische Zusammenhang lässt sich möglicherweise auch auf urbanere Gebiete übertragen. Der resultierende Anstieg der atypischen Beschäftigungsverhältnisse könnte einer- 
seits die Arbeitslosigkeit senken und die Systeme der sozialen Sicherheit entlasten, andererseits könnte es aber grössere Sicherheitsdefizite geben (Marti et al., 2007, S. 18). Davon betroffen wäre neben der Arbeitsplatz-, Beschäftigungs- und Einkommenssicherheit auch die Kombinationssicherheit, welche die Vereinbarkeit von Erwerbsarbeit mit anderen sozialen Verpflichtungen beschreibt. Das Fehlen dieser Sicherheiten führt zu prekären Arbeitsverhältnissen. Prekär sind diese für den Zukunftsforscher Gàbor Jánszky (2011) allerdings höchstens in den Augen der Politik und der Gewerkschaften, die das Bedürfnis der Menschen nach Projektarbeit bzw. nach befristeten Arbeitsverhältnissen nicht sehen. $\mathrm{Ob}$ die neuen Arbeitsformen jedoch in gleicher Weise einem Bedürfnis bei Gering- sowie Höherqualifizierten entsprechen, bleibt bisher unbelegt.

Auch der Anstieg der neuen Selbstständigkeit per se birgt Risiken für die soziale Sicherheit während und nach der Erwerbsfähigkeit. Meyer und Sidler (2009, S. 11) zeigen, dass in der Schweiz 50 Prozent der Unternehmensgründungen die ersten fünf Jahre nicht überleben und das Durchschnittsalter der gescheiterten Unternehmenden bei 45 Jahren liegt (S. 40). Sie können unter Umständen vor gravierenden finanziellen Problemen stehen, vor allem dann, wenn sie bereits abhängige Kinder und Ehepartnerinnen sowie Ehepartner haben. Da Selbstständige grundsätzlich keinen Anspruch auf Arbeitslosentaggeld haben, würden viele gescheiterte Unternehmende durch das Fehlen von Einkünften auf Sozialhilfe angewiesen sein.

Wenn für den Schritt in die Selbstständigkeit BVG-Guthaben ausbezahlt werden, kann das bei einer möglichen späteren Scheidung gravierende Folgen für die soziale Absicherung der Ehepartnerinnen und Ehepartner haben, weil das noch verbleibende Altersguthaben des Ehepaars geteilt wird. Für viele SoloSelbstständige stellt sich also nicht nur die Frage nach einem existenzsichernden Einkommen in der Gegenwart, sondern auch, ob sie eine ausreichende Altersvorsorge aufbauen können (Keller \& Seifert, 2011b, S. 42). Für den Teil der neuen Selbstständigen, die eigentlich scheinselbstständig sind und Anrecht auf eine sozialversicherungsrechtliche Gleichbehandlung wie Unselbstständige hätten, stellt sich die Frage, wie sie ihre Ansprüche gegenüber ausländischen Auftraggebenden durchsetzen, insbesondere wenn diese über international nicht einheitlich regulierte Plattformen agieren. Bei einer erfolglosen Durchsetzung droht diesen Personen, mangels ausreichender Altersersparnisse, eine verminderte und möglicherweise nicht ausreichende Altersvorsorge.

Brenke (2013) hat in Deutschland festgestellt, dass sich die geringen Einkommen der Solo-Selbstständigen drastisch auf deren Sparrate auswirkt. Fast 45 
Prozent der Solo-Selbstständigen sparen vom Monatseinkommen nichts, und die Schlussfolgerung liegt nahe, dass damit keine ausreichende Vorsorge für das Alter getroffen werden kann (S. 14). Aufgrund der sinkenden Beitragsskala der ersten Säule für die Einkommen aus selbstständiger Erwerbstätigkeit findet zwar in der Schweiz eine Umverteilung von Personen in Normalarbeitsverhältnissen auf geringverdienende Selbstständige statt, Letztere dürften jedoch überdurchschnittlich oft nur zu tiefen Renten berechtigt sein.

Die Auswirkungen der Arbeitswelt von Noah Schmid auf die Systeme der sozialen Absicherung führen in ihrer Gesamtheit zu einer deutlichen Verschlechterung der Situation. Hauptverantwortlich dafür sind die sinkenden Einkommen in Verbindung mit dem Beitragsverfahren der beruflichen Vorsorge und die fehlenden Beschäftigungs- und Arbeitsplatzsicherheiten.

Die Auswirkungen der Arbeitswelt von Andrea Burri-Lötscher auf die soziale Absicherung kommen einerseits von nicht existenzsichernden Einkommen, was sich auf das tägliche Leben der Beschäftigten auswirkt, und andererseits von der unzureichenden Altersvorsorge, welche sie in Zukunft haben werden. Als Beispiele für die Benachteiligungen bei atypischen Arbeitsverhältnissen in der Schweiz, insbesondere bei der beruflichen Vorsorge und der Arbeitslosenversicherung, nennen Marti et al. (2007, S. 1):

- $\quad$ Fehlende Unterstellung von kurzen oder befristeten Arbeitsverhältnissen bis zu drei Monaten unter das BVG-Obligatorium.

- $\quad$ Bei nicht fixierten Jahreslöhnen ist die Bestimmung des koordinierten Lohnes für BVG-Zahlungen erschwert.

- Bei unbestimmter Arbeitszeit kann eine schleichende Pensenreduktion eintreten, die nicht mehr zu einer Berechtigung zu Leistungen der Arbeitslosenversicherung führen kann.

Als Folge der beiden ersten Benachteiligungen werden, wenn überhaupt, niedrigere Beiträge während der Erwerbstätigkeit erzielt und ergänzende Sozialleistungen wie die Ergänzungsleistungen zur AHV, die aus dem allgemeinen Steueraufkommen finanziert werden müssen, stärker beansprucht. Die Ausweitung atypischer Beschäftigungsverhältnisse erhöht längerfristig also das individuelle Risiko für Altersarmut, erodiert die kollektive Beitragsbasis der AHV und verändert zudem die Struktur der öffentlichen Ausgaben (Keller \& Seifert, 2011a, S. 142). Vouchers und die Zunahme der selbstständigen Erwerbstätigkeit aus Notwendigkeit könnten möglicherweise den Druck auf die Sozialhilfe und die Arbeitslosenversicherung etwas mildern.

Die bereits stattgefundene Flexibilisierung der Arbeit veranlasst Keller und Seifert (2011b, S. 42) zur Forderung, dass die Systeme der sozialen Absicherung 
an die neuen Erwerbs- und Versicherungsbiografien angepasst werden müssten. Die Zahl der Arbeitnehmenden, die aufgrund von zu geringen Beitragsleistungen oder Erwerbsunterbrüchen nicht in der Lage sind, eine existenzsichernde Gegenwart und Zukunft aufzubauen, nimmt in Andrea Burri-Lötschers Arbeitswelt zu und belastet die Systeme der sozialen Absicherung stark.

\subsubsection{Zusammenfassung}

Die Auswirkungen der beschriebenen Arbeitswelten auf die ausgewählten Indikatoren ergeben zusammengefasst ein heterogenes Bild. Einerseits gibt es Unterschiede bei den Intensitäten und Richtungen der Auswirkungen, andererseits bei der Sicherheit, mit welcher ihr Eintritt beurteilt werden kann.

Die Arbeitswelten von Roland Müller und Sandra Könitz, erfolgsbeteiligte Projektmanagerin, sind heute bereits für eine bedeutende Anzahl Beschäftigter verwirklicht oder weisen nur geringe Unterschiede zu zeitgenössischen Arbeitsverhältnissen auf. Dies trifft insbesondere auf die Erwerbseinkommen, die kollektiven Organisationsgrade, die Arbeitslosenquote und Beschäftigungssituation sowie die Steuererträge zu, die in den beiden Arbeitswelten erwartet werden können. Negative Konsequenzen auf gesamtwirtschaftlicher Ebene sind von den beiden Arbeitswelten am ehesten mit Hinblick auf die physischen und psychischen Probleme zu gewärtigen, die in innen auftreten könnten. Das liegt vor allem daran, dass die Beschäftigten bzw. Arbeitnehmenden ein Selbstmanagement entwickeln oder soweit verbessern müssen, dass sie mit den Arbeitsanforderungen zurechtkommen können.

Dafür bieten die zwei ersten Arbeitswelten viel Potenzial für bessere Arbeitsbedingungen und grössere Zufriedenheit mit der Arbeit, insbesondere wenn Beschäftigte und Arbeitnehmende eine Präferenz für räumlich und zeitlich flexible Arbeitsformen haben. Die Entwicklung in Richtung der beiden Arbeitswelten schreitet indessen voran, da die objektiven Voraussetzungen für diese Art der Flexibilisierung gegeben sind. Bereits um die Jahrtausendwende identifizierten Harabi, Schoch und Hespeler (2001) zwei Drittel aller Beschäftigten als potenzielle "Telearbeiter" (S. 86), da sie eine geeignete berufliche Tätigkeit ausführen. Seither hat sich der technologische Wandel fortgesetzt. Auch Bereiche, die vor einigen Jahren noch nicht zu den typischen Schreibtischtätigkeiten gehört haben, werden vom Phänomen erfasst. Zusätzlich nimmt gemäss den Indikatoren zur Informationsgesellschaft des Bundesamtes zur Statistik (BFS, 2015e und 2015 f.) die Verbreitung von Hochgeschwindigkeitsinternetverbindungen sowie die Ausstattung der Haushalte und Arbeitsplätze mit Informations- und Kommunikationstechnologien zu. Die letzte objektive Voraussetzung für das 
Wachstum räumlich und zeitlich flexibilisierter Arbeit, nämlich die entsprechenden Angebote an Arbeitsplätzen vonseiten der Unternehmungen bzw. analoge Arbeitsmöglichkeiten für Selbstständigerwerbende (Haribi und Schoch, 2001, S. 79), kann ebenfalls als gegeben betrachtet werden. Da in den Arbeitswelten von Roland Müller und Sandra Könitz immer noch das Normalarbeitsverhältnis oder leichte Abwandlungen davon dominieren, sind sie bezüglich intransparenter Zahlungsströme und der Auswirkungen auf die Systeme der sozialen Absicherung am absehbarsten und mit wenig gravierenden Folgen verbunden.

Die Arbeitswelt der Teilzeitangestellten Ursula Meyerhans steht exemplarisch für mögliche Entwicklungen, die einige Aspekte der Prekarisierung für eine breitere Masse der Beschäftigten mit sich bringen könnten. Während die Arbeitsproduktivität kaum von Veränderungen betroffen sein dürfte, sind einige Sicherheiten, welche nicht prekäre Arbeitsverhältnisse heute bieten, für viele Beschäftigte schwächer ausgeprägt oder fehlen gänzlich. Mit der grösseren Verbreitung und häufigeren Kombination von Befristung und Teilzeit verschlechtern sich die Arbeitsplatz- und Beschäftigungssicherheit, d.h., insbesondere Angestellte können sich nicht immer sicher sein, nach Ablauf eines Arbeitsvertrags wieder einen gleichwertigen bzw. überhaupt einen Arbeitsplatz zu finden. Auch die Einkommenssicherheit und -höhe kann durch schwankende Arbeitsvolumina beeinträchtigt werden. Zusätzlich lässt der eher sinkende kollektive Organisationsgrad der heterogener werdenden Masse der Beschäftigten in Ursula Meyerhans' Arbeitswelt kaum optimistische Erwartungen in Bezug auf die Höhe der Erwerbseinkommen zu. Insbesondere Auftragssuchende, die wenig erfolgreich auf Crowdsourcing-Plattformen tätig sind, könnten unter kaum existenzsichernden Entgelten für ihre Arbeitsleistung leiden.

Ursula Meyerhans und ihresgleichen sind ausserdem grösseren Risiken psychischer und physischer Art ausgesetzt, als es typischerweise bei heutigen Normalarbeitsverhältnissen der Fall ist, als Folge davon, dass das traditionelle Verhältnis von Arbeitgebenden und -nehmenden zunehmend aufgeweicht wird und die Arbeitgeberpflichten durch die Outsourcing-Methoden umgangen bzw. nicht mehr wahrgenommen werden müssen. Der ökonomische Druck wird es vielen Beschäftigten nicht ermöglichen, auf einen angemessenen Gesundheitsschutz achten zu können, und generell die Arbeitsbedingungen verschärfen. Die zunehmend einfacheren Möglichkeiten für Ursula Meyerhans aber auch für ihre Auftraggebenden, nicht rückverfolgbare Zahlungen tätigen zu können, vergrössern die nicht transparenten Zahlungsströme. Der Nutzen, der dadurch entsteht, liegt im Umgehen von Abgaben an den Staat, u.a. betrifft dies die Beiträge der Arbeitgebenden und -nehmenden, welche die Systeme der sozialen Absicherung finanzieren. Da in dieser Arbeitswelt auch die Steuererträge aller 
Staatsebenen zurückgehen dürften, ist im Vergleich zu heute und zu den Arbeitswelten von Roland Müller und Sandra Könitz mit einer Verschlechterung der Situation dieser Systeme zu rechnen.

In der Arbeitswelt von Noah Schmid und sogar noch stärker in derjenigen von Andrea Burri-Lötscher treten Probleme auf gesamtwirtschaftlicher Ebene auf, die seit Längerem mit atypischer Beschäftigung in Zusammenhang stehen. Noah Schmid und Andrea Burri-Lötscher dürften beide von einer besseren Vereinbarkeit von Beruf mit anderen Lebensbereichen profitieren, jedoch unter jeweils verschiedenen Vorzeichen. Während die Beschäftigungssituation von Noah Schmid zu einem gewissen Grad noch als selbstgewählt bezeichnet werden kann, trifft dies auf Frau Burri-Lötscher nicht zu. Beide müssen sich in einer Arbeitswelt behaupten, in der eine starke Zunahme der neuen Selbstständigkeit bzw. Solo-Selbstständigkeit stattgefunden hat und deren gesamtwirtschaftliche Folgen für die Produktivität unsicher sind. Unter dieser Entwicklung leiden Arbeitsplatz- und Beschäftigungssicherheit, was sich u.a. durch höhere Arbeitslosenquoten bemerkbar macht. Da Arbeitnehmende häufig einen kurzfristig schwankenden Lohn beziehen, was das Resultat von häufig wechselnden Arbeitspensen und hohen variablen Anteilen am Lohn ist, ist die Einkommenssicherheit nicht mehr gegeben. Mindestens im Fall von Andrea Burri-Lötscher tritt eine Existenzunsicherheit ein, da sie infolge der Unterbeschäftigung zu wenig Einkommen erzielen kann. Im Weiteren verschlechtern sich u.a. aufgrund der zurückgegangenen kollektiven Organisationsgrade in beiden Fällen die Arbeitsbedingungen und es muss mit einer Zunahme der physischen und psychischen Gesundheitsrisiken durch die Arbeit gerechnet werden. Verletzungen von heute noch selbstverständlichen Schutzbestimmungen sind häufig anzutreffen, weil gesetzliche, kollektiv vereinbarte oder durch sozialpartnerschaftliche Gewohnheiten garantierte Bestimmungen wegfallen oder umgangen werden.

Die Systeme der sozialen Sicherheit können in den beiden letzten Arbeitswelten ihre Aufgabe im heutigen Sinn nicht mehr erfüllen. Die gesamtwirtschaftlich tieferen Erwerbseinkommen sowie die grössere Verbreitung und Akzeptanz auf der Nutzerseite von Kryptowährungen verschärfen das Problem weiter. Die auf Beiträgen von Arbeitgebenden und -nehmenden basierenden Systeme der sozialen Absicherung werden so infrage gestellt. 



\section{Gesamtbeurteilung und Empfeh- lungen}

Der Argumentationsgang war bisher wie folgt: Im Einleitungskapitel wurden der Projektauftrag mit den Forschungsfragen, die Gliederung der Arbeit, die Methodologie sowie die Zielgruppen der Studie erläutert. Das zweite Kapitel diente der Entwicklung des Grundlagenwissens zur flexiblen Arbeitswelt. Hier wurden Definitionen beigesteuert, die kritischen Entwicklungslinien genauer beschrieben und der für die anschliessende Personaentwicklung zentrale „morphologische Kasten" wurde erarbeitet. Dies ermöglichte bereits eine verdichtete Darstellung der Beiträge einzelner Personas zu denkbaren Zukunftsentwicklungen. Im Kapitel drei wurden dann die aktuellen Entwicklungen anhand einer rechtlichen Analyse genauer untersucht. Diese Analyse förderte zentrale Spannungsfelder zutage, die bei der weiteren Entfaltung der flexibilisierten Arbeitswelt berücksichtigt werden müssen. Kapitel vier diente dann der Ableitung von volkswirtschaftlichen Auswirkungen der aktuellen Entwicklungen und dem Ermitteln grundsätzlicher Tendenzen hinsichtlich einer Reihe zentraler Indikatoren. Diese qualitative Schätzung macht insbesondere bei der Ableitung von denkbaren Szenarien Sinn, selbst wenn durch sie keine quantitativen Informationen und Fakten zur Entwicklung zu gewinnen sind.

Dieses letzte Kapitel nun führt die Informationen der vorhergehenden Kapitel zusammen. In einem ersten Abschnitt werden denkbare Zukünfte, also Szenarien, beschrieben. Vorgenommen werden eine explizite Beantwortung der leitenden Forschungsfragen und eine bewertende Aussage der Situation anhand der Kriterien der ILO-Agenda für menschenwürdige Arbeit. Umfangreiche Handlungsempfehlungen sowie ein Fazit runden die Arbeit ab.

\subsection{Denkbare Zukunftsszenarien}

Aufgrund der erarbeiteten Grundlagen lassen sich vier grobe „Szenarien“ unterscheiden. Diese lassen sich in den Feldern der Abbildung lokalisieren und entsprechen etwa den Quadranten der Darstellung. 


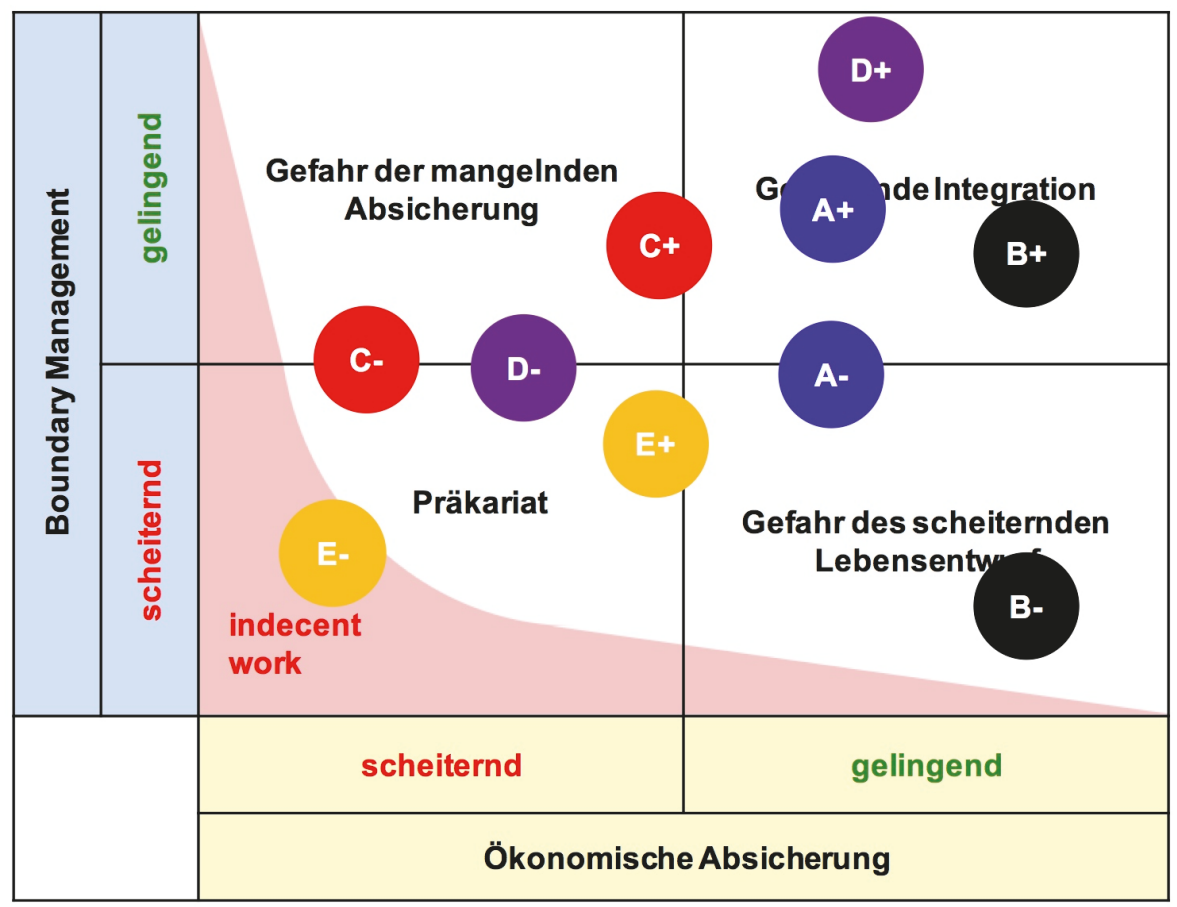

Abbildung 18: Die fünf Personas in der Entwicklungsmatrix verortet

- Szenario 1 „Lebensweltlicher Ausgleich“: Die Persona C, Ursula Meyerhans, Redaktorin, Typ Portfoliowork mit mehreren Standbeinen, entwickelt sich als dominierendes Profil. Es droht die Gefahr der mangelnden Absicherung bei gleichzeitig gelingendem Lebensentwurf. Hier verfällt der Erwerbsstatus, aber es stehen gleichzeitig (noch) Ressourcen aus der privaten Lebenswelt zur Handhabung der Situation zur Verfügung. Der Schlüssel, um eine einseitige Verschlimmerung zu vermeiden, liegt bei diesem Modell im Erhalt des Organisationsgrads dieser Personengruppe, dem Sicherstellen der Organisations- und Beschäftigungsfähigkeit der Person sowie einer Beschäftigungspolitik seitens der Unternehmen, die hier ihren Nutzen nicht auf Kosten einzelner Berufsgruppen maximieren.

- Szenario 2 „Gelingende Integration“: Die Personas A, Roland Müller, Finanzleiter im KMU (Typ Homeoffice in der Festanstellung), B, Sandra Könitz, Projektmanagerin (Typ mobiles Arbeitskraftunternehmertum), und D, Noah Schmid, Informatiker (Typ Entrepreneurship), entwickeln sich dominant und verkörpern die Integration zwischen einem gelingen- 
den Lebensentwurf und sozialer Absicherung. Der Schlüssel für eine solche Entwicklung dürfte in der wirtschaftlich gesicherten oder ausgebauten Wettbewerbsfähigkeit durch die flexiblen Arbeitsformen unter gleichzeitiger Wahrung des sozialpartnerschaftlichen Gleichgewichts zu finden sein. Diese Kombination ist fragil und erfordert grosse Aufmerksamkeit der beteiligten Akteurinnen und Akteure.

- Szenario 3 „Verschärfende Wirtschaftsdominanz“: Die Personas A, Roland Müller, und B, Sandra Könitz, entwickeln sich überproportional und bringen einen schwebend scheiternden Lebensentwurf durch berufliche Überlastung mit sich. Bereits heute ist zu beobachten, dass berufliche Leistungsträgerinnen und Leistungsträger überproportional viel arbeiten, was sich auch in den steigenden Entwicklungen im Bereich insbesondere der psychischen Berufskrankheiten zeigt. Der Schlüssel, um dieser Situation entgegenzuwirken, ist die unternehmerische Arbeitsgestaltung. Hier muss auf eine kompetente, gleichgewichtige Aushandlung nachhaltiger Beschäftigungsbeziehungen zwischen vorgesetzten Personen und Mitarbeitenden geachtet werden. Als Vorbild dienen können Berufsbereiche, die bereits heute zur Bewältigung ähnlicher Herausforderungen Strategien entwickeln müssen, beispielsweise hinsichtlich der (Nach-)Qualifikation im Selbstmanagement (siehe Sach- und Facharbeitende) oder im Umgang mit beruflichen Belastungsszenarien (Burnout-Prävention, siehe Lehrpersonen).

- Das Szenario 4 „Abschmelzung des Lebensstandards“ ist in der Optik der ILO-Agenda für menschenwürdige Arbeit als drohend unwürdig zu bezeichnen und kann sich am Profil von Persona E, Andrea Burri-Lötscher (und zum Teil auch an den Personas C und D) kristallisieren. Die beginnenden Entwicklungen im Bereich der virtuellen Arbeit (Crowdsourcing, Kryptowährungen, ...) und im Niedriglohnsektor sind hier einzuordnen. Eine unausgewogene Entwicklung in diese Richtung dürfte sich letztlich in einem Ungleichgewicht in den sozialen Sicherungssystemen niederschlagen, die auf Beiträgen von Arbeitgebenden und -nehmenden basieren. Zwar besteht generell kein „Recht auf Arbeit", aber ein sozialer Anspruch auf ausreichendes Einkommen kann doch aus den gesellschaftlichen Rahmenbedingungen plausibel abgeleitet werden. Der Schlüssel zur Bewältigung resp. Vermeidung einer solchen Situation liegt im Setzen von rechtlichen Rahmenbedingungen, um dem Extremum der potenziellen Ausbeutung begegnen zu können, bspw. mithilfe von systematischen Arbeitsmarktangeboten zur Steigerung des Qualifikationsniveau dieser Personengruppe respektive der Förderung von Beschäftigungsverhältnissen, die auch geringer Qualifi- 
zierten ein würdiges Einkommen sichern. Solche Mechanismen gibt es im Bereich der RAVs bereits heute, jedoch sind sie vermutlich nicht auf ein überdurchschnittliches Wachstum dieser Personengruppe ausgerichtet.

\subsection{Antworten auf die Forschungsfragen}

Somit sind die notwendigen Vorarbeiten geleistet und die fünf übergreifenden Forschungsfragen können zusammenfassend beantwortet werden.

\subsubsection{Stellenwert der Arbeit heute und in Zukunft}

Die Forschungsfrage lautete: „Welchen Stellenwert besitzt die Arbeit in der Gesellschaft heute und in Zukunft?" Arbeit, verstanden als Erwerbsarbeit, die einen relevanten Anteil zum Lebensunterhalt beiträgt, erhält grundsätzlich ihre relevante Position in der Alltagsgestaltung. „Die Wirtschaftsdominanz der Gesellschaft" trägt in Form der heute und in naher Zukunft möglichen Flexibilisierung der Arbeitswelt allerdings hier zu einer Zuspitzung der Situation bei. Die zunehmende Subjektivierung und die sich verschärfende Anforderung einer Grenzziehung zwischen Arbeits- und Berufswelt können in einer Kombination enden, die weder für die Gesellschaft noch die Wirtschaft, noch das Individuum verträglich ist. In einer Volkswirtschaft mit quasi Vollbeschäftigung haben weder Arbeitnehmer noch Arbeitgeber ein Interesse an einer solchen Zuspitzung. Dennoch gilt es, ein Augenmerk auf die Schaffung entsprechender Rahmenregelungen zu legen, um eine solche unerwünschte Entwicklung zu vermeiden. Die Wertigkeit der bezahlten Arbeit im Verhältnis zur unbezahlten Arbeit genau zu beurteilen, wäre eine eigene, vertiefte Studie wert.

Die in dieser Studie erarbeiteten Entwicklungen können vor dem Hintergrund der Agenda für menschenwürdige Arbeit („Agenda for Decent Work“) der International Labor Organization (ILO, 2012) interpretiert werden. Die Agenda besteht schon seit Längerem und wurde jüngst aktualisiert und ihre Wichtigkeit durch die Vereinten Nationen bekräftigt. Es ist wichtig, zu erläutern, dass die üblichen Arbeitsbedingungen in den entwickelten Industrieländern nicht das Hauptaugenmerk dieser Agenda sind. Vielmehr geht es um die Bemühungen, global akzeptable Standards für die Erwerbsarbeit zu etablieren. Dennoch sind im Zuge von Globalisierung und Digitalisierung fast alle Länder von denselben Entwicklungen betroffen, und so sollten die Überlegungen auch auf die Schweiz 
angewendet werden, um unerwünschte Auswirkungen insbesondere in neu auftretenden Arbeitsformen aufzudecken und innen entgegenzuwirken. Die Erläuterung der einzelnen Kriterien findet sich Anhang 2: Die Elemente der Decent-Work-Agenda“. Im Einzelnen umfasst die Agenda die folgenden Aspekte:

1. Beschäftigungsmöglichkeiten

2. Angemessene Entschädigung und produktive Arbeit

3. Menschenwürdige Arbeitszeiten

4. Vereinbarkeit von Arbeit, Familie und persönlichem Leben

5. Arbeit, die abgeschafft werden sollte

6. Beständigkeit und Sicherheit der Arbeit

7. Gleiche Chancen und gleiche Behandlung in der Anstellung

8. Sichere Arbeitsumgebung

9. Soziale Sicherheit

10. Sozialer Dialog, Vertretung der Arbeitnehmenden und Arbeitgebenden

11. Ökonomische und soziale Umweltbedingung für menschenwürdige Arbeit

Grundsätzlich ist zu betonen, dass sich durch die Flexibilisierung der Arbeitswelt die Erwerbsmöglichkeiten vervielfältigen. Nahezu alle Punkte der Agenda könnten verbessert werden, wenn man diese entsprechend in der Ausgestaltung berücksichtigt. Die Flexibilisierung hält also signifikante Chancen bereit, die durch ein gemeinsames Vorgehen genutzt werden sollten. Der drohenden Prekarisierung einzelner Berufsgruppen und der nachhaltigen negativen Eintrübung der Beschäftigungsfähigkeit der Erwerbsbevölkerung durch gesundheitliche Gefährdungen muss jedoch entgegengewirkt werden. Die Agenda hält für jedes Kriterium Indikatoren bereit, daher kann sie gut zum Aufbau eines regelmässigen Monitorings verwendet werden.

\subsubsection{Sind neue Arbeitsformen mit den rechtlichen Bestimmungen vereinbar?}

Grundsätzlich entsprechen die neuen Ausprägungen der Erwerbsarbeit den rechtlichen Bestimmungen. Dennoch ergeben und verschärfen sich die verschiedenen aufgezeigten Spannungsfelder. Diese sind:

Spannungsfeld I: Abgrenzung der einzelnen Arbeitsleistungen gegenüber dem Einzelarbeitsvertrag

Spannungsfeld II: Herausforderungen versus Risiken bei mobiler Arbeit 
Spannungsfeld III: Arbeitszeitregelung versus Flexibilität

Spannungsfeld IV: Regulierung durch Rahmenbedingungen versus genügend Freiraum für Flexibilität

Spannungsfeld V: Erhöhung der Arbeitsflexibilität und der Marktwirtschaftlichkeit mit gleichzeitiger Einhaltung der sozialen Sicherheit der Arbeitnehmenden

Spannungsfeld VI: Flexible Arbeit ja, aber nicht auf Kosten der Gesundheit

Spannungsfeld VII: Wie setzen wir „mobilen“ Gesundheitsschutz um?

Spannungsfeld VIII: Risiken der IT-Nutzung

Spannungsfeld IX: Rechtliche Rahmenbedingungen für Crowdsourcing schaffen

In einzelnen Aspekten könnte man nachbessern und rechtlich verbindliche Regelungen etablieren. Dabei ist jedoch grundsätzlich zu fragen, inwieweit eine rechtliche Regelung nicht besser durch eine Bekräftigung der Selbstverantwortung der Sozialpartnerinnen und Sozialpartner ersetzt wird. Rechtliche Regeln in diesen „Erwerbsarbeitsnischen“ durchzusetzen, dürfte auch aus pragmatischen Gründen schwierig und letztlich nur volkswirtschaftlich unökonomisch möglich sein.

\subsubsection{Was für volkswirtschaftliche Folgen haben neue Arbeitsformen?}

Je nach der konkreten künftigen Entwicklungslinie können die neuen Arbeitsformen unterschiedliche Ausprägungen annehmen. Die denkbaren Szenarien bieten das Potenzial, die zukünftigen Entwicklungen anhand der Indikatoren genauer eingrenzen zu können. Die rein qualitative Ableitung lässt jedoch keine angemessene Quantifizierung zu. Ein Blick auf die entsprechende Tabelle 8 scheint jedoch zu offenbaren, dass in folgenden Bereichen ein Beobachtungsbedarf besteht: Kollektiver Organisationsgrad der Beschäftigten, physische und psychische Gesundheitsrisiken, Arbeitslosigkeit, Intransparenz der Zahlungsströme sowie der sozialen Absicherung. Eine entscheidende, also richtunggebende Einflussnahme kann bei den Indikatoren Produktivität, Vereinbarkeit von Beruf mit anderen Lebensbereichen, Beschäftigte und Steuererträge geltend gemacht werden.

Beachtlich scheint die Erkenntnis, dass die Systeme der sozialen Sicherheit in den beiden letzten Arbeitswelten ( $D$ und $E$ ) ihre Aufgabe im heutigen Sinn nicht mehr erfüllen können. Die gesamtwirtschaftlich tieferen Erwerbseinkommen 
sowie die grössere Verbreitung und Akzeptanz auf der Seite der Nutzerinnen und Nutzer von Crowdsourcing und Kryptowährungen dürften das Problem noch verschärfen. Die auf Beiträgen von Arbeitgebenden und -nehmenden basierenden Systeme der sozialen Absicherung werden so infrage gestellt.

\subsubsection{Notwendige Rahmenbedingungen und Monitoring}

Die letzten beiden Forschungsfragen lauteten: „Welche Rahmenbedingungen müssten herrschen, damit möglichst viele Personen von mobil-flexiblem Arbeiten profitieren können (ohne dabei mit negativen Folgen konfrontiert zu sein)?" sowie „Wie könnten Monitoring- und Kontrollsysteme aussehen, damit die bestehende soziale Absicherung und deren staatlicher Finanzierung nicht durch mobil-flexibles Arbeiten unterlaufen werden können?" Beide Fragen werden in den folgenden Handlungsempfehlungen thematisiert.

Prinzipiell gelten die Erkenntnisse des Kapitels zum Stellenwert der Arbeit als massgebend für die Gestaltung und Ableitung entsprechender Handlungsempfehlungen, damit eine „Multiple-Win-Situation" entsteht. Ebenso werden Handlungsempfehlungen zum Monitoring der flexiblen Arbeitsformen ausgesprochen, was jedoch nicht die politische Frage beantwortet, inwieweit die Akteurinnen und Akteure die Situation überhaupt beobachten und kontrollieren sollen. Man muss zu diesem Aspekt auch erwähnen, dass vieles bereits über bestehende Statistiken erfasst wird, ohne dass dies in der betroffenen Öffentlichkeit wahrgenommen wird. Es lassen sich daher in den Handlungsempfehlungen nur wenige Vorschläge zum Monitoring finden, die in Richtung einer statistischen Optimierung zielen. Mit der Feststellung, dass flexible Arbeitsformen prinzipiell durch die rechtlichen Grundlagen erfasst werden, sind auch die Bedenken des Unterlaufs der Sozialversicherung zu relativieren. Ausgenommen hiervon sind die virtualisierten Arbeitsformen, deren Entwicklung aber aus dem Hauptfokus dieser Studie ausgenommen ist. In Anbetracht der Tatsache, dass Crowdsourcing und Kryptowährungen wohl aktuell am Beginn ihrer Entwicklung stehen, scheint eine vertiefte Analyse dieser Entwicklungen empfehlenswert.

\subsection{Handlungsempfehlungen}

Der Trend zur Flexibilisierung ist ein unumkehrbarer Teil der gesamtgesellschaftlichen Entwicklung. Diese Entwicklung birgt Chancen und Risiken für Wirtschaft und Gesellschaft. Dabei haben die gesellschaftlichen Interessengruppen zwar Gestaltungsmöglichkeiten, ein Zurückdrehen der Entwicklung ist 
aber ausgeschlossen. Flexibilisierung ist ein gesamtgesellschaftliches Phänomen, daher ist eine isolierte Lösung der damit verbundenen individuellen und gesellschaftlichen Herausforderung nicht möglich. Im Mittelpunkt dieser gesellschaftlichen Lösungssuche stehen die Sozialpartnerinnen und Sozialpartner, die sich vor der Aufgabe sehen, auf unterschiedlichen Ebenen Win-WinKonzepte entwickeln und Lösungen finden zu müssen, die den ökonomischen, sozialen und individuellen Bedürfnissen von Unternehmen und Arbeitnehmenden gerecht werden. Der Gesetzgeber kann diese Verhandlungen zwischen den Sozialpartnerinnen bzw. Sozialpartnern durch eine entsprechende Anpassung der rechtlichen Regelungen flankieren und unterstützen - oder solche Win-win-Situationen verunmöglichen. Weitere Interessengruppen, die indirekt von der Flexibilisierung betroffen sind, sollten sich zu Wort melden und an dieser gesellschaftlichen Diskussion teilnehmen:

\subsubsection{Handlungsempfehlungen auf gesellschaftspolitischer Ebene}

Die wichtigste Botschaft dieses Berichts lautet: Die individuellen und gesellschaftlichen Folgen flexibilisierter Arbeit sind ambivalent. Eine qualifizierte Grund- und Fachausbildung verbessert die Chancen erheblich, dass Arbeitnehmende die positiven Potenziale der Flexibilisierung nutzen können. In welche Richtung das Pendel in Zukunft ausschlägt, wird ohnehin nicht technisch oder ökonomisch determiniert, sondern ist abhängig von der sozialen Gestaltung neuer Arbeitsformen auf betrieblicher und überbetrieblicher Ebene. Hier empfehlen wir einen weiten Rahmen für den Umgang mit zeitlicher und örtlicher Entgrenzung der Arbeit zu setzen, für die Subjektivierung der Arbeit zu sensibilisieren, die betriebliche Flexibilität als partizipative Organisationsentwicklung zu planen und die betriebliche Mitwirkung und Selbstorganisation zu stärken.

1. Einen weiten Rahmen für den Umgang mit zeitlicher und örtlicher Entgrenzung der Arbeit setzen: Die Grenzen, die bis anhin die Arbeitszeit von der arbeitsfreien Zeit trennten und den Arbeitsort klar definierten, werden durchlässig. Dieser Strukturwandel, in welchem sich Gesellschaft und Wirtschaft befinden, zeigt sich zwar individuell, zu seiner erfolgreichen Bewältigung bedarf es aber des Dialogs zwischen den Sozialpartnerinnen bzw. Sozialpartnern sowie zwischen den Arbeitnehmenden untereinander sowie zwischen Arbeitnehmenden und Führungskräften. Dieser Dialog benötigt gesetzliche und politische Rahmenbedingungen, um den Dialogpartnern Möglichkeiten zu geben, die Risiken auf ein vernünftiges Mass reduzieren und die möglichen Vortei- 
le ausspielen zu können. Der neue Rahmen sollte dabei so weit sein, dass nur Extremfälle ausgeschlossen werden. Der vorhandenen Experimentierfreude von Arbeitgebenden und Arbeitnehmenden bei der Entfaltung gemeinsamer Vorteile sollte nichts im Wege stehen. Zielgruppe: Sozialpartnerinnen und Sozialpartner, Parlament.

2. Für die Subjektivierung der Arbeit sensibilisieren: Die Entwicklung hin zum "Arbeitskraftunternehmertum“ - also die Verlagerung unternehmerischer Risiken und Chancen auf einzelne Arbeitnehmende - hat widersprüchliche Auswirkungen. Einerseits fördert sie die Autonomie und Eigenverantwortung der Arbeitskräfte und stellt innen somit mehr Möglichkeiten zur Verfügung, die eigenen Fähigkeiten zu entfalten und die Arbeit zu gestalten. Zudem geht damit üblicherweise auch eine höhere zeitliche und örtliche Arbeitsflexibilität einher, was eine grössere Chance darstellt, Beruf und andere Lebensbereiche unter einen Hut zu bringen. Eine solche Entwicklung fördert Marktkräfte und -dynamik, um die verschiedenen Interessen im Wirtschaftssystem zum Ausgleich zu bringen. Andererseits können Arbeitnehmende marktbedingt wirtschaftliche Nachteile erfahren, die Balance zwischen Arbeit und den anderen Lebensbereichen kann empfindlich gestört werden und die Arbeitnehmenden können unter dem Druck der Verantwortung sowie der höheren Unsicherheit leiden oder sogar erkranken. Um die Arbeitnehmenden in dieser widersprüchlichen Situation zu stärken, müssen sie wirksamer als bislang dazu befähigt werden, mit dieser Übernahme von Unternehmensrisiken und -chancen kompetent umzugehen. Entscheidungsfindung, Verhandlungsstrategien, Karriere-strategien, Zeitmanagement, Selbstmanagement und Boundary-Management sind eine Auswahl von Themen, die verstärkt in die Aus- und Weiterbildung auf allen Niveaus aufgenommen werden sollten. Diese „Stärkung des Subjekts” als Antwort auf die Subjektivierung von Arbeit kann zudem auch (stärker als bisher) zu einem Anliegen der Gewerkschaften werden. Zielgruppe: Bildungspolitik, Bildungsanbieter, Gewerkschaften.

Ganz und gar „subjektivierte Arbeit” leisten Existenzgründerinnen und Existenzgründer. Solche sind meist jüngere Personen mit vergleichsweise wenigen sozialen Verpflichtungen und einem überdurchschnittlich hohen Gesundheitsniveau. Gründungen sind in der Regel sehr arbeitsintensive Zeiten und Erfahrungen, in denen die beteiligten Personen an ihre Belastungsgrenzen gehen. Das Überleben der Gründungsinitiative steht im Vordergrund und verdrängt langfristige Notwendigkeiten zur wirtschaftlichen, sozialen und gesundheitlichen Absicherung. Hier kön- 
nen Aktivitäten der Start-up-Förderung eine zentrale Rolle in der Sensibilisierung für Themen der sozialen Absicherung und betrieblichen $\mathrm{Ge}-$ sundheit spielen, wenn diese Inhalte in der entsprechenden Förderung einen Platz erhalten. Zielgruppe: Start-up-Initiativen.

3. Betriebliche Flexibilität als partizipative Organisationsentwicklung planen: Die Schweizer Organisationen und Betriebe stellen den entscheidenden Dreh- und Angelpunkt für die Gestaltung der flexiblen Arbeitswelt dar. Daher ist es von zentraler Bedeutung, dass auf betrieblicher Ebene Regelungen und Abmachungen zum Umgang mit erhöhter Flexibilität entwickelt werden:

- In die betrieblichen Regelungen zur flexiblen Arbeitszeit werden die Interessen der Beschäftigten explizit aufgenommen. Mitarbeitende und Vorgesetzte werden auf einen anderen Umgang miteinander vorbereitet.

- Die Beschäftigten qualifizieren sich dazu, ihre flexible, weitgehend selbstbestimmte Arbeitszeit effizient und belastungsarm zu organisieren (Selbstmanagement).

- Mitarbeitende und Vorgesetzte legen im persönlichen Austausch gemeinsam fest, wie sie angesichts flexibler Arbeitszeiten und Arbeitsorte arbeiten und kooperieren wollen.

- $\quad$ Flexible Arbeitszeiten geben den Mitarbeitenden viel Freiraum, sofern sie mit einer zielorientierten, indirekten Steuerung verbunden werden. Sie führen aber potenziell auch an die physischen und psychischen Leistungsgrenzen. Nachhaltige Gesundheit liegt im Interesse aller Sozialpartnerinnen und Sozialpartner und erfordert besondere Aufmerksamkeit von Betrieben und Mitarbeitenden.

- Die Flexibilisierung von Arbeitszeiten stellt neue Anforderungen an die betriebliche Personalplanung (Bestimmung der Zahl der Mitarbeitenden, Verteilung der Arbeit im Zeitverlauf, Personaleinsatzplanung). Vorgesetzte müssen lernen, bei der Bewältigung dieser Herausforderungen die Interessen von Unternehmen und Mitarbeitenden zu berücksichtigen.

- Management by Objectives“ (Führen durch Zielvereinbarung) bedeutet, dass den Mitarbeitenden nicht mehr das Wie der Produktion (oder der Erbringung von Dienstleistungen) vorgegeben wird, sondern das Was, das Ziel, das Produkt, die Dienstleistung. Damit sind die Voraussetzungen gegeben, die Qualität der Arbeit nur noch am Ergebnis, nicht aber an der dazu aufgewendeten Zeit zu messen. Arbeitsverträge für Angestellte bestehen aber weiterhin 
im Kern aus dem Tausch von Zeit gegen Geld, wobei der wöchentliche zeitliche Einsatz klar festgelegt wird. Damit es durch Management by Objectives nicht zu einer Form der Selbstausbeutung bei den Angestellten kommt, müssen in den Unternehmen Verfahren entwickelt werden, wie das Arbeitsergebnis in Relation zum zeitlichen, physischen und intellektuellen Aufwand der Leistungserbringer gesetzt und transparent und fair bewertet werden kann.

Die Einführung flexibler Arbeitsmodelle ist ein langfristiger, mit Widersprüchen behafteter Prozess (Meissner, Gentile \& Sprenger, 2015, Kissling, Meissner, Seyler, Henzen \& Gentile, 2012) und ruft häufig Ängste und Widerstände hervor: Widerstände können systematisch abgebaut oder gar vermieden werden, beispielsweise durch eine transparente Prozessgestaltung. Eine erfolgreiche Entwicklung und Einführung neuer, flexibler Systeme der Arbeitsgestaltung erfordert einen partizipativ gestalteten, transparenten Prozess der Organisationsentwicklung. Flexible Arbeitsmodelle können nicht als „Fertiglösungen“ übernommen werden, sondern müssen an die jeweils spezifischen Strukturen, Kulturen und Bedürfnisse eines Betriebes oder einer Organisation angepasst werden. Dafür braucht es oftmals auch eine Phase des gemeinsamen Ausprobierens, z.B. in Form von Pilotversuchen und Evaluationen. Sowohl die Leitung wie auch die Beschäftigten sind tendenziell nicht sicher, ob die versprochenen Vorteile der neuen Regelungen tatsächlich greifen werden. Ein Entwicklungsprojekt, in dem neue (Arbeitszeit-) Strukturen und Kulturen von allen relevanten Akteurinnen und Akteuren gleichberechtigt und gemeinsam entwickelt, erprobt und überprüft werden, nutzt die Expertise aller Beteiligten für die Entwicklung eines angepassten Modells und schafft dabei gleichzeitig Akzeptanz. Zielgruppe: Sozialpartnerinnen und Sozialpartner.

4. Betriebliche Mitwirkung und Selbstorganisation stärken: Die Schweiz zeichnet sich durch eine gute Kooperationskultur zwischen den betrieblichen Sozialpartnerinnen bzw. Sozialpartnern aus. Bei der erfolgreichen Regelung flexibler Arbeit spielt diese Kooperation eine entscheidende Rolle. Rechtlich gesehen bestimmt jedoch allein das Unternehmen oder die Organisation über den Einsatz flexibler Arbeit - es gibt z.B. keinen Anspruch auf Homeoffice. In Regionen, in denen sich Unternehmen keinem grossen Druck des Arbeitsmarktes ausgesetzt sehen, führt dieser Umstand mitunter dazu, dass allein Produktivitätsaspekte die Flexibilisierung definieren (Perch-Nielsen et al. 2013) und die Interessen der Beschäftigten an einer besseren Balance zwischen 
Arbeit und anderen Lebensbereichen wenig Berücksichtigung finden. Hier sollten die Arbeitgebenden dafür sensibilisiert werden, den Arbeitnehmenden Mitwirkung und entsprechende Gestaltungspielräume zu gewähren. Einen möglichen Weg dafür stellt die Entwicklung eines Verhaltenskodex für Arbeitgebende dar. Wenn sich allerdings betriebliche Praktiken mit negativen Konsequenzen für Arbeitnehmende häufen, sind allenfalls rechtliche Regelungen der Mitwirkung der Beschäftigten in Erwägung zu ziehen (Janssen und Nachreiner 2004, S. 133). Es soll hier auch betont werden, dass bei der Gestaltung flexibler Arbeit die Selbstorganisation in den Unternehmen an erster Stelle steht. In gewissen Bereichen können überbetriebliche Regelungen einen verlässlichen und nützlichen Rahmen darstellen. Eine sozialpartnerschaftliche Regelung, bei der z.B. in GAVs verankert wird, wie mit flexibilisierten Arbeitsformen, insbesondere flexiblen Arbeitszeiten, umzugehen ist und welche Rahmenbedingungen bei ihrer Gestaltung eingehalten werden müssen, ist dabei einer generellen gesetzlichen Regelung vorzuziehen. Das Primat bleibt jedoch der dezentralen Selbstorganisation der Sozialpartnerinnen bzw. Sozialpartner vorbehalten. Zielgruppe: Sozialpartnerinnen und Sozialpartner und deren Verbände.

\subsubsection{Handlungsempfehlungen auf rechtlicher Ebene}

Die Flexibilisierung der Arbeitswelt hat zahlreiche rechtliche Konsequenzen. Verbesserungen und Überprüfungen sind wichtig hinsichtlich der Arbeitszeitregelungen, der Sozialversicherungsdefizite bei Kurzarbeitseinsätzen, dem Umgang mit (noch) undefinierten Arbeitsformen, der Klärung von Fragen des Arbeitsortes, der Scheinselbstständigkeit, der Personalvermittlung, der Rechtsdurchsetzung, der Regelungen zur Nacherwerbsphase, und der Erstellung und Pflege einer Gesamtübersicht. Diese Massnahmen können der Nutzung der Flexibilität bei gleichzeitiger Wahrung und Förderung der Rechtsklarheit zum Vorteil dienen.

1. Regelungen zu modernen Arbeitszeitsystemen weiter anpassen: Das Arbeitsrecht führt bis heute keine der Realität angemessenen Regelungen zu modernen Arbeitszeitsystemen wie Jahresarbeitszeit, Sabbatical, Langzeitkontos u.a., während im Bereich der Arbeitszeiterfassung aktuelle gesetzliche Anpassungen die Situation angemessener darstellen. Die Rahmenbedingungen schreiben Mindestruhezeiten und Maximalarbeitszeiten vor, die von einer „normalen“, also traditionell gelebten Arbeitswoche ausgehen. In diesen Vorschriften findet sich sogar 
eine äusserst hohe Regelungsdichte. Vom klassischen Modell abweichende Arbeitszeitmodelle stehen nicht selten in Widerspruch zur rechtlichen Grundlage. Mit diesem Widerspruch sind in der praktischen Umsetzung Unsicherheiten verbunden, die als arbeitsrechtliche Hürden und Überbürokratisierung wahrgenommen werden. Zielgruppe: Parlament.

2. Sozialversicherungsdefizite bei Kurzarbeitseinsätzen thematisieren: Diverse Regelungen im Gesetz (Lohnfortzahlung bei Arbeitsverhinderung, OR 324a; gesetzliche Kündigungsfrist, OR 335c; Kündigungssperre, OR 336c, Pensionskassenansprüche) gelten nur für lang dienende Arbeitnehmende. Hier ist insbesondere der Fall des Eintritts einer Invalidität hervorzuheben, da sich das schwankende Einkommen negativ auf die Invalidenrente auswirken kann. Gleiches gilt bei längeren Krankheiten, da die obligatorische Krankentaggeldversicherung das Risiko eines länger dauernden Arbeitsausfalls nicht trägt. Es ist in Betracht zu ziehen, dass GAV häufig befristete Arbeitsverhältnisse, Teilzeitarbeiten oder Arbeit auf Abruf aus ihrem Geltungsbereich ausschliessen. Über diese Regelungslücke sollte diskutiert werden. Zielgruppen: Sozialpartnerinnen und Sozialpartner, Sozialversicherungen und Parlament.

3. Arbeitsrechtliche Praxis im Umgang mit noch undefinierten Arbeitsformen regeln: Die flexible Arbeitswelt führt zu neuartigen Beschäftigungsformen, die sich in rechtlichen Graubereichen bewegen. Durch die unklare Definition von Arbeitsformen sind spezifische Probleme nicht durch das Recht erfasst respektive abgedeckt. Dies betrifft insbesondere die Formen, welche weder eindeutig zu „Arbeitnehmenden“ noch zu den „Selbstständigerwerbenden“ gezählt werden können, z.B. die wachsende Gruppe der Arbeitenden in der Crowd. Hier entstehen Schwierigkeiten in der rechtlichen Normierung: Besteht ein Einzelarbeitsverhältnis oder doch ein Auftrag? Gibt es eine betriebliche Eingliederung oder eine Pflicht zur Befolgung von Weisungen? Diese Fragen müssen bei der Qualifizierung von Vertragsverhältnissen vorab geklärt werden. Von dieser Qualifizierung hängt letztlich auch ab, ob eine Vertragspartei dem arbeitsrechtlichen Schutz untersteht oder nicht. Primär sollten die Situationen in den Unternehmen geregelt werden. Klare Regelungen im Rahmen der Selbstorganisation zwischen den Sozialpartnerinnen bzw. Sozialpartnern sind gesetzlichen Regelungen vorzuziehen, da der flexible Arbeitsmarkt nicht rigider gemacht werden sollte. Das wäre im Endeffekt auch nicht im Interesse der Arbeitneh- 
menden, da dadurch schliesslich weniger Arbeitsstellen geschaffen werden. Im Zweifelsfall ist eine Anpassung der Gesetze in Betracht zu ziehen. Solche Regelungen wären aber nicht gänzlich neu. Bereits heute gibt es für "atypische“ Arbeitsverhältnisse (z.B. Handelsreisende, Lehrvertrag, Personalverleih) zusätzliche gesetzliche Vorschriften. Es kann auch definiert werden, dass weitere atypische Arbeitsverhältnisse sowie deren Rechte und Pflichten künftig unter bereits bestehende Normen subsumiert oder innerhalb des Unternehmens oder der Branche geklärt werden. Bis diese Klärung stattgefunden hat, besteht Unsicherheit in Lehre und Rechtsprechung und somit letztlich für die Betroffenen. An dieser Klärung können ihrerseits auch die betroffenen Parteien eines Leistungsverhältnisses mitwirken, indem sie versuchen, sich bestehenden Rechtsregelungen selbstständig unterzuordnen und undefinierte Begriffe zu vermeiden. Zielgruppen: Parlament, Parteien eines Arbeitsvertrags.

4. Aus dem Arbeitsort abgeleitete Ansprüche klären: Die Bestimmung des Arbeitsorts sollte im Sinne der Privatautonomie nach wie vor den Parteien überlassen werden. Eine begriffliche Normierung im Obligationenrecht oder in anderen Vorschriften widerspricht der sinnvollen Privatautonomie. Jedoch empfehlen wir eine Konkretisierung von Art. 327a Abs. 1 OR. Diverse Studien und Umfragen zeigen beispielsweise, dass der Auslagenersatz bei auswärtigen Arbeitsorten häufig ungenügend ausfällt. Dies kann dadurch abgeleitet werden, dass der Begriff „Unterhalt“ zu wenig konkret ist. Durch eine exemplarische Aufzählung könnte dieser Artikel transparenter gestaltet werden. Der Arbeitsort abseits vom Ort des Arbeitgebenden hat auch eine steuerliche Bedeutung. Die Differenzierung ist ungenügend, ab wann genau das Zuhause als Arbeitsort angesehen wird. Diese Differenzierung ist jedoch wichtig, da je nach Auslegung die kantonale Steuergesetzgebung zur Anwendung gelangt. Hier würde eine Sonderklausel zugunsten des Arbeitgebendenstandortes Abhilfe schaffen. Zielgruppe: Parlament.

5. Den rechtlichen Rahmen der Scheinselbstständigkeit präzisieren: Es stellt sich die Frage, in welchem rechtlichen Rahmen die Scheinselbstständigkeit zu behandeln ist. Faktisch bestehen keine Versicherungsmöglichkeiten gegen Arbeitslosigkeit, weder eine berufliche Vorsorge noch eine obligatorische Versicherung nach UVG. Ebenfalls sind Berufskrankheiten nicht abgedeckt. Eine gesetzliche Präzisierung kann Missverständnisse klären. Ansonsten ist darauf zu achten, dass dieser nicht klar definierte Bereich vertraglich präzisiert und geregelt wird. Al- 
ternativ kann der Gesetzgeber den Begriff der Arbeitnehmerin/des Arbeitnehmers ausdehnen und dadurch als Grundlage für das gesamte Arbeitsrechtfeld das Obligationenrecht zur Anwendung gelangen. Zielgruppe: Parlament, Arbeitgebende, Arbeitgebendenverbände.

6. Gesetzgebung zur Personalvermittlung überprüfen: Das Bundesgesetz über die Arbeitsvermittlung und den Personalverleih muss vollumfänglich auch auf jene Institutionen angewendet werden, die über das Internet quasi nur als „Broker” zwischen selbstständig erwerbenden "Crowdworkern" und Arbeitgebenden vermitteln. Die Herausforderung dabei ist, dass sich diese Broker mit ihrem Firmensitz und ihren Websites dem schweizerischen Rechtssystem gänzlich entziehen können. Zwar scheint es für die anspruchsvolleren der über Webplattformen vermittelten Aufträge zu gelten, dass potenzielle Arbeitgebende sich nicht gerne auf „obskure” Vermittlungsplattformen ausserhalb nationaler und europäischer Rechtsnormen begeben mögen. Dennoch wäre zu überprüfen, inwieweit die Schweiz eine europäische Regelung in diesem Bereich analog anwenden könnte. Zielgruppe: Parlament.

7. Mechanismen der Rechtsdurchsetzung überprüfen: Grundsätzlich ist festzustellen, dass sich die Sozialpartnerinnen und Sozialpartner hinsichtlich der Aushandlung ihrer Arbeitsbedingungen zumeist finden. Das hier zugrunde liegende Vertrauen sollte betont und gefördert werden. Bei Problemen hinsichtlich der Einhaltung der Arbeitszeit haben Arbeitnehmende die Möglichkeit, sich an die Arbeitsinspektorate als Aufsichtsbehörde zu wenden. Auch prüfen die Inspektorate die Einhaltung der Arbeitszeit. Insbesondere vor dem Hintergrund der aktuellen Regelungen zur Arbeitszeiterfassungspflicht ist zu überprüfen, vor welchen aktuellen Herausforderungen die Inspektorate stehen, um diese Verpflichtungen gewissenhaft wahrnehmen zu können. Beispielsweise finden neue Arbeitsformen in tendenziell kleinen Organisationen statt, welche schwieriger überprüft werden können als Grossbetriebe. Die Inspektorate sollen angemessen auf Beschwerden von Arbeitnehmenden reagieren können. Ihre Tätigkeit dient dem Interessenausgleich zwischen Betrieb und Beschäftigten. Die Tätigkeit der Inspektoren beruht häufig auf einem pragmatischen Augenmass, welches weiter eingesetzt werden sollte. Für ein effizientes Funktionieren dieser Drittparteiunterstützung müssten die Inspektorate angemessen ausgestattet sein - ihr Auftrag ist zu überprüfen und gegebenenfalls zu erweitern. Ein gewisse Rolle könnte im Rahmen der Aushandlung und Durchsetzung der Flexibilitätsparameter auch das Instrument der "tripartiten Kommissionen“ 
spielen. Diese werden im gewerblichen Bereich bereits eingesetzt und bestehen aus Vertretenden der Arbeitgebenden, Arbeitnehmenden und der öffentlichen Hand. Sie könnten Lohndumping für Selbstständige verhindern, Scheinselbstständigkeiten überprüfen, im Sinne des Arbeitgebenden für Konkurrenzschutz, für den Arbeitnehmenden für Lohnschutz sorgen, branchenweite Verhaltensstandards formulieren, Plattformen mit garantierten Standards auf geprüfte Listen setzen (White Lists), die Selbstregulierung der Fachverbände etc. begleiten und bei sozialversicherungsrechtlichen Fragestellungen mit Augenmass praktisch vor Ort helfen. Zielgruppe: Kantonale Verwaltungen, Arbeitsinspektorate, Sozialpartnerinnen und Sozialpartner.

8. Soziale Sicherung in der Nacherwerbsphase erforschen und wenn nötig politisch regeln: Die soziale Sicherung in der Nacherwerbsphase hängt nach wie vor sehr stark von der Erwerbstätigkeit ab (Äquivalenzprinzip). Das bedeutet: Je länger atypische Beschäftigungsverhältnisse im Laufe eines Erwerbslebens ausgeübt werden und je geringer das Einkommen ist, desto höher ist das Risiko der Altersarmut. Eine stärkere Entkoppelung bzw. Abschwächung des Äquivalenzprinzips könnte ebenso Teil der Lösung dieses Problems sein wie die weitere Flexibilisierung der Ruhestandsregelungen. Gesellschaftlich besteht die berechtigte Frage, wie aktive Rentner und Rentnerinnen eingebunden werden und an einem selbstbestimmten Erwerbsleben teilhaben können. Der Imperativ sollte sein, dass Rentnerinnen und Rentner arbeiten können, sofern sie dies wollen (aber nicht müssen). Ein Mindeststandard des Alterseinkommens muss gelten und ausreichend für eine befriedigende Lebensführung sein. Hier besteht Forschungsbedarf, damit für die politischen Akteurinnen und Akteure fundierte Diskussionsgrundlagen geschaffen werden können. Zielgruppen: Forschungsförderung, Forschende, Fachverbände.

9. Gesamtübersicht über rechtliche Aspekte und soziale Absicherung fördern: Die Schweiz schneidet im europäischen Vergleich hinsichtlich der sozialen Absicherung generell gut ab. Das Arbeitsgesetz beinhaltet bereits heute viele anwendbare Grundsätze, aber auch viele Ausnahmen und zahlreiche Verordnungen, was eine gewisse Unübersichtlichkeit mit sich bringt. Um Unklarheiten oder Missverständnisse im Arbeitsalltag vorzubeugen, ist zu prüfen, ob durch die Erstellung einer Gesamtübersicht bereits einige Erleichterungen erreicht werden könnten. Eine schlüssige und verständliche Übersicht wäre der Transparenz dienlich und würde die Umsetzung flexibler Arrangements positiv beein- 
flussen. Zielgruppe: Parlament, Arbeitgebendenverbände, Schweizerischer Gewerkschaftsbund, Fachverbände.

\subsubsection{Handlungsempfehlungen zur volkswirtschaftlichen Erfassung}

Die bestehenden Erhebungen der öffentlichen Statistik und die Periodizität ihrer Veröffentlichung lassen eine Beurteilung der aktuellen und kurzfristig zukünftigen Lage in vielen Fällen nicht zu. Dies ist für die Bereiche der beruflichen Mobilität der Arbeitnehmenden, der Schattenwirtschaft und der Arbeitsbedingungen der Arbeitskraftunternehmenden relevant. Da die Dynamik auf dem Arbeitsmarkt zunehmen dürfte, müsste das Monitoring vorausschauender werden, um effiziente Massnahmen auf verschiedenen Ebenen zu ermöglichen. Es sollten hier mehr und zielgenauere Indikatoren mit Frühwarnfunktion entwickelt und entsprechende Daten zur besseren Massnahmensetzung erhoben werden.

1. Berufliche Mobilität zeitnaher erfassen: Berichterstattungen über die berufliche Mobilität geben unter anderem Aufschluss über die Veränderungen auf dem Arbeitsmarkt, beispielsweise über Stellen- und Statuswechsel und die Wechselabsichten der Erwerbsbevölkerung. Bei diesem Thema ist die zeitliche Verzögerung bis zur Publikation sehr gross, weil die Datenaufbereitung teilweise fünf oder mehr Jahre benötigt. Sehr schnelle Umfragen sind die SAKE des BFS, das Schweizer Haushalt-Panel (je jährlich) und das bislang in der Regel jährlich erscheinende HR-Barometer. Eine auskunftsreiche, aber sehr langsame Befragung mit verwandtem Inhalt ist beispielsweise der mehrjährig erhobene European Working Condition Survey (EWCS). Problematisch an den schnelleren Berichterstattungen ist, dass sie keine vorausschauenden Faktoren abdecken (beispielsweise erwartete Jobwechsel) und dass Berichte, die das versuchen, zu langsam erhoben und publiziert werden. Wirtschaftspolitische Steuerungsmassnahmen, die u.a. auf den schneller erhobenen Berichten beruhen, haben in der Regel die Bewältigung aktueller, mehr oder weniger akuter Probleme zum Ziel, verfolgen aber keine längerfristige Ausrichtung. Zielgruppen: Mitglieder der Bundesstatistikkommission, Mitglieder von Fedestat und Regiostat, Expertengruppen für Wirtschaftsstatistik, Forschungsinstitutionen, Politik.

2. Virtuelle Schattenwirtschaft und Schwarzarbeit erfassen: Die zunehmende Flexibilisierung der Arbeit ist eine wichtige Ursache für das zukünftige Wachstum der Schattenwirtschaft und der daraus entstehenden Gefahr für die Stabilität der Systeme der sozialen Sicherheit. 
Schattenwirtschaft und Schwarzarbeit werden durch kausale Faktoren begünstigt, die wiederum durch virtuelle Arbeitsformen verstärkt werden könnten. Um die Schwächung der sozialen Sicherungssysteme zu vermeiden, müssen negative Entwicklungen hinsichtlich dieser Faktoren erkannt werden können. Zudem gilt es, in entsprechenden Untersuchungen auch die Besonderheiten des schweizerischen Kontextes zu berücksichtigen. Da sich eine Schattenwirtschaft im Bereich der virtuellen Arbeit durch ihren digitalen Charakter sehr schnell entfalten könnte, soll es nicht zur Beruhigung dienen, dass die Schweiz im internationalen Vergleich diesbezüglich bislang vergleichsweise gut abschneidet. Zielgruppen: Mitglieder der Bundesstatistikkommission, Mitglieder von Fedestat und Regiostat, Expertengruppen für Wirtschaftsstatistik, Forschungsinstitutionen, Politik.

3. Kontinuierliches Monitoring der Berufsbedingungen von „Arbeitskraftunternehmenden": Dieses Monitoring wird aktuell bestenfalls sporadisch durch Gewerkschaften oder Fachverbände vollzogen. Eine systematische und vollumfängliche, regelmässige Beobachtung dieser fragmentierten und anwachsenden Gruppe der Arbeitsgesellschaft zudem noch mit vorausschauenden Indikatoren existiert nicht, da auch die Gruppe der „Arbeitskraftunternehmenden“ nicht klar definiert ist. Da Unternehmen an einer leistungsfähigen und flexiblen Erwerbsbevölkerung ebenfalls interessiert sind, müssten auch Wirtschaftsvertretungen an einem solchen evtl. staatlich strukturierten Monitoring mitwirken. Zu erwähnen ist die besondere Schwierigkeit, dass diese Gruppe eine relativ grosse Veränderlichkeit aufweist und somit schwer systematisch zu erfassen ist. Es darf auch angenommen werden, dass auf individuellen Angaben beruhende vorausschauende Fragen aufgrund der häufig schwierigen Planbarkeit in dieser Gruppe mit Vorsicht genossen werden müssen. Zielgruppen: Mitglieder der Bundesstatistikkommission, Mitglieder von Fedestat und Regiostat, Expertengruppen für Wirtschaftsstatistik, Forschungsinstitutionen, Politik. 
Abschliessend die Handlungsempfehlungen im Überblick:

Tabelle 8: Überblick der Handlungsempfehlungen zur flexiblen Arbeitswelt

\begin{tabular}{|c|c|c|}
\hline Nr. & Handlungsempfehlung & Zielgruppen \\
\hline 1 & $\begin{array}{l}\text { Einen weiten Rahmen für den Umgang } \\
\text { mit zeitlicher und örtlicher Entgrenzung } \\
\text { der Arbeit setzen }\end{array}$ & $\begin{array}{l}\text { Sozialpartnerin / Sozialpartner } \\
\text { auf Ebene Betrieb } \\
\text { Parlament }\end{array}$ \\
\hline 2 & $\begin{array}{l}\text { Sensibilisierung zur Subjektivierung der } \\
\text { Arbeit }\end{array}$ & $\begin{array}{l}\text { Bildungspolitik } \\
\text { Bildungspolitikanbieterinnen } \\
\text { und -anbieter } \\
\text { Gewerkschaften } \\
\text { Start-up Initiativen }\end{array}$ \\
\hline 3 & $\begin{array}{l}\text { Betriebliche Flexibilitätsplanung als } \\
\text { partizipative Organisationsentwicklung }\end{array}$ & Sozialpartnerin / Sozialpartner \\
\hline 4 & $\begin{array}{l}\text { Breite Mitwirkung als gemeinsames } \\
\text { Interesse der Sozialpartnerinnen / } \\
\text { Sozialpartner stärken }\end{array}$ & $\begin{array}{l}\text { Sozialpartnerin / Sozialpartner } \\
\text { Arbeitnehmendenverbände }\end{array}$ \\
\hline 5 & $\begin{array}{l}\text { Regelungen zu modernen Arbeitszeit- } \\
\text { systemen weiter anpassen }\end{array}$ & Parlament \\
\hline 6 & $\begin{array}{l}\text { Sozialversicherungsdefizite bei Kurz- } \\
\text { Arbeitseinsätzen thematisieren }\end{array}$ & $\begin{array}{l}\text { Sozialpartnerin / Sozialpartner } \\
\text { Sozialversicherungen } \\
\text { Parlament }\end{array}$ \\
\hline 7 & $\begin{array}{l}\text { Arbeitsrechtliche Praxis im Umgang } \\
\text { mit noch undefinierten Arbeitsformen } \\
\text { regeln }\end{array}$ & $\begin{array}{l}\text { Parlament } \\
\text { Parteien eines Arbeitsvertrags }\end{array}$ \\
\hline 8 & $\begin{array}{l}\text { Aus dem Arbeitsort abgeleitete Ansprü- } \\
\text { che klären }\end{array}$ & Parlament \\
\hline 9 & $\begin{array}{l}\text { Den rechtlichen Rahmen der Schein- } \\
\text { selbstständigkeit präzisieren }\end{array}$ & $\begin{array}{l}\text { Parlament } \\
\text { Arbeitgebende } \\
\text { Arbeitgebendenverbände }\end{array}$ \\
\hline
\end{tabular}




\begin{tabular}{|l|l|l|}
\hline Nr. & Handlungsempfehlung & Zielgruppen \\
\hline 10 & $\begin{array}{l}\text { Gesetzgebung zur Personalvermittlung } \\
\text { überprüfen }\end{array}$ & Parlament \\
\hline 11 & $\begin{array}{l}\text { Mechanismen der Rechtsdurchsetzung } \\
\text { überprüfen }\end{array}$ & $\begin{array}{l}\text { Kantonale Verwaltungen } \\
\text { Arbeitsinspektorate } \\
\text { Sozialpartnerin / Sozialpartner }\end{array}$ \\
\hline 12 & $\begin{array}{l}\text { Soziale Sicherung in der Nacherwerbs- } \\
\text { phase erforschen und wenn nötig poli- } \\
\text { tisch regeln }\end{array}$ & Forschungsförderung \\
\hline 13 & $\begin{array}{l}\text { Gesamtübersicht über rechtliche } \\
\text { Aspekte und soziale Absicherung } \\
\text { fördern }\end{array}$ & $\begin{array}{l}\text { Fachverbände } \\
\text { Parlament } \\
\text { Arbeitgebendenverbände } \\
\text { Schweizerischer Gewerk- } \\
\text { schaftsbund } \\
\text { Fachverbände }\end{array}$ \\
\hline 14 & $\begin{array}{l}\text { Berufliche Mobilität } \\
\text { erfassen }\end{array}$ & $\begin{array}{l}\text { Mitglieder der Bundesstatistik- } \\
\text { kommission } \\
\text { Mitglieder von Fedestat und } \\
\text { Regiostat } \\
\text { Expertengruppen für Wirt- } \\
\text { schaftsstatistik } \\
\text { Forschungsinstitutionen } \\
\text { Politik }\end{array}$ \\
\hline
\end{tabular}




\begin{tabular}{|l|l|l|}
\hline Nr. & Handlungsempfehlung & Zielgruppen \\
\hline 16 & $\begin{array}{l}\text { Kontinuierliches Monitoring der Berufs- } \\
\text { bedingungen von „Arbeitskraftunter- } \\
\text { nehmenden“ }\end{array}$ & $\begin{array}{l}\text { Mitglieder der Bundesstatistik- } \\
\text { kommission } \\
\end{array}$ \\
& $\begin{array}{l}\text { Mitglieder von Fedestat und } \\
\text { Regiostat } \\
\text { Expertengruppen für Wirt- } \\
\text { schaftsstatistik } \\
\end{array}$ & $\begin{array}{l}\text { Forschungsinstitutionen } \\
\text { Politik }\end{array}$ \\
\hline
\end{tabular}

\subsection{Fazit}

Das Verständnis der traditionellen Büro- und Fabrikarbeit mit Vorgesetzten, welche die Arbeit anweisen und überwachen, scheint im Zuge der voranschreitenden Digitalisierung und strukturellen Veränderung der Arbeitswelt nicht mehr aktuell und angemessen. Und doch ist nicht ausgeschlossen, dass das tayloristische Prinzip in seinen negativen Ausprägungen im Bereich der mobilen Arbeit prominenter hervortreten könnte als bisher angenommen. Dieser Entwicklung ist (pro)aktiv zu begegnen.

Die vorliegende Studie dient diesem Ziel, in dem sie das bekannte Wissen umfangreich aufbereitet, zu Erkenntnissen bündelt und mit einer Zukunftsperspektive versieht. Sie zeigt Spannungsfelder im rechtlichen Bereich und volkswirtschaftliche Konsequenzen auf und mündet in die Entwicklung denkbarer Szenarien und angebrachter Handlungsempfehlungen.

Die vorliegende Studie zeigt, dass sich eine aktive Auseinandersetzung mit dem Phänomen lohnt und dass sie machbar ist. In die Studienerstellung flossen nicht nur signifikante Literaturbestandteile aus verschiedensten Disziplinen ein, sondern auch die Einschätzung und Expertise eines interdisziplinär arbeitenden Teams und einer engagierten Begleitgruppe, welche die Vielfalt der eingebrachten Expertise nochmals erhöhte, aber nach erfolgter Validierung auch zur Robustheit der Argumente beitrug.

Das Phänomen der flexiblen Arbeitswelt bleibt am Ende ein "fuzzy Problem“, d.h. ein schlecht definiertes, aber „echtes“ Problem. Als solches stellt es eine 
Herausforderung dar, deren Bewältigung einen grossen Unterschied machen kann, einen Unterschied hin zu einer widerstandsfähigen, resilienten Schweizer Arbeitswelt, die ihre weltweite Spitzenposition erhalten oder sogar noch ausbauen kann. Dies ist keine Selbstverständlichkeit, sondern bedarf einer gemeinschaftlichen Entwicklungsleistung aller beteiligten Parteien. Diese Studie leistet einen klaren Beitrag zur Orientierung in dieser momentan unübersichtlichen, komplexen, aber auch hochinteressanten und chancenreichen Ausgangslage. 


\section{Literaturverzeichnis}

Addison, J. T. \& Surfield, C. J. (2007): Atypical work and pay. Southern Economic Journal 73(4). S. 1038-1065.

Afuah, A. \& Tucci, C. L. (2012): Crowdsourcing As a Solution to Distant Search. Academy of Management Review 37(3). S. 355-375.

Allen, T. D., Golden, T. D. \& Shockley, K. M. (2015): How Effective Is Telecommuting? Assessing the Status of Our Scientific Findings. Psychological Science in the Public Interest 16(2). S. 40-68, doi:10.1177/1529100615593273.

Allmendinger, J., Giesecke, J., Hipp, L., Leuze, K. \& Stuth, S. (2014): Mehr Jobs oder nur mehr schlechte Jobs? Die Entwicklung atypischer Beschäftigung in Europa. WZB Brief Arbeit Nr. 13.

Andersson, P. (2008): Happiness and health: Well-being among the selfemployed. Journal of Socio-Economics 37. S. 213-236.

Angermann, A. \& Eichhorst, W. (2013): Who cares for you at home? Personal and household services in Europe. IZA policy paper No 71.

Arbeitsmarktbeobachtung Ostschweiz, Aargau und Zug (Amosa), (2008):

Chancen für Geringqualifizierte in der internationalen Bodenseeregion. Arbeitsmarktbeobachtung Ostschweiz, Aargau und Zug, Arbeitsmarktservice Liechtenstein, Arbeitsmarktservice Vorarlberg und Bundesagentur für Arbeit (Hrsg.).

http://www.amosa.net/fileadmin/user_upload/projekte/GQ/01_GQ_ Schlussbericht_DE.pdf. Abgerufen am 06.07.2015.

Åstebro, T. \& Chen, J. (2014): The entrepreneurial earnings puzzle: Mismeasurement or real? Journal of Business Venturing 29(1). S. 88-105.

Badura, B., Ducki, A., Schröder, H., Klose, J. \& Meyer, M. (Hrsg.) (2012): Fehlzeiten-Report 2012. Gesundheit in der flexiblen Arbeitswelt: Chancen nutzen - Risiken minimieren. Springer-Verlag. Berlin, Heidelberg.

Bahamondes Pavez, C., Schiml, N. \& Schüpbach, H. (2012): Stabilität und Flexibilität - Ressourcen zur nachhaltigen Erhaltung von Gesundheit und Wohlbefinden. In: Badura, B., Ducki, A., Schröder, H., Klose, J. \& Meyer, 
M. (Hrsg.): Fehlzeiten-Report 2012. (S. 169-180). Springer-Verlag. Berlin, Heidelberg.

Barnes, S.-A., de Hoyos, M., Baldauf, B., Behle, H. \& Green, A. (2013): D2:

Review of state of the art and mapping: CROWDEMPLOY. University of Warwick: Institute for Employment Research.

http://is.jrc.ec.europa.eu/pages/documents/ICT4EMPLCrowdemploymap pingforweb.pdf. Abgerufen am 19.11.2015.

Battisti, M. \& Eiselen, T. (2008): Insights Through Performative Approaches.

Forum Qualitative Social Research 9(2). Art. 44. http://nbnresolving.de/urn:nbn:de:0114-fqs0802444. Abgerufen am 19.11.2015.

Baua, Bundesanstalt für Arbeitsschutz und Arbeitsmedizin, Deutschland (2013): Im Takt? Risiken, Chancen und Gestaltung von flexiblen Arbeitszeitmodellen. 4. Auflage.

Baumgartner, D., Schulz, T. \& Seidl, I. (2012): „Quantifying entrepreneurship and its impact on local economic performance: A spatial assessment in rural Switzerland." Entrepreneurship \& Regional Development 25(3-4). S. 222-250.

Beitzer, H. (2015): Wir statten Arbeitgeber mit billigem Menschenmaterial aus. Süddeutsche Zeitung. http://www.sueddeutsche.de/wirtschaft/systemhartz-iv-wir-statten-arbeitgeber-mit-billigem-menschenmaterial-aus1.2375543. Abgerufen am 26.06.2015.

Bélanger, F. (1999): Workers' propensity to telecommute: An empirical study. Information \& Management 35. S. 139-153.

Benavides, F. G., Benach, J., Diez-Roux, A. V. \& Roman, C. (2000): How do types of employment relate to health indicators? Findings from the second European survey on working conditions. Journal of Epidemiol Community Health 2000;54. S. 495-501.

Bender, D. (2013). Mobile Arbeitsplätze als kreative Räume. CoworkingSpaces, Cafés und andere urbane Arbeitsorte. transcript Verlag. Bielefeld.

Beranek Zanon, N. (2012): Bring your own Device (BYOD) aus rechtlicher Sicht. Jusletter IT 12. September 2012.

Berglund, T., Furaker, B. \& Vulkan, P. (2013): Is job insecurity compensated for by employment and income security? Economic and industrial democracy 35(1). S. 165-184, doi:10.1177/0143831X12468904. 
Berndt, C. (2015): Menschen fürs Büro dressieren. Süddeutsche Zeitung. 14. März 2015.

Birk, S., Nelsen, M., Lackerbauer, S. \& Kirchner, C. (2015): Wie wir morgen arbeiten ... Eine Stichprobe. Institut für Arbeitsdesign und Zukunftstechnologien e.V. Parsdorf. http://www.i-faz.de/ecosystem/studie/. Abgerufen am 19.11.2015.

Bitcoin Suisse (2016): Bitcoin accepting companies in Switzerland. https://www.bitcoinsuisse.ch/en/bitcoin-switzerland/. Abgerufen am 16.01.2016.

Blockchain (2016): Gesamtzahl der Transaktionen. https://blockchain.info/de/ charts/n-transactions-total?timespan=all\&showDataPoints=false\&days AverageString $=1 \&$ show_header=true\&scale $=0$ \&address $=$. Abgerufen am 16.01.2016.

Blum, A. (2002): Strategische Bedeutung von Teilzeitbeschäftigung. In: Baillod, J. (Hrsg.): Chance Teilzeitbeschäftigung. Argumente und Materialien für Verantwortliche. (S. 35-77). vdf Hochschulverlag. Zürich.

Böhle, F. (2010): Neue Anforderungen an die Arbeitswelt - neue Anforderungen an das Subjekt. In: Keupp, H. \& Dill, H. (Hrsg.): Erschöpfende Arbeit. Gesundheit und Prävention in der flexiblen Arbeitswelt. (S. 77-95). transcript Verlag. Bielefeld.

Böhne, A. \& Breutmann, N. (2012): Flexibilisierung der Arbeitswelt aus Unternehmenssicht: Chancen und Risiken für Arbeitgeber und Arbeitnehmer. In: Badura, B., Ducki, A., Schröder, H., Klose, J. \& Meyer, M. (Hrsg.): Fehlzeiten-Report 2012. (S. 23-27). Springer-Verlag. Berlin, Heidelberg.

Bosch, G., Brügelmann, R., Breyer, F. \& Holst, E. (2014): Nebenjob. Von Steuerzahlern subventioniert. Wirtschaftsdienst 94(4). S. 236, doi:10.1007/s10273-014-1663-0.

Brandt, C. (Hrsg.) (2010): Mobile Arbeit - Gute Arbeit? Arbeitsqualität und Gestaltungsansätze bei mobiler Arbeit. Berlin: ver.di - Vereinte Dienstleistungsgewerkschaft.

Brechbühl, J. (2002): Schwarzarbeit und Sozialversicherung. Die Volkswirtschaft 2-2002. S. 9-12.

Brehmer, W. \& Seifert, H. (2008): Sind atypische Beschäftigungsverhältnisse prekär? Eine empirische Analyse sozialer Risiken. Zeitschrift Für Arbeitsmarktforschung 4. S. 501-531. 
Brenke, K. (2013): Allein tätige Selbständige: starkes Beschäftigungswachstum, oft nur geringe Einkommen. DIW Wochenbericht 7. S. 3-16.

Brenke, K. (2014): Heimarbeit: Immer weniger Menschen in Deutschland gehen ihrem Beruf von zu Hause aus nach. DIW-Wochenbericht 81(8). S. 131140. http://hdl.handle.net/10419/92922. Abgerufen am 19.11.2015.

Broughton, N. \& Ussher, K. (2013): Good for Growth: Refocusing Entrepreneurship Policy. Social Market Foundation.

http://www.smf.co.uk/publications/good-for-growth-refocusingentrepreneurship-policy/. Abgerufen am 06.07.2015.

Bundesministerium für Arbeit und Soziales (BMAS) (2015): Gewünschte und erlebte Arbeitsqualität, Forschungsbericht 456.

http://www.bmas.de/SharedDocs/Downloads/DE/PDF-

Publikationen/Forschungsberichte/fb-456.pdf?__blob=publicationFile. Abgerufen am 21.07.2015.

Bundesamt für Sozialversicherungen (BVS) (2014). Soziale Sicherheit in der Schweiz.

http://www.bsv.admin.ch/themen/ueberblick/00003/index.html?lang=de. Abgerufen am 06.07.2014.

Bundesamt für Sozialversicherungen (BVS) (2015): Statistik der Ergänzungsleistungen zur AHV und IV 2014.

http://www.bsv.admin.ch/dokumentation/zahlen/00095/00444/index.html? lang=de\&download=NHzLpZeg7t,Inp6I0NTU042I2Z6In1acy4Zn4Z2qZpn O2Yuq2Z6gpJCEdYN5fGym162epYbg2c_JjKbNoKSn6A--. Abgerufen am 06.07.2015.

Bundesamt für Statistik (BFS) (2006): Selbständige Erwerbstätigkeit in der Schweiz. Eine Untersuchung zu den Ergebnissen der Schweizerischen Arbeitskräfteerhebung. BFS Aktuell.

http://www.bfs.admin.ch/bfs/portal/de/index/news/publikationen.Documen t.83703.pdf. Abgerufen am 18.12.2014.

Bundesamt für Statistik (BFS) (2012): Armut in der Schweiz. Konzepte, Resultate und Methoden. Ergebnisse auf der Basis von SILC 2008 und 2010. Neuchâtel.

Bundesamt für Statistik (BFS) (2013): Auf dem Weg zur Gleichstellung von Mann und Frau.

http://www.ebg.admin.ch/dokumentation/00012/00193/index.html?lang= de\&download=NHzLpZeg7t,Inp6I0NTU042I2Z6In1acy4Zn4Z2qZpnO2Yu 
q2Z6gpJCDdH1_gWym162epYbg2c_JjKbNoKSn6A--. Abgerufen am 15.03.2015.

Bundesamt für Statistik (BFS) (2013b): Hochschulabsolventen und Hochschulabsolventinnen auf dem Arbeitsmarkt. Erste Ergebnisse der Längsschnittbefragung 2007.

http://www.bfs.admin.ch/bfs/portal/de/index/themen/15/22/publ.Document .111252.pdf. Abgerufen am 18.12.2014.

Bundesamt für Statistik (BFS) (2014): Teilzeitarbeit gewinnt weiter an Bedeutung.

http://www.bfs.admin.ch/bfs/portal/de/index/news/medienmitteilungen. html?pressID=9411. Abgerufen am 19.11.2015.

Bundesamt für Statistik (BFS) (2015a). Panorama: Arbeit und Erwerb. http://www.bfs.admin.ch/bfs/portal/de/index/themen/03/01/pan.Document. 118134.pdf. Abgerufen am 19.11.2015.

Bundesamt für Statistik (BFS) (2015b). SAKE in Kürze 2014: Schweizerische Arbeitskräfteerhebung (No. 360-1400).

http://www.bfs.admin.ch/bfs/portal/de/index/themen/03/22/publ.html?publi cationID=6613. Abgerufen am 19.11.2015.

Bundesamt für Statistik (BFS) (2015c): Erwerbsarbeit und Beruf. Teilzeitbeschäftigung.

http://www.bfs.admin.ch/bfs/portal/de/index/regionen/thematische_karten/ gleichstellungsatlas/erwerbsarbeit_und_beruf/teilzeitbeschaeftigung.html. Abgerufen am 08.08.2015.

Bundesamt für Statistik (BFS) (2015d): Erwerbsarbeit und Beruf. Teilzeitbeschäftigung.

http://cmsp.migration6.vbs.admin.ch/bfs/portal/de/index/themen/03/02/ blank/data/03.Document.100736.xls. Abgerufen am 08.08.2015.

Bundesamt für Statistik (BFS) (2015e): Eurostat - Einkommen, soziale Eingliederung und Lebensbedingungen. Einkommensverteilung und finanzielle Armutsgefährdung. Arbeits-Armutsgefährdungsquote nach Voll-/Teilzeitbeschäftigung.

http://appsso.eurostat.ec.europa.eu/nui/show.do?dataset=ilc_iw07\&lang= de. Abgerufen am 08.08.2015.

Bundesamt für Statistik (BFS) (2015f): Eurostat - Einkommen, soziale Eingliederung und Lebensbedingungen. Einkommensverteilung und finanzielle Armutsgefährdung. Arbeits-Armutsgefährdung nach Bildungsabschluss. 
http://appsso.eurostat.ec.europa.eu/nui/show.do?dataset=ilc_iw04\&lang= de. Abgerufen am 08.08.2015.

Bundesamt für Statistik (BFS) (2015g): Indikator 30107: HochgeschwindigkeitsInternet.

http://www.bfs.admin.ch/bfs/portal/de/index/themen/16/04/key/approche_ globale.Document.25584.xls. Abgerufen am 08.08.2015.

Bundesamt für Statistik (BFS) (2015h): Indikator 30103: IKT-Ausstattung der Haushalte (Informations- und Kommunikationstechnologien).

http://www.bfs.admin.ch/bfs/portal/de/index/themen/16/04/key/approche_ globale.Document.25552.xls. Abgerufen am 08.08.2015.

Bundesamt für Statistik (BFS) (2015i): Arbeitszeitmodelle der Arbeitnehmenden (ohne Lehrlinge) nach Nationalität.

http://www.bfs.admin.ch/bfs/portal/de/index/themen/03/02/blank/data/03. Document.100780.xls. Abgerufen am 08.08.2015.

Bundesamt für Statistik (BFS) (2015j): Arbeit auf Abruf nach Geschlecht, Nationalität, Altersgruppen, Familientyp.

http://www.bfs.admin.ch/bfs/portal/de/index/themen/03/02/blank/data/03. Document.100784.xls. Abgerufen am 09.02.2016

Bundesgerichtsentscheid (1996), BGE 116 V 136.

http://relevancy.bger.ch/php/clir/http/index.php?lang=de\&zoom=\&type= show_document\&highlight_docid=atf\%3A\%2F\%2F116-V-136\%3Ade. Abgerufen am 15.03.2015.

Bundesgerichtsentscheid (1998), BGE 124 III 251.

http://relevancy.bger.ch/php/clir/http/index.php?lang=de\&zoom=IN\&type= show_document\&highlight_docid=atf\%3A\%2F\%2F124-III-249\%3Ade. Abgerufen am 15.03.2015.

Bundesgerichtsentscheid (1998), BGE 125 III 65

http://relevancy.bger.ch/php/clir/http/index.php?lang=de\&zoom=\&type= show_document\&highlight_docid=atf\%3A\%2F\%2F125-III-65\%3Ade. Abgerufen am 15.03.2015.

Bundesministerium der Justiz und für Verbraucherschutz (1996): Altersteilzeitgesetz. http://www.gesetze-im-internet.de/alttzg_1996/index.html. Abgerufen am 06.07.2014. 
Bürgi Nägeli Rechtsanwälte (o.J.): Funktionelle und vertragliche Abgrenzungen. http://www.freier-mitarbeiter.ch/abgrenzungen. Abgerufen am 18.12.2014.

Bütler, M. (2014): Sozialstaat und Anstand. Schweiz am Sonntag. 28.12.2014.

Caicedo, E. \& Gineikytè, V. (2015): New forms of employment Crowd employment, Lithuania. Case study 16: Lingjob. http://eurofound.europa.eu/sites/default/files/page/field_ef_documents/ 16_-_ef1461_-_It_-_crowd_employment_-_lingjob_-_final.pdf. Abgerufen am 15.03.2015.

Cappellari, L., Dell'Aringa, C. \& Leonardi, M. (2012): „Temporary Employment, Job Flows and Productivity: A Tale of Two Reforms." The Economic Journal 122(562). S. 188-215.

Chanson, G. (2008): Weka Fachkongress, Arbeitsrecht I-2008. Referat Flexibel Arbeiten. Kongressunterlagen. WEKA Verlag. Zürich.

Chevalier, A. \& Kaluza, G. (2015): Psychosozialer Stress am Arbeitsplatz: Indirekte Unternehmenssteuerung, selbstgefährdendes Verhalten und die Folgen für die Gesundheit. Gesundheitsmonitor 01/15. Bertelsmann Stiftung und Barmer GEK. https://www.bertelsmannstiftung.de/fileadmin/files/Projekte/17_Gesundheitsmonitor/Newsletter_ Gesundheitsmonitor_selbstgefaehrdendes_Verhalten_20150316.pdf. Abgerufen am 06.07.2015.

Chilles, F. (2012): Umgang mit Entgrenzung aus juristischer Perspektive. In: Badura, B., Ducki, A., Schröder, H., Klose, J. \& Meyer, M. (Hrsg.): Fehlzeiten-Report 2012. (S. 125). Springer-Verlag. Berlin, Heidelberg.

Cirigliano, L. (2013): Arbeitszeiterfassung vorerst erfolgreich verteidigt. http://www.sgb.ch/index.php?id=216\&L=0\&tx_ttnews[tt_news] $=2253 \&$ cHash=2c913c12c36fc7f778bac5ed4167f76a. Abgerufen am 18.12.2014.

Clasen, J. (2012): Flexibel ohne Grenzen? - Belastungen, Anforderungen und Ressourcen von Freelancern. In: Badura, B., Ducki, A., Schröder, H., Klose, J. \& Meyer, M. (Hrsg.): Fehlzeiten-Report 2012. (S. 97-107). Springer-Verlag. Berlin, Heidelberg.

Clinton, M., Totterdell, P. \& Wood, S. (2006): „A grounded theory of portfolio working: Experiencing the smallest of small businesses". International Small Business Journal Vol. 24 No. 2. S. 179-203. 
Conerly, B. (2015): „Should Businesses Accept Bitcoin?“ Forbes. 24. April 2015. http://www.forbes.com/sites/billconerly/2015/02/24/should-businessesaccept-bitcoin/. Abgerufen am 06.07.2015.

Cooper, C. D. \& Kurland, N. B. (2002): Telecommuting, professional isolation, and employee development in public and private organizations. Journal of Organizational Behavior 23. S. 511-532.

Curtarelli, M. (2013). New forms of employment and work. First results from a Eurofound EU-28 (+ Norway) research project. Presentation given at COST meeting - Dynamics of Virtual Work - Athens 7-9 October 2013.

De Cuyper, N., De Jong, J., De Witte, H., Isaksson, K., Rigotti, T. \& Schalk, R. (2008): „Literature Review of Theory and Research on the Psychological Impact of Temporary Employment: Towards a Conceptual Model." International Journal of Management Reviews 10(1). S. 25-51.

Degenhardt, B., Gisin, L. \& Schulze, H. (2014): Schweizerische Umfrage „Homeoffice 2013“ - Teil 1: Vergleich der Rahmenbedingungen aus der Sicht der nie, unregelmässig und regelmässig alternierend im Homeoffice Arbeitenden. Hochschule für Angewandte Psychologie FHNW. Olten. http://www.fhnw.ch/aps/ifk/projekte/aktuelleprojekte/copy_of_Homeoffice--2013. Abgerufen am 19.11.2015.

Dettmers, J., Vahle-Hinz, T., Friedrich, N., Keller, M., Schulz, A. \& Bamberg, E. (2012): Entgrenzung der täglichen Arbeitszeit - Beeinträchtigungen durch ständige Erreichbarkeit bei Rufbereitschaft. In: Badura, B., Ducki, A., Schröder, H., Klose, J. \& Meyer, M. (Hrsg.): Fehlzeiten-Report 2012. (S. 53-60). Springer-Verlag. Berlin, Heidelberg.

Dixon, J., Woodman, D., Strazdins, L., Banwell, C., Broom, D. \& Burgess, J. (2013): Flexible employment, flexible eating and health risks. Critical Public Health. April 2014. S. 1-15.

Dolado, J. \& Stucchi, R. (2008): „Do Temporary Contracts Affect TFP? Evidence from Spanish Manufacturing Firms“. Discussion Paper. IZA DP No. 3832. Bonn.

Dorsemagen, C., Krause, A., Lehmann, M. \& Pekruhl, U. (2012): Flexible Arbeitszeiten in der Schweiz: Auswertung einer repräsentativen Befragung der Schweizer Erwerbsbevölkerung. Staatsekretariat für Wirtschaft SEco. Bern.

http://www.seco.admin.ch/dokumentation/publikation/00008/00022/04953 /index.html. Abgerufen am 19.11.2015. 
Dubach Spiegler, E., Muhdi, L., Stöcklin, D. \& Michahelles, F. (2011):

Crowdsourcing for "Kiosk of the Future" - A retail store case study. Proceedings of the Seventeenth Americas Conference on Information Systems.

Ducki, A. (2009): Arbeitsbedingte Mobilität und Gesundheit - Überall dabei Nirgendwo daheim. In: Badura, B., Schröder, H., Klose, J. \& Macco, K. (Hrsg.): Fehlzeiten-Report 2009. (S. 61-70). Springer-Verlag. Berlin, Heidelberg.

Dütschke, E. \& Boerner, S. (2012): Zweite Wahl oder Selbstverwirklichung? Eine empirische Studie zur Präferenz flexibler Beschäftigung. Zeitschrift für Arbeits- $u$. Organisationspsychologie 56(4). S. 173-185.

Egger, M. \& Razum, O. (Hrsg.) (2014): Public Health. Sozial- und Präventivmedizin kompakt. De Gruyter. Berlin.

Eichhorst, W. \& Tobsch, V. (2014): Flexible Arbeitswelten - Bericht an die Expertenkommission „Arbeits- und Lebensperspektiven in Deutschland“. IZA Research Report No. 59.

Eidgenössisches Büro für die Gleichstellung von Frau und Mann (EBG) (2010): Anerkennung und Aufwertung der Care-Arbeit. Impulse aus Sicht der Gleichstellung. Bern.

http://www.ebg.admin.ch/themen/00008/00486/00488/00489/index.html? download=NHzLpZeg7t,Inp6I0NTU042I2Z6In1acy4Zn4Z2qZpnO2Y uq2Z6gpJCDdXx_f2ym162epYbg2c_JjKbNoKSn6A--\&lang=de. Abgerufen am 08.08.2015.

Eidgenössisches Büro für die Gleichstellung von Mann und Frau (EBG) (2012): Unbezahlte Care-Arbeit. Grundlagen für eine bessere soziale Absicherung. Bern. Medienmitteilung.

http://www.ebg.admin.ch/dokumentation/00068/00500/00506/index.html? lang=de. Abgerufen am 08.08.2015.

Eidgenössisches Departement des Innern (EDI) (2014): Übersicht über die schweizerische soziale Sicherheit.

Ellingson, J. E., Gruys, M. L. \& Sackett, P. R. (1998): Factors related to the satisfaction and performance of temporary employees. Journal of Applied Psychology 83. S. 913-921.

Eurofound (2015a): New forms of employment. Publications Office of the European Union. Luxembourg. 
http://www.eurofound.europa.eu/sites/default/files/ef_publication/field_ef_ document/ef1461en.pdf. Abgerufen am 15.05.2015.

Eurofound (2015b): Third European Company Survey - Overview report: Workplace practices - Patterns, performance and well-being. Publications Office of the European Union. Luxembourg.

European Trade Union Confederation ETUC: Die Arbeitszeitrichtlinie und Grundrechte.

https://www.etuc.org/sites/www.etuc.org/files/A_TT_droits_fond_DE.pdf. Abgerufen am 06.07.2015.

Eurostat (2015): Erwerbstätige mit zweiter Tätigkeit. LFS Reihe. http://ec.europa.eu/eurostat/data/database?node_code=lfsa_e2ged\#. Abgerufen am 06.07.2015.

Felder, E. (2014): Die Temporär-Branche boomt. Interview Izmedien. 14. November 2014.

Feldman, D. C., Doerpinghaus, H. I. \& Turnley, W. H. (1995): Employee reactions to temporary jobs. Journal of Managerial Issues 8. S. 127-141.

Flecker, J. (2007): „Schwarzer Peter neu gezogen?“ Flexibilisierung und Weitergabe von Risiken. Theorie und Praxis 2/16.

Flick, U. (2010): Qualitative Sozialforschung. Eine Einführung. Rowohlt. Reinbek.

Frei, B. (2009): Paid Crowdsourcing Current State \& Progress toward Mainstream Business Use. http://www.smartsheet.com. Abgerufen am 19.11.2015.

Frick, K. \& Höchli, B. (2014): Die Zukunft der vernetzten Gesellschaft. Neue Spielregeln, neue Spielmacher. GDI Gottlieb Duttweiler Institute. Zürich.

Fritsch, M. (2008): „How does new business formation affect regional development? Introduction to the special issue." Small Business Economics 27. S. $245-260$.

Froschauer, U. \& Lueger, M. (2003): Das qualitative Interview. Zur Praxis interpretativer Analyse sozialer Systeme. Facultas. Wien.

Frossard, P. (2013): Temporärarbeit immer mehr auf dem Vormarsch. Panorama. 02.2013. http://www.panorama.ch/dyn/1122.aspx?id_article=307. Abgerufen am 06.07.2015. 
Fuchs, T. (o.J.): Wiki Gute Arbeit. Berlin, ver.di. Zitiert in: Paridon, H. (2012).

Berufsbedingte Mobilität. In: Badura, B., Ducki, A., Schröder, H., Klose, J. \& Meyer, M. (Hrsg.): Fehlzeiten-Report 2012. (S. 79-88). SpringerVerlag. Berlin, Heidelberg.

Gajendran, R. S. \& Harrison, D. A. (2007): The Good, the Bad, and the Unknown About Telecommuting: Meta-Analysis of Psychological Mediators and Individual Consequences. Journal of Applied Psychology 92(6). S. 1524-1541, doi:10.1037/0021-9010.92.6.1524.

Gangwisch, J. E. (2014). Work-life Balance. Commentary on Salo et al. Work time control and sleep disturbances: prospective cohort study of Finnish public sector employees (Sleep 2014;37:1217-1225). Sleep 37(7). S. $1159-1160$.

Geertz, C. (2007): Dichte Beschreibung: Beiträge zum Verstehen kultureller Systeme. 10. Auflage. Suhrkamp. Frankfurt a. M.

Geiser, T. (1997). Haftung bei neuen Arbeitsformen (Job Sharing, ComputerArbeitsplätze, Personalverleih). Aktuelle Juristische Praxis. S. 787-796.

Geiser, T. (2007): Aus der neueren bundesgerichtlichen Rechtsprechung. Aktuelle Juristische Praxis 4/2007. S. 1514-1524.

Geiser, T. (2011): Arbeitsrechtliche Rechtsprechung. Aktuelle Juristische Praxis 2/2011. S. 243-252.

Geiser, T. \& Müller, R. (2015): Arbeitsrecht in der Schweiz. Stämpfli. Bern.

Gesamtarbeitsvertrag der Maschinen-, Elektro- und Metall-Industrie. Vertragsperiode 1. Juli 2013 - 30. Juni 2018 (2013).

Gesamtarbeitsvertrag für Basler Pharma-, Chemie- und Dienstleistungsunternehmen. Gültig ab 1. Mai 2012 (2012).

Gisin, L. (2014): Boundary-Typen, Boundary-Management und Boundary Taktiken im Homeoffice. Untersuchung des Einflusses der „Boundary Theorie" auf das mobile Telearbeiten, insbesondere der Arbeit im Homeoffice. Master Thesis. Hochschule für Angewandte Psychologie FHNW. Olten.

Gisin, L., Schulze, H., Knöpfli, D. \& Degenhardt, B. (2013): Schweizerische Umfrage „Homeoffice 2012“ - Aktuelle Bedingungen sowie Vor- und Nachteile aus Sicht von Routiniers. Hochschule für Angewandte Psychologie FHNW. Olten. http://www.fhnw.ch/aps/ifk/projekte/abgeschlosseneprojekte/Homeoffice--2012/. Abgerufen am 19.11.2015. 
Glinka, H.-J. (1998): Das narrative Interview. Eine Einführung für Sozialpädagogen. Juventa. Weinheim.

Global Enterpreneurship Monitor (GEM) (2014): Global Entrepreneurship Monitor 2014 - Report on Switzerland. http://www.gemconsortium.org/report. Abgerufen am 06.07.2015.

Grebner, S., Berlowitz, I., Alvarado, V. \& Cassina, M. (2011): Stress bei Schweizer Erwerbstätigen Kurzzusammenfassung. http://www.seco.admin.ch/dokumentation/publikation/00008/00022/04731 /index.html?lang=de\&download=NHzLpZeg7t,Inp6I0NTU042I2Z6In1 acy4 Zn4Z2qZpnO2Yuq2Z6gpJCFfHx4gWym162epYbg2c_JjKbNoKSn6A--. Abgerufen am 18.12.2014.

Green, A., de Hoyos, M., Barnes, S-A., Baldauf, B. and Behle, H. (2014): Exploratory Research on Internet-enabled Work Exchanges and Employability, Analysis and synthesis of qualitative evidence on crowdsourcing for work, funding and volunteers, Report EUR 26423 EN, Editors: Stewart, J., Luxembourg: Publications Office of the European Union, European Commission Joint Research Centre Institute for Prospective Technological Studies, JRC Scientific and Policy Reports.

Greer, T. W. \& Payne, S. C. (2014): Overcoming telework challenges: outcomes of successful telework strategies. The Psychologist-Manager Journal 17(2). S. 87-111.

Grote, G. \& Staffelbach, B. (Hrsg.) (2010): Schweizer HR-Barometer 2010: Arbeitsflexibilität und Familie. Verlag Neue Zürcher Zeitung. Zürich.

Grunwald, A. (2010): Technikfolgenabschätzung - eine Einführung. Reihe: Gesellschaft - Technik - Umwelt. 2. Auflage. edition sigma.

Grüter, U. (2011): Homeoffice - Aus den Augen, aus dem Recht? WorkAnywhere Studie. Flexible Arbeitsplatzmodelle.

Guggisberg, M., Müller, B. \& Christin, T. (2012): Armut in der Schweiz. Konzepte, Resultate und Methoden. Ergebnisse auf der Basis von SILC 2008 und 2010. Eidgenössisches Departement des Innern (EDI) Bundesamt für Statistik (Hrsg.), Neuchâtel.

Gutzwiller, B. (2010): Arbeitszeitmodelle und ihre rechtlichen Folgen. Schweizer Arbeitgeber 10/2010. 
Haigner, S. D., Jenewein, S., Schneider, F. \& Wakolbinger, F. (2013): Driving forces of informal labour supply and demand in Germany. International Labour Review 152(3-4). S. 507-524.

Han, B.-C. (2010): Müdigkeitsgesellschaft. Matthes \& Seitz. Berlin.

Hans Böckler Stiftung (2007): „Kurze Arbeitszeit, hohe Produktivität“. BöcklerImpuls 17/2007.

http://www.boeckler.de/46.htm?action=newsearch\&jahr=2007\&ausgabe= 17\&searchterm=\&schlagwort=\#results. Abgerufen am 06.07.2015.

Harabi, N., Schoch, R. \& Hespeler, F. (2001): Diffusion of Telework: What is Switzerland's position in an international comparison? Results of an empirical study. http://mpra.ub.uni-muenchen.de/4443/. Abgerufen am 06.07.2015.

Harpaz, I. (2002): Advantages and disadvantages of telecommuting for the individual, organization and society. Work Study 51(2). S. 74-80.

Haupt, C. (2010): Der Zusammenhang von Arbeitsplatzunsicherheit und Gesundheitsverhalten in einer bevölkerungsrepräsentativen epidemiologischen Studie. In: Badura, B., Ducki, A. Schröder, H., Klose, J. \& Macco, K. (Hrsg.): Fehlzeiten-Report 2009. (S. 101-107). Springer-Verlag. Berlin, Heidelberg.

Hausheer, H. \& Walter, H. P. (Hrsg.). (2010). Berner Kommentar. Einleitung und Kommentar zu den Art. 319-330b OR. Stämpfli Verlag. Bern.

Helfferich, C. (2004): Qualität qualitativer Daten. Ein Schulungsmanual zur Durchführung qualitativer Einzelinterviews. 4. Auflage. Verlag für Sozialwissenschaften. Leverkusen.

Henneberger, F., Sousa-Poza, A. \& Ziegler, A. (2004): Eine ökonomische Bewertung befristeter Beschäftigungsverhältnisse. Die Schweiz im internationalen Vergleich. Nr. 102 der Reihe Diskussionspapiere des Forschungsinstituts für Arbeit und Arbeitsrecht an der Universität St. Gallen (Band 3).

Hill, E. J., Ferris, M. \& Märtinson, V. (2003): Does it matter where you work? A comparison of how three work venues (traditional office, virtual office, and Homeoffice) influence aspects of work and personal/family life. Journal of Vocational Behavior 63. S. 220-241. 
Hill, E. J., Miller, B. C., Weiner, S. P. \& Colihan, J. (1998): Influences of the virtual office on aspects of work and work/life balance. Personnel Psychology 51.

Hirsch, B. T. (2004): Why Do Part-Time Workers Earn Less? The Role of Worker and Job Skills. IZA Discussion Paper No. 1261. Bonn.

Hodson, H. (2013): „Workers of the crowd unite ...”. New Scientist. Ausgabe 2903. https://www.newscientist.com/article/mg21729036-200crowdsourcing-grows-up-as-online-workers-unite/. Abgerufen am 06.07.2015.

Höge, T. (2011): Perceived flexibility requirements at work and the entreployeework-orientation: Concept and measurement. Journal Psychologie des Alltagshandeln / Psychology of Everyday Activity 4. S. 3-21.

Honsell, H., Vogt, N. \& Wiegand, W. (Hrsg.) (2011): Basler Kommentar zum Schweizerischen Privatrecht. Obligationenrecht I. Art. 1-529 OR. (Aufl. 5). Helbling \& Lichtenhahn Verlag. Basel.

Hornberger, S. (2006): Individualisierung als ermöglichte und verordnete Selbstorganisation und ihre Anforderungen an die arbeitswissenschaftliche Analyse, Bewertung und Gestaltung von Arbeitsbedingungen. Zeitschrift Für Arbeitswissenschaft 60(2). S. 85-95. doi:10.3239/9783640702978.

Howe, J. (2006). The rise of crowdsourcing. WIRED. www.wired.com. Abgerufen am 19.11.2015.

Hupfeld, J., Brodersen, S. \& Herdegen, R. (2013): Arbeitsbedingte räumliche Mobilität und Gesundheit. iga.Report 25. Essen, Berlin.

IDEA Consult (2010): Evaluatie van het stelsel van de dienstencheques voor buurtdiensten en -banen 2009 [Evaluation of the system of service vouchers for neighborhood services and jobs 2009]. Brussels.

Initiative Neue Qualität der Arbeit (INQA) (Hrsg.) (2015): Monitor, Gewünschte und erlebte Arbeitsqualität. Bundesanstalt für Arbeitsschutz und Arbeitsmedizin, Berlin.

Institut für Arbeitsmarkt- und Berufsforschung (IAB) (2006): Teilzeitarbeit fördert Flexibilität und Produktivität. IAB Kurzbericht Nr. 7.

http://doku.iab.de/kurzber/2006/kb0706.pdf. Abgerufen am 06.07.2015. 
International Labor Organization (2012): Decent Work Indicators - Concepts and Definitions, International Labour Office.

http://www.ilo.org/wcmsp5/groups/public/---dgreports/---integration/ documents/publication/wcms_229374.pdf. Abgerufen am 1.10.2015.

Ipeirotis, P. G. (2010a): Analyzing the Amazon Mechanical Turk marketplace. XRDS: Crossroads. The Association for Computing Machinery (ACM) Magazine for Students Winter 2010 Vol. 17 No.2.

Ipeirotis, P. G. (2010b): Demographics of Mechanical Turk. Working Paper CeDER-10-01, New York University, Stern School of Business. http://hdl.handle.net/2451/29585. Abgerufen am 07.08.2014.

Jaeggi, R. (2005): Entfremdung: Zur Aktualität eines sozialphilosophischen Problems. Campus Verlag. Frankfurt a. M.

Janssen, D. \& Nachreiner, F. (2004): Flexible Arbeitszeiten. Schriftenreihe der Bundesanstalt für Arbeitsschutz und Arbeitsmedizin. Dortmund, Berlin, Dresden.

Jánszky, S. G. (2010): Zukunft der Arbeitswelt im Jahr 2020: Value Worker, Patchwork-Identitäten und HR-Management in fluiden Unternehmen. Personalführung 43 Nr. 2. S. 18-26.

Jánszky, S. G. (2011): Arbeitswelt 2020: Führung und HR sind auf der Jagd nach „freien Radikalen“. https://www.hrtoday.ch/article/arbeitswelt-2020f-hrung-und-hr-sind-auf-der-jagd-nach-freien-radikalen. Abgerufen am 07.08.2014.

Johnson, A. A., Shannon, L. L. \& Richman, A. L. (2008): Challenging common myths about workplace flexibility: Research notes from the multiorganization database. Community, Work \& Family 11(2). S. 231-242, doi:10.1080/13668800802048321.

Jost, M. \& Pletscher, C. (2013): Factsheet Berufskrankheiten. www.suva.ch/factsheet-berufskrankheiten. Abgerufen am 06.07.2015.

Kaganer, E., Carmel, E., Hirschheim, R. \& Olsen, T. (2013): Managing the Human Cloud. MIT Sloan Management Review 54(2). S. 23-32.

Kaufmann, C. (2013): Homeoffice und Arbeitsrecht. IT business 4/2013.

Kawalec, S. \& Menz, W. (2013): Die Verflüssigung von Arbeit. Crowdsourcing als unternehmerische Reorganisationsstrategie - das Beispiel IBM. Arbeits- und Industriesoziologische Studien 6/2. 
Keller, B. \& Seifert H. (2011a): Atypische Beschäftigungsverhältnisse. Stand und Lücken der aktuellen Diskussion. In: Hans-Böckler-Stiftung (Hrsg.): WSI Mitteilungen 3/2011. Düsseldorf.

Keller, B. \& Seifert H. (2011b): Atypische Beschäftigung und soziale Risiken. Entwicklung, Strukturen, Regulierung. Expertise im Auftrag der Abteilung Wirtschafts- und Sozialpolitik der Friedrich-Ebert-Stiftung. Abteilung Wirtschafts- und Sozialpolitik der Friedrich-Ebert-Stiftung (Hrsg.). Bonn.

Kelleter, K. (2009): Selbständige in Deutschland. Ergebnisse des Mikrozensus 2008. Statistisches Bundesamt (Hrsg.): Wirtschaft und Statistik (12). S. 1204-1217.

Kinkel, S., Friedewald, M., Hüsing, B., Lay, G. \& Lindner, R. (2008): Arbeiten in der Zukunft. Strukturen und Trends der Industriearbeit. Studien des Büros für Technikfolgen-Abschätzung beim Deutschen Bundestag. edition sigma. Berlin.

Kissling, I., Meissner, Jens O., Seyler, C., Henzen, C. \& Gentile, Gian-Claudio (2012): Ressourcenimpact neuer Arbeitsformen. Schlussbericht BFEProjekt SI/500689-01 im Rahmen des Forschungsprogramms EWG. BFE Bundesamt für Energie.

Kittur, A., Nickerson, J. V., Berstein, M. S., Gerber, E. M., Shaw, A., Zimmerman, J., Lease, M. \& Horton, J. J. (2013): The Future of Crowd Work. CSCW' 13. February 23-27.

Kleining, G. (1995): Lehrbuch entdeckende Sozialforschung - von der Hermeneutik zur qualitativen Heuristik. Psychologie Verlags Union. München.

Knöpfel, C. (2015): Sozialstaatliche Rahmenbedingungen in der Schweiz. In: B. Wüthrich et al. (Hrsg.): Soziale Versorgung zukunftsfähig gestalten. Springer Fachmedien. Wiesbaden.

Konicz, T. (2012): Tagelöhnertum im Internet-Zeitalter. Gegenblende - Das Debattenmagazin 14/2012.

Krause, A. \& Schulze, H. (2012): Flexible Arbeitsformen gesundheitsförderlich gestalten. HR Today 2, Special. S. 20-21.

Krause, A., Baeriswyl, S., Berset, M., Deci, N., Dettmers, J., Dorsemagen, C., Meier, W., Schraner, S., Stetter, B. \& Straub, L. (2015): Selbstgefährdung als Indikator für Mängel bei der Gestaltung mobil-flexibler Arbeit. Zur Entwicklung eines Erhebungsinstruments. Wirtschaftspsychologie 17 (1). S. 49-59. 
Krause, A., Baeriswyl, S., Berset, M., Deci, N., Dettmers, J., Dorsemagen, C., Meier, W., Schraner, S., Stetter, B. \& Straub, L. (2014): Selbstgefährdung als Indikator für Mängel bei der Gestaltung mobil-flexibler Arbeit: Zur Entwicklung eines Erhebungsinstruments. Wirtschaftspsychologie (4). S. 49-59.

Krause, A., Dorsemagen, C. \& Peters, K. (2013): Interessierte Selbstgefährdung. Hernsteiner - Fachzeitschrift für Management- und LeadershipEntwicklung (3). S. 14-15.

Krause, A., Dorsemagen, C., Stadlinger, J. \& Baeriswyl, S. (2012): Indirekte Steuerung und interessierte Selbstgefährdung: Ergebnisse aus Befragungen und Fallstudien. Konsequenzen für das Betriebliche Gesundheitsmanagement. In: Badura, B., Ducki, A., Schröder, H., Klose, J. \& Meyer, M. (Hrsg.): Fehlzeiten-Report 2012. (S. 191-202). SpringerVerlag. Berlin, Heidelberg.

Krausz, M. (2000): Effects of short- and long-term preference for temporary work upon psychological outcomes. International Journal of Manpower 21. S. 636-647.

Krausz, M., Brandwein, T. \& Fox, S. (1995): Work attitudes and emotional responses of permanent, voluntary, and involuntary temporary-help employees: An exploratory study. Applied Psychology: An International Review 44. S. 217-232.

Kreiner, G. E., Hollensbe, E. C. \& Sheep, M. L. (2009): Balancing borders and bridges: negotiating the work-home interface via boundary work tactics. Academy of Management Journal 52(4). S. 704-730.

Krieger, R. \& Graf, M. (2009): Arbeit und Gesundheit. Zusammenfassung der Ergebnisse der Schweizerischen Gesundheitsbefragung 2007. Zürich.

Krieger, R., Pekruhl, U., Lehmann, M. \& Graf, M. (2012): 5. Europäische Erhebung über die Arbeitsbedingungen 2010. Ausgewählte Ergebnisse aus Schweizer Perspektive. Staatssekretariat für Wirtschaft, Direktion für Arbeit (Hrsg.). Bern.

Lamnek, S. (1995): Qualitative Sozialforschung. Beltz Verlag. Weinheim.

Läubli, T., Schmid, K. \& Habermann-Horstmeier, L. (2014): Berufskrankheiten. In: Egger, M., Razum, O. (Hrsg.): Public Health. Sozial- und Präventivmedizin Kompakt. www.public-health-kompakt.ch/index.php/kapitel6/kap-6-2/. Abgerufen am 06.07.2015. 
Lee, J. H. (2012): Hard at Work in the Jobless Future. The Futurist March-April 2012. S. 33-35.

Lehdonvirta, V. \& Mezier, P. (2013): Identity and Self-Organization in Unstructured Work. COST Action IS 1202 Dynamics of Virtual Work: Working Paper Series Number 1. http://dynamicsofvirtualwork.com/wpcontent/uploads/2013/03/COST-Action-IS1202-Working-Paper-12.pdf. Abgerufen am 24.06.2015.

Leimeister, J. M. \& Zogaj, S. (2013): Neue Arbeitsorganisation durch Crowdsourcing. Eine Literaturstudie. Hans-Böckler-Stiftung-Arbeitspapier 287. Düsseldorf.

Leslie, L. M., Manchester, C. F., Park, T.-Y. \& Mehng, S. A. (2012): Flexible Work Practices: A Source of Career Premiums or Penalties? Academy of Management Journal 55(6). S. 1407-1428, doi:10.5465/amj.2010.0651.

Letsch, T. (2008). Rechtliche Aspekte von Work-Life-Balance. In: Portmann, W. \& Stöckli, F. (Hrsg.): Schriften zum schweizerischen Arbeitsrecht. Band/Nr. 67. (S. 21-42). Bern. Stämpfli.

Lewin, K. (1920): Die Sozialisierung des Taylor Systems: Eine grundsätzliche Untersuchung zur Arbeits- und Berufspsychologie. In: Korsch, K. (Hrsg.): Praktischer Sozialismus. Band 4. (S. 5-36). Verlag Gesellschaft und Erziehung. Berlin.

Liegl, M. (2014): Nomadicity and the Care of Place-on the Aesthetic and Affective Organization of Space in Freelance Creative Work. Computer Supported Cooperative Work 23. S. 163-183.

Lisi, D. (2013): „The impact of temporary employment and employment protection on labour productivity: evidence from an industry-level panel of EU countries." Journal for Labour Market Research 46(2). S. 119-144.

Marti, M., Sommer, H., Oleschak, R., Rissi, C., Böhringer, P., Inderhees, G. \& Stöckle, I. (2007): Flexicurity: Bedeutung für die Schweiz. Forschungsbericht Nr. 14/07. Beiträge zur Sozialen Sicherheit. Bundesamt für Sozialversicherungen, Bern.

Marti, M. \& Walker, P. (2010): Die Entwicklung atypisch-prekärer Arbeitsverhältnisse in der Schweiz. Die Volkswirtschaft. Das Magazin für Wirtschaftspolitik. 10.

Marx, I. \& Vandelannoote, D. (2014): Matthew Runs Amok: The Belgian Service Voucher Scheme. IZA Discussion Paper No. 8717. Forschungsinstitut zur 
Zukunft der Arbeit. Bonn. http://ftp.iza.org/dp8717.pdf. Abgerufen am 24.06.2015.

Masuda, A. D. et al. (2012): Flexible Work Arrangements Availability and their Relationship with Work-to-Family Conflict, Job Satisfaction, and Turnover Intentions: A Comparison of Three Country Clusters. Applied Psychology: An International Review 61(1). S. 1-29.

Mayring, P. (1996): Qualitative Inhaltsanalyse. In: Flick, U. et al. (Hrsg.): Qualitative Forschung. Ein Handbuch. 5. Auflage. (S. 468-475). Rowohlt. Reinbek bei Hamburg.

Mayring, P. (2000): Qualitative Content Analysis. Forum: Qualitative Social Research, 1, (2), Art. 20, June. Retrieved from http://www.qualitativeresearch.net/index.php/fqs/article/view/1089/2386. Abgerufen am 06.07.2015.

Mayring, P. (2002): Einführung in die Qualitative Sozialforschung. 5., überarbeitete und neu ausgestattete Auflage. Weinheim. Basel.

ME Advocat Rechtsanwälte (2013): Arbeit auf Abruf. Referat vom 12. September 2013. Staad.

Meissner, J. (2007): Herausfordernde Computerkommunikation: Eine konstruktivistische Perspektive auf organisationale Kommunikation im Kontext Neuer Medien. Verlag für Systemische Forschung. Heidelberg.

Meissner, J. O. (2007): Multi-stage Analysis for Knowledge Reflection. In: Kazi, A. S., Wohlfahrt, L. \& Wolf, P.: Hands-On Knowledge Co-Creation and Sharing: Practical Methods and Techniques. (S. 291-307). Fraunhofer IRB Verlag. Stuttgart.

Meissner, J. O. (2009): Beziehungsherausforderungen in kommunikativ hybriden Arbeitsumgebungen. Zeitschrift für Management Vol. 4, No. 4. S. 307-326.

Meissner, Jens O. (2016). Resilienz im Wandel durch Hybride Professionals? In: Geramanis, O. \& Hermann, K. (Hrsg.): Führen in ungewissen Zeiten. Impulse, Konzepte und Praxisbeispiele. (S. 265-282). uniscope, Springer Gabler. Wiesbaden.

Mewes, R., Rief, W., Martin, A., Glaesmer, H. \& Brähler, E. (2013): Arbeitsplatzunsicherheit vs. Arbeitslosigkeit: Trotz der Unterschiede im sozioökonomischen Status sind die Auswirkungen auf psychische Gesundheit und 
Inanspruchnahme von Gesundheitsleistungen ähnlich. Psychother Psych Med 2013;63. S. 138-144.

Miller, G. A. (2003): The cognitive revolution: a historical perspective. TRENDS in Cognitive Sciences Vol. 7, No. 3.

Minssen, H. (2012): Arbeit in der modernen Gesellschaft. VS Verlag für Sozialwissenschaften. Wiesbaden, doi:10.1007/978-3-531-94210-0.

Mishra, G. \& Makhija, N. (2013): Future of Money. People's Insights Vol. 2 Issue 2.

Moore, J. (2006): Homeworking and work-life balance: does it add to quality of life? Revue européenne de psychologie appliquée 56. S. 5-13.

Müller, M. (2014): Die Babyboomer sind auf dem Vormarsch. NZZ. 8. Oktober 2014.

Müller, R. (2007): Der Arbeitsort im schweizerischen Arbeitsrecht. Masterarbeit Universität Bern.

Müller, R. \& Oechsle, T. (2007): Die Pflicht zur Arbeitszeiterfassung. Aktuelle Juristische Praxis 7/2007. S. 847-855.

Neugebauer, J. \& Klebe, T. (2014): Crowdsourcing. Gegenblende - Das Debattenmagazin 26/2014.

Nielen, S. \& Schiersch, A. (2012): Befristete Beschäftigung hat keinen Einfluss auf die betriebliche Arbeitsproduktivität. DIW-Wochenbericht 79(21). S. 14-17.

http://www.diw.de/documents/publikationen/73/diw_01.c.400065.de/1221-3.pdf. Abgerufen am 06.07.2015.

Nightingale, P. \& Coad, A. (2013): Muppets and Gazelles: Political and Methodological Biases in Entrepreneurship Research. Working Paper, SPRU, Science and Technology Policy Research. University of Sussex. https://www.sussex.ac.uk/webteam/gateway/file.php?name=2013-03swps-rr-draft-v14-accepted-keywords.pdf\&site=25. Abgerufen am 06.07.2015.

Nilles, J. M. (1976): The Telecommunication-Transportation Tradeoff: Development of Policy. Wiley. New York.

Nippert-Eng, C. E. (1996): Home and work: Negotiating boundaries through everyday life. University of Chicago Press. Chicago. 
Nötzli, H. (2009): Referat „Arbeitszeit/Ferien/Feiertage“. www.arbeitsrechtfachanwalt.ch. Abgerufen am 18.12.2014.

Oesch, D. \& Rodriguez Menes, J. (2010): Upgrading or polarization? Occupational change in Britain, Germany, Spain and Switzerland, 1990-2008. Socio-Economic Review 9(3). S. 503-531.

Oesch, D. (2007): Organisationen im Umbruch: Die Gewerkschaften in der Schweiz von 1990 bis 2006. Schweizerischer Gewerkschaftsbund (Hrsg.). http://www.sgb.ch/uploads/media/51_DO_d_Gewerkschaften_CH_9006.pdf. Abgerufen am 06.07.2015.

Olsen, K. B. (2006): Productivity Impacts of Offshoring and Outsourcing: A Review. DSTI Working Paper 2006/1. Directorate for Science, Technology and Industry. OECD. Paris.

Olson, M. (2004): Die Logik des kollektiven Handelns: Kollektivgüter und die Theorie der Gruppen. 5. Auflage. Mohr Siebeck. Tübingen.

Ortega, B. \& Marchante, A. (2010): „Temporary contracts and labour productivity in Spain: a sectoral analysis“. Journal of Productivity Analysis 34(3). S. 199-212.

Österreichisches Institut für Familienforschung (ÖIF) (2011): Betriebliche Effekte von Teilzeitarbeit. Forschungsbericht Nr. 6. Universität Wien.

Pangert, B. \& Schüpbach, H. (2015): Auswirkungen arbeitsbezogener erweiterter Erreichbarkeit auf Gesundheit und Life-Domain-Balance. Wirtschaftspsychologie Heft 4-2014/1-2015. S. 73-82.

Paridon, H. \& Hupke, M. (2012). Psychosoziale Auswirkungen mobiler Arbeit: Ergebnisse einer Online-Befragung. In: Brandt, C. (Hrsg.). Mobile Arbeit - Gute Arbeit? Arbeitsqualität und Gestaltungsansätze bei mobiler Arbeit. (S. 65-80). Berlin: ver.di - Vereinte Dienstleistungsgewerkschaft.

Paridon, H. (2012): Berufsbedingte Mobilität. In: Badura, B., Ducki, A., Schröder, H., Klose, J. \& Meyer, M. (Hrsg.): Fehlzeiten-Report 2012. (S. 79-88). Springer-Verlag. Berlin, Heidelberg.

Pekruhl, U. (2013): Vertrauensarbeitszeit - Ein Gewinn für alle? Personal Schweiz Sonderausgabe 1.

Perch-Nielsen, S., von Felten, N., Henzen, C., Meissner, J., Sprenger, M., Bieri, M., Arvanitis, S., Seliger, F. \& Ley, M. (2014): Auswirkungen neuer Ar- 
beitsformen auf den Energieverbrauch und das Mobilitätsverhalten von Arbeitnehmenden. Bericht Nr. 290955. Bundesamt für Energie BFE. Bern. http://www.bfe.admin.ch/dokumentation/energieforschung/index.html? lang=de\&publication=11195. Abgerufen am 19.11.2015.

Peters, K. (2011): Indirekte Steuerung und interessierte Selbstgefährdung. In: Kratzer, N., Dunkel, W., Becker, K. \& Hinrichs, S. (Hrsg.): Arbeit und Gesundheit im Konflikt: Analysen und Ansätze für ein partizipatives Gesundheitsmanagement. (S. 105-122). edition sigma. Berlin.

Platman, K. (2004): „,Portfolio careers' and the search for flexibility in later life“. Work, Employment \& Society Vol. 18, No. 3. S. 573-599.

Post (2011): Gesamtarbeitsvertrag Post.

Pulte, P. (1987): Kapazitätsorientierte variable Arbeitszeit: KAPOVAZ. Verlag Recht und Wirtschaft. Heidelberg.

Raeder, S. (2006): Flexibilisierung der Arbeit: Zeit, Ort, Lohn. Vorlesung Arbeitspsychologie und Ergonomie. Zürich.

Regus (2011): „Why place still matters in the digital age.“ ZZA Responsive User Environments October 2011. http://www.regus.com/images/Third\%20Place\%20Whitepaper_LowRes tcm304-44973.pdf. Abgerufen am 06.07.2015.

Rehbinder, M. (2002): Schweizerisches Arbeitsrecht. Stämpfli. Bern.

Rehbinder, M. \& Stöckli, J.-F. (2010): Berner Kommentar zum schweizerischen Privatrecht. Stämpfli Verlag. Bern.

Reich, M. (2002): Das Einkommen aus selbständiger Erwerbstätigkeit. Jusletter 14. Januar 2002.

Reilly, P. A. (1998): Balancing Flexibility - Meeting the Interests of Employer and Employee. European Journal of Work and Organizational Psychology 7 . S. 243-259.

Rieder, C., Hirschi, O., Sidler, W., Sibler, W., Infanger, P., Achermann, U., Wüthrich, D., Gazzoni, G. \& Wyler, O. (2011): Informationssicherheitshandbuch für die Praxis. 7. Auflage. Gisler Druck. Altdorf.

Ritchey, T. (2011): Wicked Problems - Social Messes: Decision Support Modelling with Morphological Analysis. Springer. Berlin, Heidelberg. 
Rockmann, K. W. \& Pratt, M. G. (2015): Contagious Offsite Work and the Lonely Office: The Unintended Consequences of Distributed Work. Academy of Management Discoveries 1(2). S. 150-164, doi:10.5465/amd.2014.0016.

Rodgers, G. (1989): Precarious work in Western Europe: The state of the debate. In: Rodgers, G. \& Rodgers, J. (Hrsg.): Precarious jobs in labour market regulation: the growth of atypical employment in Western Europe. (S. 1-16). International Labour Organization, ILO. Geneva.

Rohrer, M. (2009): Stress am Arbeitsplatz - haftet der Arbeitgeber? Recht und Arbeitssicherheit 4/09.

Rosa, H. (2012): Weltbeziehungen im Zeitalter der Beschleunigung. Suhrkamp. Berlin.

Rothenberger, J. (2013): Das mobile Büro: Coworking hat sich etabliert. http://www.startwerk.ch/2013/01/07/das-mobile-buero-coworking-hatsich-etabliert/. Abgerufen am 08.08.2015.

Rudolph, R. (2011): Die neuere bundesgerichtliche Rechtsprechung zum sachlichen Kündigungsschutz. Jusletter 20. Juni 2011.

Rudolph, R. \& von Kaenel, A. (2014): Fokus Arbeitsrecht: Aktuelle Fragen zur Arbeitszeit. TREX der Treuhandexperte 03/2014.

Salas-Fumás, V. \& Sanchez-Asin, J. (2013): The management function of entrepreneurs and countries' productivity growth. Applied Economics 45:17. S. 2349-2360.

Saxton, G. D., Oh, O. \& Kishore, R. (2013): Rules of Crowdsourcing: Models, Issues, and Systems of Control. Information Systems Management 30(1). S. 2-20.

Schaltegger, C. A. \& Schneider, F. (2005): Schattenwirtschaft: Ausmass, Gründe und Konsequenzen für die Finanzpolitik. Arbeitspapier Eidgenössische Steuerverwaltung ESTV.

Schneider, F. \& Tübingen, L. (2015): Die Größe der Schattenwirtschaft - Methodik und Berechnungen für das Jahr 2015. Studie der Johannes Kepler Universität Linz und dem Institut für Angewandte Wirtschaftsforschung (IAW).

http://www.iaw.edu/tl_files/dokumente/JKU_IAW_Schattenwirtschaft_ Studie_2015.pdf. Abgerufen am 07.08.2014. 
Schneider, F. (2001): Arbeit im Schatten: Einige theoretische und empirische Überlegungen über die Schattenwirtschaft. Perspektiven der Wirtschaftspolitik 2(4). S. 425-439.

Schulze, H., Meissner, J. O. \& Weichbrodt, J. (Hrsg., 2014/2015). Special Issue "Gestaltung mobil-flexibler Arbeit“. Wirtschaftspsychologie. 4/1(16/17). Pabst Science Publishers.

Schweitzer, L. \& Duxbury, L. (2006): Benchmarking the use of telework arrangements in Canada. Canadian Journal of Administrative Sciences 23(2). S. 105-117.

Schweizer Haushalt-Panel (SHP) (2014): Welle 15. Dezember 2014. FORS. Lausanne [Datensatz].

Schweizerischer Gewerkschaftsbund (SGB) (Hrsg.) (2011): Mindestlohn Situation und Handlungsbedarf. Editions à la Carte. Zürich.

Siebecke, D. (2010): Gesundheit und Prävention in der modernen Wissensarbeit - Wettbewerbsfähigkeit und Innovationskraft durch Burn-outPrävention steigern. Personalführung 43(7). S. 20-27.

Silla, I., Gracia, F. J. \& Peiro, J. M. (2005): Job insecurity and health related outcomes among different types of temporary workers. Economic and Industrial Democracy 26. S. 89-117.

Silverman, R. E. (2014): Charities Seek Donations in Bitcoin. The Wall Street Journal. 12. April 2015. http://www.wsj.com/articles/charities-seekdonations-in-bitcoin-1428894121. Abgerufen am 07.08.2014.

Spitzley, H. (2007): Theorie und Empirie der Arbeitszeitflexibilisierung - Leitlinien zur Qualitätsverbesserung der betrieblichen Arbeitszeitgestaltung. In: Dilger, A., Gerlach, I. \& Schneider, H. (Hrsg.): Betriebliche Familienpolitik. Potenziale und Instrumente aus multidisziplinärer Sicht. (S. 125140). VS Verlag für Sozialwissenschaften. Wiesbaden.

Sprenger, M., Meissner, J. O. \& Ursprung, R. (2013): Risiko Stress: Bringen neue Arbeitsformen Abhilfe? HR Today.

Spühler, K., Tenchio, L. \& Infanger, D. (Hrsg.) (2013): Basler Kommentar zur Schweizerischen Zivilprozessordnung. (Aufl. 2). Helbling \& Lichtenhahn Verlag. Basel.

Staatssekretariat für Wirtschaft SECO (2011): Schlussbericht über das Pilotprojekt Vertrauensarbeitszeit bei Banken. 
Staatssekretariat für Wirtschaft SECO (2014): Direktion für Arbeit - Arbeitsbedingungen: Wegleitung zu den Verordnungen 3 und 4 zum Arbeitsgesetz. BBL 710.250d (Stand Mai 2014). Quelle www.seco.admin.ch. Abgerufen am 1.11.2015.

Staatssekretariat für Wirtschaft SECO (2015): Neue Verordnung zur Arbeitszeiterfassung schafft Rechtssicherheit und administrative Entlastung. http://www.seco.admin.ch/aktuell/00277/01164/01980/index.html?lang= de\&msg-id=59344. Abgerufen am 05.11.2015.

Standing, G. (2014): Understanding the Precariat through Labour and Work. Development and Change 45(5). S. 963-980.

Statistik Zürich (2011): „Frauenlöhne, Männerlöhne. Vollzeitlöhne, Teilzeitlöhne. Lohnentwicklungen in der Zürcher Privatwirtschaft 2002 bis 2008“. statistik.info 2011/02.

http://www.statistik.zh.ch/content/dam/justiz_innern/statistik/Publikationen /statistik_info/si_2011_02_lohnstudie.pdf. Abgerufen am 07.08.2014.

Stock-Homburg, R. (2013): Zukunft der Arbeitswelt 2030 als Herausforderung des Personalmanagements. In: Handbuch Strategisches Personalmanagement. (S. 604-629). Springer. Wiesbaden.

Stöckli, J.-F. (1990): Der Inhalt des Gesamtarbeitsvertrages. Stämpfli. Bern.

Streiff, U., von Kaenel, A. \& Rudolph, R. (2012): Arbeitsvertrag, Praxiskommentar zu Art. 319-362 OR. Schulthess. Zürich.

Streuli, E. \& Bauer, T. (2002): Working Poor in der Schweiz. Konzepte, Ausmass und Problemlagen aufgrund der Daten der Schweizerischen Arbeitskräfteerhebung. Neuchâtel.

http://www.bfs.admin.ch/bfs/portal/de/index/news/publikationen.Documen t.49698.pdf. Abgerufen am 07.08.2014.

Surowiecki, J. (2011): Cryptocurrency. Technology review 114(5). S. 106-107.

Sury, U. (2011): Mobiles Arbeiten und Datensicherheit: WorkAnywhere Studie. Flexible Arbeitsplatzmodelle.

Süss, S. \& Sayah, S. (2011): Work-Life-Balance von Freelancern zwischen Realität und Idealvorstellung. Zeitschrift für Personalforschung 25(3). S. 247-268. 
Süss, S. \& Sayah, S. (2013): Balance between work and life: A qualitative study of German contract workers. European Management Journal 31(3). S. 250-262.

Syndicom (2015): Entgrenzung der Arbeit. Eine Studie der Gewerkschaft Syndicom. Bern.

Tausig, M. \& Fenwick, R. (2001): „Unbinding time: alternate work schedules and work-life balance“. Journal of Family and Economic Issues Vol. 22 No. 2. S. 101-119.

Van Praag, M. \& Versloot, P. (2008): „The economic benefits and costs of entrepreneurship: a review of the research". Foundations and Trends in Entrepreneurship 4. S. 65-154.

Vartiainen, M. \& Hyrkkänen, U. (2010): Changing requirements and mental workload factors in mobile multi-locational work. New Technology, Work and Employment. 25(2). S. 11-135.

Verband SCHWEIZER PRESSE (2000): Gesamtarbeitsvertrag 2000 für Journalistinnen/Journalisten und das technische Redaktionspersonal.

Vitters $\varnothing$, J., Akselsen, S., Evjemo, B., Julsrud, T. E., Yttri, B. \& Bergvik, S. (2003): Impacts of home-based telework on quality of life for employees and their partners. Quantitative and qualitative results from a European survey. Journal of Happiness Studies 4. S. 201-233.

Voß, G. G. \& Pongratz, H. J. (1998): Der Arbeitskraftunternehmer. Eine neue Grundform der „Ware Arbeitskraft"? Kölner Zeitschrift für Soziologie und Sozialpsychologie 50(1). S. 131-158.

Walker, P., Marti, M. \& Bertschy, K. (2010). Die Entwicklung atypisch prekärer Arbeitsverhältnisse in der Schweiz. SECO Publikation Arbeitsmarktpolitik Nr. 32. Staatsekretariat für Wirtschaft SECO. Bern.

Waser, P. (2010): Die Zukunft des Arbeitens. Ein Trendreport. Stiftung Produktive Schweiz (Hrsg.). Zürich.

Weichbrodt, J. (2014): SwissFlexWork 2014: Repräsentative Befragung der Schweizer Erwerbstätigen zur Verbreitung von mobiler Arbeit und Homeoffice. Hochschule für Angewandte Psychologie FHNW. Olten. doi:10.13140/2.1.4621.1526. http://hdl.handle.net/11654/5008. Abgerufen am 19.11.2015. 
Weichbrodt, J., Schulze, H., Gisin, L., Tanner, A. \& Welge, K. (2015): Forschungsbericht „GeMobAU“: Gestaltung mobil-flexibler Arbeit in grossen und mittleren Unternehmen. Hochschule für Angewandte Psychologie FHNW. Olten. doi:10.13140/RG.2.1.1512.7524. http://hdl.handle.net/11654/4993. Abgerufen am 19.11.2015.

Weichbrodt, J., Sprenger, M., Steffen, M., Tanner, A., Meissner, J. O. \& Schulze, H. (2013): WorkAnywhere: Mehr Produktivität und Zufriedenheit der Mitarbeitenden sowie Entlastung der Verkehrsinfrastruktur dank mobilflexibler Arbeitsformen. Bern: SBB AG \& Swisscom AG. doi:10.13140/2.1.5107.4244. http://hdl.handle.net/11654/4994. Abgerufen am 19.11.2015.

WEKA Business Media AG (2014): Freelance: Verträge zur freien Mitarbeiterschaft. http://www.weka.ch/themen/recht/arbeitsrecht/speziellearbeitsvertraege/article/freelance-vertraege-zur-freien-mitarbeiterschaft/. Abgerufen am 18.12.2014.

Wennekers, S., van Stel, A., Carree, M. \& Thurik, R. (2010): The relationship between entrepreneurship and economic development: is it U-shaped? Foundations and Trends in Entrepreneurship 6(3). S. 167-237.

WHO (2014): Verfassung der Weltgesundheitsorganisation SR 0.810.1. https://www.admin.ch/opc/de/classifiedcompilation/19460131/201405080000/0.810.1.pdf. Abgerufen am 05.01.2016.

Wild, E. (2012): Time and chance: Planning and preparing for a portfolio career. Wild Search. London.

Wilkin, C. L. (2013): „I Can't Get No Job Satisfaction: Meta-Analysis Comparing Permanent and Contingent Workers". Journal of Organizational Behavior 34(1). S. 46-64.

Wittgenstein, L. (1963): Tractatus logico-philosophicus. Logisch-philosophische Abhandlung. Suhrkamp. Frankfurt a. M.

Witzel, A. (2000): The Problem-Centered Interview. Forum Qualitative Sozialforschung. 1(1).

Yin, K. (2003): Case Study Research. Design and Methods. 3rd ed. Sage. Thousand Oaks.

Zaugg, S. (2013): Das Geschäft mit Crowd-Plattformen nimmt in der Schweiz Fahrt auf. Netzwoche 02/2013. 
Zok, K. \& Dammasch, H. (2012): Flexible Arbeitswelt: Ergebnisse einer Beschäftigtenbefragung. In: Badura, B., Ducki, A., Schröder, H., Klose, J. \& Meyer, M. (Hrsg.): Fehlzeiten-Report 2012. (S. 39-52). Springer-Verlag. Berlin.

Zwicky, F. (1989): Morphologische Forschung: Wesen und Wandel materieller und geistiger struktureller Zusammenhänge. 2. Auflage. Baeschlin. Glarus. 


\section{Anhang I: Methodisches Vorgehen}

Hinter dem Vorgehen der vorliegenden Studie stehen die methodischen Gütekriterien der qualitativen Sozialforschung. In dieser wird besonderer Wert auf die Dokumentation und Nachvollziehbarkeit der Argumentation gelegt, insbesondere hinsichtlich der Transparenz des Forschungsprozesses. Die Herausforderung lag darin, die ausserordentlich vielfältige Fragestellung dieser Studie zur flexiblen neuen Arbeitswelt einer gewissen Messbarkeit zuzuführen, um daraus Trendaussagen über gewichtige und relevante Themen plausibel ableiten zu können. Diese Zuführung wurde über die volkswirtschaftlichen Konsequenzen (Kapitel 4) der Veränderungen bewerkstelligt. Die Fokussierung von der Themenvielfalt hin zur Beschreibung anhand von Kennzahlen erfolgte über die Entwicklung von Zukunftsszenarien und Personenprototypen - sogenannten „Personas“. Im Folgenden erläutern wir die methodologischen Komponenten des Studienprozesses. Hierzu gehören die Erläuterung von Herangehensweise und Methode, dem gewählten Vorgehen sowie der Diskussion von Kriterien und der Güte des verwendeten Designs.

\section{Fragestellung und Herangehensweise}

Die zentralen Fragestellungen der vorliegenden Arbeit sind:

1. Welchen Stellenwert besitzt die Arbeit in der Gesellschaft heute und in Zukunft?

2. Sind neue Arbeitsformen mit den rechtlichen Bestimmungen vereinbar?

3. Welche volkswirtschaftlichen Folgen weisen neue Arbeitsformen auf?

4. Welche Rahmenbedingungen müssten vorherrschen, damit möglichst viele Personen von mobil-flexiblem Arbeiten profitieren können (ohne dabei mit negativen Folgen konfrontiert zu sein)?

5. Wie müssten Monitoring- und Kontrollsysteme aussehen, damit die bestehende soziale Absicherung und deren staatliche Finanzierung nicht durch mobil-flexibles Arbeiten (und die damit auftretenden intransparenten Zahlungsströme) unterlaufen werden können?

Diese Fragen bringen unterschiedliche methodische Herangehensweisen mit sich. Frage 1 ist eine offene Frage, welche sich auf die neue Qualität der Arbeit sowie auf deren normative Stellung in der Gesellschaft bezieht. Für qualitative 
Fragen benötigt man ein entsprechendes Design, für normative Fragen ein Vergleichsraster, welches bereits ein normatives Programm enthält. In der vorliegenden Studie wird kein eigenes normatives Programm entwickelt, da dies Aufgabe politisch agierender Institutionen ist. Die fachliche Komponente der Frage, wie nämlich neues, flexibles Arbeiten systematisiert werden kann, macht auch eine Fachexpertise notwendig.

Frage 2 ist eine Fachfrage, die rechtliches Fachwissen erfordert. Hier ist eine Prüfung der rechtlichen Rahmengesetze notwendig.

Frage 3 ist ebenfalls eine fachliche Frage, welche eine volkswirtschaftliche Perspektive auf das Thema der flexiblen Arbeitswelt einnimmt. Die volkswirtschaftliche Konsequenz kann anhand erforschter Auswirkungen auf ausgewählte Indikatoren qualitativ und/oder quantitativ beantwortet werden. In dieser Studie werden nach Auftrag und vorgeschlagenem Vorgehen Informationen auf der Grundlage existierender Statistiken und wissenschaftlicher Arbeiten und Studien zusammengetragen und unter szenariospezifischen Annahmen generalisiert, jedoch keine neuen Datensätze erhoben. Es werden allerdings auch Hinweise darauf gegeben, welche Datenlücken für eine umfassendere Beurteilung der gesamtwirtschaftlichen Folgen der neuen Arbeitsformen vorhanden sind.

Frage 4 folgt vom Charakter her Frage 1 und beinhaltet eine sozialwissenschaftlich-qualitative sowie eine normative Komponente. Es ist schlicht offen, wer mit „möglichst vielen Personen“ genau gemeint ist und was „profitieren“ bedeutet. Wir legen hierzu bei der Beantwortung Annahmen zugrunde und erläutern diese auch.

In der fünften Frage geht es dann um die Ausgestaltung institutioneller Effizienz, d.h., wie Regeln eines gesellschaftlichen oder staatlichen Systems aussehen müssten, um möglichst effizient und effektiv zu funktionieren. Auch diese Frage erfordert eine fachliche Perspektive, gepaart mit einer imaginären Komponente, wenn es nämlich darum geht, wie Regeln und Kontrollmechanismen grundsätzlich funktionieren könnten.

Die vorliegenden Fragen lassen darauf schliessen, dass ein qualitatives Forschungsdesign angemessen ist und in Einzelfragen um besondere Fachexpertisen (Fragen 1, 2, 3 und 5), um normative Positionierung (Fragen 1 und 4) oder um quantitative Informationen (Frage 3 ) ergänzt werden muss. Während die Qualität fachlicher Expertise einleuchtend erscheint, bedarf die Güte von Erkenntnissen auf Grundlage der qualitativen Sozialforschung einer genaueren, nun folgenden Erläuterung. 


\section{Gütekriterien der qualitativen Sozialforschung}

Im vorliegenden Projekt wurden die Gütekriterien der qualitativen Sozialforschung angewandt (Mayring, 2000; 2002; Flick, 1987; 2010; Kleining 1995, Lamnek 1995). Dieses Vorgehen bedingt im Vergleich zur klassischen „szientistischen" Forschung andere und weiterführende Gütekriterien, die den Besonderheiten sozialer Phänomene gerecht werden. Daher stehen die Gütekriterien in einem gewissen Kontrast zu herkömmlichen Forschungsformaten, die sehr stark in Naturwissenschaft, Technik, Statistik oder Medizin zur Anwendung kommen.

Die klassischen Gütekriterien umfassen gemäss der Popper'schen Grundidee die drei Grundaspekte „Reliabilität“, „Objektivität" und „Validität“. Bezüglich der Reliabilität wird gefragt, ob sich die Ergebnisse wiederholt feststellen lassen. Diesem Kriterium liegt die Annahme zugrunde, dass Ergebnisse, also eine Befragung, Erhebung, Test etc., zeitlich mehr oder weniger robust rekonstruierbar wären. Das zweite Kriterium der „Objektivität“ ist dann erfüllt, wenn eine intersubjektiv geteilte und allgemein für gültig befundene Beobachtung vorliegt, beispielsweise durch ein „Vier-Augen-Prinzip“ oder eine andere bestätigende Beobachtung. Beim dritten Kriterium „Validität“ wird schliesslich gefragt, ob Methode und Erkenntnis schlüssig zusammenpassen und ob das gemessen wird, was gemessen werden soll.

Die hier verfolgte Perspektive ist eine konstruktivistische. Grund hierfür ist die Einsicht der kognitiven und linguistischen Wende in den 1970er-Jahren, dass sozialwissenschaftliche Erkenntnisse einem sozial moderierten Prozess folgen. Konstruktivistisch gesehen ist Forschung immer eine Re-Konstruktion, die aus einem Interaktionsprozess zwischen Untersuchungseinheit und Beobachter kokonstruiert wird. Es bleibt gültig, dass die Kriterien zum Vorgehen und zum Ziel einer Analyse passen müssen (Flick, 1987). Allerdings muss die Geltungsbegründung viel flexibler ausgestattet sein als bei der klassischen Herangehensweise (siehe auch Witzel, 2000; Glinka, 1998). Es müssen Belege angeführt und diskutiert werden, die die Qualität der Forschung erweisen können. Der Prozess der Begründbarkeit und Verallgemeinerbarkeit der Ergebnisse rückt in den Vordergrund (Mayring, 1996: 117 ff.), ebenso wie die Bemühungen, eine reiche, detaillierte Beschreibung von Situationen, Forschungsszenarien und den entsprechenden Ergebnissen zu liefern (Geertz, 2007; Yin, 2003). Mayring formulierte die hier nachfolgend erläuterten sechs Prinzipien der qualitativen Sozialforschung: 
1. Verfahrensdokumentation: Erkenntnisse, Ergebnisse und Zwischenergebnisse müssen festgehalten und dokumentiert sein, damit Interpretationen der Forschenden später nachvollzogen oder bei unpassender Auslegung anders formuliert werden können.

2. Argumentative Interpretationsabsicherung: Es müssen für die Forschungsfrage passende Gründe für verwendete Argumente vorliegen, reine Behauptungen sind nicht zulässig, spekulativ und zu hinterfragen.

3. Regelgeleitetheit: Der Forschungsprozess soll einem definierten Ablauf folgen. Dieser Prozess soll ausdrücklich formuliert sein, damit ein Beobachter oder Leser nachvollziehen könnte, wie die Forschenden zu ihren Interpretationen kommen. Eine Regel während des Forschungsprozesses zu ändern, muss wiederum begründet werden. Entsteht beispielsweise während einer Interviewserie der Eindruck, die Interviewende müsste Fragen in ihrem Fragebogen entfernen oder neue hinzufügen, so ist diese Änderung ordentlich zu dokumentieren, zu begründen und darf den Rahmen der Methode nicht sprengen.

4. Nähe zum Gegenstand: Die verwendete Methode muss es den Interpretierenden möglich machen, dem untersuchten Objekt möglichst nahezukommen, um die Fragestellung detailliert beantworten zu können. Ob eine Methode diese Nähe ermöglicht, muss häufig im Feld ausprobiert werden. Manche Dinge sind selbstverständlich. Beispielsweise machen geschlossene Fragebögen bei kleinsten Umfragen wenig Sinn, da sie die Feinheiten unangemessen vereinfachen. Viele Dinge ergeben sich aber im Forschungsprozess. Beispielsweise beeinträchtigt die Beziehung eines Interviewers zur ausgewählten Interviewpartnerin stark die passende Methode (Helfferich, 2004). Besteht eine Abhängigkeit, so unterliegt das Interview automatisch einem Bias, der zur Verzerrung der Interpretation führt (siehe Froschauer \& Lueger, 2003).

5. Kommunikative Validierung: Die interpretierten Ergebnisse müssen validiert, also in einer sozialen Gruppe für akzeptabel oder treffend befunden werden. Ohne diese Validierungsschlaufen durch Forschungspartner oder Gremien drohen die Ergebnisse ihre soziale Anschlussfähigkeit zu verlieren. Das Durchführen von Validierungssitzungen kann in unterschiedlichsten Formen organisiert werden (siehe Meissner, 2007). Wesentlich ist, dass eine mehr oder weniger repräsentative Gruppe den interpretierten und re-konstruierten Erkenntnissen zustimmen oder diese als treffend bewerten kann. Es ist besonders diese Komponente, die wissenschaftliches Arbeiten vom normalen Journalismus unterscheidet, bei dem die Verbreitung einer Information in der Regel vor einer sozialen Konsensfindung stattfindet. 
6. Triangulation: Wo immer möglich sollten mehrere Methoden zur Anwendung gelangen. Jede Methode hat inhärente Vor- und Nachteile. Durch das Anwenden mehrerer Methoden werden insbesondere die Nachteile ausgemerzt, sofern die Resultate der Methoden sich auf dasselbe Forschungsobjekt beziehen und untereinander vernetzt werden. Eine typische Triangulation besteht beispielsweise in der Dokumentenanalyse, die im Zusammenspiel mit teilnehmenden Beobachtungen zur Entwicklung eines Forschungsfragebogens führt.

In der vorliegenden Studie wird diesen Gütekriterien gefolgt, da die „Flexible Arbeitswelt“ mitsamt ihren Herausforderungen und den hier untersuchten Hypothesen die Anwendung eines sozialwissenschaftlichen Forschungsdesigns nahelegt.

\section{Rekonstruktion des Forschungsprozesses}

\section{Literaturanalysen und Recherchen}

Im Projekt kamen insbesondere Literaturrecherchen und Datenanalysen zum Einsatz, gepaart mit kreativ-konstruktiven Methoden zur Erzeugung der Personas und Szenarien. Folgende Literaturanalysen wurden durchgeführt:

\section{Literaturrecherche zu neuen Arbeitsformen}

Als eine (recht weitverbreitete) Form der mobil-flexiblen Arbeit wurde das "Homeoffice“, also das tageweise Arbeiten von zu Hause aus in einem ansonsten „normalen“ Angestelltenverhältnis (auch Telearbeit genannt), exemplarisch ausgewählt. Vor- und Nachteile dieser Arbeitsform sind in der Arbeitspsychologie- und Managementliteratur gut beschrieben und liessen sich einander somit auf einfache Art gegenüberstellen. Unterteilt wurden Vor- und Nachteile nach arbeitsbezogenen Aspekten (z.B. Ungestörtheit, höhere Produktivität als Vorteil und Verlust des informellen Austausches als Nachteil) sowie die Life-DomainBalance betreffenden Aspekten (z.B. mehr Schlaf, bessere Erholung als Vorteil und Abgrenzungsschwierigkeiten, Tendenz zur Mehrarbeit als Nachteil). Die Ausgangslage wurde durch Recherchen zu atypischen Arbeitsverhältnissen und weiteren Arbeitsformen (z.B. Freelancer, (Solo)-Selbstständige, befristete Anstellung, Teilzeit, Crowdworker etc., siehe auch Eurofonds, 2015) erweitert. Sämtliche Literatur wurde im Literaturverwaltungsprogramm „Mendeley“ erfasst. Die Recherche führte zu einem ganzen Katalog von Indikatoren, wie Produktivität, Arbeitszufriedenheit, Gesundheitsrisiken, Einkommen, intransparente Zah- 
lungsströme, Wirkung auf Systeme der sozialen Absicherung, Vereinbarkeit von Beruf mit anderen Lebensbereichen und noch ein paar weiteren.

\section{Literaturrecherche zu Vor- und Nachteilen von Crowdsourcing}

Analog zur Arbeitsform „Homeoffice" wurden ebenso die Vor- und Nachteile für "Crowdsourcing for paid Work" (also die Suche und Ausführung von Aufträgen vermittelt über eine Crowdsourcing-Plattform) aus der Perspektive der Arbeitenden („Verkäufer“ von Arbeitsleistung) ermittelt. Hierzu ist deutlich weniger wissenschaftliche Literatur zu finden. Dennoch konnte eine gute Aufstellung erbracht werden. Vor- und Nachteile wurden hier gruppiert in die Themen „Arbeitsverhältnis und Arbeitsmarkt" (z.B. Teilhabe am Arbeitsmarkt für benachteiligte Gruppen als Vorteil und fehlende soziale Absicherung als Nachteil), „Flexibilität und Sozialleben“ (z.B. bessere Life-Domain-Balance als Vorteil und fehlendes Feedback und Isolation als Nachteil) sowie „Arbeitsinhalte“ (z.B. eigenständige Wahl der Aufgaben als Vorteil und repetitive, tayloristische Aufgaben als Nachteil).

Eine Vorstellung der verarbeiteten Dokumente ergibt sich durch die Sichtung der Literaturdatenbasis oder das Studium des Sonderbands zum mobilen Arbeiten in der Sonderausgabe der Zeitschrift Wirtschaftspsychologie zum Thema "Gestaltung mobil-flexibler Arbeit“" (Schulze, Meissner, Weichbrodt 2015).

\section{Literaturrecherche zum Stellenwert der Arbeit}

Zur Frage, wie sich der Stellenwert der Arbeit verändert, wurde zu folgenden Schlagworten Literatur gesichtet: Zukunft der Arbeit, flexible Anstellungsverhältnisse, Entgrenzung der Arbeit, Subjektivierung der Arbeit, Arbeitskraftunternehmer.

\section{Recherche gesetzlicher Rahmenbedingungen}

Für die Recherche der gesetzlichen Rahmenbedingungen wurden die relevanten gesetzlichen Grundlagenwerke systematisch auf flexibilisierungsrelevante Aspekte hin untersucht. Das Arbeitsrecht gliedert sich inhaltlich in drei grosse Normenkomplexe: Individualarbeitsrecht (Arbeitsprivatrecht), öffentliches Arbeitsrecht und kollektives Arbeitsrecht (Rehbinder, Rz. 10). Die nachstehende Abbildung verdeutlicht diese Struktur.

Das Individualarbeitsrecht unterliegt dabei verschiedenen Rechtsquellen (Rehbinder, Schweiz. Arbeitsrecht, Rz. 30-38): 


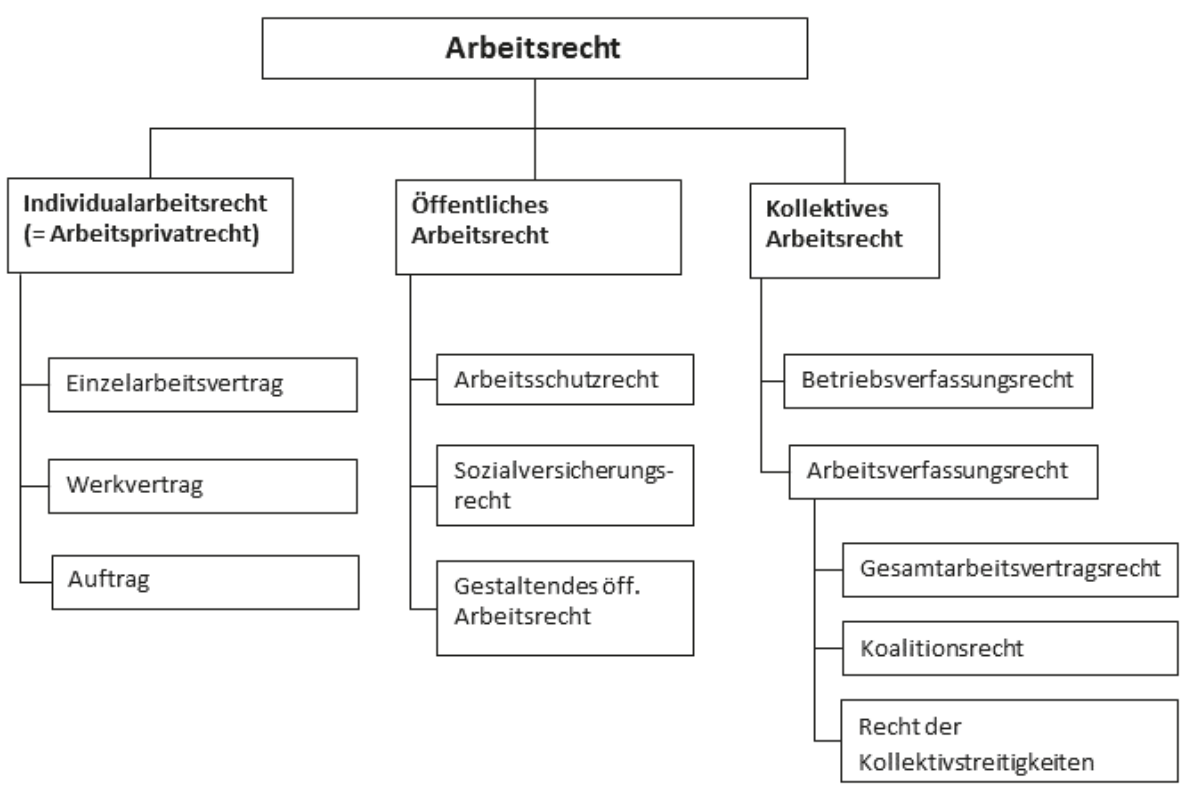

Rechtsquellen:

GesetzlicheRegelungen

Rechtsverordnungen

Normalarbeitsvertrag

Einzelvereinbarung

Direktionsrecht/Weisungsrecht
Betriebliche Übung

Betriebsordnung

Gesamtarbeitsvertrag

1. Die gesetzliche Regelung findet sich in den Artikeln 319 bis 362 des Obligationenrechts, die im Jahre 1971 grundlegend revidiert wurden, und daneben in einigen Sondergesetzen, z.B. Arbeitsvermittlungsgesetz (AVG; SR 831.10) betreffend den Personalverleih oder im Gleichstellungsgesetz (GIG; SR 151.1).

2. Die arbeitsrechtlichen Rechtsverordnungen betreffen in der Regel das (öffentlich-rechtliche) Arbeitsschutzrecht (siehe Rz. 426 ff.), z.B. die vier Verordnungen zum Arbeitsgesetz. Das Arbeitsschutzrecht hat aber gemäss Artikel 342 Absatz 2 OR in der Regel auch privatrechtliche Wirkung. Rechtsverordnung ist ferner die Allgemeinverbindlicherklärung eines Gesamtarbeitsvertrages gegenüber den Aussenseitern (siehe Rz. 558) sowie der Normalarbeitsvertrag (siehe sogleich unter 3).

3. Der Normalarbeitsvertrag ist eine besondere Form der Rechtsverordnung, nämlich ein Erlass des Bundes oder der Kantone, der dispositi- 
ves Vertragsrecht für bestimmte Typen von Arbeitsverhältnissen setzt. Inhalt des Normalarbeitsvertrages sind Bestimmungen über den Abschluss, den Inhalt und die Beendigung einzelner Arten von Arbeitsverhältnissen (Art. 359 Abs. 1 OR).

Die praktische Bedeutung der Normalarbeitsverträge liegt dort, wo sich der Gesamtarbeitsvertrag noch nicht durchsetzen konnte.

4. Die Einzelvereinbarung kann zwischen Arbeitgeber und Arbeitnehmer schriftlich oder mündlich, ausdrücklich oder stillschweigend erfolgen. Nur in einigen besonders geregelten Fällen ist für die Gültigkeit der Vereinbarung die Schriftform vorgeschrieben (vgl. Rz. 81). In die Einzelvereinbarung können betriebseinheitliche Reglemente (sog. Allgemeine Arbeitsbedingungen, entsprechend den Allgemeinen Geschäftsbedingungen im Wirtschaftsleben) einbezogen werden (vgl. Rz. 56).

5. Das Direktionsrecht oder Weisungsrecht ermöglicht dem Arbeitgeber, die Leistungspflicht des Arbeitnehmers zu konkretisieren (Art. 321d Abs. 1 OR, vgl. Rz. 166 ff.).

6. Die betriebliche Übung ist eine besondere form stillschweigender Kollektivvereinbarung, bei der aus der faktischen Handhabung im Betrieb nach einer gewissen Dauer Rechtsansprüche entstehen können (z.B. der Anspruch auf Weihnachtsgratifikation, vgl. Rz. 181).

7. Die Betriebsordnung ist eine kollektive Regelung der Arbeitsverhältnisse eines Betriebes durch einseitigen Erlass oder durch Vereinbarung mit der Arbeitnehmervertretung (Betriebskommission). Sie ist für industrielle Betriebe zwingend vorgeschrieben (ArG, vgl. Rz. $621 \mathrm{ff}$.).

8. Der Gesamtarbeitsvertrag schliesslich ist die kollektive Vereinbarung von Arbeitsbedingungen zwischen den Gewerkschaften und einzelnen Arbeitgebern oder deren Verbänden (Art. 356-358 OR, vgl. Rz. 509 ff.).

Die relevanten gesetzlichen Grundlagen im Kontext der genannten Themenfelder sind auf nationaler Ebene vollständig erhoben und sind in Form von Spannungsfeldern textlich festgehalten. Gleichermassen wurden internationale Rahmenbedingungen oder Gerichtspraxis bezüglich spezifischer Themen beigezogen, wobei hier aufgrund Ressourcen keine vertiefte Recherche erfolgt ist, was je nach Entwicklung zu einem späteren Zeitpunkt noch erfolgen könnte. 


\section{Recherchen zu den volkswirtschaftlichen Konsequenzen des flexiblen Arbeitens}

Diese an den entwickelten Personas orientierten Recherchen umfassten:

- $\quad$ Eine Recherche über die Veränderung bestimmter Basisstatistiken wie z.B. die Anzahl der selbstständig Arbeitenden in der Schweiz zeigte, dass die verfügbaren Daten des BFS nur auf einem sehr groben Niveau Antworten geben können. Auf dieser recht hohen Flughöhe liessen sich nur sehr geringe Veränderungen am Arbeitsmarkt in den letzten 20 Jahren erkennen: Beispielsweise bewegte sich der Anteil der Selbstständigen unter den Erwerbstätigen zwischen 9\% und 10\%; der Anteil der Erwerbstätigen mit befristeten Verträgen stieg leicht an von ca. $5 \%$ auf $7 \%$. Die deutlichsten Veränderungen zeigten sich beim Anteil der Teilzeitbeschäftigten mit $27 \%$ in 1993 und 35\% in 2013. Es ist erwähnenswert, dass öffentliche Statistiken viele neue Formen in Ansätzen erfassen (z.B. könnte ein Crowdworker ein Selbstständiger sein), aber nicht explizit ausweisen.

- $\quad$ Recherche des gesamtwirtschaftlichen Potenzials und der Auswirkungen von "Crowdsourcing for paid work“.

- $\quad$ Recherche des gesamtwirtschaftlichen Potenzials und der Auswirkungen von Automatisierung und Computerisierung.

- $\quad$ Recherche von Studien zum Thema atypische Beschäftigung und Ähnlichem.

- $\quad$ Suche nach möglichen Indikatoren für "Crowdsourcing for paid Work“ in European Working Conditions Survey, Household-Panel.

- $\quad$ Analyse der Daten der Schweizerischen Arbeitskräfteerhebung (SAKE) zu atypischer Beschäftigung und SECO zur Personalvermittlung. Zusammenstellung für Projektworkshop und Studie (in Kapitel 2).

- $\quad$ Zusammenstellung der Systeme der sozialen Absicherung.

Analyse der Systeme sozialer Absicherung betreffend Robustheit gegenüber intransparenten Lohnzahlungen bzw. starker Zunahme von "Selbstständigen i. S. v. Crowdworkern“. 
Im Detail wurde wie folgt vorgegangen:

1. Bestimmung der Suchbegriffe

Das Thema "flexible neue Arbeitswelt" wurde in verschiedene Teilaspekte gegliedert, um Begriffe bzw. Phrasen für die Suche in Datenbanken zu generieren. In der Arbeitsgruppe wurden dafür passende Ober- und Unterbegriffe und synonyme Begriffe gesucht, die für die zum Teil trunkierte Suche nach relevanter Literatur verwendet wurden. Die Liste umfasste folgende deutschen und englischen Begriffe (alphabetisch geordnet):

- $\quad$ atypical work

- $\quad$ atypische Arbeit

- $\quad$ atypische Beschäftigung

- crowdsourcing

- crowdwork

- flexible Arbeit

- $\quad$ flexible Beschäftigung

- $\quad$ flexible work

- $\quad$ future of work

- labor force polarization

- labor force segmentation

- neue Arbeitsformen

- non-standard employment

- Schattenwirtschaft

- Schwarzarbeit

- $\quad$ shadow economy

- Telearbeit

- $\quad$ Zukunft der Arbeit

2. Bestimmung Umfang und Art der Literatur

Für die Suche in den Datenbanken gemäss Projektausschreibung wurde nach folgender Literatur gesucht:

- $\quad$ wissenschaftliche Bücher

- Zeitschriftenartikel

- Herausgeberwerke

- $\quad$ Forschungsberichte und andere Arbeitspapiere sowie Kongressberichte

Für die ergänzende Suche im Internet wurden die folgenden zusätzlichen Arten von Literatur miteinbezogen: 
- $\quad$ Zeitungsartikel

- $\quad$ Statistiken

- Jahrbücher

- Informationen von verschiedenen Ämtern und Forschungsinstituten

- Informationen von weiteren Institutionen und Verbänden

3. Informationsquellen

Als Informationsquellen wurden berücksichtigt:

- $\quad$ IDS Luzern (gemeinsamer Katalog der Bibliotheken der Hochschule Luzern, der Zentralen Hochschulbibliothek Luzern sowie Pädagogischen Hochschule Luzern)

- $\quad$ Alle IDS-Bibliotheken in der Deutschschweiz via Swissbib

- $\quad$ Datenbank Web of Science (Zugriff auf elektronische Volltextzeitschriften) mit einer Beschränkung auf folgende „Research Areas“:

- Business Economics

- Psychology

- Social Issues

- Sociology

- Social Sciences other topics

- Internet

4. Evaluation der Treffer

Die Suche in der Datenbank „Web of Science“ im Herbst 2014 ergab zahlreiche Treffer. Die Anzahl Treffer für die Suche in den Feldern „Topic" und "Title" mit einer Begrenzung der Publikationsjahre von 1995-2014 ergab folgende Trefferzahlen:

\begin{tabular}{lll}
\hline Titel & Anzahl Treffer & Bemerkungen \\
\hline atypical work & 65 \\
\hline atypische Arbeit & 2 \\
\hline atypische Beschäftigung & 2 \\
\hline crowdsourcing & 533 \\
\hline crowdwork & 3 \\
\hline flexible Arbeit & 37 \\
\hline flexible Beschäftigung & 2 \\
\hline
\end{tabular}




\begin{tabular}{lll}
\hline flexible work & 408 & Nur Titelsuche \\
\hline future of work & 532 & 24 \\
\hline labor force polarization & 52 \\
\hline labor force segmentation & 1 \\
\hline neue Arbeitsformen & 37 \\
\hline non-standard employment & 0 \\
\hline Schattenwirtschaft & 1 \\
\hline Schwarzarbeit & 466 \\
\hline shadow economy & 1 \\
\hline Telearbeit & 88 \\
\hline Zukunft der Arbeit & \\
\hline
\end{tabular}

Im Anschluss an die Suche wurden die Abstracts (sofern vorhanden) auf Doubletten und ihre Relevanz in Bezug auf die hier aufgeführten Grössen, welche zuvor mit der TA-SWISS-Begleitgruppe festgelegt wurden, geprüft:

- $\quad$ Produktivität

- Branchenstruktur

- $\quad$ Anzahl Mitarbeiter/innen

- $\quad$ Armutsgefährdung

- $\quad$ soziale Mobilität

- Gesundheitsausgaben pro Einwohner/in

- Vereinbarkeit von Beruf und Familie/Beziehungsnetz/Freizeitaktivitäten

- $\quad$ ehrenamtliche und freiwillige Tätigkeiten, insbesondre Care-Work und Altenbetreuung

- $\quad$ Erwerbseinkommen: Median; 1. Dezil; Anteil am Gesamteinkommen; Verhältnis S80/S20

- $\quad$ Arbeitslosigkeit (nach ILO und registrierte Arbeitslose) und Unterbeschäftigung

- Beschäftigte

- Integrationsfunktion der Arbeit

- $\quad$ kollektiver Organisationsgrad der Arbeitnehmenden

- Schwarzarbeit/Schattenwirtschaft

- $\quad$ Arbeitsbedingungen (inkl. Arbeitszeiten, Verfügbarkeit etc.)

- $\quad$ physische und psychische Gesundheitsrisiken 


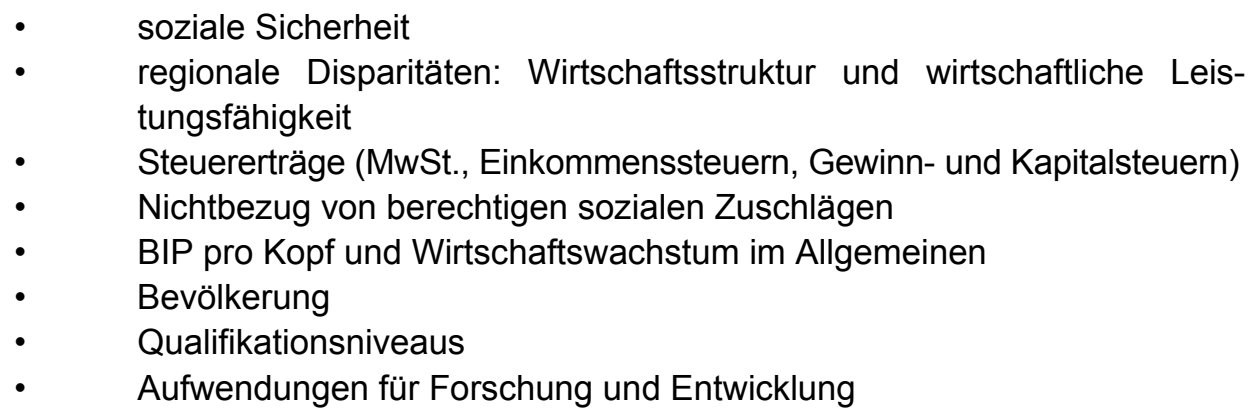

Die Durchsicht der Abstracts grenzte die Treffer stark ein. Die weitere Suche wurde dann nach dem Schneeballsystem vollzogen, d.h., über die verzeichneten Zitate und Literaturlisten der eingegrenzten Literatur wurde versucht, weitere wichtige Publikationen verschiedenen Datums zu finden. Für diesen zweiten Schritt wurde nebst in den IDS-Bibliotheken v.a. gezielt im Internet gesucht. Da dieser Schritt im Sommer 2015 erfolgte, enthält der Bericht somit Literatur mit dem Publikationsjahr 2015. Wenn sich beim Lesen und Auswerten zeigte, dass sich der Anteil der neuen Information stark verringerte, wurde die Recherche abgebrochen. Im Weiteren stellte sich heraus, dass nicht für alle oben aufgeführten Themen aktuelle und relevante Literatur zu finden war, was in der Folge zu einer Zusammenfassung bzw. Reduktion auf die elf Indikatoren für die Folgeabschätzung führte.

\section{Beschaffung der Literatur und Weiterverarbeitung der Ergebnisse}

Die als relevant eingestuften Werke und Arbeiten wurden laufend beschafft und ins Literaturverwaltungsprogram „Mendeley“ importiert. Somit konnte die Literatur einfach organisiert und in der Projektgruppe ausgetauscht werden. Als nächster Schritt des Arbeitsprozesses erfolgten die erneute Auswertung und die Verarbeitung der Literatur im Hinblick auf die fünf im Bericht vorkommenden personabasierten Arbeitswelten. Insgesamt flossen 133 Literatur- und Datensatzfunde in den Bericht ein. Das folgende Diagramm gibt einen Überblick über deren Publikationsjahre. 


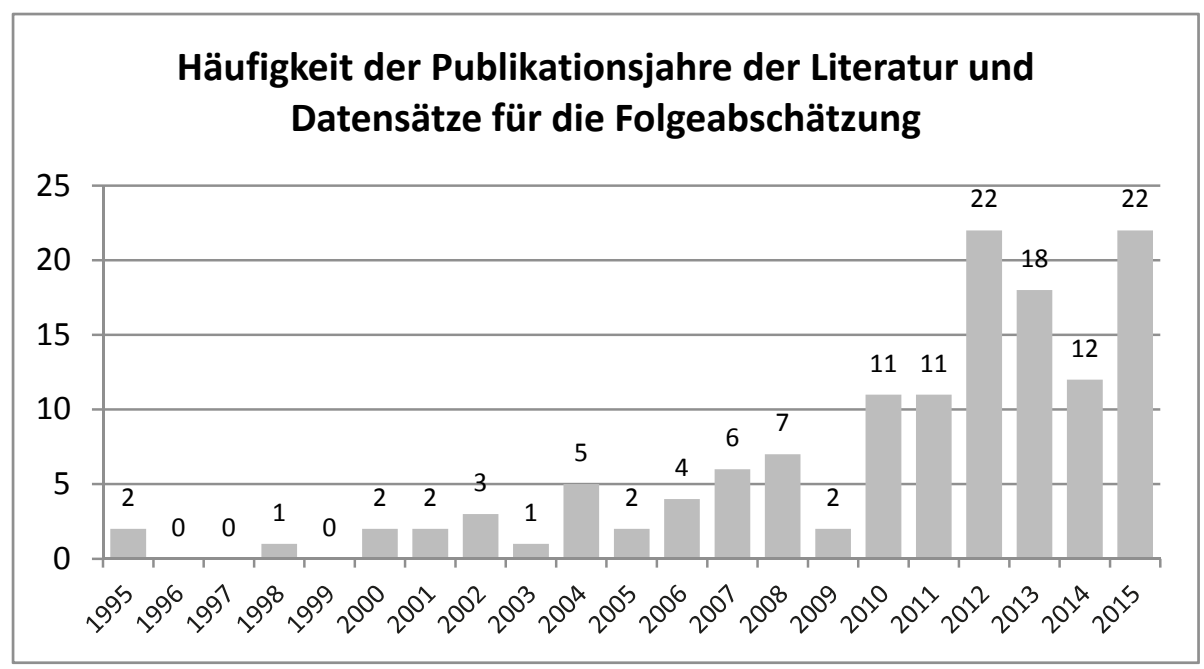

\section{Entwicklung morphologischer Kasten}

Aufgrund der umfangreichen Dokumentenrecherche wurde ein morphologischer Kasten (Ritchey, 2011; Zwicky, 1989) erarbeitet, anhand dessen man Hauptkriterien von typisierten Personen und Beschäftigungsverhältnissen in der flexiblen Arbeitswelt unterscheiden kann. Ziel der Erstellung des morphologischen Kastens war es, möglichst alle relevanten Aspekte des flexiblen Arbeitens abzubilden. Bisher gab es eine solche Entwicklung nur von Reilly (1998) - und dies auch nur für viel allgemeinere psychologische Problemstellungen. Der morphologische Kasten wurde in zwei Begleitgruppensitzungen sowie drei Teamsitzungen zur Diskussion gestellt, bis er als tragfähig angesehen wurde, also „sozial validiert“ war. Die entsprechenden Sitzungen fanden verteilt über das erste Halbjahr 2015 statt. Die Arbeitsgruppentreffen umfassten jeweils Mitglieder verschiedenen Ausbildungshintergrunds (Psychologie, Ökonomie, Recht, Staatswissenschaften, Politikwissenschaften). Ebenso waren die Begleitgruppensitzungen mit unterschiedlichsten Professionsgruppen besetzt.

\section{Entwicklung von Personenprototypen}

Der Kasten machte die Entwicklung von Personenprototypen möglich. Solche sogenannten „Personas“ stammen konzeptionell aus der Softwareentwicklung und dem Marketing und dienen der Spezifikation abstrakter Nutzerbedürfnisse bzw. Kundenwünsche anhand von typisierten Aussagen (Vartiainen \& Hyrkkä- 
nen 2010). Die Entwicklung von Personas zur Verwendung für die Technikfolgenabschätzung weist dabei die Stärke auf, dass die Schöpfer einer etwas blutleeren Ansammlung von Adjektiven (aus dem morphologischen Kasten) Leben einhauchen, in dem sie aus der Begriffswolke eine konkret vorstellbare, aber fiktive Person erzeugen. Personas sind also sehr plastische Projektionen quasi sozialpsychologische Szenarien -, die aus der vorhandenen Informationslage und der Interpretation aller einfliessenden Beobachtungen ein greifbares Nutzerbedürfnis deutlich machen. Selbstverständlich sind Personas Konstruktionen der beteiligten Akteure, hier der Projektmitarbeiter und der Begleitgruppenmitglieder, und stellen nicht "die Wirklichkeit an sich" dar, die in den Sozialwissenschaften seit der kognitiven und der linguistischen Wende (Miller, 2003; Wittgenstein, 1963) ohnehin infrage gestellt wird.

Es war fraglich, wie viele Personas entwickelt werden sollten und in welchem Detaillierungsgrad deren Charaktere auszuformulieren waren. Massgeblich wurden die Dimensionen und Ausprägungen des morphologischen Kastens kombiniert mit den Beobachtungen der Arbeitsgruppe aus der Literaturanalyse und Beobachtungspraxis. Nach der ersten Entwicklung von sieben Charakteren wurden schliesslich vier davon detailliert ausgearbeitet: zwei extreme und zwei gemässigte. Für die vier Charaktere entstand je ein Steckbrief sowie die Beschreibung eines "guten“ sowie eines „schlechten“ Tages. Über die Ausgestaltung der beiden Tagesformen wurde in der Arbeitsgruppe sehr umfassend diskutiert, insbesondere über Fragen der Abgrenzung („Was gehört noch zum typischen Profil der Persona?") und des Realitätsbezugs („Ist das nicht zu weit hergeholt?"). Letztlich konnten die Personas aber als robuste Konstruktionen entwickelt und definiert werden. Nach einer weiteren Begleitgruppensitzung wurde eine fünfte Persona gewünscht, die etwas abseits stünde, und ein nicht so ganz gut qualifiziertes Profil aufweisen sollte. Hier zeigte sich, dass die Validierung durch die Begleitgruppe funktionierte, da die Arbeitsgruppe offensichtlich einen relevanten Ausschnitt der Gesellschaft nicht abdeckte (konkret: die Gruppe der Geringqualifizierten).

\section{Entwicklung gewünschter Szenarien}

Die resultierenden Personas wurden fertiggestellt und stellen die Basis zur Ableitung von Szenarien dar. Die Szenario-Technik gehört traditionell in den Bereich der Technikfolgenabschätzung (Grunwald, 2010). In diesem Projekt stellen die Personas als sozialpsychologische Szenarien die Ausgangslage dar, um gesellschaftliche Szenarien und deren volkswirtschaftliche Folgen abschätzen zu können. Die Szenario-Technik versucht, die nähere Entwicklung einer Situa- 
tion anhand variierender Annahmen vorzuzeichnen. Dabei werden üblicherweise die drei Szenarien des besten, des wahrscheinlichsten und des schlechtesten Falls an denselben Variablen (Indikatoren) durchdekliniert. Auch denkbar ist die Entwicklung einer Typologie, die zu bestimmten, qualitativ unterschiedlichen Szenarien führt. Ein Beispiel für die Zukunft der Arbeit liefert etwa StockHoburg (2013) mit der Skizzierung der Szenarien "Organic-Trust-Szenario“, "SofTech-Szenario“, „TechFlex-Szenario“ und „Null-Variante“. Diese Szenarien sind in einer Vier-Felder-Matrix anhand der Kriterien „Bedeutung weicher Faktoren" (Y-Achse) und „Bedeutung technischer Fortschritt" (X-Achse) angeordnet. Auch bei den Szenarien wurde im Verlauf des Forschungsprozesses ein Entwurf vorgenommen, der Begleitgruppe vorgestellt und anschliessend detaillierter aufbereitet.

\section{Beurteilung des Prozesses anhand der Gütekriterien}

Der Prozess der Studienentwicklung lässt sich anhand der Einschätzung der zuvor erläuterten Gütekriterien der qualitativen Sozialforschung genauer beurteilen:

1. Verfahrensdokumentation: Das Verfahren ist dokumentiert, ebenso wie die verwendeten Methoden. Die recherchierte und aufbereitete Literatur ist abgelegt, sortiert und in Berichtsform ausgewertet.

2. Argumentative Interpretationsabsicherung: Informationen aus den aktuellen und vorangegangenen Recherchen bildeten das Fundament für die Ausgestaltung des morphologischen Kastens sowie der entwickelten Personas und Szenarien. Vorgehen und Zwischenergebnisse wurden wiederholt vor der Projektgruppe und der Begleitgruppe präsentiert sowie diskutiert und dadurch in der Argumentation geschärft. Hierfür gebührt allen Beteiligten auch ein ausdrücklicher Dank für die Offenheit und die direkte Teilnahme.

3. Regelgeleitetheit: Das gesamte Vorgehen folgt den Regeln der qualitativen Sozialforschung und der Szenario-Technik, zu der auch die Persona-Entwicklung gezählt werden kann.

4. Nähe zum Gegenstand: Um die notwendigen Details zu eruieren, wurde eine grösstmögliche Nähe zum Gegenstand über die Entwicklung der Personas vorgenommen. Hier fand die Konkretisierung an prototypischen Fällen vor dem Hintergrund umfangreicher Recherchen statt. Hinzuzufügen ist, dass die Mitglieder des Projektteams zum grossen Teil in zukunftsweisenden Arbeitsverhältnissen (Teilzeit, räumlich- 
flexible Arbeitsform, gestiegene Anforderungen an Selbstmanagement und Boundary-Management etc.) tätig sind.

5. Kommunikative Validierung: Interpretative Schritte wurden regelkonform über die Einbindung des Projektteams in den Workshops oder über die Diskussion mit der interdisziplinär besetzten Begleitgruppe (und der Dokumentation der entsprechenden Ergebnisse) sozial validiert.

6. Triangulation: Die Ergebnisse basieren auf umfangreichen Literaturund Datenrecherchen sowie Forschungs- und Feldbeobachtungen der Projektmitglieder aus umfangreichen vorangegangenen Forschungs-, Dienstleistungs- und Publikationsprojekten. Eine direkte und explizite Triangulation fand nicht statt, da verwandte Aktivitäten zeitlich früher durchgeführt wurden. Jedoch weisen früher durchgeführte Interviewstudien und Feldbeobachtungen grosse Kohärenz zu den recherchierten Informationen und den entwickelten Personas auf.

Abschliessend kann festgehalten werden, dass die Gütekriterien der qualitativen Sozialforschung in diesem Projekt eingehalten wurden. Einzig hinsichtlich der Triangulation ist eine gewisse Verzerrung festzustellen. Zudem ist zu vermerken, dass die Validierung mit der Begleitgruppe im Rahmen der Projektbegleitsitzungen stattfand und umfangreicher hätte sein können.

Zur Gesamtbeurteilung des Themas "Neue flexible Arbeitswelt" schliesslich wurden neben einem eigens entwickelten Rahmenwerk die Kriterien der International Labour Organisation (ILO) für "Decent Work" herangezogen (ILO, 2015). Diese vom ILO zur Gesamtbewertung von nationalen Arbeitsmärkten zur Verfügung gestellten Kriterien stellen ein geeignetes Referenzraster dar, das einen eigenen politischen Diskurs über akzeptable Arbeitsbedingungen unnötig macht. 



\section{Anhang 2: Die Elemente der Decent- Work-Agenda}

Die „Decent Work Agenda“, d.h. die „Agenda für menschenwürdige Arbeit“ der International Labor Organization (ILO, 2012) umfasst elf Kriterien, bietet Ansatzpunkte zur Operationalisierung und ermöglicht somit Vergleiche zwischen verschiedenen Ländern:

\section{Beschäftigungsmöglichkeiten}

Eine der Schlüsseldimensionen der Agenda ist die der „Beschäftigungsmöglichkeiten". Sie umfasst elf Indikatoren, die im Zusammenhang mit dem Angebot von und der Nachfrage nach Arbeit in einem Land stehen. Dabei ist es ein Ziel, die Arbeitsmarktbedingungen für Arbeitnehmer, potenzielle Arbeitnehmer und Arbeitgeber aufzuzeigen. Hinzu kommen Indikatoren, die die Qualität der Beschäftigung angeben. Alle Kennzahlen sollten, wenn immer möglich, getrennt werden, damit eine Unterscheidung zwischen Frauen und Männer in Bezug auf die Arbeit gemacht werden kann. Darüber hinaus ist es für die Analyse der Kennzahlen wichtig, dass diese immer im Hinblick auf Veränderungen und Trends erfolgen sollen. Rechtsrahmen bildet in diesem Zusammenhang das Engagement der Regierung in Bezug auf die Arbeiter- und Arbeitslosenversicherung.

Wichtige statistische Indikatoren für die Analyse der Beschäftigungsmöglichkeiten sind:

- Arbeitslosenquote

- Jugendliche, die weder eine Ausbildung haben noch arbeitstätig sind

- Arbeitslosenquote bei Jugendlichen

\section{Angemessene Entschädigung und produktive Arbeit}

Laut ILO steht auch die angemessene Entlohnung in einem engen Zusammenhang mit menschenwürdiger Arbeit. Mit einem adäquaten Lohn geht auch produktive Arbeit einher, was sowohl in der Decent-Work-Agenda wie auch in den Millennium-Entwicklungszielen (Millenium Development Goals) postuliert wird. Den rechtlichen Rahmen in diesem Zusammenhang bildet der Mindestlohn. Um 
den Ländern das Monitoring dieses Kriteriums zu vereinfachen, sind sieben statistische Indikatoren vorgesehen:

- Quote der erwerbstätigen Armen

- Niedriglohnquote

- Durchschnittliche Stundenlöhne in ausgewählten Berufen

- Durchschnittlicher Reallohn

- Mindestlohn in Prozent des Medianlohns

- Produktionslöhne

- Mitarbeiter mit neuer Berufsbildung

\section{Menschenwürdige Arbeitszeiten}

Ebenfalls einen wichtigen Part der Agenda stellen die angemessenen Arbeitszeiten dar. Hierbei sind zwei Arten von Indikatoren enthalten: solche, die Informationen über die Beschäftigung in einer Arbeitszeit-Bandbreite beinhalten, und solche mit Fokus auf die stündliche Zeiterfassung von Mitarbeitern. Diese beiden Indikatoren können Auskunft über die Anzahl der Personen geben, die gearbeitet haben. Darüber hinaus können auch Informationen betreffend die tatsächliche Anzahl Arbeitsstunden pro Arbeitnehmer gewonnen werden. Dazu sind auch fünf statistische Kennzahlen hinzuzuziehen:

- Beschäftigung während überlangen Arbeitszeiten

- Beschäftigung während der wöchentlichen Arbeitsstunden

- Durchschnittliche Jahresarbeitszeit pro Arbeitnehmer

- Zeitbezogene Unterbeschäftigungsquote

- Bezahlter Jahresurlaub

Auch hier sollten, wenn immer möglich, die gewonnenen statistischen Kennzahlen nach Geschlecht der Arbeitnehmenden getrennt werden. Dadurch können die Unterschiede zwischen Frauen und Männern aufgezeigt werden. Die gesetzlichen Rahmenbedingungen im Zusammenhang mit menschenwürdiger Arbeitszeit sind bezahlter Urlaub pro Jahr und maximale Arbeitszeit. Für die Interpretation der zuvor genannten Indikatoren wird empfohlen, jeweils qualitative Kennzahlen wie beispielsweise bezahlte Urlaubstage, und quantitative Indikatoren, z.B. Arbeitslosenquote, in Verbindung mit der Veränderung des BIP zu analysieren. 


\section{Vereinbarkeit von Arbeit, Familie und persönlichem Leben}

Die Elemente, die sich für die Vereinbarkeit von Arbeit, Familie und persönlichem Leben eignen, beinhalten ein paar wenige Indikatoren. Diese sind verbunden mit Normen, grundlegenden Prinzipien und Rechten bei der Arbeit und im Sozialschutz. Dafür wurden zwei Kennziffern identifiziert: asoziale bzw. ungewöhnliche Arbeitszeiten und der Mutterschutz. Die rechtlichen Rahmenbedingungen bezogen auf die statistischen Indikatoren sind der Mutterschaftsurlaub und die Elternzeit.

\section{Arbeit, die abgeschafft werden sollte}

Sowohl internationale Übereinkommen als auch die IAO fordern, dass gewisse Arbeitstypen, wie Kinder- und Zwangsarbeit, abgeschafft werden sollten. Um dieses Ziel zu erreichen, ist es gemäss der Decent-Work-Agenda wichtig, gewisse Indikatoren zu bemessen, um das Vorkommen, die Verbreitung und die Charakteristiken dieser Arbeitstypen beurteilen zu können. Für den Zweck der statistischen Messung sind die allfällige nationale Gesetzgebung und andere internationale Instrumente zu verwenden. Diese bilden somit den Ausgangspunkt für die Entwicklung von statistischen Konzepten und Definitionen für Kinder- und Zwangsarbeit. Die hier verwendeten fünf statistischen Indikatoren sind:

- Rate der Kinderarbeit

- Riskante Kinderarbeit

- Quote der schlimmsten Formen von Kinderarbeit

- Rate der Zwangsarbeit

- Forced labour rate among returned migrants

Diese stehen im Zusammenhang mit den jeweiligen rechtlichen Rahmenbedingungen in Bezug auf Kinder- und Zwangsarbeit.

\section{Beständigkeit und Sicherheit der Arbeit}

Die Decent-Work-Agenda unterscheidet zwischen Arbeitern, die ein gewisses Ausmass an Beständigkeit und Zuverlässigkeit aufweisen, und solchen, die kein Interesse an einer langwährenden Beschäftigung haben.

Dieser Punkt der Decent-Work-Agenda beinhaltet vier statistische Kennzahlen:

- Quote der prekären Beschäftigungsverhältnisse

- Beschäftigungsdauer 
- Anteil der Selbstversorgenden

- Realeinkommen der Gelegenheitsarbeiter

Die Hauptkriterien, um die Konzepte zu messen, sind die effektive Länge der Vertragsdauer oder wie einfach es ist, dem Arbeitnehmer zu kündigen, die Dauer der Beschäftigung sowie die Charakterisierung als Selbstversorger oder Gelegenheitsarbeiter. Hierbei spielt die Beendigung des Arbeitsverhältnisses als gesetzlicher Rahmen eine Rolle. Es ist darüber hinaus wichtig, dass die vier Indikatoren zusammen mit weiteren ökonomischen und sozialen Kennzahlen analysiert werden.

\section{Gleiche Chancen und gleiche Behandlung in der Anstellung}

Die Chancengleichheit und Gleichbehandlung zu fördern ist sowohl in der Decent-Work-Agenda als auch bei den Millennium-Entwicklungszielen beschrieben. Die hierfür hinzugezogenen Indikatoren können Ländern helfen, die Fortschritte zu vergleichen und das Ziel weiter zu verfolgen. Dafür wurden vier statistische Kennzahlen eingeführt:

- Berufliche Segregation

- Frauenquote im mittleren und oberen Management

- Geschlechtsspezifisches Lohngefälle

- Anteil der Frauen, die unselbstständig erwerbend sind im nicht landwirtschaftlichen Sektor

\section{Sichere Arbeitsumgebung}

Berufliche Sicherheit und Gesundheit bei der Arbeit sind essenzielle Komponenten der Decent-Work- Agenda. Die vier Kennzahlen, mit deren Hilfe das Ausmass der arbeitsbedingten Gefahren und Risiken erfasst werden, lauten:

- Quote der Arbeitsunfälle, die tödlich enden

- Häufigkeit der Arbeitsunfälle, die nicht tödlich enden

- Verlorene Zeit pro Arbeitsunfall

- Arbeitsaufsicht

\section{Soziale Sicherheit}

Die soziale Sicherheit beinhaltet alle Massnahmen zur Sicherung des Einkommens. Sie sichert Hilfe zu bei 
a) Mangel an Arbeitseinkünften, verursacht durch Krankheit, eine Behinderung, Mutterschaft, Arbeitsunfall, Arbeitslosigkeit, Alter oder Tod eines Familienmitgliedes

b) Ungenügender bzw. unerschwinglicher Zugang zur Gesundheitsversorgung

c) Unzureichende Familienunterstützung, vor allem für Kinder und unterhaltsberechtigte Erwachsene

d) Schutz vor allgemeiner Armut und sozialer Ausgrenzung.

Hierfür wurden zehn verschiedene statistische Faktoren eingeführt, um den Fortschritt zu überwachen.

- Bevölkerungsanteil über dem ordentlichen Rentenalter (65 Jahre), die von einer Alterspension profitieren

- Öffentliche Ausgaben für die soziale Sicherheit (in \% vom BIP)

- Gesundheitsausgaben nicht finanziert durch private Haushalte

- Anteil der erwerbstätigen Bevölkerung, die einen Beitrag zur Rentenversicherung leisten

- $\quad$ Anteil der förderfähigen Bevölkerung, abgedeckt durch die Gesundheitsversorgung

- Öffentliche Ausgaben für bedarfsgerechte Sozialhilfe (in \% vom BIP)

- Empfänger von Sozialhilfe

- Fehlen wegen Krankheit

- Anteil der Arbeitslosen, die regelmässig Arbeitslosengeld erhalten

- Verhältnis zwischen der durchschnittlichen Altersrente und dem Mindestlohn

Diese Indikatoren werden im Rahmen der Arbeitsunfähigkeit durch Invalidität, Krankheit und Alter analysiert.

\section{Sozialer Dialog, Vertretung der Arbeitnehmer- und der Arbeitgeber- schaft}

Das Hauptziel des sozialen Dialoges ist es, einen Konsens zwischen den Akteuren in der Arbeitswelt zu schaffen. Erfolgreiche Strukturen und Prozesse eines sozialen Dialogs haben das Potenzial, ökonomische und soziale Probleme zu lösen, Good Governance zu fördern, den sozialen und industriellen Frieden und die Stabilität voranzutreiben und darüber hinaus den ökonomischen Fortschritt zu steigern. Aus diesen Gründen spielt der soziale Dialog eine Schlüsselrolle dabei, die ILO-Kriterien zu erreichen. Das Ausmass, in dem die Rechte des sozialen Dialogs ausgeübt werden, wird erfasst, beurteilt und überwacht durch vier Indikatoren: 
- Rate der Gewerkschaftsdichte

- Unternehmen, die einem Arbeitgeberverband angehören

- Tarifverbundsrate

- Tage, an denen nicht gearbeitet wurde, im Verhältnis zu Streiks und Aussperrungen

11. Ökonomische und soziale Umweltbedingungen für menschenwürdige Arbeit

Gemäss der Definition der ILO ist es wichtig, sowohl die ökonomischen als auch die sozialen Umweltbedingungen eines Landes zu analysieren. In diesem Zusammenhang kommen elf statistische Indikatoren zur Anwendung:

- Kinder ohne Schulbildung

- Geschätzter Prozentwert der Erwerbsbevölkerung, die HIV positiv ist

- Arbeitsproduktivität

- Einkommensungleichheit bei den Haushalten

- Inflationsrate (Landesindex der Konsumentenpreise)

- Beschäftigung nach Wirtschaftszweig

- Bildung der Erwachsenen

- Arbeitsanteil am BIP

- Reales BIP pro Kopf

- Weiblicher Anteil der Beschäftigten im Industriesektor

- Einkommensungleichheit bei den Erwerbstätigen

- Massnahmen gegen Armut

Diese Indikatoren geben ein Bild über die ökonomische und soziale Situation der menschenwürdigen Arbeit ab. 


\section{Anhang 3: Beteiligte Personen und Institutionen}

\section{Trägerschaft}

Zentrum für Technologienfolgen-Abschätzung TA-SWISS

\section{Begleitgruppe}

Dr. Sergio Bellucci (Geschäftsführer TA-SWISS)

Prof. Dr. Thomas Beschorner (Universität St. Gallen)

Heidi Blattmann (Leitungsausschuss TA-SWISS)

Prof. Dr. Gudela Grote (ETH Zürich)

Dr. Christian Monn (SECO)

Prof. Katharina Prelicz-Huber (Leitungsausschuss TA-SWISS),

Präsidentin der Begleitgruppe

Dr. Lucienne Rey (Geschäftsstelle TA-SWISS)

Prof. Dr. Katja Rost (Universität Zürich)

Dr. Stefan Vannoni (Leitungsausschuss TA-SWISS)

Christine Villaret D’Anna (Msc Ergonomie - BSc Ergothérapie)

Alain Vuille (Bundesamt für Statistik)

Dr. Christoph Zanker (Verband Deutscher Maschinen und Anlagenbau e.V.)

\section{Projektverantwortung}

Prof. Dr. Jens O. Meissner, Hochschule Luzern - Wirtschaft

Dr. Johann Weichbrodt, Fachhochschule Nordwestschweiz, Hochschule für angewandte Psychologie 


\section{Projektteam:}

Sheron Baumann, Hochschule Luzern - Wirtschaft

Leila Gisin, Fachhochschule Nordwestschweiz, Hochschule für angewandte Psychologie

Alexandra Gisler, Hochschule Luzern - Wirtschaft

Bettina Hübscher, Hochschule Luzern - Wirtschaft

Prof. Ute Klotz, Hochschule Luzern - Wirtschaft

Prof. Dr. Ulrich Pekruhl, Fachhochschule Nordwestschweiz, Hochschule für Wirtschaft 


\title{
Liebe Leserin, lieber Leser
}

Wir freuen uns, dass Sie unsere Open-Access-Publikation

heruntergeladen haben. Der vdf Hochschulverlag fördert Open Access aktiv und publiziert seit 2008 Gratis-eBooks in verschiedenen Fachbereichen:

\section{Übersicht Open-Access-Titel}

\section{Möchten auch Sie Open Access publizieren?}

Der vdf Hochschulverlag stellt Ihre Publikation u.a. im eigenen Webshop sowie der ETH-Research-Collection zum Download bereit!

Kontaktieren Sie uns unter verlag@vdf.ethz.ch

Gerne informieren wir Sie auch in Zukunft über unsere

(Open-Access-)Publikationen in Ihrem Fachbereich.

\section{Newsletter abonnieren}

Auch Sie können Open Access unterstützen.

\section{Hier geht's zum Spenden-Button}

\author{
Herzlichen Dank!
}




\section{www.ta-swiss.ch}

$\mp$ Ein Kompetenzzentrum der

Akademien der Wissenschaften Schweiz

Flexible Arbeitszeiten gehören heute zum beruflichen Alltag. Dank Homeoffice oder mobiler Arbeit vergrössern sich seit einigen Jahren auch die Freiräume bei der Wabl des Arbeitsortes. Auch steigt die organisatorische Flexibilität, indem abwechslungsreiche Projektarbeit zunehmend gleichbleibende Routinearbeit ablöst.

Die Flexibilisierung der Arbeit bricht bisher fest gefügte berufliche Positionen auf. Sie führt dazu, dass vielen Erwerbstätigen erhöhte Autonomie zugestanden wird. Im Gegenzug müssen diese mehr Verantwortung übernehmen. Die zuvor klaren Grenzen zwischen Angestellten und Selbstständigen brechen auf.

Vielen Arbeitnehmenden fällt es dank flexibler Arbeit leichter, ihr individuelles Leben zu gestalten. Berufliche und private Anforderungen lassen sich besser vereinbaren. Das hat aber auch Schattenseiten, stellt es doch hohe Ansprüche an Disziplin und Selbstmanagement aller Beteiligten.

Die vorliegende Studie umreisst Chancen und Risiken flexibilisierter Arbeit für die Erwerbstätigen. Ausserdem werden absehbare Folgen für die Volkswirtschaft abgeschätzt und die rechtlichen Rahmenbedingungen beleuchtet.

\section{$\mathbf{v} / \mathbf{d} \mid \mathbf{f}$}

\section{TA-SWISS 64/2016}

ISBN 978-3-7281-3770-8

(Printausgabe)

ISBN 978-3-7281-3771-5

(E-Book)

DOI 10.3218/3771-5
Lucerne University of

Applied Sciences and Arts

\section{HOCHSCHULE LUZERN}

\author{
Wirtschaft \\ Institut für Betriebs- und \\ Regionalökonomie IBR
}

Fachhochschule Nordwestschweiz Hochschule für Angewandte Psychologie 